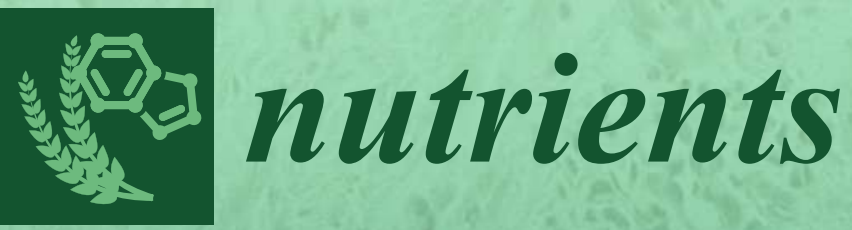

Nutraceuticals

and the skin:

\title{
Roles in Health and Disease
}

Edited by

Jean Christopher Chamcheu, Deeba Nadeem Syed and G. Kerr Whitfield Printed Edition of the Special Issue Published in Nutrients 
Nutraceuticals and the Skin: Roles in Health and Disease 



\section{Nutraceuticals and the Skin: Roles in Health and Disease}

Special Issue Editors

Jean Christopher Chamcheu

Deeba Nadeem Syed

G. Kerr Whitfield 
Special Issue Editors

$\begin{array}{ll}\begin{array}{l}\text { Jean Christopher Chamcheu } \\ \text { University of Louisiana at Monroe College of Pharmacy, } \\ \text { Monroe, LA, USA }\end{array} & \begin{array}{l}\text { Deeba Nadeem Syed } \\ \text { School of Medicine and Public Health } \\ \text { Madison, WI, USA }\end{array} \\ \begin{array}{l}\text { G. Kerr Whitfield } \\ \text { University of Arizona College of Medicine-Phoenix }\end{array} & \\ \text { Phoenix, AZ, USA } & \end{array}$

\section{Editorial Office}

MDPI

St. Alban-Anlage 66

Basel, Switzerland

This is a reprint of articles from the Special Issue published online in the open access journal Nutrients (ISSN 2072-6643) from 2017 to 2018 (available at: http:/ /www.mdpi.com/journal/nutrients/special_ issues/nutraceuticals_skin)

For citation purposes, cite each article independently as indicated on the article page online and as indicated below:

LastName, A.A.; LastName, B.B.; LastName, C.C. Article Title. Journal Name Year, Article Number, Page Range.

\section{ISBN 978-3-03897-186-3 (Pbk) \\ ISBN 978-3-03897-187-0 (PDF)}

Cover image courtesy of Jean Christopher Chamcheu.

Articles in this volume are Open Access and distributed under the Creative Commons Attribution (CC BY) license, which allows users to download, copy and build upon published articles even for commercial purposes, as long as the author and publisher are properly credited, which ensures maximum dissemination and a wider impact of our publications. The book taken as a whole is (C) 2018 MDPI, Basel, Switzerland, distributed under the terms and conditions of the Creative Commons license CC BY-NC-ND (http:/ / creativecommons.org/licenses/by-nc-nd/4.0/). 


\section{Contents}

About the Special Issue Editors $\ldots \ldots \ldots \ldots \ldots \ldots \ldots$ vii

Preface to "Nutraceuticals and the Skin: Roles in Health and Disease" . . . . . . . . . ix

Yoshihiro Tokudome, Noriomi Masutani, Shohei Uchino and Hisano Fukai

Recovery Effects of Oral Administration of Glucosylceramide and Beet Extract on Skin Barrier

Destruction by UVB in Hairless Mice

Reprinted from: Nutrients 2017, 9, 1178, doi: 10.3390/nu9111178 . . . . . . . . . . . . . 1

Montserrat Rojo de la Vega, Andrea Krajisnik, Donna D. Zhang and Georg T. Wondrak

Targeting NRF2 for Improved Skin Barrier Function and Photoprotection: Focus on the Achiote-Derived Apocarotenoid Bixin

Reprinted from: Nutrients 2017, 9, 1371, doi: 10.3390/nu9121371 . . . . . . . . . . . . 11

Hongdong Song, Siqi Zhang, Ling Zhang and Bo Li

Effect of Orally Administered Collagen Peptides from Bovine Bone on Skin Aging in Chronologically Aged Mice

Reprinted from: Nutrients 2017, 9, 1209, doi: 10.3390/nu9111209 . . . . . . . . . . . . . . 27

Simona-Roxana Georgescu, Maria-Isabela Sârbu, Clara Matei, Mihaela Adriana Ilie, Constantin Caruntu, Carolina Constantin, Monica Neagu and Mircea Tampa

Capsaicin: Friend or Foe in Skin Cancer and Other Related Malignancies?

Reprinted from: Nutrients 2017, 9, 1365, doi: 10.3390/nu9121365 . . . . . . . . . . . . . . .

Agata Jabłońska-Trypuć, Rafał Kretowski, Monika Kalinowska, Grzegorz Świderski, Marzanna Cechowska-Pasko and Włodzimierz Lewandowski

Possible Mechanisms of the Prevention of Doxorubicin Toxicity by Cichoric Acid-Antioxidant Nutrient

Reprinted from: Nutrients 2018, 10, 44, doi: 10.3390/nu10010044 . . . . . . . . . . . . .

Jonghwan Jegal, No-June Park, Sim-Kyu Bong, Hyun Jegal, Su-Nam Kim and Min Hye Yang Dioscorea quinqueloba Ameliorates Oxazolone- and 2,4-Dinitrochlorobenzene-induced Atopic Dermatitis Symptoms in Murine Models

Reprinted from: Nutrients 2017, 9, 1324, doi: 10.3390/nu9121324 . . . . . . . . . . . . . 76

Amitis Karrys, Islam Rady, Roxane-Cherille N. Chamcheu, Marya S. Sabir, Sanchita Mallick, Jean Christopher Chamcheu, Peter W. Jurutka, Mark R. Haussler and G. Kerr Whitfield

Bioactive Dietary VDR Ligands Regulate Genes Encoding Biomarkers of Skin Repair That Are Associated with Risk for Psoriasis

Reprinted from: Nutrients 2018, 10, 174, doi: 10.3390/nu10020174 _ . . . . . . . . . . . 88

Shih-Yi Chuang, Yin-Ku Lin, Chwan-Fwu Lin, Pei-Wen Wang, En-Li Chen and Jia-You Fang Elucidating the Skin Delivery of Aglycone and Glycoside Flavonoids: How the Structures Affect Cutaneous Absorption

Reprinted from: Nutrients 2017, 9, 1304, doi: 10.3390/nu9121304 . . . . . . . . . . . . . . 108

Lee M. Ashton, Kristine B. Pezdirc, Melinda J. Hutchesson, Megan E. Rollo and Clare E. Collins

Is Skin Coloration Measured by Reflectance Spectroscopy Related to Intake of Nutrient-Dense Foods? A Cross-Sectional Evaluation in Australian Young Adults

Reprinted from: Nutrients 2018, 10, 11, doi: 10.3390/nu10010011 . . . . . . . . . . . . . . 128 
Natasha Irrera, Gabriele Pizzino, Rosario D'Anna, Mario Vaccaro, Vincenzo Arcoraci, Francesco Squadrito, Domenica Altavilla and Alessandra Bitto

Dietary Management of Skin Health: The Role of Genistein

Reprinted from: Nutrients 2017, 9, 622, doi: 10.3390/nu9060622

Martina C. Meinke, Ceylan K. Nowbary, Sabine Schanzer, Henning Vollert, Jürgen Lademann and Maxim E. Darvin

Influences of Orally Taken Carotenoid-Rich Curly Kale Extract on Collagen I/Elastin Index of the Skin

Reprinted from: Nutrients 2017, 9, 775, doi: 10.3390/nu9070775 . . . . . . . . . . . . . . . . 149

Daniela Martini, Donato Angelino, Chiara Cortelazzi, Ivana Zavaroni, Giorgio Bedogni, Marilena Musci, Carlo Pruneti, Giovanni Passeri, Marco Ventura, Daniela Galli, Prisco Mirandola, Marco Vitale, Alessandra Dei Cas, Riccardo C. Bonadonna, Sergio Di Nuzzo, Maria Beatrice De Felici and Daniele Del Rio

Claimed Effects, Outcome Variables and Methods of Measurement for Health Claims Proposed Under European Community Regulation 1924/2006 in the Framework of Maintenance of Skin Function

Reprinted from: Nutrients 2018, 10, 7, doi: 10.3390/nu10010007 .

Almudena Pérez-Sánchez, Enrique Barrajón-Catalán, María Herranz-López and Vicente Micol

Nutraceuticals for Skin Care: A Comprehensive Review of Human Clinical Studies

Reprinted from: Nutrients 2018, 10, 403, doi: 10.3390/nu10040403 . . . . . . . . . . . . . . 190 


\section{About the Special Issue Editors}

Jean Christopher Chamcheu is an Assistant Professor at the University of Louisiana at Monroe (ULM) College of Pharmacy (COP), USA. He earned his PhD in Dermatology and Venereology from Uppsala University in Sweden in 2010, joined the Department of Dermatology at The University of Wisconsin-Madison, as a Post-doctoral Associate in the skin and cancer biology/disease and chemoprevention program, where he was later promoted to staff scientist in 2014. Since joining the ULM COP faculty in 2017, Dr. Chamcheu has been establishing a collaborative skin biology and disease and experimental therapeutics research program. Having extensive experience in developing tissue-engineered normal and diseased human skin and new pharmacological therapies, Dr. Chamcheu is currently investigating the interplay between genes, immunity and intervention with dietary bioactive ingredients and synthetic scaffolds in the development, prevention and treatment of hereditary, proliferative and chronic inflammatory skin diseases and cancer to promote health. He has authored several peer-reviewed publications and book chapters, supervised many students/ scientists, and is a recipient of the American Skin Association Carlson Research Scholar Award in psoriasis, and SID, PC and IJMS travel Awards.

Deeba Nadeem Syed received her medical degree from Dow Medical College, Karachi, Pakistan and her PhD in molecular and environmental toxicology from the University of Wisconsin-Madison. Dr. Syed studies bioactive compounds derived from dietary sources for the prevention or treatment of diseases, including cancer. Working on synthetic molecules, Dr. Syed helped dene a general framework for developing a class of molecules that can license transcription elongation at targeted genomic loci. Dr. Syed showed that Syn-TEF1, a synthetic transcription factor, actively enabled transcription across repressive GAA repeats that silence frataxin expression in Friedreich's ataxia, a terminal neurodegenerative disease with no effective therapy. An important focus of Dr. Syed's work is to elucidate the role of microRNAs in the regulation of gene expression in UV exposed human skin and its correlation to skin cancer. Currently, Dr. Syed studies the association of diet with cancer development in the context of changes in the microbiome.

Kerr Whitfield earned a PhD in Biochemistry from the North Carolina State University under William L. Miller, studying estrogen regulation of follicle-stimulating hormone -subunit production. His postdoctoral work at Memorial Sloan Kettering Cancer Center in New York under Ione A. Kourides and James A. Gurr, focused on the human gene for thyroid-stimulating hormone -subunit. In 1987, Dr. Whiteld joined the vitamin D research group of Dr. Mark R. Haussler, whose mentorship influenced the rest of his research career. Evolutionary studies of vitamin D action included the cloning of a vitamin D receptor from the lamprey, Petromyzon marinus. More recently, Dr. Whiteld has investigated a set of genes related to psoriasis risk that are regulated by the vitamin D receptor. Together with Peter Jurutka and Jean Christopher Chamcheu, he has studied alternative, nutritional ligands for the vitamin D receptor that have potential for the treatment of psoriasis and other skin diseases. 



\section{Preface to "Nutraceuticals and the Skin: Roles in Health and Disease"}

Natural products have a long history of use for skin ailments, including traditional medicines that date back hundreds or even thousands of years. In recent decades, some of these natural products, well recognized as sources for drugs in several human ailments, have undergone more rigorous testing, resulting in the identification of phytochemical compounds, natural plant-derived pharmaceuticals, extracts and other preparations that can suitably be termed "nutraceuticals". The current volume seeks to provide a snapshot of research into such natural dietary compounds, pharmaceuticals, extracts, and other preparations as of late 2017, with an emphasis on the transition from folk medicine or anecdotal case reports to an evidence-based approach for the prevention or treatment of skin disorders. The book is organized in three sections and contains 13 chapters, with contributions from Australia, China, Germany, Japan, Korea, Italy, Poland, Romania, Spain, Taiwan and the U.S.A. There are nine original research contributions and four reviews regarding research involving humans and rodent models, conducted in the laboratory and in the field.

The first section of this book focuses on the use of two dimensional in vitro cell culture or in vivo preclinical murine models to study exposure to agents that can lead to premature aging or cancer in the skin. This section contains five peer-reviewed articles that examine the ability of specific nutraceuticals, both of plant and animal origin, to exert preventative or therapeutic effects in skin. Tokudome et al. document how orally administered glucosylceramides from beets protect hairless mice from UVB-induced skin barrier damage. De la Vega et al. studied skin barrier function and photoprotection, focusing on the protective actions of bixin (a New World spice component) mediated via activation of the NRF2 transcription factor. Song et al. examine the effect of collagen peptides to slow skin aging in mice. The last two studies in this section deal with skin cancer. Georgescu et al. review the literature describing pathways involved in the pro- and anti-carcinogenic effects of the pungent spice capsaicin, while Jabłońska-Trypuć et al. describe how cichoric acid present in many edible fruits and vegetables including chicory or Echinacea, can ameliorate the side effects of the common cancer chemotherapy drug doxorubicin. What all of these studies have in common, is the use of objectively measured parameters of skin function, gene expression or enzyme activity to validate the impact of the nutraceutical in question on skin functional properties. Such approaches and the validation they provide are vital to set the stage for real clinical testing of nutraceuticals for disease prevention and/or treatment.

The second section deals with skin inflammation, again using skin cell cultures or mouse models. One study focuses on psoriasis (Karrys et al.) and another on atopic dermatitis (Jegal et al.). A third study (Chuang et al.) examines anti-inflammatory actions of flavonoids, including structure/function considerations of how these compounds are absorbed after application to nude mice skin. As in section one, all three of these studies examine objective parameters such as gene/protein expression of key disease markers, or direct measurements of anti-inflammatory actions, to validate any claims of therapeutic potential.

The final section of this Special Issue deals with the ultimate goal of all of these studies, namely the application of nutraceuticals or their derivatives to the improvement of human health. Ashton et al. seek to establish skin coloration as an index of diet quality in young (Australian adults); Irrera et al. review clinical studies testing the ability of genistein to promote healthy skin aging in post-menopausal women; Meinke et al. report a random controlled trial in which a carotenoid-rich 
diet (curly kale) was found to be associated with improvement in skin aging parameters; Martini et al. describe how new European regulations set guidelines for how any claims for nutraceuticals to improve skin health or treat disease must be substantiated; and finally, Pérez-S ánchez provide an up-to-date review of clinical trials of nutraceuticals for skin care and challenges in establishing a cause-and-effect relationship for health benefits in the absence of animal testing, now banned by the European Union for cosmetic products.

This Special Issue not only takes stock of the current state of research in the field of nutraceuticals and skin health and disease, but also provides an exciting glimpse of the future of nutraceuticals and their promise for improving health by utilizing nature's cornucopia of dietary compounds whose beneficial effects are only just beginning to be understood. While hoping that these articles will provide readers with useful updates in this research field, we acknowledge the superb contributions of the authors, appreciate the reviewers for contributing their time and effort in reviewing each article, and the MDPI editorial staff for their assistance in making this volume a reality.

Jean Christopher Chamcheu, Deeba Nadeem Syed, G. Kerr Whitfield Special Issue Editors 
Article

\title{
Recovery Effects of Oral Administration of Glucosylceramide and Beet Extract on Skin Barrier Destruction by UVB in Hairless Mice
}

\author{
Yoshihiro Tokudome *, Noriomi Masutani, Shohei Uchino and Hisano Fukai \\ Laboratory of Dermatological Physiology, Department of Pharmaceutical Sciences, Faculty of Pharmacy \\ and Pharmaceutical Sciences, Josai University, Saitama 350-0295, Japan \\ * Correspondence: tokudome@josai.ac.jp; Tel.: +81-49-271-8140
}

Received: 4 October 2017; Accepted: 24 October 2017; Published: 27 October 2017

\begin{abstract}
Purified glucosylceramide from beet extract (beet GlcCer) and beet extract containing an equal amount of GlcCer were administered orally to ultra violet B (UVB)-irradiated mice, and differences in the protective effects against skin barrier dysfunction caused by UVB irradiation were compared. In the beet GlcCer group, epidermal thickening and the decrease in stratum corneum (SC) ceramide content caused by UVB irradiation were reduced. In the group that was orally administered beet extract containing glucosylceramide, effects similar to those in the beet GlcCer group were observed. Oral administration of beet GlcCer had no obvious effects against an increase in TEWL or decrease in SC water content after UVB irradiation, but there was improvement in the beet extract group. Oral administration of beet GlcCer is effective in improving skin barrier function in UVB-irradiated mice. Beet extract contains constituents other than GlcCer that are also effective in improving skin barrier function.
\end{abstract}

Keywords: beet; oral administration; glucosylceramide; skin barrier; ultra violet

\section{Introduction}

The stratum corneum of skin plays a barrier function that protects organisms from external stimuli and prevents water loss. Intercellular lipids in the stratum corneum, including ceramides, cholesterol and fatty acids, are important for maintaining skin barrier function. Ceramides are the most abundant, accounting for about $40 \%$ of all intercellular lipids [1]. The number of ceramides in skin is decreased in patients with atopic dermatitis and senile xerosis, and increasing the number of ceramides is important to maintaining healthy skin [2,3]. Oral administration of plant extracts from maize [4], rice [4], konjac [5] and sugar beet [6] that contain glucosylceramide (GlcCer), a ceramide precursor, or purified GlcCer has recently been reported to be effective in improving skin barrier function not only in a hairless mouse model $[5,7,8]$, but also in humans $[5,9]$.

The mechanism by which administered GlcCer improves skin barrier function is not entirely clear. However, sphingoid bases, as in vivo metabolites of GlcCer, have recently been reported to activate ceramide synthesis $[7,10]$ promoting the formation of a cornified envelope [8], and increasing tight-junction function by induction of claudin-1 [11] in human keratinocytes. With regard to species of shingoid base, rice and maize GlcCer consist primarily of 8-cis-unsaturated bonds, such as d18:24t, ${ }^{8 t}, \mathrm{~d} 18: 1^{8 \mathrm{c}}[12]$, while beet GlcCer consists primarily of 8 -trans-unsaturated bonds, such as $\mathrm{d} 18: 2^{4 \mathrm{t}}, 8 \mathrm{c}$, $\mathrm{d} 18: 1^{8 \mathrm{t}}$, and $\mathrm{t} 18: 1^{8 \mathrm{t}}[13]$.

While there is no doubt about the effectiveness of GlcCer, it is not clear whether the potency of such plant extracts depends on GlcCer alone. In many cases, evaluated plant extracts have very low GlcCer contents and higher contents of other constituents. Furthermore, lipid constituents other than GlcCer present in plant extracts presumably differ considerably between plant species, plant parts 
(e.g., root, seeds), and method of extraction and purification. Therefore, improvement of skin barrier function by plant extracts may be related to the effects of other lipid constituents.

The present study aims to identify any differences between purified beet GlcCer and beet extract containing GlcCer and in terms of improving skin barrier function. Sugar beet is a raw material used for sugar production. The fibrous component remaining after extraction of water soluble sugar from the root is sugar beet fiber. By ethanol extraction of sugar beet fibers, beet extracts containing lipid mixtures including glucosylceramides, fatty acids and sterols can be produced as functional food materials.

This study compared differences in the effectiveness between purified GlcCer from beet extract (beet GlcCer) and beet extract containing equal amounts of GlcCer (beet extract) in preventing skin barrier dysfunction, namely, epidermal thickening, decreased stratum corneum ceramide content, increased transepidermal water loss (TEWL), and decreased stratum corneum water content, caused by UVB irradiation in hairless mice.

\section{Materials and Methods}

\subsection{Materials}

Purified glucosylceramide from beet extract (certified $\geq 99 \%$ purity) was purchased from Nagara Science Co., Ltd. (Gifu, Japan). Beet extract was provided by Nippon Beet Sugar Manufacturing Co., Ltd. (Tokyo, Japan), and was a powdered product derived from a mixture of the ethanol extract of sugar beet fiber and modified starch, containing 1.0\% glucosylceramide. Beet extract also contained free sterols, sterylglycosides and free fatty acids. The analytical data on the beet extract used in this study were as follows: $0.39 \%$ schottenol, $0.37 \% \alpha$-spinasterol, $0.23 \% \beta$-sitosterol, and $0.08 \%$ stigmastenol on HPLC; $1.3 \%$ sterylglycosides on TLC; and $0.12 \%$ palmitic acid, $0.11 \%$ linoleic acid, $0.08 \%$ oleic acid on GC (as reported by Nippon Beet Sugar Manufacturing Co., Ltd.). Ceramide NS and AS standards were purchased from Matreya LLC (Pleasant Gap, PA, USA). Ceramide NP and AP standards were obtained from Evonik Goldschmidt GmbH (Goldschmidtstrasse, Essen, Germany). Pentobarbital sodium salt was obtained from Kyoritsu Seiyaku Co. (Tokyo, Japan). Cyanoacrylate adhesives and O.C.T. compound were purchased from Daiichi Sankyo Co., Ltd. (Tokyo, Japan) and Sakura Finetek Japan Co., Ltd. (Tokyo, Japan). Phosphate-buffered saline (PBS) powder was obtained from Sigma (St. Louis, MO, USA). Silica gel 60 (Merck, Darmstadt, Germany) was used for the HPTLC plate. All other reagents were obtained commercially and used without further purification.

\subsection{Animals}

Seven-week-old male HR-1 hairless mice were purchased from Japan SLC Inc., (Hamamatsu, Shizuoka, Japan). Mice were maintained in a light- (12-h light/dark cycle) and temperature-controlled $\left(25 \pm 2{ }^{\circ} \mathrm{C}\right)$ barrier facility throughout the study. Mice were allowed free access to feed (Labo MR Stock; Nosan Corporation, Yokohama, Japan) and water. All animal experiments and maintenance were performed under conditions approved by the Animal Research Committee of Josai University. Mice were randomly assigned to four groups $(n=6$, Table 1$)$.

Table 1. Experimental groups and test samples.

\begin{tabular}{ccccc}
\hline \multirow{2}{*}{ Group } & \multirow{2}{*}{ UVB Irradiation $\left(\mathbf{m J} / \mathrm{cm}^{2}\right)$} & \multicolumn{2}{c}{ Composition of Test Sample (mg/Mouse/Day) } \\
\cline { 3 - 5 } & & GlcCer & Beet Ethanol Extract & Processed Starch \\
\hline Normal & None & 0 & 0 & 22 \\
Control & 200 & 0 & 0 & 22 \\
Beet GlcCer & 200 & 0.3 & 0 & 22 \\
Beet Extract & 200 & $(0.3)^{1}$ & 8.0 & 22 \\
\hline
\end{tabular}




\subsection{Test Sample and Schedule}

Test samples were suspended in purified water containing processed starch (starch sodium octenyl succinate) as emulsifier and $0.3 \mathrm{~mL}$ was orally administered to each mouse using a sonde every day for 14 days. All of the glucosylceramide samples were prepared on the day of administration.

A single dose of UVB $\left(200 \mathrm{~mJ} / \mathrm{cm}^{2}\right)$ irradiation was applied to the back skin of each mouse using a Philips UVB lamp (TL20W/12 RS; Philips, Amsterdam, The Netherlands) at day 7 after the first administration (only one time irradiation of UVB). Collection of stratum corneum and dorsal skin sections from half of the mice was performed under anesthesia at day 3 after UVB irradiation. Table 2 shows the experimental schedule of this manuscript.

Table 2. Experimental schedule (d: day).

\begin{tabular}{|c|c|c|c|c|c|c|c|c|c|c|c|c|c|c|c|}
\hline Events/Day & $-7 d$ & $-6 d$ & $-5 d$ & $-4 d$ & $-3 d$ & $-2 d$ & $-1 \mathrm{~d}$ & $0 \mathrm{~d}$ & $1 \mathrm{~d}$ & $2 \mathrm{~d}$ & $3 \mathrm{~d}$ & $4 \mathrm{~d}$ & $5 \mathrm{~d}$ & $6 \mathrm{~d}$ & $7 \mathrm{~d}$ \\
\hline $\begin{array}{l}\text { Sample administration } \\
\text { UV irradiation }\end{array}$ & 0 & 0 & 0 & 0 & 0 & 0 & 0 & $\begin{array}{l}0 \\
0\end{array}$ & 0 & 0 & 0 & 0 & 0 & 0 & \\
\hline Body weight & 0 & & 0 & & 0 & & 0 & 0 & 0 & 0 & 0 & 0 & 0 & 0 & 0 \\
\hline
\end{tabular}

\subsection{Measurement of Water Contents of Stratum Corneum, and TEWL}

Water contents of stratum corneum and TEWL in the back skin of mice were measured daily for 14 days. Water contents of the stratum corneum were measured with a Corneometer (Courage \& Khazaka, Cologne, Germany) and TEWL was assessed using a VAPO SCAN AS-VT100 RS (Asahi Techno Lab., Ltd., Yokohama, Kanagawa, Japan). Corneometer and VAPO SCAN data are given in terms of indicated arbitrary units (AU) and $\mathrm{g} / \mathrm{m}^{2} / \mathrm{h}$, respectively.

\subsection{Collection of Mouse Stratum Corneum}

After application of anesthesia, cyanoacrylate adhesive was dropped onto slide glass, and this was adhered to the murine back skin for one minute. Slide glass was removed to collect stratum corneum sample [2].

\subsection{Extraction Method and Lipid Analysis by HPTLC}

The slide glass used to collect stratum corneum was soaked in hexane and ethanol (95:5), and was sonicated at $37^{\circ} \mathrm{C}$ for $20 \mathrm{~min}$. After filtering the solution, it was dried under nitrogen gas. The residue was added to $100 \mu \mathrm{L}$ of chloroform:methanol (2:1). Briefly, stratum corneum samples were soaked in $4 \mathrm{~mL}$ of chloroform:methanol $(2: 1 \mathrm{v} / \mathrm{v})$ and sonicated $(70 \mathrm{~W}, 10 \mathrm{~min})$ with a probe-type sonicator (Sonifire B-12; Branson Ultrasonics, Danbury, CT, USA). The ceramide extracted solution was dried under nitrogen gas, and was resolved in $0.4 \mathrm{~mL}$ of chloroform:methanol $(2: 1 \mathrm{v} / \mathrm{v})$. Various ceramide extracts were separated using an HPTLC plate (Silica Gel 60; Merck, Darmstadt, Germany). HPTLC was developed twice with chloroform:methanol:acetic acid $=190: 9: 1(v / v)$. Ceramide molecules were visualized by treatment with $10 \% \mathrm{CuSO}_{4}, 8 \% \mathrm{H}_{3} \mathrm{PO}_{4}$ aqueous solution, and heating to $180{ }^{\circ} \mathrm{C}$ for $10 \mathrm{~min}$. The amounts of various types of ceramide (ceramide NS, NP, AS and AP) were quantitatively determined using a densitometer.

\subsection{Measurement of Stratum Corneum Mass}

The slide glass used to collect the stratum corneum was soaked in $N, N$, dimethylformamide, and was sonicated for $15 \mathrm{~min}$. The sonicated solution was passed through a filter that was weighed beforehand. The filter was dried for one week under vacuum. After dryness, the filter weighed again, and difference between the before and after measurements was taken as the stratum corneum mass. 


\subsection{Hematoxylin and Eosin Staining}

The dorsal skin of animals was detached, cut into $0.7 \mathrm{~cm} \times 0.2 \mathrm{~cm}$ strips with a scalpel, and embedded in OCT compound under rapid chilling on dry ice in order to prepare frozen sections. Frozen sections were cut at $0.1 \mu \mathrm{m}$ using the Leica CM $3050 \mathrm{~S}$ microtome (Welzlar, Germany). Sections were subsequently stained with hematoxylin and eosin, as follows. Sections were first immersed for $30 \mathrm{~min}$ in a $10 \%$ formalin solution for fixation. Sections were then rinsed with water and sequentially immersed in ethanol in order to achieve dehydration. Next, sections were rinsed with water and immersed in hematoxylin solution for $10 \mathrm{~min}$. Sections were again rinsed with water and immersed in an eosin solution containing acetic acid. Finally, sections were immersed in and penetrated with xylene 3 times, embedded, and observed under a microscope. Epidermal thickness was defined as the distance from the top of the stratum granulosum to the bottom of the stratum basale. Thickness of stratum corneum was measured from the top of the stratum corneum to the bottom.

\subsection{Measurement of Epidermis Thickness}

Thickness of the epidermis was measured at the horizontal midpoint of each visual field. Approximately 50 individual measurements were made along the wound margin for each histological section, and mean thickness was evaluated.

\subsection{Data and Statistical Analysis}

All results are expressed as means \pm standard deviation. Statistical analysis was performed using Tukey's post-hoc test (using SAS software version 9.2, SAS Institute, Cary, NC, USA).

\section{Results}

\subsection{Body Weight Change after Oral Administration of Beet GlcCer or Beet Extract in UVB-Irradiated Mice}

Figure 1 shows the body weight changes in UVB-irradiated mice. Body weight did not change in any groups after oral administration of beet GlcCer or beet extract.

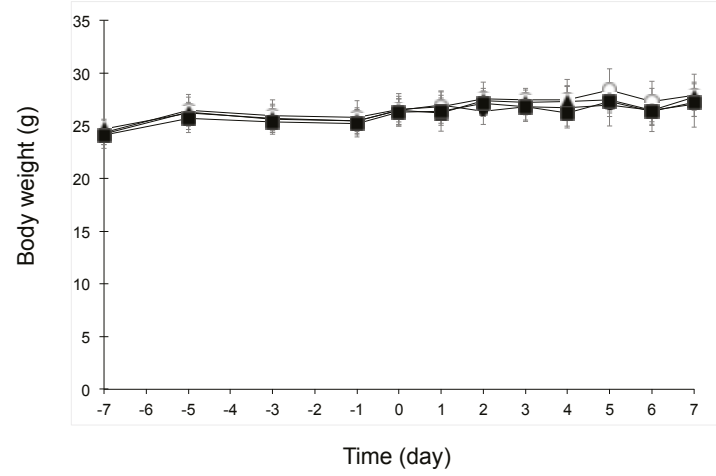

Figure 1. Body weight changes in UVB-irradiated mice after oral administration of beet GlcCer or beet extract. Symbols and bars represent means and standard deviation $(n=6$ from day -7 to day 3 , and $n=3$ from day 4 to day 7), respectively. Symbols: Closed circles, Normal group (without UVB irradiation, vehicle administration); Open circles, Control group (200 mJ $/ \mathrm{cm}^{2} \mathrm{UVB}$, vehicle administration); closed triangles, beet GlcCer group ( $200 \mathrm{~mJ} / \mathrm{cm}^{2} \mathrm{UVB}$, beet GlcCer oral administration); closed squares, beet extract group $\left(200 \mathrm{~mJ} / \mathrm{cm}^{2} \mathrm{UVB}\right.$, beet extract oral administration). 


\subsection{Epidermal Hyperplasia Following UVB Irradiation}

Frozen sections of murine dorsal skin at day 3 after UVB irradiation were prepared, and hematoxylin and eosin staining of the sections was performed (Figure 2). Skin layers were thickened by UVB irradiation (normal group, $28 \pm 4 \mu \mathrm{m}$; control group, $119 \pm 9 \mu \mathrm{m} ; p<0.01$ ). Interestingly, beet GlcCer reduced skin thickening induced by UVB irradiation, and a similar effect was also observed in the beet extract group (beet GlcCer group, $68 \pm 11 \mu \mathrm{m}$; beet extract group, $69 \pm 5 \mu \mathrm{m} ; p<0.01$ vs. control group).
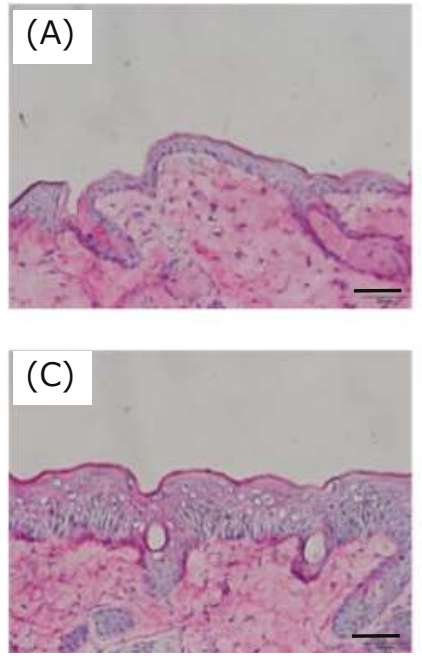
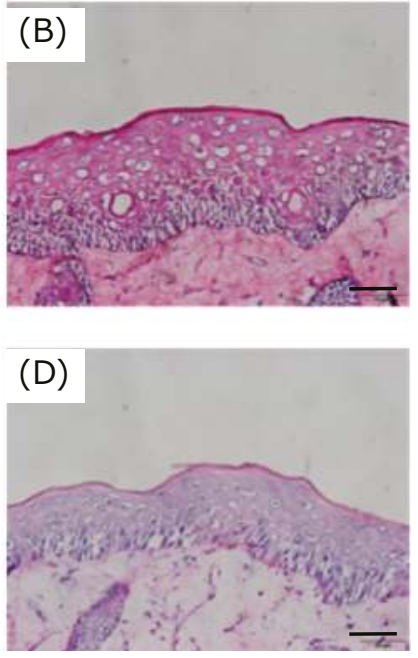

(E)

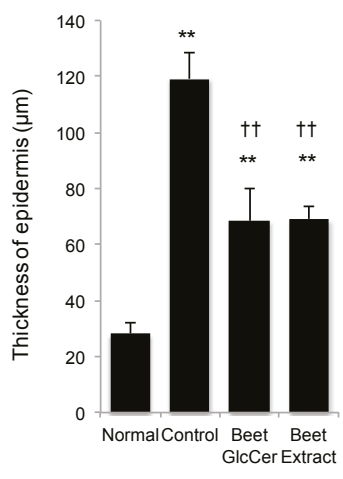

Figure 2. Hematoxylin and eosin staining, and thickness of epidermis in mouse skin. (A) Normal (without UVB irradiation, vehicle administration); (B) Control $\left(200 \mathrm{~mJ} / \mathrm{cm}^{2}\right.$ UVB irradiation, vehicle administration); (C) Beet GlcCer (200 mJ/ $\mathrm{cm}^{2}$ UVB irradiation, beet GlcCer oral administration); (D) Beet extract (200 mJ $/ \mathrm{cm}^{2}$ UVB irradiation, beet extract oral administration); and (E) Epidermal thickness in UVB irradiated mice $(n=3)$. Scale bar indicates $50 \mu \mathrm{m}$. ${ }^{* *} p<0.01$ vs. normal group, t† $p<0.01$ vs. control group, Tukey's post-hoc multiple comparison test.

\subsection{Comparison of Ceramide Contents in Stratum Corneum among Mouse Groups}

Quantitative analysis of ceramide contents in stratum corneum removed from the murine back skin at day 3 after UVB treatment was performed (Figure 3). UVB irradiation significantly decreased ceramide contents in stratum corneum. Remarkably, ceramide contents in UVB-irradiated mice orally given beet GlcCer were similar to those in the normal group. Similar results were observed between the beet extract group and the normal group. 

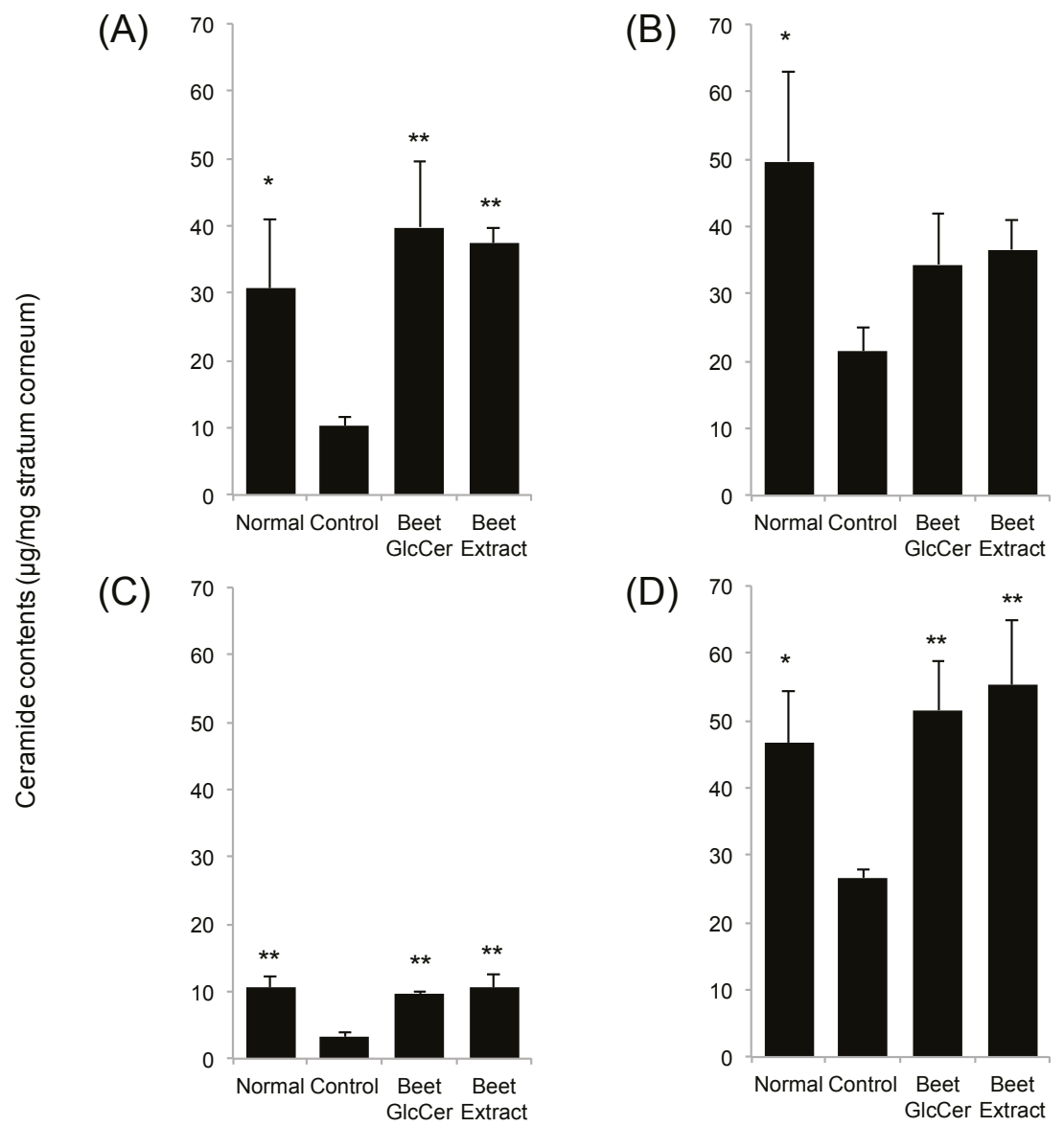

Figure 3. Ceramide contents in stratum corneum in UVB-irradiated $\left(200 \mathrm{~mJ} / \mathrm{cm}^{2}\right)$ mice after oral administration of beet GlcCer or beet extract. Ceramide NS (A); NP (B); AS (C) and AP (D); respectively. Values are given as means and standard deviation $(n=3) .{ }^{*} p<0.05,{ }^{* *} p<0.01$ vs. control group, Tukey's post-hoc multiple comparison test.

\subsection{TEWL and Water Contents in Stratum Corneum}

Figure 4 shows the TEWL and water contents in stratum corneum at days 0, 3 and 6 after UVB irradiation in each group. TEWL in UVB-irradiated groups temporarily increased at day 3 after UVB treatment, as compared with non-UVB-irradiated mice. Mice administered beet GlcCer showed slightly lower TEWL, but the difference was not significant, when compared with the control group at day 3. Increases in TEWL in the beet extract group after UVB irradiation were significantly lower than in the control group at days 3 and 6 . UVB irradiation also decreased the water content in stratum corneum in each mouse group. Although the water content in stratum corneum from the control group remained low at day 6 when compared with the normal group, early recovery of the loss of water content in the stratum corneum was observed in the beet extract group. 


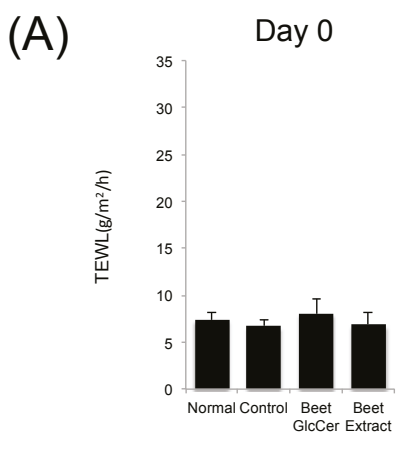

(B)

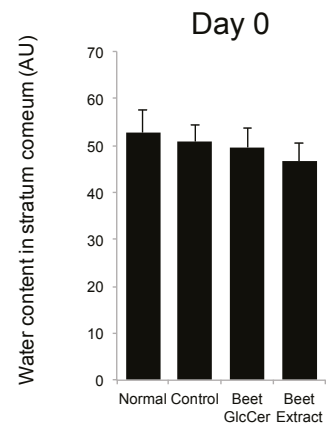

Day 3

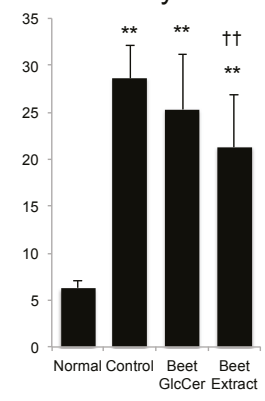

Day 3

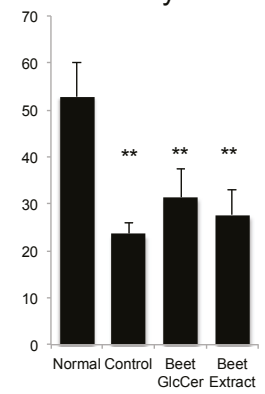

Day 6

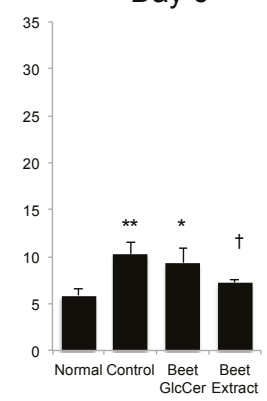

Day 6

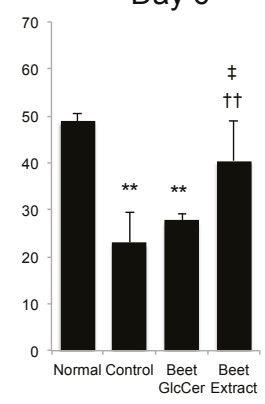

Figure 4. Effects of oral administration of beet GlcCer or beet extract on TEWL and stratum corneum after UVB irradiation. (A) Transepidermal water loss (TEWL); (B) water contents in stratum corneum. Values are shown as means \pm standard deviation $(n=6$ at day 0 and $3, n=3$ at day 6$) .{ }^{*} p<0.05$, $* * p<0.01$ vs. normal group, $\uparrow p<0.05$ vs. control group, $+\dagger p<0.01$ vs. control group, $\ddagger p<0.05$ vs. beet GlcCer group, Tukey's post-hoc multiple comparison test.

\section{Discussion}

This study showed that oral administration of purified GlcCer from beets was effective in preventing epidermal thickening and decreased stratum corneum ceramide content caused by UVB irradiation in hairless mice. In addition, administration of beet extract containing an equal amount of glucosyl ceramides was more effective than beet GlcCer in improving increased TEWL and decreased stratum corneum water content.

Initially, examination of skin sections on day 3 after UVB irradiation showed epidermal hyperplasia in controls. Epidermal thickness increased 4-fold when compared to the normal group. However, in the beet GlcCer group, epidermal thickness decreased to 50\% when compared with controls. In the beet extract group, epidermal thickness decreased to the same degree as in the beet GlcCer group. These findings strongly suggest that inhibition of epidermal hyperplasia was due to the effects of orally administered GlcCer. Inhibition of epidermal hyperplasia by beet extract [6] and purified GlcCer from corn extract has previously been reported in a hairless mouse model of skin barrier impairment induced by an Mg-deficient HR-AD diet [7]. Our study found that beet GlcCer was also effective in preventing epidermal hyperplasia induced by UVB irradiation.

Ceramides, which account for about $40 \%$ of intercellular lipids in the stratum corneum, are important in skin barrier function [1-3]. A recent study found that UVB irradiation decreased skin ceramide content and increased ceramidase gene expression [14]. With oral administration of purified GlcCer from plant extracts to hairless mice fed an HR-AD diet, a 2-fold increase in epidermal ceramide synthase gene expression has also been reported [7]. Because of these previous findings, we decided 
to focus on stratum corneum ceramide content. In our study, we analyzed stratum corneum content of ceramides NS, NP, AS and AP, for which ceramide standards were available. The results showed a decrease in all measured ceramides at 3 days after UVB irradiation, but with oral administration of beet GlcCer, all of these decreases were reduced. Moreover, similar levels of stratum corneum ceramide content were seen in both the beet GlcCer group and the beet extract group, thus strongly suggesting that GlcCer in the beet extract contributed to these observed effects.

Haratake et al. reported epidermal cell hyperplasia followed by increased TEWL in UVB-irradiated hairless mice [15]. The increase in TEWL was reduced by DNA synthesis inhibitors, and they concluded that epidermal hyperproliferation was associated with the epidermal barrier disturbance [15]. TEWL reflects water loss from the body and is commonly used a parameter of skin barrier function. In our study, epidermal hyperplasia and increased TEWL were observed in the control group at 3 days after UVB irradiation. The effectiveness of purified GlcCer from plants in preventing TEWL has previously been reported in a hairless mouse model with barrier perturbation induced by single-dose UVB irradiation [8], in a hairless mouse model with sodium dodecyl sulfate-induced skin roughness [5], in a Mg-deficient diet-induced atopic dermatitis-like model [7], and in a tape-stripped injured skin mouse model with regard to GlcCer from konjac and maize [7]. However, there are few reports on GlcCer from beet. Haruta-Ono et al. reported on orally administered of sphingomyelin to the improvement of epidermal function in hairless mice [16]. TEWL and stratum corneum water content of sphingomyelin-administered mice showed better values than the control group. Ceramide also increased. From these results, oral application of GlcCer and sphingomyelin may have a good effect on epidermal function.

In our study, although TEWL was slightly lower in the beet GlcCer group when compared with the control group, the difference was not statistically significant. Interestingly, however, oral administration of beet extract containing an equal amount of GlcCer, when compared to the control group, significantly reduced the increase in TEWL caused by UVB irradiation. In addition, there was earlier recovery of decreased stratum corneum water content. These results strongly suggest that constituents other than GlcCer in beet extract help protect against or restore UVB irradiation-induced skin barrier dysfunction.

For example, beet-derived steryl glycosides, phytosterols and free fatty acids are present in beet extracts (described in Materials and Methods). Recent studies have reported that steryl glycosides and phytosterols inhibit UVB irradiation-induced inflammatory reactions (cytokines, matrix metalloproteinase production) in skin cells $[17,18]$. In UVB-irradiated skin tissue, inflammatory cytokines and chemokines such as IL-1 $\alpha, \mathrm{TNF} \alpha$ and IL-8 are released from keratinocytes [19-21]. This is followed by activated lymphocyte infiltration into skin tissue, leading to an inflammatory reaction. Oral administration of beet extract may reduce skin barrier dysfunction after UVB irradiation more so than administration of GlcCer alone because phytosterols in beet extract prevent expansion of the inflammatory reaction in skin tissue after UVB irradiation. Confirmation of this hypothesis will require further studies.

\section{Conclusions}

Oral administration of beet GlcCer prevented epidermal hyperplasia and decreased stratum corneum ceramide content caused by UVB irradiation. In addition, our findings suggest that beet extract contains constituents other than GlcCer that are also effective in improving skin barrier function.

Author Contributions: Y.T. designed the research, N.M., S.U. and H.F. performed the experiments, Y.T. and N.M. analyzed the data, and Y.T. wrote the manuscript. All authors have read and approved the final manuscript.

Conflicts of Interest: The authors declare no conflicts of interest. 


\section{References}

1. Gray, G.M.; Yardley, H.J. Different populations of pig epidermal cells: Isolation and lipid composition. J. Lipid Res. 1975, 16, 441-447. [PubMed]

2. Imokawa, G.; Abe, A.; Jin, K.; Higaki, Y.; Kawashima, M.; Hidano, A. Decreased level of ceramides in stratum corneum of atopic dermatitis: An etiologic factor in atopic dry skin? J. Investig. Dermatol. 1991, 96, 523-526. [CrossRef] [PubMed]

3. Yamamoto, A.; Serizawa, S.; Ito, M.; Sato, Y. Stratum corneum lipid abnormalities in atopic dermatitis. Arch. Dermatol. Res. 1991, 283, 219-223. [CrossRef] [PubMed]

4. Tsuji, K.; Mitsutake, S.; Ishikawa, J.; Takagi, Y.; Akiyama, M.; Shimizu, H.; Tomiyama, T.; Igarashi, Y. Dietary glucosylceramide improves skin barrier function in hairless mice. J. Dermatol. Sci. 2006, 44, 101-107. [CrossRef] [PubMed]

5. Uchiyama, T.; Nakano, Y.; Ueda, O.; Mori, H.; Nakashima, M.; Noda, A.; Ishizaki, C.; Mizoguchi, M. Oral Intake of Glucosylceramide Improves Relatively Higher Level of Transepidermal Water Loss in Mice and Healthy Human Subjects. J. Health Sci. 2008, 54, 559-566. [CrossRef]

6. Kawano, K.-I.; Umemura, K. Oral intake of beet extract provides protection against skin barrier impairment in hairless mice. Phytother. Res. 2013, 27, 775-783. [CrossRef] [PubMed]

7. Duan, J.; Sugawara, T.; Hirose, M.; Aida, K.; Sakai, S.; Fujii, A.; Hirata, T. Dietary sphingolipids improve skin barrier functions via the upregulation of ceramide synthases in the epidermis. Exp. Dermatol. 2012, 21, 448-452. [CrossRef] [PubMed]

8. Hasegawa, T.; Shimada, H.; Uchiyama, T.; Ueda, O.; Nakashima, M.; Matsuoka, Y. Dietary glucosylceramide enhances cornified envelope formation via transglutaminase expression and involucrin production. Lipids 2011, 46, 529-535. [CrossRef] [PubMed]

9. Hori, M.; Kishimoto, S.; Tezuka, Y.; Nishigori, H.; Nomoto, K.; Hamada, U.; Yonei, Y. Double-blind study on effects of glucosyl ceramide in beet extract on skin elasticity and fibronectin production in human dermal fibroblasts. Anti-Aging Mag. 2010, 7, 129-142. [CrossRef]

10. Shirakura, Y.; Kikuchi, K.; Matsumura, K.; Mukai, K.; Mitsutake, S.; Igarashi, Y. 4,8-Sphingadienine and 4-hydroxy-8-sphingenine activate ceramide production in the skin. Lipids Health Dis. 2012, 11, 108. [CrossRef] [PubMed]

11. Kawada, C.; Hasegawa, T.; Watanabe, M.; Nomura, Y. Dietary glucosylceramide enhances tight junction function in skin epidermis via induction of claudin-1. Biosci. Biotechnol. Biochem. 2013, 77, 867-869. [CrossRef] [PubMed]

12. Aida, K.; Takakuwa, N.; Kinoshita, M.; Sugawara, H.; Imai, H.; Ono, J.; Ohnishi, M. Properties and physiological effects of plant cerebroside species as functional lipids. Adv. Res. Plant Lipids 2003, 233-236. [CrossRef]

13. Takakuwa, N.; Saito, K.; Oda, Y. Content of Functional Lipid Ceramide in Crop Tissues and By-Products from Their Processing. Atarashii Kenkyu Seika 2005, 173-177.

14. Ra, J.; Lee, D.E.; Kim, S.H.; Jeong, J.W.; Ku, H.K.; Kim, T.Y.; Choi, I.D.; Jeung, W.; Sim, J.H.; Ahn, Y.T. Effect of oral administration of Lactobacillus plantarum HY7714 on epidermal hydration in ultraviolet B-irradiated hairless mice. J. Microbiol. Biotechnol. 2014, 24, 1736-1743. [CrossRef] [PubMed]

15. Haratake, A.; Uchida, Y.; Schmuth, M.; Tanno, O.; Yasuda, R.; Epstein, J.H.; Elias, P.M.; Holleran, W.M. UVB-induced alterations in permeability barrier function: Roles for epidermal hyperproliferation and thymocyte-mediated response. J. Investig. Dermatol. 1997, 108, 769-775. [CrossRef] [PubMed]

16. Haruta-Ono, Y.; Ueno, H.; Ueda, N.; Kato, K.; Yoshioka, T. Investigation into the dosage of dietary sphingomyelin concentrate in relation to the improvement of epidermal function in hairless mice. Anim. Sci. J. 2012, 83, 178-183. [CrossRef] [PubMed]

17. Lee, T.H.; Lee, S.M.; Lee, D.Y.; Son, Y.; Chung, D.K.; Baek, N.I.; Kim, J. A glycosidic spinasterol from Koreana stewartia promotes procollagen production and inhibits matrix metalloproteinase-1 expression in UVB-irradiated human dermal fibroblasts. Biol. Pharm. Bull. 2011, 34, 768-773. [CrossRef] [PubMed]

18. Hwang, E.; Park, S.Y.; Sun, Z.W.; Shin, H.S.; Lee, D.G.; Yi, T.H. The protective effects of fucosterol against skin damage in UVB-irradiated human dermal fibroblasts. Mar. Biotechnol. 2014, 16, 361-370. [CrossRef] [PubMed] 
19. Kupper, T.S.; Chua, A.O.; Flood, P.; McGuire, J.; Gubler, U. Interleukin 1 gene expression in cultured human keratinocytes is augmented by ultraviolet irradiation. J. Clin. Investig. 1987, 80, 430-436. [CrossRef] [PubMed]

20. Kondo, S.; Kono, T.; Sauder, D.N.; McKenzie, R.C. IL-8 gene expression and production in human keratinocytes and their modulation by UVB. J. Investig. Dermatol. 1993, 101, 690-694. [CrossRef] [PubMed]

21. Bashir, M.M.; Sharma, M.R.; Werth, V.P. UVB and proinflammatory cytokines synergistically activate TNF-alpha production in keratinocytes through enhanced gene transcription. J. Investig. Dermatol. 2009, 129, 994-1001. [CrossRef] [PubMed]

(c) 2017 by the authors. Licensee MDPI, Basel, Switzerland. This article is an open access article distributed under the terms and conditions of the Creative Commons Attribution (CC BY) license (http:/ / creativecommons.org/licenses/by/4.0/). 


\title{
Targeting NRF2 for Improved Skin Barrier Function and Photoprotection: Focus on the Achiote-Derived Apocarotenoid Bixin
}

\author{
Montserrat Rojo de la Vega, Andrea Krajisnik, Donna D. Zhang and Georg T. Wondrak * \\ Department of Pharmacology and Toxicology, College of Pharmacy \& Arizona Cancer Center, \\ University of Arizona, Tucson, AZ 85724, USA; emrvg@email.arizona.edu (M.R.d.l.V.); \\ akrajisnik@email.arizona.edu (A.K.); dzhang@pharmacy.arizona.edu (D.D.Z.) \\ * Correspondence: wondrak@pharmacy.arizona.edu; Tel.: +1-520-626-9009
}

Received: 15 November 2017; Accepted: 15 December 2017; Published: 18 December 2017

\begin{abstract}
The transcription factor NRF2 (nuclear factor-E2-related factor 2) orchestrates major cellular defense mechanisms including phase-II detoxification, inflammatory signaling, DNA repair, and antioxidant response. Recent studies strongly suggest a protective role of NRF2-mediated gene expression in the suppression of cutaneous photodamage induced by solar UV (ultraviolet) radiation. The apocarotenoid bixin, a Food and Drug Administration (FDA)-approved natural food colorant (referred to as 'annatto') originates from the seeds of the achiote tree native to tropical America, consumed by humans since ancient times. Use of achiote preparations for skin protection against environmental insult and for enhanced wound healing has long been documented. We have recently reported that (i) bixin is a potent canonical activator of the NRF2-dependent cytoprotective response in human skin keratinocytes; that (ii) systemic administration of bixin activates NRF2 with protective effects against solar UV-induced skin damage; and that (iii) bixin-induced suppression of photodamage is observable in $\mathrm{Nrf}^{+/+}$but not in $\mathrm{Nrf2} 2^{-/-}$ SKH-1 mice confirming the NRF2-dependence of bixin-induced antioxidant and anti-inflammatory effects. In addition, bixin displays molecular activities as sacrificial antioxidant, excited state quencher, PPAR (peroxisome proliferator-activated receptor) $\alpha / \gamma$ agonist, and TLR (Toll-like receptor) $4 / \mathrm{NF} K \mathrm{~B}$ (nuclear factor kappa-light-chain-enhancer of activated B cells) antagonist, all of which might be relevant to the enhancement of skin barrier function and environmental stress protection. Potential skin photoprotection and photochemoprevention benefits provided by topical application or dietary consumption of this ethno-pharmacologically validated phytochemical originating from the Americas deserves further preclinical and clinical examination.
\end{abstract}

Keywords: skin photodamage; skin barrier function; solar ultraviolet (UV); NRF2; PPAR $\alpha$; bixin; achiote

\section{Introduction: Solar Radiation, Photodamage, Photoaging, and Skin Photocarcinogenesis}

Exposure to solar ultraviolet (UV) radiation is a causative factor in acute skin photodamage, chronic photoaging, and photocarcinogenesis [1-4]. More recently, a causative role of solar photons in the visible and infrared spectral range contributing to skin photodamage has been substantiated [5-8]. Moreover, cutaneous exposure to other environmental stressors including combustion pollutants, heavy metals, metalloids, and ozone has been shown to contribute to skin damage and carcinogenesis. Remarkably, nonmelanoma skin cancer (NMSC; also referred to as keratinocyte cancers (KC)) is the most common malignancy in the United States, and skin cancer incidence is increasing rapidly, presenting a public health burden of considerable magnitude [9]. Even though sunscreen-based photoprotection is an effective component of a sun-safe strategy to reduce cumulative lifetime exposure 
to UV light, much effort has been directed towards the development of more effective molecular strategies acting through mechanisms different from (or synergistic with) photon absorption [9-14].

\section{NRF2: A Master Regulator of Skin Barrier Function, Cellular Defense Mechanisms against Environmental Stress, and Solar Radiation Response}

The redox-sensitive transcription factor NRF2 (nuclear factor-E2-related factor 2) orchestrates major cellular defense mechanisms including phase-II detoxification, inflammatory signaling, DNA repair, and antioxidant response, and recent experimental evidence supports an important role of NRF2 in skin barrier function. NRF2 has therefore emerged as a promising molecular target for the pharmacological prevention of human pathologies resulting from exposure to environmental toxicants including solar UV-induced damage and carcinogenesis [15-18]. Moreover, the potential of NRF2 for modulation of skin chronological and photodamage-associated aging has attracted considerable attention $[9,19,20]$.

\section{NRF2: Molecular Biology and Pharmacological Modulation}

NRF2 is ubiquitously expressed in all tissues, including the skin, but its protein levels and consequently its activity are tightly regulated (Figure 1). Under basal (homeostatic) conditions, NRF2 resides in the cytosol, where it binds to its negative regulator Kelch-ECH associated protein 1 (KEAP1), a substrate adaptor for a cullin 3-RING box protein 1 (CUL3-RBX1) E3 ubiquitin ligase complex [21]. Thus, NRF2 is ubiquitylated and degraded by the $26 \mathrm{~S}$ proteasome [22]. However, upon exposure to reactive oxygen species (ROS) or to electrophilic compounds, key sensor cysteine residues in KEAP1 (cysteine 151 in particular) are chemically modified, causing a conformational change in KEAP1 that prevents degradation of NRF2, which remains complexed to KEAP1 [23,24]. This allows newly synthesized NRF2 to accumulate and translocate to the nucleus, where it heterodimerizes with small MAF (musculoaponeurotic fibrosarcoma) proteins and binds to the antioxidant response elements (AREs) in the regulatory regions of its downstream genes [25]. This mode of canonical NRF2 regulation has been extensively studied in the context of skin protection and pathogenesis. In addition, other modes of NRF2 regulation, such as the p62-dependent non-canonical pathway that activates NRF2 in an autophagy-dependent manner [26,27] or the GSK3- $\beta \operatorname{TrCP}$ (glycogen synthase kinase $3 / \beta$-transducin repeat containing protein) degradation pathway $[28,29]$, have been described. However, the involvement of these other modes of NRF2 regulation in skin barrier function and environmental stress protection remain to be determined.

Many natural chemopreventive compounds that have antioxidant properties exert their cytoprotective function through NRF2 activation. Classic examples of NRF2 inducers are sulforaphane (from cruciferous vegetables) [16], curcumin (from Curcuma longa) [30], cinnamaldehyde (from cinnamon) [31,32], and tanshinones (from Salvia miltiorrhiza) [33], among many others. These compounds are promiscuous electrophilic molecules that also react with cysteine 151 of KEAP1, induce NRF2, and confer protection against a number of chemical insults or radiation damage (including UV) observable in vitro and in vivo [34-36]. Recently, a synthetic triterpenoid NRF2 modulator and bardoxolone-derivative, RTA 408, has been tested for topical NRF2 activation in rat, murine, and human skin $[37,38]$, but limited data on skin protection properties are available. Taken together, a significant opportunity for the development of cutaneous NRF2-dependent skin protection strategies using nutrient-derived molecular entities remains to be explored. 


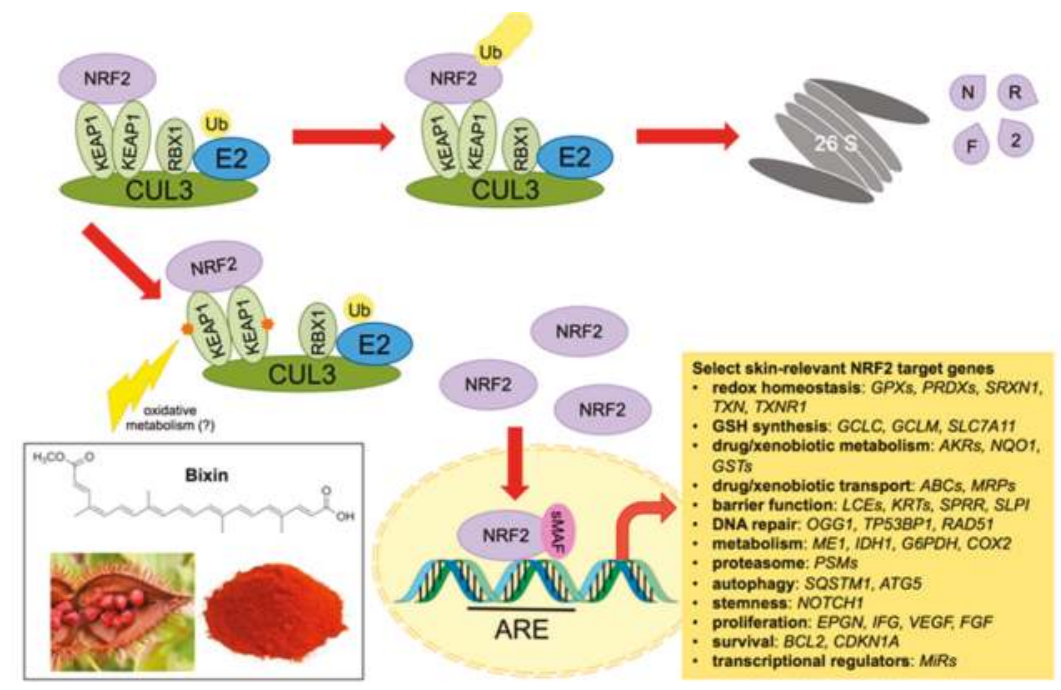

Figure 1. The nuclear factor-E2-related factor 2 (NRF2) pathway with a focus on skin barrier function and environmental stress protection. The transcription factor NRF2 binds to Kelch-ECH associated protein 1 (KEAP1), the substrate adaptor protein for the cullin 3-RING box protein 1 (CUL3-RBX1) E3 ubiquitin ligase complex. Under basal conditions, NRF2 is ubiquitylated and degraded by the 26S proteasome. Upon modification of reactive cysteines in KEAP1 by reactive oxygen species (ROS) and electrophiles (including bixin), NRF2 is no longer ubiquitylated. This allows for newly synthesized NRF2 to accumulate, translocate to the nucleus, and activate the transcription of antioxidant response element (ARE)-containing target genes by dimerizing with small MAF (sMAF) proteins. Select skin-relevant NRF2 target genes are displayed according to the cellular function they perform. GPXs, glutathione peroxidases; PRDXs, peroxiredoxins; SRXN1, sulfiredoxin 1; TXN, thioredoxin; TXNR1, thioredoxin reductase 1; GCLC, glutamate cysteine ligase, catalytic subunit; GCLM, glutamate cysteine ligase, modifier subunit; SLC7A11, glutamate/cystine antiporter (xCT); AKRs, aldoketoreductases; NQO1, NAD(P)H:quinone oxidoreductase 1; GSTs, glutathione S-transferases; $A B C$, ATP-binding cassette family proteins; $M R P_{S}$, multidrug resistance-associated proteins; $L C E s$, late cornified envelope family members; KRTs, keratins; $S P R R$, small proline rich proteins; OGG1, 8-oxo-guanine glycosylase; $T P 53 B P 1$, p53 binding protein 1; RAD51, DNA repair protein RAD51 homolog 1; ME1, malic enzyme; $I D H 1$, isocitrate dehydrogenase 1; G6PDH, glucose-6-phosphate dehydrogenase; COX2, cytochrome c oxidase subunit 2; PSM, proteasome subunit proteins; SQSTM1, sequestosome 1 (p62); ATG5, autophagy-related gene 5; NOTCH1, Notch homolog 1, translocation-associated; EPGN, epigen; IGF, insulin-like growth factor; VEGF, vascular endothelial growth factor; $F G F$, fibroblast growth factor; $B C L 2$, B cell lymphoma 2; CDKN1A, cyclin dependent kinase inhibitor 1A (p21); MiR, microRNAs.

\section{NRF2 Control of Skin Barrier Structure and Function}

Recently, it has been shown that numerous genes encoding skin barrier structural and functional components are under NRF2 transcriptional control, including late cornified envelope 1 (LCE1) family members (LCE1B, LCE1C, LCE1E, LCE1G, LCE1H, LCE1M), keratins (KRT6A, KRT16, KRT17), small proline rich proteins $(S P R R 2 D, S P R R 2 H)$, secretory leukocyte protease inhibitor (SLPI), and the EGF family member epigen (EPGN), some of which contain a validated ARE [39-43]. Moreover, a novel role of NRF2 in skin barrier and desmosome function has been attributed to transcriptional control of MiR-encoding genes (MIR29AB1 and MIR29B2C) in keratinocytes, substantiating a novel NRF2-miR29-DSC2 (desmocollin-2) axis in control of desmosome function and cutaneous homeostasis [44]. In addition, much research has substantiated a role of NRF2 in epidermal redox 
control, stress response regulation, terminal differentiation, and barrier homeostasis, and a crucial role of NRF2 in the control of a cytoprotective glutathione gradient throughout the epidermis has been demonstrated $[13,35,40,41,45]$.

Additional functional implications of NRF2 relevant to skin barrier maintenance, repair, and rejuvenation have recently emerged, including a role in metabolic control and mitochondrial homeostasis, proteasomal function and autophagy, and stem cell renewal and pluripotency [46-48].

Moreover, abundant functional crosstalk exists between NRF2 and other cutaneous stress response pathways including AhR (arylhydrocarbon receptor) and NFkB [49-51]. For example, the co-occurrence of ARE- and xenobiotic response element- (XRE-)sequences in the promoter region of several AhR-controlled genes (including NQO1 (NAD(P)H quinone oxidoreductase 1) and GST (glutathione-S-transferase) indicates mechanistic crosstalk between NRF2 and AhR at the gene expression level [52]. Likewise, direct AhR binding to XREs located in the NRF2 promoter region has been confirmed by immunoprecipitation analysis, enabling AhR agonists to induce NRF2 expression at the mRNA and protein levels. It has also been demonstrated that protease-activated receptor-2 (PAR-2), an important mediator of inflammation and immune responses by serine proteinases, activates NQO1 via NRF2 stabilization in keratinocytes, suggesting that in addition to induction of inflammation, PAR-2 can play a cytoprotective role that depends on NRF2 [53].

\section{NRF2 in Skin Pathology}

A substantial body of experimental evidence indicates that NRF2 dysregulation, either due to insufficient adaptive activation in response to environmental stressors or due to constitutive hyperactivation as a result of genetic alterations that may also involve KEAP1, has detrimental effects compromising skin barrier function and stress responses. Seminal research has documented that constitutive epidermal NRF2 overactivation through permanent genetic deletion of KEAP1-caused hyperkeratosis in murine skin [54]. It has also been demonstrated that forced constitutive NRF2 overactivation causes chloracne-like skin disease characterized by acanthosis, hyperkeratosis, and cyst formation in mice [43]. Likewise, oncogenic NRF2 mutations have been detected in squamous cell carcinomas of the esophagus and skin [55-57]. In contrast to compromised skin structure and function that may originate from both impaired NRF2 activation as well as forced hyperactivation, NRF2 activation in healthy skin is transient and subject to extensive feedback regulation and modulatory crosstalk. Pharmacological modulation of NRF2 in skin aiming at a therapeutic, preventive, or regenerative benefit must therefore be performed without causing prolonged hyperactivation of the pathway as has been discussed before $[56,58]$.

Wound healing. Recent research indicates that a glutathione-NRF2-thioredoxin cross-talk enables keratinocyte survival and wound repair through modulation of inflammation, apoptosis, and oxidative stress [59]. Importantly, substantial research has identified an essential role of NRF2 in diabetic wound healing, amenable to therapeutic intervention using small molecule NRF2 activators such as sulforaphane and cinnamaldehyde $[32,60]$.

Psoriasis. In psoriasis, NRF2 is an important driver of keratinocyte proliferation with up-regulation of Keratin 6, Keratin 16, and Keratin 17 [61]. However, NRF2-directed intervention in psoriasis is efficacious since the anti-psoriatic drug monomethylfumarate increases NRF2 levels and induces aquaporin-3 mRNA and protein expression, important for keratinocyte differentiation [62].

Allergic dermatitis. NRF2 activation has been identified as a key event triggered by common skin sensitizers known be cysteine-directed electrophiles [63-66]. However, pharmacological NRF2 activation using ginger-derived 6-shogaol has shown efficacy in allergic dermatitis-like skin lesions through anti-inflammatory redox modulation [67].

Atopic dermatitis. Redox dysregulation is an emerging causative factor contributing to compromised skin barrier function in atopic dermatitis, and pharmacological intervention targeting NRF2 has shown promise targeting atopic dermatitis-like skin lesions in 2,4-dinitrochlorobenzene (DNCB)-sensitized and challenged mice $[68,69]$. 
Melanocytic dysfunction. It is now understood that NRF2 also plays an essential role in the maintenance of melanocyte responses to environmental stressors. NRF2 has been implicated in cutaneous pigmentation disorders resulting from redox alterations relevant to vitiligo and stress-induced and chronological hair greying [70-73]. Interestingly, recent evidence suggests that NRF2 plays a role in facilitating glutathione-dependent chemoresistance of malignant melanoma cells [74].

Chronological aging and progeria. Increasing evidence indicates a role of NRF2 in the control of chronological cellular aging [75-77]. Recently, an unanticipated mechanistic role of NRF2 dysfunction as a key contributor to premature aging has been proposed in the genetic premature aging disorder Hutchinson-Gilford progeria syndrome (HGPS), attributed to increased chronic oxidative stress [78,79]. In HGPS, a de novo LMNA (lamin A/C) gene mutation encodes for progerin, a dysfunctional nuclear architectural protein variant of lamin A lacking 50 amino acids. Progerin formation is also observed during normal cellular aging, and chronic UVA exposure has been shown to induce progerin in cultured human dermal fibroblasts [80]. Recent experimental evidence suggests that progerin sequesters NRF2 and thereby causes its subnuclear mislocalization, resulting in impaired NRF2 transcriptional activity and consequently increased chronic oxidative stress. Importantly, reactivation of NRF2 activity in HGPS patient cells reverses progerin-associated nuclear aging defects, suggesting that progerin-dependent repression of NRF2-mediated antioxidant responses is a key factor underlying HGPS-type premature aging with potential relevance to chronological aging and UVA-induced photoaging.

NRF2 in skin photodamage. Recent studies strongly suggest a protective role of NRF2-mediated gene expression in the suppression of cutaneous photodamage induced by solar UV radiation (as evidenced by suppression of UV-induced apoptosis and inflammatory signaling), and NRF2 activation has been shown to protect cutaneous keratinocytes and fibroblasts against the cytotoxic effects of UVA and UVB [16,18,19,31,33,81-88]. Importantly, research performed in SKH-1 mice documents that genetic NRF2 activation protects mice against acute photodamage and photocarcinogenesis $[36,89]$. Therefore, pharmacological modulation of NRF2 has now attracted considerable attention as a novel approach to skin photoprotection, cancer photochemoprevention, and suppression of skin photoaging $[13,33,34,86]$. Indeed, protection of primary human keratinocytes from UVB-induced cell death by novel drug-like NRF2 activators has been reported, a photoprotective effect attributed in part to NRF2-dependent elevation of cellular glutathione levels [40,87,90].

Our own studies have demonstrated the photoprotective effects of pharmacological NRF2 activation in cultured human skin cells and reconstructed epidermal skin models $[13,31,33]$. Topical application of NRF2 inducers, e.g., the synthetic NRF2-activator TBE-31, has shown pronounced photoprotective and photochemopreventive activity in murine skin, and suppression of solar UV-induced human skin erythema was achieved by topical application of a standardized broccoli extract delivering the NRF2 inducer sulforaphane [36]. However, little research has explored the concept of cutaneous photoprotection and photochemoprevention achievable by systemic administration of NRF2 inducers $[13,91]$.

\section{Systemic Photoprotection by Dietary NRF2 Activators: Focus on the Apocarotenoid Bixin, an FDA-Approved Food Colorant and Spice Native to Tropical America}

The dietary origin of numerous photochemopreventive factors suggests the possibility of achieving efficient skin delivery through oral systemic administration, an emerging concept referred to as 'nutritional' or 'systemic photoprotection' [9]. Indeed, clinical studies document feasibility of human skin photoprotection by dietary intake of lycopene from processed tomato and flavonoid-rich cocoa $[10,92-94]$. In an attempt to test for the first time the feasibility of NRF2-dependent systemic photoprotection by dietary constituents, we focused our photoprotection studies on the apocarotenoid bixin (Figures 1 and 2), an FDA-approved natural food colorant from the seeds of the achiote tree (Bixa orellana) native to tropical America $[13,95,96]$. A native spice derived from the Americas, annatto 
is an orange-red condiment and food coloring used to impart a yellow or orange color to signature foods of Latin America and the Caribbean.

Consumed by human populations in the Americas since ancient times, this apocarotenoid, derived from lycopene through oxidative cleavage, is now used worldwide as a spice, food colorant, and cosmetic and pharmaceutical ingredient (referred to as 'annatto'; E160b). Due to its unusual (linear/noncyclic) chemical structure, the apocarotenoid bixin displays characteristics different from all other carotenoids. Specifically, bixin is water soluble, does not display provitamin A activity, and is distinguished by an excellent safety record as well as established systemic bioavailability and pharmacokinetic profile upon oral administration as documented extensively in mice and humans [97-99]. Indeed, bixin is now one of the most consumed food colorants in the world distinguished by a long record of dietary and ethno-pharmacological use $[95,96,100]$. Chemical activities of bixin as sacrificial antioxidant, free radical scavenger, and efficient physical quencher of photoexcited states including singlet oxygen (surpassed only by lycopene) are documented [101]. Topical preparations of annatto extract have been in ethno-pharmacological use showing therapeutic efficacy for wound healing, mouth ulcers, and other pathologies associated with impaired epithelial barrier function [100,102]. It is also interesting that translational research documents the efficacy of bixin-loaded polycaprolactone nanofibers as an innovative delivery system accelerating wound healing and reducing scar tissue formation in diabetic mice [103]. Moreover, bixin-based systemic protection against environmental toxicants including methylmercury and carbon tetrachloride has been documented in vivo $[104,105]$.

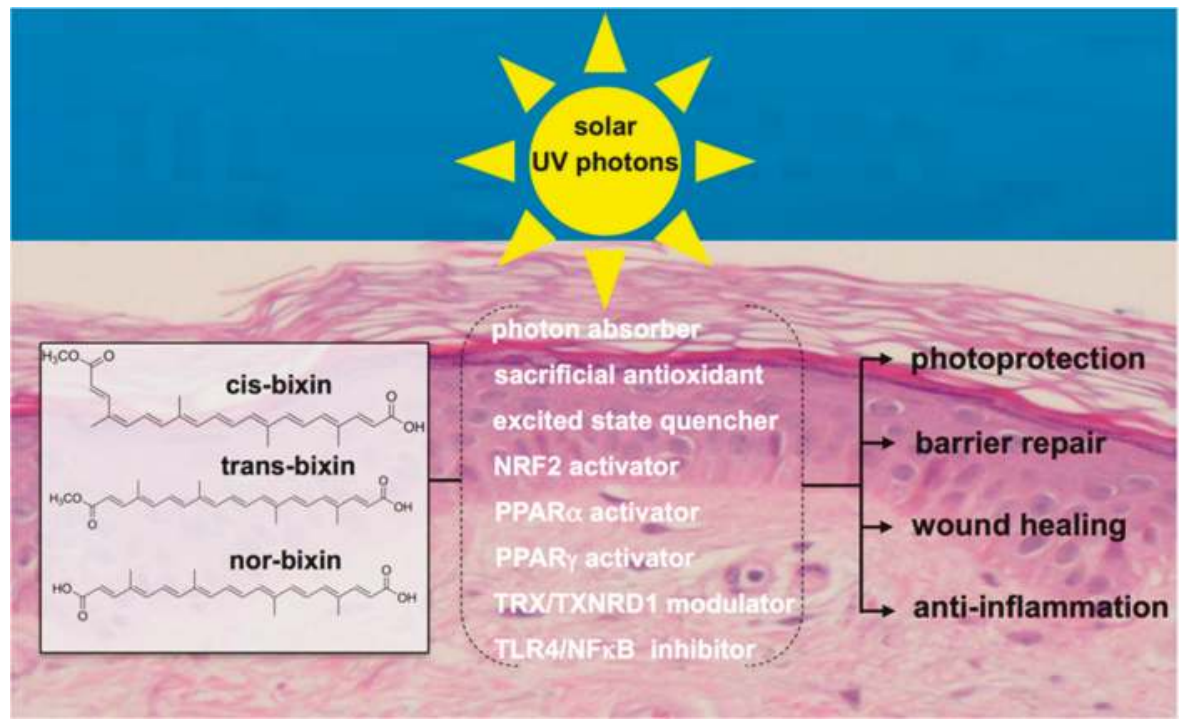

Figure 2. Bixin for improved skin barrier function and photoprotection. Based on pleiotropic activities including direct chemical and NRF2-dependent antioxidant modulation, cis-bixin and its physiologically relevant derivatives trans-bixin and nor-bixin enhance skin barrier structure and function with photoprotective and potentially photochemopreventive efficacy; thioredoxin (TRX), thioredoxin reductase 1 (TXNRD1).

In prior studies, bixin has demonstrated antigenotoxic and antioxidant cytoprotective activities, and systemic availability of oral bixin and its demethylated metabolite norbixin has been documented in rodent studies and healthy human subjects $[97,98,106,107]$. In long term murine feeding experiments, supplementation levels up to $5 \%(w / w$ food) were well tolerated. Importantly, acceptable daily 
intake (ADI) over a lifetime without an appreciable health risk (http:/ / apps.who.int/ food-additivescontaminants-jecfa-database/search.aspx) surpasses that of any other carotenoid approved as a food additive [ADI (bixin): $12 \mathrm{mg} / \mathrm{kg}$ body weight/day] [108].

\section{Bixin for NRF2-Dependent Systemic Skin Photoprotection}

Bixin was identified as the result of a screen for diet-derived small molecule NRF2 activators targeting oxidative stress and redox dysregulation in epithelial cells $[13,109]$. Using activity guided fractionation and bio-analytical tools for the quantitative detection of bixin and other small molecule constituents in annatto extracts, we were able to demonstrate that bixin is the active molecular entity in annatto total organic extracts responsible for NRF2 activation. Recently, we have reported for the first time that (i) bixin is a potent activator of the NRF2-dependent cytoprotective response in cultured human skin keratinocytes; (ii) systemic administration of bixin activates cutaneous NRF2 with potent protective effects against solar UV-induced skin damage in SKH-1 mice; and (iii) bixin-induced suppression of photodamage is observable in $\mathrm{Nrf2} 2^{+/+}$but not in $\mathrm{Nrf2^{-/- }} \mathrm{SKH}-1$ mice confirming the NRF2-dependence of bixin-based antioxidant and anti-inflammatory cutaneous effects [13]. Based on its unique status as a FDA-approved food additive with an established safety profile and potent NRF2-inducing activity, we also have investigated and established efficacy of systemic NRF2 activation using intraperitoneal administration of bixin for lung protection against ventilation-induced oxidative stress [110]. Importantly, dietary carotenoids (including $\beta$-carotene, lycopene, lutein, 3,3'-dihydroxyisorenieratene, zeaxanthin, astaxanthin) and their biosynthetic precursor molecules (such as phytoene) have been under investigation for epithelial chemoprevention and cutaneous photoprotection before [10,92,111-113], and the systemic photoprotective activity of carotenoids, displayed only after dietary uptake and cutaneous accumulation, has largely been attributed to their activity as photon absorbers, sacrificial antioxidants, and excited state/singlet oxygen quenchers [101,113,114].

Interestingly, it has been shown that astaxanthin and its analogs (such as adonixanthin) activate NRF2, preventing light-induced ocular photoreceptor degeneration [115]. Moreover, fucoxanthin, another marine carotenoid from seaweed, has been shown to enhance the level of reduced glutathione via NRF2 in human keratinocytes [116]. Indeed, prior research has examined the specific mechanism of NRF2 activation by carotenoids, and oxidative metabolism leading to the generation of electrophilic unsaturated mono- and dialdehydes (such 10,10'-diapocarotene-10,10'-dial) has been identified as the mechanistic basis underlying upregulated antioxidant responses [117-119]. The specific structure-activity relationship of NRF2 upregulation by carotenoid-derived electrophilic metabolites has been explored before, and it is therefore likely that bixin-dependent NRF2 activation requires similar oxidative transformation to electrophilic intermediates, a subject of ongoing investigation. However, even though the concept of cutaneous photoprotection achieved by systemic administration of specific carotenoids and other phytochemicals has been explored in the past $[10,92,111,112,120-122]$, prior to our own investigations, no research had investigated the NRF2-dependence of carotenoid-based systemic photoprotection [13]. However, the biological effects of prolonged cutaneous NRF2 activation as a consequence of oral/systemic delivery of a pharmacological molecular agent that may also affect NRF2 regulation in non-cutaneous tissue remain to be elucidated.

\section{Other Molecular Targets of Bixin with Relevance to Skin Barrier Function and Protection}

Beyond NRF2-directed activities, bixin has been demonstrated to cause specific modulation of the following molecular targets potentially relevant to skin barrier function and environmental stress responses (Figure 2). 


\subsection{PPAR $\alpha$ and PPAR $\gamma$}

Interestingly, peroxisome proliferator-activated receptors (PPARs) have now been recognized as important determinants of keratinocyte responses to skin injury regulating skin homeostasis, epithelial repair, and morphogenesis $[123,124]$. Specifically, PPAR $\alpha$ is a ligand-activated transcription factor that regulates the expression of genes involved in fatty acid oxidation.

Recently, it has been demonstrated that oral administration of bixin improves obesity-induced abnormalities of carbohydrate and lipid metabolism in mice, an affect attributed to PPAR $\alpha$ activation confirmed by luciferase reporter assays [125]. Specifically, treatment with bixin- and norbixin-induced PPAR $\alpha$ target gene expression upstream of fatty acid oxidation in PPAR $\alpha$-expressing HepG2 hepatocytes. Likewise, in obese KK-Ay mice, chronic nutritional supplementation using bixin suppressed the development of hyperlipidemia and hepatic lipid accumulation with improvement of hyperglycemia, hyperinsulinemia, and hypoadiponectinemia. This effect is consistent with upregulated mRNA expression levels of adiponectin (ADIPOQ), an adipocyte-derived adipokine with multiple beneficial effects such as anti-obesity and anti-insulin resistance roles as well as anti-apoptotic, anti-oxidative, and anti-inflammatory activities in skin [124]. Likewise, experimental evidence suggests that bixin also enhances adipocyte insulin sensitivity downstream of PPAR $\gamma$ activation [126]. It is therefore tempting to speculate that the documented beneficial effects of bixin on cutaneous barrier function and wound healing may be in part attributable to PPAR $\alpha / \gamma$-directed agonism operative in addition to NRF2 activation as discussed above. However, the effects of prolonged pharmacological $\operatorname{PPAR} \alpha$ - or $\gamma$-directed agonism on skin barrier function remain to be explored.

\subsection{Thioredoxin/Thioredoxin Reductase}

One of the key cellular antioxidant systems is regulated by the selenoproteins thioredoxin (TRX) and thioredoxin reductase (TXNRD1), which use NADPH as an electron donor to reduce oxidized substrates. TXNRD1 contains a very reactive selenocysteine in its active site that is prone to electrophilic or oxidative attack, making it another important sensor of the cellular redox state in addition to KEAP1 [127]. Thus, electrophilic compounds that typically activate NRF2 by KEAP1 cysteine modifications will also inhibit TXNRD1 [127]. The TRX/TXNRD1 system is essential for keratinocyte survival, UV protection, and wound healing [59]. Interestingly, one report indicates that at high $(200 \mu \mathrm{M})$ concentrations bixin generates ROS, inhibiting both TRX and TXNRD1 with induction of cell death [128]. This could be due to an exacerbated redox imbalance caused by the inability of TRX/TXNRD1 to reduce their substrates, such as peroxiredoxins (PRX), as well as de-repression of proapoptotic proteins, such as apoptosis signaling kinase 1 (ASK1), apoptosis inducing factor (AIF), and caspase 3. Other important substrates of the TRX/TXNRD1 system are PTEN (phosphatase and tensin homolog), NF- $\mathrm{KB}, \mathrm{AP} 1$ (activator protein 1), and p53 (tumor protein 53), with important implications for regulation of cell survival in response to TRX/TXNRD1 dysruption [129]. Interestingly, it has been proposed that the TRX/TXNRD1 system might reduce the oxidized cysteine residues in KEAP1 to restore its functionality [130]. Dual inactivation of these reactive proteins (KEAP1 and TRX/TXNRD1) could contribute to pronounced NRF2 activation achieved by bixin. However, since TRX and TXNRD1 are NRF2 target genes, reduced proteins might be restored by de novo synthesis and GSH synthesis.

\subsection{TLR4/NFKB}

It has been observed that nutrional bixin attenuates cardiac injury progression through inhibition of fibrosis, inflammation, and redox dysregulation, cytoprotective effects that were attributed to Toll-like receptor 4/nuclear factor kappa B (TLR4/NF- $\mathrm{kB}$ ) antagonism in mice [131]. Likewise, bixin antagonized lipopolysaccharide (LPS)-induced pro-inflammatory cytokine over-expression in cultured cardiac muscle cells. Given the emerging importance of TLR4 signaling in skin inflammation 
and UV-induced photodamage, it is therefore tempting to speculate that nutritional bixin regimens may benefit human skin through TLR4 antagonism operative in addition to NRF2 activation [132,133].

\section{Conclusions}

The promising concept of achieving cutaneous solar protection through dietary intake of NRF2 activators remains largely unexplored, representing an innovative molecular strategy that deserves further exploration. Building on its excellent safety record as an FDA-approved natural food colorant and additive, its systemic availability upon oral administration in humans, and ability to activate NRF2 in skin, dietary consumption of bixin, an ethno-pharmacologically validated phytochemical originating from the Americas, warrants future preclinical and clinical evaluation for improved skin barrier function and photoprotection.

Acknowledgments: Funding through the following NIH grants contributed to this review: R03CA167580, R03CA212719, ES007091, ES006694.

Conflicts of Interest: The authors declare no conflict of interest.

\section{References}

1. Chen, H.; Weng, Q.Y.; Fisher, D.E. UV signaling pathways within the skin. J. Investig. Dermatol. 2014, 134, 2080-2085. [CrossRef] [PubMed]

2. Natarajan, V.T.; Ganju, P.; Ramkumar, A.; Grover, R.; Gokhale, R.S. Multifaceted pathways protect human skin from UV radiation. Nat. Chem. Biol. 2014, 10, 542-551. [CrossRef] [PubMed]

3. Brash, D.E. UV signature mutations. Photochem. Photobiol. 2015, 91, 15-26. [CrossRef] [PubMed]

4. Park, S.L.; Justiniano, R.; Williams, J.D.; Cabello, C.M.; Qiao, S.; Wondrak, G.T. The Tryptophan-Derived Endogenous Aryl Hydrocarbon Receptor Ligand 6-Formylindolo(3,2-b)Carbazole Is a Nanomolar UVA Photosensitizer in Epidermal Keratinocytes. J. Investig. Dermatol. 2015, 135, 1649-1658. [CrossRef] [PubMed]

5. Liebel, F.; Kaur, S.; Ruvolo, E.; Kollias, N.; Southall, M.D. Irradiation of skin with visible light induces reactive oxygen species and matrix-degrading enzymes. J. Investig. Dermatol. 2012, 132, 1901-1907. [CrossRef] [PubMed]

6. Nakashima, Y.; Ohta, S.; Wolf, A.M. Blue light-induced oxidative stress in live skin. Free Radic. Biol. Med. 2017, 108, 300-310. [CrossRef] [PubMed]

7. Schroeder, P.; Calles, C.; Benesova, T.; Macaluso, F.; Krutmann, J. Photoprotection beyond ultraviolet radiation-Effective sun protection has to include protection against infrared A radiation-induced skin damage. Skin Pharmacol. Physiol. 2010, 23, 15-17. [CrossRef] [PubMed]

8. Zastrow, L.; Groth, N.; Klein, F.; Kockott, D.; Lademann, J.; Renneberg, R.; Ferrero, L. The missing link—Light-induced (280-1600 nm) free radical formation in human skin. Skin Pharmacol. Physiol. 2009, 22, 31-44. [PubMed]

9. Wondrak, G.T. Sunscreen-Based Skin Protection Against Solar Insult: Molecular Mechanisms and Opportunities. In Fundamentals of Cancer Prevention; Alberts, D., Hess, L.M., Eds.; Springer: Berlin/Heidelberg, Garmany, 2014; pp. 301-320.

10. Gonzalez, S.; Astner, S.; An, W.; Goukassian, D.; Pathak, M.A. Dietary lutein/zeaxanthin decreases ultraviolet B-induced epidermal hyperproliferation and acute inflammation in hairless mice. J. Investig. Dermatol. 2003, 121, 399-405. [CrossRef] [PubMed]

11. Wondrak, G.T.; Jacobson, M.K.; Jacobson, E.L. Endogenous UVA-photosensitizers: Mediators of skin photodamage and novel targets for skin photoprotection. Photochem. Photobiol. Sci. 2006, 5, $215-237$. [CrossRef] [PubMed]

12. Nichols, J.A.; Katiyar, S.K. Skin photoprotection by natural polyphenols: Anti-inflammatory, antioxidant and DNA repair mechanisms. Arch. Dermatol. Res. 2010, 302, 71-83. [CrossRef] [PubMed]

13. Tao, S.; Park, S.L.; de la Vega, M.R.; Zhang, D.D.; Wondrak, G.T. Systemic administration of the apocarotenoid bixin protects skin against solar UV-induced damage through activation of NRF2. Free Radic. Biol. Med. 2015, 89, 690-700. [CrossRef] [PubMed] 
14. Diffey, B.L.; Norridge, Z. Reported sun exposure, attitudes to sun protection and perceptions of skin cancer risk: A survey of visitors to Cancer Research UK's SunSmart campaign website. Br. J. Dermatol. 2009, 160, 1292-1298. [CrossRef] [PubMed]

15. Suzuki, T.; Motohashi, H.; Yamamoto, M. Toward clinical application of the Keap1-Nrf2 pathway. Trends Pharmacol. Sci. 2013, 34, 340-346. [CrossRef] [PubMed]

16. Saw, C.L.; Huang, M.T.; Liu, Y.; Khor, T.O.; Conney, A.H.; Kong, A.N. Impact of Nrf2 on UVB-induced skin inflammation/photoprotection and photoprotective effect of sulforaphane. Mol. Carcinog. 2011, 50, 479-486. [CrossRef] [PubMed]

17. Ma, Q. Role of nrf2 in oxidative stress and toxicity. Annu. Rev. Pharmacol. Toxicol. 2013, 53, 401-426. [CrossRef] [PubMed]

18. Schafer, M.; Werner, S. Nrf2-A regulator of keratinocyte redox signaling. Free Radic. Biol. Med. 2015, 88, 243-252. [CrossRef] [PubMed]

19. Hirota, A.; Kawachi, Y.; Yamamoto, M.; Koga, T.; Hamada, K.; Otsuka, F. Acceleration of UVB-induced photoageing in nrf2 gene-deficient mice. Exp. Dermatol. 2011, 20, 664-668. [CrossRef] [PubMed]

20. Bosch, R.; Philips, N.; Suarez-Perez, J.A.; Juarranz, A.; Devmurari, A.; Chalensouk-Khaosaat, J.; Gonzalez, S. Mechanisms of Photoaging and Cutaneous Photocarcinogenesis, and Photoprotective Strategies with Phytochemicals. Antioxidants 2015, 4, 248-268. [CrossRef] [PubMed]

21. Kobayashi, A.; Kang, M.I.; Okawa, H.; Ohtsuji, M.; Zenke, Y.; Chiba, T.; Igarashi, K.; Yamamoto, M. Oxidative stress sensor Keap1 functions as an adaptor for Cul3-based E3 ligase to regulate proteasomal degradation of Nrf2. Mol. Cell. Biol. 2004, 24, 7130-7139. [CrossRef] [PubMed]

22. Zhang, D.D.; Lo, S.C.; Cross, J.V.; Templeton, D.J.; Hannink, M. Keap1 is a redox-regulated substrate adaptor protein for a Cul3-dependent ubiquitin ligase complex. Mol. Cell. Biol. 2004, 24, 10941-10953. [CrossRef] [PubMed]

23. Zhang, D.D.; Hannink, M. Distinct cysteine residues in Keap1 are required for Keap1-dependent ubiquitination of $\mathrm{Nrf} 2$ and for stabilization of $\mathrm{Nrf} 2$ by chemopreventive agents and oxidative stress. Mol. Cell. Biol. 2003, 23, 8137-8151. [CrossRef] [PubMed]

24. Baird, L.; Lleres, D.; Swift, S.; Dinkova-Kostova, A.T. Regulatory flexibility in the Nrf2-mediated stress response is conferred by conformational cycling of the Keap1-Nrf2 protein complex. Proc. Natl. Acad. Sci. USA 2013, 110, 15259-15264. [CrossRef] [PubMed]

25. Itoh, K.; Chiba, T.; Takahashi, S.; Ishii, T.; Igarashi, K.; Katoh, Y.; Oyake, T.; Hayashi, N.; Satoh, K.; Hatayama, I.; et al. An Nrf2/small Maf heterodimer mediates the induction of phase II detoxifying enzyme genes through antioxidant response elements. Biochem. Biophys. Res. Commun. 1997, 236, 313-322. [CrossRef] [PubMed]

26. Komatsu, M.; Kurokawa, H.; Waguri, S.; Taguchi, K.; Kobayashi, A.; Ichimura, Y.; Sou, Y.S.; Ueno, I.; Sakamoto, A.; Tong, K.I.; et al. The selective autophagy substrate p62 activates the stress responsive transcription factor Nrf2 through inactivation of Keap1. Nat. Cell. Biol. 2010, 12, 213-223. [CrossRef] [PubMed]

27. Lau, A.; Wang, X.J.; Zhao, F.; Villeneuve, N.F.; Wu, T.; Jiang, T.; Sun, Z.; White, E.; Zhang, D.D. A noncanonical mechanism of Nrf2 activation by autophagy deficiency: Direct interaction between Keap1 and p62. Mol. Cell. Biol. 2010, 30, 3275-3285. [CrossRef] [PubMed]

28. Salazar, M.; Rojo, A.I.; Velasco, D.; de Sagarra, R.M.; Cuadrado, A. Glycogen synthase kinase-3beta inhibits the xenobiotic and antioxidant cell response by direct phosphorylation and nuclear exclusion of the transcription factor Nrf2. J. Biol. Chem. 2006, 281, 14841-14851. [CrossRef] [PubMed]

29. Chowdhry, S.; Zhang, Y.; McMahon, M.; Sutherland, C.; Cuadrado, A.; Hayes, J.D. Nrf2 is controlled by two distinct beta-TrCP recognition motifs in its Neh6 domain, one of which can be modulated by GSK-3 activity. Oncogene 2013, 32, 3765-3781. [CrossRef] [PubMed]

30. Li, H.; Gao, A.; Jiang, N.; Liu, Q.; Liang, B.; Li, R.; Zhang, E.; Li, Z.; Zhu, H. Protective Effect of Curcumin Against Acute Ultraviolet B Irradiation-induced Photo-damage. Photochem. Photobiol. 2016, 92, 808-815. [CrossRef] [PubMed]

31. Wondrak, G.T.; Cabello, C.M.; Villeneuve, N.F.; Zhang, S.; Ley, S.; Li, Y.; Sun, Z.; Zhang, D.D. Cinnamoyl-based Nrf2-activators targeting human skin cell photo-oxidative stress. Free Radic. Biol. Med. 2008, 45, 385-395. [CrossRef] [PubMed] 
32. Long, M.; Rojo de la Vega, M.; Wen, Q.; Bharara, M.; Jiang, T.; Zhang, R.; Zhou, S.; Wong, P.K.; Wondrak, G.T.; Zheng, H.; et al. An Essential Role of NRF2 in Diabetic Wound Healing. Diabetes 2016, 65, 780-793. [CrossRef] [PubMed]

33. Tao, S.; Justiniano, R.; Zhang, D.D.; Wondrak, G.T. The Nrf2-inducers tanshinone I and dihydrotanshinone protect human skin cells and reconstructed human skin against solar simulated UV. Redox Biol. 2013, 1, 532-541. [CrossRef] [PubMed]

34. Chun, K.S.; Kundu, J.; Kundu, J.K.; Surh, Y.J. Targeting Nrf2-Keap1 signaling for chemoprevention of skin carcinogenesis with bioactive phytochemicals. Toxicol. Lett. 2014, 229, 73-84. [CrossRef] [PubMed]

35. Mathew, S.T.; Bergstrom, P.; Hammarsten, O. Repeated Nrf2 stimulation using sulforaphane protects fibroblasts from ionizing radiation. Toxicol. Appl. Pharmacol. 2014, 276, 188-194. [CrossRef] [PubMed]

36. Knatko, E.V.; Ibbotson, S.H.; Zhang, Y.; Higgins, M.; Fahey, J.W.; Talalay, P.; Dawa, R.; Ferguson, J.; Huang, J.T.; Clarke, R.; et al. Nrf2 activation protects against solar-simulated ultraviolet radiation in mice and humans. Cancer Prev. Res. 2015, 8, 475-486. [CrossRef] [PubMed]

37. Reisman, S.A.; Goldsberry, A.R.; Lee, C.Y.; O'Grady, M.L.; Proksch, J.W.; Ward, K.W.; Meyer, C.J. Topical application of RTA 408 lotion activates Nrf2 in human skin and is well-tolerated by healthy human volunteers. BMC Dermatol. 2015, 15, 10. [CrossRef] [PubMed]

38. Nakagami, Y.; Masuda, K. A novel Nrf2 activator from microbial transformation inhibits radiation-induced dermatitis in mice. J. Radiat. Res. 2016, 57, 567-571. [CrossRef] [PubMed]

39. Ishitsuka, Y.; Huebner, A.J.; Rice, R.H.; Koch, P.J.; Speransky, V.V.; Steven, A.C.; Roop, D.R. Lce1 Family Members Are Nrf2-Target Genes that Are Induced to Compensate for the Loss of Loricrin. J. Investig. Dermatol. 2016, 136, 1656-1663. [CrossRef] [PubMed]

40. Schafer, M.; Farwanah, H.; Willrodt, A.H.; Huebner, A.J.; Sandhoff, K.; Roop, D.; Hohl, D.; Bloch, W.; Werner, S. Nrf2 links epidermal barrier function with antioxidant defense. EMBO Mol. Med. 2012, 4, 364-379. [CrossRef] [PubMed]

41. Kumar, V.; Bouameur, J.E.; Bar, J.; Rice, R.H.; Hornig-Do, H.T.; Roop, D.R.; Schwarz, N.; Brodesser, S.; Thiering, S.; Leube, R.E.; et al. A keratin scaffold regulates epidermal barrier formation, mitochondrial lipid composition, and activity. J. Cell Biol. 2015, 211, 1057-1075. [CrossRef] [PubMed]

42. Huebner, A.J.; Dai, D.; Morasso, M.; Schmidt, E.E.; Schafer, M.; Werner, S.; Roop, D.R. Amniotic fluid activates the nrf2/keap1 pathway to repair an epidermal barrier defect in utero. Dev. Cell 2012, 23, 1238-1246. [CrossRef] [PubMed]

43. Schafer, M.; Willrodt, A.H.; Kurinna, S.; Link, A.S.; Farwanah, H.; Geusau, A.; Gruber, F.; Sorg, O.; Huebner, A.J.; Roop, D.R.; et al. Activation of Nrf2 in keratinocytes causes chloracne (MADISH)-like skin disease in mice. EMBO Mol. Med. 2014, 6, 442-457. [CrossRef] [PubMed]

44. Kurinna, S.; Schafer, M.; Ostano, P.; Karouzakis, E.; Chiorino, G.; Bloch, W.; Bachmann, A.; Gay, S.; Garrod, D.; Lefort, K.; et al. A novel Nrf2-miR-29-desmocollin-2 axis regulates desmosome function in keratinocytes. Nat. Commun. 2014, 5, 5099. [CrossRef] [PubMed]

45. Piao, M.S.; Park, J.J.; Choi, J.Y.; Lee, D.H.; Yun, S.J.; Lee, J.B.; Lee, S.C. Nrf2-dependent and Nrf2-independent induction of phase 2 detoxifying and antioxidant enzymes during keratinocyte differentiation. Arch. Dermatol. Res. 2012, 304, 387-395. [CrossRef] [PubMed]

46. Jang, J.; Wang, Y.; Kim, H.S.; Lalli, M.A.; Kosik, K.S. Nrf2, a regulator of the proteasome, controls self-renewal and pluripotency in human embryonic stem cells. Stem Cells 2014, 32, 2616-2625. [CrossRef] [PubMed]

47. Holmstrom, K.M.; Kostov, R.V.; Dinkova-Kostova, A.T. The multifaceted role of Nrf2 in mitochondrial function. Curr. Opin. Toxicol. 2016, 1, 80-91. [CrossRef] [PubMed]

48. Hawkins, K.E.; Joy, S.; Delhove, J.M.; Kotiadis, V.N.; Fernandez, E.; Fitzpatrick, L.M.; Whiteford, J.R.; King, P.J.; Bolanos, J.P.; Duchen, M.R.; et al. NRF2 Orchestrates the Metabolic Shift during Induced Pluripotent Stem Cell Reprogramming. Cell Rep. 2016, 14, 1883-1891. [CrossRef] [PubMed]

49. Haarmann-Stemmann, T.; Abel, J.; Fritsche, E.; Krutmann, J. The AhR-Nrf2 pathway in keratinocytes: On the road to chemoprevention? J. Investig. Dermatol. 2012, 132, 7-9. [CrossRef] [PubMed]

50. Wakabayashi, N.; Slocum, S.L.; Skoko, J.J.; Shin, S.; Kensler, T.W. When NRF2 talks, who's listening? Antioxid. Redox Signal. 2010, 13, 1649-1663. [CrossRef] [PubMed]

51. Takei, K.; Hashimoto-Hachiya, A.; Takahara, M.; Tsuji, G.; Nakahara, T.; Furue, M. Cynaropicrin attenuates UVB-induced oxidative stress via the AhR-Nrf2-Nqo1 pathway. Toxicol. Lett. 2015, 234, 74-80. [CrossRef] [PubMed] 
52. Miao, W.; Hu, L.; Scrivens, P.J.; Batist, G. Transcriptional regulation of NF-E2 p45-related factor (NRF2) expression by the aryl hydrocarbon receptor-xenobiotic response element signaling pathway: Direct cross-talk between phase I and II drug-metabolizing enzymes. J. Biol. Chem. 2005, 280, 20340-20348. [CrossRef] [PubMed]

53. Kim, J.Y.; Kim, D.Y.; Son, H.; Kim, Y.J.; Oh, S.H. Protease-activated receptor-2 activates NQO-1 via Nrf2 stabilization in keratinocytes. J. Dermatol. Sci. 2014, 74, 48-55. [CrossRef] [PubMed]

54. Wakabayashi, N.; Itoh, K.; Wakabayashi, J.; Motohashi, H.; Noda, S.; Takahashi, S.; Imakado, S.; Kotsuji, T.; Otsuka, F.; Roop, D.R.; et al. Keap1-null mutation leads to postnatal lethality due to constitutive Nrf2 activation. Nat. Genet. 2003, 35, 238-245. [CrossRef] [PubMed]

55. Kim, Y.R.; Oh, J.E.; Kim, M.S.; Kang, M.R.; Park, S.W.; Han, J.Y.; Eom, H.S.; Yoo, N.J.; Lee, S.H. Oncogenic NRF2 mutations in squamous cell carcinomas of oesophagus and skin. J. Pathol. 2010, 220, 446-451. [CrossRef] [PubMed]

56. Lau, A.; Villeneuve, N.F.; Sun, Z.; Wong, P.K.; Zhang, D.D. Dual roles of Nrf2 in cancer. Pharmacol. Res. 2008, 58, 262-270. [CrossRef] [PubMed]

57. Wang, X.J.; Sun, Z.; Villeneuve, N.F.; Zhang, S.; Zhao, F.; Li, Y.; Chen, W.; Yi, X.; Zheng, W.; Wondrak, G.T.; et al. Nrf2 enhances resistance of cancer cells to chemotherapeutic drugs, the dark side of Nrf2. Carcinogenesis 2008, 29, 1235-1243. [CrossRef] [PubMed]

58. Harder, B.; Jiang, T.; Wu, T.; Tao, S.; Rojo de la Vega, M.; Tian, W.; Chapman, E.; Zhang, D.D. Molecular mechanisms of Nrf2 regulation and how these influence chemical modulation for disease intervention. Biochem. Soc. Trans. 2015, 43, 680-686. [CrossRef] [PubMed]

59. Telorack, M.; Meyer, M.; Ingold, I.; Conrad, M.; Bloch, W.; Werner, S. A Glutathione-Nrf2-Thioredoxin Cross-Talk Ensures Keratinocyte Survival and Efficient Wound Repair. PLoS Genet. 2016, 12, e1005800. [CrossRef] [PubMed]

60. Zheng, H.; Whitman, S.A.; Wu, W.; Wondrak, G.T.; Wong, P.K.; Fang, D.; Zhang, D.D. Therapeutic potential of Nrf2 activators in streptozotocin-induced diabetic nephropathy. Diabetes 2011, 60, 3055-3066. [CrossRef] [PubMed]

61. Yang, L.; Fan, X.; Cui, T.; Dang, E.; Wang, G. Nrf2 Promotes Keratinocyte Proliferation in Psoriasis through Up-Regulation of Keratin 6, Keratin 16, and Keratin 17. J. Investig. Dermatol. 2017, 137, 2168-2176. [CrossRef] [PubMed]

62. Helwa, I.; Choudhary, V.; Chen, X.; Kaddour-Djebbar, I.; Bollag, W.B. Anti-Psoriatic Drug Monomethylfumarate Increases Nuclear Factor Erythroid 2-Related Factor 2 Levels and Induces Aquaporin-3 mRNA and Protein Expression. J. Pharmacol. Exp. Ther. 2017, 362, 243-253. [CrossRef] [PubMed]

63. Natsch, A. The Nrf2-Keap1-ARE toxicity pathway as a cellular sensor for skin sensitizers-Functional relevance and a hypothesis on innate reactions to skin sensitizers. Toxicol. Sci. 2010, 113, 284-292. [CrossRef] [PubMed]

64. Delaine, T.; Niklasson, I.B.; Emter, R.; Luthman, K.; Karlberg, A.T.; Natsch, A. Structure-activity relationship between the in vivo skin sensitizing potency of analogues of phenyl glycidyl ether and the induction of Nrf2-dependent luciferase activity in the KeratinoSens in vitro assay. Chem. Res. Toxicol. 2011, 24, 1312-1318. [CrossRef] [PubMed]

65. Natsch, A.; Emter, R. Nrf2 activation as a key event triggered by skin sensitisers: The development of the stable KeratinoSens reporter gene assay. Altern. Lab. Anim. 2016, 44, 443-451. [PubMed]

66. El Ali, Z.; Delomenie, C.; Botton, J.; Pallardy, M.; Kerdine-Romer, S. Dendritic cells' death induced by contact sensitizers is controlled by Nrf2 and depends on glutathione levels. Toxicol. Appl. Pharmacol. 2017, 322, 41-50. [CrossRef] [PubMed]

67. Park, G.; Oh, D.S.; Lee, M.G.; Lee, C.E.; Kim, Y.U. 6-Shogaol, an active compound of ginger, alleviates allergic dermatitis-like skin lesions via cytokine inhibition by activating the Nrf2 pathway. Toxicol. Appl. Pharmacol. 2016, 310, 51-59. [CrossRef] [PubMed]

68. Akram, M.; Shin, I.; Kim, K.A.; Noh, D.; Baek, S.H.; Chang, S.Y.; Kim, H.; Bae, O.N. A newly synthesized macakurzin C-derivative attenuates acute and chronic skin inflammation: The Nrf2/heme oxygenase signaling as a potential target. Toxicol. Appl. Pharmacol. 2016, 307, 62-71. [CrossRef] [PubMed]

69. Choi, J.H.; Jin, S.W.; Han, E.H.; Park, B.H.; Kim, H.G.; Khanal, T.; Hwang, Y.P.; Do, M.T.; Lee, H.S.; Chung, Y.C.; et al. Platycodon grandiflorum root-derived saponins attenuate atopic dermatitis-like skin 
lesions via suppression of NF-kappaB and STAT1 and activation of Nrf2/ARE-mediated heme oxygenase-1. Phytomedicine 2014, 21, 1053-1061. [CrossRef] [PubMed]

70. Marrot, L.; Jones, C.; Perez, P.; Meunier, J.R. The significance of Nrf2 pathway in (photo)-oxidative stress response in melanocytes and keratinocytes of the human epidermis. Pigment Cell Melanoma Res. 2008, 21, 79-88. [CrossRef] [PubMed]

71. Denat, L.; Kadekaro, A.L.; Marrot, L.; Leachman, S.A.; Abdel-Malek, Z.A. Melanocytes as instigators and victims of oxidative stress. J. Investig. Dermatol. 2014, 134, 1512-1518. [CrossRef] [PubMed]

72. Jadkauskaite, L.; Coulombe, P.A.; Schafer, M.; Dinkova-Kostova, A.T.; Paus, R.; Haslam, I.S. Oxidative stress management in the hair follicle: Could targeting NRF2 counter age-related hair disorders and beyond? Bioessays 2017, 39. [CrossRef] [PubMed]

73. Jian, Z.; Li, K.; Song, P.; Zhu, G.; Zhu, L.; Cui, T.; Liu, B.; Tang, L.; Wang, X.; Wang, G.; Gao, T.; Li, C. Impaired activation of the Nrf2-ARE signaling pathway undermines $\mathrm{H}_{2} \mathrm{O}_{2}$-induced oxidative stress response: A possible mechanism for melanocyte degeneration in vitiligo. J. Investig. Dermatol. 2014, 134, 2221-2230. [CrossRef] [PubMed]

74. Rocha, C.R.; Kajitani, G.S.; Quinet, A.; Fortunato, R.S.; Menck, C.F. NRF2 and glutathione are key resistance mediators to temozolomide in glioma and melanoma cells. Oncotarget 2016, 7, 48081-48092. [CrossRef] [PubMed]

75. Kubo, E.; Chhunchha, B.; Singh, P.; Sasaki, H.; Singh, D.P. Sulforaphane reactivates cellular antioxidant defense by inducing Nrf2/ARE/Prdx6 activity during aging and oxidative stress. Sci. Rep. 2017, 7, 14130. [CrossRef] [PubMed]

76. Bruns, D.R.; Drake, J.C.; Biela, L.M.; Peelor, F.F., 3rd; Miller, B.F.; Hamilton, K.L. Nrf2 Signaling and the Slowed Aging Phenotype: Evidence from Long-Lived Models. Oxid. Med. Cell. Longev. 2015, 2015, 732596. [CrossRef] [PubMed]

77. Zhang, H.; Davies, K.J.A.; Forman, H.J. Oxidative stress response and Nrf2 signaling in aging. Free Radic. Biol. Med. 2015, 88, 314-336. [CrossRef] [PubMed]

78. Kubben, N.; Zhang, W.; Wang, L.; Voss, T.C.; Yang, J.; Qu, J.; Liu, G.H.; Misteli, T. Repression of the Antioxidant NRF2 Pathway in Premature Aging. Cell 2016, 165, 1361-1374. [CrossRef] [PubMed]

79. Gorbunova, V.; Rezazadeh, S.; Seluanov, A. Dangerous Entrapment for NRF2. Cell 2016, 165, 1312-1313. [CrossRef] [PubMed]

80. Takeuchi, H.; Runger, T.M. Longwave UV light induces the aging-associated progerin. J. Investig. Dermatol. 2013, 133, 1857-1862. [CrossRef] [PubMed]

81. Hirota, A.; Kawachi, Y.; Itoh, K.; Nakamura, Y.; Xu, X.; Banno, T.; Takahashi, T.; Yamamoto, M.; Otsuka, F. Ultraviolet A irradiation induces NF-E2-related factor 2 activation in dermal fibroblasts: Protective role in UVA-induced apoptosis. J. Investig. Dermatol. 2005, 124, 825-832. [CrossRef] [PubMed]

82. Dinkova-Kostova, A.T.; Jenkins, S.N.; Fahey, J.W.; Ye, L.; Wehage, S.L.; Liby, K.T.; Stephenson, K.K.; Wade, K.L.; Talalay, P. Protection against UV-light-induced skin carcinogenesis in SKH-1 high-risk mice by sulforaphane-containing broccoli sprout extracts. Cancer Lett. 2006, 240, 243-252. [CrossRef] [PubMed]

83. Benedict, A.L.; Knatko, E.V.; Dinkova-Kostova, A.T. The indirect antioxidant sulforaphane protects against thiopurine-mediated photooxidative stress. Carcinogenesis 2012, 33, 2457-2466. [CrossRef] [PubMed]

84. Gruber, F.; Mayer, H.; Lengauer, B.; Mlitz, V.; Sanders, J.M.; Kadl, A.; Bilban, M.; de Martin, R.; Wagner, O.; Kensler, T.W.; et al. NF-E2-related factor 2 regulates the stress response to UVA-1-oxidized phospholipids in skin cells. FASEB J. 2010, 24, 39-48. [CrossRef] [PubMed]

85. Tian, F.F.; Zhang, F.F.; Lai, X.D.; Wang, L.J.; Yang, L.; Wang, X.; Singh, G.; Zhong, J.L. Nrf2-mediated protection against UVA radiation in human skin keratinocytes. Biosci. Trends 2011, 5, 23-29. [CrossRef] [PubMed]

86. Kalra, S.; Knatko, E.V.; Zhang, Y.; Honda, T.; Yamamoto, M.; Dinkova-Kostova, A.T. Highly potent activation of Nrf2 by topical tricyclic bis(cyano enone): Implications for protection against UV radiation during thiopurine therapy. Cancer Prev. Res. 2012, 5, 973-981. [CrossRef] [PubMed]

87. Schafer, M.; Dutsch, S.; auf dem Keller, U.; Navid, F.; Schwarz, A.; Johnson, D.A.; Johnson, J.A.; Werner, S. Nrf2 establishes a glutathione-mediated gradient of UVB cytoprotection in the epidermis. Genes Dev. 2010, 24, 1045-1058. [CrossRef] [PubMed] 
88. Rolfs, F.; Huber, M.; Kuehne, A.; Kramer, S.; Haertel, E.; Muzumdar, S.; Wagner, J.; Tanner, Y.; Bohm, F.; Smola, S.; et al. Nrf2 Activation Promotes Keratinocyte Survival during Early Skin Carcinogenesis via Metabolic Alterations. Cancer Res. 2015, 75, 4817-4829. [CrossRef] [PubMed]

89. Knatko, E.V.; Higgins, M.; Fahey, J.W.; Dinkova-Kostova, A.T. Loss of Nrf2 abrogates the protective effect of Keap1 downregulation in a preclinical model of cutaneous squamous cell carcinoma. Sci. Rep. 2016, 6, 25804. [CrossRef] [PubMed]

90. Lieder, F.; Reisen, F.; Geppert, T.; Sollberger, G.; Beer, H.D.; auf dem Keller, U.; Schafer, M.; Detmar, M.; Schneider, G.; Werner, S. Identification of UV-protective activators of nuclear factor erythroid-derived 2-related factor 2 (Nrf2) by combining a chemical library screen with computer-based virtual screening. J. Biol. Chem. 2012, 287, 33001-33013. [CrossRef] [PubMed]

91. Dinkova-Kostova, A.T.; Fahey, J.W.; Benedict, A.L.; Jenkins, S.N.; Ye, L.; Wehage, S.L.; Talalay, P. Dietary glucoraphanin-rich broccoli sprout extracts protect against UV radiation-induced skin carcinogenesis in SKH-1 hairless mice. Photochem. Photobiol. Sci. 2010, 9, 597-600. [CrossRef] [PubMed]

92. Sies, H.; Stahl, W. Nutritional Protection Against Skin Damage From Sunlight. Annu. Rev. Nutr. 2004, 24, 173-200. [CrossRef] [PubMed]

93. Heinrich, U.; Neukam, K.; Tronnier, H.; Sies, H.; Stahl, W. Long-term ingestion of high flavanol cocoa provides photoprotection against UV-induced erythema and improves skin condition in women. J. Nutr. 2006, 136, 1565-1569. [PubMed]

94. Stahl, W.; Heinrich, U.; Wiseman, S.; Eichler, O.; Sies, H.; Tronnier, H. Dietary tomato paste protects against ultraviolet light-induced erythema in humans. J. Nutr. 2001, 131, 1449-1451. [PubMed]

95. Ulbricht, C.; Windsor, R.C.; Brigham, A.; Bryan, J.K.; Conquer, J.; Costa, D.; Giese, N.; Guilford, J.; Higdon, E.R.; Holmes, K.; et al. An evidence-based systematic review of annatto (Bixa orellana L.) by the Natural Standard Research Collaboration. J. Diet. Suppl. 2012, 9, 57-77. [CrossRef] [PubMed]

96. Stohs, S.J. Safety and efficacy of Bixa orellana (achiote, annatto) leaf extracts. Phytother. Res. 2014, 28, 956-960. [CrossRef] [PubMed]

97. Levy, L.W.; Regalado, E.; Navarrete, S.; Watkins, R.H. Bixin and norbixin in human plasma: Determination and study of the absorption of a single dose of Annatto food color. Analyst 1997, 122, 977-980. [CrossRef] [PubMed]

98. Junior, A.C.; Asad, L.M.; Oliveira, E.B.; Kovary, K.; Asad, N.R.; Felzenszwalb, I. Antigenotoxic and antimutagenic potential of an annatto pigment (norbixin) against oxidative stress. Genet. Mol. Res. 2005, 4, 94-99. [PubMed]

99. World Health Organization. Evaluation of certain food additives and contaminants. World Health Organ. Tech. Rep. Ser. 2013, 983, 1-75.

100. Vilar Dde, A.; Vilar, M.S.; de Lima e Moura, T.F.; Raffin, F.N.; de Oliveira, M.R.; Franco, C.F.; de Athayde-Filho, P.F.; Diniz Mde, F.; Barbosa-Filho, J.M. Traditional uses, chemical constituents, and biological activities of Bixa orellana L.: A review. Sci. World J. 2014, 2014, 857292.

101. Di Mascio, P.; Kaiser, S.; Sies, H. Lycopene as the most efficient biological carotenoid singlet oxygen quencher. Arch. Biochem. Biophys. 1989, 274, 532-538. [CrossRef]

102. Piva, R.M.; Johann, A.C.; Costa, C.K.; Miguel, O.G.; Rosa, E.R.; de Azevedo-Alanis, L.R.; Trevilatto, P.C.; Ignacio, S.A.; Bettega, P.V.; Gregio, A.M. Bixin action in the healing process of rats mouth wounds. Curr. Pharm. Biotechnol. 2013, 14, 785-791. [CrossRef] [PubMed]

103. Pinzon-Garcia, A.D.; Cassini-Vieira, P.; Ribeiro, C.C.; de Matos Jensen, C.E.; Barcelos, L.S.; Cortes, M.E.; Sinisterra, R.D. Efficient cutaneous wound healing using bixin-loaded PCL nanofibers in diabetic mice. J. Biomed. Mater. Res. B Appl. Biomater. 2017, 105, 1938-1949. [CrossRef] [PubMed]

104. Barcelos, G.R.; Grotto, D.; Serpeloni, J.M.; Aissa, A.F.; Antunes, L.M.; Knasmuller, S.; Barbosa, F., Jr. Bixin and norbixin protect against DNA-damage and alterations of redox status induced by methylmercury exposure in vivo. Environ. Mol. Mutagen. 2012, 53, 535-541. [CrossRef] [PubMed]

105. Moreira, P.R.; Maioli, M.A.; Medeiros, H.C.; Guelfi, M.; Pereira, F.T.; Mingatto, F.E. Protective effect of bixin on carbon tetrachloride-induced hepatotoxicity in rats. Biol. Res. 2014, 47, 49. [CrossRef] [PubMed]

106. Somacal, S.; Figueiredo, C.G.; Quatrin, A.; Ruviaro, A.R.; Conte, L.; Augusti, P.R.; Roehrs, M.; Denardin, I.T.; Kasten, J.; da Veiga, M.L.; et al. The antiatherogenic effect of bixin in hypercholesterolemic rabbits is associated to the improvement of lipid profile and to its antioxidant and anti-inflammatory effects. Mol. Cell. Biochem. 2015, 403, 243-253. [CrossRef] [PubMed] 
107. Roehrs, M.; Figueiredo, C.G.; Zanchi, M.M.; Bochi, G.V.; Moresco, R.N.; Quatrin, A.; Somacal, S.; Conte, L.; Emanuelli, T. Bixin and norbixin have opposite effects on glycemia, lipidemia, and oxidative stress in streptozotocin-induced diabetic rats. Int. J. Endocrinol. 2014, 2014, 839095. [CrossRef] [PubMed]

108. WHO (World Health Organization). Evaluation of Certain Food Additives and Contaminants. Thirty-fifth report of the Joint FAO/WHO Expert Committee on Food Additives. Tech. Rep. Ser. 1990, 789, 1-48.

109. Long, M.; Tao, S.; Rojo de la Vega, M.; Jiang, T.; Wen, Q.; Park, S.L.; Zhang, D.D.; Wondrak, G.T. Nrf2-dependent suppression of azoxymethane/dextran sulfate sodium-induced colon carcinogenesis by the cinnamon-derived dietary factor cinnamaldehyde. Cancer Prev. Res. 2015, 8, 444-454. [CrossRef] [PubMed]

110. Tao, S.; Rojo de la Vega, M.; Quijada, H.; Wondrak, G.T.; Wang, T.; Garcia, J.G.; Zhang, D.D. Bixin protects mice against ventilation-induced lung injury in an NRF2-dependent manner. Sci. Rep. 2016, 6, 18760. [CrossRef] [PubMed]

111. Astner, S.; Wu, A.; Chen, J.; Philips, N.; Rius-Diaz, F.; Parrado, C.; Mihm, M.C.; Goukassian, D.A.; Pathak, M.A.; Gonzalez, S. Dietary lutein/zeaxanthin partially reduces photoaging and photocarcinogenesis in chronically UVB-irradiated Skh-1 hairless mice. Skin Pharmacol. Physiol. 2007, 20, 283-291. [CrossRef] [PubMed]

112. Stahl, W.; Sies, H. beta-Carotene and other carotenoids in protection from sunlight. Am. J. Clin. Nutr. 2012, 96, 1179S-1184S. [CrossRef] [PubMed]

113. Fernandez-Garcia, E. Skin protection against UV light by dietary antioxidants. Food Funct. 2014, 5, 1994-2003. [CrossRef] [PubMed]

114. Di Mascio, P.; Devasagayam, T.P.; Kaiser, S.; Sies, H. Carotenoids, tocopherols and thiols as biological singlet molecular oxygen quenchers. Biochem. Soc. Trans. 1990, 18, 1054-1056. [CrossRef] [PubMed]

115. Inoue, Y.; Shimazawa, M.; Nagano, R.; Kuse, Y.; Takahashi, K.; Tsuruma, K.; Hayashi, M.; Ishibashi, T.; Maoka, T.; Hara, H. Astaxanthin analogs, adonixanthin and lycopene, activate Nrf2 to prevent light-induced photoreceptor degeneration. J. Pharmacol. Sci. 2017, 134, 147-157. [CrossRef] [PubMed]

116. Zheng, J.; Piao, M.J.; Kim, K.C.; Yao, C.W.; Cha, J.W.; Hyun, J.W. Fucoxanthin enhances the level of reduced glutathione via the Nrf2-mediated pathway in human keratinocytes. Mar. Drugs 2014, 12, 4214-4230. [CrossRef] [PubMed]

117. Ben-Dor, A.; Steiner, M.; Gheber, L.; Danilenko, M.; Dubi, N.; Linnewiel, K.; Zick, A.; Sharoni, Y.; Levy, J. Carotenoids activate the antioxidant response element transcription system. Mol. Cancer Ther. 2005, 4, 177-186. [PubMed]

118. Linnewiel, K.; Ernst, H.; Caris-Veyrat, C.; Ben-Dor, A.; Kampf, A.; Salman, H.; Danilenko, M.; Levy, J.; Sharoni, Y. Structure activity relationship of carotenoid derivatives in activation of the electrophile/antioxidant response element transcription system. Free Radic. Biol. Med. 2009, 47, 659-667. [CrossRef] [PubMed]

119. Linnewiel-Hermoni, K.; Khanin, M.; Danilenko, M.; Zango, G.; Amosi, Y.; Levy, J.; Sharoni, Y. The anti-cancer effects of carotenoids and other phytonutrients resides in their combined activity. Arch. Biochem. Biophys. 2015, 572, 28-35. [CrossRef] [PubMed]

120. Vayalil, P.K.; Mittal, A.; Hara, Y.; Elmets, C.A.; Katiyar, S.K. Green tea polyphenols prevent ultraviolet light-induced oxidative damage and matrix metalloproteinases expression in mouse skin. J. Investig. Dermatol. 2004, 122, 1480-1487. [CrossRef] [PubMed]

121. Gonzalez, S.; Gilaberte, Y.; Philips, N. Mechanistic insights in the use of a Polypodium leucotomos extract as an oral and topical photoprotective agent. Photochem. Photobiol. Sci. 2010, 9, 559-563. [CrossRef] [PubMed]

122. Chen, A.C.; Damian, D.L.; Halliday, G.M. Oral and systemic photoprotection. Photodermatol. Photoimmunol. Photomed. 2014, 30, 102-111. [CrossRef] [PubMed]

123. Icre, G.; Wahli, W.; Michalik, L. Functions of the peroxisome proliferator-activated receptor (PPAR) alpha and beta in skin homeostasis, epithelial repair, and morphogenesis. J. Investig. Dermatol. Symp. Proc. 2006, 11, 30-35. [CrossRef] [PubMed]

124. Kim, E.J.; Lee, D.H.; Kim, Y.K.; Eun, H.C.; Chung, J.H. Adiponectin Deficiency Contributes to Sensitivity in Human Skin. J. Investig. Dermatol. 2015, 135, 2331-2334. [CrossRef] [PubMed]

125. Goto, T.; Takahashi, N.; Kato, S.; Kim, Y.I.; Kusudo, T.; Taimatsu, A.; Egawa, K.; Kang, M.S.; Hiramatsu, T.; Sakamoto, T.; et al. Bixin activates PPARalpha and improves obesity-induced abnormalities of carbohydrate and lipid metabolism in mice. J. Agric. Food Chem. 2012, 60, 11952-11958. [CrossRef] [PubMed] 
126. Takahashi, N.; Goto, T.; Taimatsu, A.; Egawa, K.; Katoh, S.; Kusudo, T.; Sakamoto, T.; Ohyane, C.; Lee, J.Y.; Kim, Y.I.; et al. Bixin regulates mRNA expression involved in adipogenesis and enhances insulin sensitivity in 3T3-L1 adipocytes through PPARgamma activation. Biochem. Biophys. Res. Commun. 2009, 390, 1372-1376. [CrossRef] [PubMed]

127. Cebula, M.; Schmidt, E.E.; Arner, E.S. TrxR1 as a potent regulator of the Nrf2-Keap1 response system. Antioxid. Redox Signal. 2015, 23, 823-853. [CrossRef] [PubMed]

128. Tibodeau, J.D.; Isham, C.R.; Bible, K.C. Annatto constituent cis-bixin has selective antimyeloma effects mediated by oxidative stress and associated with inhibition of thioredoxin and thioredoxin reductase. Antioxid. Redox Signal. 2010, 13, 987-997. [CrossRef] [PubMed]

129. Zhang, J.; Li, X.; Han, X.; Liu, R.; Fang, J. Targeting the Thioredoxin System for Cancer Therapy. Trends Pharmacol. Sci. 2017, 38, 794-808. [CrossRef] [PubMed]

130. Dinkova-Kostova, A.T.; Kostov, R.V.; Canning, P. Keap1, the cysteine-based mammalian intracellular sensor for electrophiles and oxidants. Arch. Biochem. Biophys. 2017, 617, 84-93. [CrossRef] [PubMed]

131. Xu, Z.; Kong, X.Q. Bixin ameliorates high fat diet-induced cardiac injury in mice through inflammation and oxidative stress suppression. Biomed. Pharmacother. 2017, 89, 991-1004. [CrossRef] [PubMed]

132. Dickinson, S.E.; Wondrak, G.T. TLR4-directed Molecular Strategies Targeting Skin Photodamage and Carcinogenesis. Curr. Med. Chem. 2017. [CrossRef] [PubMed]

133. Janda, J.; Burkett, N.B.; Blohm-Mangone, K.; Huang, V.; Curiel-Lewandrowski, C.; Alberts, D.S.; Petricoin, E.F., 3rd; Calvert, V.S.; Einspahr, J.; Dong, Z.; et al. Resatorvid-based Pharmacological Antagonism of Cutaneous TLR4 Blocks UV-induced NF-kappaB and AP-1 Signaling in Keratinocytes and Mouse Skin. Photochem. Photobiol. 2016, 92, 816-825. [CrossRef] [PubMed]

(C) 2017 by the authors. Licensee MDPI, Basel, Switzerland. This article is an open access article distributed under the terms and conditions of the Creative Commons Attribution (CC BY) license (http:/ / creativecommons.org/licenses/by/4.0/). 


\title{
Article \\ Effect of Orally Administered Collagen Peptides from Bovine Bone on Skin Aging in Chronologically Aged Mice
}

\author{
Hongdong Song ${ }^{1}$, Siqi Zhang ${ }^{1}$, Ling Zhang ${ }^{1}$ and Bo Li ${ }^{1,2, *}$ \\ 1 Beijing Advanced Innovation Center for Food Nutrition and Human Health, College of Food Science and \\ Nutritional Engineering, China Agricultural University, Beijing 100083, China; songhd@cau.edu.cn (H.S.); \\ zsq199312@163.com (S.Z.); zhanglingys@outlook.com (L.Z.) \\ 2 Beijing Higher Institution Engineering Research Center of Animal Product, Beijing 100083, China \\ * Correspondence: libo@cau.edu.cn; Tel./Fax: +86-10-6273-7669
}

Received: 19 September 2017; Accepted: 31 October 2017; Published: 3 November 2017

\begin{abstract}
Collagen peptides (CPs) have demonstrated to exert beneficial effects on skin photoaging. However, little has been done to evaluate their effects on chronologically aged skin. Here, the effects of CPs from bovine bone on skin aging were investigated in chronologically aged mice. 13-month-old female Kunming mice were administered with CPs from bovine bone (200, 400 and $800 \mathrm{mg} / \mathrm{kg}$ body weight/day) or proline ( $400 \mathrm{mg} / \mathrm{kg}$ body weight/day) for 8 weeks. Mice body weight, spleen index (SI) and thymus index (TI), degree of skin laxity (DSL), skin components, skin histology and antioxidant indicators were analyzed. Ingestion of CPs or proline had no effect on mice skin moisture and hyaluronic acid content, but it significantly improved the skin laxity, repaired collagen fibers, increased collagen content and normalized the ratio of type I to type III collagen in chronologically aged skin. CPs prepared by Alcalase performed better than CPs prepared by collagenase. Furthermore, CPs intake also significantly improved the antioxidative enzyme activities in skin. These results indicate that oral administration of $\mathrm{CPs}$ from bovine bone or proline can improve the laxity of chronologically aged skin by changing skin collagen quantitatively and qualitatively, and highlight their potential application as functional foods to combat skin aging in chronologically aged process.
\end{abstract}

Keywords: collagen peptides; bovine bone; proline; skin aging; chronologically aged mice; antioxidative enzymes

\section{Introduction}

The impact of aging on the appearance and function of skin has received increasing attention in recent decades. It is widely accepted that skin aging is distinguished into chronological skin aging and skin photoaging [1]. Skin photoaging is caused by solar radiation and it is common in sunlight-exposed skin, especially in the face [2]. Therefore, skin photoaging could be prevented or decreased by photo-protection. The common clinical signs of photoaged skin include deep and coarse wrinkles, dryness, sallowness and laxity [2,3]. In contrast, chronological skin aging is caused by passage of time and it takes place all the time in whole-body skin, including facial skin. Chronologically aged skin is characterized by fine wrinkling and laxity [3]. Chronological skin aging accounts for a great part of skin aging and it is more common than skin photoaging in dark skinned individuals and females [2]. A youthful appearance is considered to play an important role in keeping self-esteem and social relations [4]. Therefore, there is increasing demand for anti-aging interventions to delay or even reverse signs of skin aging.

The use of diet supplements to improve the appearance and function of aged skin has received growing attention. Many dietary components, such as polyphenols [5], vitamins [6], fatty acids [7], 
trace minerals [8] and proteins [9], have reported to exert beneficial effects on aged skin and have been used as nutraceuticals or functional foods in many counties and regions. Recently, researchers have paid much attention to protein hydrolysates as potential dietary supplements. Collagen is the main structural protein of the different connective tissues, such as skin, bone, cartilage and tendons, and has been widely used in the medicine and food industries. Collagen peptides (CPs) are the enzymolysis product of collagen or gelatin and they are used as important active components because of their various bioactivities, high bioavailability and good biocompatibility [10-12]. Several studies have demonstrated the beneficial effects of CPs ingestion on skin photoaging. Oral administration of CPs from fish skin had obvious protective effects on photoaging skin, including improving moisture retention ability, repairing the endogenous collagen and elastin protein fibers [13-15]. In addition, clinical trials have also demonstrated that the beneficial effects of CPs intake on facial skin, including improving facial skin elasticity, reduce skin dryness and wrinkles, and increase the collagen content of the skin dermis $[16,17]$. However, little work was performed to evaluate the effects of CPs intake on chronologically aged skin.

Bovine bone is the main by-products in the bovine processing industry and has been widely used as raw material to obtain high-quality gelatin [18]. Although there are some concerns with mad cow disease in Europe and the United States, bovine bone is still one of the most abundant sources of gelatin and accounts for $23.1 \%$ of the gelatin production [19]. Therefore, bovine bone is an abundant and high-quality raw material used to prepare CPs. The biological effect of CPs from bovine bone is mainly concentrated on its beneficial effect on bone metabolism, including inhibition of bone loss and improvement of osteoarthritis [20,21]. However, there is limited knowledge about the effect of CPs from bovine bone on skin aging. Therefore, preparing CPs from bovine bone and further evaluating its effect on skin aging is a good way to utilize the by-products for an economical and environmental advantage.

The functional activities of protein-derived hydrolysates or peptides are greatly impacted by their molecular structure and weight, which are highly affected by their processing conditions and especially enzyme specificity $[15,22]$. Alcalase is a common protease and widely used to prepare protein hydrolysate or peptides. It is a typical endoprotease and preferentially cleaves sites containing hydrophobic residues, such as Ala, Leu, Val and Phe. Bacterial collagenase is a protease that hydrolysates collagen. It has a preference for X-Gly ( $\mathrm{X}$ is usually a neutral amino acid) bond of the -Gly-Pro-X-Gly-Pro-X-repeating sequence in the collagen molecule $[23,24]$. Collagenase has a great promise in collagen processing industry. Considering enzyme specificity, the molecular structure or sequences of peptides produced by these two enzymes may be different, which may greatly impact their effects on chronologically aged skin.

The objective of the present study is to investigate the effects of CPs from bovine bone on skin aging based on the chronologically aged model. Bovine bone was employed as a raw material to prepare different $\mathrm{CPs}$ using Alcalase and collagenase. Then, the effects of $\mathrm{CPs}$ from bovine bone on chronologically aged skin were investigated in chronologically aged mice by analyzing the skin histology, skin components and antioxidative indicators. The results showed that oral administration of CPs from bovine bone has beneficial effects on chronologically aged skin by improving the skin laxity, but it had no on moisture retention of skin. CPs prepared by Alcalase performed better than CPs prepared by collagenase.

\section{Materials and Methods}

\subsection{Materials and Chemicals}

Alcalase was purchased from Novozymes (Beijing, China). Proline (food grade) and bacterial collagenase were purchased from Sigma-Aldrich (St. Louis, MO, USA). The bicinchoninic acid (BCA) protein assay kit was purchased from Beijing Solarbio Science and Technology Co., Ltd. (Beijing, China). Commercial kits used for determining hydroxyproline (Hyp), type I and type III 
collagen, hyaluronic acid (HA), superoxide dismutase (SOD), catalase (CAT), and malondialdehyde (MDA) were purchased from Jiancheng Inst. of Biotechnology (Nanjing, China). All other chemicals used in the study were of analytical grade or better.

\subsection{Collagen Peptides ( $C P s)$ Preparation}

Gelatin was extracted from bovine bone with hot water. Briefly, the bovine bone was treated in boiling water for $6 \mathrm{~h}$, followed by removing bone using gauze filter. The filtrate was cooled, defatted and centrifuged at $4500 \times g$ for 15 min with a refrigerated centrifuge (TGL-185, Pingfan Co., Ltd., Changsha, China). After centrifugation, the upper soluble fractions were collected and freeze-dried to obtain the gelatin. The gelatin was enzymatically hydrolyzed by the Alcalase at $\mathrm{pH} 8.0$ for $4.0 \mathrm{~h}$ to obtain collagen peptides (named $\mathrm{ACP}$ ), and the collagenase at $\mathrm{pH} 7.5$ for $3.0 \mathrm{~h}$ to obtain collagen peptides (named CCP). Finally, the hydrolysates were dialyzed to discard salt and free amino acids, freeze-dried and stored at $-80{ }^{\circ} \mathrm{C}$ until use.

\subsection{Molecular Weight Distribution}

The molecular weight distribution of CPs was measured using a Shimadzu LC-15C high performance liquid chromatography (HPLC) system (Shimadzu, Tokyo, Japan) equipped with a TSK gel G2000 SWXL column $(7.8 \times 300 \mathrm{~mm}$, Tosoh, Tokyo, Japan). Samples were loaded onto the column and eluted with $45 \%(v / v)$ acetonitrile containing $0.1 \%(v / v)$ trifluoroacetic acid at a flow rate of $0.5 \mathrm{~mL} / \mathrm{min}$ and monitored at $214 \mathrm{~nm}$ at room temperature. A molecular weight calibration curve $\left(y=-0.1881 x+6.5867, y\right.$ : $\log \mathrm{MW}, x$ : time, $\left.R^{2}=0.9954\right)$ was obtained from the average retention times of the following standards: Gly-Ser (146 Da), Asn-Cys-Ser (322 Da), Trp-Pro-Trp-Trp (674 Da), bacitracin (1423 Da) and aprotinin (6512 Da) [15].

\subsection{Amino Acid Composition}

The samples were hydrolyzed in $6.0 \mathrm{M} \mathrm{HCl}$ at $110{ }^{\circ} \mathrm{C}$ for $24 \mathrm{~h}$. After phenylisothiocyanate (PITC) derivatization reaction, the amino acid composition was analyzed by a Shimadzu LC-15C high performance liquid chromatography (HPLC) system (Shimadzu, Tokyo, Japan) equipped with a reverse Zorbax SB-C18 column $(4.6 \times 250 \mathrm{~mm}$, Agilent, Santa Clara, CA, USA). The mobile phase consisted of (A) $10 \mathrm{mM}$ phosphate buffer solution ( $\mathrm{pH}$ 6.9) and (B) 100\% acetonitrile and the flow rate was $1.0 \mathrm{~mL} / \mathrm{min}$. The gradient was programmed as follows: $0-5 \mathrm{~min}, 5-10 \% \mathrm{~B} ; 5-25 \mathrm{~min}, 10-17 \% \mathrm{~B}$; $25-45 \mathrm{~min}, 17-35 \%$ B; $45-48 \mathrm{~min}, 35-100 \%$ B; $48-50 \mathrm{~min}, 100 \%$ B; $50-58 \mathrm{~min}, 100-5 \%$ B; and $58-60 \mathrm{~min}$, $5 \%$ B. The detection wavelength was set at $254 \mathrm{~nm}$ [15].

\subsection{Animals, Diets, and Treatments}

Animal experiments were carried out under the protocols approved by the Committee for Animal Research of Peking University and followed the Guide for the Care and Use of Laboratory Animals (NIH publication No. 86-23, revised 1996). The present experiment was approved by the Animal Experimental Welfare \& Ethical Inspection Committee, the Supervision, Inspection and Testing Center of Genetically Modified Organisms, Ministry of Agriculture (Beijing, China), and was performed in the Experimental Animal Center, Supervision and Testing Center for GMOs Food Safety, Ministry of Agriculture (SPF grade, Beijing, China).

Two-month-old (young mice, $28 \pm 2 \mathrm{~g}$, specific pathogen free (SPF) grade) and thirteen-month-old (old mice, $45 \pm 5 \mathrm{~g}$, SPF grade) female Kunming mice were purchased from Sibeifu (Beijing) Laboratory Animal Science and Technology Co., Ltd. (Beijing, China). The two-month-old mice were set as young controls $(n=10)$ and were given $0.2 \mathrm{~mL}$ normal saline. The thirteen-month-old mice were divided, based on body weight, into 6 groups ( $n=10$ /group), including the model group and CPs treatment groups. The model group was given $0.2 \mathrm{~mL}$ normal saline; whereas the CPs treatment groups were given $0.2 \mathrm{~mL}$ ACP at doses of 200 (ACP-200), 400 (ACP-400) and $800 \mathrm{mg} / \mathrm{kg}$ body weight (ACP-800), respectively, and $0.2 \mathrm{~mL} \mathrm{CCP}$ at a dose of $400 \mathrm{mg} / \mathrm{kg}$ body weight (ACP-400). In addition to free 
access to normal AIN-93M purified diet and water, each group was intragastrically administrated with $0.2 \mathrm{~mL}$ of normal saline or CPs once a day for eight weeks. After eight weeks, mice were sacrificed and samples were collected for further treatment and analysis.

\subsection{Measurement of Degree of Skin Laxity (DSL)}

During the period of this study, mice backs were epilated with $6 \%(w / w)$ sodium sulfide 2 days before measuring degree of skin laxity each time. Briefly, the dorsal skin, about $1 \mathrm{~cm}$ away from the tail root, was gently stretched by left hand with mice hind limbs off the table top slightly. Right hand controls mouse movement by pulling tail. The stretch length was measured immediately when mice were immobile. The DSL was defined as the following equation: DSL $(\mathrm{mm})=$ stretch length of dorsal skin.

\subsection{Measurement of Spleen Index (SI) and Thymus Index (TI)}

The mice were weighed and sacrificed. Spleen and thymus were excised from the mice and weighed immediately. The spleen index (SI) and thymus index (TI) were calculated according to the following equation: SI or TI (mg/g) = (weight of spleen or thymus)/body weight.

\subsection{Histological Analysis}

After eight weeks, mice were sacrificed and dorsal skin samples were dissected out immediately. 4 skin samples (About $1 \mathrm{~cm}^{2}$ ) in each group were fixed in $4 \%$ buffered neutral formalin solution for $24 \mathrm{~h}$, and embedded in paraffin. Serial sections $(7 \mu \mathrm{m})$ were put onto silane-coated slides and stained with haematoxylin-eosin (HE). The stained sections were further analyzed using an optical microscope. 1 representative image of HE-stained dorsal skin section in each group was presented in part of results.

\subsection{Measurement of Moisture Content}

Mice backs were epilated with $6 \%(w / w)$ sodium sulfide 2 days before sacrificing mice. Dorsal skins were collected after mice were sacrifice and skin moisture was measured immediately. The moisture content of skin sample was determined according to GB/T5009.3-2010, a national standard of China for measuring moisture content. This method was employed to measure moisture content of skin in several previous reports $[13,14]$. Briefly, about $0.1 \mathrm{~g}$ of powdered skin sample was put into weighing bottle and dried in an oven at $105^{\circ} \mathrm{C}$ for $4 \mathrm{~h}$. The moisture content was calculated according to the following equation:

$$
\text { Moisture content }=\left(m_{1}-m_{2}\right) /\left(m_{1}-m_{3}\right) \times 100
$$

$m_{1}, m_{2}$ and $m_{3}$ is the weight of weighing bottle plus skin sample, weighting bottle plus dry finished skin sample and weighing bottle, respectively.

\subsection{Determination of Hyaluronic Acid (HA) Content}

About $0.1 \mathrm{~g}$ skin tissue was powdered in a liquid nitrogen bath and homogenized in pre-cooling saline. After centrifugation at $14,000 \times g$ for $15 \mathrm{~min}$ at $4{ }^{\circ} \mathrm{C}$ with a refrigerated centrifuge (TGL-185, Pingfan Co., Ltd., Changsha, China), the supernatant was collected to analyze the hyaluronic acid (HA) content using a commercial HA measurement kit (Nanjing Jiancheng Bio Inst., Nanjing, China).

\subsection{Determination of Collagen Content}

A commercial hydroxyproline assay kit (Nanjing Jiancheng Bio Inst., Nanjing, China) was used to analyze the Hyp content. Briefly, about $0.05 \mathrm{~g}$ skin tissue was totally hydrolyzed, oxidized and reacted with dimethyl-amino-benzaldehyde. The end product has a maximal absorption at $550 \mathrm{~nm}$. The Hyp content in the skin was finally determined by comparison with the absorbance of the Hyp 
standard. The collagen content was calculated according to the Hyp content using a conversion factor of 8.00 [25].

\subsection{Ratio of Type I to Type III Collagen}

Commercial type I and type III collagen assay kits (Nanjing Jiancheng Bio Inst., Nanjing, China) were used to analyze the relative content of type I and type III collagen. The ratio of type I to type III collagen was calculated according to the following equation: ratio of type I to type III collagen = content of type I collagen/content of type I collagen.

\subsection{Antioxidant Indicators Analysis}

Skin tissue were powdered in a liquid nitrogen bath and homogenized with 9 weights of pre-cooling saline. Homogenate was centrifuged at $14,000 \times \mathrm{g}$ for $15 \mathrm{~min}$ at $4{ }^{\circ} \mathrm{C}$ with a refrigerated centrifuge (TGL-185, Pingfan Co., Ltd., Changsha, China) to collect the supernatants. Total protein concentration was determined using a bicinchoninic acid (BCA) assay kit (Solarbio, Beijing, China). The SOD activity, CAT activity and malondialdehyde MDA content (expressed as MDA equivalents) were analyzed using the corresponding enzyme-linked immunosorbent assay (ELISA) kit (Nanjing Jiancheng Bio Inst., Nanjing, China) according to the manufacturer's instructions and the results were expressed in $\mathrm{U} / \mathrm{mg}$ protein or $\mathrm{nmol} / \mathrm{mg}$ protein.

\subsection{Statistical Analysis}

Results are expressed by the means \pm standard deviation (SDs). Comparisons between two groups were analyzed by Student's $t$-test. Differences between the means of the individual groups were analyzed using the analysis of variance (ANOVA) with Duncan's multiple range tests. A difference was considered statistically significant when $p<0.05$. All computations were performed with SPSS Statistics 19 (IBM, Chicago, IL, USA).

\section{Results}

\subsection{Characterization of Collagen Peptides}

Alcalase and collagenase (two optimized enzymes in our prior study) were used for producing different collagen peptides (named ACP and CCP, respectively). The molecular weight distributions of ACP and CCP are shown in Figure 1. ACP and CCP had a similar molecular weight distribution. Both ACP and CCP mainly consisted of peptides in molecular weight ranges of $<500 \mathrm{Da}$ (more than $50 \%$ ), and the peptides of $<1000 \mathrm{Da}$ accounted for approximately $70 \%$ and $74 \%$, respectively.

The amino acid compositions of ACP and CCP are shown in Table 1. ACP and CCP had similar amino acid compositions. Gly is the most dominant amino acid in ACP and CCP, which is consistent with the Gly-X-Y repeating sequence in the collagen macromolecule. In addition, $\mathrm{ACP}$ and $\mathrm{CCP}$ are also rich in Pro, Glu, Phe, Arg and Thr. 


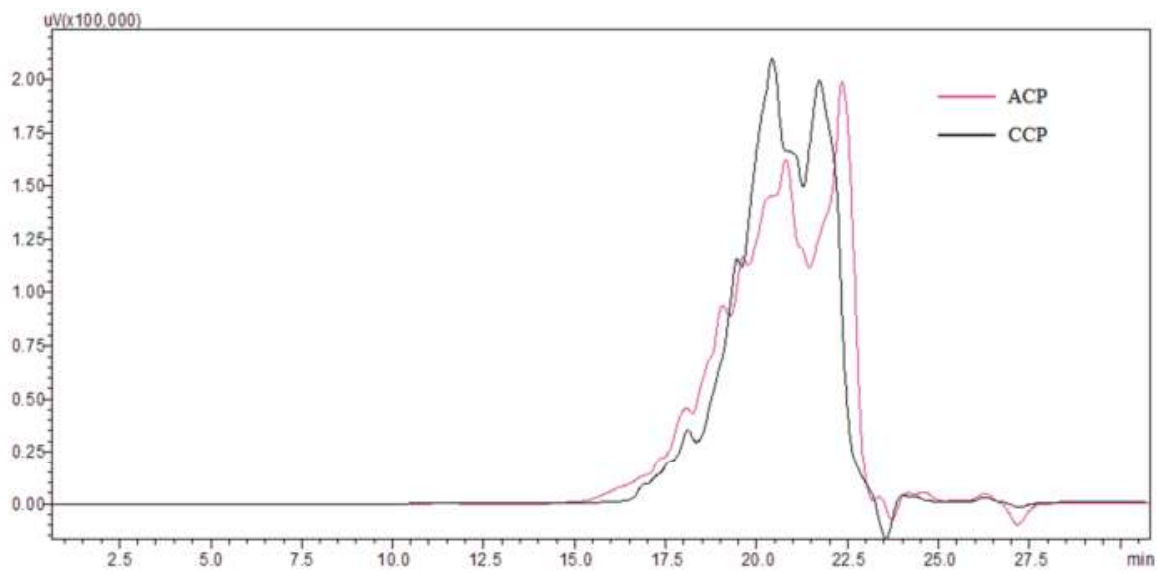

Figure 1. The molecular weight distributions of collagen peptides.

Table 1. Amino acid compositions of collagen peptides.

\begin{tabular}{ccc}
\hline \multirow{2}{*}{ Amino Acid } & \multicolumn{2}{c}{ Relative Content $(\mathbf{g} / \mathbf{1 0 0} \mathbf{g})^{\mathbf{a}, \mathbf{b}}$} \\
\cline { 2 - 3 } & ACP & CCP \\
\hline Asp & 5.68 & 5.17 \\
Glu & 10.51 & 11.53 \\
Ser & 3.38 & 3.17 \\
Gly & 19.84 & 21.28 \\
His & 3.27 & 2.97 \\
Thr & 7.90 & 8.51 \\
Ala & 4.09 & 4.68 \\
Pro & 12.47 & 12.18 \\
Arg & 8.61 & 8.47 \\
Tyr & 2.28 & 1.79 \\
Val & 3.06 & 2.90 \\
Met & 1.79 & 1.27 \\
Cys & 2.39 & 2.08 \\
Ile & 4.11 & 3.93 \\
Leu & 0.70 & 0.08 \\
Phe & 9.31 & 9.36 \\
Lys & 0.61 & 0.62 \\
Total & 100.00 & 100.00
\end{tabular}

${ }^{\mathrm{a}}$ Expressed as $\mathrm{g} / 100 \mathrm{~g}$ total amino acids; ${ }^{\mathrm{b}} \mathrm{ACP}$, collagen peptides prepared by Alcalase; $\mathrm{CCP}$, collagen peptides prepared by collagenase.

\subsection{Degree of Skin Laxity}

As summarized in Table 2, degree of skin laxity (DSL) of young (Y) group was increased during the experiment period but significantly lower than that of model group (old mice), which indicated that skin laxity was increased in an age-dependent manner. During the 8 weeks, the DSL of mice in CPs-treated groups (ACP and CCP groups) decreased over time compared with that in week 0 . Significant differences in DSL were seen between ACP-400 group and time-matched model group at week $6(p<0.05)$, and the DSL of ACP-400 had no significant difference with that of young group. Furthermore, when the time of oral intake of ACP was as long as 8 weeks, the DSL of all ACP-fed groups decreased to the level of young group $(p>0.05)$, and some of groups (ACP-800 and CCP-400) were even better than the young group. Similarly, oral administration of proline at a dose of 
$400 \mathrm{mg} / \mathrm{kg}$ body weight also reduced the DSL with a significant difference observed compared with the time-matched model group at week $8(p<0.05)$.

Table 2. Degree of skin laxity (DSL) of chronologically aged mice after the administration of collagen peptides for 8 weeks.

\begin{tabular}{cccccc}
\hline \multirow{2}{*}{ Group $^{\text {a }}$} & \multicolumn{5}{c}{ Degree of Skin Laxity (DSL, mm) } \\
\cline { 2 - 5 } & Week 0 & Week 2 & Week 4 & Week 6 & Week 8 \\
\hline Y & $14.90 \pm 2.32 *$ & $19.15 \pm 1.57 *$ & $19.75 \pm 1.03^{*}$ & $19.60 \pm 0.91 *$ & $20.40 \pm 1.48^{*}$ \\
M & $22.25 \pm 2.40$ & $22.40 \pm 1.67$ & $23.80 \pm 2.25$ & $22.50 \pm 1.30$ & $23.00 \pm 1.26$ \\
ACP-200 & $23.05 \pm 0.56$ & $23.50 \pm 1.64$ & $23.11 \pm 1.26$ & $22.00 \pm 1.31$ & $21.22 \pm 1.47^{*}$ \\
ACP-400 & $22.45 \pm 1.88$ & $21.55 \pm 1.78$ & $21.65 \pm 1.57$ & $20.15 \pm 1.34 *$ & $20.25 \pm 1.47^{*}$ \\
ACP-800 & $22.30 \pm 1.81$ & $22.72 \pm 2.06$ & $22.05 \pm 2.26$ & $21.60 \pm 1.78$ & $19.80 \pm 0.90^{*}$ \\
CCP-400 & $22.40 \pm 1.57$ & $22.35 \pm 1.41$ & $21.25 \pm 2.03$ & $22.10 \pm 1.92$ & $19.95 \pm 1.65^{*}$ \\
Pro-400 & $21.65 \pm 1.23$ & $21.30 \pm 2.28$ & $22.80 \pm 0.81$ & $21.70 \pm 1.00$ & $19.75 \pm 1.44^{*}$ \\
\hline
\end{tabular}

a Y, young group; M, model group (old group); $\mathrm{ACP}$, administrated by collagen peptides which was prepared by Alcalase; CCP, administrated by collagen peptides which was prepared by collagenase; Pro, proline group. 200, 400 and 800 represent administration doses of 200,400 and $800 \mathrm{mg} / \mathrm{kg}$ body weight, respectively. The values are shown as the means \pm SDs ( $n=10$ mice/group). A significant difference was observed at $* p<0.05$ compared to the time-matched model group.

\subsection{Body Weight, Spleen Index (SI) and Thymus Index (TI)}

The body weight of young group was increased during the experiment period, whereas that of model group remained stable (Table 3). Treatment with ACP (200, 400 and $800 \mathrm{mg} / \mathrm{kg}$ body weight), $\mathrm{CCP}$ and proline ( $400 \mathrm{mg} / \mathrm{kg}$ body weight) for 8 weeks caused no statistically significant differences in the body weight compared with the untreated model group. Furthermore, the SI and TI of ACP groups, CCP and proline groups also had no significant difference compared to that of the model group. The body weight and organ indices could be measured to preliminarily determine whether a sample or sample dose had obvious toxicological effects on the animal subjects [26]. There was no obvious atrophy, hyperplasia or swelling of spleen and thymus after CPs and proline intake. Based on these results, it was concluded that oral administration of CPs from bovine bone at doses of $200-800 \mathrm{mg} / \mathrm{kg}$ body weight, or proline at $400 \mathrm{mg} / \mathrm{kg}$ body weight for 8 weeks, had no obvious toxicological effects.

Table 3. Body weight, spleen index (SI) and thymus index (TI) of chronologically aged mice after the administration of collagen peptides for 8 weeks.

\begin{tabular}{|c|c|c|c|c|c|c|c|}
\hline \multirow{2}{*}{ Group $^{a}$} & \multicolumn{5}{|c|}{ Body Weight (g) } & \multirow{2}{*}{$\begin{array}{c}\text { Spleen Index } \\
(\mathrm{mg} / \mathrm{g})\end{array}$} & \multirow{2}{*}{$\begin{array}{c}\text { Thymus Index } \\
(\mathrm{mg} / \mathrm{g})\end{array}$} \\
\hline & Week 0 & Week 2 & Week 4 & Week 6 & Week 8 & & \\
\hline$Y$ & $28.28 \pm 0.73$ & $30.27 \pm 1.62$ & $31.71 \pm 1.30$ & $32.63 \pm 1.81$ & $32.70 \pm 1.52$ & $3.48 \pm 0.69$ & $1.82 \pm 0.39$ \\
\hline ACP-200 & $47.41 \pm 5.47$ & $46.28 \pm 6.07$ & $46.44 \pm 4.41$ & $47.41 \pm 4.13$ & $46.83 \pm 3.34$ & $3.72 \pm 0.06$ & $1.71 \pm 0.65$ \\
\hline ACP- 400 & $47.50 \pm 5.57$ & $46.32 \pm 4.91$ & $46.90 \pm 4.48$ & $46.64 \pm 4.90$ & $46.35 \pm 4.90$ & $3.24 \pm 1.24$ & $2.06 \pm 0.80$ \\
\hline ACP-800 & $47.75 \pm 5.67$ & $46.88 \pm 4.68$ & $48.36 \pm 7.42$ & $49.40 \pm 6.63$ & $47.39 \pm 6.57$ & $3.86 \pm 1.39$ & $1.55 \pm 0.62$ \\
\hline
\end{tabular}

${ }^{a} \mathrm{Y}$, young group; $\mathrm{M}$, model group (old group); $\mathrm{ACP}$, administrated by collagen peptides which was prepared by Alcalase; CCP, administrated by collagen peptides which was prepared by collagenase; Pro, proline group. 200,400 and 800 represent administration doses of 200,400 and $800 \mathrm{mg} / \mathrm{kg}$ body weight, respectively. The values are shown as the means \pm SDs ( $n=10$ mice/group). No significant difference between each administration group and time-matched model group was observed $(p>0.05)$. ${ }^{\mathrm{b}}$ Indicates values at week 8.

\subsection{Skin Histology}

The results of morphological examination of mice dorsal skin are illustrated in Figure 2. Skin collagen fibers in dermis were stained a light red with haematoxylin-eosin (HE). In the model group (old mice), lighter red and more space (green arrow) were observed in the dermis tissue than were those of the young group. There were thinner dermis and less sebaceous gland (red arrow) in the 
model group compared with the young group. After ACP and CCP intake, the space in the dermis tissue was decreased and the fibers appeared to be denser and more organized compared with the model group. Besides, the number of sebaceous gland was increased when treated by ACP, especially at dose of $800 \mathrm{mg} / \mathrm{kg}$ body weight. These results indicated that ACP improved the aged collagen fibers in skin dermis in a dose-dependent manner. Similarly, the sparse, fragmented, and disorganized fibers were also obviously improved and the number of sebaceous gland was greatly increased by the oral administration of proline at a dose of $400 \mathrm{mg} / \mathrm{kg}$ body weight for 8 weeks.

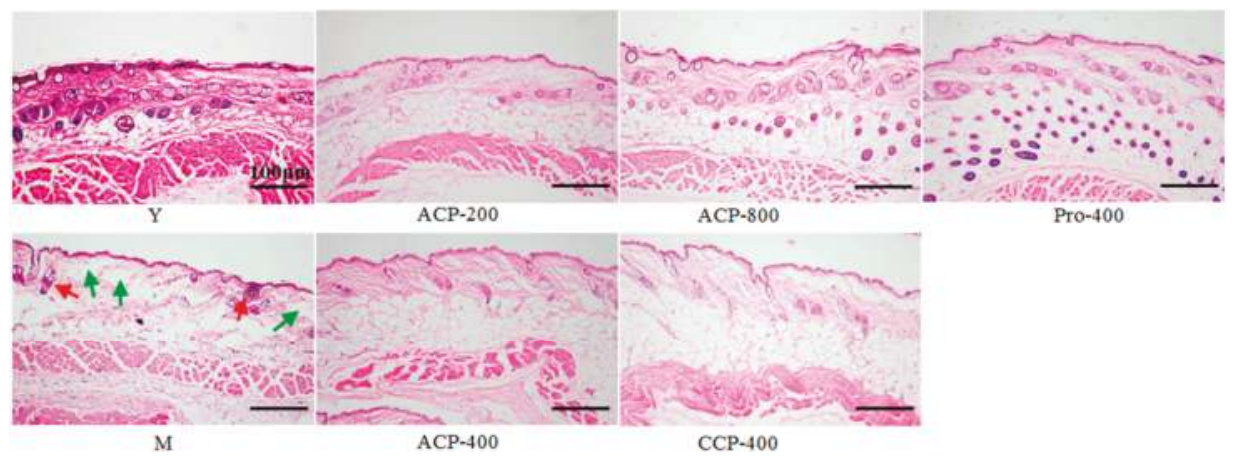

Figure 2. Representative images of haematoxylin-eosin (HE)-stained dorsal skin section from all groups, Y, young group; $\mathrm{M}$, model group (old group); ACP, administrated by collagen peptides which was prepared by Alcalase; CCP, administrated by collagen peptides which was prepared by collagenase; Pro, proline group. 200, 400 and 800 represent administration doses of 200, 400 and $800 \mathrm{mg} / \mathrm{kg}$ body weight, respectively. Sebaceous glands and space in dermis tissue are shown as red arrows and green arrows, respectively. Scale bars, $100 \mu \mathrm{m}$.

\subsection{Skin Components}

The results of skin moisture, hyaluronic acid (HA), collagen content and ratio of type I to type III collagen are shown in Figure 3A-D. Skin moisture content and ratio of type I to type III collagen in the model group (old mice) were significantly lower than that in the young group (all $p<0.05$ ), indicating that skin moisture content and ratio of type I to type III collagen were decreased with age. Skin HA and collagen contents were also lower than that in the young group, although there was no significant difference observed compared with the model group. Ingestion of $\mathrm{CPs}$ (both ACP and $\mathrm{CCP}$ ) and proline had no significant effect on skin moisture and HA contents compared with the model group. In contrast, oral administration of ACP (200, 400 and $800 \mathrm{mg} / \mathrm{kg}$ body weight) caused a dose-dependent increase in the collagen content, and there was a significant difference in the collagen content between the group receiving $800 \mathrm{mg} / \mathrm{kg}$ of ACP and the model group $(p<0.05)$. Ingestion of $\mathrm{CCP}$ at a dose of $400 \mathrm{mg} / \mathrm{kg}$ body weight also increased the collagen content in skin $(p<0.05 \mathrm{vs}$. the model group). However, proline intake at a dose of $400 \mathrm{mg} / \mathrm{kg}$ body weight had no significant effect on collagen content compared with the model group. A dose-dependent increase was also observed for ratio of type I to type III collagen in the ACP-fed groups, and there were significant differences between the groups receiving 400 and $800 \mathrm{mg} / \mathrm{kg}$ of ACP and the model group (all $p<0.05$ ); whereas CCP ingestion at a dose of $400 \mathrm{mg} / \mathrm{kg}$ body weight had no significant effect on ratio of type I to type III collagen compared to the model group. Oral administration of proline also significantly increased ratio of type I to type III collagen in skin ( $p<0.05 \mathrm{vs}$. the model group). 

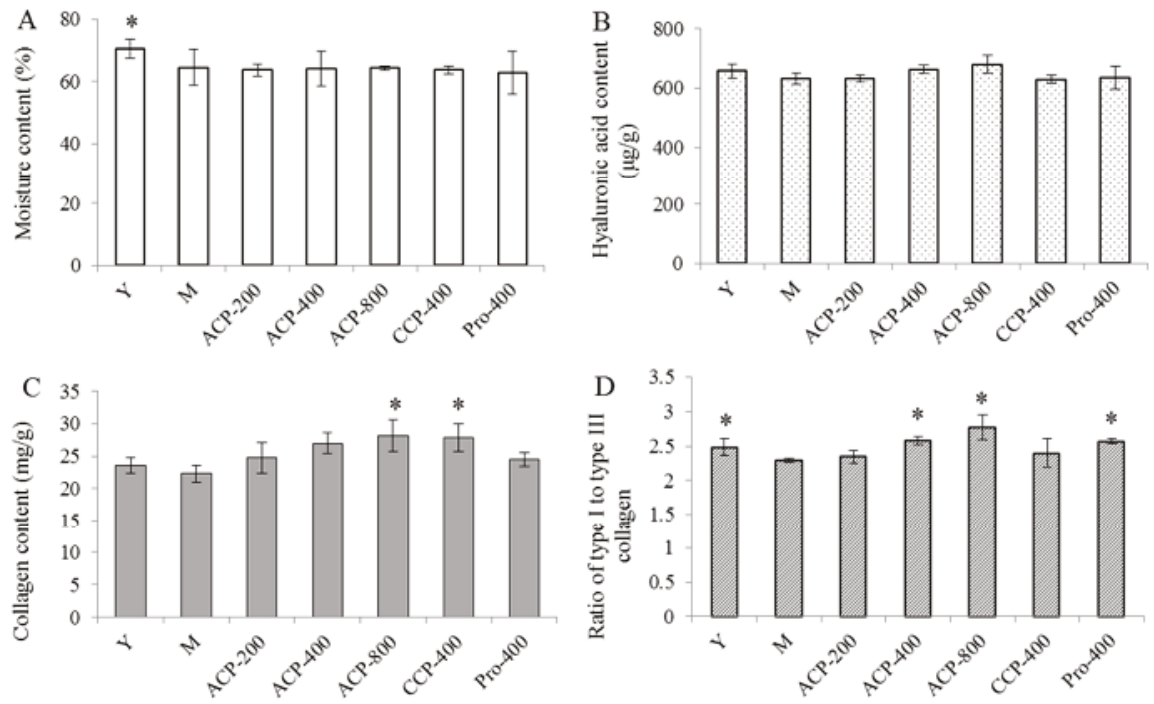

Figure 3. Moisture content (A), hyaluronic acid content (B), hydroxyproline content (C) and ratio of type I to type III collagen (D) of chronologically aged mice after the administration of collagen peptides for 8 weeks. Y, young group; M, model group (old group); ACP, administrated by collagen peptides which was prepared by Alcalase; CCP, administrated by collagen peptides which was prepared by collagenase; Pro, proline group. 200, 400 and 800 represent administration doses of 200, 400 and $800 \mathrm{mg} / \mathrm{kg}$ body weight, respectively. The values are shown as the means \pm SDs ( $n=10$ mice/group). A significant difference was observed at $p<0.05$ compared to the time-matched model group.

\subsection{Antioxidant Indicators}

The superoxide dismutase (SOD), catalase (CAT) and malondialdehyde (MDA) content in skin are shown in Table 4 . The SOD and CAT activities in the M group were significantly lower in the skin compared to the $\mathrm{Y}$ group $(p<0.05)$; whereas the MDA level in the model group was higher than that in the young group $(p<0.05)$. Oral administration of CPs (both ACP and CCP) significantly increased the SOD and CAT activities and reduced the MDA level (all $p<0.05$ vs. the model group). Besides, the increase of SOD and CAT activities and the decrease of MDA level in ACP-fed groups showed a dose-dependent manner. In contrast, proline ingestion had no obvious significant effect on these three antioxidant indicators.

Table 4. SOD and CAT activities and MDA content in dorsal skin of chronologically aged mice after administration of collagen peptides for 8 weeks.

\begin{tabular}{cccc}
\hline Group $^{\text {a }}$ & SOD (U/mg Protein) & CAT (U/mg Protein) & MDA Equivalents (nmol/mg Protein) \\
\hline Y & $36.594 \pm 1.142^{*}$ & $10.412 \pm 1.143^{*}$ & $2.209 \pm 0.278^{*}$ \\
M & $26.877 \pm 3.880$ & $4.650 \pm 1.582$ & $3.135 \pm 0.302$ \\
ACP-200 & $38.746 \pm 0.753^{*}$ & $8.324 \pm 0.890^{*}$ & $2.347 \pm 0.209^{*}$ \\
ACP-400 & $39.823 \pm 3.410^{*}$ & $11.327 \pm 1.096^{*}$ & $2.261 \pm 0.107^{*}$ \\
ACP-800 & $40.036 \pm 4.820^{*}$ & $12.012 \pm 0.752^{*}$ & $2.154 \pm 0.325^{*}$ \\
CCP-400 & $39.796 \pm 1.211^{*}$ & $9.354 \pm 1.856^{*}$ & $2.204 \pm 0.201^{*}$ \\
Pro-400 & $32.646 \pm 1.691$ & $3.318 \pm 0.665$ & $2.456 \pm 0.316$ \\
\hline
\end{tabular}

${ }^{a}$ Y, young group; $\mathrm{M}$, model group (old group); $\mathrm{ACP}$, administrated by collagen peptides which was prepared by Alcalase; CCP, administrated by collagen peptides which was prepared by collagenase.; Pro, proline group. 200, 400 and 800 represent administration doses of 200,400 and $800 \mathrm{mg} / \mathrm{kg}$ body weight, respectively. The values are shown as the means $\pm \operatorname{SDs}(n=10$ mice/group). A significant difference was observed at $* p<0.05$ compared to the time-matched model group. 


\section{Discussion}

Skin aging is consisted of chronological aging and photoaging. There are some difference in clinical signs and underlying mechanisms for these two processes $[27,28]$. Collagen peptides (CPs) have been widely reported to exert beneficial effects on photoaging skin, but few studies was carried out to evaluate their effect on chronologically aged skin. In present study, 13-month-old Kunming mice, equivalent to 45 years old of human life [29], were employed to investigate the effect of CPs on chronologically aged skin. In several previous clinical trials, a daily dose of $2.5 \mathrm{~g}$ or $5 \mathrm{~g}$ of CPs has been employed in adult subjects, and these doses were considered to be safe $[16,17,30]$. According to the conversion of animal doses to human equivalent dose (HED) based on the body surface area (BSA) [31], about daily dose of 500 or $1000 \mathrm{mg} / \mathrm{kg}$ body weight could be used in mice. In addition, daily doses of 50-200 $\mathrm{mg} / \mathrm{kg}$ body weight have also been used in several animal experiments [13-15]. Therefore, doses of 200, 400 and $800 \mathrm{mg} / \mathrm{kg}$ body weight/day were employed in the present study.

Skin laxity is a main feature of natural skin aging and is increased with age [32]. Therefore, skin laxity was dynamically evaluated by measuring the degree of skin laxity (DSL) to observe the effect of CPs intake on chronologically aged skin. An obvious beneficial effect was observed after 8 weeks of CPs intake. Therefore, mice were sacrificed and samples were collected for further treatment and analysis after 8 weeks. Unexpectedly, proline (abundant in collagen) ingestion also significantly improved skin laxity. These results provided guidance for the application of CPs or proline against chronological skin aging. The 8-week duration of CPs ingestion might be equivalent to several years old of human life in terms of life span. But it does not mean that the beneficial effects of CPs could be observed only after several years duration of CPs intake, because several studies have reported that significant beneficial effects of CPs on aging skin could be observed after 6 to 12 weeks in both clinical trials and animal experiments [13-17].

As the main component of the skin dermis, collagen has been reported to be beneficial in improving skin laxity and decreasing the appearance of wrinkles and its reduction in the quantity and quality is a major cause of laxity and wrinkles [33,34]. In the chronologically aged skin, dermal collagen fiber became sparse, fragmented and disorganized [35,36]. However, intake of CPs (both ACP and $\mathrm{CCP}$ ) repaired collagen fibers and the fibers appeared to be denser and more organized compared to the aged skin. Collagen in skin mainly consists of type I and type III collagen. Collagen production and the ratio of type I to type III collagen is decreased gradually with age $[14,28,37,38]$. Type I collagen tend to form broader bundles of fibers, while type III collagen forms narrow bundles. A decrease in the diameter and number of the collagen bundles is correlated with the decrease in load and tensile strength reported in aging skin [38]. Oral administration of CPs increased the collagen content and ratio of type I to type III collagen in a dose-dependent manner, which suggested that CPs improved skin laxity by changing skin collagen quantitatively and qualitatively. In contrast, the skin moisture and hyaluronic acid (HA) were not affected by CPs ingestion. HA is a key molecule involved in skin moisture, because it has a unique capacity to bind and retain water molecules [39]. Taken together with the results of the current study, it was concluded that oral administration of CPs had beneficial effect on chronologically aged skin by improving skin laxity, but it had no influence on moisture retention of skin.

Interestedly, ingestion of proline also had beneficial effect on chronologically aged skin in terms of skin laxity, collagen content and ratio of type I to type III collagen. This result of in vivo study was consistent with that of a previous in vitro experiment which demonstrated that proline could increase the collagen synthesis of confluent fibroblasts, but it did not stimulate the proliferation of fibroblasts $[40,41]$. Watanabe-Kamiyama and coworkers have reported that proline could reach the skin after proline intake [42]. Therefore, we speculated that proline intake exerted beneficial effect on chronologically aged skin by increasing the collagen synthesis of skin fibroblasts.

It has been widely accepted that oxidative stress plays a critical role in initiating and driving the signaling events that result in skin aging. Study has reported that the production of reactive oxygen species (ROS) was increased in photoaged and chronologically aged skin [43]. In addition to 
directly attacking macromolecules, such as proteins, lipids, DNA and RNA, the excessive ROS also initiate several signaling pathways, including mitogen-activated protein kinases (MAPKs) and nuclear factor kappa-light-chain-enhancer of activated B cells (NF- $\mathrm{KB}$ ), and further activate transcription factor activator protein-1 (AP-1) [44]. AP-1 induces collagen degradation by upregulating collagen-degraded enzymes such as matrix metalloprotease (MMP)-1, MMP-3 and MMP-9 and downregulating the biosynthesis of collagen. These changes in the skin lead to the phenotype of aged skin [45]. Therefore, antioxidants or free radical scavengers, such as ascorbic acid and polyphenols were reported to improve skin aging by scavenging excessive ROS. Normally, endogenous antioxidant enzymes are able to scavenge the excessive ROS to protect skin tissues from oxidative injuries. SOD and CAT are two antioxidant enzymes that inactivate superoxide anions and hydrogen peroxide, respectively. MDA is a product of lipid peroxidation and is usually quantified to estimate the lipid peroxidation extent induced by ROS. The SOD and CAT activities were decreased and MDA content was increased with age. However, CPs (ACP and CCP) intake could increase SOD and CAT activities and decrease MDA content, indicating that ingestion of collagen peptides from bovine bone had the ability to decrease ROS in skin. The decreased ROS in skin might help to increase the biosynthesis of collagen and decrease the collagen degradation by reducing the MMPs production. Indeed, several previous studies have reported that collagen hydrolysate ingestion could increases skin collagen expression and suppresses MMP-1 and MMP-2 [46,47]. In vitro study had demonstrated that ACP and CCP had high antioxidant capacity base on the hydroxyl radicals and ABTS + scavenging assays (data not shown). In addition, it has been widely reported that nuclear factor E2-related factor 2 (Nrf2)-antioxidant response element (ARE) pathway plays a central role in regulating antioxidant enzymes against oxidative stress [48,49]. Therefore, we speculate that CPs exerted their antioxidant effect in a direct and/or indirect manner. It is also possible that CPs exerted their beneficial effects on chronological aged skin in other ways, as previous studies have reported that Pro-Hyp in human blood after oral ingestion of CPs stimulates fibroblast growth [41,50]. It should be noted that proline intake did not have an obvious effect on skin antioxidant capacity. These results suggested that CPs had more complex action mechanisms underlying anti-aging effect than proline.

Bovine bone is an abundant source of gelatin. The CPs from bovine bone is mainly concentrated on its beneficial effect on bone metabolism. However, the current study found that the CPs from bovine bone also had beneficial effect on skin aging. The CPs prepared in this study mainly consist of oligopeptides ( $<1000 \mathrm{Da})$. It was reported that small peptides, especially the di-and tripeptides, are more easily absorbed in the intestinal tract than larger molecules, and oligopeptides are more bioactive than proteins, polypeptides and free amino acids [51,52]. Therefore, we speculated that ACP and CCP are readily absorbed and might exhibit potential biological effects once they are orally administered. Another purpose of this study is to preliminary investigate whether different CPs prepared by Alcalase and collagenase have different effects on chronologically aged skin. Based on the present results, it can be drawn that the beneficial effects of ACP were slightly better than those of CCP. Therefore, Alcalase is a favorable enzyme to produce $\mathrm{CPs}$ with beneficial effects on skin aging in food and medical industries. The present result of molecular weight distribution provides a guide for testing and controlling the quality of CPs. Besides, CPs should be protected from oxygen and light because of its easy oxidation.

\section{Conclusions}

In summary, the present study demonstrated oral administration of collagen peptides from bovine bone could improve the laxity of chronologically aged skin by increasing skin collagen content and ratio of type I to type III collagen, but it had no effect on moisture retention of skin. The beneficial effects of collagen peptides prepared by Alcalase (ACP) were slightly better than those of collagen peptides prepared by collagenase (CCP). Another action mechanism underlying the beneficial effects on aged skin of collagen peptides may be involved in increasing the antioxidant properties in the body. Proline intake also improved the laxity of chronologically aged skin but it did not affect the skin 
antioxidant capacity. These results suggest that collagen peptides from bovine bone and proline are potential dietary supplements for use against skin aging in chronologically aged process.

Acknowledgments: This study was supported by the earmarked fund from China Agriculture Research System (CARS-46) and National Natural Science Foundation of China (NSFC, No. 31271846).

Author Contributions: Bo Li conceived and designed the experiments; Hongdong Song, Siqi Zhang and Ling Zhang performed the experiments and analyzed the data; Hongdong Song and Bo Li wrote the paper.

Conflicts of Interest: The authors declare no conflict of interest.

\section{References}

1. Rittié, L.; Fisher, G.J. Natural and sun-induced aging of human skin. Cold Spring Harb. Perspect. Med. 2015, 5, a015370. [CrossRef] [PubMed]

2. Durai, P.C.; Thappa, D.M.; Kumari, R.; Malathi, M. Aging in elderly: Chronological versus photoaging. Indian J. Dermatol. 2012, 57, 343-352. [PubMed]

3. Helfrich, Y.R.; Sachs, D.L.; Voorhees, J.J. Overview of skin aging and photoaging. Dermatol. Nurs. 2008, 20, 177-183. [PubMed]

4. Dobos, G.; Lichterfeld, A.; Blume-Peytavi, U.; Kottner, J. Evaluation of skin ageing: A systematic review of clinical scales. Brit. J. Dermatol. 2015, 172, 1249-1261. [CrossRef] [PubMed]

5. Chen, J.; Li, Y.; Zhu, Q.; Li, T.; Lu, H.; Wei, N.; Huang, Y.; Shi, R.; Ma, X.; Wang, X.; et al. Anti-skin-aging effect of epigallocatechin gallate by regulating epidermal growth factor receptor pathway on aging mouse model induced by d-Galactose. Mech. Ageing Dev. 2017, 164, 1-7. [CrossRef] [PubMed]

6. Tran, D.; Townley, J.P.; Barnes, T.M.; Greive, K.A. An antiaging skin care system containing alpha hydroxy acids and vitamins improves the biomechanical parameters of facial skin. Clin. Cosmet. Investig. Dermatol. 2015, 8, 9-17. [PubMed]

7. Latreille, J.; Kesse-Guyot, E.; Malvy, D.; Andreeva, V.; Galan, P.; Tschachler, E.; Hercberg, S.; Guinot, C.; Ezzedine, K. Association between dietary intake of $n-3$ polyunsaturated fatty acids and severity of skin photoaging in a middle-aged Caucasian population. J. Dermatol. Sci. 2013, 72, 233-239. [CrossRef] [PubMed]

8. Fanian, F.; Mac-Mary, S.; Jeudy, A.; Lihoreau, T.; Messikh, R.; Ortonne, J.P.; Sainthillier, J.M.; Elkhyat, A.; Guichard, A.; Kenari, K.H.; et al. Efficacy of micronutrient supplementation on skin aging and seasonal variation: A randomized, placebo-controlled, double-blind study. Clin. Interv. Aging 2013, 8, 1527-1537. [CrossRef] [PubMed]

9. Murata, M.; Satoh, T.; Wakabayashi, H.; Yamauchi, K.; Abe, F.; Nomura, Y. Oral administration of bovine lactoferrin attenuates ultraviolet B-induced skin photodamage in hairless mice. J. Dairy Sci. 2014, 97, 651-658. [CrossRef] [PubMed]

10. Zhuang, Y.; Hou, H.; Zhao, X.; Zhang, Z.; Li, B. Effects of collagen and collagen hydrolysate from jellyfish (Rhopilema esculentum) on mice skin photoaging induced by UV irradiation. J. Food Sci. 2009, 74, H183-H188. [CrossRef] [PubMed]

11. Zague, V. A new view concerning the effects of collagen hydrolysate intake on skin properties. Arch. Dermatol. Res. 2008, 300, 479-483. [CrossRef] [PubMed]

12. Oesser, S.; Adam, M.; Babel, W.; Seifert, J. Oral administration of $14 \mathrm{C}$ labeled gelatin hydrolysate leads to an accumulation of radioactivity in cartilage of mice (C57/BL). J. Nutr. 1999, 129, 1891-1895. [PubMed]

13. Fan, J.; Zhuang, Y.; Li, B. Effects of collagen and collagen hydrolysate from jellyfish umbrella on histological and immunity changes of mice photoaging. Nutrients 2013, 5, 223-233. [CrossRef] [PubMed]

14. Hou, H.; Li, B.; Zhang, Z.; Xue, C.; Yu, G.; Wang, J.; Bao, Y.; Bu, L.; Sun, J.; Peng, Z. Moisture absorption and retention properties, and activity in alleviating skin photodamage of collagen polypeptide from marine fish skin. Food Chem. 2012, 135, 1432-1439. [CrossRef] [PubMed]

15. Song, H.; Meng, M.; Cheng, X.; Li, B.; Wang, C. The effect of collagen hydrolysates from silver carp (Hypophthalmichthys molitrix) skin on UV-induced photoaging in mice: Molecular weight affects skin repair. Food Funct. 2017, 8, 1538-1546. [CrossRef] [PubMed]

16. Proksch, E.; Segger, D.; Degwert, J.; Schunck, M.; Zague, V.; Oesser, S. Oral supplementation of specific collagen peptides has beneficial effects on human skin physiology: A double-blind, placebo-controlled study. Skin Pharmacol. Physiol. 2014, 27, 47-55. [CrossRef] [PubMed] 
17. Schwartz, S.R.; Park, J. Ingestion of BioCell Collagen ${ }^{\circledR}$, a novel hydrolyzed chicken sternal cartilage extract; enhanced blood microcirculation and reduced facial aging signs. Clin. Interv. Aging 2012, 7, 267-273. [PubMed]

18. Nur, A.T.; Che, M.Y.; Rn, R.M.H.; Aina, M.A.; Amin, I. Use of principal component analysis for differentiation of gelatine sources based on polypeptide molecular weights. Food Chem. 2014, 151, 286-292.

19. Ali, M.E.; Sultana, S.; Hamid, S.B.; Hossain, M.A.; Yehya, W.A.; Kader, M.A.; Bhargava, S.K. Gelatin controversies in food, pharmaceuticals and personal care products: Authentication methods, current status and future challenges. Crit. Rev. Food Sci. Nutr. 2016, 29, 1-17. [CrossRef] [PubMed]

20. Liu, J.; Wang, Y.; Song, S.; Wang, X.; Qin, Y.; Si, S.; Guo, Y. Combined oral administration of bovine collagen peptides with calcium citrate inhibits bone loss in ovariectomized rats. PLoS ONE 2015, 10, e0135019. [CrossRef] [PubMed]

21. Kumar, S.; Sugihara, F.; Suzuki, K.; Inoue, N.; Venkateswarathirukumara, S. A double-blind, placebo-controlled, randomised, clinical study on the effectiveness of collagen peptide on osteoarthritis. J. Sci. Food Agric. 2015, 95, 702-707. [CrossRef] [PubMed]

22. Humiski, L.M.; Aluko, R.E. Physicochemical and bitterness properties of enzymatic pea protein hydrolysates. J. Food Sci. 2007, 72, S605-S611. [CrossRef] [PubMed]

23. Liang, Q.; Ren, X.; Ma, H.; Li, S.; Xu, K.; Oladejo, A.O. Effect of low-frequency ultrasonic-assisted enzymolysis on the physicochemical and antioxidant properties of corn protein hydrolysates. J. Food Qual. 2017, 2017, 1-10. [CrossRef]

24. Kanth, S.V.; Venba, R.; Madhan, B.; Chandrababu, N.K.; Sadulla, S. Studies on the influence of bacterial collagenase in leather dyeing. Dyes Pigment. 2008, 76, 338-347. [CrossRef]

25. Cheng, F.Y.; Hsu, F.W.; Chang, H.S.; Lin, L.C.; Sakata, R. Effect of different acids on the extraction of pepsin-solubilised collagen containing melanin from silky fowl feet. Food Chem. 2009, 113, 563-567. [CrossRef]

26. Zhang, X.D.; Wu, H.Y.; Wu, D.; Wang, Y.Y.; Chang, J.H.; Zhai, Z.B.; Meng, A.M.; Liu, P.X.; Zhang, L.A.; Fan, F.Y. Toxicologic effects of gold nanoparticles in vivo by different administration routes. Int. J. Nanomed. 2010, 5, 771-781. [CrossRef] [PubMed]

27. Jenkins, G. Molecular mechanisms of skin ageing. Mech. Ageing Dev. 2002, 123, 801-810. [CrossRef]

28. Chung, J.H.; Seo, J.Y.; Choi, H.R.; Lee, M.K.; Youn, C.S.; Rhie, G.; Cho, K.H.; Kim, K.H.; Park, K.C.; Eun, H.C. Modulation of skin collagen metabolism in aged and photoaged human skin in vivo. J. Investig. Dermatol. 2001, 117, 1218-1224. [CrossRef] [PubMed]

29. Song, H.; Zhang, L.; Luo, Y.; Zhang, S.; Li, B. Effects of collagen peptides intake on skin ageing and platelet release in chronologically aged mice revealed by cytokine array analysis. J. Cell. Mol. Med. 2017. [CrossRef] [PubMed]

30. Ohara, H.; Ito, K.; Iida, H.; Matsumoto, H. Improvement in the moisture content of the stratum corneum following 4 weeks of collagen hydrolysate ingestion. Nippon Shokuhin Kogaku Kaishi 2009, 56, 137-145. [CrossRef]

31. Reagan-Shaw, S.; Nihal, M.; Ahmad, N. Dose translation from animal to human studies revisited. FASEB J. 2008, 22, 659-661. [CrossRef] [PubMed]

32. Quan, T.; Fisher, G.J. Role of age-associated alterations of the dermal extracellular matrix microenvironment in human skin aging: A mini-review. Gerontology 2015, 61, 427-434. [CrossRef] [PubMed]

33. Tanaka, Y.; Nakayama, J. Upregulated expression of La ribonucleoprotein domain family member 6 and collagen type I gene following water-filtered broad-spectrum near-infrared irradiation in a 3-dimensional human epidermal tissue culture model as revealed by microarray analysis. Australas. J. Dermatol. 2017. [CrossRef] [PubMed]

34. Sadick, N.S.; Harth, Y. A 12-week clinical and instrumental study evaluating the efficacy of a multisource radiofrequency home-use device for wrinkle reduction and improvement in skin tone, skin elasticity, and dermal collagen content. J. Cosmet. Laser Ther. 2016, 18, 422-427. [CrossRef] [PubMed]

35. Demaria, M.; Desprez, P.Y.; Campisi, J.; Velarde, M.C. Cell autonomous and non-autonomous effects of senescent cells in the skin. J. Investig. Dermatol. 2015, 135, 1722-1726. [CrossRef] [PubMed]

36. Zouboulis, C.C.; Boschnakow, A. Chronological and photoaging of the human sebaceous gland. Clin. Exp. Dermatol. 2001, 26, 600-607. [CrossRef] [PubMed] 
37. Varani, J.; Dame, M.K.; Rittie, L.; Fligiel, S.E.; Kang, S.; Fisher, G.J.; Voorhees, J.J. Decreased collagen production in chronologically aged skin: Roles of age-dependent alteration in fibroblast function and defective mechanical stimulation. Am. J. Pathol. 2006, 168, 1861-1868. [CrossRef] [PubMed]

38. Lovell, C.R.; Smolenski, K.A.; Duance, V.C.; Light, N.D.; Young, S.; Dyson, M. Type I and III collagen content and fibre distribution in normal human skin during ageing. Br. J. Dermatol. 1987, 117, 419-428. [CrossRef] [PubMed]

39. Papakonstantinou, E.; Roth, M.; Karakiulakis, G. Hyaluronic acid: A key molecule in skin aging. Dermato-Endocrinology 2012, 4, 253-258. [CrossRef] [PubMed]

40. Haratake, A.; Watase, D.; Fujita, T.; Setoguchi, S.; Matsunaga, K.; Takata, J. Effects of oral administration of collagen peptides on skin collagen content and its underlying mechanism using a newly developed low collagen skin mice model. J. Funct. Foods 2015, 16, 174-182. [CrossRef]

41. Ohara, H.; Ichikawa, S.; Matsumoto, H.; Akiyama, M.; Fujimoto, N.; Kobayashi, T.; Tajima, S. Collagen-derived dipeptide, proline-hydroxyproline, stimulates cell proliferation and hyaluronic acid synthesis in cultured human dermal fibroblasts. J. Dermatol. 2010, 37, 330-338. [CrossRef] [PubMed]

42. Watanabe-Kamiyama, M.; Shimizu, M.; Kamiyama, S.; Taguchi, Y.; Sone, H.; Morimatsu, F.; Shirakawa, H.; Furukawa, Y.; Komai, M. Absorption and effectiveness of orally administered low molecular weight collagen hydrolysate in rats. J. Agric. Food Chem. 2010, 58, 835-841. [CrossRef] [PubMed]

43. Callaghan, T.M.; Wilhelm, K.P. A review of ageing and an examination of clinical methods in the assessment of ageing skin. Part I: Cellular and molecular perspectives of skin ageing. Int. J. Cosmet. Sci. 2008, 30, 313-322. [CrossRef] [PubMed]

44. Kammeyer, A.; Luiten, R.M. Oxidation events and skin aging. Ageing Res. Rev. 2015, 21, 16-29. [CrossRef] [PubMed]

45. Xu, Y.; Fisher, G.J. Ultraviolet (UV) light irradiation induced signal transduction in skin photoaging. J. Dermatol. Sci. 2015, 1, S1-S8. [CrossRef]

46. Liang, J.; Pei, X.; Zhang, Z.; Wang, N.; Wang, J.; Li, Y. The protective effects of long-term oral administration of marine collagen hydrolysate from chum salmon on collagen matrix homeostasis in the chronological aged skin of sprague-dawley male rats. J. Food Sci. 2010, 75, H230-H238. [CrossRef] [PubMed]

47. Zague, V.; de Freitas, V.; Rosa, M.D.C.; de Castro, G.Á.; Jaeger, R.G.; Machado-Santelli, G.M. Collagen hydrolysate intake increases skin collagen expression and suppresses matrix metalloproteinase 2 activity. J. Med. Food 2011, 14, 618-624. [CrossRef] [PubMed]

48. Maltese, G.; Psefteli, P.; Rizzo, B.; Srivastava, S.; Gnudi, L.; Mann, G.E.; Siow, R.C. The anti-ageing hormone klotho induces Nrf2-mediated antioxidant defences in human aortic smooth muscle cells. J. Cell. Mol. Med. 2017, 21, 621-627. [CrossRef] [PubMed]

49. Sun, Z.; Park, S.Y.; Hwang, E.; Zhang, M.; Seo, S.A.; Lin, P.; Yi, T.H. Thymus vulgaris alleviates UVB irradiation induced skin damage via inhibition of MAPK/AP-1 and activation of Nrf2-ARE antioxidant system. J. Cell. Mol. Med. 2017, 21, 336-348. [CrossRef] [PubMed]

50. Shigemura, Y.; Iwai, K.; Morimatsu, F.; Iwamoto, T.; Mori, T.; Oda, C.; Taira, T.; Park, E.Y.; Nakamura, Y.; Sato, K. Effect of Prolyl-hydroxyproline (Pro-Hyp), a food-derived collagen peptide in human blood, on growth of fibroblasts from mouse skin. J. Agric. Food Chem. 2009, 57, 444-449. [CrossRef] [PubMed]

51. Bouglé, D.; Bouhallab, S. Dietary bioactive peptides: Human studies. Crit. Rev. Food Sci. Nutr. 2017, 57, 335-343. [CrossRef] [PubMed]

52. Jia, J.; Zhou, Y.; Lu, J.; Chen, A.; Li, Y.; Zheng, G. Enzymatic hydrolysis of Alaska pollack (Theragra chalcogramma) skin and antioxidant activity of the resulting hydrolysate. J. Sci. Food Agric. 2010, 90, 635-640. [CrossRef] [PubMed] 


\title{
Capsaicin: Friend or Foe in Skin Cancer and Other Related Malignancies?
}

\author{
Simona-Roxana Georgescu ${ }^{1}$, Maria-Isabela Sârbu ${ }^{1}$, Clara Matei ${ }^{1}$, Mihaela Adriana Ilie ${ }^{2}$, \\ Constantin Caruntu ${ }^{3,4, *}$, Carolina Constantin ${ }^{5}$, Monica Neagu ${ }^{5,6}$ and Mircea Tampa ${ }^{1}$ \\ 1 Department of Dermatology, Carol DavilaUniversity of Medicine and Pharmacy, 020021 Bucharest, Romania; \\ simonaroxanageorgescu@yahoo.com (S.-R.G.); isabela_sarbu@yahoo.com (M.-I.S.); \\ matei_clara@yahoo.com (C.M.); tampa_mircea@yahoo.com (M.T.) \\ 2 Department of Biochemistry, Carol Davila University of Medicine and Pharmacy, 020021 Bucharest, \\ Romania; mihaelaadriana2005@yahoo.com \\ 3 Department of Physiology, Carol Davila University of Medicine and Pharmacy, 050474 Bucharest, Romania \\ 4 Department of Dermatology, Prof. N.C. Paulescu National Institute of Diabetes, Nutrition and Metabolic \\ Diseases, 011233 Bucharest, Romania \\ 5 Immunology Department, Victor Babes National Institute of Pathology, 050096 Bucharest, Romania; \\ caroconstantin@gmail.com (C.C.); neagu.monica@gmail.com (M.N.) \\ 6 Faculty of Biology, University of Bucharest, 76201 Bucharest, Romania \\ * Correspondence: costin.caruntu@gmail.com; Tel.: +40-745-086-978
}

Received: 15 November 2017; Accepted: 12 December 2017; Published: 16 December 2017

\begin{abstract}
Capsaicin is the main pungent in chili peppers, one of the most commonly used spices in the world; its analgesic and anti-inflammatory properties have been proven in various cultures for centuries. It is a lipophilic substance belonging to the class of vanilloids and an agonist of the transient receptor potential vanilloid 1 receptor. Taking into consideration the complex neuro-immune impact of capsaicin and the potential link between inflammation and carcinogenesis, the effect of capsaicin on muco-cutaneous cancer has aroused a growing interest. The aim of this review is to look over the most recent data regarding the connection between capsaicin and muco-cutaneous cancers, with emphasis on melanoma and muco-cutaneous squamous cell carcinoma.
\end{abstract}

Keywords: capsaicin; skin; neurogenic inflammation; cancer; carcinogenesis; squamous cell carcinoma; melanoma

\section{Introduction}

Chili peppers belong to the genus Capsicum of the Solanaceae family and are some of the most used condiments in the world being consumed on daily basis by almost $25 \%$ of the population [1-6]. The chili extract has been long used in traditional medicine. Alcoholic hot pepper extract was used as a counterirritant analgesic and helped treat burning sensations and pruritus. In tropical countries it was administrated to induce vasodilatation and to increase heat loss [7].

The main pungent component in chili peppers is capsaicin and this plant component is probably produced as a defense mechanism against herbivores and fungi [6]. Capsaicin, an alkylamide, is the most abundant capsaicinoid found in chili peppers $(69 \%)$ but dihydrocapsaicin $(22 \%)$, nordihydrocapsaicin $(7 \%)$, homocapsaicin $(1 \%)$ and homodihydrocapsaicin (1\%) are also present [1]. The history of capsaicin goes back to the 19thcentury. In 1816, Bucholtz managed for the first time the extraction as a solution of the pungent component from the chili pepper [8]. In 1846, Thresh named this component capsaicin and achieved for the first time its isolation in pure, crystalline form [9]. Another important moment is the identification of the exact structure of capsaicin, which was communicated in 1919 by Nelson [10]. There are still recent studies that try to improve the isolation and purification 
of capsaicin from the capsaicinoid extract [11] reinforced by studies that reveal that there are clear regulations of the composition during fruit ripening [12]. In 1930, Späth and Darling synthesized capsaicin for the first time [13]. The 20th century has thus established capsaicin as a compound with various actions besides being a natural food additive $[14,15]$.

\section{Capsaicin and Neurogenic Inflammation}

Capsaicin (trans-8-methyl-N-vanillyl-6-noneamide) is a lipophilic substance, belonging to the class of vanilloids [16]; its molecular formula is $\mathrm{C}_{18} \mathrm{H}_{27} \mathrm{NO}_{3}$ and its molecular weight is $305.4 \mathrm{Da}$. Capsaicin is an agonist of the transient receptor potential vanilloid 1 receptor (TRPV1) which is a member of the transient receptor potential (TRP) family of cation channels [17].

Besides capsaicin, TRPV1 can be activated by temperatures of $43{ }^{\circ} \mathrm{C}$ or higher, by acidity $(\mathrm{pH}<6)$, endocanabinoids such as anandamide, metabolites of polyunsaturated fatty acids or other vanilloids [18]. Its function can also be modulated by inflammatory mediators, such as bradykinin and prostaglandin E2 with a facilitatory effect induced probably by protein kinases (PKC or PKA) -mediated receptor phosphorylation [19-21]. Other agents like nerve growth factor (NGF), catecholamines, histamine can also increase TRPV1 responses [22-24].

TRPV1 receptors are expressed in the central nervous system and in sensory neurons of the dorsal root ganglion, but also in non-neuronal tissues [25]. In the skin, TRPV1 is present in the unmyelinated type $\mathrm{C}$ and thin myelinated A-delta sensory nerve fibres, keratinocytes, mast cells, dermal blood vessels, fibroblasts, hair follicles, vascular smooth muscle cells, sebocytes and eccrine sweat glands [26-28]. TRPV1 might therefore play the role of extraneuronal receptor [29]. To date, it has been suggested that TRPV1 might play a role in mastocyte activation [30], release of proinflammatory mediators from keratinocytes [31] and modulation of proliferation, differentiation and apoptosis of keratinocytes from the outer root sheath [32].

Applied on the skin or oral mucosa, capsaicin induces initially a local burning sensation [26], followed by allodynia and hyperesthesia to mechanical and heat stimulation [33]. These nociceptive effects are associated with a transient local wheal and flare response known as neurogenic inflammation, triggered by the release of neuropeptides from the cutaneous sensory nerve endings (see Figure 1) [34,35]. Substance P (SP) and calcitonin-gene related peptide (CGRP) are recognized as the most important neuropeptides within neurogenic inflammation [36]. SP acts upon micro vascularization through its neurokinin-1 receptor (NK-1R) and has vasodilatory effects, increases vascular permeability and favors the release of pro-inflammatory cytokines [37], whilst CGRP induces microvascular dilatation resulting in increased blood flow [38]. Besides the neuropeptides release from nerve fibers, activation of mast cells has an important role in the capsaicin-induced inflammatory reaction [39]. Neuropeptides, with SP having the most significant effects, induce mast cell degranulation and synthesis of pro-inflammatory cytokines [40,41]. Mast cell mediators in turn activate nociceptors and further amplify the release of neuropeptides from the sensory nerves [39].

On the other hand, capsaicin blocks the axoplasmic transport of substance P and somatostatin in sensory neurons, thus depleting the neuropeptides $[6,42,43]$ and progressively reducing the initial local inflammatory effect, explaining the potential use of capsaicin in the treatment of chronic inflammatory skin diseases [28].

Moreover, subsequent applications of capsaicin lead to desensitization which is responsible for the analgesic effect of topical capsaicin [6,44] and its wide use in the treatment of neuropathic pain [45], post-herpetic neuralgia, diabetic neuropathy, post-surgical neuralgia, post-traumatic neuropathy and musculoskeletal pain [6,46].

Capsaicin can also have neurotoxic effects and can induce a gradual degeneration of cutaneous nerve fibers when used in high concentrations or for a long period of time [47-49].

Thus, capsaicin, depending on the duration and intensity of stimulation, can induce opposite effects, and the study of capsaicin-induced reactions has aroused the interest of both researchers and clinicians from a broad range of specialties. 


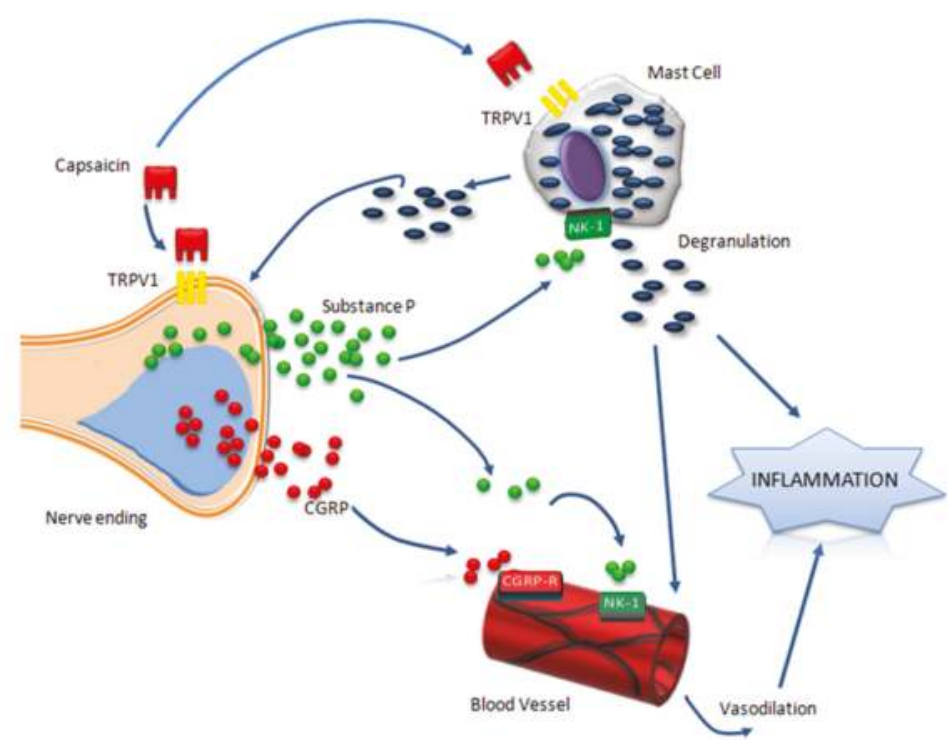

Figure 1. Capsaicin-induced inflammatory response is initiated by activation of transient receptor potential vanilloid 1 receptor(TRPV1) followed by the release of pro-inflammatory neuropeptides from nerve endings. Substance $\mathrm{P}(\mathrm{SP})$ and calcitonin-gene related peptide(CGRP), by activation of neurokinin-1 receptor (NK-1) and CGRP receptors, induce vasodilation, increased vascular permeability and release of pro-inflammatory cytokines. The released neuropeptides can induce degranulation of mast cells that play an important role in amplification of capsaicin-induced neurogenic inflammation.

\section{Capsaicin and Cancer}

Various studies have suggested a potential pro-carcinogenic role of capsaicin use [3] further supported by the potential connection between inflammation and tumorigenesis. In some cases, pro-inflammatory cytokines/chemokines can trigger malignant transformation and tumor associated inflammation in turn can promote proliferation and survival of malignant cells [50,51].

However, other recent studies indicate more to a protective effect against various types of cancer via different pathways, mostly unrelated to TRPV1 [3,52-60]. Thus, we will elaborate further on the capsaicin involvement in muco-cutaneous squamous cell carcinoma and melanoma, as the main malignancies where capsaicin has proven its involvement (see Table 1).

Table 1. Summarizing the carcinogenic and anti-carcinogenic effects of capsaicin, the primary pathway through which the effect is occurring, and the experimental model used to demonstrate the effect.

\begin{tabular}{cccc}
\hline Effect of Capsaicin & $\begin{array}{c}\text { Primary Pathway through Which } \\
\text { the Effect Is Occurring }\end{array}$ & $\begin{array}{c}\text { Model Used to Demonstrate the } \\
\text { Effect }\end{array}$ & References \\
\hline & $\begin{array}{c}\text { Mitochondrial pathway-dependent } \\
\text { apoptosis: } \downarrow \text { Bcl-2 } \uparrow \text { Bax, } \uparrow \text { Bad }\end{array}$ & $\begin{array}{c}\text { human pharyngeal SCC cells } \\
\text { (FaDu) }\end{array}$ & Le et al. [61] \\
\cline { 2 - 4 } Anticarcinogenic & $\begin{array}{c}\text { Induction of reactive oxygen species; } \\
\text { apoptosis independent oftransient } \\
\text { receptor potential vanilloid 1 receptor } \\
\text { (TRPV1) }\end{array}$ & $\begin{array}{c}\text { oral squamous cell carcinoma } \\
\text { (OSCC) cell lines }\end{array}$ & Gonzales et al. [62] \\
\cline { 2 - 4 } & $\begin{array}{c}\text { Nuclear factor kappaB (NF-kB), } \\
\text { activator protein 1 (AP-1) }\end{array}$ & $\begin{array}{c}\text { ICR mouse model; } \\
\text { humanpromyelocytic } \\
\text { leukemiaHL-60 cells }\end{array}$ & Han et al. [63] \\
\hline
\end{tabular}


Table 1. Cont.

\begin{tabular}{|c|c|c|c|}
\hline Effect of Capsaicin & $\begin{array}{l}\text { Primary Pathway through Which } \\
\text { the Effect Is Occurring }\end{array}$ & $\begin{array}{l}\text { Model Used to Demonstrate the } \\
\text { Effect }\end{array}$ & References \\
\hline & $\begin{array}{c}\text { Inhibition of the cytochrome P-450 } \\
\text { IIE1 isoform }\end{array}$ & ICR mouse model & Surh et al. [64] \\
\hline & $\begin{array}{l}\downarrow \text { nicotinamide adenine dinucleotide } \\
\text { (NADH) oxidase activity; } \uparrow \text { apoptosis }\end{array}$ & $\begin{array}{l}\text { A375, SK-MEL-28 human } \\
\text { melanoma cell lines; B16 murine } \\
\text { melanoma cell line }\end{array}$ & Morré et al. [65] \\
\hline & $\begin{array}{l}\downarrow \text { nicotinamide adenine dinucleotide } \\
\text { phosphate-reduced(NAD(P)H): } \\
\text { quinone oxidoreductase ; } \downarrow \text { NF- } \kappa \mathrm{B}\end{array}$ & $\begin{array}{l}\text { CRL } 1585 \text { and CRL } 1619 \text { human } \\
\text { melanoma cell lines }\end{array}$ & Brar et al. [66] \\
\hline & $\begin{array}{l}\text { لactivation of constitutive and } \\
\text { IL-1beta-induced NF- } \mathrm{KB}\end{array}$ & Human melanoma cells & Patel et al. [67] \\
\hline & $\begin{array}{c}\uparrow p 53 \text {, induces apoptosis via Bcl-2, Bax, } \\
\text { caspases } 3,8,9\end{array}$ & A375 human melanoma cell line & Kim [68] \\
\hline & $\begin{array}{l}\text { Down-regulation of PI3-K/Akt } \\
\text { pathway }\end{array}$ & B16-F10 mouse melanoma cells & Shin et al. [69] \\
\hline & $\begin{array}{l}\text { Downregulation of Bcl-2; induction of } \\
\text { apoptosis }\end{array}$ & B16-F10 mouse melanoma cells & Jun et al. [60] \\
\hline & $\begin{array}{c}\downarrow \text { caspase-activated DNase } \\
\text { inhibitor(ICAD)expression; induction } \\
\text { of apoptosis }\end{array}$ & $\begin{array}{l}\text { human melanoma A375-S2 cell } \\
\text { line }\end{array}$ & Gong et al. [70] \\
\hline & Induction of apoptosis & $\begin{array}{l}\text { melanocytes and HBL, A375SM, } \\
\text { C8161 melanoma cell lines }\end{array}$ & Marques et al. [71] \\
\hline & Delays tumor growth & melanoma B16-F10; mouse model & Schwartz et al. [72] \\
\hline \multirow{4}{*}{ Cocarcinogenic } & $\begin{array}{l}\text { Epidermal growth factor } \\
\text { receptor(EGFR) pathway }\end{array}$ & DMBA/TPA mouse model & Bode et al. [73] \\
\hline & $\begin{array}{c}\text { EGFR pathway; } \uparrow \text { cyclo-oxygenase- } 2 \\
\text { (COX-2) }\end{array}$ & DMBA/TPA mouse model & Hwang et al. [25] \\
\hline & EGFR/Akt/mTOR signaling pathway & DMBA/TPA mouse model & Li et al. [74] \\
\hline & Erk/p38 signaling pathway & DMBA/TPA mouse model & Liu et al. [75] \\
\hline
\end{tabular}

\subsection{The Impact of Capsaicin on Muco-Cutaneous Squamous Cell Carcinoma}

Muco-cutaneous squamous cell carcinoma is one of the most frequent malignancies among Caucasians and its incidence has increased in the last decades, probably due to lifestyle changes and the increased proportion of aged populations [76-79]. Muco-cutaneous squamous cell carcinoma is responsible for most deaths associated with non-melanoma muco-cutaneous cancer. It may generate major defects both aesthetically and functionally and require a complex therapeutic approach, depending on the stage of the disease and the general status of the patient [76-81]. For that reason, muco-cutaneous squamous cell carcinoma is an important public health problem and new therapeutic approaches are necessary [82-90].

The most important risk factors for the development of muco-cutaneous squamous cell carcinoma are fair skin type, chronic exposure to ultraviolet radiation (UVR), exposure to ionizing radiation, smoking, exposure to chemical carcinogens, human papillomavirus (HPV) infections and genetic predisposition [80,81,87-89,91-93].

Moreover, various studies have shown that neuroendocrine factors might play a role in the development of muco-cutaneous squamous cell carcinoma [94]. The release of CGRP and substance P, as well as other neuropeptides, from unmyelinated c-fibres and myelinated A delta-fibres of sensory nerves, a well-known effect triggered by capsaicin is also induced by UVR exposure and may contribute to induction of carcinogenesis [94,95]. CGRP has important vasodilatory effects on small and large vessels, potentiates microvascular permeability and edema caused by SP, enhances in vitro keratinocyte and melanocyte proliferation and is a potent immunomodulator [94-97]. By impairing the function of 
cutaneous macrophages and Langerhans cells, CGRP is a potent inhibitor of acute and delayed type hypersensitivity reactions [95] but also interferes with anti-tumoral immune response initiation [94].

SP is a member of the tachykinin family which has vasodilatory effects, induces protein extravasation, lymphocyte proliferation, chemotaxis, activates macrophages and promotes the secretion of interleukin 1 (IL-1), IL-6and TNF- $\alpha[94,95,98]$. It has been associated with stress induced mast cell activation [41]. The effects of SP are mediated through NK-1R, which is widely expressed in the brain, skin, intestine, lung and immune cells [94,95]. There is some evidence that SP and NK1-R might be involved in the development and progression of cancer. Thus, SP has been associated with cell proliferation and migration in esophageal squamous cell carcinoma (SCC) [99], melanoma [100,101], retinoblastoma [102], neuroblastoma and glioma [103]. Brener et al. investigated the presence of SP and NK-1R in 93 oral SCC from 73 patients and concluded that the SP/NK-1R system might have a role in tumor development and progression [104]. Other authors studied the distribution of SP and NK-1R in esophageal SCC and found a higher density of SP positive nerve fibres and NK-1R expression in carcinoma cells, thus concluding that SP and NK-1R promote growth and migration of esophageal SCC cells [99]. Considering the evidence regarding the role of SP in the development of the disease some authors suggested that NK-1R antagonists might be useful in the treatment of oral cancer [104].

Taking into consideration the complex neuro-immune impact of capsaicin and the potential link between inflammation and carcinogenesis, the effect of capsaicin on muco-cutaneous cancer has aroused a growing interest. Since several reports indicated that the consumption of chili peppers might be associated with an increased risk of cancer [105], some authors studied the effect of long term capsaicin treatment. Toth and Gannett found that, after a lifelong diet with capsaicin, $22 \%$ of female mice and $14 \%$ of male mice had tumors of the cecum. In the control group only $8 \%$ of mice had cecum tumors [106]. Chanda et al. assessed the oncogenic potential of topical trans-capsaicin applied for 26 weeks in Tg.AC mice. The Tg.AC mice received trans-capsaicin dissolved in diethylene glycol monoethyl ether (DGME). Mice from the positive control group received tetradecanoylphorbol-13-acetate (TPA) dissolved in DGME and controls received lidocaine. The authors found that topical capsaicin was not associated with an increased incidence of preneoplastic and neoplastic lesions as compared to the concurrent vehicle or lidocaine while the TPA treated mice had multiple skin papillomas. The authors therefore concluded that trans-capsaicin, lidocaine and DGME should be considered non-oncogenic [107].

Le et al. studied the effect of capsaicin on human pharyngeal SCC cells ( $\mathrm{FaDu})$ and found that capsaicin inhibits growth and proliferation in a time and dose dependent manner and induces apoptosis via mitochondrial pathways [61]. The authors also analyzed the expression of the anti-apoptotic Bcl-2 gene and the pro-apoptotic Bax and Bad genes and found a reduction of Bcl-2 gene and enhanced expression of Bax and Bad genes [61]. Gonzales et al. studied the anti-tumor effect of capsaicin, a TRPV1 agonist, and capsazepine, a TRPV1 antagonist, on oral squamous cell carcinoma (OSCC) cell lines; the authors found that capsaicin alone reduced cell viability [62]. The association of capsazepine and capsaicin not only did not reverse the effect of capsaicin but capsazepine alone was also cytotoxic to tumor cells; the authors therefore concluded that the antiapoptotic effect of vanilloids is independent of TRPV1 and suggested that the induction of reactive oxygen species is responsible for apoptosis [62].

Han et al. showed in a study published in 2002 that topical application of capsaicin on the skin of female ICR mice suppresses phorbol ester-induced activation of nuclear factor kappaB (NF- $\mathrm{KB}$ ) and activator protein 1 (AP-1) and concluded that this might be responsible for the chemopreventive effects of capsaicin [63]. These results are congruent with findings previously reported by another group of authors [108-111]. Surh et al. studied the chemoprotective effect of capsaicin against tumorigenesis and mutagenesis produced by vinyl carbamate (VC) and N-nitrosodimethylamine(NDMA) also on female ICR mice [64]. The authors found that topical capsaicin pre-treatment lowered the number of VC-induced tumors by $60 \%$ and hypothesized that capsaicin suppresses tumorigenesis and mutagenesis by inhibiting cytochrome P-450 IIE1 isoform [64]. 


\subsection{The Effect of Capsaicin on Melanoma}

Melanoma is a malignant tumor that arises from melanocytes; melanocytes are melanin-producing cells situated in the basal layer of epidermis, in uveal structures of the eye and in the meninges; of all possible sites, skin is the most frequent location of melanoma $[87,88]$. Even though melanoma is less frequent than most malignant cutaneous tumors (i.e., basal cell carcinoma, squamous cell carcinoma), it has the most aggressive course, accounting for more than $75 \%$ of all skin cancer deaths. Melanoma can occur at any age but it is more frequent between 30 and 70 years; females are more frequently affected than males (male:female ratio 1:1.5) [88,89]. The incidence of melanoma has been on the rise worldwide in the last decades. Excessive ultraviolet radiation exposure (from both sun and artificial sources-e.g., tanning beds) especially under the age of 20, skin phototypes I and II (light skin pigmentation), genetic predisposition, increased number of melanocytic nevi and the presence of atypical nevi are the main risk factors for developing melanoma. Most melanomas occur de novo $[90,112,113]$.

The treatment of melanoma varies depending on the stage of the disease. Surgical excision is the mainstay treatment for primary melanoma. Metastatic melanomas however require chemotherapy, immunotherapy or palliative treatment. These are usually associated with severe adverse reactions and low response rates $[87,88]$. Therefore, new drugs as well as new ways of investigating their efficacy have been elaborated [114]. The prognosis of patients with metastatic melanoma was improved after the introduction of BRAF(B-Rafenzyme) inhibitors (vemurafenib, dabrafenib), mitogen-activated protein/extracellular signal-regulated kinase kinase(MEK) inhibitors (trametinib) and immune checkpoint inhibitors (nivolumab, ipilimumab) $[113,115]$. These therapies however are very expensive and are not available for all the patients $[113,115]$.

Under these circumstances, there is a real need to identify new therapeutic targets in order to develop cheaper, but efficient, treatment options. Hence, the mechanisms behind the development and progression of melanoma were intensely studied and recent reports showed that neuro-endocrine factors might be involved [100,101,116,117]. Several studies have investigated the potential role of NK-1R and SP, one of the main neuropeptides involved in capsaicin-induced inflammatory reaction. A recent study performed on canine melanoma tissues and cell lines found that 11 of 15 tumors revealed NK-1R immunoreactivity [118]. The expression of SP in malignant melanoma and melanoma precursors was also studied and the authors showed that $68 \%$ of primary invasive melanomas, $40 \%$ of metastatic melanomas, $60 \%$ of in situ melanomas and $58 \%$ of dysplastic nevi express the neuropeptide [119]. SP and NK-1R are also involved in melanogenesis [120]. B16-F10 melanoma cells treatment with SP results in activation of NK-1R, phosphorylation of p70 S6K1, inhibition of p38mitogen-activated protein kinase(MAPK), down-regulation in tyrosinase activity and suppression of melanogenesis [121]. There is increasing evidence regarding the involvement of SP and NK-1R in melanoma cells proliferation $[100,101,122,123]$. For that reason, NK-1R is now regarded as a target in melanoma treatment and NK-1R antagonists are being intensely studied [100,101,122,123].

The direct role of capsaicin in the treatment of melanoma was investigated in several studies, as explained further [65-72,124-129]. Morré et al. studied the effect of capsaicin on nicotinamide adenine dinucleotide(NADH) oxidase activity of plasma membranes and cell growth of human primary melanocytes and melanoma cells (A-375 and SK-MEL-28 cell cultures) [65]. The authors found that capsaicin inhibits plasma membrane NADH oxidase activity preferentially in melanoma cells thus inhibiting growth and increasing apoptosis [65]. Brar et al. also showed in a study performed on human melanoma cell lines that reactive oxygen species produced endogenously from nicotinamide adenine dinucleotide phosphate-reduced( $\mathrm{NAD}(\mathrm{P}) \mathrm{H})$ :quinone oxidoreductase activate $\mathrm{NF}-\mathrm{\kappa B}$ in melanoma cells in an autocrine fashion and that capsaicin significantly reduces proliferation of melanoma cells [66].

Patel et al. showed in a study published in 2002 that the NF- $\mathrm{kB}$ activation regulates the expression of IL-8 in melanoma cells and that the addition of capsaicin determines the inhibition of constitutive and IL1-beta and TNF- $\alpha$ induced IL-8 expression in melanoma cells [67]. In melanoma, IL-8 over-expression is associated with the transition from radial growth phase to vertical growth phase and with the 
development of metastases [124,125]. Capsaicin is a potent inhibitor of NF-kB. It suppresses the activation of NF- $\kappa B$ by inhibiting $I \kappa B \alpha$ (nuclear factor of kappa light polypeptide gene enhancer in B-cells inhibitor, alpha) degradation and blocking the translocation of p65 in human promyelocytic leukaemia HL-60 cells $[63,126]$.

In a study published in 2012, Kim aimed to explain the mechanism by which capsaicin induces apoptosis in melanoma cells [68]. The author therefore studied the role of nitric oxide (NO) during apoptosis induced by capsaicin and resveratrol on A375 human melanoma cells and found that NO stimulates p53 and induces conformational changes in Bax and Bcl-2 and activates caspases 3, 8 and 9 . The authors concluded that capsaicin and resveratrol activate the mitochondrial and death receptor pathways [68].

In a study published in 2008 , Shin et al. evaluated the effects of capsaicin on highly metastatic B16-F10 mouse melanoma cells and found that capsaicin inhibits migration of melanoma cells in a dose-dependent manner [69]. The authors also found that capsaicin decreases the phosphorylation of the p85 regulatory subunit of phosphatidylinositol 3-kinase (PI3-K) and Akt and concluded that capsaicin down-regulates the PI3-K/Akt pathway. Furthermore, the authors found that capsaicin inhibits the Rac1 activity [69]. The PI3-K/Akt pathway is one of the main signaling networks in cancer and plays an important role in melanoma initiation and in therapeutic resistance $[127,128]$. Rac1 is involved in cell migration and metastasis [129]. Jun et al. also studied the effect of capsaicin on B16-F10 murine melanoma cells. The authors found that capsaicin determines release of mitochondrial cytochrome c, activation of caspase-3 and cleavage of poly (ADP-ribose) polymerase and finally induces apoptosis of melanoma cells through down regulation of Bcl-2 [60]. Other studies have observed induction of apoptosis by capsaicin in melanoma cells, as well: Gong et al. showed, in a study performed on human melanoma A375-S2 cells, that capsaicin induces melanoma cell death in a time and dose dependent manner by reducing the expression of inhibitor of caspase activated DNase (ICAD); ICAD expression was decreased over the lapse of time, as cell treated with capsaicin progressed into apoptotic stages [70]. Some authors studied the combined effect of capsaicin and other agents on melanoma cells [71,72]. Marques et al. investigated the apoptotic effect of capsaicin and HA14-1, a small molecular compound that inhibits the anti-apoptotic effect of Bcl-2, on melanoma cells, melanocytes and fibroblasts [71]. The authors found that capsaicin induces apoptosis in melanocytes and HBL, A375SM and C8161 melanoma cell lines at lower concentrations than in fibroblasts and that the capsaicin and HA14-1 combination shows additive inhibitory effect on melanoma and melanocyte viability, inducing apoptosis in two of the three studied melanoma cell lines [71]. The authors concluded that capsaicin can be associated with other organic compounds as a pro-apoptotic agent to reduce toxicity and adverse reactions [71]. Schwartz et al. studied the combined effect of hydroxycitrate, lipoic acid and capsaicin on lung cancer cells, bladder cancer cells and melanoma cells and found that the association of these drugs is effective in inducing tumor regression and lacks toxicity [72].

Taking into account the increasing evidence regarding its anti-carcinogenic role, expanding the research on capsaicin actions may lead to identification of potential new therapeutic pathways.

\subsection{Capsaicin's Involvement in Carcinogenesis}

A potential co-carcinogenic role of capsaicin has aroused the interest of various researchers. A study published in 2009, showed that TRPV1 interacts with the epidermal growth factor receptor (EGFR) and determines its degradation though the lysosomal pathway [73]. EGFR is a receptor tyrosine kinase with an important role in the development of the epidermis, which is overexpressed in many epithelial cancers. Using a skin carcinogenesis model with 7,12-dimethyl benz(a)anthracene (DMBA) and TPA in TRPV1 ${ }^{-/-}$(knockout) and TRPV1 ${ }^{+/+}$(wild type) mice, authors have shown that TRPV1 ${ }^{-/-}$mice developed significantly more skin tumors than TRPV1 ${ }^{+/+}$mice [73]. Moreover, to assess to role of EGFR in skin carcinogenesis, the authors performed the same experiment, except that some of the mice received an EGFR inhibitor; the scientists discovered that carcinogenesis was substantially more suppressed in TRPV1 ${ }^{-/}$mice, after EGFR inhibitor was administered [73]. 
Another study published in 2010 showed that topical application of capsaicin on the skin of TRPV1 wildtype mice and TRPV1 knockout mice, which were previously subjected to the two-stage skin carcinogenesis experiment with DMBA (9,10-Dimethyl-1,2-benzanthracene) and TPA, was associated with significantly more and larger tumors than TPA treatment alone [25]. TRPV1 knockout mice were more affected than TRPV1 wildtype mice. Mice treated with capsaicin alone however have not developed any tumors. These findings suggest that carcinogenesis has a TRPV1 independent mechanism. Further research revealed higher levels of COX-2(cyclo-oxygenase-2) in mice treated with capsaicin and TPA than in mice treated with TPA alone thus suggesting that capsaicin induces an increased COX-2 expression in the presence of TPA. COX-2 expression was increased in EGFR wildtype cells but not in EGFR knockout cells. The authors therefore suggest that capsaicin acts as a co-carcinogen through EGFR dependent mechanisms/pathways [25].

The link between capsaicin receptor and skin tumorigenesis was the subject of an experimental in vivo research which found that topical application of TRPV1-antagonist AMG9810[(E)-3-(4-t-Butylphenyl)-N-(2,3-dihydrobenzo[b][1,4] dioxin-6-yl)acrylamide]promotes tumor development in mice previously treated with DMBA. The levels of EGFR were also higher in these mice as compared to the control group. Moreover, the phosphorylation level of EGFR was significantly increased in AMG9810 treated mice compared to the control groups. EGFR phosphorylation activates the Akt/mTOR-signaling pathway which has an important role in tumorigenesis. It was therefore concluded that the TRPV1 antagonist induces carcinogenesis by activating the EGFR/Akt/mTOR signaling pathway [74].

Liu et al. also studied the effect of topical applications of capsaicin on the dorsal skin of mice in which carcinogenesis was induced by DMBA/TPA. The authors showed that capsaicin led to the appearance of more numerous and larger skin tumors as compared to the control group and suggested that Erk, p38 and inflammation may play an important role in the cancer-promoting effect of capsaicin [75].

All these findings suggest that, even though capsaicin itself is not a carcinogen, long-term application of capsaicin for pain relief might increase the risk of carcinogenesis when it is associated with a tumor promoter [73].

\section{Conclusions}

Capsaicin is one of the most commonly used spices in the world and its analgesic and anti-inflammatory properties have been known for centuries. Short term administration of capsaicin has the ability to trigger the release of neuropeptides like SP and CGRP which might play a role in tumorigenesis. However, chronic administration of capsaicin progressively reduces the initial inflammatory reaction, leading to desensitization or even to neurotoxic effects, depending on the duration and intensity of applications.

In recent years various studies have focused on the potential impact of capsaicin on tumorigenesis, investigating both the anti-carcinogenic and carcinogenic actions of capsaicin. Data available so far regarding the effect of capsaicin on various types of skin cancers suggests that capsaicin has a chemopreventive role. Sinceseveral authors showed that under certain circumstances capsaicin can have a pro-tumorgenic potential, caution is mandatory when capsaicin is administered in conditions that favor tumorigenesis as it might have a co-carcinogenic effect.

Acknowledgments: This paper was partially supported by grant COP A 1.2.3., ID: P_40_197/2016, grant of the Romanian National Authority for Scientific Research and Innovation and by Young Researchers Grants from the Carol Davila University of Medicine and Pharmacy, No. 33884/11.11.2014 and 33897/11.11.2014.

Author Contributions: All authors have equally contributed in the design and preparation of the manuscript.

Conflicts of Interest: The authors declare no conflict of interest. 


\section{References}

1. Barceloux, D.G. Pepper and capsaicin (Capsicum and Piper species). Dis. Mon. 2009, 55, 380-390. [CrossRef] [PubMed]

2. Kiple, K.F. The Cambridge World History of Food.(2-Volume Set); Cambridge University Press: Cambridge, UK, 2001; Volume 2.

3. Bode, A.M.; Dong, Z. The two faces of capsaicin. Cancer Res. 2011, 71, 2809-2814. [CrossRef] [PubMed]

4. Srivastava, S.K. Role of Capsaicin in Oxidative Stress and Cancer; Springer Science \& Business Media: Berlin/Heidelberg, Germany, 2013; Volume 3.

5. Dasgupta, P.; Fowler, C.J. Chillies: From antiquity to urology. BJU Int. 1997, 80, 845-852. [CrossRef]

6. Sharma, S.K.; Vij, A.S.; Sharma, M. Mechanisms and clinical uses of capsaicin. Eur. J. Pharmacol. 2013, 720, 55-62. [CrossRef] [PubMed]

7. Abdel-Salam, O.M. Capsaicin as a Therapeutic Molecule; Springer Science \& Business Media: Berlin/Heidelberg, Germany, 2014; Volume 68.

8. Papoiu, A.D.; Yosipovitch, G. Topical capsaicin. The fire of a 'hot' medicine is reignited. Expert Opin. Pharmacother. 2010, 11, 1359-1371. [CrossRef] [PubMed]

9. Thresh, L.T. Isolation of capsaicin. Pharm. J. 1846, 6, 941-942.

10. Nelson, E.K. The constitution of capsaicin, the pungent principle of capsicum. J. Am. Chem. Soc. 1919, 41, 1115-1121. [CrossRef]

11. Fan, Y.; Lu, Y.M.; Yu, B.; Tan, C.P.; Cui, B. Extraction and purification of capsaicin from capsicum oleoresin using an aqueous two-phase system combined with chromatography. J. Chromatogr. B. 2017, 1063, 11-17. [CrossRef] [PubMed]

12. Fayos, O.; De Aguiar, A.C.; Jiménez-Cantizano, A.; Ferreiro-González, M.; Garcés-Claver, A.; Martínez, J.; Mallor, C.; Ruiz-Rodríguez, A.; Palma, M.; Barroso, C.G.; et al. Ontogenetic Variation of Individual and Total Capsaicinoids in Malagueta Peppers (Capsicum frutescens) during Fruit Maturation. Molecules 2017, 22, 736. [CrossRef] [PubMed]

13. Späth, E.; Darling, S.F. Synthese des capsaicins. Eur. J. Inorg. Chem. 1930, 63, 737-743. [CrossRef]

14. Zheng, J.; Zhou, Y.; Li, Y.; Xu, D.P.; Li, S.; Li, H.B. Spices for Prevention and Treatment of Cancers. Nutrients 2016, 8, 495. [CrossRef] [PubMed]

15. Qin, Y.; Ran, L.; Wang, J.; Yu, L.; Lang, H.D.; Wang, X.L.; Mi, M.T.; Zhu, J.D. Capsaicin Supplementation Improved Risk Factors of Coronary Heart Disease in Individuals with Low HDL-C Levels. Nutrients 2017, 9, 1037. [CrossRef] [PubMed]

16. Reyes-Escogido, M.D.L.; Gonzalez-Mondragon, E.G.; Vazquez-Tzompantzi, E. Chemical and pharmacological aspects of capsaicin. Molecules 2011, 16, 1253-1270. [CrossRef] [PubMed]

17. Caterina, M.J.; Schumacher, M.A.; Tominaga, M.; Rosen, T.A.; Levine, J.D.; Julius, D. The capsaicin receptor: A heat-activated ion channel in the pain pathway. Nature 1997, 389, 816-824. [PubMed]

18. Tominaga, M.; Tominaga, T. Structure and function of TRPV1. Pflügers. Arch. 2005, 451, 143-150. [CrossRef] [PubMed]

19. Premkumar, L.S.; Ahern, G.P. Induction of vanilloid receptor channel activity by protein kinase C. Nature 2000, 408, 985-990. [CrossRef] [PubMed]

20. Sugiura, T.; Tominaga, M.; Katsuya, H.; Mizumura, K. Bradykinin lowers the threshold temperature for heat activation of vanilloid receptor 1. J. Neurophysiol. 2002, 88, 544-548. [PubMed]

21. Schnizler, K.; Shutov, L.P.; Van Kanegan, M.J.; Merrill, M.A.; Nichols, B.; McKnight, G.S.; Usachev, Y.M. Protein kinase A anchoring via AKAP150 is essential for TRPV1 modulation by forskolin and prostaglandin E2 in mouse sensory neurons. J. Neurosci. 2008, 28, 4904-4917. [CrossRef] [PubMed]

22. Filippi, A.; Caruntu, C.; Gheorghe, R.O.; Deftu, A.; Amuzescu, B.; Ristoiu, V. Catecholamines reduce transient receptor potential vanilloid type 1 desensitization in cultured dorsal root ganglia neurons. J. Physiol. Pharmacol. 2016, 67, 843-850. [PubMed]

23. Shu, X.; Mendell, L.M. Nerve growth factor acutely sensitizes the response of adult rat sensory neurons to capsaicin. Neurosci. Lett. 1999, 274, 159-162. [CrossRef]

24. Kajihara, Y.; Murakami, M.; Imagawa, T.; Otsuguro, K.; Ito, S.; Ohta, T. Histamine potentiates acid-induced responses mediating transient receptor potential V1 in mouse primary sensory neurons. Neuroscience 2010, 166, 292-304. [CrossRef] [PubMed] 
25. Hwang, M.K.; Bode, A.M.; Byun, S.; Song, N.R.; Lee, H.J.; Lee, K.W.; Dong, Z. Cocarcinogenic effect of capsaicin involves activation of EGFR signaling but not TRPV1. Cancer Res. 2010, 70, 6859-6869. [CrossRef] [PubMed]

26. Căruntu, C.; Negrei, C.; Ghiţă, M.A.; Căruntu, A.; Bădărău, A.I.; Buraga, I.; Brănişteanu, D. Capsaicin, a hot topic in skin pharmacology and physiology. Farmacia 2015, 63, 487-491.

27. Inoue, K.; Koizumi, S.; Fuziwara, S.; Denda, S.; Inoue, K.; Denda, M. Functional vanilloid receptors in cultured normal human epidermal keratinocytes. Biochem. Biophys. Res. Commun. 2002, 291, 124-129. [CrossRef] [PubMed]

28. Ständer, S.; Moormann, C.; Schumacher, M.; Buddenkotte, J.; Artuc, M.; Shpacovitch, V.; Metze, D. Expression of vanilloid receptor subtype 1 in cutaneous sensory nerve fibers, mast cells, and epithelial cells of appendage structures. Exp. Dermatol. 2004, 13, 129-139. [CrossRef] [PubMed]

29. Roosterman, D.; Goerge, T.; Schneider, S.W.; Bunnett, N.W.; Steinhoff, M. VI. Role of Capsaicin and transient receptor potential ion channels in the skin. Physiol. Rev. 2006, 86, 1338-1380.

30. Bíró, T.; Maurer, M.; Modarres, S.; Lewin, N.E.; Brodie, C.; Ács, G.; Blumberg, P.M. Characterization of functional vanilloid receptors expressed by mast cells. Blood 1998, 91, 1332-1340. [PubMed]

31. Southall, M.D.; Li, T.; Gharibova, L.S.; Pei, Y.; Nicol, G.D.; Travers, J.B. Activation of epidermal vanilloid receptor-1 induces release of proinflammatory mediators in human keratinocytes. J. Pharmacol. Exp. Ther. 2003, 304, 217-222. [CrossRef] [PubMed]

32. Bodó, E.; Bíró, T.; Telek, A.; Czifra, G.; Griger, Z.; Tóth, B.I.; Paus, R. A hot new twist to hair biology: Involvement of vanilloid receptor-1 (VR1/TRPV1) signaling in human hair growth control. Am. J. Pathol. 2005, 166, 985-998.

33. Geppetti, P.; Nassini, R.; Materazzi, S.; Benemei, S. The concept of neurogenic inflammation. BJU Int. 2008, 101, 2-6. [CrossRef] [PubMed]

34. Căruntu, C.; Boda, D. Evaluation through in vivo reflectance confocal microscopy of the cutaneous neurogenic inflammatory reaction induced by capsaicin in human subjects. J. Biomed. Opt. 2012, 17, 0850031-0850037. [CrossRef] [PubMed]

35. Ghita, M.A.; Caruntu, C.; Lixandru, D.; Pitea, A.; Batani, A.; Boda, D. The Quest for Novel Biomarkers in Early Diagnosis of Diabetic Neuropathy. Curr. Proteom. 2017, 14, 86-99. [CrossRef]

36. Holzer, P. Local effector functions of capsaicin-sensitive sensory nerve endings: Involvement of tachykinins, calcitonin gene-related peptide and other neuropeptides. Neuroscience 1998, 24, 739-768. [CrossRef]

37. Schmelz, M.; Petersen, L.J. Neurogenic inflammation in human and rodent skin. News Physiol. Sci. 2001, 16, 33-37. [PubMed]

38. Brain, S.D.; Tippins, J.R.; Morris, H.R.; Maclntyre, I.; Williams, T.J. Potent vasodilator activity of calcitonin gene-related peptide in human skin. J. Investig. Dermatol. 1986, 87, 533-536. [CrossRef] [PubMed]

39. Ansel, J.C.; Kaynard, A.H.; Armstrong, C.A.; Olerud, J.; Bunnett, N.; Payan, D. Skin-nervous system interactions. J. Investig. Dermatol. 1996, 106, 198-204. [CrossRef] [PubMed]

40. Ansel, J.C.; Brown, J.R.; Payan, D.G.; Brown, M.A. Substance P selectively activates TNF-alpha gene expression in murine mast cells. J. Immunol. 1993, 150, 4478-4485. [PubMed]

41. Căruntu, C.; Boda, D.; Musat, S.; Căruntu, A.; Mandache, E. Stress-induced mast cell activation in glabrous and hairy skin. Mediat. Inflamm. 2014, 2014, 105950. [CrossRef] [PubMed]

42. Anand, P.; Bley, K. Topical capsaicin for pain management: Therapeutic potential and mechanisms of action of the new high-concentration capsaicin 8\% patch. Br. J. Anaesth. 2011, 107, 490-502. [CrossRef] [PubMed]

43. Frias, B.; Merighi, A. Capsaicin, nociception and pain. Molecules 2016, 21, 797. [CrossRef] [PubMed]

44. Smutzer, G.; Devassy, R.K. Integrating TRPV1 receptor function with capsaicin psychophysics. Adv. Pharmacol. Sci. 2016, 2016, 1512457. [CrossRef] [PubMed]

45. Peppin, J.F.; Pappagallo, M. Capsaicinoids in the treatment of neuropathic pain: A review. Ther. Adv. Neurol. Disord. 2014, 7, 22-32. [CrossRef] [PubMed]

46. Fattori, V.; Hohmann, M.S.; Rossaneis, A.C.; Pinho-Ribeiro, F.A.; Verri, W.A. Capsaicin: Current understanding of its mechanisms and therapy of pain and other pre-clinical and clinical uses. Molecules 2016, 21, 844. [CrossRef] [PubMed]

47. Holzer, P. Capsaicin: Cellular targets, mechanisms of action, and selectivity for thin sensory neurons. Pharmacol. Rev. 1991, 43, 143-201. [PubMed]

48. Simone, D.A.; Nolano, M.; Wendelschafer-Crabb, G.; Kennedy, W.R. Intradermal injection of capsaicin in humans: Diminished pain sensation associated with rapid degeneration of intracutaneous nerve fibers. Soc. Neurosci. Abstr. 1996, 22, 1802. 
49. Simone, D.A.; Nolano, M.; Johnson, T.; Wendelschafer-Crabb, G.; Kennedy, W.R. Intradermal injection of capsaicin in humans produces degeneration and subsequent reinnervation of epidermal nerve fibers: Correlation with sensory function. J. Neurosci. 1998, 18, 8947-8959. [PubMed]

50. Neagu, M.; Caruntu, C.; Constantin, C.; Boda, D.; Zurac, S.; Spandidos, D.A.; Tsatsakis, A.M. Chemically induced skin carcinogenesis: Updates in experimental models. Oncol. Rep. 2016, 35, 2516-2528. [CrossRef] [PubMed]

51. Neagu, M.; Constantin, C.; Dumitrascu, G.R.; Lupu, A.R.; Caruntu, C.; Boda, D.; Zurac, S. Inflammation markers in cutaneous melanoma-edgy biomarkers for prognosis. Discoveries 2015, 3, e38. [CrossRef]

52. Mori, A.; Lehmann, S.; O’Kelly, J.; Kumagai, T.; Desmond, J.C.; Pervan, M.; Koeffler, H.P. Capsaicin, a component of red peppers, inhibits the growth of androgen-independent, p53 mutant prostate cancer cells. Cancer Res. 2006, 66, 3222-3229. [CrossRef] [PubMed]

53. Zhang, J.H.; Lai, F.J.; Chen, H.; Luo, J.; Zhang, R.Y.; Bu, H.Q.; Lin, S.Z. Involvement of the phosphoinositide 3-kinase/Akt pathway in apoptosis induced by capsaicin in the human pancreatic cancer cell line PANC-1. Oncol. Lett. 2013, 5, 43-48. [PubMed]

54. Lee, Y.S.; Kang, Y.S.; Lee, J.S.; Nicolova, S.; Kim, J.A. Involvement of NADPH oxidase-mediated generation of reactive oxygen species in the apototic cell death by capsaicin in HepG2 human hepatoma cells. Free Radic. Res. 2004, 38, 405-412. [CrossRef] [PubMed]

55. Lu, H.F.; Chen, Y.L.; Yang, J.S.; Yang, Y.Y.; Liu, J.Y.; Hsu, S.C.; Chung, J.G. Antitumor activity of capsaicin on human colon cancer cells in vitro and colo 205 tumor xenografts in vivo. J. Agric. Food Chem. 2010, 58, 12999-13005. [CrossRef] [PubMed]

56. Chang, H.C.; Chen, S.T.; Chien, S.Y.; Kuo, S.J.; Tsai, H.T.; Chen, D.R. Capsaicin may induce breast cancer cell death through apoptosis-inducing factor involving mitochondrial dysfunction. Hum. Exp. Toxicol. 2011, 30, 1657-1665. [CrossRef] [PubMed]

57. Huh, H.C.; Lee, S.Y.; Lee, S.K.; Park, N.H.; Han, I.S. Capsaicin induces apoptosis of cisplatin-resistant stomach cancer cells by causing degradation of cisplatin-inducible Aurora-A protein. Nutr. Cancer 2011, 63, 1095-1103. [CrossRef] [PubMed]

58. Wang, H.M.; Chuang, S.M.; Su, Y.C.; Li, Y.H.; Chueh, P.J. Down-regulation of tumor-associated NADH oxidase, tNOX (ENOX2), enhances capsaicin-induced inhibition of gastric cancer cell growth. Cell Biochem. Biophys. 2011, 61, 355-366. [CrossRef] [PubMed]

59. Moon, D.O.; Kang, C.H.; Kang, S.H.; Choi, Y.H.; Hyun, J.W.; Chang, W.Y.; Kim, G.Y. Capsaicin sensitizes TRAIL-induced apoptosis through Sp1-mediated DR5 up-regulation: Involvement of Ca2+influx. Toxicol. Appl. Pharmacol. 2012, 259, 87-95. [CrossRef] [PubMed]

60. Jun, H.S.; Park, T.; Lee, C.K.; Kang, M.K.; Park, M.S.; Kang, H.I.; Kim, O.H. Capsaicin induced apoptosis of B16-F10 melanoma cells through down-regulation of Bcl-2. Food Chem. Toxicol. 2007, 45, 708-715. [CrossRef] [PubMed]

61. Le, T.D.; Jin, D.C.; Rho, S.R.; Kim, M.S.; Yu, R.; Yoo, H. Capsaicin-induced apoptosis of FaDu human pharyngeal squamous carcinoma cells. Yonsei Med. J. 2012, 53, 834-841. [CrossRef] [PubMed]

62. Gonzales, C.B.; Kirma, N.B.; Jorge, J.; Chen, R.; Henry, M.A.; Luo, S.; Hargreaves, K.M. Vanilloids induce oral cancer apoptosis independent of TRPV1. Oral Oncol. 2014, 50, 437-447. [CrossRef] [PubMed]

63. Han, S.S.; Keum, Y.S.; Chun, K.S.; Surh, Y.J. Suppression of phorbol ester-induced NF-kB activation by capsaicin in cultured human promyelocytic leukemia cells. Arch. Pharm. Res. 2002, 25, 475-479. [CrossRef] [PubMed]

64. Surh, Y.J.; Lee, R.C.J.; Park, K.K.; Mayne, S.T.; Liem, A.; Miller, J.A. Chemoprotective effects of capsaicin and diallyl sulfide against mutagenesis or tumorigenesis by vinyl carbamate and $N$-nitrosodiinethylamine. Carcinogenesis 1995, 16, 2467-2471. [CrossRef] [PubMed]

65. Morré, D.J.; Sun, E.; Geilen, C.; Wu, L.Y.; De Cabo, R.; Krasagakis, K.; Morre, D.M. Capsaicin inhibits plasma membrane NADH oxidase and growth of human and mouse melanoma lines. Eur. J. Cancer 1996, 32, 1995-2003. [CrossRef]

66. Brar, S.S.; Kennedy, T.P.; Whorton, A.R.; Sturrock, A.B.; Huecksteadt, T.P.; Ghio, A.J.; Hoidal, J.R. Reactive oxygen species from $\mathrm{NAD}(\mathrm{P}) \mathrm{H}$ : Quinone oxidoreductase constitutively activate NF- $\mathrm{kB}$ in malignant melanoma cells. Am. J. Physiol Cell. Physiol. 2001, 280, C659-C676. [PubMed] 
67. Patel, P.S.; Varney, M.L.; Dave, B.J.; Singh, R.K. Regulation of Constitutive and Induced NF-кB Activation in Malignant Melanoma Cells by Capsaicin Modulates Interleukin-8 Production and Cell Proliferation. J. Interferon Cytokine Res. 2002, 22, 427-435. [CrossRef] [PubMed]

68. Kim, M.Y. Nitric oxide triggers apoptosis in A375 human melanoma cells treated with capsaicin and resveratrol. Mol. Med. Rep. 2012, 5, 585-591. [CrossRef] [PubMed]

69. Shin, D.H.; Kim, O.H.; Jun, H.S.; Kang, M.K. Inhibitory effect of capsaicin on B16-F10 melanoma cell migration via the phosphatidylinositol 3-kinase/Akt/Rac1 signal pathway. Exp. Mol. Med. 2008, 40, 486-494. [CrossRef] [PubMed]

70. Gong, X.F.; Wang, M.W.; Ikejima, T. Mechanisms of capsaicin-induced apoptosis of human melanoma A375-S2 cells. Chin. J. Oncol. 2005, 27, 401-403.

71. Marques, C.M.; Dibden, C.; Danson, S.; Haycock, J.W.; MacNeil, S. Combined effects of capsaicin and HA14-1 in inducing apoptosis in melanoma cells. J. Cosmet. Dermatol. Sci. Appl. 2013, 3, 175-189. [CrossRef]

72. Schwartz, L.; Guais, A.; Israël, M.; Junod, B.; Steyaert, J.M.; Crespi, E.; Abolhassani, M. Tumor regression with a combination of drugs interfering with the tumor metabolism: Efficacy of hydroxycitrate, lipoic acid and capsaicin. Investig. New Drugs 2013, 31, 256-264. [CrossRef] [PubMed]

73. Bode, A.M.; Cho, Y.Y.; Zheng, D.; Zhu, F.; Ericson, M.E.; Ma, W.Y.; Dong, Z. Transient receptor potential type vanilloid 1 suppresses skin carcinogenesis. Cancer Res. 2009, 69, 905-913. [CrossRef] [PubMed]

74. Li, S.; Bode, A.M.; Zhu, F.; Liu, K.; Zhang, J.; Kim, M.O.; Langfald, A.K. TRPV1-antagonist AMG9810 promotes mouse skin tumorigenesis through EGFR/Akt signaling. Carcinogenesis 2011, 32, 779-785. [CrossRef] [PubMed]

75. Liu, Z.; Zhu, P.; Tao, Y.; Shen, C.; Wang, S.; Zhao, L.; Zhu, Z. Cancer-promoting effect of capsaicin on DMBA/TPA-induced skin tumorigenesis by modulating inflammation, Erk and p38 in mice. Food Chem. Toxicol. 2015, 81, 1-8. [CrossRef] [PubMed]

76. Alam, M.; Ratner, D. Cutaneous squamous-cell carcinoma. N. Engl. J. Med. 2001, 344, 975-983. [CrossRef] [PubMed]

77. Rudolph, R.; Zelac, D. Squamous cell carcinoma of the skin. Plast. Reconstr. Surg. 2004, 114, 82e-94e. [CrossRef] [PubMed]

78. Weinberg, A.S.; Ogle, C.A.; Shim, E.K. Metastatic cutaneous squamous cell carcinoma: An update. Dermatol. Surg. 2007, 33, 885-899. [CrossRef] [PubMed]

79. Ferlay, J.; Shin, H.R.; Bray, F.; Forman, D.; Mathers, C.; Parkin, D.M. Estimates of worldwide burden of cancer in 2008: GLOBOCAN 2008. Int. J. Cancer 2008, 127, 2893-2917. [CrossRef] [PubMed]

80. Shah, J.P.; Gil, Z. Current concepts in management of oral cancer-surgery. Oral Oncol. 2009, 45, 394-401. [CrossRef] [PubMed]

81. Feller, L.; Lemmer, J. Oral squamous cell carcinoma: Epidemiology, clinical presentation and treatment. J. Cancer Ther. 2012, 3, 263-268. [CrossRef]

82. Matei, C.; Caruntu, C.; Ion, R.M.; Georgescu, S.R.; Dumitrascu, G.R.; Constantin, C.; Neagu, M. Protein microarray for complex apoptosis monitoring of dysplastic oral keratinocytes in experimental photodynamic therapy. Biol. Res. 2014, 47, 33. [CrossRef] [PubMed]

83. Monta, G.; Caracò, C.; Simeone, E.; Grimaldi, A.M.; Marone, U.; Marzo, M.; Mozzillo, N. Electrochemotherapy efficacy evaluation for treatment of locally advanced stage III cutaneous squamous cell carcinoma: A 22-cases retrospective analysis. J. Transl. Med. 2017, 15, 82. [CrossRef] [PubMed]

84. Tampa, M.; Matei, C.; Popescu, S.; Georgescu, S.R.; Neagu, M.; Constantin, C.; Ion, R.M. Zinc trisulphonatedphthalocyanine used in photodynamic therapy of dysplastic oral keratinocytes. Rev. Chim. 2013, 64, 639-645.

85. Matei, C.; Tampa, M.; Ion, R.M.; Neagu, M.; Constantin, C. Photodynamic properties of aluminiumsulphonatedphthalocyanines in human displazic oral keratinocytes experimental model. Dig. J. Nanomater. Biostruct. (DJNB) 2012, 7, 1535-1547.

86. Yu, C.C.; Hung, S.K.; Lin, H.Y.; Chiou, W.Y.; Lee, M.S.; Liao, H.F.; Su, Y.C. Targeting the PI3K/AKT/mTOR signaling pathway as an effectively radiosensitizing strategy for treating human oral squamous cell carcinoma in vitro and in vivo. Oncotarget 2017, 8, 68641-68653. [CrossRef] [PubMed]

87. Goldsmith, L.A.; Katz, S.I.; Gilchrest, B.A.; Paller, A.S.; Leffell, D.J.; Wolff, K. Fitzpatrick's Dermatology in General Medicine, 8th ed.; McGrawHill: New York, NY, USA, 2012; ISBN 978-00-7-166904-7. 
88. Bolognia, J.L.; Joseph, L.J.; Schaffer, J.V. Dermatology, 3th ed.; Elsevier: Amsterdam, the Netherlands, 2012; ISBN 978-07-2-343571-6.

89. Burns, T.; Breathnach, S.; Cox, N.; Griffiths, C. Rook's Textbook of Dermatology, 8th ed.; Wiley Blackwell: Hoboken, NJ, USA, 2010.

90. Apalla, Z.; Nashan, D.; Weller, R.B.; Castellsagué, X. Skin cancer: Epidemiology, disease burden, pathophysiology, diagnosis, and therapeutic approaches. Dermatol. Ther. (Heidelb) 2017, 7, 5-19. [CrossRef] [PubMed]

91. Petti, S. Lifestyle risk factors for oral cancer. Oral Oncol. 2009, 45, 340-350. [CrossRef] [PubMed]

92. Boda, D.; Neagu, M.; Constantin, C.; Voinescu, R.N.; Caruntu, C.; Zurac, S.; Tsatsakis, A.M. HPV strain distribution in patients with genital warts in a female population sample. Oncol. Lett. 2016, 12, 1779-1782. [CrossRef] [PubMed]

93. Wang, J.; Aldabagh, B.; Yu, J.; Arron, S.T. Role of human papillomavirus in cutaneous squamous cell carcinoma: A meta-analysis. J. Am. Acad. Dermatol. 2014, 70, 621-629. [CrossRef] [PubMed]

94. Lupu, M.; Caruntu, A.; Caruntu, C.; Papagheorghe, L.M.L.; Ilie, M.A.; Voiculescu, V.; Boda, D.; Constantin, C.; Tanase, C.; Sifaki, M.; et al. Neuroendocrine factors: The missing link in non-melanoma skin cancer. Oncol. Rep. 2017, 38, 1327-1340. [CrossRef] [PubMed]

95. Scholzen, T.; Armstrong, C.A.; Bunnett, N.W.; Luger, T.A.; Olerud, J.E.; Ansel, J.C. Neuropeptides in the skin: Interactions between the neuroendocrine and the skin immune systems. Exp. Dermatol. 1998, 7, 81-96. [CrossRef] [PubMed]

96. Hara, M.; Toyoda, M.; Yaar, M.; Bhawan, J.; Avila, E.M.; Penner, I.R.; Gilchrest, B.A. Innervation of melanocytes in human skin. J. Exp. Med. 1996, 184, 1385-1395. [CrossRef] [PubMed]

97. Seiffert, K.; Granstein, R.D. Neuropeptides and neuroendocrine hormones in ultraviolet radiation-induced immunosuppression. Methods 2002, 28, 97-103. [CrossRef]

98. Weidner, C.; Klede, M.; Rukwied, R.; Lischetzki, G.; Neisius, U.; Schmelz, M.; Petersen, L.J. Acute effects of substance $\mathrm{P}$ and calcitonin gene-related peptide in human skin-a microdialysis study. J. Investig. Dermatol. 2000, 115, 1015-1020. [CrossRef] [PubMed]

99. Dong, J.; Feng, F.; Xu, G.; Zhang, H.; Hong, L.; Yang, J. Elevated SP/NK-1R in esophageal carcinoma promotes esophageal carcinoma cell proliferation and migration. Gene 2015, 560, 205-210. [CrossRef] [PubMed]

100. Muñoz, M.; Rosso, M.; González-Ortega, A.; Coveñas, R. The NK-1 receptor antagonist L-732,138 induces apoptosis and counteracts substance P-related mitogenesis in human melanoma cell lines. Cancers 2010, 2, 611-623. [CrossRef] [PubMed]

101. Muñoz, M.; Rosso, M.; Robles-Frias, M.J.; Salinas-Martín, M.V.; Rosso, R.; González-Ortega, A.; Coveñas, R. The NK-1 receptor is expressed in human melanoma and is involved in the antitumor action of the NK-1 receptor antagonist aprepitant on melanoma cell lines. Lab. Investig. 2010, 90, 1259-1269. [CrossRef] [PubMed]

102. Muñoz, M.; Rosso, M.; Robles-Frías, M.J.; Coveñas, R.; Salinas-Martín, M.V. Immunolocalization of the Neurokinin-1 Receptor: A new target in the treatment of the human primary retinoblastoma. In Eye Cancer Res. Progress; Nova Science Publishers: Hauppauge, NY, USA, 2008; pp. 157-178. ISBN 978-1-60456-045-9.

103. Muñoz, M.; Pérez, A.; Coveñas, R.; Rosso, M.; Castro, E. Antitumoural action of L-733,060 on neuroblastoma and glioma cell lines. Arch. Italiennes Biol. 2004, 142, 105-112.

104. Brener, S.; González-Moles, M.A.; Tostes, D.; Esteban, F.; Gil-Montoya, J.A.; Ruiz-Avila, I.; Munoz, M. A role for the substance $\mathrm{P} / \mathrm{NK}-1$ receptor complex in cell proliferation in oral squamous cell carcinoma. Anticancer Res. 2009, 29, 2323-2329. [PubMed]

105. Serra, I.; Yamamoto, M.; Calvo, A.; Cavada, G.; Baez, S.; Endoh, K.; Tajima, K. Association of chili pepper consumption, low socioeconomic status and longstanding gallstones with gallbladder cancer in a Chilean population. Int. J. Cancer 2002, 102, 407-411. [CrossRef] [PubMed]

106. Toth, B.; Gannett, P. Carcinogenicity of lifelong administration of capsaicin of hot pepper in mice. In Vivo 1992, 6, 59-63. [PubMed]

107. Chanda, S.; Erexson, G.; Frost, D.; Babbar, S.; Burlew, J.A.; Bley, K. 26-Week dermal oncogenicity study evaluating pure trans-capsaicin in Tg. AC hemizygous mice (FBV/N). Int. J. Toxicol. 2007, 26, 123-133. [CrossRef] [PubMed] 
108. Surh, Y.J.; Lee, S.S. Capsaicin, a double-edged sword: Toxicity, metabolism, and chemopreventive potential. Life Sci. 1995, 56, 1845-1855. [CrossRef]

109. Park, K.K.; Surh, Y.J. Effects of capsaicin on chemically-induced two-stage mouse skin carcinogenesis. Cancer Lett. 1997, 114, 183-184. [CrossRef]

110. Park, K.K.; Chun, K.S.; Yook, J.I.; Surh, Y.J. Lack of tumor promoting activity of capsaicin, a principal pungent ingredient of red pepper, in mouse skin carcinogenesis. Anticancer Res. 1998, 18, 4201-4205. [PubMed]

111. Surh, Y.J.; Han, S.S.; Keum, Y.S.; Seo, H.J.; Lee, S.S. Inhibitory effects of curcumin and capsaicin on phorbol ester-induced activation of eukaryotic transcription factors, NF-kappaBand AP-1. Biofactors 2000, 12, 107-112. [CrossRef] [PubMed]

112. Braun-Falco, O.; Plewig, G.; Wolff, H.H.; Landthaler, M. Braun-Falco's Dermatology, 3rd ed.; Springer: Berlin/Heidelberg, Germany, 2009; ISBN 978-3-540-29312-5.

113. Sterry, W.; Paus, R.; Burgdorf, W.; Holtermann, H. Thieme Clinical Companions Dermatology; Thieme: Stuttgart, Germany, 2006.

114. Tampa, M.; Matei, C.; Caruntu, C.; Poteca, T.; Mihaila, D.; Paunescu, C.; Neagu, M. Cellular impedance measurement-novel method for in vitro investigation of drug efficacy. Farmacia 2016, 5, 430-434.

115. Niezgoda, A.; Niezgoda, P.; Czajkowski, R. Novel approaches to treatment of advanced melanoma: A review on targeted therapy and immunotherapy. BioMed Res. Int. 2015, 2015, 851387. [CrossRef] [PubMed]

116. Caruntu, C.; Mirica, A.; Roca, A.E.; Mirica, R.; Caruntu, A.; Matei, C.; Moraru, L. The Role of Estrogens and Estrogen Receptors in Melanoma Development and Progression. ActaEndocrinol. 2016, 12, $234-241$. [CrossRef]

117. Caruntu, C.; Boda, D.; Constantin, C.; Caruntu, A.; Neagu, M. Catecholamines Increase in vitro Proliferation of Murine B16F10 Melanoma Cells. ActaEndocrinol. 2014, 10, 545-558. [CrossRef]

118. Borrego, J.F.; Huelsmeyer, M.K.; Pinkerton, M.E.; Muszynski, J.L.; Miller, S.A.K.; Kurzman, I.D.; Vail, D.M. Neurokinin-1 receptor expression and antagonism by the NK-1R antagonist maropitant in canine melanoma cell lines and primary tumour tissues. Vet. Comp. Oncol. 2016, 14, 210-224. [CrossRef] [PubMed]

119. Khare, V.K.; Albino, A.P.; Reed, J.A. The neuropeptide/mast cell secretagogue substance P is expressed in cutaneous melanocytic lesions. J. Cutan. Pathol. 1998, 25, 2-10. [CrossRef] [PubMed]

120. Zhou, J.; Geng, K.K.; Ping, F.F.; Gao, Y.Y.; Liu, L.; Feng, B.N. Cross-talk between 5-hydroxytryptamine and substance $\mathrm{P}$ in the melanogensis and apoptosis of B16F10 melanoma cells. Eur. J. Pharmacol. 2016, 775, 106-112. [CrossRef] [PubMed]

121. Ping, F.; Shang, J.; Zhou, J.; Song, J.; Zhang, L. Activation of neurokinin-1 receptor by substance P inhibits melanogenesis in B16-F10 melanoma cells. Int. J. Biochem. Cell Biol. 2012, 44, 2342-2348. [CrossRef] [PubMed]

122. Muñoz, M.; Bernabeu-Wittel, J.; Coveñas, R. NK-1 as a melanoma target. Expert Opin. Ther. Targets 2011, 15, 889-897. [CrossRef] [PubMed]

123. Muñoz, M.; Pérez, A.; Rosso, M.; Zamarriego, C.; Rosso, R. Antitumoral action of the neurokinin-1 receptor antagonist L-733 060 on human melanoma cell lines. Melanoma Res. 2004, 14, 183-188. [PubMed]

124. Ueda, Y.; Richmond, A. NF-кB activation in melanoma. Pigment. Cell. Melanoma Res. 2006, 19, 112-124. [CrossRef] [PubMed]

125. Neagu, M.; Constantin, C.; Longo, C. Chemokines in the melanoma metastasis biomarkers portrait. J. Immunoass. Immunochem. 2015, 36, 559-566. [CrossRef] [PubMed]

126. Oyagbemi, A.A.; Saba, A.B.; Azeez, O.I. Capsaicin: A novel chemopreventive molecule and its underlying molecular mechanisms of action. Indian J. Cancer 2010, 47, 53-58. [CrossRef] [PubMed]

127. Davies, M.A. The role of the PI3K-AKT pathway in melanoma. Cancer J. 2012, 18, 142-147. [CrossRef] [PubMed]

128. Kwong, L.N.; Davies, M.A. Navigating the therapeutic complexity of PI3K pathway inhibition in melanoma. Clin. Cancer Res. 2013, 19, 5310-5319. [CrossRef] [PubMed]

129. Price, L.S.; Collard, J.G. Regulation of the cytoskeleton by Rho-family GTPases: Implications for tumour cell invasion. Semin. Cancer Biol. 2001, 11, 167-173. [CrossRef] [PubMed]

(c) 2017 by the authors. Licensee MDPI, Basel, Switzerland. This article is an open access article distributed under the terms and conditions of the Creative Commons Attribution (CC BY) license (http:/ / creativecommons.org/licenses/by/4.0/). 
Article

\title{
Possible Mechanisms of the Prevention of Doxorubicin Toxicity by Cichoric Acid-Antioxidant Nutrient
}

\author{
Agata Jabłońska-Trypuć ${ }^{1, *}$, Rafał Krętowski ${ }^{2}$, Monika Kalinowska ${ }^{1}$, Grzegorz Świderski ${ }^{1}$, \\ Marzanna Cechowska-Pasko ${ }^{2}$ and Włodzimierz Lewandowski ${ }^{1}$ \\ 1 Department of Chemistry, Biology and Biotechnology, Faculty of Civil Engineering and Environmental \\ Engineering, Białystok University of Technology, Wiejska 45E Street, 15-351 Białystok, Poland; \\ m.kalinowska@pb.edu.pl (M.K.); g.swiderski@pb.edu.pl (G.Ś.); w.lewandowski@pb.edu.pl (W.L.) \\ 2 Department of Pharmaceutical Biochemistry, Medical University of Bialystok, 15-222 Białystok, Poland; \\ r.kretowski@umb.edu.pl (R.K.); mapasko@gmail.com (M.C.-P.) \\ * Correspondence: a.jablonska@pb.edu.pl; Tel.: +48-85-746-9000; Fax: +48-85-746-9015
}

Received: 10 November 2017; Accepted: 3 January 2018; Published: 5 January 2018

\begin{abstract}
Skin is the largest organ in the human body, and which protects organism against unfavorable external factors e.g., chemicals, environment pollutants, allergens, microorganisms, and it plays a crucial role in maintaining general homeostasis. It is also an important target of oxidative stress due to the activity of oxygen reactive species (ROS), which are constantly generated in the fibroblasts in response to exogenous or endogenous prooxidant agents. An example of such compound with proved prooxidant activity is Doxorubicin (DOX), which is an effective anticancer agent belongs in anthracycline antibiotic group. Increasingly frequent implementation of various strategies to reduce undesirable DOX side effects was observed. Very promising results come from the combination of DOX with dietary antioxidants from the polyphenol group of compounds, such as cichoric acid (CA) in order to lower oxidative stress level. The aim of this work was to evaluate the influence of CA combined with DOX on the oxidative stress parameters in fibroblasts, which constitute the main cells in human skin. We also wanted to examine anti-apoptotic activity of CA in fibroblasts treated with selected concentrations of DOX. Results obtained from the combination of DOX with CA revealed that CA exhibits cytoprotective activity against DOX-induced damage by lowering oxidative stress level and by inhibiting apoptosis. The present finding may indicate that CA may serve as antioxidative and anti-apoptotic agent, active against DOX-induced damage.
\end{abstract}

Keywords: cichoric acid; doxorubicin; fibroblasts; skin; oxidative stress

\section{Introduction}

Skin is the largest organ in the human body. It protects the organism against unfavorable external factors e.g., chemicals, environment pollutants, allergens, microorganisms, and it plays a crucial role in maintaining general homeostasis [1]. Therefore, it is also an important target of oxidative stress due to the activity of reactive oxygen species (ROS), which are constantly generated in the fibroblasts and keratinocytes in response to exogenous or endogenous prooxidant agents [2]. Furthermore, ROS produced during normal skin metabolism, constitute a part of proper skin functionality and usually cause a little damage due to the activity of intracellular mechanisms, which reduce their harmful properties. It is worth noting that intensive or prolonged oxidative stress may overwhelm antioxidant defense mechanisms of the human skin and contribute to the skin disorders development, e.g.,: skin aging, dermatitis, allergic reactions or even skin neoplasms [3]. Main constituents of skin are fibroblasts, which arise during embryogenesis from embryonic mesenchyme. A certain amount of 
spindle-shaped mesenchymal cells differentiate into supporting cells-fibroblasts. They are usually elongated, spindle-shaped, with several projections lying in one plane. They have one cell nucleus, round or oval with distinct nucleoli. Sometimes several nucleoli within the nucleus can be observed. Fibroblasts are the main cells that build up the dermis. Skin consists of three layers: epidermis, dermis and subcutaneous tissue. The epidermis forms a surface layer that stays in contact with the external environment and is built principally of keratinocytes that synthesize keratin-a component of the stratum corneum of the epidermis. The skin is composed of two layers: papillary and reticular $[4,5]$.

Free radicals as atoms or compounds, which have at least one unpaired electron in the outer orbit, are mainly produced in the mitochondria during electron flow through the mitochondrial respiratory chain, and they increase their reactivity through oxidation-reduction mechanisms. About $2 \%$ of oxygen consumed by the respiratory chain undergoes simultaneous reduction causing the formation of ROS. As a result of these processes, superoxide anion radicals are formed, that initiate a whole cascade of reactive oxygen species, such as hydroxyl radical, hydrogen peroxide and singlet oxygen. ROS are generated in cells in different ways, but their main source are mitochondria, which consume about $90 \%$ of the oxygen needed by the cells. Mitochondria are also most often damaged by ROS, and with age they degenerate fairly quickly. Under normal conditions ROS are permanently removed by a complex antioxidant system, but in pathological conditions there is an imbalance between prooxidants and antioxidants. This imbalance can lead to oxidative stress and subsequently overproduction of ROS, which modify cellular components such as DNA, proteins and lipids [6,7]. Oxidative stress largely affects the skin, which occupies the largest area of the human body and is a protective coating for the internal organs. The skin structure allows it to perform physical and biochemical protection functions. Due to its functions and localization in the human body, skin is the most exposed for oxidative stress tissue. It is most susceptible to oxidative damage, which results from the presence of potential biological targets for free radical reactions in its components.

Air pollution, naturally occurring airborne gases such as ozone or high concentrations of oxygen, ionizing and non-ionizing radiation, bacteria, viruses and various exogenous toxins and chemicals can be considered as exogenous sources of factors that promote oxidative stress in the skin. Oxidative stress can also be caused by physical damage to the skin, such as burns or injuries [8,9]. On the other hand, endogenous sources of oxidative damage are generated by enzymes involved in the autoxidation of endo- and exogenous compounds, as well as by lipoxygenases and cyclooxygenases during eicosanoid metabolism. Nitric oxide synthase is an example of an enzyme that produces NO (nitric oxide) radicals directly in the skin. The source of ROS may also be neutrophils activated by a bacterial invasion in which a respiratory burst is observed.

One of the classes of compounds, which activity in the human organism is based on the generation of oxidative stress, mainly in cancer cells, are anticancer drugs. An example of such compound with proved prooxidant activity is Doxorubicin (DOX). DOX is an effective anticancer agent from anthracycline antibiotic group, which has a large spectrum of activity and it is being used alone or in combination to treat a variety of tumors, both haematological and solid, such as breast cancer $[10,11]$. It inhibits cell proliferation, induces oxidative stress, inhibits topoisomerase II, and finally leads to the cell death mainly through apoptosis [12-14]. DOX tends to accumulate in mitochondria, causing changes in their structure and function. However, the main cause of DOX side effects observed in human is an extremely high level of oxidative stress within the cell, eventually causing apoptotic cell death $[15,16]$. A generation of free radicals caused by DOX can be described in two ways. The first one is an enzymatic mechanism consisting of the formation of semiquinone free radicals by the activity of NADPH-dependent reductases, which cause a one-electron reduction of DOX to DOX-semiquinone. Redox transformations of DOX-derived quinone/semiquinone provide superoxide radicals in the presence of oxygen $[17,18]$. The second mechanism has non-enzymatic background and it involves reaction with an iron $\left(\mathrm{Fe}^{3+}\right)$, which produces $\mathrm{Fe}^{2+}$-doxorubicin free radical complex. This Fe-DOX complex is able to reduce oxygen to hydrogen peroxide and other free radicals. In our previous 
experiment regarding DOX influence on breast cancer cells we revealed that DOX-metal complexes are even more efficient than a parent drug [10,17].

Increasingly frequent implementation of various strategies to reduce undesirable DOX side effects was observed. Besides dose regulation, combined therapies with antioxidants, especially plant origin substances, are being used. Very promising results come from the combination of DOX with dietary antioxidants from the polyphenol group of compounds in order to lower oxidative stress level [19]. One of the antioxidative agents with promising properties is cichoric acid. Cichoric acid (CA), a dicaffeyltartaric acid, was identified in a variety of edible plants and vegetables, such as: iceberg lettuce, basil, Cichorium intybus L., dandelion, Echinacea purpurea and Orthosiphon stamineus. Many of above-mentioned plants are being used in folk medicine. CA exhibits many biological properties including anti-hyaluronidase activity, protection of collagen from possible free radical damage, antiviral activity, promoting phagocyte activity and free radical scavenger properties [20,21]. According to the literature, CA also inhibits HIV (human immunodeficiency virus) integrase, which is responsible for the HIV DNA copy integration with the DNA of the host cell [22]. It should be also mentioned that CA has the ability to influence an immune response in chronically stressed mice, causing a significant decrease in stress level [23,24]. In addition recent research revealed that CA enhances glucose uptake in muscle cells and subsequently stimulates Langerhans islets to the secretion of the insulin [25]. Despite so many beneficial activities of CA described in literature, no studies exist regarding its antioxidative properties against DOX-induced oxidative stress in normal human fibroblasts.

Several studies have shown that CA reveals its general antioxidative properties, which may counteract doxorubicin-induced cytotoxicity, but they were conducted on cancer cell lines and they have not clarified the mechanisms of its action. Therefore, with our study, we wanted to determine if CA acts as an antioxidant against doxorubicin-induced oxidative stress and if it acts via antioxidative and/or antiapoptotic pathway. We tested it in human normal fibroblasts cell line, because fibroblasts are very responsive to chemical signals and changes made to this signals can affect other cells. Fibroblasts constitute also main cell type of the skin, which is exposed for anticancer drugs side effects. Therefore strategies to attenuate the toxic effects of doxorubicin, such as combined therapy with dietary antioxidants (e.g., CA) are implemented.

\section{Materials and Methods}

\subsection{Reagents}

Dulbecco's modified Eagle's medium (DMEM), containing glucose at $4.5 \mathrm{mg} / \mathrm{mL}(25 \mathrm{mM})$, penicillin, streptomycin, trypsin-EDTA (ethylenediaminetetraacetic acid), FBS (Fetal Bovine Serum) and PBS (Phosphate-buffered saline) (without $\mathrm{Ca}$ and $\mathrm{Mg}$ ) were provided by Gibco (San Diego, CA, USA), GSH/GSSG-Glo ${ }^{\text {TM }}$ Assay kit, Caspase-Glo ${ }^{\circledR}$ 3/7 Assay kit, Caspase-Glo ${ }^{\circledR} 9$ Assay kit were provided by Promega, Madison, WI, USA. MTT reagent was purchased from Sigma-Aldrich (St. Louis, MO, USA). Acridine orange and ethidium bromide were obtained from Sigma-Aldrich (St. Louis, MO, USA). Dichlorodihydrofluorescein diacetate assay (DCFH-DA) was provided by Sigma-Aldrich, St. Louis, MO, USA. SDS (Sodium dodecyl sulfate), TCA (Trichloroacetic acid), TBA (Thiobarbituric acid), Folin-Ciocalteu reagent were provided by Sigma-Aldrich and DTNB (Ellman's Reagent) (5,5-dithio-bis-(2-nitrobenzoic acid)) by Serva. Cichoric acid, chlorogenic acid, caffeic acid, quercetin, DPPH (2,2-diphenyl-1-picrylhydrazyl), trolox, $\mathrm{H}_{2} \mathrm{O}_{2}$, horseradish peroxidase, phosphate buffer $\mathrm{pH}=7$ were purchased from Sigma-Aldrich Co. and used without purification. Methanol was purchased from Merck (Darmstadt, Germany).

\subsection{Anti-/Pro-Oxidant Activity}

Antiradical activity was determined according to the DPPH assay described by [26]. The methanolic solutions of cichoric, chlorogenic and caffeic acids, quercetin and DPPH were prepared just before analysis. $1 \mathrm{~mL}$ of tested compound and $2 \mathrm{~mL}$ of DPPH was added to each tube, vortexed 
and incubated in the darkness for $1 \mathrm{~h}$ at $23^{\circ} \mathrm{C}$. The final concentrations of phenolic compounds were 8 , 5,1 and $0.5 \mu \mathrm{M}$, and DPPH was $40 \mu \mathrm{M}$. The absorbance of solutions was measured at $516 \mathrm{~nm}$ against methanol as the blank using Agilent Carry 5000 spectrophotometer (Agilent, Santa Clara, CA, USA). The control sample consisted of $2 \mathrm{~mL}$ of DPPH solution and $1 \mathrm{~mL}$ of methanol. The antiradical activity of phenolic compounds was calculated as the percent of DPPH inhibition according to the equation:

$$
\% \mathrm{I}=\frac{\mathrm{A}_{\text {control }}^{516}-\mathrm{A}_{\text {sample }}^{516}}{\mathrm{~A}_{\text {control }}^{516}} \times 100 \%
$$

where: \%I-\% inhibition of DPPH radical, $\mathrm{A}_{\text {conrol }}^{516}$-absorbance of the control, $\mathrm{A}_{\text {sample }}^{516}$-absorbance of the sample.

The pro-oxidant activity of tested compounds was determined as the rate of oxidation of trolox according to the procedure described by Zeraik et al. [27]. $100 \mu \mathrm{M}$ trolox, $50 \mu \mathrm{M} \mathrm{H}_{2} \mathrm{O}_{2}, 0.01 \mu \mathrm{M}$ horseradish peroxidase in phosphate buffer $(\mathrm{pH}=7)$ and $0.25 \mu \mathrm{M}$ tested substances were incubated at $25^{\circ} \mathrm{C}$. The measurement was conducted at $272 \mathrm{~nm}$ using Agilent Carry 5000 spectrophotometer.

\subsection{Cell Culture}

The effect of CA, DOX and CA-DOX was examined in normal human skin fibroblast cell line (CRL1474), which was obtained from American Type Culture Collection (ATCC). Cells were maintained in DMEM (Gibco) supplemented with 10\% FBS (Gibco), penicillin (100 U/mL), and streptomycin $(100 \mu \mathrm{g} / \mathrm{mL})$ at $37^{\circ} \mathrm{C}$ in a humidified atmosphere of $5 \% \mathrm{CO}_{2}$ in air. The viability of cells was estimated at CA concentrations of: $0.5 \mu \mathrm{M}, 1 \mu \mathrm{M}, 10 \mu \mathrm{M}, 50 \mu \mathrm{M}, 100 \mu \mathrm{M}, 200 \mu \mathrm{M}$ and $300 \mu \mathrm{M}$; DOX concentrations of: $0.09 \mu \mathrm{M}, 0.18 \mu \mathrm{M}, 0.38 \mu \mathrm{M}, 0.75 \mu \mathrm{M}, 1.5 \mu \mathrm{M}, 3 \mu \mathrm{M}$ and $6 \mu \mathrm{M}$; mix of CA and DOX in concentrations: $0.5 \mu \mathrm{M} \mathrm{CA}+3 \mu \mathrm{M}$ DOX, $1 \mu \mathrm{M} \mathrm{CA}+3 \mu \mathrm{M}$ DOX, $10 \mu \mathrm{M}$ CA $+3 \mu \mathrm{M}$ DOX, $50 \mu \mathrm{M}$ CA + $3 \mu \mathrm{M}$ DOX, $100 \mu \mathrm{M}$ CA $+3 \mu \mathrm{M}$ DOX, $200 \mu \mathrm{M} \mathrm{CA}+3 \mu \mathrm{M}$ DOX and $300 \mu \mathrm{M}$ CA + $3 \mu \mathrm{M}$ DOX. Caspases $3 / 7$ activity, caspase 9 activity, $\mathrm{SH}$ (thiol) group content, TBARS (Thiobarbituric acid reactive substances) content, GSH/GSSG (Glutathione/Oxidized Glutathione) ratio, ROS content and apoptosis were examined at CA concentrations of $300 \mu \mathrm{M}$, DOX concentration of $3 \mu \mathrm{M}$ and the mixture of these two compounds (CA + DOX, concentrations: $300 \mu \mathrm{M}+3 \mu \mathrm{M}$, respectively). Fibroblasts were seeded in 96-well plates at a density $2 \times 10^{4}$ cells /well. The cells were cultured for $24 \mathrm{~h}$ and $48 \mathrm{~h}$ and were treated with DOX in a concentration of $3 \mu \mathrm{M}, \mathrm{CA}$ in a concentration of $300 \mu \mathrm{M}$ and with a mixture of DOX and CA $(3 \mu \mathrm{M}$ $+300 \mu \mathrm{M}$, respectively). Cells were seeded in: (a) transparent plates for cytotoxicity tests; (b) white plates for determination of caspase 3/7 and 9 activity, determination of GSH/GSSG ratio; (c) black plates for intracellular ROS detection.

\subsection{Chemical Treatment of Cells}

$\mathrm{CA}$ was stored in a refrigerator at temperature $4{ }^{\circ} \mathrm{C}$. CA was dissolved in TrisHCl buffer. The compound was added to the cultured cells for a final concentration in the range of $0.5 \mu \mathrm{M}$ to $300 \mu \mathrm{M}$. DOX was stored in a freezer at temperature $-20^{\circ} \mathrm{C}$. The compound was added to the culture cells for a final concentration in the range of $0.09 \mu \mathrm{M}$ to $6 \mu \mathrm{M}$. CA and DOX were both added to the cultured cells in selected concentrations. The control cells were incubated without the tested compounds.

\subsection{CA, DOX and CA-DOX Cytotoxicity}

CA, DOX and CA-DOX cytotoxicity were measured according to the method of Carmichael using 3-(4,5-dimethylthiazol-2-yl)-2,5-diphenyltetrazolium bromide (MTT) [28]. The cells cultured for $24 \mathrm{~h}$ and $48 \mathrm{~h}$ were treated with: 1 st $-\mathrm{CA}$ in the concentration range from $0.5 \mu \mathrm{M}$ to $300 \mu \mathrm{M} ; 2 \mathrm{nd}-\mathrm{DOX}$ in the concentration range from $0.09 \mu \mathrm{M}$ to $6 \mu \mathrm{M}$; 3rd-CA combined with DOX: CA in the concentration range from $0.5 \mu \mathrm{M}$ to $300 \mu \mathrm{M}$ combined with DOX in the $3 \mu \mathrm{M}$ concentration. After $24 \mathrm{~h}$ and $48 \mathrm{~h}$ cells were washed 3 times with PBS and subsequently incubated with $10 \mu \mathrm{L}$ of MTT solution $(5 \mathrm{mg} / \mathrm{mL}$ in 
PBS) for $2 \mathrm{~h}$ at $37^{\circ} \mathrm{C}$ in $5 \% \mathrm{CO}_{2}$ in an incubator. Subsequently, $100 \mu \mathrm{L}$ of DMSO was added and cells were incubated in the dark for the next $2 \mathrm{~h}$. The absorbance was measured at $570 \mathrm{~nm}$ in a microplate reader GloMax ${ }^{\circledR}$-Multi Microplate Multimode Reader (Promega Corporation, Madison, WI, USA). The viability of fibroblast cells was calculated as a percentage of control cells, incubated without tested compounds. All the experiments were done in triplicates.

\subsection{Determination of Caspase 3/7 Activity}

After intended time of incubation medium was removed and Assay reagents were added to the wells. The activity of caspases 3/7 was measured using luminescent assay based on the substrate which contains the tetrapeptide sequence DEVD in a reagent optimized for caspase activity, luciferase activity and cell lysis. The cell lysis was followed by the caspase cleavage of the substrate and generation of the luminescent signal. The luminescence is proportional to the amount of caspase activity present in the sample. Assay is based on the thermostable luciferase activity, which generates stable, "glow-type" luminescent signal. The luminescence was measured in a microplate reader GloMax ${ }^{\circledR}$-Multi Microplate Multimode Reader. All the experiments were done in triplicates.

\subsection{Determination of Caspase 9 Activity}

After intended time of incubation medium was removed and Assay reagents were added to the wells. The activity of caspase 9 was measured by using luminescent assay based on a luminogenic caspase-9 substrate in a buffer system provided for caspase activity, luciferase activity and cell lysis. The addition of a special Caspase-Glo ${ }^{\circledR} 9$ Reagent results in cell lysis, followed by caspase cleavage of the substrate, and generation of a "glow-type" luminescent signal. The signal generated is proportional to the amount of caspase activity present in the sample. The assay is based on thermostable luciferase, which generates the stable "glow-type" luminescent signal. The luminescence was measured in a microplate reader GloMax ${ }^{\circledR}$-Multi Microplate Multimode Reader (Promega Corporation, Madison, WI, USA). All the experiments were done in triplicates.

\subsection{Total Protein Content in Cells}

Fibroblasts $\left(1 \times 10^{5}\right.$ cells $\left./ \mathrm{mL}\right)$ were incubated in $2 \mathrm{~mL}$ of culture medium with tested compounds in tissue culture 6-well plates. The determination of protein concentration was performed spectrophotometrically as per Lowry et al. (1951) as described previously [29]. All the experiments were done in triplicates.

\subsection{Determination of SH Groups}

SH-groups were measured using the method of Rice-Evans (1991) as described previously [29]. Fibroblasts $\left(1 \times 10^{5}\right.$ cells $\left./ \mathrm{mL}\right)$ were incubated in $2 \mathrm{~mL}$ of culture medium with tested compounds in tissue culture 6-well plates. All the experiments were done in triplicates.

\subsection{Determination of TBA Reactive Species (TBARS) Levels}

The method of Rice-Evans (1991) was applied in order to measure the level of membrane lipid-peroxidation products (TBARS), as described previously [29]. Fibroblasts $\left(1 \times 10^{5}\right.$ cells $\left./ \mathrm{mL}\right)$ were incubated in $2 \mathrm{~mL}$ of culture medium with test compounds in tissue culture 6-well plates. All the experiments were done in triplicates.

\subsection{Determination of GSH/GSSG}

Total glutathione and GSH/GSSG ratio were each assayed in triplicate via GSH/GSSG-Glo ${ }^{\mathrm{TM}}$ kit (Promega, Madison, WI, USA) following manufacturer's instructions. Prior to the assay growth media were removed and cells washed with PBS. Assay is based on a luminescence measurement and detects and quantifies total glutathione (GSH + GSSG), GSSG and GSH/GSSG ratios in cultured 
cells. Stable luminescent signals are correlated with either the GSH or GSSG concentration of a sample. In this method GSH-dependent conversion of a GSH probe, Luciferin-NT, to luciferin by a glutathione S-transferase enzyme is coupled to a firefly luciferase reaction. Light from luciferase depends on the amount of luciferin formed, which in turn depends on the amount of GSH present. Thus, the luminescent signal is proportional to the amount of GSH. GSH/GSSG ratios are calculated directly from luminescence measurements.

\subsection{Intracellular ROS Detection}

The intracellular ROS level was measured according to Krętowski R. et al. [30]. After diffusion through the cell membrane, DCFH-DA is deacetylated by cellular esterases to a non-fluorescent compound, which is later oxidized by intracellular ROS into a fluorescent $2^{\prime}, 7^{\prime}$-dichlorofluorescein (DCF). After $24 \mathrm{~h}$, the medium was removed, the cells were stained with $10 \mu \mathrm{M}$ of DCFH-DA in $200 \mu \mathrm{L}$ PBS at $37^{\circ} \mathrm{C}, 5 \% \mathrm{CO}_{2}$ incubator, for $45 \mathrm{~min}$. Next, the dye was removed and replaced with DOX in a concentration of $3 \mu \mathrm{M}, \mathrm{CA}$ in a concentration of $300 \mu \mathrm{M}$ and with a mixture of DOX $+\mathrm{CA}$ $(3 \mu \mathrm{M}+300 \mu \mathrm{M}$, respectively) and incubated for $24 \mathrm{~h}$ and $48 \mathrm{~h}$. Then, the DCF fluorescence intensity was measured using the GloMax ${ }^{\circledR}$-Multi Detection System (Promega Corporation, Madison, WI, USA) at the excitation wavelength of $485 \mathrm{~nm}$ and the emission wavelength of $535 \mathrm{~nm}$. The intracellular ROS generation in fibroblasts cells was shown as the intensity of fluorescence of the DCF. All the experiments were done in triplicates.

\subsection{Intracellular ROS Detection by Flow Cytometry}

The level of intracellular reactive oxygen species (ROS) was determined using dichlorodihydrofluorescein diacetate (DCFH-DA), (Sigma, St. Louis, MO, USA). After diffusion through the cell membrane, DCFH-DA is deacetylated by cellular esterases to a non-fluorescent compound, which is later oxidized by intracellular ROS into a fluorescent $2^{\prime}, 7^{\prime}$-dichlorofluorescein (DCF). The cells $\left(2.5 \times 10^{5}\right)$ were seeded in $2 \mathrm{~mL}$ of growth medium in 6-well plates. After $24 \mathrm{~h}$, the medium was removed, the cells were stained with $10 \mu \mathrm{M}$ of DCFH-DA in $2 \mathrm{~mL}$ PBS at $37^{\circ} \mathrm{C}$, $5 \% \mathrm{CO}_{2}$ incubator, for $30 \mathrm{~min}$. Next, the dye was removed and replaced with DOX in a concentration of $3 \mu \mathrm{M}, \mathrm{CA}$ in a concentration of $300 \mu \mathrm{M}$ and with a mixture of DOX and CA $(3 \mu \mathrm{M}+300 \mu \mathrm{M}$, respectively) and incubated for $24 \mathrm{~h}$ and $48 \mathrm{~h}$. Then, the cells were trypsinized, resuspended in DMEM and then in PBS. The DCF fluorescence intensity was measured according to flow cytometry method, by using FACSCanto II cytometer (Becton Dickinson, Franklin Lakes, NJ, USA). Data were analysed using FACSDiva software (version 6, Becton Dickinson, Franklin Lakes, NJ, USA). The intracellular ROS generation in fibroblasts was depicted as the $\%$ of DCF positive cells.

\subsection{Fluorescent Microscopy Assay}

For apoptotic and necrotic cells nuclear morphology evaluation fluorescent dyes, such as ethidium bromide $(10 \mu \mathrm{M})$ and acridine orange $(10 \mu \mathrm{M})$ were used. The MCF-7 cells were seeded on cell imaging dishes with coverglass bottom with DOX in a concentration of $3 \mu \mathrm{M}, \mathrm{CA}$ in a concentration of $300 \mu \mathrm{M}$ and with a mixture of DOX and CA ( $3 \mu \mathrm{M}+300 \mu \mathrm{M}$, respectively) and without tested compound as a control, for $24 \mathrm{~h}$ and $48 \mathrm{~h}$. After incubation the cells were washed twice with PBS and then stained with dyes solution in the dark in room temperature, for $10 \mathrm{~min}$. After incubation, staining mixture was removed and the cells were washed with PBS in order to analyze under fluorescent microscope (200× magnification). Ethidium bromide stains only cells that have lost their membrane integrity, while acridine orange stains live and dead cells. The cells were analyzed and photographed via using Leica DM IL fluorescent microscope (Leica Microsystems, Wetzlar, Germany). The following criteria were used: living cells—have regularly distributed green chromatin nucleus, early apoptotic cells characterized by bright green nucleus with condensed or often fragmented chromatin, late apoptotic cells-orange nuclei with chromatin condensation or fragmentation, while necrotic cells showed 
orange-stained cell nuclei. The cells were counted and percentage of apoptotic cells was the sum of early apoptotic and late apoptotic cells percent.

\subsection{Statistical Analysis}

For parametric data one-way analysis of variance (ANOVA) followed Tukey's test was applied. Results from five independent experiments were expressed as mean \pm standard deviation (SD) of mean for parametric data. Significance was considered when $p \leq 0.05$. Statistica 13.0 was used.

\section{Results}

\subsection{Antioxidant (DPPH Assays) and Pro-Oxidant (Trolox Assay) Activity}

The anti- and pro-oxidant activity of cichoric acid (CA) was determined and compared with the activity of caffeic acid (CFA), chlorogenic acid (CGA) and quercetin (Q). In Figure 1 the antioxidant properties of selected polyphenolics (determined as antiradical properties against $\mathrm{DPPH} \bullet$ radical) are shown. The percent of $\mathrm{DPPH}^{\bullet}$ radical inhibition (\%I) was the highest for cichoric acid for four out of six studied concentrations and it was equal: $88.44 \pm 0.53,82.37 \pm 0.70,52.52 \pm 0.99,31.81 \pm 1.46$, $13.60 \pm 1.45$ and $6.67 \pm 0.91 \%$, for the concentrations $8,5,3,2,1$ and $0.5 \mu \mathrm{M}$, respectively. Caffeic acid shows slightly lower antioxidant properties than cichoric acid, $\% \mathrm{I}=85.37 \pm 0.41,80.11 \pm 1.67$, $42.57 \pm 0.48,9.66 \pm 0.76$ and $5.45 \pm 1.37$ for $8,5,1$ and $0.5 \mu \mathrm{M}$, respectively. Quercetin possess similar antioxidant activity as cichoric and caffeic acid, except the 5 and $3 \mu \mathrm{M}$ concentrations where the $\% \mathrm{I}=65.96 \pm 2.53$ and $62.77 \pm 0.98$, respectively. Chlorogenic acid shows the lowest antioxidant properties among studied compounds, for all studied concentrations (the $\% \mathrm{I}=49.78$ $\pm 0.14,36.00 \pm 1.98,26.93 \pm 0.38,21.18 \pm 0.59,5.73 \pm 0.63$ and $3.01 \pm 1.08$, respectively; Figure 1). Therefore, taking into account the increasing value of \% as a measure of the antioxidant activity, the studied compounds may be ordered as follows: cichoric acid $>$ caffeic acid $\sim$ quercetin $>$ chlorogenic acid (for the concentrations $8,5,2,1$ and $0.5 \mu \mathrm{M}$ ).
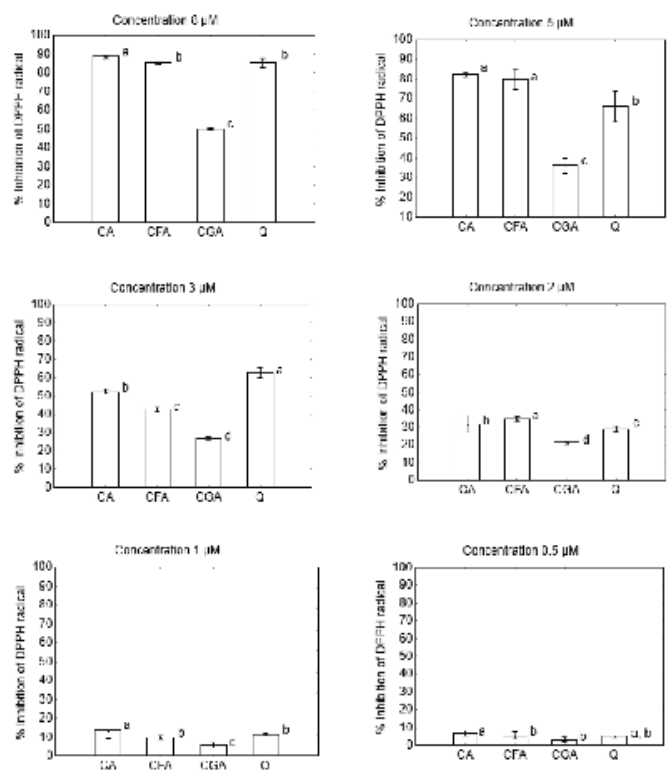

Figure 1. DPPH radical scavenging activity (\%) of cichoric acid (CA), caffeic acid (CFA), chlorogenic acid (CGA) and quercetin (Q) for the concentrations $8,5,2,1$ and $0.5 \mu \mathrm{M}$. The same letter near the means indicate no significant difference (Tukey test, $p<0.05$ ). 
The pro-oxidant property of phenolic compounds was studied for the concentration $0.25 \mu \mathrm{M}$ (Figure 2). The rate of oxidation of trolox depends on the type of compounds. For the first $20 \mathrm{~min}$. of measurement chlorogenic acid shows the highest pro-oxidant properties whereas cichoric acid is the lowest (the pro-oxidant activity increases in the series: cichoric acid $<$ quercetin $<$ caffeic acid $<$ chlorogenic acid). For the next $30 \mathrm{~min}$. the pro-oxidant capacity increases as follows: quercetin $<$ cichoric acid < caffeic acid < chlorogenic acid), and after $60 \mathrm{~min}$. all tested compounds reveal similar pro-oxidant properties.

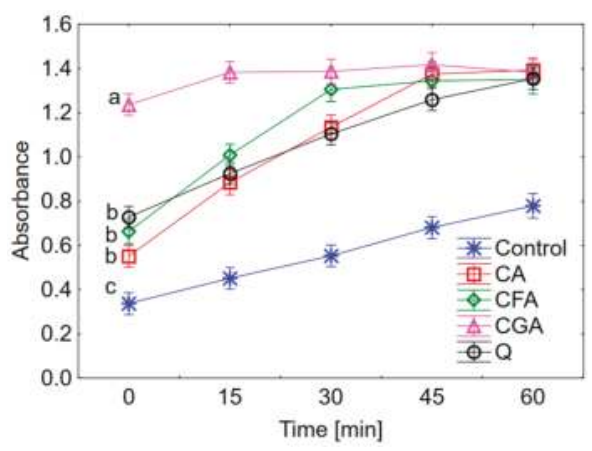

Figure 2. The effect of selected phenolic compounds $(0.25 \mu \mathrm{M})$ on the oxidation of trolox. CA: cichoric acid, CFA: caffeic acid, CGA: chlorogenic acid, Q: quercetin. The same letters for particular compounds indicate no significant difference (Tukey test, $p<0.05$ ).

\section{2. $C A, D O X$ and $C A-D O X$ Cytotoxicity}

Cell viability was determined using MTT assay. CA significantly increased cell proliferation especially after $24 \mathrm{~h}$ of exposure (Figure 3A-C). None of the tested CA concentration resulted in a decrease below the control level. The concentration of $1 \mu \mathrm{M}$ of CA increased the viability of cells by $46 \%$ after $24 \mathrm{~h}$ and by about 50\% after $48 \mathrm{~h}$ treatment. Treatment with $100 \mu \mathrm{M}$ of CA also increased fibroblast cells proliferation by about $40 \%$ in both tested incubation times (Figure 3A). DOX treatment caused decrease in fibroblast viability (Figure 3B). The most significant decrease by about $53 \%$ was observed under the influence of $3 \mu \mathrm{M}$ DOX after $48 \mathrm{~h}$ treatment. We didn't observe any increases above the control level in viability of cells under the influence of DOX. Therefore we selected one concentration of DOX, which is $3 \mu \mathrm{M}$ and combined it with all of the tested concentrations of CA (ranged from $0.5 \mu \mathrm{M}$ to $300 \mu \mathrm{M}$ ). We observed significant increase in viability of cells after $24 \mathrm{~h}$ and $48 \mathrm{~h}$ treatment with 200 and $300 \mu \mathrm{M}$ CA combined with $3 \mu \mathrm{M}$ DOX (Figure 3C). Simultaneous treatment with $300 \mu \mathrm{M}$ CA and $3 \mu \mathrm{M}$ DOX for $48 \mathrm{~h}$ increased cell proliferation by about $200 \%$ as compared to untreated controls. Based on that experiment we chose one combination of DOX and CA, which was $300 \mu \mathrm{M}$ of CA and $3 \mu \mathrm{M}$ of DOX for studying oxidative stress and apoptosis. 

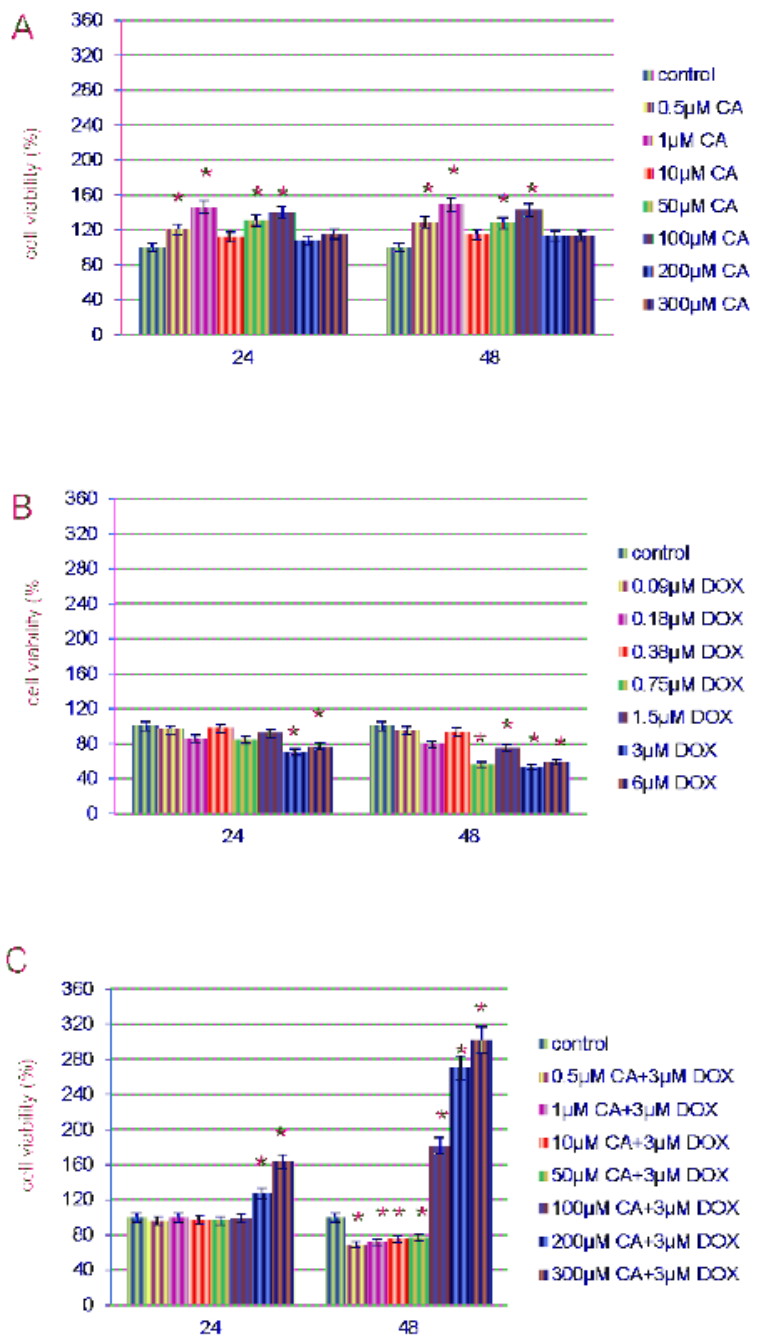

Figure 3. The effect of CA, DOX and CA + DOX on cell viability of fibroblasts. The cells were incubated with $0.5 \mu \mathrm{M}, 1 \mu \mathrm{M}, 10 \mu \mathrm{M}, 50 \mu \mathrm{M}, 100 \mu \mathrm{M}, 200 \mu \mathrm{M}$ and $300 \mu \mathrm{M}$ CA for $24 \mathrm{~h}$ and $48 \mathrm{~h}(\mathrm{~A}) ; 0.09 \mu \mathrm{M}$, $0.18 \mu \mathrm{M}, 0.38 \mu \mathrm{M}, 0.75 \mu \mathrm{M}, 1.5 \mu \mathrm{M}, 3 \mu \mathrm{M}$ and $6 \mu \mathrm{M}$ DOX for $24 \mathrm{~h}$ and $48 \mathrm{~h}(\mathrm{~B})$; mix of CA and DOX in concentrations: $0.5 \mu \mathrm{M} \mathrm{CA}+3 \mu \mathrm{M}$ DOX, $1 \mu \mathrm{M} \mathrm{CA}+3 \mu \mathrm{M}$ DOX, $10 \mu \mathrm{M} \mathrm{CA}+3 \mu \mathrm{M}$ DOX, $50 \mu \mathrm{M}$ CA + $3 \mu \mathrm{M}$ DOX, $100 \mu \mathrm{M}$ CA $+3 \mu \mathrm{M}$ DOX, $200 \mu \mathrm{M}$ CA $+3 \mu \mathrm{M}$ DOX and $300 \mu \mathrm{M}$ CA $+3 \mu \mathrm{M}$ DOX for $24 \mathrm{~h}$ and $48 \mathrm{~h}(\mathrm{C})$. Mean values from three independent experiments $\pm \mathrm{SD}$ are shown. Significant alterations are expressed relative to control, untreated cells, and marked with asterisks. Statistical significance was considered if $* p<0.05$. CA: cichoric acid, DOX: doxorubicin.

\subsection{Determination of Caspase $3 / 7$ Activity}

Caspase-Glo ${ }^{\circledR}$ 3/7 Assay was used to assess apoptosis level under the influence of the tested substances on fibroblast cell line. The cells were subjected to $3 \mu \mathrm{M} \mathrm{DOX}, 300 \mu \mathrm{M}$ CA and DOX combined with CA in the above-mentioned concentrations for $24 \mathrm{~h}$ and $48 \mathrm{~h}$ (Figure 4). $300 \mu \mathrm{M}$ of $\mathrm{CA}$ was added to the cell culture $2 \mathrm{~h}$ before adding $3 \mu \mathrm{M}$ DOX. An increased level of apoptosis was 
observed in the presence of $3 \mu \mathrm{M}$ DOX and in case of simultaneous treatment with DOX and CA, however when CA was added to the DOX-treated culture the level of apoptosis was decreased as compared to DOX—treated cells. Exposure of fibroblast cells to $300 \mu \mathrm{M}$ CA significantly decreased caspase $3 / 7$ activity and therefore apoptosis.

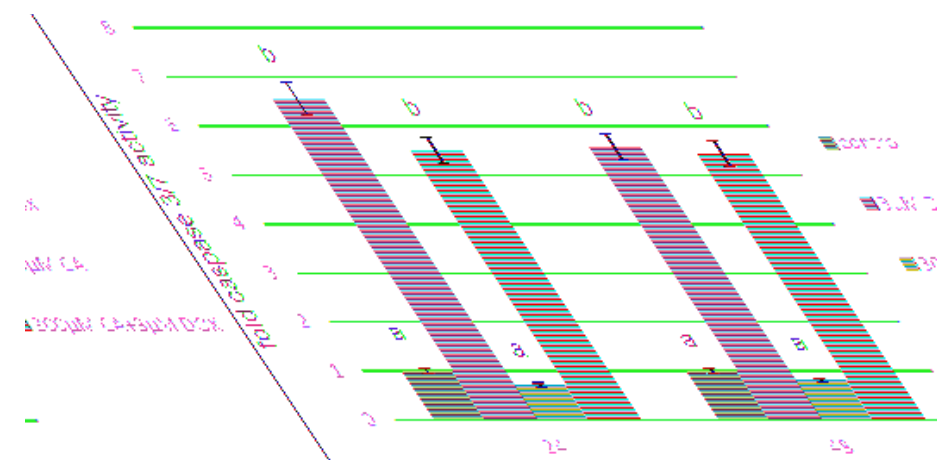

Figure 4. The effect of DOX, CA and CA + DOX on caspase 3/7 activity in fibroblast cells. The cells were incubated with $3 \mu \mathrm{M}$ DOX, $300 \mu \mathrm{M}$ CA and DOX $(3 \mu \mathrm{M})$ combined with CA $(300 \mu \mathrm{M})$ for $24 \mathrm{~h}$ and $48 \mathrm{~h}$ Mean values from three independent experiments \pm SD are shown. Different letters $(\mathrm{a}, \mathrm{b})$ indicate statistical differences $(p \leq 0.05)$ between control, $3 \mu \mathrm{M} \mathrm{DOX}, 300 \mu \mathrm{M} \mathrm{CA}$ and DOX combined with CA estimated by Tukey's test.

\subsection{Determination of Caspase 9 Activity}

For the measurement of the caspase 9 activity Caspase-Glo ${ }^{\circledR} 9$ Assay was applied. Similarly as in case of caspase $3 / 7$ activity results, a significant increase in caspase 9 activity was observed under the influence of $3 \mu \mathrm{M}$ DOX (Figure 5). CA in $300 \mu \mathrm{M}$ concentration caused a significant decrease in caspase 9 activity. However, the most interesting and significant result is statistically significant decrease in studied caspase activity under the influence of $300 \mu \mathrm{M}$ CA combined with $3 \mu \mathrm{M}$ DOX. The level of apoptosis in this case is even lower than in control, untreated cells, which may mean the CA is effective in counteracting the effect of DOX on healthy cell apoptosis.

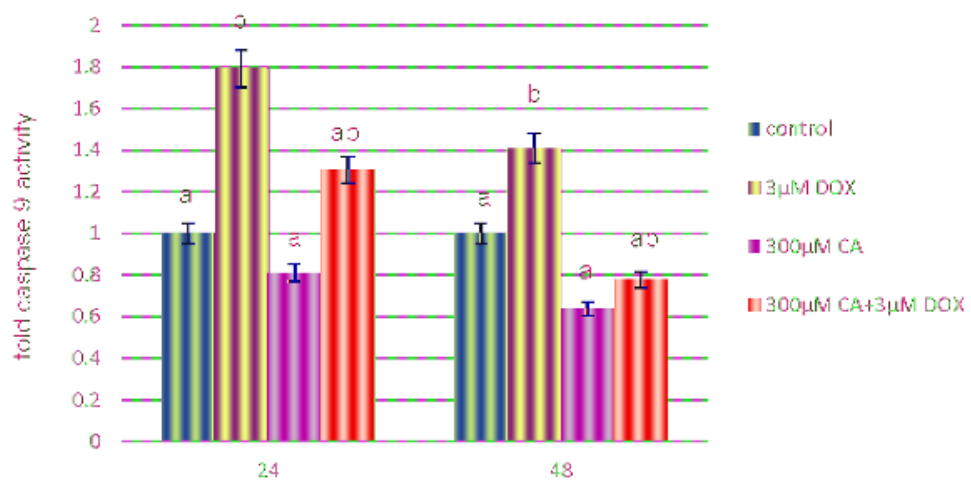

Figure 5. The effect of DOX, CA and CA+DOX on caspase 9 activity in fibroblast cells. The cells were incubated with $3 \mu \mathrm{M}$ DOX, $300 \mu \mathrm{M}$ CA and DOX $(3 \mu \mathrm{M})$ combined with CA $(300 \mu \mathrm{M})$ for $24 \mathrm{~h}$ and $48 \mathrm{~h}$ Mean values from three independent experiments \pm SD are shown. Different letters $(\mathrm{a}, \mathrm{b})$ indicate statistical differences $(p \leq 0.05)$ between control, $3 \mu \mathrm{M}$ DOX, $300 \mu \mathrm{M}$ CA and DOX combined with CA estimated by Tukey's test. 


\subsection{Determination of SH Groups}

The effect of DOX and CA on SH group content is shown in Figure 6. To determine the oxidation of the SH group, a spectrophotometric assay with Ellman's reagent was used. Thiol group content was evaluated as a marker of protein oxidation. A significant decrease in thiol group content of $\sim 25 \%$ compared to the control was observed especially after $48 \mathrm{~h}$ of treatment at a concentration of $3 \mu \mathrm{M}$ DOX. Exposure to CA resulted in $~ 50 \%$ increase in the total cellular content of the thiol groups after $48 \mathrm{~h}$ of treatment. Obtained results revealed that $300 \mu \mathrm{M}$ concentration of CA eliminated pro-oxidative effect of DOX on fibroblasts, because we observed a significant increase in thiol group content under the influence of $3 \mu \mathrm{M}$ DOX in culture pretreated with $300 \mu \mathrm{M}$ CA. An observed SH group level after $48 \mathrm{~h}$ treatment with DOX combined with CA was even higher than it was in control untreated cells. These data indicate that CA is an effective agent in elimination of the oxidative stress, mainly oxidative damage of proteins, caused by DOX.

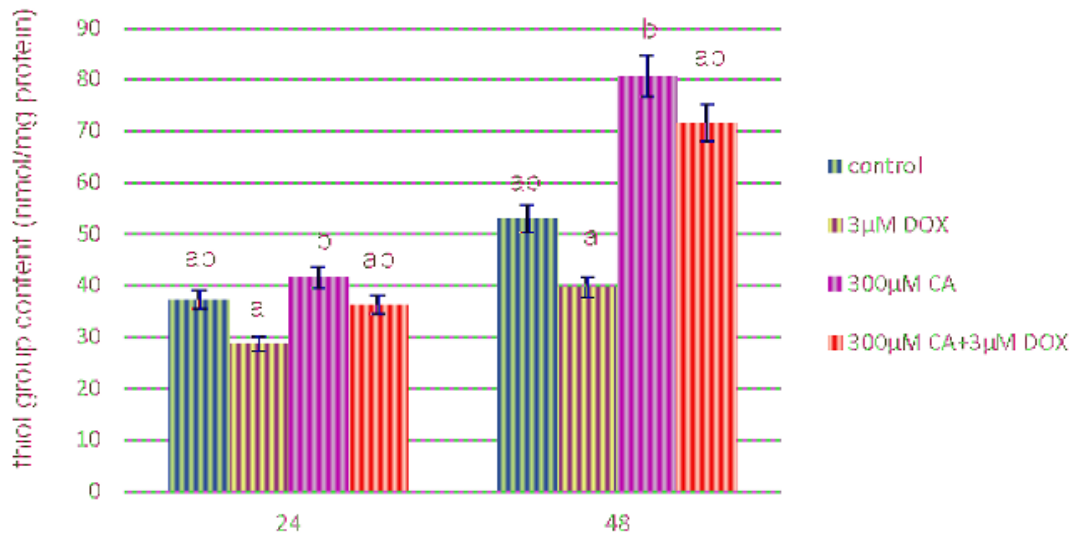

Figure 6. The effect of DOX, CA and CA+DOX on SH group content in fibroblasts. The cells were incubated with $3 \mu \mathrm{M}$ DOX, $300 \mu \mathrm{M}$ CA and DOX $(3 \mu \mathrm{M})$ combined with CA $(300 \mu \mathrm{M})$ for $24 \mathrm{~h}$ and $48 \mathrm{~h}$ Mean values from three independent experiments $\pm \mathrm{SD}$ are shown. Different letters $(\mathrm{a}, \mathrm{b})$ indicate statistical differences $(p \leq 0.05)$ between control, $3 \mu \mathrm{M}$ DOX, $300 \mu \mathrm{M} \mathrm{CA}$ and DOX combined with CA estimated by Tukey's test.

\subsection{Determination of TBA Reactive Species (TBARS) Levels}

Lipid peroxidation is a process connected with a variety of cellular dysfunctions, which result from the inappropriate modifications of lipid-protein complexes. TBARS content was measured as an index of lipid peroxidation. The results showed significant differences between TBARS levels in the control, DOX, CA and DOX-CA - treated cells (Figure 7). The addition of $3 \mu \mathrm{M}$ DOX to the cells induced a significant increase in TBARS content of $\sim 95 \%$ compared to the control after 24 h treatment. CA at a concentration of $300 \mu \mathrm{M}$ induced a decrease of $\sim 14 \%$ compared to the control observed after $24 \mathrm{~h}$ of incubation. CA in $300 \mu \mathrm{M}$ concentration caused a reduction in TBARS content, which was raised because of DOX influence. The obtained results suggest that CA demonstrates protective properties against TBARS production caused by DOX, and as a consequence it decreases membrane phospholipid peroxidation. 


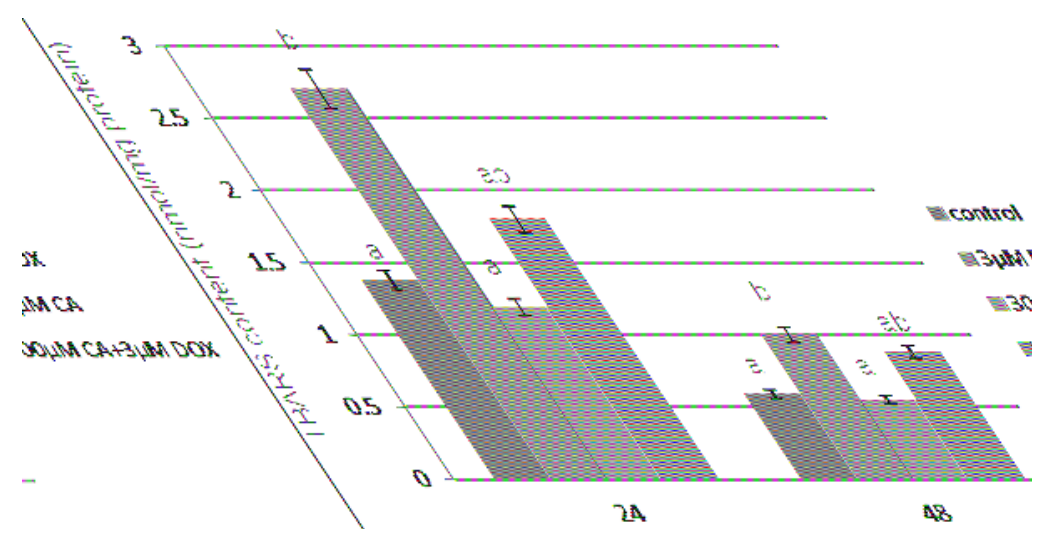

Figure 7. The effect of DOX, CA and CA + DOX on TBARS content in fibroblast cells. The cells were incubated with $3 \mu \mathrm{M}$ DOX, $300 \mu \mathrm{M}$ CA and DOX $(3 \mu \mathrm{M})$ combined with CA $(300 \mu \mathrm{M})$ for $24 \mathrm{~h}$ and $48 \mathrm{~h}$ Mean values from three independent experiments \pm SD are shown. Different letters $(\mathrm{a}, \mathrm{b})$ indicate statistical differences $(p \leq 0.05)$ between control, $3 \mu \mathrm{M}$ DOX, $300 \mu \mathrm{M} \mathrm{CA}$ and DOX combined with CA estimated by Tukey's test.

\subsection{Determination of GSH/GSSG}

Reduced glutathione is one of the most important low mass antioxidants, therefore the estimation of GSH/GSSG ratio constitute an essential study in oxidative stress parameters research. DOX in $3 \mu \mathrm{M}$ concentration treatment caused a significant decreases in GSH/GSSG ratio after $24 \mathrm{~h}$ and $48 \mathrm{~h}$ of incubation, while $300 \mu \mathrm{M}$ of CA treatment resulted in significant increases in both incubation times as compared to the control, untreated cells. Cell culture pre-treatment with CA in $300 \mu \mathrm{M}$ concentration resulted in an elevated ration of GSH/GSSG after $24 \mathrm{~h}$ and $48 \mathrm{~h}$ of incubation even after addition of DOX. After $24 \mathrm{~h}$ of incubation with the addition of DOX and CA mixture the level of GSH was elevated even as compared to control untreated cells. The effect of DOX, CA and DOX combined with CA on GSH/GSSG ratio is shown in Figure 8. Obtained results revealed an inhibitory influence of DOX and stimulatory effect of CA on GSH amount in fibroblast cell line.

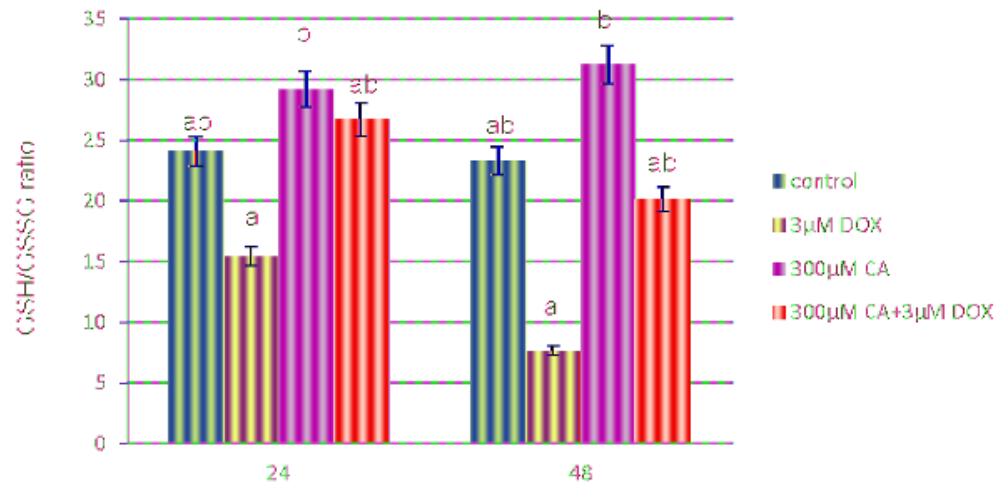

Figure 8. The effect of DOX, CA and CA + DOX on GSH/GSSG ratio in fibroblasts. The cells were incubated with $3 \mu \mathrm{M}$ DOX, $300 \mu \mathrm{M}$ CA and DOX $(3 \mu \mathrm{M})$ combined with CA $(300 \mu \mathrm{M})$ for $24 \mathrm{~h}$ and $48 \mathrm{~h}$ Mean values from three independent experiments \pm SD are shown. Different letters $(a, b)$ indicate statistical differences $(p \leq 0.05)$ between control, $3 \mu \mathrm{M}$ DOX, $300 \mu \mathrm{M}$ CA and DOX combined with CA estimated by Tukey's test. 


\subsection{Intracellular ROS Detection}

Figure 9 shows the relative fluorescence intensity of $2^{\prime}, 7^{\prime}$-dichlorofluorescein (DCF) as a percent of control in fibroblast cells incubated with $3 \mu \mathrm{M}$ of DOX or $300 \mu \mathrm{M}$ of CA or $3 \mu \mathrm{M}$ of DOX and $300 \mu \mathrm{M}$ of CA for 24 and $48 \mathrm{~h}$. An increase in the intracellular ROS production resulted in higher intensity of DCF fluorescence and it was dependent on the concentration of studied compounds. After $24 \mathrm{~h}$ incubation of fibroblast cells with $3 \mu \mathrm{M}$ DOX, the intracellular ROS generation was about $50 \%$ higher in comparison to control, untreated cells. The $48 \mathrm{~h}$ incubation with DOX resulted in $35 \%$ increase in ROS production. CA treatment caused non-significant decrease in intracellular ROS production. Pre-treatment with $300 \mu \mathrm{M}$ CA significantly reduced ROS content in DOX treated cell culture. These data show inhibitory and protective effect of CA on ROS formation.

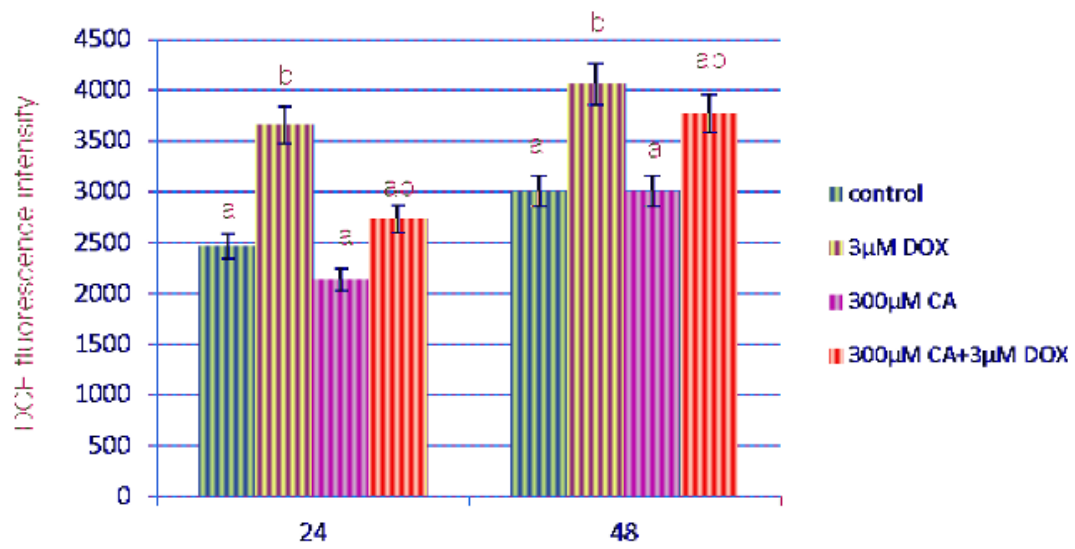

Figure 9. The effect of DOX, CA and CA + DOX on the level of intracellular ROS in fibroblasts. The cells were incubated with $3 \mu \mathrm{M}$ DOX, $300 \mu \mathrm{M}$ CA and DOX $(3 \mu \mathrm{M})$ combined with CA $(300 \mu \mathrm{M})$ for $24 \mathrm{~h}$ and $48 \mathrm{~h}$ Mean values from three independent experiments \pm SD are shown. Different letters $(\mathrm{a}, \mathrm{b})$ indicate statistical differences $(p \leq 0.05)$ between control, $3 \mu \mathrm{M}$ DOX, $300 \mu \mathrm{M} \mathrm{CA}$ and DOX combined with CA estimated by Tukey's test.

Obtained results were confirmed by using flow cytometry analysis. Figure 10 indicates the effect of DOX, CA and DOX combined with CA on intracellular ROS generation in fibroblasts. It shows the $\%$ of DCF positive fibroblast cells incubated with $3 \mu \mathrm{M}$ of DOX, $300 \mu \mathrm{M}$ of CA and the mixture of two tested compounds for $24 \mathrm{~h}$ and $48 \mathrm{~h}$. Obtained results indicate that DOX in $3 \mu \mathrm{M}$ concentration is efficient in enhancing oxidative stress level in fibroblasts. The amount of ROS under the influence of DOX was significantly elevated by about $20 \%$ after $24 \mathrm{~h}$ of incubation and by about $45 \%$ after $48 \mathrm{~h}$ of incubation as compared to control, non-treated cells. Presented results are statistically significant. Pretreatment with $300 \mu \mathrm{M}$ of CA caused a significant reduction in ROS content in DOX-treated culture. The difference in ROS formation in cultures treated with CA mixed with DOX and cultures treated with DOX only amounted approximately $40 \%$ after $48 \mathrm{~h}$ of incubation. This results confirmed protective properties of CA against ROS generation in DOX-treated fibroblasts. 


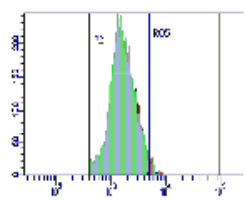

$24 h$, control

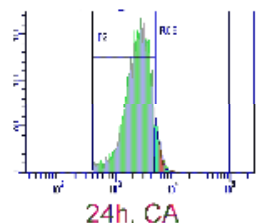

$24 h, C A$

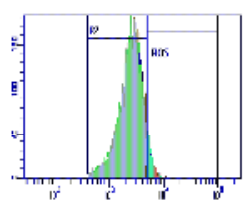

$48 \mathrm{~h}$, control

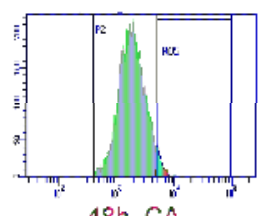

48h, CA

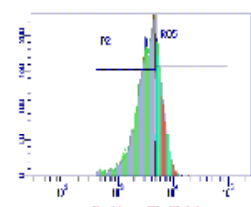

24h, DOX

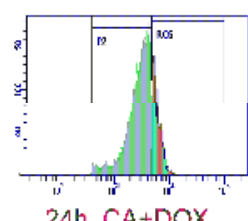

$24 \mathrm{~h}, \mathrm{CA}+\mathrm{DOX}$

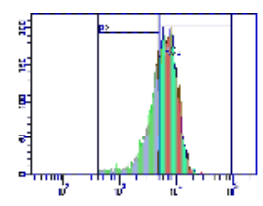

$48 \mathrm{~h}, \mathrm{DOX}$

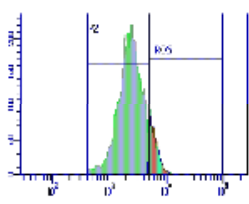

48h. CA+DOX

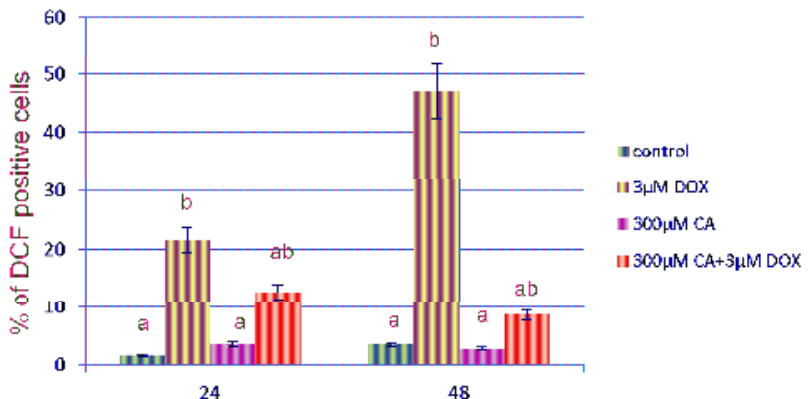

Figure 10. The effect of DOX, CA and CA + DOX on the level of intracellular ROS examined by flow cytometry in fibroblast cells. This Figure shows representative histograms of fibroblasts FACS analysis and the percentage of fibroblast cells generating intracellular ROS. The cells were incubated with $3 \mu \mathrm{M}$ DOX, $300 \mu \mathrm{M}$ CA and DOX $(3 \mu \mathrm{M})$ combined with CA $(300 \mu \mathrm{M})$ for $24 \mathrm{~h}$ and $48 \mathrm{~h}$ Mean values from three independent experiments \pm SD are shown. Different letters $(a, b)$ indicate statistical differences $(p \leq 0.05)$ between control, $3 \mu \mathrm{M}$ DOX, $300 \mu \mathrm{M}$ CA and DOX combined with CA estimated by Tukey's test

\subsection{Fluorescent Microscopy Assay}

In order to evaluate the apoptotic and necrotic cells morphology and for the confirmation of the apoptosis process measured in above described methods, fluorescent staining was used (Figure 11). Similar to luminescence analysis we observed changes between the control, DOX and CA-treated cells. In control cells lack of chromatin condensation and a few to over a dozen of bright green stained nucleoli were observed. At a $3 \mu \mathrm{M}$ concentration of DOX, numerous of apoptotic cells with visible chromatin condensation and apoptotic bodies especially after $48 \mathrm{~h}$ treatment were seen. DOX treatment also caused chromatin marginalization. However, CA treated cells did not reveal any significant apoptotic changes and CA pretreatment in cells before addition of DOX caused a significant reduction in apoptosis level. 


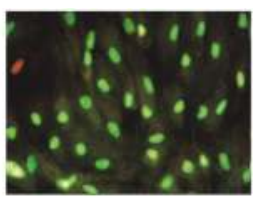

$24 h$, control

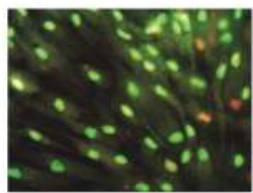

$24 h, C A$

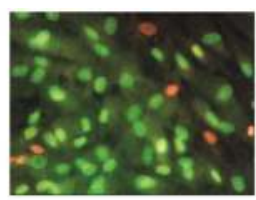

$48 \mathrm{~h}$, control

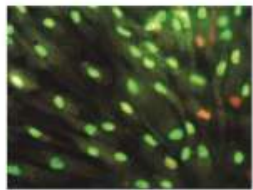

48h, CA

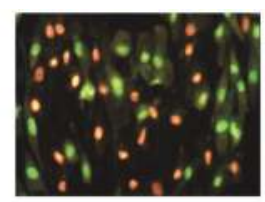

24h, DOX

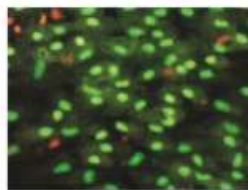

$24 h, C A+D O X$

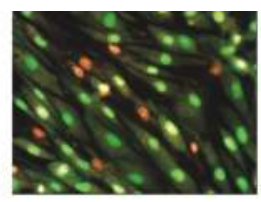

48h, DOX

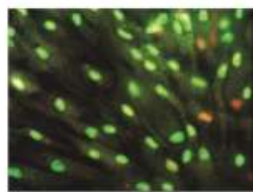

$48 h, C A+D O X$

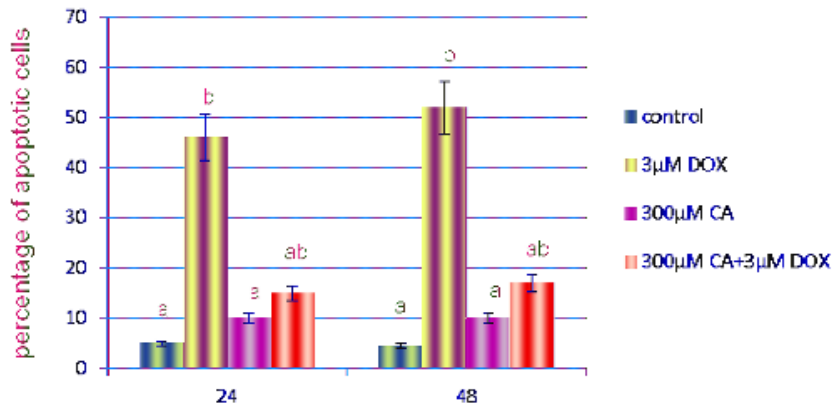

Figure 11. The effect of DOX, CA and CA + DOX on apoptosis and necrosis in the fibroblasts evaluated by fluorescence microscope assay. The cells were incubated in $3 \mu \mathrm{M}$ DOX, $300 \mu \mathrm{M} \mathrm{CA}$ and DOX $(3 \mu \mathrm{M})$ combined with CA $(300 \mu \mathrm{M})$ for 24 , and $48 \mathrm{~h}$ and stained with acridine orange and ethidium bromide. The cells were photographed under a fluorescence microscope at $200 \times$ magnification. We presented representative images from one of three independent experiments and the percentage of apoptotic cells on the graph. Different letters $(\mathrm{a}, \mathrm{b})$ indicate statistical differences $(p \leq 0.05)$ between control, $3 \mu \mathrm{M}$ DOX, $300 \mu \mathrm{M}$ CA and DOX combined with CA estimated by Tukey's test

\section{Discussion}

The selected natural phenolic compounds are common components of human diet and their antioxidant and as well as anti-inflammatory and anticancer properties are widely described, e.g., CA could be beneficial for preventing dermal injury in the setting of DOX treatment [31-34]. Cichoric acid is a compound with many potentially beneficial properties. It can be obtained from isolated and purified plant and vegetables and it has been described as a one daily nutraceutical which enhances antioxidant activity [35]. The consumption of CA, its derivatives and CA containing plants may significantly influence redox balance in human organism and provide excellent health benefits. CA has been reported as potential anti-diabetic and anti-obesity compound [36]. However, what is important in the context of nutrition in pathological conditions, its oral intake alleviates severe side effects of an excessive alcohol consumption, which was studied in case of acute alcohol-induced hepatic steatosis in mice. Mechanism of CA action in above described study resulted from oxidative stress inhibition and CA anti-inflammation properties [37]. 
The anti- and pro-oxidant activity of cichoric acid was determined and compared with the activity of caffeic acid, chlorogenic acid and quercetin. Cichoric acid is the derivative of caffeic acid and tartaric acid. The presence of the two caffeic acid moieties in the cichoric acid molecule determines its high antioxidant properties. Therefore the caffeic acid was chosen in this comparative study. Chlorogenic acid is another chosen phenolic compound which is an ester of caffeic and quinic acids. It was selected in this study in order to estimate whether the number of caffeic acid moieties or the present of additional structure (e.g., quinic acid fragment) affect the anti-/pro-oxidant properties of molecules. Quercetin was chosen as one of the most intensively studied antioxidants. Obtained results indicate that according to the increasing antioxidant activity the studied compounds may be ordered as follows: cichoric acid $>$ caffeic acid $\sim$ quercetin $>$ chlorogenic acid. The selected compounds were studied for their pro-oxidative effect on trolox oxidation. In this assay the radicals of polyphenolics are produced in their reaction with $\mathrm{H}_{2} \mathrm{O}_{2}$ catalysed by horseradish peroxidase. Then the phenoxyl radicals react with trolox and cause its oxidation to trolox radicals and trolox quinones. In the same time the phenoxyl radicals are transformed to phenolic compounds. The maximum absorption for trolox quinone is $272 \mathrm{~nm}$. This is a general procedure for study the pro-oxidant activity of phenolic compounds [27]. Obtained results showed that the pro-oxidant activity increases in the series: cichoric acid < quercetin < caffeic acid < chlorogenic acid. Cichoric acid was selected for this study because of its high antioxidant properties and relatively lower pro-oxidant activity compared to compounds which display structural similarities.

An increasing interest in the evaluation of clinical effectiveness of natural extracts, dietary supplements, plant origin antioxidants, especially from the polyphenol group of compounds is being observed. The understanding of the molecular mechanisms underlying dietary antioxidants action in diseases prevention and treatment is important to effectively complete these improvements with healthy lifestyle changes. The aim of this actions is mainly to improve human health, prevent diseases and eliminate some severe side effects of the commonly used chemotherapeutics, especially in the cancer treatment. An example of such chemotherapeutic, widely used in the treatment of a variety of cancers, both hematological malignancies and solid tumors is DOX. However, the clinical application of DOX is limited, which results from its high, cumulative dose-dependent toxicity, especially cardiotoxicity, leading to cardiomyopathy. An observed DOX-induced toxicity may be a result of DOX-induced mitochondrial dysfunction and accumulation of oxidative stress products, such as lipid peroxidation products and/or protein oxidation products $[17,38]$.

In order to study possible protective properties of CA on DOX-treated normal human fibroblasts we decided to choose, based on prior experiments, a range of DOX [39] and CA [40] concentrations and estimate the most effective concentrations for both tested compounds. MTT assay revealed that in normal conditions, without any stress factors, CA is efficient in the stimulation of fibroblasts proliferation especially in $0.5 \mu \mathrm{M}, 1 \mu \mathrm{M}, 50 \mu \mathrm{M}$ and $100 \mu \mathrm{M}$ concentration. On the other hand, DOX inhibits fibroblasts viability most effectively at the concentration of $3 \mu \mathrm{M}$. Therefore, this DOX concentration was chosen for further analysis. The third part of MTT assay showed that pretreatment of fibroblasts with CA $(0.5-300 \mu \mathrm{M})$ for $2 \mathrm{~h}$ before stimulate with DOX was the most effective at $300 \mu \mathrm{M}$ of CA. Because of that reason, this combination of DOX with CA was applied for the evaluation of the anti-apoptotic and antioxidative effect. Literature data indicate the fact that CA has positive influence on normal cells, e.g., human dermal fibroblasts, where it induces Hepatocyte Growth Factor production on hepatocytes and where it may act as an anti-viral agent [41,42].

Reactive oxygen species (ROS) occurring due to too high level of oxidative stress are chemically reactive molecules that may damage main cellular macromolecules, such as DNA, lipids and proteins, subsequently causing genetic mutations. An excessive exposure to ROS may be seriously deleterious because of oxidative damage to important molecules in cell [43]. To assess the effect of CA on proteins in this work we used one of the most widely studied markers of protein oxidation-protein thiol groups content. We observed the oxidative action of DOX, which conforms that DOX exhibits pro-oxidant properties against proteins and this action is connected with its cytotoxic activity. On the other hand 
stimulation with CA caused a significant increase in thiol group content, which means that tested compound act as an antioxidant. Especially after $48 \mathrm{~h}$ of treatment we can observe that prooxidative activity of DOX was effectively reversed by CA treatment. Study by Tsai KL et al. confirmed that pretreatment with CA may inhibit oxidative stress caused by external factors [44].

Many natural compounds from the polyphenol group, which also include CA, are characterized by high antioxidant capacities and have been considered as a potential protectors against DOX-induced toxicity. This protective effect may be also connected with the reduction of lipid peroxidation process [19]. In support of this report, we observed that CA does not cause oxidative alterations of membrane lipids and protects against DOX-induced TBARS production, which is probably related with antioxidant properties of CA. Already after $24 \mathrm{~h}$ we noticed significant increase in TBARS content in DOX-treated cells and a decrease in cells pretreated with $300 \mu \mathrm{M}$ of CA. CA activity as a compound that prevents lipid peroxidation process was confirmed by several studies [45-47]. Polyphenols exhibit antioxidative properties at several levels, among others they prevent the lipid peroxidation of biological membranes and inhibit the oxidation of lipoproteins and polyunsaturated fatty acids, which is in consistence with our results [48].

Low ROS concentration within the cell is essential for keeping redox balance and for the stimulation of cell proliferation rate. However, an excessive level of accumulated in cell ROS may cause serious damage in protein, lipid and DNA structures through their oxidation and subsequently apoptosis and cell death $[49,50]$. According to the literature CA, which belongs to the polyphenols group of compounds, may provoke ROS generation in cancer cells and also in preadipocytes, but in normal cells CA was a potent ROS scavenger, reducing ROS accumulation under basal as well as oxidative stress conditions [51]. It is in accordance with our results regarding ROS generation in fibroblast cells. We observed a significant increase in ROS production under the influence of DOX and a decrease in ROS content caused by CA. Pretreatment of fibroblast cells with CA $(300 \mu \mathrm{M})$ for $2 \mathrm{~h}$ before stimulate with DOX significantly inhibited the formation of ROS. Flow cytometry results confirm that intervention with CA mitigated DOX-caused oxidative stress and reduced ROS formation in fibroblasts stimulated with DOX. Therefore we presume that the primary mechanism through which CA alleviates DOX-facilitated cell death of fibroblast cells is its antioxidant function. An observed, DOX-induced significant increase in ROS content was accompanied by a decrease in GSH/GSSG ratio, especially after $48 \mathrm{~h}$ of treatment. Similarly as in other tested oxidative stress parameters, GSH/GSSG ratio was significantly reduced after exposure to DOX and these results were effectively reversed by CA treatment. Our results are in accordance with literature data indicating positive influence of selected polyphenols on GSH content. One of the main phenolic compounds of curry spice turmeric is curcumin, the consumption of which brings beneficial effects. It was shown that curcumin plays a protective role against adriamycin-induced toxicity through three main mechanisms: first-it inhibits lipid peroxidation, second - it stabilizes cell membranes and third-it raises glutathione level [52]. Therefore we presume that CA likewise influences GSH level in fibroblast cells. According to Fauser JK et al. a reduced level of the main GSH antioxidant may result in apoptosis and it also may be connected with a reduction of G0/G1 and arrest of the S and M cell cycle phases, together with increased ROS generation [53]. Apoptosis is a programmed cell death that is extremely important for human organism homeostasis maintaining, for tissue development and malignancies treatment. Regulation of this process is main target for anticancer drugs, however chemotherapeutics, such as anthracycline antibiotics, e.g., DOX, may also induce apoptosis in normal, non-cancerous cells. At least two pathways have been described by which an apoptosis occurs: first is an extrinsic death-receptor dependent apoptosis and the second-intrinsic mitochondrial dependent apoptosis. In both pathways selected caspases activation occurs, which means activation of initiator caspases (e.g., caspase- 8 and -9) and effector caspases (e.g., caspase-3, -6, and -7) [54,55]. A variety of evidence support also the role of reactive oxygen species in apoptotic cell death. It has been shown that oxygen free radicals are directly involved in the pathogenesis of apoptosis. Caspase 3/7 assay, caspase 9 assay and fluorescent microscopy assay showed that at $3 \mu \mathrm{M}$ of DOX concentration, numerous apoptotic cells were seen, 
while pretreatment with $300 \mu \mathrm{M}$ of CA reduced the number of positive fluorescent cells and decreased the activity of studied caspases significantly. We observed an increase in caspase 3, 7 and 9 activity under the influence of DOX as compared to control non-treated cells, which was accompanied by a decrease in GSH/GSSG ratio and a significant increase in ROS content. Apoptosis is particularly related to the cascade of caspases. Literature data indicated that the release of cytochrome $\mathrm{c}$ from the mitochondria into the cytosol, may result in the caspase-9 activation, which subsequently activates effector caspase, such as caspase-3, which causes DNA fragmentation and eventually-cell death [56]. In our study, we found that CA significantly prevented DOX-induced activation of caspase 3 and 7, which is in accordance with literature data that confirms such CA activity. It may thus be hypothesized that this anti-apoptotic effect of CA might be attributed to observed increase in the levels of antioxidant glutathione (reduced GSH) and subsequently to a decrease in oxidative stress level. Tsai KL et al. revealed that $C A$ precluded the activation of caspase 3 and subsequent DNA strand breaks, through the inhibition of the translocation of NF- $\mathrm{KB}$ from the cytosol to the nucleus, suppression of Bax and promotion the Bcl-2 expression [44]. In our experiment pretreatment with CA was associated with the inhibition of the downstream apoptotic signaling pathways, finally preventing activation of caspase-3, caspase- 7 and caspase -9 induced by DOX. It is suggested that CA is a natural food-derived compound that inhibits apoptosis [57]. Our findings confirm this hypothesis, because under the influence of CA we showed a significant increase in cells proliferation and an decrease in caspase $3 / 7$ and 9 level, which is accompanied by an increase in GSH content and a decrease in ROS content. Anti-apoptotic effect of CA in DOX-treated fibroblasts was confirmed by fluorescence microscopy assay, which indicated that tested compound was efficient both in time and dose-dependent manner.

\section{Conclusions}

In conclusion, our findings suggest that CA mitigates DOX-induced oxidative stress and inhibits DOX-facilitated ROS formation. Chicoric acid treatment also inhibited DOX-facilitated apoptosis. It is likely that these beneficial effects contribute to the overall antioxidative and anti-apoptotic function of chicoric acid. However, only in vitro investigations were used to test the cytoprotective effects of chicoric acid from DOX-caused fibroblasts dysfunction. Above results may be the first step in explaining important mechanisms underlying the protective effects of CA observed in DOX cytotoxicity. However, additional studies are indispensable to evaluate long-term effect of CA on dermal injuries caused by DOX treatment. Further analysis must also be conducted on the other skin cell types, such as keratinocytes and melanocytes. Further analysis must also be conducted on the molecular level of the anti-oxidative and anti-apoptotic effects of CA, which may be considered as lead compounds for the development of functional food.

Acknowledgments: This work was financially supported by National Science Centre, Poland, under the research project number 2015/17/B/NZ9/03581.

Author Contributions: Agata Jabłońska-Trypuć-corresponding author, wrote the paper, planned experiments; performed experiments; analysed data; Rafał Krẹtowski-planned flow cytometry experiments; performed flow cytometry experiments; analysed data; Monika Kalinowska-planned chemical experiments, performed chemical experiment, analysed data; Grzegorz Świderski-analyzed the data; Marzanna Cechowska-Pasko-analyzed data; Włodzimierz Lewandowski-analyzed the data.

Conflicts of Interest: The authors declare no conflict of interest.

Compliance with ethical standards: The manuscript does not contain clinical studies or patient data.

\section{References}

1. Shroff, A.; Mamalis, A.; Jagdeo, J. Oxidative Stress and Skin Fibrosis. Curr. Pathobiol. Rep. 2014, 2, $257-267$. [CrossRef] [PubMed]

2. Bickers, D.R.; Athar, M. Oxidative stress in the pathogenesis of skin disease. J. Investig. Dermatol. 2006, 126, 2565-2575. [CrossRef] [PubMed] 
3. Ji, H.; Li, X.K. Oxidative Stress in Atopic Dermatitis. Oxid. Med. Cell. Longev. 2016, 2016, 2721469. [CrossRef] [PubMed]

4. Komuro, T. Re-evaluation of fibroblasts and fibroblast-like cells. Anat. Embryol. 1990, 182, 103-112. [CrossRef] [PubMed]

5. Atkins, F.M.; Friedman, M.M.; Rao, P.V.S.; Metcalfe, D.D. Interactions between mast cells, fibroblasts and connective tissue components. Int. Arch. Allergy Appl. Immunol. 1985, 77, 96-102. [CrossRef] [PubMed]

6. Gebicki, J.M.; Bartosz, G. The role of proteins in propagation of damage induced by reactive oxygen species in vivo. Postepy Biochem. 2010, 56, 115-123. [PubMed]

7. Bartosz, G. Total antioxidant capacity. Adv. Clin. Chem. 2003, 37, 219-292. [PubMed]

8. Menzel, D.B. The toxicity of air pollution in experimental animals and humans: The role of oxidative stress. Toxicol. Lett. 1994, 72, 269-277. [CrossRef]

9. Kohen, R.; Gati, I. Skin low molecular weight antioxidants and their role in aging and in oxidative stress. Toxicology 2000, 148, 149-157. [CrossRef]

10. Jabłońska-Trypuć, A.; Świderski, G.; Krętowski, R.; Lewandowski, W. Newly Synthesized Doxorubicin Complexes with Selected Metals-Synthesis, Structure and Anti-Breast Cancer Activity. Molecules 2017, 22, 1106. [CrossRef] [PubMed]

11. Coldwell, K.; Cutts, S.M.; Ognibene, T.J.; Henderson, P.T.; Phillips, D.R. Detection of adriamycin-DNA adducts by accelerator mass spectrometry. Met. Mol. Biol. 2010, 613, 103-118. [CrossRef]

12. Sauter, K.A.; Magun, E.A.; Iordanov, M.S.; Magun, B.E. ZAK is required for doxorubicin, a novel ribotoxic stressor, to induce SAPK activation and apoptosis in HaCaT cells. Cancer Biol. Ther. 2010, 10, 258-266. [CrossRef] [PubMed]

13. Cao, B.; Li, M.; Zha, W.; Zhao, Q.; Gu, R.; Liu, L.; Shi, J.; Zhou, J.; Zhou, F.; Wu, X.; et al. Metabolomic approach to evaluating adriamycin pharmacodynamics and resistance in breast cancer cells. Metabolomics 2013, 9, 960-973. [CrossRef] [PubMed]

14. Ta, H.Q.; Thomas, K.S.; Schrecengost, R.S.; Bouton, A.H. A novel association between p130Cas and resistance to the chemotherapeutic drug adriamycin in human breast cancer cells. Cancer Res. 2008, 68, 8796-8804. [CrossRef] [PubMed]

15. Taskin, E.; Dursun, N. Recovery of adriamycin induced mitochondrial dysfunction in liver by selenium. Cytotechnology 2015, 67, 977-986. [CrossRef] [PubMed]

16. Yapislar, H.; Taskin, E.; Ozdas, S.; Akin, D.; Sonmez, E. Counteraction of apoptotic and inflammatory effects of adriamycin in the liver cell culture by clinopitolite. Biol. Trace Elem. Res. 2016, 170, 373-381. [CrossRef] [PubMed]

17. De Beer, E.L.; Bottone, A.E.; Voest, E.E. Doxorubicin and mechanical performance of cardiac trabeculae afteracute and chronic treatment: A review. Eur. J. Pharmacol. 2001, 415, 1-11. [CrossRef]

18. Singal, P.K.; Li, T.; Kumar, D.; Danelisen, I.; Iliskovic, N. Adriamycin-induced heart-failure: Mechanism and modulation. Mol. Cell. Biochem. 2000, 207, 77-85. [CrossRef] [PubMed]

19. Quiles, J.L.; Huertas, J.R.; Battino, M.; Mataix, J.; Ramírez-Tortosa, M.C. Antioxidant nutrients and adriamycin toxicity. Toxicology 2002, 180, 79-95. [CrossRef]

20. Pellati, F.; Benvenuti, S.; Magro, L.; Melegari, M.; Soragni, F. Analysis of phenolic compounds and radical scavenging activity of Echinacea spp. J. Pharm. Biomed. Anal. 2004, 35, 289-301. [CrossRef]

21. Barnes, J.; Anderson, L.A.; Gibbons, S.; Phillipson, J.D. Echinacea species (Echinacea angustifolia (DC.) Hell., Echinacea pallida (Nutt.) Nutt., Echinacea purpurea (L.) Moench): A review of their chemistry, pharmacology and clinical properties. J. Pharm. Pharmacol. 2005, 57, 929-954. [CrossRef] [PubMed]

22. Robinson, W.E.; Reinecke, M.G.; Abdel-Malek, S.; Jia, Q.; Chow, S.A. Inhibitors of HIV-1 replication that inhibit HIV integrase. Proc. Natl. Acad. Sci. USA 1996, 93, 6326-6331. [CrossRef] [PubMed]

23. Kour, K.; Bani, S. Chicoric acid regulates behavioral and biochemical alterations induced by chronic stress in experimental Swiss albino mice. Pharmacol. Biochem. Behav. 2011, 99, 342-348. [CrossRef] [PubMed]

24. Kour, K.; Bani, S. Augmentation of immune response by chicoric acid through the modulation of CD28/CTLA-4 and Th1 pathway in chronically stressed mice. Neuropharmacology 2011, 60, 852-860. [CrossRef] [PubMed]

25. Zhu, D.; Wang, Y.; Du, Q.; Liu, Z.; Liu, X. Cichoric Acid Reverses Insulin Resistance and Suppresses Inflammatory Responses in the Glucosamine-Induced HepG2 Cells. J. Agric. Food Chem. 2015, 63, 10903-10913. [CrossRef] [PubMed] 
26. Rice-Evans, C.A.; Diplock, A.T.; Symons, M.C.R. Techniques in Free Radical Research; Elsevier: Amsterdam, The Netherlands, 1991.

27. Zeraik, M.L.; Petrono, M.S.; Coelho, D.; Regasini, L.O.; Silva, D.H.S.; da Fonseca, L.M.; Machado, S.A.S.; Bolzani, V.S.; Ximenes, V.F. Improvement of pro-oxidant capacity of protocatechuic acid by esterification. PLoS ONE 2014, 9, e110277. [CrossRef] [PubMed]

28. Carmichael, J.; DeGraff, W.G.; Gazdar, A.F.; Minna, J.D.; Mitchell, J.B. Evaluation of a tetrazolium-based semiautomated colorimetric assay: Assessment of chemosensitivity testing. Cancer Res. 1987, 47, 936-942. [PubMed]

29. Jabłońska-Trypuć, A.; Pankiewicz, W.; Czerpak, R. Traumatic acid reduces oxidative stress and enhances collagen biosynthesis in cultured human skin fibroblasts. Lipids 2016, 51, 1021-1035. [CrossRef] [PubMed]

30. Krętowski, R.; Kusaczuk, M.; Naumowicz, M.; Kotyńska, J.; Szynaka, B.; Cechowska-Pasko, M. The Effects of Silica Nanoparticles on Apoptosis and Autophagy of Glioblastoma Cell Lines. Nanomaterials 2017, 7, 230. [CrossRef] [PubMed]

31. Kalinowska, M.; Bielawska, A.; Lewandowska-Siwkiewicz, H.; Priebe, W.; Lewandowski, W. Apple: Phenolic compounds, extraction and health benefits-A review. Plant Phys. Biochem. 2014, 84, 169-188. [CrossRef] [PubMed]

32. Lewandowska, H.; Kalinowska, M.; Lewandowski, W.; Stępkowski, T.M.; Brzóska, K. The role of natural polyphenols in cell signaling and cytoprotection against cancer development. J. Nutr. Biochem. 2016, 32, 1-19. [CrossRef] [PubMed]

33. Bajko, E.; Kalinowska, M.; Borowski, P.; Siergiejczyk, L.; Lewandowski, W. 5-O-Caffeoylquinic acid: A spectroscopic study and biological screening for antimicrobial activity. LWT Food Sci. Technol. 2016, 65, 471-479. [CrossRef]

34. Facino, R.M.; Carini, M.; Aldini, G.; Saibene, L.; Pietta, P.; Mauri, P. Echinacoside and caffeoyl conjugates protect collagen from free radical-induced degradation: A potential use of Echinacea extracts in the prevention of skin photodamage. Planta Med. 1995, 61, 510-514. [CrossRef] [PubMed]

35. Azay-Milhau, J.; Ferrare, K.; Leroy, J.; Aubaterre, J.; Tournier, M.; Lajoix, A.D.; Tousch, D. Antihyperglycemic effect of a natural chicoric acid extract of chicory (Cichorium intybus L.): A comparative in vitro study with the effects of caffeic and ferulic acids. J. Ethnopharmacol. 2013, 150, 755-760. [CrossRef] [PubMed]

36. Xiao, H.; Wang, J.; Yuan, L.; Xiao, C.; Wang, Y.; Liu, X. Chicoric acid induces apoptosis in 3T3-L1 preadipocytes through ROS-mediated PI3K/Akt and MAPK signaling pathways. J. Agric. Food Chem. 2013, 61, 1509-1520. [CrossRef] [PubMed]

37. Landmann, M.; Kanuri, G.; Spruss, A.; Stahl, C.; Bergheim, I. Oral intake of chicoric acid reduces acute alcohol-induced hepatic steatosis in mice. Nutrition 2014, 30, 882-889. [CrossRef] [PubMed]

38. Wouters, K.A.; Kremer, L.C.; Miller, T.L.; Herman, E.H.; Lipshultz, S.E. Protecting against anthracycline-induced myocardial damage: A review of the most promising strategies. Br. J. Haematol. 2005, 131, 561-578. [CrossRef] [PubMed]

39. Guerriero, E.; Sorice, A.; Capone, F.; Storti, G.; Colonna, G.; Ciliberto, G.; Costantini, S. Combining doxorubicin with a phenolic extract from flaxseed oil: Evaluation of the effect on two breast cancer cell lines. Int. J. Oncol. 2017, 50, 468-476. [CrossRef] [PubMed]

40. Tsai, Y.L.; Chiu, C.C.; Chen, J.Y.F.; Chan, K.C.; Lin, S.D. Cytotoxic effects of Echinacea purpurea flower extracts and cichoric acid on human colon cancer cells through induction of apoptosis. J. Ethnopharmacol. 2012, 143, 914-919. [CrossRef] [PubMed]

41. Kurisu, M.; Nakasone, R.; Miyamae, Y.; Matsuura, D.; Kanatani, H.; Yano, S.; Shigemori, H. Induction of hepatocyte growth factor production in human dermal fibroblasts by caffeic acid derivatives. Biol. Pharm. Bull. 2013, 36, 2018-2021. [CrossRef] [PubMed]

42. Zhang, H.L.; Dai, L.H.; Wu, Y.H.; Yu, X.P.; Zhang, Y.Y.; Guan, R.F.; Liu, T.; Zhao, J. Evaluation of hepatocyteprotective and anti-hepatitis B virus properties of Cichoric acid from Cichorium intybus leaves in cell culture. Biol. Pharm. Bull. 2014, 37, 1214-1220. [CrossRef]

43. Rucinska, A.; Roszczyk, M.; Gabryelak, T. Cytotoxicity of the isoflavone genistein in NIH 3 T3 cells. Cell Biol. Int. 2008, 32, 1019-1023. [CrossRef] [PubMed]

44. Tsai, K.L.; Kao, C.L.; Hung, C.H.; Cheng, Y.H.; Lin, H.C.; Chu, P.M. Chicoric acid is a potent anti-atherosclerotic ingredient by anti-oxidant action and anti-inflammation capacity. Oncotarget 2017, 8, 29600-29612. [CrossRef] [PubMed] 
45. Dalby-Brown, L.; Barsett, H.; Landbo, A.K.; Meyer, A.S.; Mølgaard, P. Synergistic antioxidative effects of alkamides, caffeic acid derivatives, and polysaccharide fractions from Echinacea purpurea on in vitro oxidation of human low-density lipoproteins. J. Agric. Food Chem. 2005, 53, 9413-9423. [CrossRef] [PubMed]

46. Sloley, B.D.; Urichuk, L.J.; Tywin, C.; Coutts, R.T.; Pang, P.K.; Shan, J.J. Comparison of chemical components and antioxidants capacity of different Echinacea species. J. Pharm. Pharmacol. 2001, 53, 849-857. [CrossRef] [PubMed]

47. Ahn, H.R.; Lee, H.J.; Kim, K.A.; Kim, C.Y.; Nho, C.W.; Jang, H.; Pan, C.H.; Lee, C.Y.; Jung, S.H. Hydroxycinnamic acids in Crepidiastrum denticulatum protect oxidative stress-induced retinal damage. J. Agric. Food Chem. 2014, 62, 1310-1323. [CrossRef] [PubMed]

48. Granados-Principal, S.; Quiles, J.L.; Ramirez-Tortosa, C.L.; Sanchez-Rovira, P.; Ramirez-Tortosa, M.C. New advances in molecular mechanisms and the prevention of adriamycin toxicity by antioxidant nutrients. Food Chem. Toxicol. 2010, 48, 1425-1438. [CrossRef] [PubMed]

49. Tsai, C.W.; Lin, C.Y.; Lin, H.H.; Chen, J.H. Carnosic acid, a rosemary phenolic compound, induces apoptosis through reactive oxygen species-mediated p38 activation in human neuroblastoma IMR-32 cells. Neurochem. Res. 2011, 36, 2442-2451. [CrossRef] [PubMed]

50. Lee, Y.S. Role of NADPH oxidase-mediated generation of reactive oxygen species in the mechanism of apoptosis induced by phenolic acids in HepG2 human hepatoma cells. Arch. Pharm. Res. 2005, 28, 1183-1189. [CrossRef] [PubMed]

51. Schlernitzauer, A.; Oiry, C.; Hamad, R.; Galas, S.; Cortade, F.; Chabi, B.; Casas, F.; Pessemesse, L.; Fouret, G.; Feillet-Coudray, C.; et al. Chicoric acid is an antioxidant molecule that stimulates AMP kinase pathway in L6 myotubes and extends lifespan in Caenorhabditis elegans. PLoS ONE 2013, 8, e78788. [CrossRef] [PubMed]

52. Quiles, J.L.; Ochoa, J.J.; Huertas, J.R.; López-Frías, M.; Mataix, J. Olive oil and mitochondrial oxidative stress: Studies on adriamycin toxicity, physical exercise and ageing. In Olive Oil and Health; Quiles, J.L., Ramirez-Tortosa, M.C., Yaqoob, P., Eds.; CABI Publishing: Oxford, UK, 2006; pp. 119-151.

53. Fauser, J.K.; Matthews, G.M.; Cummins, A.G.; Howarth, G.S. Induction of apoptosis by the medium-chain length fatty acid lauric acid in colon cancer cells due to induction of oxidative stress. Chemotherapy 2013, 59, 214-224. [CrossRef] [PubMed]

54. Jacobson, M.D.; Weil, M.; Raff, M.C. Programmed cell death in animal development. Cell 1997, 88, 347-354. [CrossRef]

55. Wong, R.S. Apoptosis in cancer: From pathogenesis to treatment. J. Exp. Clin. Cancer Res. 2011, $30,87$. [CrossRef] [PubMed]

56. Kumar, D.; Kirshenbaum, L.; Li, T.; Danelisen, I.; Singal, P. Apoptosis in isolated adult cardiomyocytes exposed to adriamycin. Ann. N. Y. Acad. Sci. 1999, 874, 156-168. [CrossRef] [PubMed]

57. Zhu, D.; Zhang, X.; Niu, Y.; Diao, Z.; Ren, B.; Li, X.; Liu, Z.; Liu, X. Cichoric acid improved hyperglycaemia and restored muscle injury via activating antioxidant response in MLD-STZ-induced diabetic mice. Food Chem. Toxicol. 2017, 107, 138-149. [CrossRef] [PubMed]

(C) 2018 by the authors. Licensee MDPI, Basel, Switzerland. This article is an open access article distributed under the terms and conditions of the Creative Commons Attribution (CC BY) license (http:/ / creativecommons.org/licenses/by/4.0/). 
Article

\title{
Dioscorea quinqueloba Ameliorates Oxazolone- and 2,4-Dinitrochlorobenzene-induced Atopic Dermatitis Symptoms in Murine Models
}

\author{
Jonghwan Jegal ${ }^{1,+}{ }^{+}$, No-June Park ${ }^{2,+}{ }^{+}$Sim-Kyu Bong ${ }^{2}$, Hyun Jegal ${ }^{2}$, Su-Nam Kim ${ }^{2, *}$ and \\ Min Hye Yang ${ }^{1, *}$ \\ 1 College of Pharmacy, Pusan National University, Busan 46241, Korea; puhahaha2027@naver.com \\ 2 Natural Products Research Institute, Korea Institute of Science and Technology, Gangneung 25451, Korea; \\ parknojune1@naver.com (N.-J.P.); 115044@kist.re.kr (S.-K.B.); 116524@kist.re.kr (H.J.) \\ * Correspondence: snkim@kist.re.kr (S.-N.K.); mhyang@pusan.ac.kr (M.H.Y.); \\ Tel.: +82-33-650-3503 (S.-N.K.); +82-51-510-2811 (M.H.Y.); \\ Fax: +82-33-650-3419 (S.-N.K.); +82-51-513-6754 (M.H.Y.) \\ + These authors contributed equally to this work.
}

Received: 26 October 2017; Accepted: 29 November 2017; Published: 5 December 2017

\begin{abstract}
Dioscorea quinqueloba has been used for food substances, as well as in herbal medicines for allergic diseases such as asthma. This study aimed to investigate the anti-atopic dermatitis (AD) effects of the total extract of D. quinqueloba rhizomes and active fractionson murine oxazoloneand 2,4-dinitrochlorobenzene-induced models of AD. Specific AD symptoms, such as erythema, ear swelling, and epidermis thickening, were significantly reduced in the oxazolone-mediated AD BALB/c mice upon topical application of D. quinqueloba rhizomes $95 \% \mathrm{EtOH}$ extract (DQ). DQEA (D. quinqueloba rhizomes EtOAc fraction) was beneficial for protecting the skin barrier against $\mathrm{AD}$ in DNCB-sensitized SKH-1 hairless mice. Decreased total serum IgE and IL-4 levels could be observed in atopic dorsal skin samples of the DQEA-treated group. On the basis of the phytochemical analysis, DQEA was found to contain dioscin and gracillin as its main compounds. Therapeutic applications with $D$. quinqueloba might be useful in the treatment of $\mathrm{AD}$ and related inflammatory skin diseases.
\end{abstract}

Keywords: Dioscorea quinqueloba; atopic dermatitis; transepidermal water loss; skin hydration; interleukin 4; immunoglobulin E

\section{Introduction}

Atopic dermatitis (AD) is a pruritic chronic inflammatory skin disease [1]. Worldwide, AD occurs with a prevalence of $2-10 \%$ in adults, and up to $15-30 \%$ in children [2,3]. Atopic disease is triggered by a variety of allergic factors, including irritants, food, and stress factors [4]. The symptoms of AD include atopic eczema, itching, and dryskin [5]. Intense pruritus is the most common dermatologic feature of $\mathrm{AD}$, thus negatively impacting the health-related quality of life in patients suffering from $\operatorname{AD}[5,6]$.

Atopic dermatitis can be categorized into two types, including an extrinsic type (environmental or allergic $\mathrm{AD}$ ) and an intrinsic type (genetic or non-allergic $\mathrm{AD}$ ) [7]. Extrinsic $\mathrm{AD}$ is the classical type of $\mathrm{AD}$, while the incidence of intrinsic $\mathrm{AD}$ is approximately $20 \%$ of patients [7,8]. Extrinsic or environmental triggers enhance IgE-mediated sensitization and allergic reaction, thus further contributing to severe forms of skin inflammation in AD [9]. Diverse inflammatory cytokines orchestrate atopic skin inflammation, and the concomitant activation of IL-4 is thought to play a critical role in the pathogenesis of $\mathrm{AD}$ [10]. An impaired epidermal skin barrier is also a characteristic feature and causative factor of $\mathrm{AD}[10]$. Skin barrier damage contributes to the high serum IgE level, reduced 
skin surface hydration, and enhanced transepidermal water loss in patients with extrinsic $\operatorname{AD}[8,11,12]$. Therefore, improvements in skin barrier function with natural moisturizing agents show great potential as a pharmacological target in atopic diseases.

Dioscorea quinqueloba Thunb. belongs to the Dioscoreaceae family, and has been cultivated in China, Japan, and Korea as a food. The rhizomes of D. quinqueloba have been used in traditional Korean medicine as an alternative therapy for cardiovascular disease, as well as various medical conditions, mainly for arthrosclerosis, myocardial infarction, and asthma [12-14]. Analysis of the chemical composition of Dioscorea species indicates that the main metabolites are steroidal saponins such as diosin and diosgenin, which have convulsive, local anesthetic and antidiuretic effects $[15,16]$. Recent reports have shown that the genus Dioscorea exhibits various biological properties, including anti-inflammatory, antitumor, and anti-adipogenic activities [17-19]. However, studies on the D. quinqueloba are comparatively few in number, and furthermore, there have not been any attempts to reveal its anti-atopic effect. This study aimed to investigate the potential therapeutic effects of D. quinqueloba on oxazolone- and 2,4-dinitrochlorobenzene (DNCB)-induced murine AD models. Histopathological examination and blood serum analysis, including total IL-4 and IgE levels, were performed to observe anti-atopic properties of D. quinqueloba.

\section{Material and Methods}

\subsection{Plant Material and Extraction}

Dried rhizomes of D. quinqueloba were purchased from JirisanHanbang Food ${ }^{\circledR}$ (Sancheong, Gyeongnam, South Korea) and identified by Eun Ju Jeong of Department of Agronomy and Medicinal Plant Resources, Gyeongnam National University of Science and Technology. A voucher specimen (PNU-0023) has been deposited in the Medicinal Herb Garden, Pusan National University. Dioscorea quinqueloba sample $(20 \mathrm{~kg}$ ) was extracted with $95 \% \mathrm{EtOH}$ and evaporated under reduced pressure to yield D. quinqueloba EtOH extract (DQ) $(670 \mathrm{~g})$. The DQ was suspended in distilled water (2 L) and partitioned with EtOAc (4 L) and $n-\mathrm{BuOH}(4 \mathrm{~L})$ to yield D. quinqueloba EtOAc fraction (DQEA) $(150 \mathrm{~g})$ and $n-\mathrm{BuOH}$ fraction (DQB) $(300 \mathrm{~g})$.

\subsection{Animals}

Six-week-old female BALB/c and SKH-1 hairless mice were purchased from the animal facility of Orient Bio Inc. (Seongnam, Republic of Korea) and housed in an air-conditioned animal room at a temperature of $25 \pm 5{ }^{\circ} \mathrm{C}$ and $55 \pm 5 \%$ humidity. Mice were given access to a standard laboratory diet and water ad libitum. All animal experiments were conducted in accordance with the Guide for the Care and Use of Laboratory Animals of the National Institutes of Health (NIH publication No. 85-23, revised 1996) and were approved by the Institutional Animal Care and Use Committee of the KIST (Certification No. KIST-2016-011).

\subsection{Induction of Topical AD-Like Skin Dermatitis in Mice by Oxazolone and Treatment with $D Q$}

4-Ethoxymethylene-2-phenyl-2-oxazolin-5-one (oxazolone) (1\%) dissolved in vehicle (propylene glycol:EtOH $=7: 3$ ) was used as sensitizer to induce AD in BALB/c mouse ears according to the previously described method [20]. In brief, the ears of BALB/c mice were sensitized with $20 \mu \mathrm{L}$ of $1 \%$ oxazolone on the first day. After the first challenge, $20 \mu \mathrm{L}$ of $0.1 \%$ oxazolone was repeatedly applied to ears for an additional 3 weeks at 2-day intervals. At the same time, the ears of the BALB/c mice were exposed to $20 \mu \mathrm{L}$ of $1 \%$ DQ daily in the oxazolone-DQ-group for 3 weeks, and the application of $1 \% \mathrm{DQ}$ was separated by $4 \mathrm{~h}$ from that of oxazolone. The normal control animals (CON) were treated with distilled water alone. No substances were applied to the skin surface on the last day of the experiment. On the last day, measurements of skin inflammation signs, including ear swelling and erythema, were carried out. 


\subsection{Induction of AD-Like Skin Lesions in Mice by DNCB and Treatment with DQEA}

2,4-dinitrochlorobenzenedissolved in acetone was used to induce AD in SKH-1 hairless mice as previously described with small modification [21,22]. Briefly, the dorsal skin of hairless mice was sensitized by painting $100 \mu \mathrm{L}$ of $1 \%$ DNCB daily for 7 days. After the first challenge, the mice were challenged with $100 \mu \mathrm{L}$ of $0.1 \%$ DNCB for an additional 2 weeks at 3-day intervals. The DNCB-DQEA group animals were painted with $100 \mu \mathrm{L}$ of $1 \%$ DQEA for 2 weeks, and the application of DQEA was separated by $4 \mathrm{~h}$ from that of DNCB. The normal control animals (CON) were treated with distilled water alone. No substances were applied to the skin surface on the last day of the experiment. On the last day, mice were sacrificed, and dorsal skin and blood samples were collected for further analysis.

\subsection{Histology}

For histologic examination, the ear or dorsal skin from mice were fixed in $10 \%$ formalin and processed for paraffin embedding. Tissue sections $(2-3 \mathrm{~mm})$ were then stained with hematoxylin and eosin. Histopathological changes were examined by light microscopy (Olympus CX31/BX51, Olympus Optical Co., Tokyo, Japan) and photographed (TE-2000U, Nikon Instruments Inc., Melville, NY, USA).

\subsection{Measurement of Transepidermal Water Loss, Skin Hydration, and Skin Surface pH}

Skin barrier repair was monitored by estimating transepidermal water loss (TEWL), skin hydration, and skin surface $\mathrm{pH}$. Tewameter TM210 device (Courage and Khazaka, Cologne, Germany) and SKIN-O-MAT (Cosmomed, Ruhr, Germany) were used to evaluate the skin surface of the hairless mice according to the manufacturer's instructions. TEWL, skin hydration, and skin surface $\mathrm{pH}$ were measured once per week after the twice-daily application of DQEA or vehicle.

\subsection{Measurement of Serum IgE and IL-4 Levels}

Blood samples were centrifuged at $10,000 \mathrm{rpm}$ for $15 \mathrm{~min}$ at $4{ }^{\circ} \mathrm{C}$, and then serum was collected and stored at $-80^{\circ} \mathrm{C}$ for further investigations. Total IgE and IL-4 concentration in mouse serum were measured via enzyme-linked immunosorbent assay (eBioscience, San Diego, CA, USA) according to the manufacturer's instructions.

\subsection{Measurement of IL-4 mRNA Expression in RBL-2H3 Cells}

The rat basophilic leukemia cell line, RBL-2H3, was obtained from the American Type Culture Collection (CRL-2256, Bethesda, MD, USA) and grown in minimum essential medium with Eagle's salt, supplemented with $10 \%$ fetal bovine serum (FBS), $2 \mathrm{mM}$ L-glutamine, $100 \mathrm{U} / \mathrm{mL}$ penicillin, and $100 \mu \mathrm{g} / \mathrm{mL}$ streptomycin at $37^{\circ} \mathrm{C}$ in a humidified incubator with a $5 \% \mathrm{CO}_{2} / 95 \%$ air atmosphere. The RBL-2H3 cells were treated with dimethylsulfoxide (DMSO), DQ, DQEA, and DQB $(10 \mu \mathrm{g} / \mathrm{mL})$ $30 \mathrm{~min}$ before the induction of inflammation with phorbol 12-myristate 13-acetate/ionomycin (PI), which induced a state similar to AD. Controls were treated with DMSO without PMA/ionomycin (PI). After $16 \mathrm{~h}$ of treatment, cells were harvested to synthesize cDNA, and mRNA of IL-4 was measured with quantitative real-time PCR (qPCR). Total RNA from the treated cells was prepared with RNAiso Reagent (TaKaRa, Shiga, Japan) according to the manufacturer's protocol and stored at $-80^{\circ} \mathrm{C}$ until use. Accumulated PCR products were detected directly by monitoring the increase in the reporter dye (SYBR). The expression levels of cytokines in the exposed cells were compared to the expression levels in control cells at each collection time point using the comparative cycle threshold (Ct) method. The sequences of the primers used in this study were: IL- 4 forward: $5^{\prime}$-ACC TTG CTG TCA CCC TGT TC-3'; IL-4 reverse: $5^{\prime}$-TTG TGA GCG TGG ACTCAT TC-3'; $\beta$-actin forward: $5^{\prime}$-TCA TCA CCA TCG GCA ACG-3', $\beta$-actin reverse: $5^{\prime}$-TTC CT GAT GTC CAC GTC GC-3'. The quantity of each transcript was calculated as described in the instrument manual and normalized to the amount of $\beta$-actin. 


\subsection{Chromatographic Conditions}

The DQEA sample was analyzed using an Agilent 6530 Accurate-Mass Q-TOF LC/MS system (Agilent Technologies, Palo Alto, CA, USA) for phytochemical characterization. A Poroshell 120 EC-C18 column $(3.0 \times 100 \mathrm{~mm}, 2.7 \mu \mathrm{m}$, Agilent, Palo Alto, CA, USA) was used for analysis at a flow rate of $0.3 \mathrm{~mL} / \mathrm{min}$. The mobile phase consisted of acetonitrile (solvent $\mathrm{A}$ ) and water (solvent $\mathrm{B}$ ), using a linear gradient elution: 5-95\% A (0-20 min); 10\% A (20-30 min). All acquisitions were performed under positive ionization mode. Mass spectra were recorded across the range $m / z=100-1500$ with accurate mass measurement of all mass peaks.

\subsection{Statistical Analysis}

Data are expressed as the mean \pm standard deviation (S.D.). The values were expressed as percent changes from the mean value of the control experiment. Statistical analyses were performed by a one-way analysis of variance (ANOVA) using Statistical Package (SPSS, Inc., Chicago, IL, USA). $p$-values less than 0.05 were considered statistically significant.

\section{Results}

\subsection{Effects of $D Q$ on $A D$ Symptoms Induced by Oxazolone in BALB/c Mouse Ears}

Pathological reactions of AD such as increased ear thickness, erythema, and dryness were observed in the ears of oxazolone-challenged BALB/c mice (Figure 1a). According to the phenotypic observation, ear swelling and erythematic intensity caused by oxazolone were significantly reduced when ears were exposed to 1\% DQ for 21 days (Figure 1b). Histologic evaluation also showed that the DQ was efficient at improving AD-like skin lesions in the oxazolone-sensitized mouse ears. Skin thickening and the number of infiltrating lymphocytes were significantly increased in AD mice compared with those in normal mice, and these changes were attenuated by DQ treatment (Figure 1c).

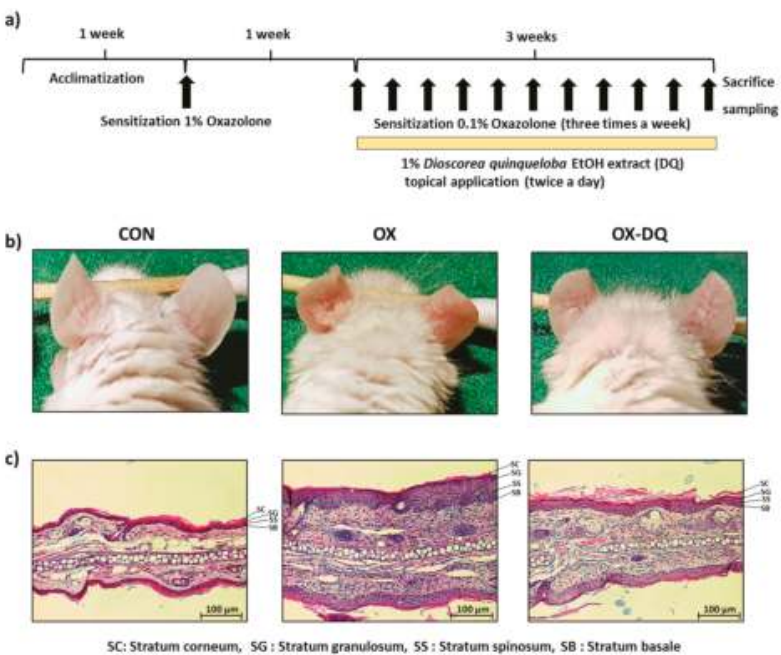

Figure 1. Effects of DQ on the development of oxazolone-induced AD-like symptoms in BALB/c mouse ears and histopathological analysis. CON: control group, OX: oxazolone-treated group, OX-DQ: oxazolone and $1 \%$ D. quinqueloba EtOH extract-treated group. (a) Schematic representation of the experiment; (b) Clinical features of AD-like skin lesions; (c) Histopathological features of skin lesions. Tissues were excised, fixed in $10 \%$ formaldehyde, embedded in paraffin, and sectioned. The sections were stained with hematoxylin and eosin (H\&E) (magnification, $100 \times g)$. 
3.2. Effects of DQ on Ear Thickness and Serum IgE and IL-4 Levels in Oxazolone-Induced AD BALB/c Mouse Ears

Increased ear thickness (2.3-fold) and epidermis thickness (2.5-fold) were observed after application of $1 \%$ oxazolone to the ears of mice, but these were reversed by treatment with DQ. The ear and epidermal thickness were reduced by $25 \%$ and $79 \%$, respectively, on day 28 compared with the oxazolone-treated group (Figure 2a,b). Serum levels of IL-4 and Ig-E were markedly lower in mice of OX-DQ group as compared to the OX group. The $1 \%$ DQ application noticeably attenuated total IgE concentration (CON: $52.1 \mathrm{ng} / \mathrm{mL}$, OX: $190.9 \mathrm{ng} / \mathrm{mL}$, and OX-DQ: $118.5 \mathrm{ng} / \mathrm{mL}$ ) (Figure 2c) and IL-4 concentration (CON: 12.6 pg/mL, OX: $28.6 \mathrm{pg} / \mathrm{mL}$, and OX-DQ: $16.9 \mathrm{pg} / \mathrm{mL}$ ) (Figure 2d).

a)

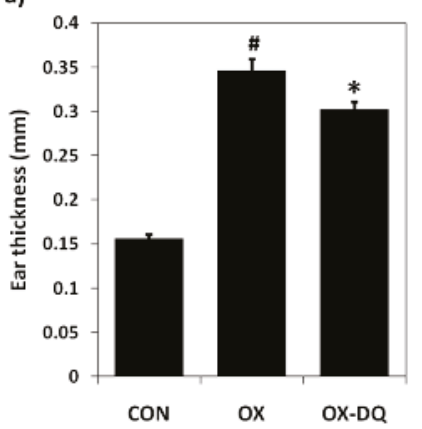

c)

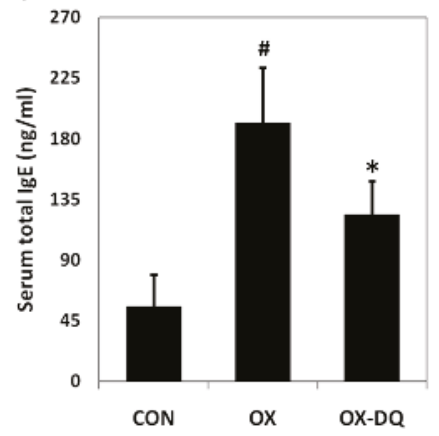

b)

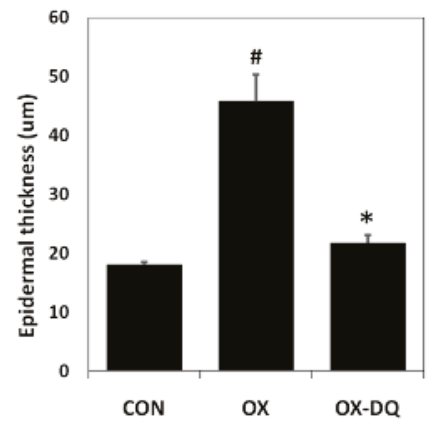

d)

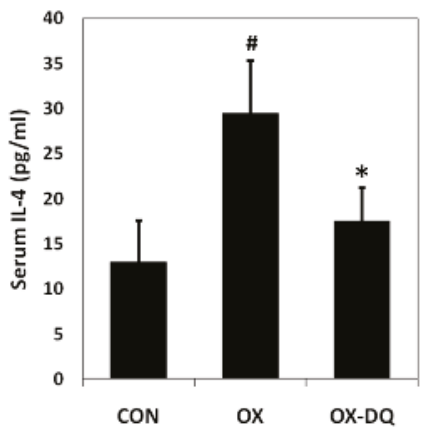

Figure 2. Effects of DQ on the ear thickness and serum IgE and IL-4 levels in oxazolone-induced AD-like symptoms in BALB/c mouse ears. CON: control group, DNCB: DNCB-treated group, DNCB-DQ: oxazolone and $1 \%$ D. quinqueloba $\mathrm{EtOH}$ extract-treated group. (a) Ear thickness; (b) epidermal thickness; (c) Serum total IgE levels; (d) Serum total IL-4 levels (D). Results are expressed as the mean \pm SEM (standard error of the mean) $(n=7)$. The means \pm SEM of two independent experiments are shown. ${ }^{\#} p<0.05$ vs. control; ${ }^{*} p<0.05$ vs. oxazolone-treated group.

\subsection{Effects of DQEA on AD Symptoms Induced by DNCB in Hairless Mice}

Out of two fractions from DQ (DQEA and DQB), the DQEA exhibited a dramatic drop in mRNA expression of IL-4 genes in RBL-2H3 cells stimulated by PMA/ionomycin (PI) A further experiment was performed to investigate anti-atopic properties of DQEA on AD skin lesions induced by DNCB. For DNCB sensitization, mice were given paintings of 1\% DNCB for 3 weeks (Figure 3a). Treatment of 1\% DQEA for 2 weeks markedly alleviated DNCB-induced atopy-like dermatitis such as definite erythema, papula, and vesiculation in SKH-1 hairless mice (Figure 3b). Histopathological features of the dorsal skin lesions from DQEA-treated AD hairless mice were shown in Figure 3c. Epidermal 
thickening by cell hyperplasia, slight spongiosis, and lymphocyte infiltration in the dermis were observed in DNCB-treated control mice, but these were reversed by treatment with DQEA.

a)

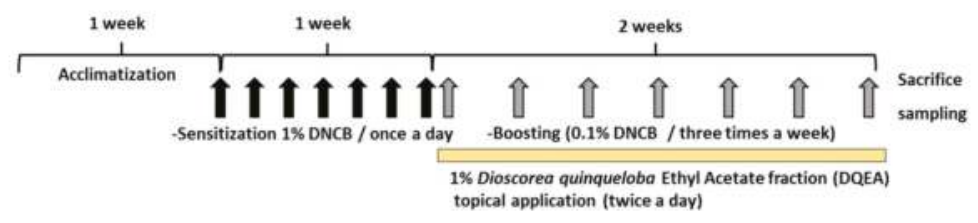

b)

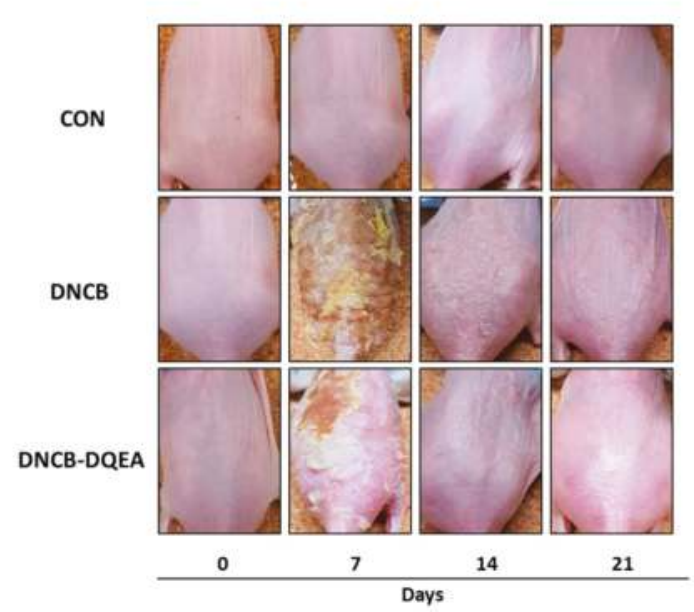

c)

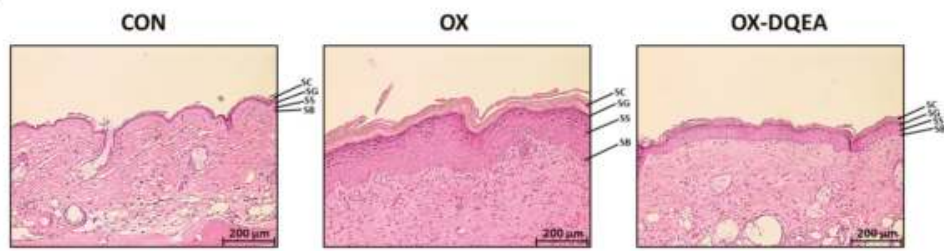

SC: Stratum corneum, SG : Stratum granulosum, SS : Stratum spinosum, SB : Stratum basale

Figure 3. Effects of DQEA on the development of DNCB-induced AD-like symptoms in hairless mice and histopathological analysis. CON: control group, DNCB: DNCB-treated group, DNCB-DQEA: $\mathrm{DNCB}$ and $1 \%$ D. quinqueloba EtOAc fraction-treated group. (a) Schematic representation of the experiment; (b) Clinical features of AD-like skin lesions; (c) Histopathological features of skin lesions. Tissues were excised, fixed in $10 \%$ formaldehyde, embedded in paraffin, and sectioned. The sections were stained with hematoxylin and eosin $(\mathrm{H \& E})$ (magnification, $100 \times g)$.

\subsection{Effects of DQEA on Ear Thickness and Serum IgE and IL-4 Levels in DNCB-Induced AD Hairless Mice}

DNCB-sensitized mice showed a dramatic increase in epidermis thickness, which was significantly reduced in mice treated with DQEA (Figure 4a). DQEA inhibited the DNCB-induced epidermal hyperplasia by $67 \%$ in $\mathrm{AD}$ hairless mice. The results of serum testing showed that the levels of IgE and IL-4 were increased in the DNCB-stimulated group. The 1\% DQEA application noticeably decreased the DNCB-induced serum IgE levels by $65 \%$ and IL-4 levels by $57 \%$ of DNCB-treated controls (Figure $4 b, c$ ). 
a)

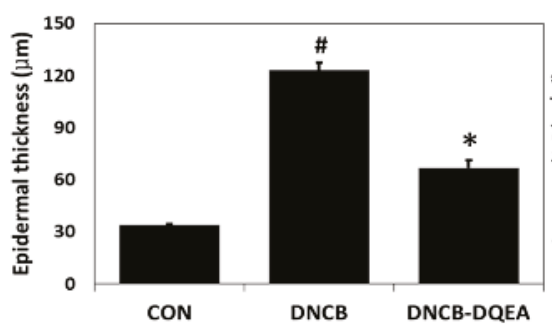

c)

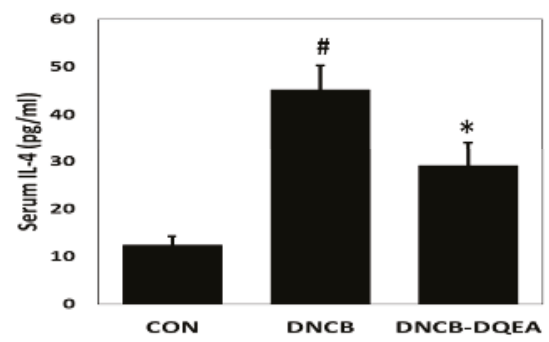

e)

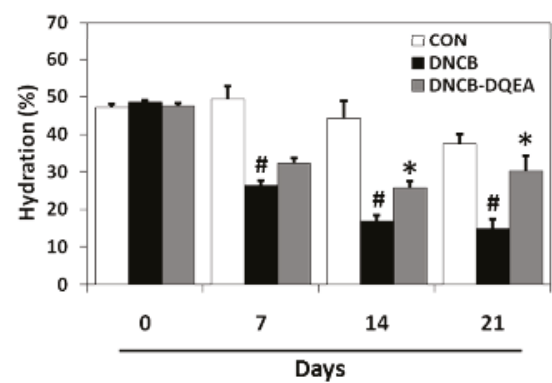

b)

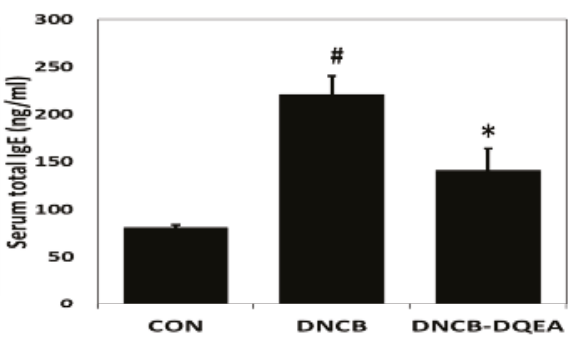

d)

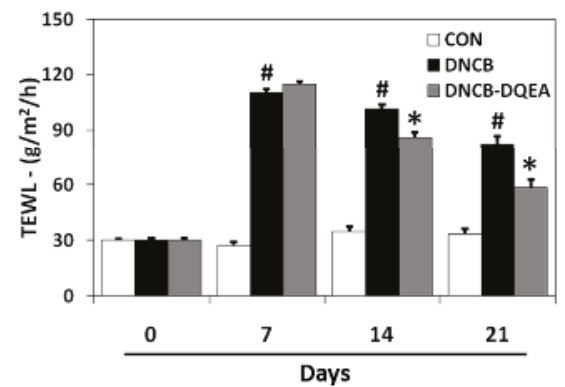

f)

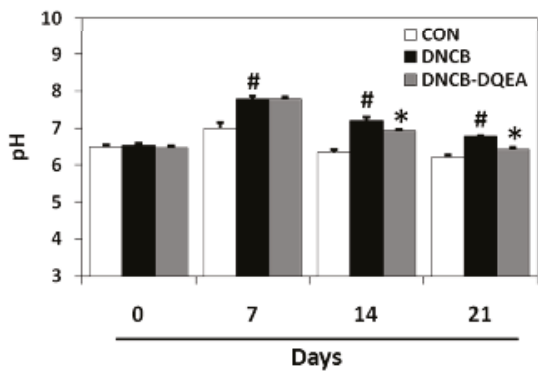

Figure 4. Effects of DQEA on the epidermal thickness, serum IgE and IL-4 levels, and skin barrier function in DNCB-induced AD-like symptoms in hairless mice. CON: control group, DNCB: DNCB-treated group, DNCB-DQEA: DNCB and 1\% D. quinqueloba EtOAc fraction-treated group. (a) Epidermal thickness; (b) Serum total IgE levels (c) Serum total IL-4 levels; (d) TWEL; (e) Skin hydration; (f) Skin surface $\mathrm{pH}$. Results are expressed as the mean \pm SEM $(n=7)$. The means \pm SEM of two independent experiments are shown. ${ }^{\#} p<0.05$ vs. control; ${ }^{*} p<0.05$ vs. DNCB-treated group.

\subsection{Effects of DQEA on Skin Barrier Function in DNCB-Induced AD Hairless Mice}

Severe skin barrier damage, such as increased TEWL and skin surface $\mathrm{PH}$, and decreased epidermal hydration, was detected in the DNCB-treated control group. This AD-like skin barrier dysfuction was reversed by treatment with 1\% DQEA. After 21 days of treatment, DNCB greatly increased TEWL to $79.8 \mathrm{~J}\left(\mathrm{~g} /\left(\mathrm{m}^{2} \mathrm{~h}\right)\right)$, whereas it was markedly reduced to $59.5 \mathrm{~J}\left(\mathrm{~g} /\left(\mathrm{m}^{2} \mathrm{~h}\right)\right)$ by the 
transdermal application of DQEA (Figure 4d). Consistent with this finding, skin hydration level was decreased in the DNCB-induced group (65.8\% decrease) compared with the control group, whereas DQEA increased the hydration level to $68 \%$ (Figure $4 \mathrm{e}$ ). Skin surface $\mathrm{pH}$ value was significantly increased in lesional skin, but not in non-lesional skin of AD. Figure $4 \mathrm{f}$ revealed that DQEA treatment normalized the altered $\mathrm{pH}$ of DNCB-sensitized hairless mouse skin.

\subsection{The Standardization of DQEA Using the High-Performance Liquid Chromatography/Mass Spectrometry} (HPLC/MS)

For the simultaneous determination of the major constituents of DQEA, the optimized chromatographic condition was investigated. The optimal mobile phase, which consisted of acetonitrile/water, was subsequently employed for the analysis of DQEA, and led to a good resolution and satisfactory peak shape. The presence of two compounds, 1 : dioscin $\left(\mathrm{m} / z 868.08\right.$ at $\left.t_{\mathrm{R}} 18.66 \mathrm{~min}\right)$ and 2: gracillin $\left(\mathrm{m} / z 884.08\right.$ at $\left.t_{\mathrm{R}} 19.78 \mathrm{~min}\right)$ in DQEA was verified by comparing each retention time and UV spectrum with those of each standard compound and spiking with authentic standards (Figure $5 \mathrm{a}, \mathrm{b}$ ). DQEA was found to contain dioscin $(319 \mathrm{mg} / \mathrm{g})$ and gracillin $(91.6 \mathrm{mg} / \mathrm{g})$ as major compounds.

a)

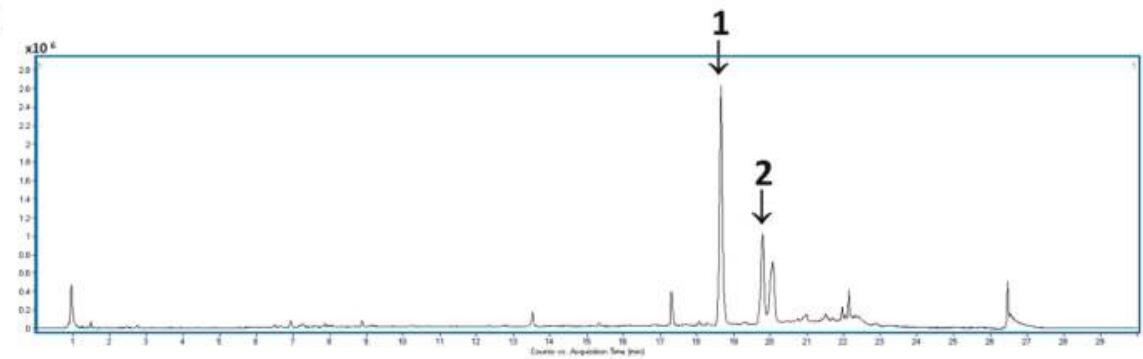

b)

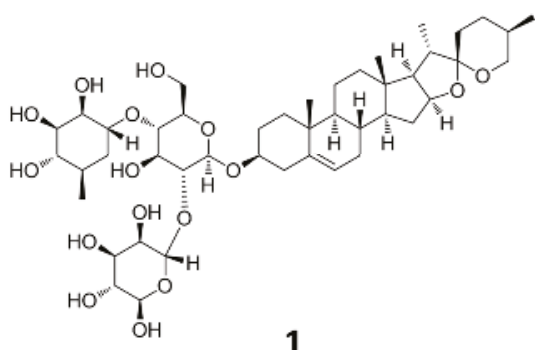

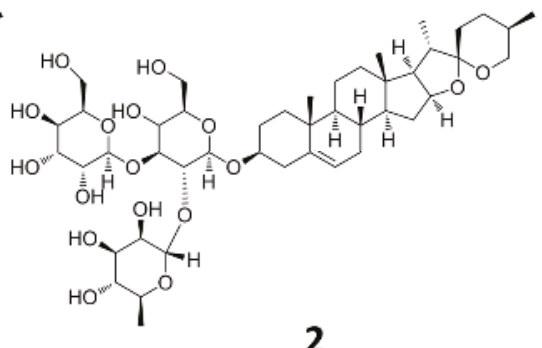

Figure 5. (a) HPLC chromatogram of DQEA (a) and chemical structures of major components (b). Fingerprint analysis of D. quinqueloba EtOAc fraction was performed in positive ion mode using HPLC/MS (high-performance liquid chromatography/mass spectrometry). 1: dioscin, 2: gracillin.

\section{Discussion}

Skin barrier dysfunction is one of the primary causes of allergic disorders, and is crucially involved in the pathogenesis of $\mathrm{AD}$ [23]. The skin barrier function is mainly disturbed in contact and extrinsic $\mathrm{AD}$, the classical type of $\mathrm{AD}$, which has high prevalence [24]. Impaired permeability barrier function of skin in $\mathrm{AD}$ patients enhances penetration of environmental allergens into the skin, thus triggering immunological reactions and inflammation [24,25]. Use of proper moisturizers/emollients to enhance skin hydration is likely to play a key part in management of AD [26]. Natural products are a rich source of medicinal agents, and natural product-related drugs account for over $50 \%$ of 
the most-prescribed drugs in the USA [27]. Substances of natural origin have been widely used for treatment of skin problems due to their therapeutic efficacies in dermatology, which include anti-inflammatory, antimicrobial, and cell-stimulating properties [28]. In addition, plants-including extracts, pure compounds, and phytochemical combinations-are commonly added to moisturizers to improve dry skin conditions in AD patients [28,29]. For this reason, naturally occurring moisturizing agents can be useful in the treatment of at allergic and atopic diseases through improvement skin barrier function [30].

In our preliminary research to find anti-inflammatory materials from plant extracts, the $95 \% \mathrm{EtOH}$ extract of D. quinqueloba (DQ) showed strong IL-4 inhibition in RBL-2H3 cells. The anti-atopic property of $\mathrm{DQ}$ was studied using an experimental animal model of AD induced by oxazolone. BALB/c mouse ears were sensitized with oxazolone and treated with DQ subcutaneously for 2 weeks. The results indicated that the topical application of DQ could improve atopic damage in oxazolone-induced $\mathrm{BALB} / \mathrm{c}$ mouse ears. This finding strongly suggested that the specific fraction from total extract of D. quinqueloba is responsible for the anti-atopic effect. For this reason, the DQ was fractionated in order to find the most active principles. Out of two fractions of DQ, the production of IL-4 in RBL-2H3 was only affected by the EtOAc fraction of DQ (DQEA). IL-4 has been considered one of the key proximal cytokines of type 2 inflammation in atopic disease. Impaired barrier function induced by skin sensitizers develops into a pruritic inflammatory skin disease characterized by hyperactivated cytokines of helper T cells [22]. The hyperproduction of IL-4, a key regulatory cytokine for IgE synthesis, is generally detected in various experimental animal models to study AD [31]. In addition, transgenic mice expressing epidermal IL-4 have been reported to develop skin inflammation reproducing all key features of human AD [27,32]. Broad application of DQEA to dorsal skins of DNCB-sensitized SKH-1 hairless mice attenuated severe atopic symptoms such as erythema and lichenification. Taken together, it was concluded that D. quinqueloba improves severe AD symptoms in both oxazolone- and DNCB-induced AD mice as a potent IL-4 inhibitor.

Stratum corneum hydration and transepidermal water loss are considered as a marker of the inside-outside skin barrier [33]. The water-holding capacity of the D. quinqueloba sample in atopic skin was evaluated by measuring both epidermal hydration and TEWL. Barrier repair was delayed and skin hydration was impaired in DNCB-sensitized mice. Treatment with DQEA significantly increased skin hydration and decreased TWEL and skin surface $\mathrm{pH}$ in DNCB-induced AD hairless mice. Atopic skin normally shows a defective skin barrier function, both in rough and in clinically healthy skin [34]. Emollient enhancement of the skin barrier from DQEA-applied AD animals was observed after 3 weeks of treatment. Therefore, DQEA might be an appropriate material for improving barrier function and dry skin as a skin therapeutic agent for AD. An elevated serum level of IgE is a main feature of AD and IL-4 acts as a key cytokine in the process of atopic inflammation [35].DQEA also attenuated IgE hyperproduction and epidermal overexpression of IL-4, resulting in the prevention and treatment of Th2-dominated inflammation in AD-like skin lesions. Based on the results, we propose that DQEA corrects skin barrier dysfunction and early inflammation in murine AD models.

Standardization of $D$. quinqueloba is necessary to provide information on quality standards of natural product-derived drug development. Phytochemical screening, using steroidal saponins as bioactive marker compounds, was performed to standardize the DQEA sample. Steroidal saponins have long attracted scientific attention, due to their structural diversity and significant biological activities [36]. Dioscin and disogenin, steroidal saponins obtained from Dioscorea species, are currently being considered as an important starting material for the industrial production of steroid drugs [37]. Phytochemical analysis of the DQEA resulted in the presence of dioscin and gracillin as major components.

\section{Conclusions}

In conclusion, topical treatment with $D$. quinqueloba total extract was protective against oxazolone-induced AD-like lesions in mice ears. Bioassay-guided fractionation of the DQ led to the finding of the most biologically active fraction, DQEA. Clinical symptoms of AD, such as 
pruritus, erythema, fissuring, and lichenification (skin thickening), were significantly reduced in the DQEA-treated mice. The DQEA improved skin barrier dysfunction and suppressed the overproduction of serum IgE and IL-4 in murine DNCB-sensitized atopic models. Based on the fingerprint analysis, dioscin, and gracillin were confirmed to be the major active constituents of DQEA. Further study is warranted to identify the therapeutic effects of DQEA against AD symptoms in human skin.

Acknowledgments: This study was supported by a grant from the Korea Healthcare Technology R\&D Project, the Ministry of Health \& Welfare, Republic of Korea. (Grant No. HI14C2687), the Korea Institute of Science and Technology, Republic of Korea (Grant No. 2Z04930 and 2Z04950), the National Research Foundation of Korea (NRF) grant funded by the Ministry of Science, ICT \& Future Planning (NRF-2016R1C1B2007694 and NRF-2016K1A1A8A01938595), and the "Cooperative Research Program for Agriculture Science and Technology Development (Project No. PJ01282301)" Rural Development Administration, Republic of Korea.

Author Contributions: S.-N.K. and M.H.Y. designedthe research; J.J., N.-J.P., S.-K.B. and H.J. performed the experiments; J.J., N.-J.P., S.-N.K. and M.H.Y. analyzed the data, and interpreted the results of the experiments. J.J., S.-N.K. and M.H.Y. drafted the manuscript. All authors read and approved the final manuscript.

Conflicts of Interest: The authors declare no conflict of interest.

\section{References}

1. Williams, H.C. Is the prevalence of atopic dermatitis increasing? Clin. Exp. Dermatol. 1992, 17, 385-391. [CrossRef] [PubMed]

2. Kapoor, R.; Menon, C.; Hoffstad, O.; Bilker, W.; Leclerc, P.; Margolis, D.J. The prevalence of atopic triad in children with physician-confirmed atopic dermatitis. J. Am. Acad. Dermatol. 2008, 58, 68-73. [CrossRef] [PubMed]

3. Larsen, F.S.; Hanifin, J.M. Epidemiology of atopic dermatitis: A review. Allergy Asthma Proc. 2002, 22, 1-24. [CrossRef]

4. Morren, M.A.; Przybilla, B.; Bamelis, M.; Heykants, B.; Reynaers, A.; Degreef, H. Atopic dermatitis: Triggering factors. J. Am. Acad. Dermatol. 1994, 31, 467-473. [CrossRef]

5. Guttman-Yassky, E.; Nograles, K.E.; Krueger, J.G. Contrasting pathogenesis of atopic dermatitis and psoriasis-part I: Clinical and pathologic concepts. J. Allergy Clin. Immunol. 2011, 127, 1110-1118. [CrossRef] [PubMed]

6. Ständer, S.; Steinhoff, M. Pathophysiology of pruritus in atopic dermatitis: An overview. Exp. Dermatol. 2002, 11, 12-24. [CrossRef] [PubMed]

7. Charman, C.; Williams, H. Outcome measures of disease severity in atopic eczema. Arch. Dermatol. 2000, 136, 763-769. [CrossRef] [PubMed]

8. Tokura, Y. Extrinsic and intrinsic types of atopic dermatitis. J. Dermatol. Sci. 2010, 58, 1-7. [CrossRef] [PubMed]

9. Darlenski, R.; Kazandjieva, J.; Hristakieva, E.; Fluhr, J.W. Atopic dermatitis as a systemic disease. Clin. Dermatol. 2014, 32, 409-413. [CrossRef] [PubMed]

10. Renz, H.; Jujo, K.; Bradley, K.L.; Domenico, J.; Gelfand, E.W.; Leung, D.Y. Enhanced IL-4 production and IL-4 receptor expression in atopic dermatitis and their modulation by interferon-gamma. J. Investig. Dermatol. 1992, 99, 403-408. [CrossRef] [PubMed]

11. Mori, T.; Ishida, K.; Mukumoto, S.; Yamada, Y.; Imokawa, G.; Kabashima, K.; Kobayashi, M.; Bito, T.; Nakamura, M.; Ogasawara, K.; et al. Comparison of skin barrier function and sensory nerve electric current perception threshold between IgE-high extrinsic and IgE-normal intrinsic types of atopic dermatitis. $\mathrm{Br}$. J. Dermatol. 2010, 162, 83-90. [CrossRef] [PubMed]

12. Kim, S.J.; Jung, J.Y.; Kim, H.W.; Park, T. Anti-obesity effects of Juniperus chinensis extract are associated with increased AMP-activated protein kinase expression and phosphorylation in the visceral adipose tissue of rats. Biol. Pharm. Bull. 2008, 31, 1415-1421. [CrossRef] [PubMed]

13. Ali, A.M.; Mackeen, M.M.; Intan-Safinar, I.; Hamid, M.; Lajis, N.H.; el-Sharkawy, S.H.; MurakoshI, M. Antitumour-promoting and antitumour activities of the crude extract from the leaves of Juniperus chinensis. J. Ethnopharmacol. 1996, 53, 165-169. [CrossRef]

14. Viruel, J.; Catalan, P.; Segarra-Moragues, J.G. New microsatellite loci in the dwarf yams Dioscorea group Epipetrum (Dioscoreaceae). Am. J. Bot. 2010, 97, e121-e123. [CrossRef] [PubMed] 
15. Broadbent, J.L.; Schnieden, H. A comparison of some pharmacological properties of dioscorine and dioscine. Br. J. Pharmacol. Chemother. 1958, 13, 213-215. [CrossRef] [PubMed]

16. Karnick, C.R. Dioscorea (YAMS)-The Food of the Slaves, with Potentials for Newer Drugs: A review. Q. J. Crude Drug Res. 1969, 9, 1372-1391. [CrossRef]

17. Yang, M.H.; Yoon, K.D.; Chin, Y.W.; Park, J.H.; Kim, J. Phenolic compounds with radical scavenging and cyclooxygenase-2 (COX-2) inhibitory activities from Dioscoreaopposita. Bioorg. Med. Chem. 2009, 17, 2689-2694. [CrossRef] [PubMed]

18. Wang, J.M.; Ji, L.L.; Branford-White, C.J.; Wang, Z.Y.; Shen, K.K.; Liu, H.; Wang, Z.T. Antitumor activity of Dioscorea bulbifera L. rhizome in vivo. Fitoterapia 2012, 83, 388-394. [CrossRef] [PubMed]

19. Yang, M.H.; Chin, Y.W.; Chae, H.S.; Yoon, K.D.; Kim, J. Anti-adipogenic Constituents from Dioscoreaopposita in 3T3-L1 Cells. Biol. Pharm. Bull. 2014, 37, 1683-1688. [CrossRef] [PubMed]

20. Blaylock, B.L.; Newsom, K.K.; Holladay, S.D.; Shipp, B.K.; Bartow, T.A.; Mehendale, H.M. Topical exposure to chlordane reduces the contact hypersensitivity response to oxazolone in BALB/c mice. Toxicol. Lett. 1995, 81, 205-211. [CrossRef]

21. Matsumoto, K.; Mizukoshi, K.; Oyobikawa, M.; Ohshima, H.; Sakai, Y.; Tagami, H. Objective evaluation of the efficacy of daily topical applications of cosmetics bases using the hairless mouse model of atopic dermatitis. Skin Res. Technol. 2005, 11, 209-217. [CrossRef] [PubMed]

22. Matsumoto, K.; Mizukoshi, K.; Oyobikawa, M.; Ohshima, H.; Tagami, H. Establishment of an atopic dermatitis-like skin model in a hairless mouse by repeated elicitation of contact hypersensitivity that enables to conduct functional analyses of the stratum corneum with various non-invasive biophysical instruments. Skin Res. Technol. 2004, 10, 122-129. [CrossRef] [PubMed]

23. Aioi, A.; Tonogaito, H.; Suto, H.; Hamada, K.; Ra, C.; Ogawa, H.; Maibach, H.; Matsuda, H. Impairment of skin barrier function in NC/Nga Tnd mice as a possible model for atopic dermatitis. Br. J. Dermatol. 2001, 144, 12-18. [CrossRef] [PubMed]

24. Proksch, E.; Fölster-Holst, R.; Jensen, J.M. Skin barrier function, epidermal proliferation and differentiation in eczema. J. Dermatol. Sci. 2006, 43, 159-169. [CrossRef] [PubMed]

25. Boguniewicz, M.; Leung, D.Y. Atopic dermatitis: A disease of altered skin barrier and immune dysregulation. Immunol. Rev. 2011, 242, 233-246. [CrossRef] [PubMed]

26. Leung, D.Y.; Boguniewicz, M.; Howell, M.D.; Nomura, I.; Hamid, Q.A. New insights into atopic dermatitis. J. Clin. Investig. 2004, 113, 651-657. [CrossRef] [PubMed]

27. Newman, D.J.; Cragg, G.M.; Snader, K.M. The influence of natural products upon drug discovery. Nat. Prod. Rep. 2000, 17, 215-234. [CrossRef] [PubMed]

28. Bodeker, G.; Ryan, T.J.; Volk, A.; Harris, J.; Burford, G. Integrative Skin Care: Dermatology and traditional and complementary medicine. J. Altern. Complement. Med. 2017, 23, 479-486. [CrossRef] [PubMed]

29. Loden, M. The clinical benefit of moisturizers. J. Eur. Acad. Dermatol. Venereol. 2005, 19, 672-688. [CrossRef] [PubMed]

30. Loden, M.; Andersson, A.C.; Lindberg, M. Improvement in skin barrier function in patients with atopic dermatitis after treatment with a moisturizing cream (Canoderm). Br. J. Dermatol. 1999, 140, $264-267$. [CrossRef] [PubMed]

31. Poulsen, L.K.; Reimert, C.M.; Bindslev-Jensen, C.; Hansen, M.B.; Bendtzen, K. Biomolecular regulation of the IgE immune response. II. In vitro IgE synthesis and spontaneous production of cytokines. Int. Arch. Allergy Immunol. 1995, 106, 55-61. [CrossRef] [PubMed]

32. Chan, L.S.; Robinson, N.; Xu, L. Expression of interleukin-4 in the epidermis of transgenic mice results in a pruritic inflammatory skin disease: An experimental animal model to study atopic dermatitis. J. Investig. Dermatol. 2001, 117, 977-983. [CrossRef] [PubMed]

33. Jensen, J.M.; Pfeiffer, S.; Witt, M.; Bräutigam, M.; Neumann, C.; Weichenthal, M.; Schwarz, T.; Fölster-Holst, R.; Proksch, E. Different effects of pimecrolimus and betamethasone on the skin barrier in patients with atopic dermatitis. J. Allergy Clin. Immunol. 2009, 124, R19-R28. [CrossRef] [PubMed]

34. Sator, P.G.; Schmidt, J.B.; Hönigsmann, H. Comparison of epidermal hydration and skin surface lipids in healthy individuals and in patients with atopic dermatitis. J. Am. Acad. Dermatol. 2003, 48, 352-358. [CrossRef] [PubMed]

35. Ricci, M. IL-4: A key cytokine in atopy. Clin. Exp. Allergy 1994, 24, 801-812. [CrossRef] [PubMed] 
36. Mahato, S.B.; Ganguly, A.N.; Sahu, N.P. Steroid saponins. Phytochemistry 1982, 21, 959-978. [CrossRef]

37. Qi, S.S.; Dong, Y.S.; Zhao, Y.K.; Xiu, Z.L. Qualitative and quantitative analysis of microbial transformation of steroidal saponins in Dioscoreazingiberensis. Chromatographia 2009, 69, 865-870. [CrossRef]

(c)

(C) 2017 by the authors. Licensee MDPI, Basel, Switzerland. This article is an open access article distributed under the terms and conditions of the Creative Commons Attribution (CC BY) license (http:/ / creativecommons.org/licenses/by/4.0/). 
Article

\title{
Bioactive Dietary VDR Ligands Regulate Genes Encoding Biomarkers of Skin Repair That Are Associated with Risk for Psoriasis
}

\author{
Amitis Karrys ${ }^{1,2,+}$, Islam Rady ${ }^{3,4,+}$, Roxane-Cherille N. Chamcheu ${ }^{3}$, Marya S. Sabir ${ }^{5}$, \\ Sanchita Mallick ${ }^{5}$, Jean Christopher Chamcheu ${ }^{3,6, *}$, Peter W. Jurutka ${ }^{2,5}$, Mark R. Haussler ${ }^{2}$ and \\ G. Kerr Whitfield ${ }^{2}$ \\ 1 School of Life Sciences, Arizona State University, Tempe, AZ 85281, USA; tkarrys12@gmail.com \\ 2 Department of Basic Medical Sciences, University of Arizona College of Medicine-Phoenix, \\ Phoenix, AZ 85004, USA; peter.jurutka@asu.edu (P.W.J.); haussler@email.arizona.edu (M.R.H.); \\ gkw@email.arizona.edu (G.K.W.) \\ 3 Department of Dermatology, School of Medicine and Public Health, University of Wisconsin, \\ Madison, WI 53706, USA; irady@dermatology.wisc.edu (I.R.); roxanechamcheu@gmail.com (R.-C.N.C.) \\ 4 Department of Zoology, Faculty of Science, Al-Azhar University, P.O. Box 11884 Nazr City, Cairo, Egypt \\ 5 School of Mathematical and Natural Sciences, Arizona State University, Phoenix, AZ 85306, USA; \\ msabir@asu.edu (M.S.S.); smallic5@asu.edu (S.M.) \\ 6 Department of Basic Pharmaceutical Sciences, School of Pharmacy, College of Health \& Pharmaceutical \\ Sciences, University of Louisiana at Monroe, 1800 Bienville Drive, Bienville 362, Monroe, LA 71201, USA \\ * Correspondence: chamcheu@ulm.edu; Tel.: +1-318-342-6820 \\ $\dagger$ These authors contributed equally to this work.
}

Received: 20 November 2017; Accepted: 30 January 2018; Published: 4 February 2018

\begin{abstract}
Treatment with 1,25-dihydroxyvitamin $\mathrm{D}_{3}(1,25 \mathrm{D})$ improves psoriasis symptoms, possibly by inducing the expression of late cornified envelope $(L C E) 3$ genes involved in skin repair. In psoriasis patients, the majority of whom harbor genomic deletion of $L C E 3 B$ and $L C E 3 C$ ( $L C E 3 C \_L C E 3 B-d e l$ ), we propose that certain dietary analogues of $1,25 \mathrm{D}$ activate the expression of residual $L C E 3 A / L C E 3 D / L C E 3 E$ genes to compensate for the loss of $L C E 3 B / L C E 3 C$ in the deletant genotype. Herein, human keratinocytes (HEKn) homozygous for LCE3C_LCE3B-del were treated with docosahexaenoic acid (DHA) and curcumin, two low-affinity, nutrient ligands for the vitamin D receptor (VDR). DHA and curcumin induce the expression of LCE3A/LCE3D/LCE3E mRNAs at concentrations corresponding to their affinity for VDR. Moreover, immunohistochemical quantitation revealed that the treatment of keratinocytes with DHA or curcumin stimulates LCE3 protein expression, while simultaneously opposing the tumor necrosis factor-alpha (TNF $\alpha$ )-signaled phosphorylation of mitogen activated protein (MAP) kinases, p38 and Jun amino-terminal kinase (JNK), thereby overcoming inflammation biomarkers elicited by TNF $\alpha$ challenge. Finally, DHA and curcumin modulate two transcription factors relevant to psoriatic inflammation, the activator protein-1 factor Jun B and the nuclear receptor NR4A2/NURR1, that is implicated as a mediator of VDR ligand-triggered gene control. These findings provide insights into the mechanism(s) whereby dietary VDR ligands alter inflammatory and barrier functions relevant to skin repair, and may provide a molecular basis for improved treatments for mild/moderate psoriasis.
\end{abstract}

Keywords: vitamin D receptor; late cornified envelope genes; docosahexaenoic acid; curcumin; epidermis; keratinocytes; psoriasis treatment; nutraceuticals; differentiation; activator protein-1 


\section{Introduction}

Psoriasis is a skin disease of largely unknown etiology with an estimated prevalence of over three percent in the United States [1]. Prominent features include overproliferation and incomplete differentiation of epidermal keratinocytes, as well as epidermal inflammation. Chemical analogs of 1,25 dihydroxyvitamin $\mathrm{D}_{3}(1,25 \mathrm{D})$, the hormonal metabolite of vitamin $\mathrm{D}$, are routinely used as topical agents to treat mild/moderate psoriasis. The success of $1,25 \mathrm{D}$-based agents is presumably related to the reported role of vitamin D in skin biology, in particular its role in regulating keratinocyte proliferation and differentiation, but also its known ability to regulate components of the immune system in skin (reviewed in [2]). In support of the role of vitamin D (or lack thereof) in psoriasis pathogenesis, several studies have associated low vitamin D status with psoriasis (e.g., [3,4]). However, $1,25 \mathrm{D}$ analog therapy, even when combined with an anti-inflammatory drug (e.g., betamethasone), is not effective in approximately $40 \%$ of patients with mild to moderate psoriasis [5]. Severe cases of psoriasis can be treated effectively with injectable anti-inflammatory agents such as etanercept, adalimumab, infliximab, or secukinumab [6]; however, these agents are not approved for patients with mild/moderate cases [7]. Thus, developing improved topical agents for mild/moderate psoriasis remains an important goal.

The currently accepted paradigm of $1,25 \mathrm{D}$ action is that $1,25 \mathrm{D}$, or an analog thereof, binds with high affinity to the nuclear vitamin $\mathrm{D}$ receptor (VDR), which then forms a heterodimer with a retinoid $X$ receptor (RXR) isoform on vitamin D-responsive elements (VDREs) in chromosomal DNA, modulating the expression of nearby target genes [8]. Although 1,25D and its analogs are known to improve psoriasis symptoms in many patients, presumably by serving as VDR ligands, the key epidermal genes that are regulated to ameliorate psoriasis are poorly characterized. One approach to enhancing topical therapies for psoriasis is therefore to identify key genes affected by treatment as well as VDR analogs or other ligands that may optimize these effects. Our approach has been to examine those genes for which genetic variations have been shown to confer risk for psoriasis as potential targets for VDR-mediated regulation.

Psoriasis susceptibility loci (PSORS) include the PSORS4 locus, which is contained within the epidermal differentiation complex (EDC), a large assemblage of over 60 genes expressed during the process of epithelial differentiation (Figure 1). Five closely related late cornified envelope genes ( LCE3A-E) exist in a cluster within the EDC and have been reported to play a role in skin repair [9]. The PSORS4 risk allele is a deletion of two of these genes, LCE3C_LCE3B-del, leading to the hypothesis that the loss of two (of five) LCE3 genes might reduce the ability of psoriatic lesions to heal [9]. This deletion is very common and studies have indicated that the frequency of LCE3C_LCE3B-del is over $50 \%$ in many human populations, and is significantly overrepresented in patients with psoriasis, where its frequency increases to $65-75 \%$ of the patient population [10].

The endocrine VDR ligand (1,25D), as well as novel VDR ligands delphinidin and cyanidin, have previously been shown to upregulate LCE3 gene expression to some extent [11,12]. Further, a VDRE has been identified adjacent to the $L C E 3 A$ gene [11] and is not affected by the $L C E 3 C \_L C E 3 B$ deletion (Figure 1). We have proposed that activation by liganded VDR from this VDRE could coordinately upregulate the expression of the $L C E 3 A / L C E 3 D / L C E 3 E$ genes to compensate for the LCE3C_LCE3B deletion under the assumption that the highly homologous LCE3 genes have overlapping functions [13]. We have additionally suggested that delphindin and cyanidin represent candidate lead compounds that could be developed into agents to treat mild to moderate psoriasis in a similar manner to how $1,25 \mathrm{D}$ was utilized as a lead compound to create therapeutically effective analogs such as calcipotriol [14].

For the current study, we analyzed the ability of two additional bioactive lipids of nutritional origin, namely docosahexaenoic acid (DHA) and curcumin, to upregulate LCE3 mRNAs as well as proteins. These two compounds were chosen not only for their demonstrated affinity for the vitamin D receptor [15], but also for their reported beneficial effects in skin. Curcumin has been used since ancient times to treat skin inflammation and other ailments, and its anti-inflammatory actions have been studied in the context of various skin conditions, including pruritus, facial photoaging, 
radiodermatitis, and diabetic microangiopathy (reviewed in [16]). DHA and other omega-3 fatty acids have also been studied for their anti-inflammatory effects, mediated via a variety of mechanisms (reviewed in [17]), including serving as the precursor for the anti-inflammatory molecule resolvin D1 that was recently shown to improve inflammatory symptoms in a mouse imiquimod-induced model of psoriasis [18]. Both DHA [19,20] and curcumin [21,22] have been examined as treatments for psoriasis, but corresponding studies enrolled only a relatively small number of patients and the molecular mechanism(s) accounting for the positive effects observed in a subset of patients were not investigated.

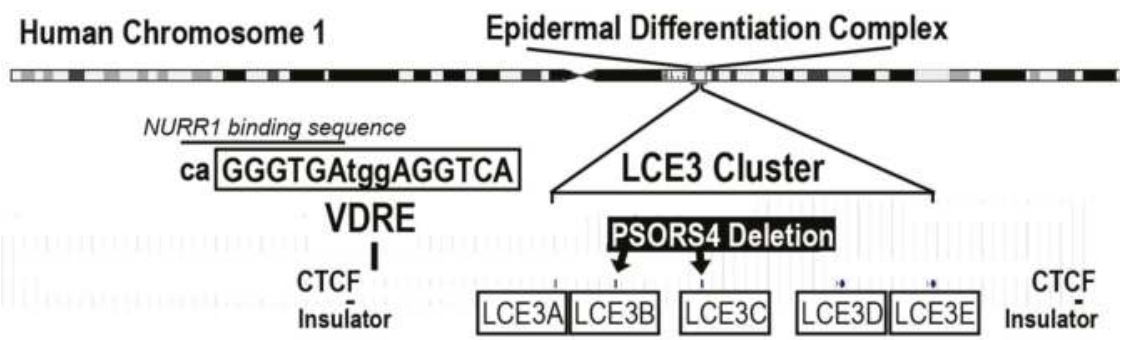

Figure 1. Location of the LCE3 gene cluster (exact location of each gene indicated by a tiny bars above the boxed LCE3 designation) and the common PSORS4 gene deletion within the epidermal differentiation complex on human chromosome 1 that also contains other skin genes such as filaggrin, loricrin, involucrin, and S100A (not shown). The PSORS4 deletion eliminates two LCE3 genes (LCE3C_LCE3B-del), but leaves LCE3A/LCE3D/LCE3E intact. A putative vitamin D-responsive element (VDRE) is located adjacent to the $L C E 3 A$ gene, but within the boundaries established on either side of the LCE3 gene cluster by sites for the CCCTC-binding factor (CTCF) that may serve to insulate this gene cluster from other nearby genes. This VDRE has been shown to confer responsiveness to both $1,25 \mathrm{D}$ and delphinidin in transfection experiments using a heterologous reporter gene construct [11]. Shown above the VDRE sequence is the location of an overlapping recognition sequence for the nuclear receptor subfamily 4 group A member 2 (NR4A2), also known as NURR1 (see text for explanation).

We also examined the ability of $1,25 \mathrm{D}$, curcumin, and DHA to upregulate the expression of two other disease biomarkers, the activator protein-1 factor Jun B and the nuclear receptor NR4A2, to illuminate additional pathways by which 1,25D, curcumin, and/or DHA might improve psoriasis symptoms. Jun B was selected since the Jun B gene is localized in psoriasis susceptibility locus 6 (PSORS6). Additionally, an inducible epidermal knockout of Jun B in adult mice was reported to yield a phenotype possessing the histological and molecular hallmarks of psoriasis [23]. Further, Jun B expression, similar to LCE3 gene expression, is associated with skin repair [24]. NR4A2, also known as NURR1, nuclear receptor of T cells (NOT), or transcriptionally inducible nuclear receptor (TINUR), was selected for study because a putative VDRE located near the LCE3 gene cluster overlaps with a consensus NR4A2 binding site (Figure 1, caGGGTGA). An additional reason why NR4A2 was of interest is our previously published hypothesis that part of the actions of liganded VDR might be mediated via the upregulation of NR4A2 [25]. Based on the current study, we conclude that both Jun B and NR4A2 may play a role in the action of 1,25D and DHA, and to some extent of curcumin, on skin, but higher doses of ligands are required to demonstrate a statistically significant effect on mRNA levels.

Finally, several literature reports have implied that the effects of DHA in the skin are mediated, not by VDR as we have hypothesized, but rather via peroxisome proliferator-activated receptor (PPAR) isoforms, with PPAR $\delta$ being the dominant isoform in human skin [26]. To assess whether PPAR $\delta$ is a major regulator of LCE genes, we probed GW501516, a selective ligand for PPAR $\delta$, for its ability to upregulate $L C E 3$ genes in keratinocytes. 


\section{Materials and Methods}

\subsection{Source of Ligands and Reagents}

Crystalline 1,25D was obtained from Roche Diagnostics (Indianapolis, IN, USA). Docosahexaenoic acid was purchased from Sigma Aldrich Corporation (St. Louis, MO, USA). GW510516 was secured from Santa Cruz Biotechnology (Dallas, TX, USA). GW501516 and 1,25D were dissolved in ethanol at 1000 times the concentrations needed for cell culture experiments $(1000 \times)$ and stored at $-20{ }^{\circ} \mathrm{C}$. Curcumin was obtained from Cayman Chemical Co. (Ann Arbor, MI, USA), dissolved in dimethyl sulfoxide (DMSO) at $1000 \times$, and stored at $-20^{\circ} \mathrm{C}$. Figure $2 \mathrm{~A}$ depicts the structures of these ligands. An antibody against NR4A2/NURR1 (LS-C99204) was purchased from LifeSpan BioSciences, Inc. (LSBio, Seattle, WA, USA). Antibodies to LCE3B-E (clone C-14, sc-138974), Jun B (clone N-17X, sc-46X), p-JNK (clone G-7, sc-6254), p-p38 (clone D-8, sc-7973), and filaggrin (clone AKH1, sc-66192) were purchased from Santa Cruz Biotechnology (Santa Cruz, CA, USA). Additional antibodies to LCE3B-E proteins were a kind gift from M. Narita in the laboratory of J. Shalkwijk [27]. Alexa Fluor 488-conjugated goat anti-rabbit IgG and Texas Red-conjugated goat anti-mouse IgG secondary antibodies were purchased from Invitrogen Molecular Probes (Eugene, OR, USA). Horseradish peroxidase (HRP) conjugated to anti-mouse IgG and anti-rabbit IgG secondary antibodies were purchased from Cell Signaling Technology (Danvers, MA, USA). The Pierce BCA Protein Assay Kit was from Thermo Scientific (Waltham, MA, USA), and Novex precast Tris-Glycine gels were purchased from Invitrogen (Carlsbad, CA, USA) or Bio-Rad Laboratories (Hercules, CA, USA). Recombinant human Tumor Necrosis Factor-alpha (rhTNF $\alpha$ ) was purchased from R \& D Systems (Minneapolis, MN, USA). ProLong ${ }^{\circledR}$ Gold Anti-fade Reagent containing 4',6-diamidino-2-phenylindole (DAPI) for nuclear staining was obtained from Invitrogen (Carlsbad, CA, USA) and Life Technologies (Grand Island, NY, USA).

A

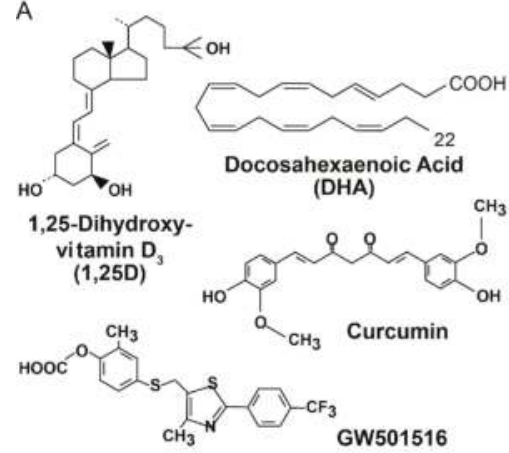

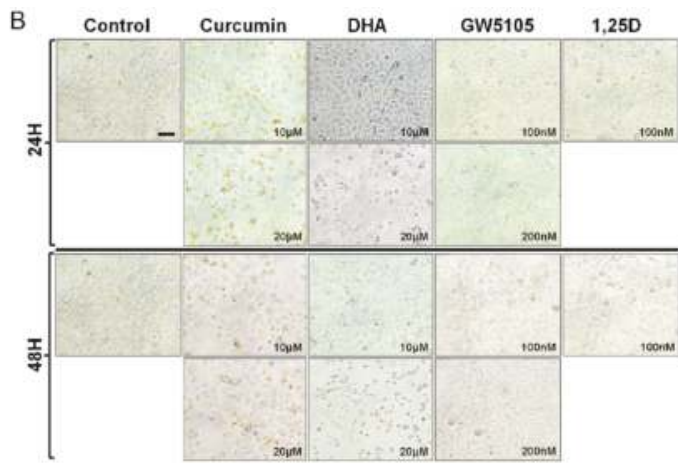

Figure 2. (A) Ligands used in the current study. 1,25D is the natural high affinity ligand for vitamin D receptor (VDR). Curcumin and docosahexaenoic acid (DHA) are natural, low-affinity ligands for VDR, and GW501516 is a specific synthetic ligand for peroxisome proliferator-activated receptor (PPAR) $\delta$, with the latter nuclear receptor also having a reported affinity for DHA. (B) Morphology of normal human epidermal keratinocytes (NHEKs) treated without ligand (control) or with various ligands at the indicated concentrations and time of incubation. Magnification $=300 \times$.

\subsection{Cell Culture and Treatment}

Human primary neonatal keratinocytes (HEKn) from single donors were purchased from Invitrogen Corp. (Carlsbad, CA, USA) and cultured in low calcium serum-free EpiLife medium supplemented with the Human Keratinocytes Growth Supplement Kit (HKGS Kit; Cat\# S-001-K, Thermo Fisher Scientific, Rockford, IL, USA) along with gentamicin and amphotericin from GIBCO (Carlsbad, CA, USA). Cells were re-fed every 2-4 days and split as necessary. Cells were plated at 
550,000 cells per $60-\mathrm{mm}$ dish and treated with $1000 \times$ stocks of calcium chloride (dissolved in water) and/or the ligands of interest (Figure 2A). Rat osteosarcoma cells (UMR-106) were obtained from the American Type Culture Collection (Manassas, VA, USA) and maintained in Dulbecco's Modified Eagles Medium (Hyclone, GE Healthcare, Logan, UT, USA) supplemented with gentamicin/amphotericin in a humidified atmosphere at $37^{\circ} \mathrm{C}$ and $5 \%$ carbon dioxide. For immunofluorescence and Western blot analyses, cells were treated with different concentrations of agents at different time points (24-48 h) prior to harvest and analysis. In some experiments, cells were first pre-incubated or pre-treated for $48 \mathrm{~h}$ with respective ligands/agents and then treated with or without TNF $\alpha(15 \mathrm{ng} / \mathrm{mL})$ for $30 \mathrm{~min}$ prior to harvest.

\subsection{Morphology, Immunocytochemistry, and Immunofluorescence Analysis}

Keratinocytes were seeded in four-chamber tissue culture glass slides and pre-treated with concentrations of each ligand as follows: curcumin $(10-20 \mu \mathrm{M}), 1,25 \mathrm{D}(0.1 \mu \mathrm{M}), \mathrm{DHA}(10-20 \mu \mathrm{M})$, and GW501516 (100-200 nM), for 24-48 h and processed as earlier described [28]. Briefly, after treatment with (or without) VDR ligands for $48 \mathrm{~h}$, the cells were stimulated for $30 \mathrm{~min}$ before harvest with or without $15 \mathrm{ng} / \mathrm{mL}$ TNF $\alpha$ prior to being washed twice with $1 \times$ phosphate-buffered saline (PBS) $\left(\mathrm{Ca}^{2+} / \mathrm{Mg}^{2+}\right.$-free). Subsequently, cells were fixed in $2 \%$ paraformaldehyde in a 1:1 mix of cold acetone/methanol in PBS for $20 \mathrm{~min}$ at room temperature, followed by $15 \mathrm{~min}$ at $4{ }^{\circ} \mathrm{C}$, followed by three washes in $1 \times$ PBS. Cells were then permeabilized for $5 \mathrm{~min}$ at room temperature with Triton stabilization buffer $(0.5 \%$ Triton X-100, $100 \mathrm{mM}$ piperazine-N,N'-bis(2-ethanesulfonic acid (PIPES) buffer ( $\mathrm{K}^{+}$-free), 4\% PEG, and $1 \mathrm{mM}$ ethyleneglycol-bis(aminoethylether)-tetraacetic acid (EGTA)) and washed three times in $1 \times$ PBS. Nonspecific epitopes were blocked with blocking solution (10\% normal goat serum, $2.5 \%$ bovine serum albumin) in PBS for $20 \mathrm{~min}$. Samples were blotted and subsequently incubated overnight at $4{ }^{\circ} \mathrm{C}$ with one of the following monoclonal or polyclonal primary antibodies diluted in blocking buffer: NR4A2/NURR1 (1:100 dilution), LCE3B-E (cross-reacts with four of the five LCE3 protein isoforms [27]) (1:100 dilution), Jun B (1:100 dilution), p-JNK (1:40 dilution), p-p38 (1:100 dilution), or filaggrin (1:50 dilution). Hereafter, the cells were washed three times and incubated with goat anti-rabbit IgG Alexa Fluor 488 or goat anti-mouse IgG-Texas Red secondary antibodies conjugated to HRP, all at 1:600 dilution in blocking buffer, for $45 \mathrm{~min}$ at $37^{\circ} \mathrm{C}$. Slides were washed twice in PBS for 10 min each followed by washing once in double-distilled water, and the cover slips were mounted on glass slides using ProLong ${ }^{\circledR}$ Gold Anti-fade Reagent containing DAPI from Thermo Fisher Scientific (Cat\# P36941, Rockford, IL, USA) for nuclear counter-staining. The mounted slides were allowed to cure overnight in the dark at room temperature. Automated images were acquired using the Nuance Imaging system with a camera equipped on a light microscope as described below.

\subsection{Nuance Multispectral Imaging System FX-Software}

Automated immunofluorescence images were acquired using an Olympus BX43 light microscope (Olympus America Inc., Center Valley, PA, USA) equipped with a CRI camera on a Nuance ${ }^{\mathrm{TM}}$ Imaging FX system version 3.0.2 (Perkin Elmer, Inc., Waltham, MA, USA) using $20 \times / 0.5$ or $40 \times / 0.75$ objectives connected to a computer and an X-Cite ${ }^{\circledR}$ Series 120 Q Sport light source. Data acquisition and image analysis using Nuance software technology plate-form were conducted as previously described [29,30]. Briefly, a spectral library was created using image cubes to define distinctive spectral curves for each fluorophore, and counterstained to adjust for background effects and to accurately quantify the positive staining of biomarkers using InForm version 1.4.0 software (Perkin Elmer Inc., Waltham, MA, USA), which allows for an objective analysis of biomarkers with increased accuracy. Isotype controls were used for immunostaining (proportion of green/red pixels for antigen staining) with values averaged from at least five fields for each slide sample. 


\subsection{PSORS4 Genotyping of HEKn Cell Lots}

Genomic DNA was isolated from cells using a DNAeasy kit (Qiagen Corp., Valencia, CA, USA) according to the manufacturer's protocol. A triple primer set was utilized to genotype cells: LCE3CF 5'-TCACCCTGGAACTAGACCTCA-3'; LCE3CR 5'-CTCCAACCACTTGTTCTTCTCA-3'; LCE3CR2D 5'-CATCCCAGGGATGCTGCATG-3' [31]. PCR reactions contained approximately $130 \mathrm{ng} / \mu \mathrm{L}$ of HEKn genomic DNA from a single lot of cells, $0.5 \mu \mathrm{L}$ of an $18 \mu \mathrm{M}$ stock of the above three primers $(0.9 \mu \mathrm{M}$ final concentration of each primer), and $5 \mu \mathrm{L}$ of Fast Start Universal SYBR Green Master Mix from Roche Applied Science (Indianapolis, IN, USA) in a total volume of $10 \mu \mathrm{L}$. An Applied Biosystems 2400 machine was programmed for 35 cycles: $94{ }^{\circ} \mathrm{C}$ for $30 \mathrm{~s}, 60{ }^{\circ} \mathrm{C}$ for $30 \mathrm{~s}$, and $72{ }^{\circ} \mathrm{C}$ for $1 \mathrm{~min}$, followed by a $72{ }^{\circ} \mathrm{C}$ step for $10 \mathrm{~min}$. PCR products were resolved on $3 \%$ agarose gels. A single band at 199 bp indicates that cells harbor a homozygous LCE3C_LCE3B deletion (LCE3C_LCE3B-del), a single band at the position $240 \mathrm{bp}$ indicates a homozygous intact locus, and the presence of both bands signifies a heterozygote.

\subsection{Transient Transfection and Treatment of UMR-106 Cells}

UMR-106 cells were plated at 650,000 cells/well in a 6-well plate. After $24 \mathrm{~h}$ of incubation, the cells were transfected with PolyJet reagent (SignaGen Laboratories, Gaithersburg, MD, USA) according to the manufacturer's protocol. Briefly, each well received $20 \mu \mathrm{L} /$ well PolyJet and $500 \mathrm{ng} /$ well of pSG5-VDR M4, an expression plasmid containing the human VDR cDNA with translation starting at codon 4 (one of the common polymorphic variants of VDR [32]). After $24 \mathrm{~h}$ of incubation, the cells were treated with ethanol vehicle or 1, 10, or $100 \mathrm{nM} 1,25 \mathrm{D}$. RNA was harvested after 22-24 h of incubation.

\subsection{Cell Harvesting and Total RNA Preparation}

Cultured cells were harvested by trypsinization using standard techniques and cell pellets were washed with sterile phosphate-buffered saline. RNA isolation was performed using an Aurum Total RNA Mini Kit (Bio-Rad Corp., Hercules, CA, USA) from HEKn cells seeded at 550,000 cells per 60-mm plate and grown to a final confluency of approximately $65-70 \%$. The quantity and purity of prepared RNAs were assessed by UV absorbance at 260 vs. $280 \mathrm{~nm}$. Similar procedures were employed for the UMR-106 cells.

\subsection{Primer Design and Testing}

The UC Santa Cruz Genome Browser [33] was utilized to determine the coding sequence of the genes to be investigated. Unless otherwise referenced, Primer3Plus [34] was used to design primers that spanned an intro-exon junction. For the detection of human LCE3 transcripts, the following primers were used: LCE3A forward primer $5^{\prime}$-CTGAGTCACCACAGATGCCG-3' and reverse primer 5'-CTTGCTGACCACTTCCCCTG-3'; LCE3B forward primer 5'-CTC CTGCTGTGCTCCAAGAC- $3^{\prime}$ and reverse primer $5^{\prime}$-ATCTTGCTGACCACTGCCTC-3'; LCE3C forward primer $5^{\prime}$-GGTCTG AGGGTTCTGTGCTC-3' and reverse primer $5^{\prime}$-ACACTTGGGT GAGGGACAAC-3'; LCE3D forward primer $5^{\prime}$-CCCCAAAGAGCCCAGTACAG-3' and reverse primer 5'-CTGTGGTGGTTCAGGAAGCA-3'; LCE3E forward primer 5'-CCCAAGTGTCCCCCAAAGAA-3' and reverse primer $5^{\prime}$-CTGTGGTGGTTCAGGAAGCA- $3^{\prime}$. For the detection of human Jun $B$ and NR4A2 transcripts, the following primers were used: human Jun $B$ forward primer 5'-CGGCAGCTACTTTTCTGGTC-3' and reverse primer 5'-GAAGAGGCGAGCTTGAGAGA-3'; human NR4A2 (NURR1) forward primer 5'-CTACGACGTCAAGCCACCTT-3' and reverse primer $5^{\prime}$-TCATCTCCTCAGACTGGGGG-3'. Human glyceraldehyde 3-phosphate dehydrogenase (GAPDH) mRNA was amplified using forward primer $5^{\prime}$-TGACAACTTTGGTATCGTGGAAGG-3' and reverse primer $5^{\prime}$-AGGGATGATGTTCTGGAGAGCC- $3^{\prime}$. For the detection of rat transcripts, the following primers were used: rat Nr4a2 forward primer $5^{\prime}$-CTACGCTTAGCATACAGGTC-3' and reverse primer 5'-TTCCTTGAGCCCGTGTCT-3' [35]. Rat GAPDH was amplified using forward primer 
5'-AGGTCGGTGTGAACGGATTTG-3' and reverse primer 5'-CATTCTCAGCCTTGACTGTGC-3'. All primer pairs were prepared as $18 \mu \mathrm{M}$ stocks and stored at $-20^{\circ} \mathrm{C}$.

\subsection{Real-Time $P C R$}

First strand cDNA was synthesized using a Bio-Rad iScript kit from total RNA isolated from HEKn cells. Quantitative real-time PCR was performed with Fast Start Universal SYBR Green Master Mix (Roche Applied Science) in an ABI 7500 Fast thermal cycler, or a BioRad CFX96 thermal cycler for data in Figure 7B only. Each GAPDH PCR well contained $0.5 \mu \mathrm{L}$ of primers, $0.25 \mu \mathrm{L}$ cDNA, and $5 \mu \mathrm{L}$ of SYBR Green reagent mixed in a total volume of $10 \mu \mathrm{L}$. Wells for the detection of other gene products contained $0.5 \mu \mathrm{L}$ of primers, $0.75 \mu \mathrm{L}$ of cDNA, and $5 \mu \mathrm{L}$ of SYBR Green. The temperature profile included 40 cycles with a melting step of $15 \mathrm{~s}$ at $95^{\circ} \mathrm{C}$ and an annealing/elongation step of $1 \mathrm{~min}$ at $60^{\circ} \mathrm{C}$. Real-time PCR data were analyzed via the comparative $C_{t}$ method and normalized to GAPDH. Fold effects for ligand treatments were calculated in relation to the samples treated with ethanol or DMSO vehicle.

\subsection{Protein Extraction and Immunoblotting}

After treatment with different concentrations of ligands at different time points, normal human keratinocyte cells were harvested and whole cell lysates were prepared for Western blot analysis. Briefly, cells were homogenized by sonication in ice-cold $1 \times$ RIPA lysis buffer $(50 \mathrm{mM}$ Tris-HCl, $\mathrm{pH}$ 7.4, $150 \mathrm{mM} \mathrm{NaCl}, 1 \mathrm{mM}$ EGTA, 1 mM EDTA, $20 \mathrm{mM} \mathrm{NaF}, 100$ mM Na 3 VO4, 0.5\% NP-40, $1 \%$ Triton $\mathrm{X}-100,1 \mathrm{mM}$ PMSF) with freshly added protease inhibitor cocktail (Protease Inhibitor Cocktail Set III, Calbiochem, La Jolla, CA, USA). The homogenate was then centrifuged at 14,000 $\times g$ for $25 \mathrm{~min}$ at $4{ }^{\circ} \mathrm{C}$, and the supernatant was collected, aliquoted, and stored at $-80^{\circ} \mathrm{C}$. For immunoblotting, $10-20 \mu \mathrm{g}$ of protein was resolved on $8-12 \%$ SDS polyacrylamide (SDS-PAGE) gels and transferred onto nitrocellulose membranes. Blots were incubated in blocking buffer $(5 \%$ non-fat dry milk $/ 1 \%$ Tween 20; in $20 \mathrm{mM}$ Tris-buffered saline (TBS), $\mathrm{pH}$ 7.6) for $45 \mathrm{~min}$ at room temperature, followed by incubation with a primary antibody directed against either LCE3B-E, Jun B, or NR4A2/NURR1 in blocking buffer overnight at $4{ }^{\circ} \mathrm{C}$. Following several washes, membranes were incubated with the appropriate HRP-conjugated secondary antibody and detected by enhanced chemiluminescence (ECL) and autoradiography using a Bio-Rad Gel-Doc System (Bio-Rad Laboratories Inc., Hercules, CA, USA). Densitometric measurements of the bands were performed with image analysis software using the Biorad ChemiDoc MP imaging system (Bio-Rad, Hercules, CA, USA). To ensure equal protein loading, membranes were re-probed with antibodies to appropriate house-keeping proteins (GAPDH or vinculin) and processed as above. GAPDH or vinculin data were then used as normalization factors.

\section{Results}

To examine the effects of DHA and curcumin on human neonatal epidermal keratinocytes, we initially monitored the morphology of HEKn cells treated with different concentrations of ligands or vehicle control over time using phase contrast microscopy. As depicted in Figure 2B, we observed that these ligands differentially induced changes in cellular morphology reminiscent of keratinocyte differentiation.

We hypothesized that many of the effects of both DHA and curcumin on keratinocytes are mediated via the vitamin $\mathrm{D}$ receptor (VDR) acting to upregulate the expression of the $L C E 3 A / L C E 3 D / L C E 3 E$ genes. First, we determined if the single-patient sample of HEKn cells harbored the PSORS4 deletion and, if so, whether the deletion was homozygous or heterozygous, using a PCR protocol that incorporated a triple set of primers (see Methods Section 2.5). This reaction yielded a single PCR product at $199 \mathrm{bp}$ (data not shown), indicative of a homozygous LCE3C_LCE3B deletion (LCE3C_LCE3B-del). Utilizing a homozygous deletant allowed for a direct test of the hypothesis that DHA and/or curcumin upregulate LCE3A/LCE3D/LCE3E mRNA expression without interfering background from PCR primers annealing to the similar $L C E 3 B$ and/or LCE3C mRNAs. 
The first experiment was performed by treating HEKn cells homozygous for LCE3C_LCE3B-del with two concentrations of DHA. The selection of concentrations was based on a previously published competition binding assay [15] which indicated that DHA competes with radioactively labeled $1,25 \mathrm{D}$ for VDR binding with an $\mathrm{IC}_{50}$ of approximately $10 \mu \mathrm{M}$. As shown in Figure 3A, DHA indeed upregulates LCE3 mRNA expression in a dose-dependent manner, with $20 \mu \mathrm{M}$ DHA eliciting 11-fold, 5.5-fold, and 7-fold increases in mRNAs for $L C E 3 A, L C E 3 D$, and $L C E 3 E$, respectively. These mRNA effects are superior to the respective 3.6-fold, 4.5-fold, and 4.7-fold increases in mRNAs for LCE3A, LCE3D, and LCE3E reported previously by our group for HEKn cells treated with 100 nM 1,25D [11], which serves as a published positive control (employing the hormonal VDR ligand) for the present experiments. To confirm this effect of DHA at the protein level, cells were treated in the presence or absence of single doses of DHA $(10 \mu \mathrm{M})$ with or without activation by recombinant human TNF $\alpha$. Protein expression of LCE3 gene products was monitored by double immunofluorescent microscopy as described in the Methods section; the antibody for LCE3 expression recognizes LCE3B, LCE3C, LCE3D, and LCE3E. As shown in Figure 3B, treatment with $10 \mu \mathrm{M}$ DHA alone for 48 h strongly induced the expression of LCE3 proteins compared to vehicle control cells (see LCE3B-E column, comparing top two panels), with no discernible effect on the levels of phosphorylated p38 MAP kinase (p-p38). Phospho-p38 was included in the study because it is a pro-inflammatory factor that is activated in psoriasis [36]. Because the pro-inflammatory cytokine TNF $\alpha$ is critically involved in the early phase of psoriasis [37], we next examined the effect of TNF $\alpha$ activation on the response to DHA ligands. As shown in Figure 3B, treatment with TNF $\alpha$ did not discernibly modulate LCE3B-E protein expression, whether in the presence or absence of DHA (LCE3B-E column, compare bottom two panels with top two panels), but strongly induced the phosphorylation of p38 (see p-p38 column, second panel from the bottom). Additionally, the pre-treatment of keratinocytes with DHA for $48 \mathrm{~h}$, prior to activation with TNF $\alpha$ for $30 \mathrm{~min}$, appeared to block the effect of TNF $\alpha$ on p38 phosphorylation (Figure 3B; p-p38 column, bottom panel). A subset of these results was further corroborated by Western blot analyses (see last section of Results below).

A second dietary agent, namely curcumin, a turmeric derivative and bioactive polyphenol, was similarly evaluated in cultured keratinocytes. Because curcumin exhibits a comparable VDR competition profile to DHA (an $\mathrm{IC}_{50}$ of approximately $5-10 \mu \mathrm{M}$ [15]), and has been reported to be a bona fide VDR ligand [38] with beneficial effects on skin repair [24], we treated HEKn cells with concentrations of curcumin similar to those used for DHA, namely 6.7 and $10 \mu \mathrm{M}$ for mRNA studies and 5, 10, and $20 \mu \mathrm{M}$ for immunocytochemistry. Also, DMSO rather than ethanol was employed as the solvent vehicle for curcumin. The results in Figure $4 \mathrm{~A}$ reveal that curcumin is capable of upregulating LCE3A, LCE3D, and LCE3E mRNAs 3- to 4-fold in a dose-dependent manner at concentrations corresponding closely to the concentrations capable of competing with $1,25 \mathrm{D}$ for binding to the VDR. This magnitude of induction of LCE3 genes is quite comparable to that of 3.6- to 4.7-fold achieved by $100 \mathrm{nM}$ 1,25D as previously published [12]. Immunostaining for protein expression in human keratinocytes confirmed that curcumin treatment alone strongly induced the protein expression of LCE3B-E (Figure 4B; LCE3B-E column, compare top two panels). Analogous to the DHA study shown in Figure 3B, a 30-min treatment with TNF $\alpha$ ( \pm curcumin) was also included (lower two rows of Figure $4 \mathrm{~B}$ ), and there is a suggestion that the combination of curcumin and $\mathrm{TNF} \alpha$ yields a higher induction of LCE3B-E protein expression than curcumin alone (Figure 3B, LCE3D-E column, compare second and fourth panels). Finally, the phosphorylation of MAPK p38 in response to the various treatments was also included, with curcumin showing effects similar to DHA, namely an ability to block p38 phosphorylation in response to TNF $\alpha$ (Figure 4B, p-p38 column, compare third and fourth panels). A subset of these results was further corroborated by Western blot analyses (see last section of Results). 

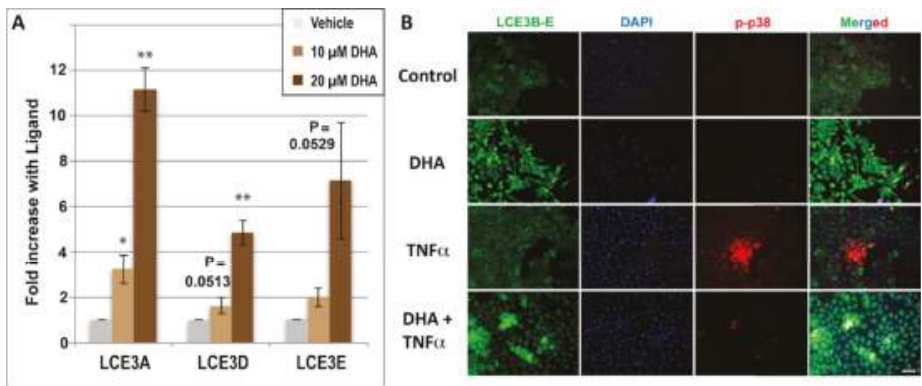

Figure 3. (A) Regulation by docosahexaenoic acid (DHA) of the LCE3A, LCE3D and LCE3E genes spared by the PSORS4 deletion. Human primary neonatal keratinocytes (HEKn) cells homozygous for the deletion were treated with ethanol vehicle (negative control) or DHA (10 or $20 \mu \mathrm{M}$ ), and total RNA was prepared and analyzed for the expression of mRNA using specific primers for each LCE3 isoform. Bar graphs represent the average of three independent experiments \pm STDEV. Asterisks denote results with ligands that are significantly different from ethanol controls as determined by the Student's two-tailed $t$-test: ${ }^{*} p<0.05 ;{ }^{* *} p<0.01$. $p$-Values approaching 0.05 are given above the corresponding bar. (B) Regulation of LCE3 proteins (and p-p38) by DHA and tumor necrosis factor-alpha (TNF $\alpha$ ) as monitored by immunohistochemistry using an antibody that cross-reacts with LCE3B, LCE3C, LCE3D, and LCE3E proteins. Cells were cultured in four chamber tissue culture glass slides and treated for $48 \mathrm{~h}$ with $(10 \mu \mathrm{M})$ or without DHA as indicated. Treatment with TNF $\alpha(15 \mathrm{ng} / \mathrm{mL})$ occurred $30 \mathrm{~min}$ prior to fixing and binding of permeabilized cells to the indicated antibodies. Image acquisition is described in Methods and representative images are shown. Images in right-hand column represent a merging of all three signals (LCE3B-3E antibody (green), 4',6-diamidino-2-phenyl (DAPI) (blue), and p-p38 antibody (red)) at a magnification of $\times 200$.
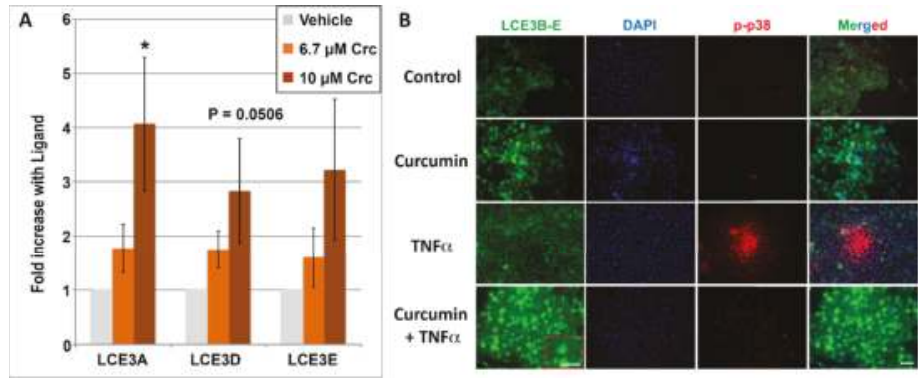

Figure 4. (A) Regulation of $L C E 3 A, L C E 3 D$, and $L C E 3 E$ mRNA by curcumin (Crc). HEKn cells were treated with ETOH vehicle (negative control) or curcumin (at 6.7 or $10 \mu \mathrm{M}$ concentration). Bar graphs show real-time PCR results, which are the average of four independent experiments \pm STDEV. An asterisk denotes ligand-treated averages that are significantly different from ethanol controls as determined by two-tailed Student's $t$-test, ${ }^{*} p<0.05$. One of the four replicates of this experiment was performed with HEKn cells heterozygous for the PSORS4 deletion, which yielded results very similar to the three replicates from homozygous cells. (B) Regulation of LCE3 and p-p38 proteins by curcumin and TNF $\alpha$ as monitored by immunohistochemistry. Cells were cultured in four chamber tissue culture glass slides and treated for $48 \mathrm{~h}$ with $(15 \mu \mathrm{M})$ or without curcumin as indicated. Treatment with $\mathrm{TNF} \alpha(15 \mathrm{ng} / \mathrm{mL})$ occurred $30 \mathrm{~min}$ prior to fixing and binding of permeabilized cells to the indicated antibodies. Image acquisition is described in Methods and representative images are shown. Images in right-hand column represent a merging of all three signals (LCE3B-3E (green), DAPI (blue), and p-p38 antibody (red)) at a magnification of $\times 200$. Insets in red boxes indicate a higher power magnification $(\times 400)$ of the selected section. 
Although the present data are consistent with the conclusion that DHA is acting as a VDR ligand, literature reports indicate that DHA effects in skin may be mediated by a peroxisome proliferator-activated receptor (PPAR), presumably PPAR $\delta$, which is the predominant isoform expressed in skin [26]. We and others have recently shown that PPAR $\delta$ is overexpressed in human psoriatic as well as in murine psoriasis-like skin lesions [39], and that treatment with delphinidin, another VDR ligand, normalized the expression in a preclinical mouse model of psoriasis [30]. To determine whether PPAR $\delta$ is capable of upregulating LCE3 mRNAs in human keratinocytes, a selective ligand for PPAR $\delta$ (GW501516) was examined at a single $100 \mathrm{nM}$ concentration which, according to published reports, represents a saturating dose for activating PPAR $\delta$ [40]. The results (data not shown) indicated a nonsignificant trend toward a very slight $(<1.5$-fold) upregulation of $L C E 3 A, L C E 3 D$, and LCE3E mRNA expression. Moreover, Western blot data from keratinocytes treated for 24 to $48 \mathrm{~h}$ with two doses of GW501516 (100 and $200 \mathrm{nM}$ ) yielded no significant increase in the expression of LCE3 proteins (see last section of Results). In this same experiment, 10-20 $\mu \mathrm{M}$ DHA or curcumin elicited a statistically significant enhancement in LCE3 protein levels, providing a positive control and indicating that liganded PPAR $\delta$ is not an inducer of LCE3 protein in HEKn cells. Based on these data, we conclude that liganded PPAR plays little or no role in LCE3 gene expression, and this result allows us to distinguish the relative contributions of VDR and PPAR $\delta$ in mediating the actions of DHA with respect to LCE3 maintenance. The conclusion is that VDR alone executes the function of DHA to induce LCE3 gene and protein expression. This does not eliminate potential cross-talk between the signaling of the VDR and PPAR systems in skin, at least with regard to the regulation of genes other than those of the LCE3 class. For example, GW501516 is a strong inducer of NR4A2 protein in HEKn cells, as are 1,25D and curcumin (see last part of Results).

We next assessed whether DHA upregulates Jun B, for which depressed expression or gene deletion has been noted to be associated with psoriasis. Quantitative real-time PCR results (Figure 5A) indicate that DHA significantly upregulates Jun B mRNA expression, but the effect is minimal (2-fold) after $24 \mathrm{~h}$ of treatment, and is not evident until DHA is present at the higher $20 \mu \mathrm{M}$ concentration. Nevertheless, we previously demonstrated Jun B induction by 1,25D in KERTr human keratinocytes, utilizing microarray technology to quantitate mRNA [8]. In the present report, utilizing immunofluorescence to determine the effects of $48 \mathrm{~h}$ of DHA treatment, we observed an increase in Jun B protein expression (Figure 5B) in both the presence and absence of TNF $\alpha$ treatment. The immunofluorescence experiment also included the monitoring of JNK phosphorylation using a specific phospho-JNK antibody (see p-JNK column in Figure 5B). As expected, JNK is phosphorylated in response to $30 \mathrm{~min}$ of treatment with $\mathrm{TNF} \alpha$, an effect that is largely abolished when $\mathrm{TNF} \alpha$-treated cells are pretreated with DHA (Figure 5B, p-JNK column, compare bottom two panels). The upregulation of Jun B by DHA was confirmed by Western blot analysis (see last section of Results), exhibiting a significant, dose-dependent upregulation of Jun B protein at both 24 and $48 \mathrm{~h}$, approaching the dramatic action of $100 \mathrm{nM} 1,25 \mathrm{D}$ to enhance Jun B protein levels.

The ability of curcumin $\pm \mathrm{TNF} \alpha$ to upregulate Jun B expression was also investigated, and Figure 6 illustrates the immunofluorescence results using an anti-Jun B antibody. Treatment with curcumin increased levels of Jun B (Figure 6, Jun B column, compare top two panels), an effect that was confirmed with Western blotting (Figure 8B), which demonstrated a significant and dose-dependent increase in Jun B protein after both 24 and $48 \mathrm{~h}$ of treatment using doses of 5, 10, and $20 \mu \mathrm{M}$. Curcumin also appears to dampen the Jun B-induced phosphorylation of p-JNK by TNF $\alpha$ (Figure 6, p-JNK column, compare bottom two panels). Thus, two low-affinity VDR ligands (DHA and curcumin) evaluated in the present study are able to induce Jun B, as well as to oppose TNF $\alpha$-induced p-JNK phosphorylation.

Finally, we explored the possibility that VDR ligands, including 1,25D, DHA, and curcumin, regulate the expression of the nuclear receptor NURR1, also known as NR4A2, pursuing a notion previously derived conceptually from the recent demonstration that some VDR actions follow a secondary induction mechanism, whereby liganded VDR first induces NURR1, which in turn activates the expression of the gene(s) of interest [25]. Moreover, as noted above, the LCE3 VDRE shown in 
Figure 1 contains within its $5^{\prime}$ half-element a consensus binding sequence for the NR4A2 monomer, caGGGTGA. For these reasons, we examined the level of NURR1 transcripts in DHA-, 1,25D-, and vehicle-treated HEKn cells. The results (Figure 7A) demonstrate that both 1,25D and DHA upregulate NURR1 mRNA in HEKn cells, although there exists no indication of a classic dose-response relationship for DHA, and the results are variable for treatment with 1,25D and $10 \mu \mathrm{M}$ DHA. To definitively demonstrate that NURR1 can be induced in a VDR and ligand dose-dependent fashion, UMR-106 osteoblast-like cells were transfected with human VDR and treated with concentrations of 1,25D from 1 to $100 \mathrm{nM}$. Data for NURR1 mRNA levels compared with vehicle controls, as displayed in Figure 7B, exhibit clear 1,25D dose-dependency for a 3- to 4-fold induction of NURR1 by the hormonal vitamin $\mathrm{D}$. We followed up these mRNA studies by investigating the effect of a single dose of DHA or curcumin treatment using both immunofluorescence as well as Western blotting on the protein expression of NURR1 as well as filaggrin, another biomarker of skin inflammatory disease. Immunofluorescence after $48 \mathrm{~h}$ of treatment with ligands reveals a striking ability of both DHA alone and curcumin alone to upregulate both NURR1 and filaggrin (see NURR1 and filaggrin columns in Figure 7C, comparing second and fourth panels to top control panel). These results were confirmed by Western blotting (Figure $8 \mathrm{C}$ ). Although the DHA effects pictured in Figure $8 \mathrm{C}$ are statistically significant only at the higher $(20 \mu \mathrm{M})$ dose, effects with both DHA and curcumin displayed a dose-response at both time points. Importantly, 1,25D exerts a dramatic positive induction of NURR1 protein in HEKn cells (Figure 8C). Taken together, these results are consistent with the intermediary function of NURR1 as a secondary mediator of at least part of the action of VDR ligands in osteoblasts and skin, with putative occupation of the NURR1 site embedded within the VDRE identified in the LCE gene region being of potential mechanistic significance.
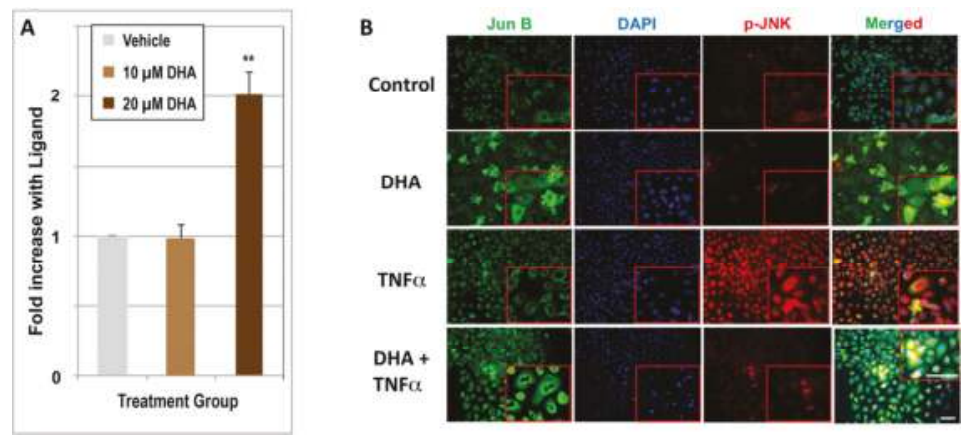

Figure 5. (A) Regulation of Jun B mRNA by DHA. HEKn cells were treated with ethanol vehicle (control), or with either $10 \mu \mathrm{M}$ DHA or $20 \mu \mathrm{M}$ DHA. RNA isolation, synthesis of first strand DNA, and real-time PCR are described in Methods. Results are means from three independent experiments \pm STDEV. The double asterisk $\left({ }^{* *}\right)$ denotes an average that is statistically significant by Student's $t$-test from the ethanol control, $p<0.01$. (B) Regulation of Jun B and p-JNK proteins by DHA and TNF $\alpha$ as monitored by immunohistochemistry. Cells were cultured in four chamber tissue culture glass slides and treated for $48 \mathrm{~h}$ with $(10 \mu \mathrm{M})$ or without DHA as indicated. Treatment with TNF $\alpha(15 \mathrm{ng} / \mathrm{mL})$ occurred $30 \mathrm{~min}$ prior to fixing and binding of immobilized proteins to the indicated antibodies. Image acquisition is described in Methods and representative images are shown. Images in the right-hand column represent a merging of all three signals (Jun B antibody, DAPI, and p-JNK antibody), measured using Nuance software as described in Methods. (Magnification $\times 200$ ). Insets in red boxes indicate a higher power magnification $(\times 400)$ of the selected section. The yellow color indicates co-localization of both markers. 


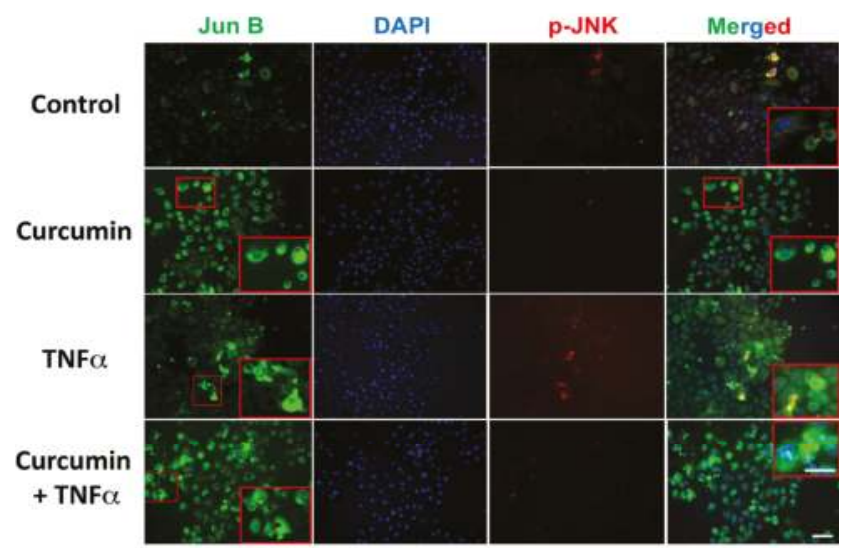

Figure 6. Regulation of Jun B and p-JNK proteins by curcumin and $\mathrm{TNF} \alpha$ as monitored by immunohistochemistry. Cells were cultured in four chamber tissue culture glass slides and treated for $48 \mathrm{~h}$ with or without curcumin $(15 \mu \mathrm{M})$ as indicated. Treatment with TNF $\alpha(15 \mathrm{ng} / \mathrm{mL})$ occurred $30 \mathrm{~min}$ prior to fixing in $2 \%$ paraformaldehyde and binding of immobilized proteins to the indicated antibodies. Image acquisition is described in Methods and representative images are shown. Images in right-hand column represent a merging of all three signals (Jun B antibody, DAPI and p-JNK antibody), measured using Nuance software as described in Methods. (Magnification $\times 200$ ). Insets in red boxes indicate a higher power magnification $(\times 400)$ of the selected section.
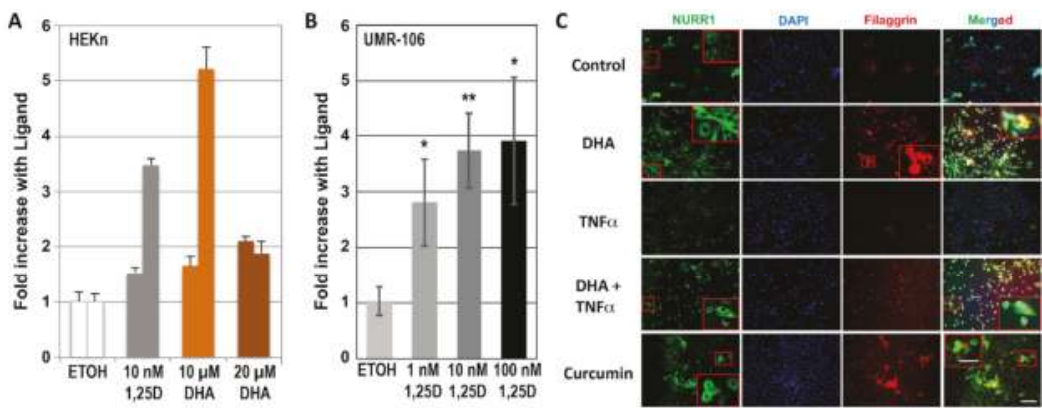

Figure 7. Regulation of NURR1 (NR4A2) mRNA by 1,25D. (A) Response of NURR1 mRNA to $1,25 \mathrm{D}$ and DHA in HEKn cells. Cells were plated as described in Methods and dosed with the indicated concentrations of 1,25D or DHA for 22-24 h. Total RNA and first strand cDNA were then prepared, and real-time qPCR was performed using primers to human NURR1 as described in Methods. Error bars represent STDEV of triplicate real-time PCR wells from each of two independent experiments. (B) A similar experiment to (A), but performed using increasing doses of 1,25D in rat UMR-106 cultures. Results are means of three independent experiments \pm STDEV, ${ }^{*} p<0.05,{ }^{* *} p<0.01$ compared to control by Student's t-test. (C) Regulation of NURR1 and filaggrin proteins by DHA and TNF $\alpha$ as monitored by immunohistochemistry. Cells were cultured in tissue culture glass slides and treated for $48 \mathrm{~h}$ with $(10 \mu \mathrm{M})$ or without DHA as indicated. Treatment with TNF $\alpha(15 \mathrm{ng} / \mathrm{mL})$ occurred $30 \mathrm{~min}$ prior to fixing in $2 \%$ paraformaldehyde and binding of immobilized proteins to the indicated antibodies. Image acquisition is described in Methods and representative images are shown. Images in right-hand column represent a merging of all three signals (NURR1 antibody, DAPI and filaggrin antibody), measured using Nuance software as described in Methods. (Magnification $\times 200$ ). Insets in red boxes indicate a high power magnification $(\times 400)$ of the highlighted section. 
A
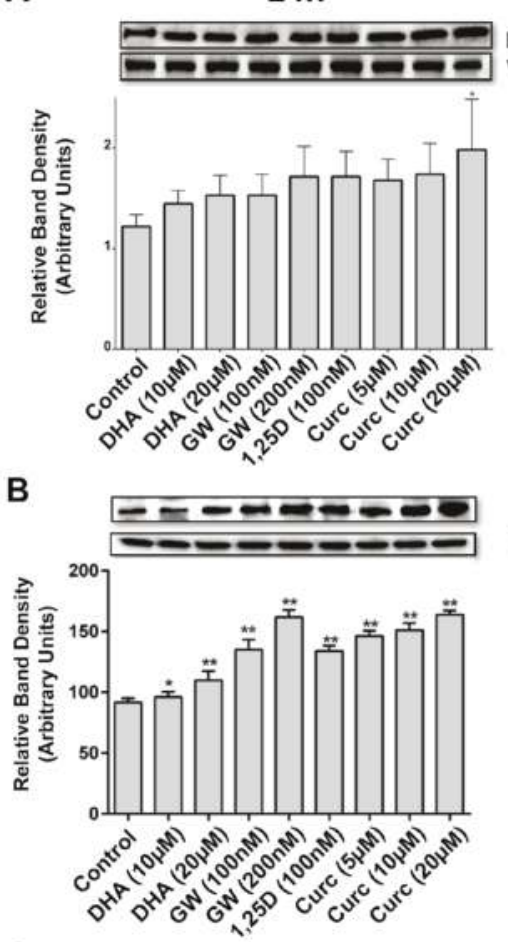

C

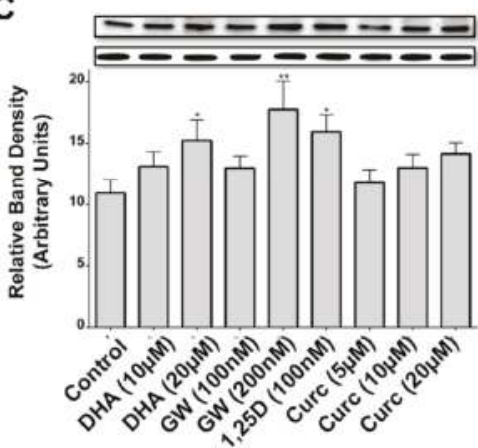

48h
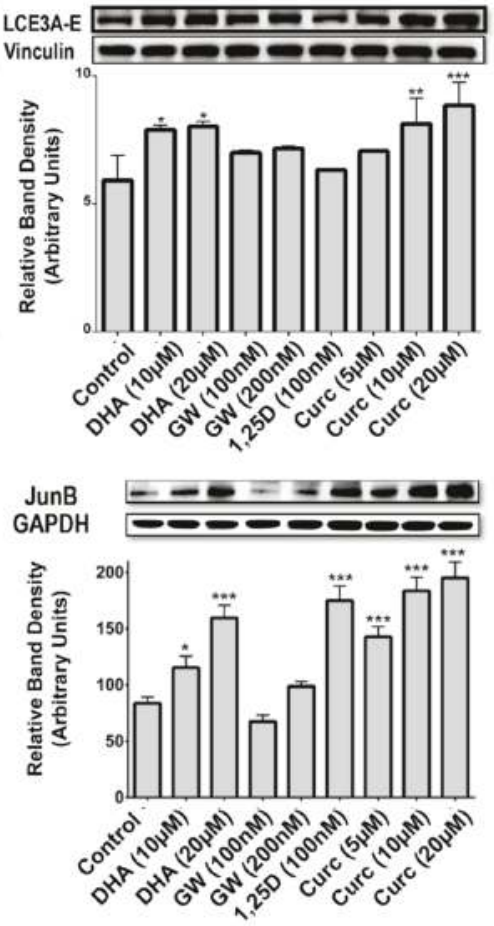

Figure 8. Dose-dependency of protein expression in response to 1,25D, curcumin (Curc) (ligand for the vitamin D receptor), GW501516 (GW; ligand for PPAR $\delta$ ), and DHA (ligand for both receptors). (A) Protein expression for LCE3 proteins as monitored by an antibody that recognizes four LCE3 isoforms (LCE3B/LCE3C/LCE3D/LCE3E), using Vinculin expression as an unregulated control to normalize for protein loading. (B) Protein expression of Jun B, using GAPDH as an unregulated control. (C) Protein expression of NR4A2 (NURR1), using Vinculin as an unregulated control. All bars represent an average of at least five fields for each sample (blots were normalized using Chemidoc quantification analysis software as described in Methods) \pm STDEV. ${ }^{*} p<0.5,{ }^{* *} p<0.01,{ }^{* * *} p<0.001$.

\section{Discussion and Conclusions}

Analogs of $1,25 \mathrm{D}$ are used routinely for psoriasis treatment, often in combination with an anti-inflammatory steroid [41]. Previous studies from our laboratory have pursued the hypothesis 
that 1,25D and other VDR ligands improve symptoms of psoriasis by upregulating skin repair genes, and in two recent publications by our group, we reported that the VDR ligands 1,25D, delphinidin, and cyanidin upregulate $L C E 3 A / L C E 3 D / L C E 3 E$ gene expression, potentially to compensate for the common genomic deletion of $L C E 3 B$ and LCE3C [11,12].

The dietary lipids DHA (an omega-3 fatty acid) and curcumin (a bioactive component of turmeric spice) were selected for investigation as both have been subjects of clinical trials for the treatment of psoriasis (NCT01351805 and NCT00235625). In addition to these clinical trials, dietary studies have concluded that psoriatic patients typically consume diets low in omega- 3 fatty acids, including DHA [42], and conversely, that a diet low in calories but high in omega-3 fatty acids appears to confer a better response to immunomodulatory drugs in a cohort of obese patients with plaque-type psoriasis [43]. Another dietary study found that adherence to a Mediterranean diet was inversely related to psoriasis area severity index (PASI) score, and that high consumption of fish (which contain omega-3 fatty acids including DHA) was independently negatively associated with a high PASI score [44]. Although it is difficult to separate the potential effects of DHA from those of other dietary components, these studies, taken together, suggest that consumption of omega- 3 fatty acids, including DHA, may represent a useful adjuvant to other psoriasis therapies.

In addition to other modes of action, the current study pursues the notion that DHA and curcumin may act as VDR ligands to regulate genes involved in psoriasis pathology. Both DHA and curcumin have been shown, like other alternative, low-affinity VDR ligands such as delphinidin and cyanidin, to compete with 1,25D for direct, low-affinity binding to VDR [15]. The data shown in Figure 3 indicate that DHA significantly upregulates LCE3 gene expression in cells homozygous for LCE3C_LCE3B-del. We propose that this induction of LCE3 gene products compensates for the loss of $L C E 3 B$ and $L C E 3 C$ in skin repair, under the assumption that the highly similar LCE3 gene products are functionally redundant [27]. Curcumin was also demonstrated to be capable of upregulating $L C E 3 A, L C E 3 D$, and $L C E 3 E$ genes (Figure 4). Given that the effective concentrations of DHA and curcumin employed in these experiments coincide with those that competitively bind to VDR, we postulate that these ligands upregulate LCE3 gene expression, at least in part, by binding to, and activating the VDR-RXR heterodimer. Our previous work identified a VDRE adjacent to the LCE3A gene (Figure 1) that is capable of conferring regulation onto a heterologous reporter gene by $1,25 \mathrm{D}$, delphinidin, or cyanidin in transfected CCD-1106 KERTr human keratinocyte cells [11], lending further support to the notion that liganded VDR may induce the expression of these genes in vivo.

Recent reports reveal that the presence of LCE3C_LCE3B-del within the LCE3 gene cluster has a lengthy evolutionary history in the hominid lineage. The deletion appears to have arisen in the common ancestor to modern humans and Denisovans $[45,46]$. Based on an alysis of genomic DNA from ancient hominins as well as modern humans, including a haplotype analysis of the deletion as well as flanking regions in the LCE3 locus, the authors of these studies conclude that the $32 \mathrm{~kb} L C E 3 C_{-} L C E 3 B$ deletion has been maintained under balancing selection in the human lineage. In considering the potential reasons as to why both LCE3 haplotypes are currently found in all tested human populations, including those from Eurasia, Africa, and the Americas [46,47], these authors quoted Bergboer et al. [48], who hypothesized that LCE3C_LCE3B-del, by delaying skin repair, could allow for greater penetration of microbial antigens, which then serve as a natural "vaccine" against future infections (while also posing an increased risk for an autoimmune response). Further, the hypothesis explaining the retention of the intact LCE3 cluster is that the full complement of five LCE3 genes confers a superior ability to support skin repair. Whatever the exact explanation, it appears that an interplay between these two sets of advantages/disadvantages has led to the preservation of both alleles in a state of balance over hundreds of thousands of years.

The extent to which DHA upregulates LCE3 mRNA expression exceeds 10-fold in the case of $L C E 3 A$, which is noticeably greater than the approximate 4 -fold achieved by $10^{-8} \mathrm{M} 1,25 \mathrm{D}$ in prior studies [11,12]. This comparison suggests that, at least for the upregulation of LCE3 gene expression, DHA may be superior to the currently used therapeutic agent 1,25D (or analogs thereof), taking into 
account that higher concentrations of DHA are required [19] to achieve this effect due to the lower affinity of DHA for the receptor. This result positions both DHA and the previously tested cyanidin compound [11] as two dietary, non-toxic nutrients that are each capable of upregulating the expression of LCE3 skin repair genes in a fashion superior to that of $1,25 \mathrm{D}$, the currently used anti-psoriatic agent. Moreover, curcumin is yet another candidate alternative to $1,25 \mathrm{D}$ chemical analogs for psoriasis treatment, as its 3- to 4-fold action to induce LCE3A mRNA (Figures $4 \mathrm{~A}$ and $8 \mathrm{~A}$ ) is equivalent in

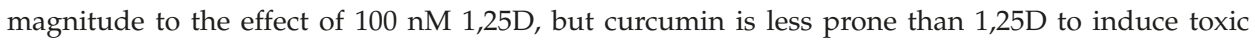
hypercalcemia and likely is endowed with beneficial influences not intrinsic to 1,25D.

The results presented herein (Figures 3-5) do not rule out the possibility that DHA, curcumin, or other VDR ligands may act on LCE3 gene transcription via additional mechanisms. Indeed, literature reports have suggested that DHA effects in the skin are mediated, not by VDR, but rather via peroxisome proliferator-activated receptor (PPAR) isoforms such as PPAR $\delta$ [26]. Furthermore, the doses of DHA that are capable of activating PPAR $\delta$ are similar to doses necessary for the activation of VDR [49]. By observing that treatment with a saturating concentration of the PPAR $\delta$ ligand GW501516 only modestly (and not statistically significantly) upregulated LCE3A, LCE3D, and LCE3E mRNAs (data not shown), and did not significantly enhance LCE3 protein expression (Figure 8A), we conclude that the effect of DHA on LCE3 gene expression is exerted predominantly via the activation of VDR. Thus, DHA appears to function via a mechanism involving the association of liganded VDR with VDREs in target genes. To prove conclusively that liganded VDR docks on the postulated LCE3 gene region VDRE (Figure 1) will require in vivo ChIP-seq experiments in skin, a technical challenge which is beyond the scope of the current investigation, but is warranted for future studies.

The involvement of TNF $\alpha$ in the pathogenesis of psoriasis is well documented [50] and is the target of biological therapies that have proven effectiveness in severe cases [51]. The involvement of the MAP kinase p38 in the pathogenesis of psoriasis and its activation by the TNF $\alpha$ pathway are also well established [52]. We therefore investigated whether the VDR ligands DHA and curcumin could impact p38 phosphorylation in response to TNF $\alpha$ treatment of keratinocytes. Our finding that both ligands inhibit TNF $\alpha$-induced p38 phosphorylation (Figures 3B and 4B) suggests that these VDR ligands have anti-psoriatic actions besides the induction of skin repair involving $L C E 3$ gene products. The effects of both ligands on TNF $\alpha$ signaling are similar to those observed in the imiquimod-induced mouse models of psoriasis by curcumin [53], and in other tissues, such as rat endothelial cells, by DHA [54]. In the case of DHA, the involvement of PPAR receptors such as PPAR $\gamma$ in these effects cannot be excluded (see [55]). Similarly, the effect of TNF $\alpha$ on JNK phosphorylation is also relevant to psoriasis [56]. Again, the pretreatment of keratinocytes with either DHA or curcumin inhibited the TNF $\alpha$-induced phosphorylation of JNK (Figures 5B and 6), another indication that the anti-psoriatic mechanisms of these ligands extend to the TNF $\alpha$ pathway, which is successfully targeted by biologic treatments for moderate to severe psoriasis.

As a concluding experiment in this study, we evaluated whether VDR ligands, including DHA, 1,25D, and curcumin, regulate the expression of the nuclear receptor NURR1, also known as NR4A2, based on recent insight that specific VDR actions appear to involve a secondary mechanism whereby liganded VDR upregulates NURR1 expression, which in turn activates the expression the gene(s) of interest [25]. Moreover, increased expression of NURR1 mRNA and protein occurs in involved psoriasis skin compared with uninvolved and normal skin [57], which is consistent with another recent report showing that NURR1 expression is crucial for the development of mature, fully functional Th17 cells [58] that have, in turn, been shown to play an important role in psoriasis pathogenesis via the production of IL-17 and IL-23 cytokines [59]. For these reasons, it was of interest to investigate the potential ability of DHA, curcumin, and 1,25D to control NURR1 expression. The results (Figures 7 and $8 \mathrm{C}$ ) revealed that DHA, curcumin, and 1,25D induce, rather than repress, NURR1 expression, consistent with the hypothesis that NURR1 acts as a secondary mediator of at least part of the function of VDR ligands to induce skin repair genes such as the LCEs. However, because NURR1 is apparently a pro-psoriatic transcription factor in human skin [57], its induction by VDR 
ligands complicates our understanding of the mechanism whereby vitamin $\mathrm{D}$ analogs are effective in suppressing mild to moderate psoriasis symptoms. In other words, by inducing NURR1, VDR ligands could conceivably aggravate inflammation and proliferation as collateral effects to inducing skin repair genes, although one could argue that NURR1 is actually a trigger for normal skin remodeling in place of psoriatic pathology. It is also possible that the relative timing of NURR1 upregulation might be important with respect to its effects on inflammation and/or proliferation versus skin repair, an issue that could be addressed in future studies. In conclusion, identified herein is a secondary induction mechanism whereby VDR ligands increase the expression of NURR1, which in turn may function as the primary inducer of skin repair genes such as the LCE3 ensemble. Again, proof of this concept will require in vivo ChIP-seq experiments in skin, determining whether NURR1 is the transcription factor actually docked on the composite response element for VDR and NURR1 identified in the LCE3 gene region (Figure 1).

Given the lack of understanding of how 1,25D analogs improve symptoms of psoriasis, the effect of these compounds, as well as low-affinity VDR ligands such as DHA, curcumin, cyanidin, delphinidin, and others, could be examined on the expression of genes harbored in different loci. One of many possible examples is secreted mammalian Ly6/urokinase plasminogen activator receptor-related protein (SLURP-2), a gene that is strongly induced in psoriatic skin lesions [60]. Finally, since 14,21-dihydroxy-DHA has been shown to be a dramatic wound healing lipid in murine models [61], it is tempting to speculate that cellular metabolites of DHA, and possibly other low-affinity VDR ligands, could be discovered as high-affinity nuclear receptor ligands. These novel super-bioactive metabolites, if identified, could prove vastly more efficacious than their low-affinity VDR ligand precursors or even than 1,25D itself. In conclusion, the present investigation reveals the ability of nutrient or diet-derived VDR ligands to upregulate specific skin-expressed genes and proteins with relevance for psoriasis, and sets the stage for future studies examining the regulation of psoriasis-related skin-expressed genes by novel metabolites of low-affinity VDR ligands such as DHA and curcumin, potentially leading to drug discovery of new molecular-based treatments for mild/moderate psoriasis. Further studies beyond the scope of the current manuscript are warranted to critically examine these observations and to decipher their detailed molecular mechanisms.

Acknowledgments: This work was supported in part by grants NIH DK033351 to M.R.H., NIH CA140285 to P.W.J., a POHOFI Inc., Pre-College Intern Scholarship Award to R.C.N.C., a American Skin Association (ASA) Carson Research Scholar Award in Psoriasis to J.C.C., a UW-Madison Skin Disease Research Center (SDRC) Pilot and Feasibility Research Award to J.C.C. from NIH/NIAMS grant P30 AR066524, funding from the University of Louisiana at Monroe School of Pharmacy to J.C.C., and a grant from the University of Arizona, Department of Basic Medical Sciences to G.K.W.

Author Contributions: A.K., I.R. and R.C.N.C. performed most of the experimental work, and A.K. wrote the first draft of the manuscript. J.C.C. and G.K.W. designed and supervised the work, and performed most of the data analysis and manuscript editing. M.S.S. and S.M. performed the experiment and data analysis for Figure 7B as well as aided in manuscript editing. P.W.J. and M.R.H. provided laboratory and grant support for the project, as well as key insights into the planning of the project, interpretation of the data, and placing the conclusions into the context of the broader field. All authors read and approved the final submitted version of this manuscript.

Conflicts of Interest: The authors declare no conflict of interest.

\section{References}

1. Rachakonda, T.D.; Schupp, C.W.; Armstrong, A.W. Psoriasis prevalence among adults in the United States. J. Am. Acad. Dermatol. 2014, 70, 512-516. [CrossRef] [PubMed]

2. Barrea, L.; Savanelli, M.C.; Somma, C.D.; Napolitano, M.; Megna, M.; Colao, A.; Savastano, S. Vitamin D and its role in psoriasis: An overview of the dermatologist and nutritionist. Rev. Endocr. Metab. Disord. 2017, 18, 195-205. [CrossRef] [PubMed]

3. Gisondi, P.; Rossini, M.; Di Cesare, A.; Idolazzi, L.; Farina, S.; Beltrami, G.; Peris, K.; Girolomoni, G. Vitamin D status in patients with chronic plaque psoriasis. Br. J. Dermatol. 2012, 166, 505-510. [CrossRef] [PubMed] 
4. Orgaz-Molina, J.; Buendia-Eisman, A.; Arrabal-Polo, M.A.; Ruiz, J.C.; Arias-Santiago, S. Deficiency of serum concentration of 25-hydroxyvitamin D in psoriatic patients: A case-control study. J. Am. Acad. Dermatol. 2012, 67, 931-938. [CrossRef] [PubMed]

5. Devaux, S.; Castela, A.; Archier, E.; Gallini, A.; Joly, P.; Misery, L.; Aractingi, S.; Aubin, F.; Bachelez, H.; Cribier, B.; et al. Topical vitamin D analogues alone or in association with topical steroids for psoriasis: A systematic review. J. Eur. Acad. Dermatol. Venereol. 2012, 26, 52-60. [CrossRef] [PubMed]

6. Kim, I.H.; West, C.E.; Kwatra, S.G.; Feldman, S.R.; O'Neill, J.L. Comparative efficacy of biologics in psoriasis: A review. Am. J. Clin. Dermatol. 2012, 13, 365-374. [CrossRef] [PubMed]

7. Crow, J.M. Therapeutics: Silencing psoriasis. Nature 2012, 492, S58-S59. [CrossRef] [PubMed]

8. Haussler, M.R.; Whitfield, G.K.; Kaneko, I.; Haussler, C.A.; Hsieh, D.; Hsieh, J.C.; Jurutka, P.W. Molecular mechanisms of vitamin D action. Calcif. Tissue Int. 2013, 92, 77-98. [CrossRef] [PubMed]

9. Bergboer, J.G.; Tjabringa, G.S.; Kamsteeg, M.; van Vlijmen-Willems, I.M.; Rodijk-Olthuis, D.; Jansen, P.A.; Thuret, J.Y.; Narita, M.; Ishida-Yamamoto, A.; Zeeuwen, P.L.; et al. Psoriasis risk genes of the late cornified envelope-3 group are distinctly expressed compared with genes of other LCE groups. Am. J. Pathol. 2011, 178, 1470-1477. [CrossRef] [PubMed]

10. Riveira-Munoz, E.; He, S.M.; Escaramis, G.; Stuart, P.E.; Huffmeier, U.; Lee, C.; Kirby, B.; Oka, A.; Giardina, E.; Liao, W.; et al. Meta-analysis confirms the LCE3C_LCE3B deletion as a risk factor for psoriasis in several ethnic groups and finds interaction with HLA-CW6. J. Investig. Dermatol. 2011, 131, 1105-1109. [CrossRef] [PubMed]

11. Austin, H.R.; Hoss, E.; Batie, S.F.; Moffet, E.W.; Jurutka, P.W.; Haussler, M.R.; Whitfield, G.K. Regulation of late cornified envelope genes relevant to psoriasis risk by plant-derived cyanidin. Biochem. Biophys. Res. Commun. 2014, 443, 1275-1279. [CrossRef] [PubMed]

12. Hoss, E.; Austin, H.R.; Batie, S.F.; Jurutka, P.W.; Haussler, M.R.; Whitfield, G.K. Control of late cornified envelope genes relevant to psoriasis risk: Upregulation by 1,25-dihydroxyvitamin D3 and plant-derived delphinidin. Arch. Dermatol. Res. 2013, 305, 867-878. [CrossRef] [PubMed]

13. Jackson, B.; Tilli, C.M.; Hardman, M.J.; Avilion, A.A.; MacLeod, M.C.; Ashcroft, G.S.; Byrne, C. Late cornified envelope family in differentiating epithelia-response to calcium and ultraviolet irradiation. J. Investig. Dermatol. 2005, 124, 1062-1070. [CrossRef] [PubMed]

14. Reichrath, J.; Müller, S.M.; Kerber, A.; Baum, H.P.; Bahmer, F.A. Biologic effects of topical calcipotriol (MC 903) treatment in psoriatic skin. J. Am. Acad. Dermatol. 1997, 36, 19-28. [CrossRef]

15. Haussler, M.R.; Haussler, C.A.; Bartik, L.; Whitfield, G.K.; Hsieh, J.C.; Slater, S.; Jurutka, P.W. Vitamin D receptor: Molecular signaling and actions of nutritional ligands in disease prevention. Nutr. Rev. 2008, 66, S98-S112. [CrossRef] [PubMed]

16. Vaughn, A.R.; Branum, A.; Sivamani, R.K. Effects of turmeric (Curcuma longa) on skin health: A systematic review of the clinical evidence. Phytother. Res. 2016, 30, 1243-1264. [CrossRef] [PubMed]

17. Calder, P.C. Omega-3 fatty acids and inflammatory processes: From molecules to man. Biochem. Soc. Trans. 2017, 45, 1105-1115. [CrossRef] [PubMed]

18. Xu, J.; Duan, X.; Hu, F.; Poorun, D.; Liu, X.; Wang, X.; Zhang, S.; Gan, L.; He, M.; Zhu, K.; et al. Resolvin D1 attenuates imiquimod-induced mice psoriasiform dermatitis through mapks and nf-kappab pathways. J. Dermatol. Sci. 2017. [CrossRef]

19. Mayser, P.; Mrowietz, U.; Arenberger, P.; Bartak, P.; Buchvald, J.; Christophers, E.; Jablonska, S.; Salmhofer, W.; Schill, W.B.; Kramer, H.J.; et al. Omega-3 fatty acid-based lipid infusion in patients with chronic plaque psoriasis: Results of a double-blind, randomized, placebo-controlled, multicenter trial. J. Am. Acad. Dermatol. 1998, 38, 539-547. [CrossRef]

20. Rahman, M.; Beg, S.; Ahmad, M.Z.; Kazmi, I.; Akhter, S.; Ahmed, A. Omega-3 fatty acids as pharmacotherapeutics in psoriasis: Current status and future of nanomedicine in its effective delivery. Curr. Drug Targets 2013, 14, 708-722. [CrossRef] [PubMed]

21. Thangapazham, R.L.; Sharma, A.; Maheshwari, R.K. Beneficial role of curcumin in skin diseases. Adv. Exp. Med. Biol. 2007, 595, 343-357. [PubMed]

22. Kurd, S.K.; Smith, N.; VanVoorhees, A.; Troxel, A.B.; Badmaev, V.; Seykora, J.T.; Gelfand, J.M. Oral curcumin in the treatment of moderate to severe psoriasis vulgaris: A prospective clinical trial. J. Am. Acad. Dermatol. 2008, 58, 625-631. [CrossRef] [PubMed] 
23. Zenz, R.; Eferl, R.; Kenner, L.; Florin, L.; Hummerich, L.; Mehic, D.; Scheuch, H.; Angel, P.; Tschachler, E.; Wagner, E.F. Psoriasis-like skin disease and arthritis caused by inducible epidermal deletion of Jun proteins. Nature 2005, 437, 369-375. [CrossRef] [PubMed]

24. Florin, L.; Knebel, J.; Zigrino, P.; Vonderstrass, B.; Mauch, C.; Schorpp-Kistner, M.; Szabowski, A.; Angel, P. Delayed wound healing and epidermal hyperproliferation in mice lacking JunB in the skin. J. Investig. Dermatol. 2006, 126, 902-911. [CrossRef] [PubMed]

25. Kaneko, I.; Saini, R.K.; Griffin, K.P.; Whitfield, G.K.; Haussler, M.R.; Jurutka, P.W. FGF23 gene regulation by 1,25-dihydroxyvitamin D: Opposing effects in adipocytes and osteocytes. J. Endocrinol. 2015, 226, 155-166. [CrossRef] [PubMed]

26. Kuenzli, S.; Saurat, J.H. Peroxisome proliferator-activated receptors in cutaneous biology. Br. J. Dermatol. 2003, 149, 229-236. [CrossRef] [PubMed]

27. Niehues, H.; van Vlijmen-Willems, I.M.; Bergboer, J.G.; Kersten, F.F.; Narita, M.; Hendriks, W.J.; van den Bogaard, E.H.; Zeeuwen, P.L.; Schalkwijk, J. Late cornified envelope (LCE) proteins: Distinct expression patterns of LCE2 and LCE3 members suggest nonredundant roles in human epidermis and other epithelia. Br. J. Dermatol. 2016, 174, 795-802. [CrossRef] [PubMed]

28. Syed, D.N.; Lall, R.K.; Chamcheu, J.C.; Haidar, O.H.M. Involvement of ER stress and activation of apoptotic pathways in fisetin induced cytotoxicity in human melanoma. Arch. Biochem. Biophys. 2014, 563, 108-117. [CrossRef] [PubMed]

29. Huang, W.; Hennrick, K.; Drew, S. A colorful future of quantitative pathology: Validation of Vectra technology using chromogenic multiplexed immunohistochemistry and prostate tissue microarrays. Hum. Pathol. 2013, 44, 29-38. [CrossRef] [PubMed]

30. Chamcheu, J.C.; Adhami, V.M.; Esnault, S.; Sechi, M.; Siddiqui, I.A.; Satyshur, K.A.; Syed, D.N.; Dodwad, S.M.; Chaves-Rodriquez, M.I.; Longley, B.J.; et al. Dual inhibition of PI3K/AKT and mTOR by the dietary antioxidant, delphinidin, ameliorates psoriatic features in vitro and in an imiquimod-induced psoriasis-like disease in mice. Antioxid. Redox Signal 2017, 26, 49-69. [CrossRef] [PubMed]

31. De Cid, R.; Riveira-Munoz, E.; Zeeuwen, P.L.; Robarge, J.; Liao, W.; Dannhauser, E.N.; Giardina, E.; Stuart, P.E.; Nair, R.; Helms, C.; et al. Deletion of the late cornified envelope LCE3B and LCE3C genes as a susceptibility factor for psoriasis. Nat. Genet. 2009, 41, 211-215. [CrossRef] [PubMed]

32. Jurutka, P.W.; Remus, L.S.; Whitfield, G.K.; Thompson, P.D.; Hsieh, J.C.; Zitzer, H.; Tavakkoli, P.; Galligan, M.A.; Dang, H.T.; Haussler, C.A.; et al. The polymorphic N terminus in human vitamin D receptor isoforms influences transcriptional activity by modulating interaction with transcription factor IIB. Mol. Endocrinol. 2000, 14, 401-420. [CrossRef] [PubMed]

33. Speir, M.L.; Zweig, A.S.; Rosenbloom, K.R.; Raney, B.J.; Paten, B.; Nejad, P.; Lee, B.T.; Learned, K.; Karolchik, D.; Hinrichs, A.S.; et al. The UCSC genome browser database: 2016 update. Nucleic Acids Res. 2016, 44, D717-D725. [CrossRef] [PubMed]

34. Untergasser, A.; Nijveen, H.; Rao, X.; Bisseling, T.; Geurts, R.; Leunissen, J.A. Primer3plus, an enhanced web interface to primer3. Nucleic Acids Res. 2007, 35, W71-W74. [CrossRef] [PubMed]

35. Misund, K.; Selvik, L.K.; Rao, S.; Norsett, K.; Bakke, I.; Sandvik, A.K.; Laegreid, A.; Bruland, T.; Prestvik, W.S.; Thommesen, L. Nr4a2 is regulated by gastrin and influences cellular responses of gastric adenocarcinoma cells. PLoS ONE 2013, 8, e76234. [CrossRef] [PubMed]

36. Johansen, C.; Kragballe, K.; Westergaard, M.; Henningsen, J.; Kristiansen, K.; Iversen, L. The mitogen-activated protein kinases P38 and ERK1/2 are increased in lesional psoriatic skin. Br. J. Dermatol. 2005, 152, $37-42$. [CrossRef] [PubMed]

37. Ettehadi, P.; Greaves, M.W.; Wallach, D.; Aderka, D.; Camp, R.D. Elevated tumour necrosis factor-alpha (TNF- $\alpha$ ) biological activity in psoriatic skin lesions. Clin. Exp. Immunol. 1994, 96, 146-151. [CrossRef] [PubMed]

38. Bartik, L.; Whitfield, G.K.; Kaczmarska, M.; Lowmiller, C.L.; Moffet, E.W.; Furmick, J.K.; Hernandez, Z.; Haussler, C.A.; Haussler, M.R.; Jurutka, P.W. Curcumin: A novel nutritionally derived ligand of the vitamin D receptor with implications for colon cancer chemoprevention. J. Nutr. Biochem. 2010, 21, 1153-1161. [CrossRef] [PubMed] 
39. Chamcheu, J.C.; Chaves-Rodriquez, M.I.; Adhami, V.M.; Siddiqui, I.A.; Wood, G.S.; Longley, B.J.; Mukhtar, H. Upregulation of PI3K/AKT/MTOR, FABP5 and PPARBETA/DELTA in human psoriasis and imiquimod-induced murine psoriasiform dermatitis model. Acta Derm. Venereol. 2016, 96, 854-856. [PubMed]

40. Ham, S.A.; Hwang, J.S.; Yoo, T.; Lee, H.; Kang, E.S.; Park, C.; Oh, J.W.; Lee, H.T.; Min, G.; Kim, J.H.; et al. Ligand-activated PPAR $\delta$ inhibits UVB-induced senescence of human keratinocytes via PTEN-mediated inhibition of superoxide production. Biochem. J. 2012, 444, 27-38. [CrossRef] [PubMed]

41. Van de Kerkhof, P.C. An update on topical therapies for mild-moderate psoriasis. Derm. Clin. 2015, 33, 73-77. [CrossRef] [PubMed]

42. Barrea, L.; Macchia, P.E.; Tarantino, G.; Di Somma, C.; Pane, E.; Balato, N.; Napolitano, M.; Colao, A.; Savastano, S. Nutrition: A key environmental dietary factor in clinical severity and cardio-metabolic risk in psoriatic male patients evaluated by 7-day food-frequency questionnaire. J. Transl. Med. 2015, 13, 303. [CrossRef] [PubMed]

43. Guida, B.; Napoleone, A.; Trio, R.; Nastasi, A.; Balato, N.; Laccetti, R.; Cataldi, M. Energy-restricted, $n-3$ polyunsaturated fatty acids-rich diet improves the clinical response to immuno-modulating drugs in obese patients with plaque-type psoriasis: A randomized control clinical trial. Clin. Nutr. 2014, 33, 399-405. [CrossRef] [PubMed]

44. Barrea, L.; Balato, N.; Di Somma, C.; Macchia, P.E.; Napolitano, M.; Savanelli, M.C.; Esposito, K.; Colao, A.; Savastano, S. Nutrition and psoriasis: Is there any association between the severity of the disease and adherence to the Mediterranean diet? J. Transl. Med. 2015, 13, 18. [CrossRef] [PubMed]

45. Lin, Y.L.; Pavlidis, P.; Karakoc, E.; Ajay, J.; Gokcumen, O. The evolution and functional impact of human deletion variants shared with archaic hominin genomes. Mol. Biol. Evol. 2015, 32, 1008-1019. [CrossRef] [PubMed]

46. Pajic, P.; Lin, Y.L.; Xu, D.; Gokcumen, O. The psoriasis-associated deletion of late cornified envelope genes $L C E 3 B$ and $L C E 3 C$ has been maintained under balancing selection since human denisovan divergence. BMC Evol. Biol. 2016, 16, 265. [CrossRef] [PubMed]

47. Bassaganyas, L.; Riveira-Munoz, E.; Garcia-Aragones, M.; Gonzalez, J.R.; Caceres, M.; Armengol, L.; Estivill, X. Worldwide population distribution of the common LCE3C-LCE3B deletion associated with psoriasis and other autoimmune disorders. BMC Genom. 2013, 14, 261. [CrossRef] [PubMed]

48. Bergboer, J.G.; Zeeuwen, P.L.; Schalkwijk, J. Genetics of psoriasis: Evidence for epistatic interaction between skin barrier abnormalities and immune deviation. J. Investig. Dermatol. 2012, 132, 2320-2331. [CrossRef] [PubMed]

49. Nakanishi, A.; Tsukamoto, I. n-3 polyunsaturated fatty acids stimulate osteoclastogenesis through PPAR $\gamma$-mediated enhancement of c-Fos expression, and suppress osteoclastogenesis through PPAR $\gamma$-dependent inhibition of NFKB activation. J. Nutr. Biochem. 2015, 26, 1317-1327. [CrossRef] [PubMed]

50. Victor, F.C.; Gottlieb, A.B. TNF-alpha and apoptosis: Implications for the pathogenesis and treatment of psoriasis. J. Drugs Dermatol. 2002, 1, 264-275. [PubMed]

51. Menter, A.; Gordon, K.B.; Leonardi, C.L.; Gu, Y.; Goldblum, O.M. Efficacy and safety of adalimumab across subgroups of patients with moderate to severe psoriasis. J. Am. Acad. Dermatol. 2010, 63, 448-456. [CrossRef] [PubMed]

52. Arthur, J.S.; Darragh, J. Signaling downstream of p38 in psoriasis. J. Investig. Dermatol. 2006, 126, 1689-1691. [CrossRef] [PubMed]

53. Varma, S.R.; Sivaprakasam, T.O.; Mishra, A.; Prabhu, S.M.R.P.R. Imiquimod-induced psoriasis-like inflammation in differentiated human keratinocytes: Its evaluation using curcumin. Eur. J. Pharmacol. 2017, 813, 33-41. [CrossRef] [PubMed]

54. Yamagata, K.; Suzuki, S.; Tagami, M. Docosahexaenoic acid prevented tumor necrosis factor alpha-induced endothelial dysfunction and senescence. Prostaglandin. Leukot Essent Fatty Acids 2016, 104, 11-18. [CrossRef] [PubMed]

55. Bernardo, A.; Giammarco, M.L.; De Nuccio, C.; Ajmone-Cat, M.A.; Visentin, S.; De Simone, R.; Minghetti, L. Docosahexaenoic acid promotes oligodendrocyte differentiation via PPAR- $\gamma$ signalling and prevents tumor necrosis factor-alpha-dependent maturational arrest. Biochim. Biophys. Acta 2017, 1862, 1013-1023. [CrossRef] [PubMed] 
56. Kim, B.E.; Howell, M.D.; Guttman-Yassky, E.; Gilleaudeau, P.M.; Cardinale, I.R.; Boguniewicz, M.; Krueger, J.G.; Leung, D.Y. TNF- $\alpha$ downregulates filaggrin and loricrin through c-Jun N-terminal kinase: Role for TNF- $\alpha$ antagonists to improve skin barrier. J. Investig. Dermatol. 2011, 131, 1272-1279. [CrossRef] [PubMed]

57. O'Kane, M.; Markham, T.; McEvoy, A.N.; Fearon, U.; Veale, D.J.; FitzGerald, O.; Kirby, B.; Murphy, E.P. Increased expression of the orphan nuclear receptor NURR1 in psoriasis and modulation following TNF- $\alpha$ inhibition. J. Investig. Dermatol. 2008, 128, 300-310. [CrossRef] [PubMed]

58. Raveney, B.J.; Oki, S.; Yamamura, T. Nuclear receptor NR4A2 orchestrates Th17 cell-mediated autoimmune inflammation via IL-21 signalling. PLoS ONE 2013, 8, e56595. [CrossRef] [PubMed]

59. Deng, Y.; Chang, C.; Lu, Q. The inflammatory response in psoriasis: A comprehensive review. Clin. Rev. Allergy Immunol. 2016, 50, 377-389. [CrossRef] [PubMed]

60. Moriwaki, Y.; Takada, K.; Tsuji, S.; Kawashima, K.; Misawa, H. Transcriptional regulation of SLURP2, a psoriasis-associated gene, is under control of IL-22 in the skin: A special reference to the nested gene LYNX1. Int. Immunopharmacol. 2015, 29, 71-75. [CrossRef] [PubMed]

61. Lu, Y.; Tian, H.; Hong, S. Novel 14,21-dihydroxy-docosahexaenoic acids: Structures, formation pathways, and enhancement of wound healing. J. Lipid Res. 2010, 51, 923-932. [CrossRef] [PubMed]

(C) 2018 by the authors. Licensee MDPI, Basel, Switzerland. This article is an open access article distributed under the terms and conditions of the Creative Commons Attribution (CC BY) license (http:/ / creativecommons.org/licenses/by/4.0/). 
Article

\title{
Elucidating the Skin Delivery of Aglycone and Glycoside Flavonoids: How the Structures Affect Cutaneous Absorption
}

\author{
Shih-Yi Chuang ${ }^{1,+}$, Yin-Ku Lin ${ }^{2,3,+}$, Chwan-Fwu Lin ${ }^{1,4}$, Pei-Wen Wang ${ }^{5}$, En-Li Chen ${ }^{6}$ \\ and Jia-You Fang 1,6,7,8,* \\ 1 Research Center for Food and Cosmetic Safety and Research Center for Chinese Herbal Medicine, \\ Chang Gung University of Science and Technology, Kweishan, Taoyuan 333, Taiwan; \\ clemencechuang@gmail.com (S.-Y.C.); cflin@mail.cgust.edu.tw (C.-F.L.) \\ 2 Department of Traditional Chinese Medicine, Chang Gung Memorial Hospital, Keelung 204, Taiwan; \\ lin1266@cgmh.org.tw \\ 3 School of Traditional Chinese Medicine, Chang Gung University, Kweishan, Taoyuan 333, Taiwan \\ 4 Department of Cosmetic Science, Chang Gung University of Science and Technology, Kweishan, \\ Taoyuan 333, Taiwan \\ 5 Department of Medical Research, China Medical University Hospital, China Medical University, \\ Taichung 404, Taiwan; pwwang@hotmail.com \\ 6 Pharmaceutics Laboratory, Graduate Institute of Natural Products, Chang Gung University, Kweishan, \\ Taoyuan 333, Taiwan; fajy5521@gmail.com \\ 7 Chinese Herbal Medicine Research Team, Healthy Aging Research Center, Chang Gung University, \\ Kweishan, Taoyuan 333, Taiwan \\ 8 Department of Anesthesiology, Chang Gung Memorial Hospital, Kweishan, Taoyuan 333, Taiwan \\ * Correspondence: fajy@mail.cgu.edu.tw; Tel.: +886-3-211-8800; Fax: +886-3-211-8236 \\ $\dagger$ These authors contributed equally to this work.
}

Received: 1 November 2017; Accepted: 28 November 2017; Published: 30 November 2017

\begin{abstract}
Flavonoids are bioactive phytochemicals that exhibit protective potential against cutaneous inflammation and photoaging. We selected eight flavonoid aglycones or glycosides to elucidate the chemistry behind their skin absorption capability through experimental and computational approaches. The skin delivery was conducted using nude mouse and pig skins mounted on an in vitro Franz cell assembly. The anti-inflammatory activity was examined using the $\mathrm{O}_{2}{ }^{\bullet-}$ and elastase inhibition in activated human neutrophils. In the equivalent dose $(6 \mathrm{mM})$ application on nude mouse skin, the skin deposition of naringenin and kaempferol was 0.37 and $0.11 \mathrm{nM} / \mathrm{mg}$, respectively, which was higher than that of the other flavonoids. Both penetrants were beneficial for targeted cutaneous therapy due to their minimal diffusion across the skin. The absorption was generally greater for topically applied aglycones than glycosides. Although naringenin could be classified as a hydrophilic flavonoid, the flexibility of the chiral center in the $\mathrm{C}$ ring of this flavanone could lead to better skin transport than the flavonols and flavones with a planar structure. An optimized hydrophilic and lipophilic balance of the flavonoid structure was important for governing the cutaneous delivery. The hydrogen bond acceptor and stratum corneum lipid docking estimated by molecular modeling showed some relationships with the skin deposition. The interaction with cholesteryl sulfate could be a factor for predicting the cutaneous absorption of aglycone flavonoids (correlation coefficient $=0.97$ ). Baicalin $(3 \mu \mathrm{M})$ showed the highest activity against oxidative burst with an $\mathrm{O}_{2}{ }^{- \text {- }}$ inhibition percentage of $77 \%$. Although naringenin displayed an inhibition efficiency of only $20 \%$, this compound still demonstrated an impressive therapeutic index because of the high absorption. Our data are advantageous to providing the information on the structure-permeation relationship for topically applied flavonoids.
\end{abstract}


Keywords: flavonoid; cutaneous absorption; structure-permeation relationship; anti-inflammation; molecular modeling

\section{Introduction}

Strong evidence links oxidative stress with several pathologies [1]. The skin, the largest organ in the body, is a main target of oxidative stress because of its size [2]. Reactive oxygen species (ROS) play a key role in some cutaneous diseases, including photoaging, psoriasis, dermatitis, acne, rosacea, alopecia, and skin cancer [3]. Flavonoids are antioxidants ubiquitously found in vegetables and fruits. The structure of flavonoids is associated with the derivatives of chalcone. They can prevent or treat cutaneous inflammation and malignancy by maintaining the skin's homeostasis [4]. Some flavonoids, such as naringenin and quercetin, have been reported to exhibit anti-aging activity that slows down the skin's natural senescence [5]. Flavonoids have been largely used in dermatology and cosmeceuticals in the form of crude extracts.

Topical application provides an efficient method for facilitating the local action of drugs on the skin [6]. For an active ingredient to exhibit bioactivity, its delivery into the targeted skin must be successful. It is evidenced that the absorption rate and scavenging activity of some flavonoids depend on their chemical structure [7]. The exploration of the structure-permeation relationship (SPR) offers insights into the understanding of how the physicochemical properties of chemicals influence cutaneous absorption or targeting. The establishment of SPR is useful for predicting the skin delivery of the compounds and for facilitating the development of new topically applied actives. In addition to the aglycone form, flavonoids are present in the form of glycoside in nature. Some aglycones and glycosides, such as quercetin/rutin and baicalein/baicalin, are paired. Glycosides also show potent bioactivity in many cases. For example, baicalin is proven to protect the skin from UV radiation, burning, and aging [8]. Although many reports have dealt with the biological effect of glycoside flavonoids, investigation of how the sugar moiety affects cutaneous absorption is still lacking. In a continuing attempt to elucidate SPR, the objective of this study was to evaluate the physicochemical characteristics and the skin absorption of a series of aglycone flavonoids and their corresponding glycosides. These included myricetin, naringenin, quercetin, baicalein, kaempferol, naringin, rutin, and baicalin (Figure 1).

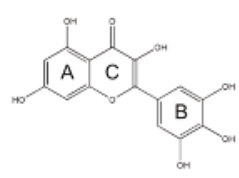

Myricetin

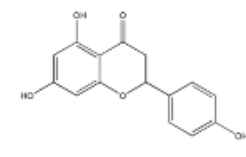

Naringenin

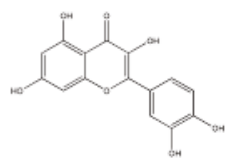

Quercetin

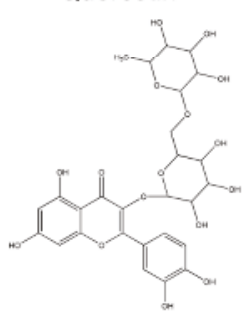

Rutin

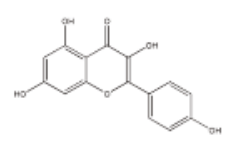

Kaempferol

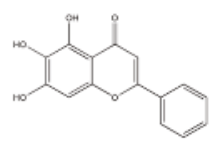

Baicalein

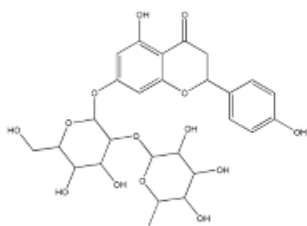

Naringin

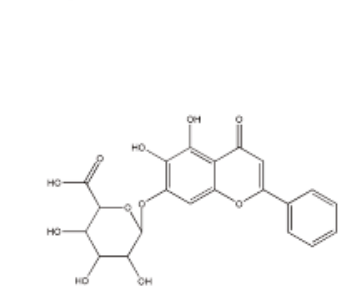

Baicalin

Figure 1. The chemical structures of flavonoids tested in this study. 
In the present study, the in vitro Franz cell model was utilized to assess the skin absorption of flavonoids. Both nude mouse skin and baby pig skin were employed as the permeation barriers. In inspecting the permeation behavior of a penetrant, it is important to identify the possible transport pathways [9]. We examined flavonoid permeation via the skin after removal of the stratum corneum (SC), lipid, sebum, and protein in order to understand the delivery routes. Data collected for the capacity factor $\left(\log K^{\prime}\right)$, the partition coefficient $(\log P)$, and the aqueous solubility allowed us to distinguish the relative contribution to cutaneous absorption. The anti-inflammatory activity of these compounds was determined by the capacity of inhibiting superoxide anion $\left(\mathrm{O}_{2}{ }^{-}\right)$and elastase of formyl-methionyl-leucyl phenylalanine (fMLF)-activated human neutrophils. A wealth of information on the hydrogen bond acceptor, the hydrogen bond donor, the total polarity surface, and the molecular volume was obtained by the computation of molecular modeling. We also investigated the predicted interaction between flavonoids and SC lipid components to explain the skin permeation trend. The present study sheds light on the effect of the flavonoid structure on skin absorption and targeting, broadening the comprehension of SPR in natural antioxidants.

\section{Materials and Methods}

\subsection{Materials}

All flavonoids tested in this study were purchased from Sigma-Aldrich (St. Louis, MO, USA). All other chemicals and solvents were of reagent grade without further purification.

\subsection{Capacity Factor $\left(\log K^{\prime}\right)$}

The HPLC setup for the calculation of $\log K^{\prime}$ was an HPLC system (7-series, Hitachi, Tokyo, Japan) with a LiChrospher ${ }^{\circledR} \mathrm{C} 18$ column $(200 \times 4.6 \mathrm{~mm}$, Merck, Darmstadt, Germany). The mobile phase consisted of a mixture of methanol and $\mathrm{pH} 2$ phosphate buffer solution (1:1). The flow rate and wavelength for determination were $1 \mathrm{~mL} / \mathrm{min}$ (L-7110 pump) and $256 \mathrm{~nm}$ (L-7455 diode array detector), respectively. The retention times of flavonoids were detected, and the $\log K^{\prime}$ was computed as $\log \left[\left(t_{r}-t_{0}\right) / t_{0}\right]$, where $t_{r}$ and $t_{0}$ were the retention time of the flavonoid and the nonretained solvent peak, respectively.

\subsection{Partition Coefficient $(\log P)$}

Flavonoids in methanol $(0.5 \mathrm{mg} / \mathrm{mL})$ were pipetted into a tube at a volume of $1 \mathrm{~mL}$. Methanol was then evaporated under vacuum. In the amount of $1 \mathrm{~mL}$ of each, $n$-Octanol and water were incorporated into the tube. After being shaken for $24 \mathrm{~h}$ at $37^{\circ} \mathrm{C}$, the tube was centrifugated at $10,000 \times \mathrm{g}$ for $10 \mathrm{~min}$. The flavonoid content in both phases was analyzed by HPLC. The $\log P$ was calculated as $\log$ (flavonoid content in $n$-octanol/flavonoid content in water).

\subsection{Saturated Solubility in $20 \%$ PEG400 Aqueous Solution}

The saturated solubility of flavonoids in 20\% PEG400/pH 7.4 buffer was measured by loading $20 \mathrm{mM}$ compounds in the vehicle and shaking them at $37^{\circ} \mathrm{C}$ for $2 \mathrm{~h}$. The suspension was then centrifuged at $10,000 \times \mathrm{g}$ for $10 \mathrm{~min}$. The supernatant was filtered across the polyvinylidene fluoride membrane with a pore size of $0.45 \mu \mathrm{m}$. The resulting supernatant was analyzed by HPLC to record the solubility $(\mathrm{mM})$.

\subsection{Animals}

Eight-week-old female nude mice were provided by the National Laboratory Animal Center (Taipei, Taiwan). One-week-old specific-pathogen-free pigs were obtained from the Animal Technology Institute Taiwan (Miaoli, Taiwan). All animals were treated in strict accordance with the recommendations set forth in the Guidelines for the Institutional Animal Care and Use Committee of Chang Gung University (CGU15-083). 


\subsection{Preparation of SC-Disrupted Skin}

The full-thickness dorsal skin of the nude mouse was excised after sacrifice. The SC-stripped skin was obtained by stripping the skin surface 20 times with adhesive tape $\left(\operatorname{Scotch}^{\circledR}, 3 \mathrm{M}\right.$, Maplewood, MN, USA). The delipidized skin was achieved by incubating the skin surface onto a chloroform/methanol solution (2:1) for $2 \mathrm{~h}$ [10]. To prepare the desebumed skin, the SC side of the skin was washed with cold hexane $\left(4^{\circ} \mathrm{C}\right)$ five times, based on the procedure of the previous study [11]. The skin's surface was then treated with a $40 \%$ ethanol/water solution for $2 \mathrm{~h}$, a process that successfully denatured the skin's proteins.

\subsection{In Vitro Franz Cell Assembly}

The cutaneous absorption of flavonoids was assessed using a Franz diffusion cell system. The excised animal skin or cellulose membrane was mounted between the donor and receptor compartments with the SC facing up toward the donor. The receptor was filled with 30\% ethanol/pH 7.4 buffer and placed on a magnetic stirring plate. The effective permeation area of the cell was $0.785 \mathrm{~cm}^{2}$. The stirring rate and temperature of the receptor were $600 \mathrm{rpm}$ and $37{ }^{\circ} \mathrm{C}$, respectively. Saturated solution or a $6 \mathrm{mM}$ flavonoid suspension was prepared in 20\% PEG400/pH 7.4 buffer to be loaded into the donor compartment. A 300- $\mu \mathrm{L}$ aliquot in the receptor was collected at determined durations, followed by an immediate replacement with fresh receptor medium. The amount of flavonoid in the receptor was determined by HPLC. The skin was removed from the cell after a 24-h application. After being washed with water, the skin sample was weighed and positioned in a vial with $1 \mathrm{~mL}$ methanol. MagNA Lyser (Roche) was used to homogenize the skin. The homogenate was centrifuged at $10,000 \times g$ for $10 \mathrm{~min}$. The supernatant was analyzed by HPLC to quantify the flavonoid deposition in the skin reservoir.

\subsection{Inhibition of Neutrophilic Inflammation by Flavonoids}

The protocol was approved by the Institutional Review Board at Chang Gung Memorial Hospital, and written informed consent was obtained from all volunteers (201600500B0C101). Whole blood was withdrawn from healthy volunteers between 20 and 30 years of age. Human neutrophils were isolated using a typical method of dextran sedimentation prior to centrifugation in a Ficoll-Hypaque gradient and hypotonic lysis of erythrocytes [12]. The granulocyte layer was harvested and suspended in calcium-free HBSS at $\mathrm{pH} 7.4$, which was maintained at $4{ }^{\circ} \mathrm{C}$ until use.

The reduction of ferricytochrome $c$ was utilized for measuring the superoxide anion $\left(\mathrm{O}_{2}{ }^{\bullet-}\right)$ release from the neutrophils [13]. The human neutrophils $\left(6 \times 10^{5}\right.$ cells $\left./ \mathrm{mL}\right)$ were incubated with $0.5 \mathrm{mg} / \mathrm{mL}$ ferricytochrome $c$ and $1 \mathrm{mM} \mathrm{CaCl}_{2}$ at $37^{\circ} \mathrm{C}$, and were then treated with flavonoids $(3 \mu \mathrm{M})$ in DMSO for $10 \mathrm{~min}$. Neutrophils were stimulated by adding $0.1 \mu \mathrm{M}$ fMLF with cytochalasin B $(1 \mu \mathrm{g} / \mathrm{mL})$. The reduction of ferricytochrome $c$ was monitored by the absorbance at $550 \mathrm{~nm}$ using a U3010 ultraviolet/visible spectrophotometer (Hitachi, Tokyo, Japan). The human neutrophils were equilibrated with an elastase substrate (MeO-Suc-Ala-Ala-Pro-Val-p-nitroanilide, $100 \mu \mathrm{M}$ ) at $37^{\circ} \mathrm{C}$ for $2 \mathrm{~min}$. Flavonoids at a concentration of $3 \mu \mathrm{M}$ were added into the neutrophil suspension for $10 \mathrm{~min}$. The cells were activated by fMLF and cytochalasin B for a further $10 \mathrm{~min}$. Elastase release was detected by measuring the change of absorbance at $405 \mathrm{~nm}$.

\subsection{Molecular Modeling}

The structures of the flavonoids were sketched using Discovery Studio ${ }^{\circledR}$ version 4.1 workstation (Accelrys, San Diego, CA, USA). The hydrogen bond acceptor or donor number, total polarity surface, and molecular volume of the flavonoids were estimated. The superimposition of flavonoids with ceramides, palmitic acid, cholesteryl sulfate, and cholesterol was computed to observe the conformation and ligand-binding activity. The negative CDOCKER energy was calculated after conducting the molecular docking simulation of the flavonoids with these SC lipids. 


\subsection{In Vivo Cutaneous Tolerance of Flavonoids}

The $20 \%$ PEG400/pH 7.4 buffer loaded with flavonoids at $6 \mathrm{mM}$ was topically applied daily $(0.6 \mathrm{~mL})$ on the nude mouse back for 7 days. The flavonoid-containing vehicle was replaced with a new one each day. After removal of the vehicle, the treated skin region was evaluated by Tewameter TM300 (Courage and Khazaka, Köln, Germany) to determine the transepidermal water loss (TEWL). Stained by hematoxylin and eosin (H\&E), the treated skin was excised for histological examination after a 7-day flavonoid application.

\subsection{Data Analysis}

In the cutaneous absorption experiment, flavonoid deposition in the skin was estimated as the molar amount per mg of skin (nM/mg). In the case of the skin deposition from the saturated solution, the calibrated skin deposition (CSD) was measured as flavonoid deposition divided by the applied dose (saturated solubility). The flux $\left(\mathrm{nM} / \mathrm{cm}^{2} / \mathrm{h}\right)$ was estimated by the slope of the penetrated amount-time curve. The permeability coefficient (PC) was calculated from the flux divided by the saturated solubility of the flavonoids. The dermal/transdermal selectivity index ( $S$ value) was calculated using an equation of skin deposition/flux (for equivalent dose application) or CSD/PC (for saturated dose application).

The data in the present study were presented as mean and standard deviation (S.D.). The statistical difference in the data of different experimental groups was evaluated using the Kruskal-Wallis test. The post hoc test for checking individual differences was Dunn's test. A 0.05 level of probability was taken as the statistical significance. The software used for statistical comparison was WINKS (Texasoft, Cedar Hill, TX, USA).

\section{Results}

\subsection{Physicochemical Properties of Flavonoids}

To understand the relationship between skin permeation and physicochemical properties, the molecular weight (MW), lipophilicity $\left(\log K^{\prime}\right.$ and $\log P$ ), and aqueous solubility of flavonoids were considered as presented in Table 1. Figure 1 demonstrates that the aglycone flavonoids tested in this study have different hydroxyl groups from 3 to 6 , with myricetin showing the most abundant hydroxyl moieties and the highest MW. The structures of most aglycones belong to flavonols, with the exception of the flavanone naringenin and flavone baicalein. Naringin, rutin, and baicalin are the glycosides with sugar residue in the structures of naringenin, quercetin, and baicalein, respectively. The glycosides had a higher MW than their corresponding aglycones. Both $\log K^{\prime}$ and $\log P$ are the indicators of lipophilicity. The capacity factor was increased following the decrease of hydroxyl moieties for flavonols and flavones. The trend of $\log P$ correlated well with that of $\log K^{\prime}$. Although kaempferol exhibited a higher partition coefficient compared to baicalein (3.71 versus 3.59 ), no statistically significant difference was found $(p>0.05)$. Naringenin, a flavanone, revealed a lower lipophilicity than baicalein, though both compounds possessed the same hydroxyl group number (3). The lipophilicity of glycosides was less than that of aglycones because of the presence of sugar. As expected, the compounds with lower lipophilicity displayed higher solubility in PEG400/pH 7.4 buffer solution. The flavanone showed much higher solubility compared to the flavonols and flavones. Naringenin was 470-fold more soluble than baicalein. The same result was achieved with naringin, which showed an aqueous solubility of $>20 \mathrm{mM}$. 
Table 1. Physicochemical properties of aglycone and glycoside flavonoids.

\begin{tabular}{cccccc}
\hline Category & Compound & MW (Da) & $\log \boldsymbol{K}^{\prime}$ & $\log \boldsymbol{P}$ & Solubility in 20\% PEG400 (mM) \\
\hline \multirow{5}{*}{ Aglycone } & Myricetin & 318.24 & 0.34 & $3.18 \pm 0.07$ & $0.53 \pm 0.01$ \\
& Naringenin & 272.25 & 0.51 & $3.21 \pm 0.04$ & $3.62 \pm 0.28$ \\
& Quercetin & 302.24 & 0.51 & $3.32 \pm 0.09$ & $0.38 \pm 0.0025$ \\
& Kaempferol & 286.24 & 0.65 & $3.71 \pm 0.57$ & $0.19 \pm 0.0047$ \\
& Baicalein & 270.24 & 0.73 & $3.59 \pm 0.08$ & $0.0077 \pm 0.0019$ \\
\hline \multirow{5}{*}{ Glycoside } & Naringin & 580.54 & 0.19 & $0.15 \pm 0.04$ & $>20$ \\
& Rutin & 610.52 & 0.24 & $0.76 \pm 0.22$ & $1.84 \pm 0.04$ \\
& Baicalin & 446.36 & 0.42 & $0.89 \pm 0.02$ & $5.32 \pm 0.15$
\end{tabular}

MW, molecular weight; $\log P$, partition coefficient measured by $n$-octanol/water partitioning; $\log K^{\prime}$, logarithm of $\left(t_{r}-t_{0}\right) / t_{0}, t_{r}$ is the retention time of compound peak, $t_{0}$ is the retention time of solvent peak. Each value represents the mean and S.D $(n=4)$.

\subsection{Cutaneous Absorption of Flavonoids}

The cutaneous absorption of the penetrants was compared using a Franz cell assembly. Nude mouse and baby pig dorsal skins were used in this experiment due to their close similarity to human skin. The donor dose was first set at an equivalent concentration (6 mM). This dose surpassed the saturated solubility. This led to a condition of suspension in the donor except for naringin, which showed a solution type because of the extremely high solubility in the aqueous vehicle. Both flavonoid deposition within the skin and the flux across the skin were examined in this study. Dermal delivery should be addressed in the skin deposition that is carried out with the aim of cutaneous targeting with minimizing systemic absorption. Flux is a factor to use in predicting how well the penetrant will reach the systemic circulation. Figure 2A,B depicts the skin permeation profiles of flavonoids via nude mouse skin and pig skin, respectively. Both animal skins demonstrated similar trends of cutaneous absorption. Among the aglycones tested, naringenin and kaempferol showed the greatest skin deposition. Our results showed that myricetin, which had the lowest lipophilicity among the flavonoids examined, was the aglycone with the least deposition. The glycosides may be susceptible to enzymatic hydrolysis in skin tissue with aglycone release. After HPLC analysis, we found no aglycone in the skin deposition and receptor after topical application of glycosides. This indicated that the glycosides were not metabolized in this case. The skin deposition of the glycosides was minor compared to that of the corresponding aglycones.

Naringenin revealed a high cutaneous delivery not only in skin deposition but also in flux. The highest flux of aglycones was observed with naringenin, followed by baicalein. Myricetin and quercetin levels in the receptor were negligible or even below the HPLC detection limit, giving a flux of near zero. Contrary to the result with skin deposition, glycosylation generally increased the flux, except in the case of naringin diffusion across nude mouse skin. The flux of naringin and baicalin was comparable and significantly higher than that of rutin. Cutaneous targeting is a strategy for local skin prevention or therapy with reduced systemic effects. As shown in Figure 2, we calculated $S$ value as an index of selectivity between cutaneous targeting and transdermal delivery. Kaempferol demonstrated the highest $S$ value (2.7 and 1.5 for nude mouse skin and pig skin); Myricetin and quercetin were absorbed into the skin reservoir without reaching the receptor, leading to the infinity of $S$ value $(\infty)$. Rutin was the compound with the highest $S$ value (1.3 and 0.4 for nude mouse skin and pig skin, respectively) among the glycosides. 

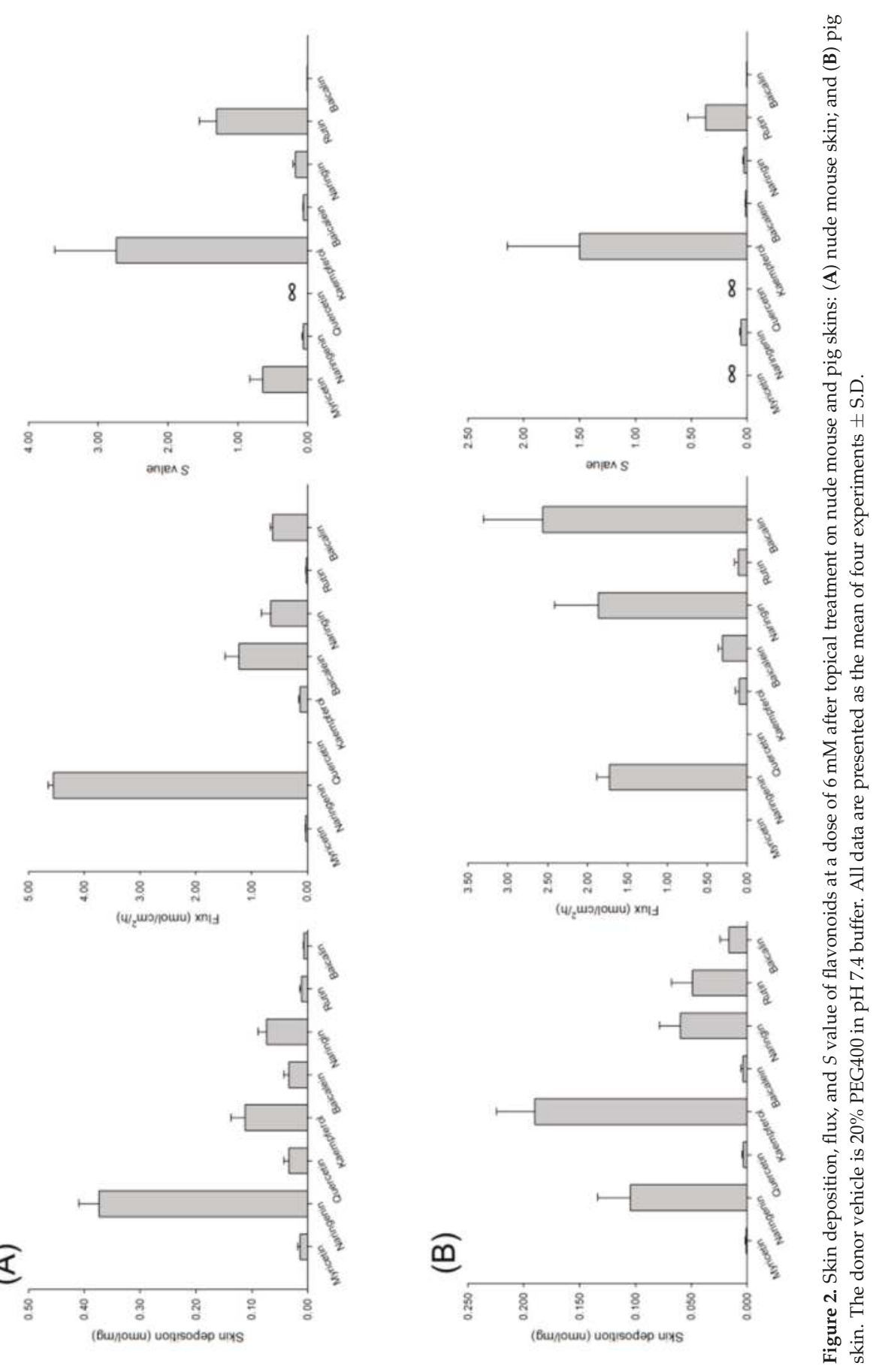
The flavonoids in the donor were also dosed with saturated solubility to achieve the maximum thermodynamic equivalents for all penetrants. Naringin was excluded in this experiment because we could not determine the saturated solubility of this compound $(>20 \mathrm{mM})$. The extremely high solubility would lead to skin damage, complicating the discussion of SPR. The trend of the cutaneous absorption profiles was similar for nude mouse skin and pig skin after topical application of saturated solution, as shown in Figure 3A,B, respectively. Equivalent doses of naringenin and kaempferol showed the highest skin deposition in saturated solubility. The CSD of baicalein was below the quantification limit, which may have been due to the negligible solubility $(0.0077 \mathrm{mM})$. Among the topically applied aglycones, only naringenin and baicalein could penetrate across the skin to the receptor. The neglected PC of myricetin, quercetin, and kaempferol led to the infinity of $S$ value. The $S$ value of baicalein was 0 . The $S$ value of baicalin was greater than that of rutin. This result was opposite to the $S$ value of glycosides vehiculated at the equivalent dose.

\subsection{Flavonoid Permeation via SC-Disrupted Skin}

The SC is the outermost layer of the skin, consisting of the main barrier of most penetrants. Effective permeation through the SC is necessary to obtain successful skin targeting. The SC-disrupted skin was prepared to explore the possible permeation pathways of flavonoids. These included SC-stripped, delipidized, desebumed, and deproteinized nude mouse skins. The penetrants at the equimolar dose $(6 \mathrm{mM})$ were used as the donor in this experiment. Figure $4 \mathrm{~A}$ demonstrates the released percentage of flavonoids that penetrated the cellulose membrane at $24 \mathrm{~h}$. The release rate was related to the penetrant escape from the vehicle. The release was a delivery stage before penetrant entrance into the SC. The glycosides showed a higher release percentage compared to the aglycones. Naringenin and kaempferol were the two penetrants with greater release than the other aglycones. Figure 4B-I summarizes the cutaneous deposition of eight flavonoids in different SC-disrupted skins. The deposition in SC-stripped skin and delipidized skin was comparable for all flavonoids tested, indicating that intercellular lipid bilayers are an important route for flavonoid transport. The removal of the SC could evoke skin deposition of myricetin and quercetin by $>30$ fold compared to the intact skin. Myricetin deposition in desebumed skin was 140-fold higher than that in untreated skin. The other flavonoids showed a 2-6-fold increase in skin deposition after sebum removal. The enhancement of skin deposition by protein denaturation was lower than that by lipid removal. The deproteinization process even failed to change the skin deposition of quercetin and its corresponding glycoside. The result of protein removal suggests the minor role of the intracellular route compared to the intercellular route for flavonoid diffusion across the SC. 

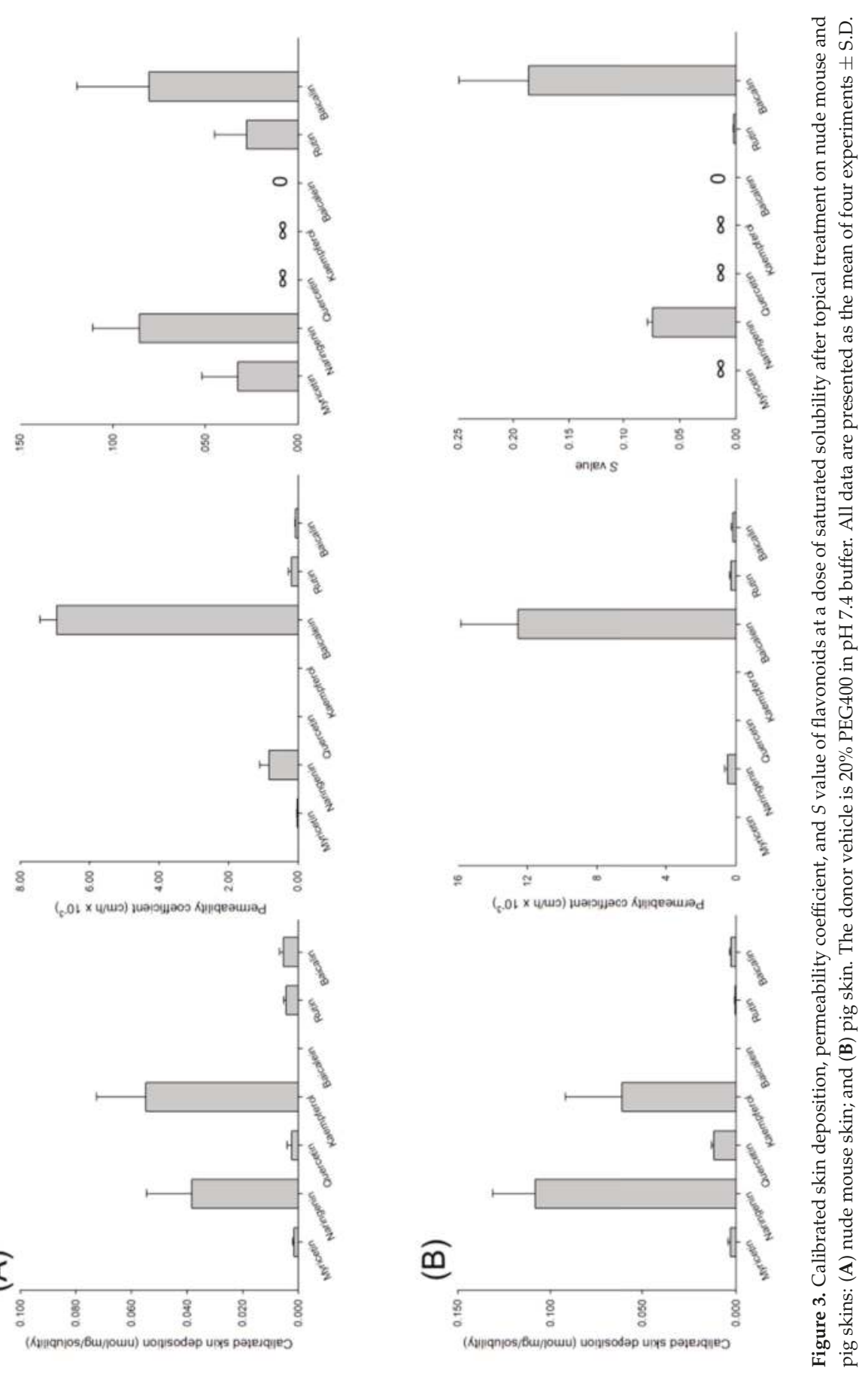

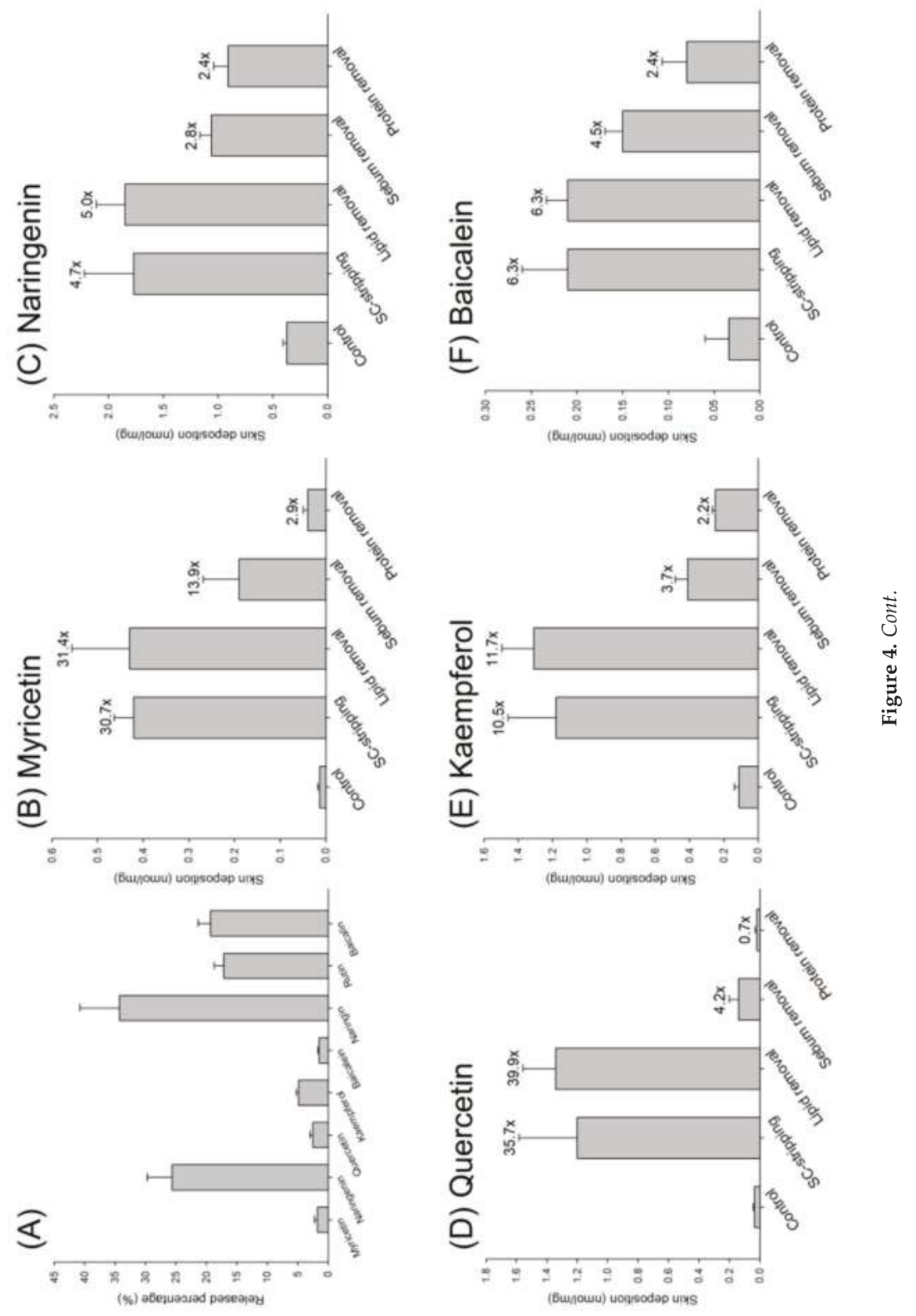


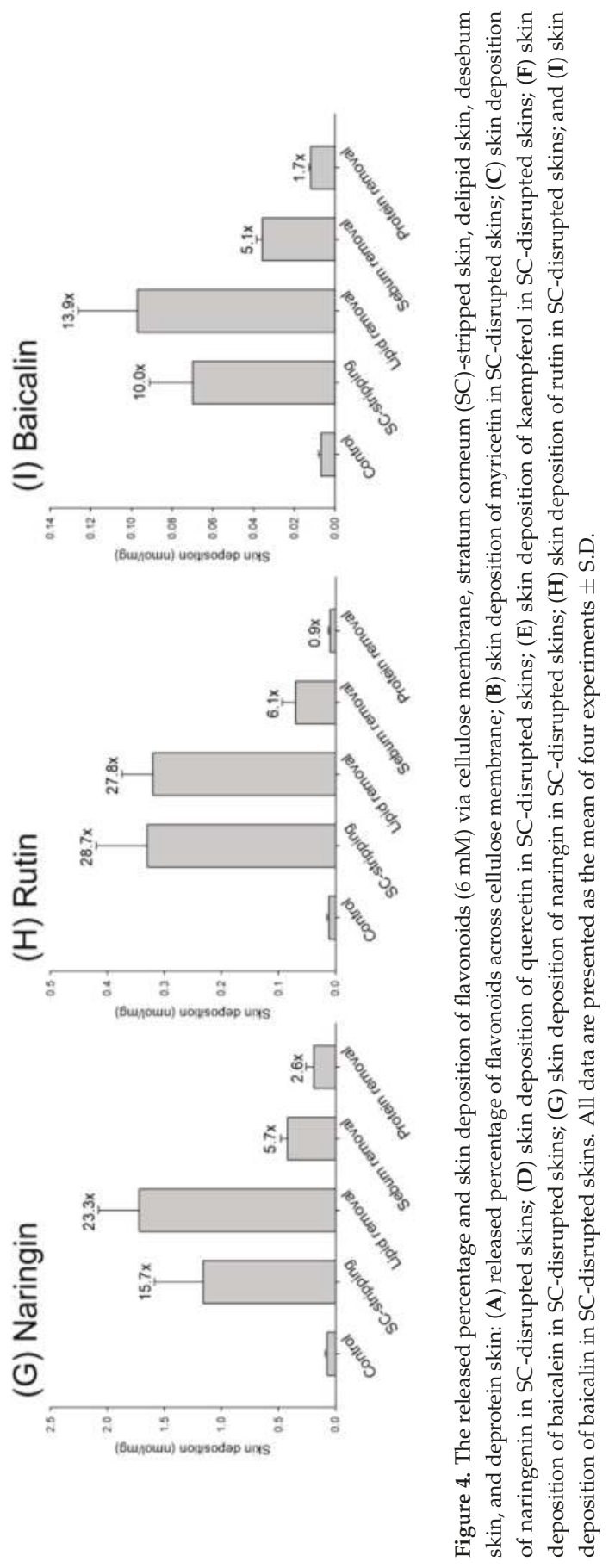




\subsection{Inhibition of Neutrophil Inflammation by Flavonoids}

The $\mathrm{O}_{2}{ }^{\bullet-}$ production and elastase release in stimulated neutrophils are indicators reflecting a massive neutrophil infiltration into the inflamed skin. The flavonoids were rated for the inhibition percentage of $\mathrm{O}_{2}{ }^{\circ-}$ and elastase in response to fMLF, as shown in Table 2. Incubation of fMLF-activated neutrophils in the presence of flavonoids resulted in an inhibition on superoxide anion, with baicalein and baicalin exhibiting the greatest activity $(77 \%$ and $76 \%)$. The $\mathrm{O}_{2}{ }^{-}{ }^{-}$inhibition effect was comparable for the aglycones and the corresponding glycosides. The flavonoids generated less inhibition on elastase than superoxide. Quercetin was among the most potent flavonoids to repress elastase $(29 \%)$. The elastase inhibition ranged between $10 \%$ and $20 \%$ for most of the flavonoids. The glycosides had a lower effect on elastase inhibition than the corresponding aglycones. Baicalin did not change the elastase release of fMLF-stimulated neutrophils. To estimate the possible bioactivity after topical delivery, we calculated TI based on multiplying the nude mouse skin deposition and the inflammatory inhibition percentage as shown in Table 2. Although the $\mathrm{O}_{2}{ }^{-{ }^{-}}$inhibition of naringenin was weak, this flavanone showed the highest TI because of the preferred delivery into the skin reservoir. The potent activity of baicalein on $\mathrm{O}_{2}{ }^{\bullet-}$ inhibition had led to a TI of 2.60, which approximated the TI level of kaempferol. The compound with the highest TI for elastase inhibition was also found to be naringenin, followed by kaempferol. Naringin was the penetrant with a higher TI than the other glycosides. Naringin demonstrated less TI value compared to naringenin.

Table 2. The inhibition percentage (\%) of superoxide anion $\left(\mathrm{O}_{2}{ }^{--}\right)$and elastase and the therapeutic index (TI) of aglycone and glycoside flavonoids at $3 \mu \mathrm{M}$.

\begin{tabular}{cccccc}
\hline Category & Compound & $\mathbf{O}_{\mathbf{2}}{ }^{\bullet-}$ Inhibition & TI $_{\text {superoxide }}$ & Elastase Inhibition & TI $_{\text {elastase }}$ \\
\hline \multirow{5}{*}{ Aglycone } & Myricetin & $42.07 \pm 6.22$ & 0.58 & $16.81 \pm 4.76$ & 0.23 \\
& Naringenin & $19.72 \pm 8.23$ & 7.36 & $17.97 \pm 6.62$ & 6.71 \\
& Quercetin & $26.44 \pm 8.07$ & 0.89 & $28.67 \pm 8.51$ & 0.96 \\
& Kaempferol & $26.49 \pm 8.41$ & 2.97 & $18.11 \pm 9.01$ & 2.03 \\
& Baicalein & $77.48 \pm 5.27$ & 2.60 & $12.25 \pm 2.15$ & 0.41 \\
\hline \multirow{3}{*}{ Glycoside } & Naringin & $21.51 \pm 2.53$ & 1.59 & $13.89 \pm 9.67$ & 1.02 \\
& Rutin & $33.87 \pm 8.67$ & 0.39 & $13.16 \pm 3.39$ & 0.15 \\
& Baicalin & $75.73 \pm 6.21$ & 0.53 & 0 & 0 \\
\hline
\end{tabular}

TI is calculated by the multiplication of inhibition percentage at $3 \mu \mathrm{M}$ and nude mouse skin deposition at equivalent dose $(6 \mathrm{mM})$. Each value represents the mean and S.D $(n=3)$.

\subsection{Molecular Modeling}

The informative explanation of the effect of molecular structure on cutaneous delivery is based on the hydrogen bond number, the total polarity surface, and the molecular volume. The parameters for flavonoids are computed by Discovery Studio ${ }^{\circledR} 4.1$ as presented in Table 3. The number of hydrogen bond acceptors and donors increased following the increase of hydroxyl groups in the structure. The hydrogen bond number of glycosides was much higher than that of aglycones due to the presence of hydroxyl moieties in the sugar. Rutin exhibited the highest hydrogen bond numbers among all flavonoids examined. The total polarity surface correlated well with the hydrogen bond number, with the glycosides showing a greater polarity surface than aglycones. This indicates more polar interactions of the glycosides in aqueous medium. The molecular volume is a reflection of MW. We found a high positive correlation between molecular volume and MW (correlation coefficient $=0.9979$ ).

To study the cutaneous transport characteristics of the flavonoids in greater detail, we applied computational molecular docking to analyze the possible interaction of the penetrants to SC lipids. The three-dimensional lipid model was generated for their posterior use as the target structure in the in silico calculation. Table 4 summarizes the best docking score (negative CDOCKER) of the flavonoids interacting with SC lipids, including ceramides II, III, and VI, palmitic acid, cholesteryl sulfate, and cholesterol. The flavonoids were docked into conformationally minimized SC lipids. The cooperativity 
of the interaction included van der Waals, hydrogen bonding, and lipophilic and electrostatic forces. The greater negative energy dictated a stronger binding interaction. The glycosides revealed greater interaction to ceramides than aglycones, with rutin showing the highest interaction. The discrepancy of the negative CDOCKER between ceramides and different aglycones was not large ( -24 to -18$)$.

Table 3. The hydrogen bond number, total polarity surface and molecular volume of aglycone and glycoside flavonoids determined by molecular modeling.

\begin{tabular}{cccccc}
\hline Category & Compound & $\begin{array}{c}\text { Hydrogen Bond } \\
\text { Acceptor Number }\end{array}$ & $\begin{array}{c}\text { Hydrogen Bond } \\
\text { Donor Number }\end{array}$ & $\begin{array}{c}\text { Total Polarity } \\
\text { Surface }\end{array}$ & $\begin{array}{c}\text { Molecular } \\
\text { Volume }\end{array}$ \\
\hline \multirow{5}{*}{ Aglycone } & Myricetin & 8 & 6 & 147.68 & 225.35 \\
& Naringenin & 5 & 3 & 86.99 & 204.42 \\
& Quercetin & 7 & 5 & 127.45 & 217.11 \\
& Kaempferol & 6 & 4 & 107.22 & 203.74 \\
& Baicalein & 5 & 3 & 86.99 & 195.85 \\
\hline \multirow{3}{*}{ Glycoside } & Naringin & 14 & 8 & 225.05 & 437.66 \\
& Rutin & 16 & 10 & 265.52 & 444.18 \\
& Baicalin & 11 & 6 & 183.21 & 316.93 \\
\hline
\end{tabular}

Table 4. The negative CDOCKER energy of aglycone and glycoside flavonoids to interact with the stratum corneum components determined by molecular modeling.

\begin{tabular}{ccccccc}
\hline Compound & Ceramide II & Ceramide III & Ceramide VI & Palmitic Acid & Cholesteryl Sulfate & Cholesterol \\
\hline Myricetin & -19.454 & -25.852 & -22.285 & -19.048 & -117.792 & - \\
Naringenin & -19.143 & -25.670 & -24.231 & -18.155 & -121.188 & - \\
Quercetin & -18.928 & -21.850 & -22.063 & -18.032 & -118.533 & - \\
Kaempferol & -21.053 & -20.831 & -19.639 & -17.399 & -119.485 & - \\
Baicalein & -18.841 & -20.637 & -20.427 & -18.073 & -118.632 & - \\
Naringin & -27.144 & -28.937 & -37.266 & -17.326 & -109.196 & - \\
Rutin & -33.007 & -35.341 & -38.940 & -23.502 & -109.365 & -47.1637 \\
Baicalin & -24.573 & -30.721 & -28.555 & -19.751 & -112.425 & -53.8485 \\
\hline
\end{tabular}

- Means no interaction.

Rutin showed the highest negative CDOCKER level with palmitic acid among the flavonoids detected. A relatively higher negative CDOCKER energy was found for cholesteryl sulfate than for the other lipids. It appears that the flavonoids preferentially interacted with cholesteryl sulfate. In the case of cholesteryl sulfate, the negative CDOCKER of aglycones was higher compared to the corresponding glycosides. Naringenin $(-121)$ and kaempferol $(-119)$ exhibited a more potent interaction with cholesteryl sulfate than the other penetrants. No interaction was detected between cholesterol and the compounds except with rutin and baicalin. Figure 5 illustrates the best docking poses of the flavonoids interacting with cholesteryl sulfate. The flavonoids displayed the ligand binding activity to cholesteryl sulfate but in different conformations.
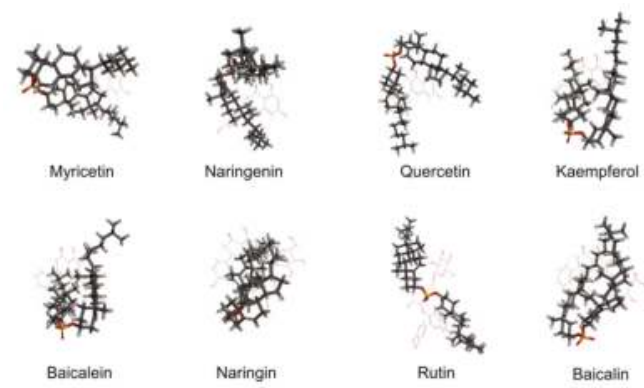

Figure 5. Superimposition of the computed poses for flavonoids with cholesteryl sulfate. 


\subsection{In Vivo Cutaneous Tolerance of Flavonoids}

The tolerance at the equivalent dose to the skin was determined by in vivo topical exposure for seven days. We examined $\triangle \mathrm{TEWL}$, as shown in Figure 6A. $\triangle \mathrm{TEWL}$ was calculated by the TEWL value of the treated skin area minus the value of the aqueous vehicle control. Most of the flavonoids exhibited a near-zero point $\triangle T E W L$ profile. This suggests that these compounds did not induce a barrier defect of SC. Naringenin and kaempferol even reduced TEWL as compared to the vehicle control. This may imply a protective capability of both compounds on barrier function. Figure $6 \mathrm{~B}-\mathrm{K}$ reveals the histology of flavonoid-treated skin after a seven-day administration. We could see a decrease in the SC layers of the vehicle control compared to the sham group (Figure 6B versus Figure 6C), indicating an interruption of barrier position. The SC layers could be recovered through the application of naringenin, kaempferol, and naringin. The flavonoid treatment did not alter the viable skin morphology, expressing a negligible irritation.

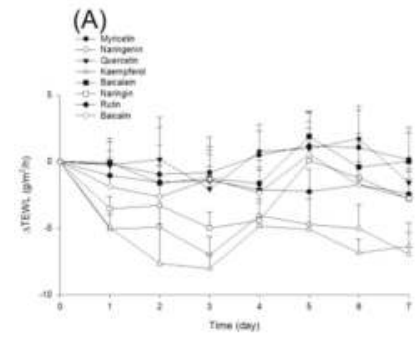

(D) Myricetin

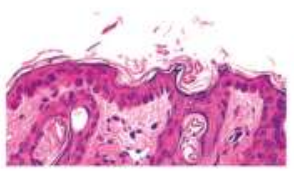

(G) Kaempferol

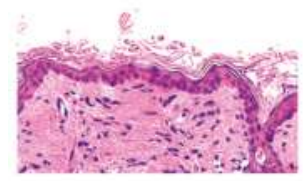

(J) Rutin
(B) Sham

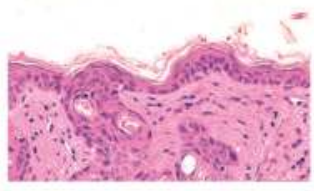

(C) Medium control

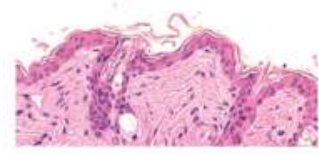

(E) Naringenin

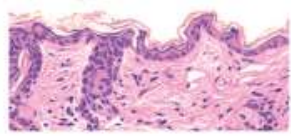

(H) Baicalein

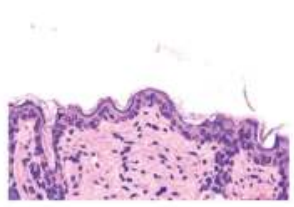

(K) Baicalin

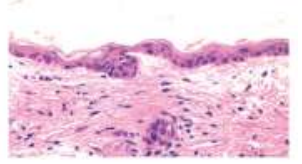

(I) Naringin

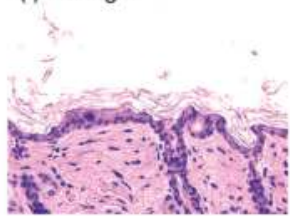

(F) Quercetin

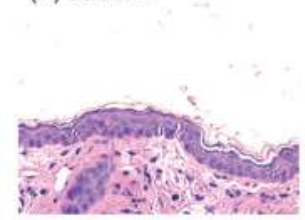

Figure 6. The in vivo safety of topically applied flavonoids on skin after a 7-day exposure: (A) $\triangle$ TEWL-time curves; (B) the histology of sham control skin; (C) the histology of aqueous medium-treated skin; (D) the histology of myricetin-treated skin; (E) the histology of naringenin-treated skin; (F) the histology of quercetin-treated skin; (G) the histology of kaempferol-treated skin; $(\mathbf{H})$ the histology of baicalein-treated skin; (I) the histology of naringin-treated skin; (J) the histology of rutin-treated skin; and (K) the histology of baicalin-treated skin. The TEWL data are presented as the mean of six experiments \pm S.D. 


\section{Discussion}

Sustained inflammation contributes to the pathogenesis of some skin disorders, including photoaging, psoriasis, and atopic dermatitis. Flavonoids exert potent anti-inflammatory effects on the skin. Nevertheless, whether the cutaneous absorption of flavonoids is sufficient to trigger the bioactivity is questionable. An ideal topical administration has a much higher absorption accomplished in the cutaneous reservoir compared to that in the systemic circulation to achieve efficient targeting and escape systemic toxicity [14]. We examined the skin delivery and anti-inflammatory activity of aglycone and glycoside flavonoids to select the potential candidates for topical application with the aim of prevention or therapy. The aqueous vehicle was used as the flavonoid donor since most attempts for modeling SPR had been based on the data of aqueous solution application [15]. Naringenin and kaempferol were efficacious for topical application with respect to targeting into the skin and possible treatment capability based on the data of $S$ value and TI, respectively. The cutaneous permeation of flavonoids exhibited a similar trend either at an equivalent dose or at the saturated solubility. Both aglycones also produced a protective effect on skin-barrier function. The glycosides generally showed lower absorption than the corresponding aglycones. Previous study [16] also suggests a low skin bioavailability of flavonoid glycosides such as baicalin.

The physicochemical properties of flavonoids from the experimental data and molecular modeling prediction were beneficial to discussing the SPR. The aglycone lipophilicity was decreased following the increase of the hydroxyl group number. An exception was naringenin, which had only 3 hydroxyl groups but low lipophilicity. Different from the flavonols and flavones, naringenin is a flavanone with fewer double bonds in the $\mathrm{C}$ ring. The flavonols were more lipophilic and assumed a planar structure [17]. The flexibility of the chiral center in the $\mathrm{C}$ ring of naringenin could fold to form a three-dimensional structure. The approximation of the $\mathrm{B}$ ring to the $\mathrm{A}$ and $\mathrm{C}$ rings increased the possibility of the production of intramolecular hydrogen bonds, leading to the decrease of lipophilicity and enhancement of aqueous solubility. This effect did not occur with flavonols and flavones due to the rigidity of the structure.

Some physicochemical factors of penetrants can influence the skin permeation. These include lipophilicity, solubility, release rate, MW, and hydrogen bonding. The penetrants with higher lipophilicity can increase the skin-absorption capacity due to the facile partitioning and entrance into the SC lipids [18]. Both $\log K^{\prime}$ and $\log P$ are the parameters of lipophilicity. Another parameter for anticipating the skin absorption is the total polarity surface, which correlates inversely with lipophilicity and biomembrane absorption [19]. The correlation coefficient between $\log P$ and the total polarity surface for the flavonoids was 0.9003 . The polar surface area is defined as the total sum of the surface of the polar atoms [20]. The highest total polarity surface of myricetin could explain the low skin absorption of this compound. However, the lipophilicity could not explain the high skin deposition of naringenin and kaempferol. There was no correlation between lipophilicity and skin absorption of the flavonoids. The penetrants should first partition into the SC before diffusion across the SC. The sebum spread on the SC surface contributes to a capacity-governing penetrant partitioning from the vehicle to the SC [21]. Theoretically, this partitioning is highly dependent on the lipophilicity [22]. Our results showed that the skin deposition enhancement after sebum removal was less for naringenin and kaempferol than for the other flavonoids. This suggests an ease in the partitioning of naringenin and kaempferol to the SC layer, and sebum was a barrier for the permeation of the other flavonoids. From these data, it can be concluded that the cutaneous absorption of flavonoids cannot be solely ascribed to their lipophilicity. The skin more facilely absorbed the flavonoids with moderate hydrophilicity and aqueous solubility.

Although a lipophilic nature is required for transport across the SC, a hydrophilic property is also needed for entry into the viable skin [23]. The penetrants with the $\log P>3$ show a decrease of viable-skin diffusion following the increase of lipophilicity [24]. Both the SC and the viable epidermis/dermis are the permeation barriers. The role of viable skin in flavonoid absorption could be understood by the permeation via SC-stripped skin. Myricetin and quercetin were the flavonoids with 
low skin absorption. The SC removal largely promoted the deposition of both flavonoids by $>30$ fold. This indicates a significant barrier function of the SC for the two penetrants. The skin deposition of myricetin and quercetin was 0.4 and $1.2 \mathrm{nM} / \mathrm{mg}$ after the SC's removal, respectively. This value was still lower than the naringenin deposition after stripping. Baicalein was a lipophilic flavonoid with facile entrance into the SC because of the limited enhancement of skin deposition after stripping. However, the baicalein deposition in the stripped skin was only $0.2 \mathrm{nM} / \mathrm{mg}$. These results demonstrate that the viable skin could be a barrier to the flavonoid permeation. The cellulose membrane is like viable skin due to its hydrophilic property. We found that the released percentage across the cellulose membrane was lower for myricetin, quercetin, and baicalein than for naringenin and kaempferol, confirming the diffusion barrier of viable skin. Naringenin and kaempferol could conquer the viable-skin barrier, resulting in the effortless absorption. The much higher aqueous solubility of naringenin compared to the other penetrants was the reason for the easy diffusion into the viable skin. Although kaempferol exerted a comparable lipophilicity to baicalein, the considerable solubility of kaempferol had led to greater absorption than with baicalein. Because of the existence of sugar moiety, the glycoside flavonoids had good solubility in the aqueous vehicle. Although the glycosides might easily permeate into viable skin according to the high percentage of release, the absorption of glycosides was less compared to that of the aglycones. The SC was the main barrier to retard glycoside delivery since SC removal could greatly enhance their deposition.

The SC layer contains lipids and corneocytes cross-linked by keratin. The SC lipids and keratin contribute to the nonpolar and polar pathways for permeation. Similar absorption should be observed for the permeation into SC-stripped skin and delipidized skin if the penetrants predominantly transport via the nonpolar route [25]. Our data supported the lipid pathway being the main diffusion mechanism for the flavonoids. Although proteins occupy $80 \%$ of the SC constituents, the polar pathway is not the major route for flavonoids because of the limited skin deposition enhancement after protein denaturation. A high affinity of the penetrants to the SC lipids can generate a reservoir in the skin for plentiful absorption [26]. To obtain detailed information on the absorption of flavonoids, we established the possible flavonoid-lipid interaction employing molecular docking. The lipid content of the SC basically comprises ceramides, fatty acids, cholesteryl sulfate, and cholesterol [27]. There are nine classes of ceramide identified in the SC [28]. We chose ceramides II, III, and VI for docking because of their abundance in human SC [29]. Palmitic acid was used as the model fatty acid. Cholesteryl sulfate presented much stronger interaction with the flavonoids than the other SC lipids. Naringenin and kaempferol showed the highest negative CDOCKER for interacting with cholesteryl sulfate. This result coincided with the highest skin deposition of both compounds. The correlation coefficient between nude mouse skin deposition and CDOCKER of aglycones was 0.9673. The negative CDOCKER of glycosides was inferior to that of aglycones with respect to interacting with cholesteryl sulfate. A contrary result was detected for the other lipids. The aglycones even showed no interaction with cholesterol. Thus, cholesteryl sulfate could offer the most suitable flavonoid-lipid interaction for predicting flavonoid absorption. The CDOCKER of other lipids such as ceramides are infeasible to be the indicator of flavonoid absorption because of the low correlation between the energy and skin deposition. For example, rutin had the highest negative CDOCKER with ceramides and fatty acid but a low skin delivery capability.

The hydrogen bond acceptor is another factor that inversely correlates with cutaneous absorption. Since the SC is the hydrogen bond acceptor [30], an increase in the number of hydrogen bonds reduces the permeation across the SC [31]. Myricetin is the compound with a higher hydrogen bond acceptor number than the other aglycones. The hydrogen bond acceptor number of glycosides was much higher than that of aglycones. Myricetin and glycosides were inferred to have less interaction with the SC according to hydrogen bonding and cholesteryl sulfate docking. Molecular size rather than lipophilicity would impact skin absorption if less interaction was detected between the penetrants and the SC components [32]. The skin diffusion is size-dependent, with the larger penetrants demonstrating lower skin delivery. Both MW and molecular volume illustrated a larger size of myricetin and 
glycoside structures. The consequence was the low absorption of these compounds. The molecules partially associated with the solvent cage when they traversed the skin from the aqueous vehicle. The permeation rate involved not only the molecule itself but also the entire solvated complex [33]. Myricetin and the glycosides with a higher hydrogen bond number might attract more water molecules to produce a large solvation complex, causing a detrimental effect on skin transport. Although the MW and molecular volume of naringenin were not the lowest, the folding character of this flavanone might generate a smaller size than the flavonols and flavones with a planar structure. The small molecular size after bending favored the diffusion into the skin reservoir.

The aim of topically applied flavonoids was to offer better targeting to the skin with minimal systemic absorption. The $S$ value is a parameter for judging the skin-targeting efficacy. With respect to aglycones, naringenin and kaempferol showed efficient targeting to the skin tissue. The high $S$ value of myricetin and quercetin was due to there being no or negligible flux. The low cutaneous deposition of myricetin and quercetin might not elicit significant bioactivity on the skin. Rutin presented a high $S$ value in the case of the equivalent dose, which could favor the cutaneous efficacy. In the case of a saturated dose, baicalin had the highest $S$ value among the glycosides. As with myricetin, the low deposition has limited the topical application of glycosides for therapeutic use.

Neutrophils act as the predominant phagocytic cells for the first line of defense. Although the activation of neutrophils enhances immunity to retard xenobiotic invasion, the overwhelming stimulation contributes to inflammatory disorders and adaptive immune responses [34]. Chronic inflammatory skin diseases such as psoriasis and photoaging can be characterized by neutrophil infiltration [35]. The activated neutrophils damage the skin by the production of reactive oxygen species (ROS). A respiratory burst of neutrophils is an oxygen-dependent process leading to the formation of ROS. The protective effect of flavonoids on the skin is directly linked to the antiradical potency [7]. Our results revealed a flavonoid-driven mitigation of $\mathrm{O}_{2}{ }^{-\boldsymbol{}}$ in fMLF-stimulated neutrophils. The $\mathrm{O}_{2}{ }^{\bullet-}$ inhibition was the highest for baicalein. Although baicalein reduced $\mathrm{O}_{2}{ }^{\bullet-}$ more effectively, it only exerted moderate TI due to the low efficiency of cutaneous absorption. The lowest superoxide inhibition was found for naringenin. However, this flavanone still exhibited the highest TI because of the efficacious skin targeting. The intramolecular hydrogen bond can suppress antioxidant activity [36]. This is the reason for the low $\mathrm{O}_{2}{ }^{\bullet-}$ inhibition by the flavanone.

The number of hydroxyl groups in the structure of the antioxidants is a factor influencing the antiradical effect, with the presence of more hydroxyl groups resulting in stronger activity [37]. This was not the case in our study since no correlation was observed between the hydroxyl moiety number and the scavenging capacity. The $\mathrm{O}_{2}{ }^{\bullet-}$ inhibition of glycosides was similar to their corresponding aglycones. The lower skin deposition of glycosides had resulted in the lower TI of $\mathrm{O}_{2}{ }^{\bullet-}$ compared to the aglycones. Both neutrophil trafficking and elastase release are elevated in skin photoaging. Elastase plays a role in wrinkling after photoaging [38]. The experimental data provided evidence that the flavonoids showed less inhibition on elastase than $\mathrm{O}_{2}{ }^{\bullet}$. The glycosylation decreased the inhibitory activity on elastase. The same as $\mathrm{O}_{2}{ }^{\bullet-}$, the greatest TI of elastase proved to be topically applied naringenin.

The $\triangle$ TEWL data demonstrated that the flavonoids did not damage the skin barrier as compared to the aqueous vehicle control. No SC disruption was observed for naringenin and kaempferol, though the skin deposition was very high. It is surprising that naringenin and kaempferol even alleviated $\triangle \mathrm{TEWL}$. Water contact with the SC in high content can disturb the barrier's nature [39]. The aqueous vehicle used in this study could increase TEWL. The skin histology demonstrated fewer SC layers of the aqueous medium control than the sham group. Naringenin and kaempferol with high cutaneous absorption might protect the skin to reduce the impairment generated by the water. Further work is needed to elucidate the protection mechanism. Naringenin, extensively contained in lemons, oranges, and grapes, is reported to be a potent active of attenuating skin inflammation and oncogenesis $[4,40]$. Kaempferol has been demonstrated to restrain photoaging and UV-induced carcinogenesis [41]. The synthetic drugs such as indomethacin and celecoxib provide protection 
against photoaging and skin cancers but have severe adverse effects. Topically applied naringenin and kaempferol can be promising candidates as alternatives for skin-inflammation treatment.

\section{Conclusions}

This study examined the cutaneous absorption and anti-inflammatory activity of flavonoids with the goal of choosing the optimal candidates for topical delivery. The results revealed that the glycosides showed less absorption and targeting than the aglycones. The low lipophilicity and large molecular size of glycosides contributed to the unsatisfied absorption. Naringenin and kaempferol were the compounds with the greatest deposition in the skin. They also protected the skin from the barrier disruption induced by the aqueous medium. Both flavonoids are potential candidates for topical application to treat skin inflammation. Both the SC and viable skin were the permeation barriers for flavonoid transport. The SPR demonstrated the importance of the hydrophilic and lipophilic balance of the flavonoid structure in order to achieve feasible skin delivery. The hydrogen bond acceptor and docking calculated from molecular modeling described the cutaneous absorption trend. We had utilized the aqueous vehicle as the flavonoid formulation. It is expected that the different compositions of vehicle may largely affect the skin permeation of the penetrants. The flavonoids may exhibit different permeation trend in other vehicles such as oil and nanoparticles. Further study is needed to elucidate the impact of different vehicles on flavonoid absorption. Our results offer essential information for the development of a topically applied flavonoid formulation. The experimental profiles in this study also provide the direction to design or synthesize new compounds for facilitating skin delivery.

Acknowledgments: The authors are grateful to the financial support by Ministry of Science and Technology of Taiwan (MOST-105-2320-B-182-010-MY3) and Chang Gung Memorial Hospital (CMRPG2F0491-3).

Author Contributions: S.-Y.C. and J.-Y.F. conceived the topic. S.-Y.C., Y.-K.L. and E.-L.C. performed the experimental works. Y.-K.L. outlined the paper and mainly wrote it. P.-W.W. and J.-Y.F. contributed to the drafting of the manuscript.

Conflicts of Interest: The authors declare no conflict of interest.

\section{References}

1. Petrou, A.L.; Terzidaki, A. A meta-analysis and review examining a possible role for oxidative stress and singlet oxygen in diverse diseases. Biochem. J. 2017, 474, 2713-2731. [CrossRef] [PubMed]

2. Rinnerthaler, M.; Bischof, J.; Streubel, M.K.; Trost, A.; Richter, K. Oxidative stress in aging human skin. Biomolecules 2015, 5, 545-589. [CrossRef] [PubMed]

3. Ascenso, A.; Ribeiro, H.M.; Marques, H.C.; Simões, S. Topical delivery of antioxidants. Curr. Drug Deliv. 2011, 8, 640-660. [CrossRef] [PubMed]

4. George, V.C.; Vijesh, V.V.; Amararathna, D.I.M.; Lakshmi, C.A.; Anbarasu, K.; Kumar, D.R.N.; Ethiraj, K.R.; Kumar, R.A.; Rupasinghe, H.P.V. Mechanism of action of flavonoids in prevention of inflammation-associated skin cancer. Curr. Med. Chem. 2016, 23, 1-20.

5. Shen, C.Y.; Jiang, J.G.; Yang, L.; Wang, D.W.; Zhu, W. Anti-ageing active ingredients from herbs and nutraceuticals used in traditional Chinese medicine: Pharmacological mechanisms and implications for drug discovery. Br. J. Pharmacol. 2017, 174, 1395-1425. [CrossRef] [PubMed]

6. Abd, E.; Roberts, M.S.; Grice, J.E. A comparison of the penetration and permeation of caffeine into and through human epidermis after application in various vesicle formulations. Skin Pharmacol. Physiol. 2016, 29, 24-30. [CrossRef] [PubMed]

7. Arct, J.; Pytkowska, K. Flavonoids as components of biologically active cosmeceuticals. Clin. Dermatol. 2008, 26, 347-357. [CrossRef] [PubMed]

8. Zhou, B.R.; Liu, W.L.; Luo, D. Protective effect of baicalin against multiple ultraviolet b exposure-mediated injuries in C57BL/6 mouse skin. Arch. Pharm. Res. 2011, 34, 261-268. [CrossRef] [PubMed]

9. Dayan, N. Pathways for skin penetration. Cosmet. Toilet. 2005, 120, 67-76. 
10. Liu, K.S.; Chen, Y.W.; Aljuffali, I.A.; Chang, C.W.; Wang, J.J.; Fang, J.Y. Topically applied mesoridazine exhibits the strongest cutaneous analgesia and minimized skin disruption among tricyclic antidepressants: The skin absorption assessment. Eur. J. Pharm. Biopharm. 2016, 105, 59-68. [CrossRef] [PubMed]

11. Campbell, C.S.J.; Contreras-Rojas, L.R.; Delgado-Charro, M.B.; Guy, R.H. Objective assessment of nanoparticle disposition in mammalian skin after topical exposure. J. Control. Release 2012, 162, 201-207. [CrossRef] [PubMed]

12. Boyum, A.; Lovhaug, D.; Tresland, L.; Nordlie, E.M. Separation of leucocytes: Improved cell purity by fine adjustments of gradient medium density and osmolality. Scand. J. Immunol. 1991, 34, 697-712. [CrossRef] [PubMed]

13. Yang, S.C.; Chung, P.J.; Ho, C.M.; Kuo, C.Y.; Hung, M.F.; Huang, Y.T.; Chang, W.Y.; Chang, Y.W.; Chan, K.H.; Hwang, T.L.; et al. Propofol inhibits superoxide production, elastase release, and chemotaxis in formyl peptide-activated human neutrophils by blocking formyl peptide receptor 1. J. Immunol. 2013, 190, 6511-6519. [CrossRef] [PubMed]

14. Sawamura, R.; Sakurai, H.; Wada, N.; Nishiya, Y.; Honda, T.; Kazui, M.; Kurihara, A.; Shinagawa, A.; Izumi, T. Bioactivation of loxoprofen to a pharmacologically active metabolite and its disposition kinetics in human skin. Biopharm. Drug Dispos. 2015, 36, 352-363. [CrossRef] [PubMed]

15. Riviere, J.E.; Brooks, J.D. Predicting skin permeability from complex chemical mixtures: Dependency of quantitative structure permeation relationships on biology of skin model used. Toxicol. Sci. 2011, 119, 224-232. [CrossRef] [PubMed]

16. Mir-Palomo, S.; Nácher, A.; Díez-Sales, O.; Busó, O.V.; Caddeo, C.; Manca, M.L.; Manconi, M.; Fadda, A.M.; Saurí, A.R. Inhibition of skin inflammation by baicalin ultradeformable vesicles. Int. J. Pharm. 2016, 511, 23-29. [CrossRef] [PubMed]

17. Lin, C.F.; Leu, Y.L.; Al-Suwayeh, S.A.; Ku, M.C.; Hwang, T.L.; Fang, J.Y. Anti-inflammatory activity and percutaneous absorption of quercetin and its polymethoxylated compound and glycosides: The relationships to chemical structures. Eur. J. Pharm. Sci. 2012, 47, 857-864. [CrossRef] [PubMed]

18. Lin, C.F.; Hung, C.F.; Aljuffali, I.A.; Huang, Y.L.; Liao, W.C.; Fang, J.Y. Methylation and esterification of magnolol for ameliorating cutaneous targeting and therapeutic index by topical application. Pharm. Res. 2016, 33, 2152-2167. [CrossRef] [PubMed]

19. Schaftenaar, G.; De Vlieg, J. Quantum mechanical polar surface area. J. Comput. Aided Mol. Des. 2012, 26, 311-318. [CrossRef] [PubMed]

20. Ertl, P.; Rohde, B.; Selzer, P. Fast calculation of molecular polar surface area as a sum of fragment-based contributions and its application to the prediction of drug transport properties. J. Med. Chem. 2000, 43, 3714-3717. [CrossRef] [PubMed]

21. Liu, K.S.; Hsieh, P.W.; Aljuffali, I.A.; Lin, Y.K.; Chang, S.H.; Wang, J.J.; Fang, J.Y. Impact of ester promoieties on transdermal delivery of ketorolac. J. Pharm. Sci. 2014, 103, 974-986. [CrossRef] [PubMed]

22. Liu, X.; Testa, B.; Fahr, A. Lipophilicity and its relationship with passive drug permeation. Pharm. Res. 2011, 28, 962-977. [CrossRef] [PubMed]

23. Song, K.; An, S.M.; Kim, M.; Koh, J.S.; Boo, Y.C. Comparison of the antimelanogenic effects of $p$-coumaric acid and its methyl ester and their skin permeabilities. J. Dermatol. Sci. 2011, 63, 17-22. [CrossRef] [PubMed]

24. Schneider, M.; Stracke, F.; Hansen, S.; Schaefer, U.F. Nanoparticles and their interactions with the dermal barrier. Dermatoendocrinology 2009, 1, 197-206. [CrossRef]

25. Yan, Y.D.; Sung, J.H.; Lee, D.W.; Kim, J.S.; Jeon, E.M.; Kim, D.D.; Kin, D.W.; Kim, J.O.; Piao, M.G.; Li, D.X.; et al. Evaluation of physicochemical properties, skin permeation and accumulation profiles of salicylic acid amide prodrugs as sunscreen agent. Int. J. Pharm. 2011, 419, 154-160. [CrossRef] [PubMed]

26. Pierre, M.B.R.; Lopez, R.F.V.; Bentley, M.V.L.B. Influence of ceramide 2 on in vitro skin permeation and retention of 5-ALA and its ester derivatives, for photodynamic therapy. Braz. J. Pharm. Sci. 2009, 45, 109-116. [CrossRef]

27. Mojumdar, E.H.; Gooris, G.S.; Barlow, D.J.; Lawrence, M.J.; Deme, B.; Bouwstra, J.A. Skin lipids: Localization of ceramide and fatty acid in the unit cell of the long periodicity phase. Biophys. J. 2015, 108, 2670-2679. [CrossRef] [PubMed]

28. Kessner, D.; Ruettinger, A.; Kiselev, M.A.; Wartewig, S.; Neubert, R.H.H. Properties of ceramides and their impact on the stratum corneum structure: A review. Skin Pharmacol. Physiol. 2008, 21, 58-74. [CrossRef] [PubMed] 
29. Paige, D.G.; Morse-Fisher, N.; Harper, J.I. Quantification of stratum corneum ceramides and lipid envelope ceramides in the hereditary ichthyoses. Br. J. Dermatol. 1994, 131, 23-27. [CrossRef] [PubMed]

30. N'Da, D.D. Prodrug strategies for enhancing the percutaneous absorption of drugs. Molecules 2014, 19, 20780-20787. [CrossRef] [PubMed]

31. Alikhan, A.; Farahmand, S.; Maibach, H.I. Correlating percutaneous absorption with physicochemical parameters in vivo in man: Agricultural, steroid, and other organic compounds. J. Appl. Toxicol. 2009, 29, 590-596. [CrossRef] [PubMed]

32. Yamaguchi, K.; Mitsui, T.; Aso, Y.; Sugibayashi, K. Structure-permeability relationship analysis of the permeation barrier properties of the stratum corneum and viable epidermis/dermis of rat skin. J. Pharm. Sci. 2008, 97, 4391-4403. [CrossRef] [PubMed]

33. Mohammed, D.; Matts, P.J.; Hadgraft, J.; Lane, M.E. In vitro-in vivo correlation in skin permeation. Pharm. Res. 2014, 31, 394-400. [CrossRef] [PubMed]

34. Nauseef, W.M.; Borregaard, N. Neutrophils at work. Nat. Immunol. 2014, 15, 602-611. [CrossRef] [PubMed]

35. Kim, J.; Krueger, J.G. The immunopathogenesis of psoriasis. Dermatol. Clin. 2015, 33, 13-23. [CrossRef] [PubMed]

36. Amorati, R.; Zotova, J.; Baschieri, A.; Valgimigli, L. Antioxidant activity of magnolol and honokiol: Kinetic and mechanistic investigations of their reaction with peroxyl radicals. J. Org. Chem. 2015, 80, 10651-10659. [CrossRef] [PubMed]

37. Alonso, C.; Lucas, R.; Barba, C.; Marti, M.; Rubio, L.; Comelles, F.; Morales, J.C.; Coderch, L.; Parra, J.L. Skin delivery of antioxidant surfactants based on gallic acid and hydroxytyrosol. J. Pharm. Pharmacol. 2015, 67, 900-908. [CrossRef] [PubMed]

38. Takeuchi, H.; Gomi, T.; Shishido, M.; Watanabe, H.; Suenobu, N. Neutrophil elastase contributes to extracellular matrix damage induced by chronic low-dose UV irradiation in a hairless mouse photoaging model. J. Dermatol. Sci. 2010, 60, 151-158. [CrossRef] [PubMed]

39. Lombardi Borgia, S.; Schlupp, P.; Mehnert, W.; Schäfer-Korting, M. In vitro skin absorption and drug release-A comparison of six commercial prednicarbate preparations for topical use. Eur. J. Pharm. Biopharm. 2008, 68, 380-389. [CrossRef] [PubMed]

40. Al-Roujayee, A.S. Naringenin improves the healing process of thermally-induced skin damage in rats. J. Intern. Med. Res. 2017, 45, 570-582. [CrossRef] [PubMed]

41. Yao, K.; Chen, H.; Liu, K.; Langfald, A.; Yang, G.; Zhang, Y.; Yu, D.H.; Kim, M.O.; Lee, M.H.; Li, H.; et al. Kaempferol targets RSK2 and MSK1 to suppress UV radiation-induced skin cancer. Cancer Prev. Res. 2014, 7, 958-967. [CrossRef] [PubMed]

(C) 2017 by the authors. Licensee MDPI, Basel, Switzerland. This article is an open access article distributed under the terms and conditions of the Creative Commons Attribution (CC BY) license (http:/ / creativecommons.org/licenses/by/4.0/). 
Article

\title{
Is Skin Coloration Measured by Reflectance Spectroscopy Related to Intake of Nutrient-Dense Foods? A Cross-Sectional Evaluation in Australian Young Adults
}

\author{
Lee M. Ashton ${ }^{1,2}$, Kristine B. Pezdirc ${ }^{1,2}$, Melinda J. Hutchesson ${ }^{1,2}$, Megan E. Rollo ${ }^{1,2}$ and \\ Clare E. Collins ${ }^{1,2, *}$ \\ 1 School of Health Sciences, Faculty of Health and Medicine, University of Newcastle, Callaghan 2308, \\ Australia; lee.ashton@newcastle.edu.au (L.M.A.); Kristine.pezdirc@newcastle.edu.au (K.B.P.); \\ Melinda.hutchesson@newcastle.edu.au (M.J.H.); Megan.rollo@newcastle.edu.au (M.E.R.) \\ 2 Priority Research Centre in Physical Activity and Nutrition, University of Newcastle, \\ Callaghan 2308, Australia \\ * Correspondence: clare.collins@newcastle.edu.au; Tel.: +61-2-4921-5646
}

Received: 10 November 2017; Accepted: 20 December 2017; Published: 23 December 2017

\begin{abstract}
The current study examines associations between the dietary intakes of nutrient-dense foods, measured using brief indices and skin coloration, measured using reflectance spectroscopy in young adults. This is a cross-sectional analysis of 148 young Australian males and females ( $55 \%$ female) aged 18 to 25 years. Dietary intake was assessed using a validated food frequency questionnaire, with responses used to calculate two dietary indices: (i) the Australian Recommended Food Score (ARFS); and (ii) the Fruit And Vegetable VAriety Score (FAVVA). Skin yellowness was measured at three body locations using reflectance spectroscopy. Associations were assessed using Spearman's correlation coefficients, regression analysis, and agreement using weighted kappa $\left(\mathrm{K}_{\mathrm{w}}\right)$. Significant, moderate correlations were found between skin yellowness and diet index scores for the ARFS $(\rho=0.30, p<0.001)$ and FAVVA score $(\rho=0.39, p<0.001)$. These remained significant after adjustment for confounders (total fat intake, sex, skin lightness) and for agreement based on categorical rankings. Results suggest that measurement of skin coloration by reflectance spectroscopy can be used as an indicator of overall dietary quality and variety in young adults. Further exploration in diverse populations is required.
\end{abstract}

Keywords: cross-sectional study; carotenoids; skin color; diet quality

\section{Introduction}

Carotenoids are fat-soluble, yellow, orange, and red pigments found primarily in fruit and vegetables [1]. Small amounts of dietary carotenoids are also found in animal food sources, including fish, eggs and dairy products [1]. Carotenoids have been classified as antioxidants due to their ability to neutralize free radicals [2]. Human skin is directly exposed to ultraviolet (UV) radiation, tobacco smoke and ozone, which contribute to the production of free radicals [2]. Carotenoids accumulate in all layers of the skin, where they serve a protective role through neutralizing free radicals via the protective antioxidant chain in tissues [2].

Dietary sources of carotenoids are absorbed via the intestinal epithelial cells and enter the blood stream to be delivered to target tissues and organs, including all layers of human skin in particular the stratum corneum [3,4]. Carotenoids can be assayed using biochemical methods in blood samples or by non-invasive optical methods in human skin, such as reflectance spectroscopy or resonance raman spectroscopy to quantify the carotenoids present [5]. Both of these methods have been validated against 
plasma carotenoid concentrations $[4,6,7]$. Resonance raman spectroscopy detects skin carotenoids using a laser spectroscopy which probes the vibrational energy levels of a molecule [5]. Several studies have found positive correlations between diet (in particular fruit and vegetables) and skin carotenoids using this method $[6,8,9]$. Reflectance spectroscopy measures skin color using Commission Internationale de $l^{\prime}$ Eclairage (CIE) L* $\mathrm{a}^{*} \mathrm{~b}^{*}$ color space (where $\mathrm{L}^{*}$ represents skin lightness and positive values of $\mathrm{a}^{*}$ and $\mathrm{b}^{*}$ represent degrees of redness and yellowness, respectively) [3]. The accumulation of dietary carotenoids in the skin contributes to the appearance of skin yellowness ( $\left.b^{*}\right)$ specifically [3]. Several studies have shown positive associations between skin yellowness $\left(\mathrm{b}^{*}\right)$ and fruit and vegetable intake using reflectance spectroscopy [10-12]. A recent Randomized Controlled Trial (RCT) reported significant correlations between skin yellowness $\left(b^{*}\right)$, plasma carotenoid concentrations and the reported intake of high-carotenoid fruit and vegetables over a four-week period [13]. The findings from this study suggested that reflectance spectroscopy can be utilized as a quick non-invasive method for measuring dietary carotenoid intake and/or identifying low fruit and vegetable intake [13].

Diet quality scoring indices are commonly used as a method to identify both healthful and unhealthy dietary patterns. They have been designed to compare the nutritional adequacy of an individual's dietary intake and how closely it aligns with the current guidelines [14]. The relationship between diet quality indices and nutrition-related health outcomes has been reviewed, which indicates that diet quality can predict biomarkers of disease and the risk of health outcomes including cardiovascular disease, some cancers, and all-cause and disease-specific mortality [14]. The validity of using dietary indices has been compared with objective measures, such as plasma carotenoids [15]. Plasma carotenoids are a biological marker of recent fruit and vegetable intake [16]. Plasma carotenoid concentrations have been shown to have positive correlations with sub-scale scores for fruit and vegetables within the Australian Recommend Food Score (AFRS) [15]. However, evaluation of plasma carotenoids as a biomarker of dietary intake is burdensome, expensive and invasive [15]. Reflectance spectroscopy offers an alternative objective measure of carotenoid intake that is non-invasive, rapid and less burdensome. However, this method has not been assessed or validated relative to brief dietary indices. Therefore, the aim of the current study was to evaluate the association between dietary intakes of nutrient dense foods, measured using brief indices and skin coloration, measured using reflectance spectroscopy in Australian adults aged 18 to 25 years.

\section{Materials and Methods}

\subsection{Study Design}

This secondary analysis uses cross-sectional data sets from two separate studies in young adults. Study one was a cross-sectional sample of 98 young men and women $(n=91,93 \%$ female) [10], and study two was the baseline data from 50 young men who were recruited into a RCT targeting improved diet, physical activity and wellbeing [17]. The methods and primary analyses of both studies are published in detail elsewhere [10,17]. Study protocols for study one (H-2012-0217) and two (H-2015-0445) were approved by the University of Newcastle Human Research Ethics committee with the RCT also registered on the Australian New Zealand Clinical Trials Registry (ACTRN12616000350426). Written informed consent was obtained from all participants. Data from the two studies were combined in order to achieve a study population of young adults that was inclusive of both sexes. Data collection methods for the key outcome variables used in the current analysis were identical in both studies [10,17].

\subsection{Participants}

Complete participant demographics are published in detail elsewhere [10,17]. Briefly, the population samples in the current analysis were adults aged 18-25 years, recruited from the Hunter region of New South Wales, Australia and had completed both the Australian Eating Survey (AES) and reflectance spectroscopy measurement of skin coloration. Data were collected from October 2012 to 
June 2013 in study one and from March 2016 to May 2016 in study two. Eligibility criteria for study one were young adults that were non-smokers, and for study two were adults over 18 years old [10]. Key eligibility criteria for study two were being a young male (18-25 years), partaking in $<300 \mathrm{~min} /$ week of combined moderate to vigorous physical activity, and consuming less than the age- and sex-specific national recommendations for fruit and vegetables intake [17]. However, as 97\% of young Australian adults aged 18 to 24 years fail to meet national recommendations of two serves fruit/day and five-six serves of vegetable/day [18], only a very small proportion of men were excluded based on this.

\subsection{Skin Coloration Measurement Using Reflectance Spectroscopy}

The decision to use the reflectance spectroscopy method was due to its advantageous properties for skin carotenoid measurement including; its ability for quick and non-invasive assessments, high signal levels and its self-calibrating properties [5]. Despite previous concerns of reflectance spectroscopy having lower sensitivity in detecting carotenoids [5], recent research has found carotenoids characteristically absorb light in the $400-540 \mathrm{~nm}$ region of the spectrum and reflect back longer (yellow) wavelengths [10]. Therefore, wavelengths were set accordingly, to ensure greater specificity to various carotenoids and distinction from melanin and blood. In-person measurement of skin coloration was conducted using a handheld spectrophotometer at the University of Newcastle by researchers trained in standardized operating procedures for all measurements. KP undertook measurements in study one and trained LA, who undertook measurements in study two. Participants were advised prior to assessment not to wear make-up. All skin sites for reflectance spectroscopy assessment were cleaned using alcohol wipes with time allowed for the skin to dry. Skin coloration was measured using a hand-held CM700D spectrophotometer (Konica Minolta, Osaka, Japan) with an $8 \mathrm{~mm}$ diameter aperture, 2-degree observer angle and illuminant D65. The spectrophotometer was white point calibrated at each measurement session. Skin color (CIE L*a*b values) was recorded for each participant at three body locations on the left-hand side of the body unless stated otherwise. The three body locations included the inner arm (radiale), outer arm (medial humeral epicondyle) and palm (thenar muscle). The measurements were repeated three times at each site and the average recorded. Body locations were selected according to anatomical landmarks as specified in the ISAK International standards for anthropometric assessment [19]. Dietary carotenoids influence skin yellowness ( $\left.b^{*}\right)$ values and change in skin color [3]. As skin $\mathrm{a}^{*}$ (redness) values are associated with skin blood perfusion [20] participants were asked to refrain from all physical activity for two hours prior to data collection. Since melanin affects both skin yellowness $\left(b^{*}\right)$ and lightness $\left(L^{*}\right)$ [21], this was adjusted for in the current analyses.

\subsection{Dietary Indices}

Data from the Australian Eating Survey food frequency questionnaire (AES FFQ) [22] were used to calculate two brief dietary indices: (i) The Australian Recommended Food Score (ARFS), previously shown to be a reliable and valid indicator of overall diet quality and variety [23]; and (ii) the Fruit And Vegetable VAriety Score (FAVVA), which assesses variety and frequency of fruit and vegetable intake. The AES FFQ is a self-administered 120 item semi-quantitative FFQ which assesses usual dietary intake over the past 6 months [22], previously shown to be valid and reliable in adults for assessing usual dietary intake [22].

Australian Recommended Food Score (ARFS): uses a sub-set of 70 questions from AES FFQ related to core nutrient-dense foods. The total ARFS score is calculated by summing points within eight sub-scales (Supplementary file 1), based on usual weekly intake of specific foods and beverages whose consumption aligns with the Australian Guide to Healthy Eating (AGHE) within the Australian Dietary Guidelines [24]. There are 20 questions related directly to vegetable intake, 12 to fruit, 13 to protein foods (seven to meat and six to vegetarian sources of protein), 12 to breads/cereals, 10 to dairy foods, one to water, and two to spreads/sauces. The total score ranges from zero to a maximum of 73 points. Briefly, most items in the AES FFQ frequency response options are collapsed into two 
categories 'once per week or more' or 'less than once per week or never'. For most foods, respondents were awarded one point for a reported consumption of 'once per week or more', but differed for some items depending on national dietary guideline recommendations with consideration of the AGHE [24]. A higher total score is indicative of more optimal nutrient intakes, greater variety within the core food groups and alignment with Australian Dietary Guidelines [23,25]. Some of the food items for meat (i.e., beef, lamb) and dairy (i.e., ice-cream, frozen yoghurt) had a limit placed on their score for higher intakes, due to higher intakes being associated with potentially higher saturated fat or disease risk. Additional points were awarded for greater consumption of vegetables with evening meals, and healthier choices for bread and milk. Table S1 summarizes the detailed scoring method for items in the ARFS.

Fruit and Vegetable VAriety Score: uses a sub-set of 35 questions from AES FFQ related to usual intake frequency of a variety of vegetables and fruits across a comprehensive range of those consumed by the general Australian population. The FAVVA score was developed and modeled based on a previous fruit and vegetable index [26]. This total FAVVA score is calculated by summing points awarded from the fruit and vegetable sub-scales with 23 questions related directly to usual intake frequency of vegetables and legumes, and 12 questions about fruit intake. The FAVVA score uses all the fruit and vegetable questions from the AES FFQ except for the two vegetable questions relating to intake of 'hot chips'. The total score ranges from 0 to a maximum of 190 points. Table S2 summarizes the detailed scoring method for items in the FAVVA. Briefly, for most items, 0 points were awarded for a report of 'Never' consumed, and then points were awarded incrementally for more frequent intake, with 1 point for '<once per month', 2 points for ' $1-3$ times per month', 3 points for 'once per week', 4 points for ' $2-4$ times per week' and 5 points for those reporting ' $\geq 5$ times per week'. A number of frequently consumed fruit (apples, bananas and oranges) and vegetable (peas, broccoli, carrots and lettuce) items had a scoring range to reflect more frequent consumption to account for this, with 6 points awarded for 'once per day' and 7 points for ' 2 or more times per day'.

Total energy intake (kJ/day) and total fat intake (g/day) was calculated from the AES FFQ. Total servings of fruit and vegetables/day were calculated by summing the weight of relevant food items estimated by the AES FFQ, divided by the standard serving size dictated by the Australian Guide to Healthy Eating (fruit serving $150 \mathrm{~g}$, vegetable serving $75 \mathrm{~g}$ ) [24]. Height was measured using a portable BSM370 stadiometer correct to $0.1 \mathrm{~cm}$ using the stretch stature method and weight was measured using the Inbody720 Body Composition Analyzer (Biospace Co., Ltd., Seoul, Korea). Body mass index (BMI) was calculated using the standard equation (weight $\mathrm{kg} /$ height $\mathrm{m}^{2}$ ). Age and sex were recorded by questionnaire.

\subsection{Statistical Analysis}

Data were analyzed using Stata Version 12 (StataCorp. 2011. Stata Statistical Software: StataCorp LP, College Station, TX, USA) using an alpha level of 0.05 . The relationship between skin yellowness due to dietary carotenoid (overall $\mathrm{b}^{*}$ value calculated as the average across the three body sites at the radiale, medial humeral epicondyle and palmar thenar muscle) and the two dietary indices (ARFS and FAVVA) were evaluated in three ways. Firstly, Spearman's correlation coefficients, due to the non-normal distribution of dietary intake, were used to compare the strength of the linear relationship between overall $b^{*}$ and diet index scores. Correlation strength was described as poor $<0.20$, moderate $0.2-0.6$, or strong $>0.6$, as previously identified within dietary validation studies $[27,28]$. Secondly, linear regression models were used to examine how much of the variation in skin yellowness (overall $b^{*}$ value) was explained by scores for each diet index. This included an unadjusted model and an adjusted model. The unadjusted model did not account for any confounders, while the adjusted model included the following potential confounders in the model; sex, skin lightness $\left(\mathrm{L}^{*}\right)$ —as melanin affects both skin yellowness $\left(b^{*}\right)$ and lightness $\left(\mathrm{L}^{*}\right)[21]$ —and total fat intake (g/day) —as carotenoids are fat soluble and bioavailability is affected by dietary fat [29]. The regression models were bootstrapped [30] to obtain estimates of the standard errors of the coefficients with replications in the order of 100 [31]. 
$R^{2}$ values and regression coefficients (95\% Confidence Intervals) are also reported. Lastly, the precision of agreement between categorical ranking of skin yellowness $\left(b^{*}\right)$ and diet index score by tertiles was tested using weighted Kappa $\left(\mathrm{K}_{\mathrm{w}}\right)$ statistics.

\section{Results}

\subsection{Study Population}

A total of 148 adults completed the AES FFQ and had their skin coloration measured. The key characteristics of these participants are summarized in Table 1.

Table 1. Characteristics of participants $(n=148)$.

\begin{tabular}{|c|c|}
\hline Characteristic & Mean \pm SD or $n(\%)$ \\
\hline Age (years) & $21.7 \pm 2.2$ \\
\hline Female & $82(55.4 \%)$ \\
\hline Weight (kg) & $70.9 \pm 15.8$ \\
\hline Height $(\mathrm{cm})$ & $171.7 \pm 9.6$ \\
\hline $\operatorname{BMI}\left(\mathrm{kg} / \mathrm{m}^{2}\right)$ & $23.9 \pm 4.1$ \\
\hline Energy intake (kJ/day) & $9238.0 \pm 3004.9$ \\
\hline Fat intake (g/day) & $78.4 \pm 28.6$ \\
\hline Fruit serves/day & $1.8 \pm 1.5$ \\
\hline Vegetable serves/day & $4.4 \pm 2.4$ \\
\hline \multicolumn{2}{|l|}{ ARFS (total possible score) } \\
\hline Total Score (73) & $32.5 \pm 9.8$ \\
\hline Vegetables (21) & $11.7 \pm 5.0$ \\
\hline Fruit (12) & $5.1 \pm 3.2$ \\
\hline Protein-Meat (7) & $2.3 \pm 1.4$ \\
\hline Protein-Vegetarian sources (6) & $2.0 \pm 1.4$ \\
\hline Breads/cereals-Grains (13) & $5.5 \pm 2.2$ \\
\hline Dairy (11) & $4.2 \pm 1.8$ \\
\hline Spreads/Sauces (2) & $1.0 \pm 0.8$ \\
\hline \multicolumn{2}{|l|}{ FAVVA (total possible score) } \\
\hline Total Score (190) & $85.1 \pm 25.4$ \\
\hline Vegetables (122) & $56.3 \pm 17.2$ \\
\hline Fruit (68) & $28.8 \pm 11.6$ \\
\hline \multicolumn{2}{|l|}{ Skin coloration reflectance spectroscopy } \\
\hline Overall L* & $64.3 \pm 3.6$ \\
\hline Overall $a^{*}$ & $8.6 \pm 1.5$ \\
\hline Overall b* & $16.7 \pm 2.4$ \\
\hline
\end{tabular}

$\mathrm{L}^{*}$ values represent skin lightness, $\mathrm{a}^{*}$ values represent skin redness and $\mathrm{b}^{*}$ values represent skin yellowness. Overall values represent average skin color across the three body sites (radiale, medial humeral epicondyle and palmar thenar muscle). Abbreviations: $\mathrm{SD}=$ Standard Deviation, $\mathrm{BMI}=$ Body Mass Index, ARFS = Australian Recommended Food Score, FAVVA= Fruit And Vegetable VAriety Score.

\subsection{Association between Skin Coloration Overall b* (Skin Yellowness) and Diet Indices}

Table 2 summarizes the spearman's correlations and linear regression analyses (unadjusted and adjusted) for all associations. Table 3 summarizes the extent of agreement between tertiles of skin yellowness $\left(b^{*}\right)$, and each dietary index and sub-scale variable using weighted Kappa statistics. 


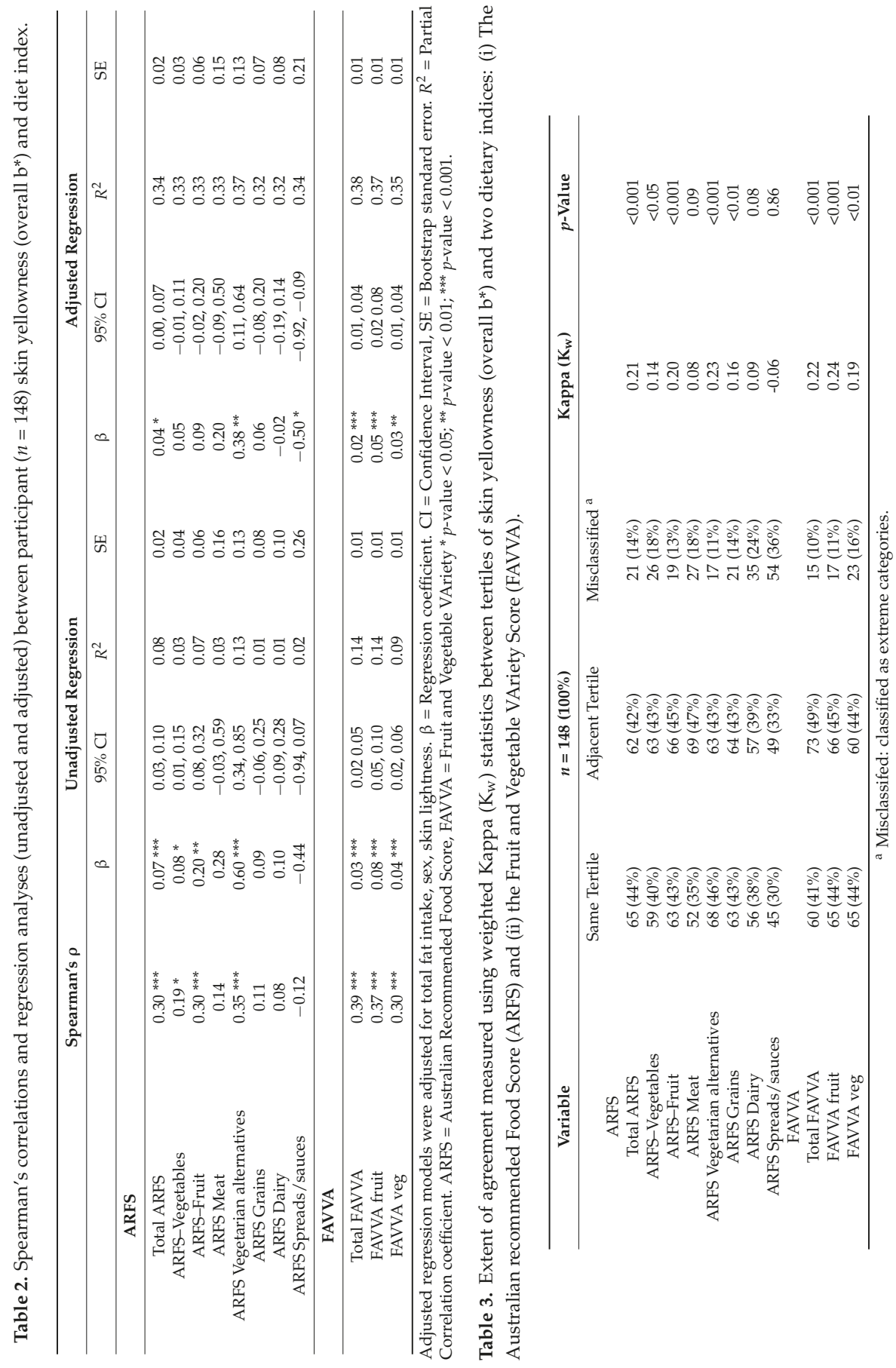




\section{(i) Spearman's Correlation}

ARFS: There was a statistically significant moderate positive correlation between total ARFS and skin yellowness $\mathrm{b}^{*}(\rho=0.30, p<0.001)$, and for the ARFS sub-scales of fruit $(\rho=0.30, p<0.001)$, and vegetarian alternatives $(\rho=0.35, p<0.001)$. Although the vegetable sub-scale was significantly correlated $(p<0.05)$ with skin yellowness $b^{*}$, this association was classified as poor $(\rho<0.2)$. No other associations with sub-scales reached statistical significance.

FAVVA: For the total FAVVA score there were statistically significant, moderate positive correlations with skin yellowness $b^{*}(\rho=0.39, p<0.001)$, and the sub-scales of fruit $(\rho=0.37, p<0.001)$ and vegetables $(\rho=0.30, p<0.001)$ (Table 2$)$.

(ii) Linear Regression Analysis

ARFS: In the unadjusted regression model, there were statistically significant positive associations between total ARFS and skin yellowness $b^{*}(\beta=0.07, p<0.001)$, and for the ARFS sub-scales of fruit ( $\beta=0.20, p<0.01)$ vegetables $(\beta=0.08, p<0.05)$, and vegetarian alternatives $(\beta=0.60, p<0.001)$. The significant associations between skin yellowness $\left(b^{*}\right)$ and total ARFS $(\beta=0.04, p<0.05)$ and vegetarian alternatives $(\beta=0.38, p<0.001)$ remained statistically significant in the fully adjusted regression model. In this fully adjusted model, the spreads/sauces sub-scale was negatively associated with skin yellowness $b^{*}(\beta=-0.50, p<0.05)$.

FAVVA: In the unadjusted regression model, there were statistically significant positive associations between total FAVVA and skin yellowness $b^{*}(\beta=0.03, p<0.001)$, and for the FAVVA sub-scales of fruit $(\beta=0.08, p<0.001)$ and vegetables $(\beta=0.04, p<0.001)$, all of which remained significant in the fully adjusted regression analyses (Table 2 ).

(iii) Agreement Using Weighted Kappa $\left(\mathrm{K}_{\mathrm{W}}\right)$ Statistics

ARFS: Level of agreement based on categorical rankings indicated significant agreement by tertile of total ARFS and skin yellowness $b^{*}\left(K_{W}=0.21, p<0.001\right)$, and also for the sub-scales of vegetables $\left(\mathrm{K}_{\mathrm{W}}=0.14, p<0.05\right)$, fruit $\left(\mathrm{K}_{\mathrm{W}}=0.20, p<0.001\right)$, vegetarian alternatives $\left(\mathrm{K}_{\mathrm{W}}=0.23, p<0.001\right)$ and grains $\left(\mathrm{K}_{\mathrm{W}}=0.16, p<0.01\right)$ (Table 3$)$.

FAVVA: Level of agreement based on categorical rankings was significant for total FAVVA score $\left(\mathrm{K}_{\mathrm{W}}=0.22, p<0.001\right)$, FAVVA fruit sub-scale $\left(\mathrm{K}_{\mathrm{W}}=0.24, p<0.001\right)$ and FAVVA vegetable sub-scale $\left(K_{W}=0.19, p<0.01\right)$ when compared to tertiles of skin yellowness ( $\left.b^{*}\right)$ (Table 3$)$.

\section{Discussion}

This is the first study to explore the associations between brief dietary indices reflecting intakes of nutrient-dense, healthy foods and skin coloration, measured objectively using skin reflectance spectroscopy. Results indicate that in young adults aged 18 to 25 years, a higher diet quality score and a high fruit and vegetable variety scores, as assessed using the ARFS and the FAVVA indices, was related to higher skin yellowness $\left(b^{*}\right)$. This was demonstrated through statistically significant positive correlations between dietary index scores and skin yellowness $\left(b^{*}\right)$, and also by agreement (Kappa) across quantiles, which remained significant in linear regression analyses adjusted for sex, total fat intake (g/day), and skin lightness $\left(\mathrm{L}^{*}\right)$. The total ARFS score and sub-scales for fruit and vegetarian alternatives were moderately correlated with skin yellowness $\left(b^{*}\right)$. While the total FAVVA score and sub-scales for fruit and vegetables were also moderately correlated with skin yellowness $\left(b^{*}\right)$. This suggests that measurement of skin yellowness using reflectance spectroscopy could potentially be used as an efficient and objective way of predicting overall dietary quality and intakes of carotenoid-rich foods. However, the moderate correlations in the current study may not be considered large enough to have confidence in using the reflectance spectroscopy as a diagnostic tool and further research is required to explore these associations in larger and more diverse populations.

For ARFS, the association with vegetarian alternative foods was not expected, but may be due to the high carotenoid content of foods in this category, specifically lutein, zeaxanthin and $\beta$-carotene, 
found in legumes such as lentils, beans and chickpeas [32]. Previous research has consistently found higher intakes of foods containing dietary carotenoids are associated with higher skin yellowness $\left(b^{*}\right)$. For example a cross-sectional study in young men and women in the UK found significant positive correlations between skin yellowness and dietary $\beta$-carotene intake $(\rho=0.29, p=0.013)$ after controlling for exercise [11]. A recent RCT in young Australian women that examined the impact of consuming foods high versus low in dietary carotenoids found significant positive correlations between the change in skin yellowness $\left(b^{*}\right)$ and change in plasma carotenoid concentrations of $\alpha$-carotene $(\rho=0.29$, $p<0.05), \beta$-carotene $(\rho=0.35, p<0.001)$ and total carotenoids $(\rho=0.27, p<0.05)[13]$.

The negative relationship between ARFS spreads/sauces sub-scale with skin yellowness was not expected, and may have reduced the association with the total ARFS score. For each additional point scored for spreads/sauces sub-scale, there was a decrease in 0.50 units in overall skin yellowness. This sub-scale was included in the diet quality score because yeast extract spread and tomato ketchup/barbecue sauce contain a large amount of B-group vitamins and $\beta$-carotene (respectively) [33], which have been shown to be associated with skin color [11,13]. This suggested that higher scores in this population potentially reflect co-consumption of foods low in dietary carotenoids that may displace nutrient-dense foods. An example of this would be tomato sauce with a meat pie, but without vegetables. Further research into the dietary patterns in this specific age group is warranted. In addition, the fat-soluble nature of dietary carotenoids may help explain the negative relationship with spreads/sauces sub-scale [29].

Total FAVVA score and sub-scales for fruit and vegetables were all significantly associated with skin yellowness $\left(\mathrm{b}^{*}\right)$. The FAVVA score reflects frequency and variety of fruit and vegetables consumed, and therefore these results were expected, given that previous studies have also reported higher fruit and vegetable consumption to be associated with higher skin yellowness $\left(b^{*}\right)$ [11-13]. The correlation coefficients in the current analysis are comparable to a cross-sectional study in 82 young men and women in the UK, which demonstrated a significant correlation $(\rho=0.25, p=0.03)$ between usual fruit and vegetable intakes and skin yellowness after controlling for exercise [11]. Another study that examined change in fruit and vegetable intakes in 35 young men and women over a six-week period [12] found a modest increase in intake was associated with a significant increase in skin yellowness $(b=0.31, p=0.05)$. These significant relationships highlight the sensitivity of reflectance spectroscopy in detecting carotenoids in skin, which is important, given young adults generally have low fruit and vegetables intakes [18]. In Australia; the most recent National Health Survey reported that $97 \%$ of young Australian adults aged 18 to 24 years fail to meet national recommendations of two serves fruit/day and 5-6 serves of vegetable/day [18]. Reported intakes of vegetables and fruit were higher in the current study and although some of the differences could be explained by the different dietary methods used to quantify intake, the majority of young adults still failed to achieve national recommendations ( $n=124 / 148,84 \%$ ). An important insight from the current analysis is the potential for reflectance spectroscopy to predict diet intake of individuals across a range of differing intakes, and the ability to identify those who consume a greater variety of vegetables and fruit more frequently.

\subsection{Implications for Research}

Traditional methods of dietary assessment can be burdensome and resource intensive [34]. Results from the current study highlight that a rapid, non-invasive assessment of skin coloration, measured objectively using reflectance spectroscopy at three body sites may be sufficient to estimate overall diet quality and frequency and variety of usual dietary intake, particularly for fruit and vegetables. This is important for population groups where recall of dietary intake is challenging, or where resources mean that only a brief assessment of diet is warranted.

Diet quality has consistently been shown to have an inverse relationship with all-cause morbidity and mortality [14,35], while consuming a greater variety of fruit and vegetables is associated with lower odds of developing metabolic syndrome, obesity, hypercholesterolemia and hypertension [36,37]. As such, the associations between skin yellowness measured using reflectance spectroscopy and the 
dietary indices evaluated in the current study may therefore be indicative of disease risk. Future research should examine whether skin coloration, measured using reflectance spectroscopy, can predict health status and disease risk in diverse populations.

\subsection{Strengths and Limitations}

A key limitation of the current study is the cross-sectional design, and hence only associations between intake and skin yellowness could be evaluated. In addition, the diversity of the samples of dietary intakes across the two datasets due to differences in eligibility criteria may have affected the ability to identify stronger associations. In addition, responses from the AES FFQ were self-reported and therefore subject to reporting bias [38]. While other studies have explored relationships between carotenoids and skin redness [12,39], this was not assessed in the current study, due to the greater contribution of dietary carotenoids in the skin on the appearance of skin yellowness $\left(b^{*}\right)$ [3]. Strengths include use of an objective measure of skin color using reflectance spectroscopy with assessments taken at standardized anthropometric sites and operating procedure. In addition, to the best of the authors' knowledge, this is the first evaluation of skin coloration in relation to brief dietary indices in a relatively large number of adults.

\section{Conclusions}

The present study indicates that overall diet quality and fruit and vegetable variety, assessed using two brief dietary indices (ARFS and FAVVA), were significantly associated with skin yellowness $\left(b^{*}\right)$, although those associations were weaker after adjustment for skin lightness $\left(\mathrm{L}^{*}\right)$, sex, and total fat intake. This suggests that measurement of skin coloration using reflectance spectroscopy could potentially be used as a predictor of overall dietary quality and variety. Future research should examine these associations in more diverse populations and also examine whether skin coloration can predict health status and disease risk.

Supplementary Materials: The following are available online at http:/ /www.mdpi.com/2072-6643/10/1/11/s1, Table S1: Scoring method for items in the ARFS, Table S2: Scoring method for items in the FAVVA index.

Acknowledgments: The research from data set one was supported by a scholarship top-up grant from the Hunter Medical Research Institute (HMRI). The research from data set two was funded by a HMRI (14-30) project grant. CC is supported by a National Health and Medical Research Council of Australia Senior Research Fellowship and a University of Newcastle, Faculty of Health and Medicine Gladys M. Brawn Senior Research Fellowship. MH is supported by a University of Newcastle Gladys M Brawn Career Development Fellowship (Teaching Assistance).

Author Contributions: K.P., C.C., and M.H. conceived and designed the experiments in the research for data set one, while L.A., C.C., M.R. and M.H. conceived and designed the experiments in the research for data set two; L.A. analyzed the data. All authors contributed to data interpretation, commented on drafts and approved the final manuscript; L.A. and K.P. wrote the paper.

Conflicts of Interest: The authors declare no conflict of interest.

\section{References}

1. Arscott, S. Food sources of carotenoids. In Carotenoids and Human Health; Tanumihardjo, S., Ed.; Humana Press: Totowa, NJ, USA, 2013.

2. Darvin, M.E.; Sterry, W.; Lademann, J.; Vergou, T. The role of carotenoids in human skin. Molecules 2011, 16, 10491-10506. [CrossRef]

3. Alaluf, S.; Heinrich, U.; Stahl, W.; Tronnier, H.; Wiseman, S. Dietary carotenoids contribute to normal human skin color and UV photosensitivity. J. Nutr. 2002, 132, 399-403. [PubMed]

4. Mayne, S.T.; Cartmel, B.; Scarmo, S.; Lin, H.Q.; Leffell, D.J.; Welch, E.; Ermakov, I.; Bhosale, P.; Bernstein, P.S.; Gellermann, W. Noninvasive assessment of dermal carotenoids as a biomarker of fruit and vegetable intake. Am. J. Clin. Nutr. 2010, 92, 794-800. [CrossRef] [PubMed]

5. Ermakov, I.; Gellermann, W. Optical detection methods for carotenoids in human skin. Arch. Biochem. Biophys. 2015, 572, 101-111. [CrossRef] [PubMed] 
6. Jahns, L.; Johnson, L.K.; Mayne, S.T.; Cartmel, B.; Picklo, M.J.; Ermakov, I.; Gellermann, W.; Whigham, L.D. Skin and plasma carotenoid response to a provdied intervention diet high in vegetables and fruit: Uptake and depletion kinettics. Am. J. Clin. Nutr. 2014, 100, 930-937. [CrossRef] [PubMed]

7. Scarmo, S.; Cartmel, B.; Lin, H.; Leffell, D.J.; Welch, E.; Bhosale, P.; Bernstein, P.S.; Mayne, S.T. Significant correlations of dermal total carotenoids and lycopene with their respective plasma levels in healthy adults. Arch. Biochem. Biophys. 2010, 504, 34-39. [CrossRef] [PubMed]

8. Aguilar, S.S.; Wengreen, H.J.; Lefevre, M.; Madden, G.J.; Gast, J. Skin carotenoids: A biomarker of fruit and vegetable intake in children. J. Acad. Nutr. Diet. 2014, 114, 1174-1180. [CrossRef] [PubMed]

9. Aguilar, S.S.; Wengreen, H.J.; Dew, J. Skin carotenoid response to a high-carotenoid juice in children: A randomized clinical trial. J. Acad. Nutr. Diet. 2015, 115, 1771-1778. [CrossRef] [PubMed]

10. Pezdirc, K.; Hutchesson, M.J.; Whitehead, R.; Ozakinci, G.; Perrett, D.; Collins, C.E. Fruit, vegetable and dietary carotenoid intakes explain variation in skin-color in young caucasian women: A cross-sectional study. Nutrients 2015, 7, 5800-5815. [CrossRef] [PubMed]

11. Stephen, I.D.; Coetzee, V.; Perrett, D.I. Carotenoid and melanin pigment coloration affect perceived human health. Evol. Hum. Behav. 2011, 32, 216-227. [CrossRef]

12. Whitehead, R.D.; Re, D.; Xiao, D.; Ozakinci, G.; Perrett, D.I. You are what you eat: Within-subject increases in fruit and vegetable consumption confer beneficial skin-color changes. PLoS ONE 2012, 7, e32988. [CrossRef] [PubMed]

13. Pezdirc, K.; Hutchesson, M.J.; Williams, R.L.; Rollo, M.E.; Burrows, T.L.; Wood, L.G.; Oldmeadow, C.; Collins, C.E. Consuming high-carotenoid fruit and vegetables influences skin yellowness and plasma carotenoids in young women: A single-blind randomized crossover trial. J. Acad. Nutr. Diet. 2016, 116, 1257-1265. [CrossRef] [PubMed]

14. Wirt, A.; Collins, C.E. Diet quality-What is it and does it matter? Public Health Nutr. 2009, 12, $2473-2492$. [CrossRef] [PubMed]

15. Ashton, L.M.; Williams, R.L.; Wood, L.; Schumacher, T.; Burrows, T.; Rollo, M.; Pezdirc, K.; Collins, C. Comparison of australian recommended food score (ARFS) and plasma carotenoid concentrations: A validation study in adults. Nutrients 2017, 9, 888. [CrossRef] [PubMed]

16. Burrows, T.; Williams, R.; Rollo, M.; Wood, L.G.; Garg, M.; Jensen, M.; Collins, C. Plasma carotenoid levels as biomarkers of dietary carotenoid consumption: A systematic review of the validation studies. J. Nutr. Intermed. Metab. 2015, 2, 15-64. [CrossRef]

17. Ashton, L.M.; Morgan, P.J.; Hutchesson, M.J.; Rollo, M.E.; Collins, C.E. Feasibility and preliminary efficacy of the 'heyman' healthy lifestyle program for young men: A pilot randomised controlled trial. Nutr. J. 2017, 16, 2. [CrossRef] [PubMed]

18. Australian Bureau of Statistics. Australian Bureau of Statistics. Australian Health Survey: First Results 2014-2015. Available online: http:/ / www.abs.gov.au/AUSSTATS/abs@.nsf/DetailsPage/4364.0.55.001201415?OpenDocument (accessed on 4 January 2017).

19. Marfell-Jones, M.J.; Stewart, A.; De Ridder, J. International Standards for Anthropometric Assessment; International Society for the Advancement of Kinanthropometry: Wellington, New Zealand, 2012.

20. Stephen, I.D.; Coetzee, V.; Smith, M.L.; Perrett, D.I. Skin blood perfusion and oxygenation colour affect perceived human health. PLoS ONE 2009, 4, e5083. [CrossRef] [PubMed]

21. Stamatas, G.N.; Zmudzka, B.Z.; Kollias, N.; Beer, J.Z. Non-invasive measurements of skin pigmentation in situ. Pigment Cell Melanoma Res. 2004, 17, 618-626. [CrossRef] [PubMed]

22. Collins, C.E.; Boggess, M.M.; Watson, J.F.; Guest, M.; Duncanson, K.; Pezdirc, K.; Rollo, M.; Hutchesson, M.J.; Burrows, T.L. Reproducibility and comparative validity of a food frequency questionnaire for australian adults. Clin. Nutr. 2014, 33, 906-914. [CrossRef] [PubMed]

23. Collins, C.E.; Burrows, T.L.; Rollo, M.E.; Boggess, M.M.; Watson, J.F.; Guest, M.; Duncanson, K.; Pezdirc, K.; Hutchesson, M.J. The comparative validity and reproducibility of a diet quality index for adults: The australian recommended food score. Nutrients 2015, 7, 785-798. [CrossRef] [PubMed]

24. National Health and Medical Research Council (NHMRC). Eat for Health: Australian Dietary Guidelines; Department of Health and Ageing, Research Council Canberra: Canberra, Australia, 2013.

25. Collins, C.E.; Young, A.F.; Hodge, A. Diet quality is associated with higher nutrient intake and self-rated health in mid-aged women. J. Am. Coll. Nutr. 2008, 27, 146-157. [CrossRef] [PubMed] 
26. Aljadani, H.M.; Patterson, A.; Sibbritt, D.; Hutchesson, M.J.; Jensen, M.E.; Collins, C.E. Diet quality, measured by fruit and vegetable intake, predicts weight change in young women. J. Obes. 2013, 2013. [CrossRef] [PubMed]

27. McNaughton, S.; Hughes, M.; Marks, G. Validation of a FFQ to estimate the intake of PUFA using plasma phospholipid fatty acids and weighed foods records. Br. J. Nutr. 2007, 97, 561-568. [CrossRef] [PubMed]

28. Schumacher, T.; Burrows, T.; Rollo, M.; Wood, L.; Callister, R.; Collins, C. Comparison of fatty acid intakes assessed by a cardiovascular-specific food frequency questionnaire with red blood cell membrane fatty acids in hyperlipidaemic australian adults: A validation study. Eur. J. Clin. Nutr. 2016, 70, 1433-1438. [CrossRef] [PubMed]

29. Van het Hof, K.H.; West, C.E.; Weststrate, J.A.; Hautvast, J.G. Dietary factors that affect the bioavailability of carotenoids. J. Nutr. 2000, 130, 503-506. [PubMed]

30. Efron, B.; Tibshirani, R. An Introduction to the Bootstrap; Chapman \& Hall/CRC: New York, NY, USA, 1994.

31. Poi, B. From the help desk; some bootstrapping techniques. Stata J. 2004, 4, 312-328.

32. EL-Qudah, J.M. Estimation of carotenoid contents of selected mediterranean legumes by HPLC. World J. Med. Sci. 2014, 10, 89-93.

33. FSANZ. Nuttab 2006-Australian Food Composition Tables. Available online: http:/ / www.foodstandards. gov. $\mathrm{au} /$ science/monitoringnutrients/nutrientables/nuttab/pages/default.aspx (accessed on 14 October 2017).

34. Ashman, A.M.; Collins, C.E.; Brown, L.J.; Rae, K.M.; Rollo, M.E. A brief tool to assess image-based dietary records and guide nutrition counselling among pregnant women: An evaluation. JMIR mHealth uHealth 2016, 4. [CrossRef] [PubMed]

35. Lassale, C.; Gunter, M.J.; Romaguera, D.; Peelen, L.M.; Van der Schouw, Y.T.; Beulens, J.W.; Freisling, H.; Muller, D.C.; Ferrari, P.; Huybrechts, I. Diet quality scores and prediction of all-cause, cardiovascular and cancer mortality in a pan-european cohort study. PLoS ONE 2016, 11, e0159025. [CrossRef] [PubMed]

36. Azadbakht, L.; Mirmiran, P.; Azizi, F. Dietary diversity score is favorably associated with the metabolic syndrome in tehranian adults. Int. J. Obes. 2005, 29, 1361-1367. [CrossRef] [PubMed]

37. Azadbakht, L.; Mirmiran, P.; Esmaillzadeh, A.; Azizi, F. Dietary diversity score and cardiovascular risk factors in tehranian adults. Public Health Nutr. 2006, 9, 728-736. [CrossRef] [PubMed]

38. Calvert, C.; Cade, J.; Barrett, J.; Woodhouse, A.; Group, U.S. Using cross-check questions to address the problem of mis-reporting of specific food groups on food frequency questionnaires. Eur. J. Clin. Nutr. 1997, 51, 708-712. [CrossRef] [PubMed]

39. Tan, K.; Graf, B.; Mitra, S.; Stephen, I. Daily consumption of a fruit and vegetable smoothie alters facial skin color. PLoS ONE 2015, 10, e0133445. [CrossRef] [PubMed]

(C) 2017 by the authors. Licensee MDPI, Basel, Switzerland. This article is an open access article distributed under the terms and conditions of the Creative Commons Attribution (CC BY) license (http:/ / creativecommons.org/licenses/by/4.0/). 


\title{
Dietary Management of Skin Health: The Role of Genistein
}

\author{
Natasha Irrera ${ }^{1, \dagger}{ }^{\text {, Gabriele Pizzino }}{ }^{1, \dagger}{ }^{,}$, Rosario D’Anna ${ }^{2}$, Mario Vaccaro ${ }^{1}$, Vincenzo Arcoraci ${ }^{1}$, \\ Francesco Squadrito ${ }^{1, *}$, Domenica Altavilla ${ }^{3}$ and Alessandra Bitto ${ }^{1}$ \\ 1 Department of Clinical and Experimental Medicine, University of Messina, Messina 98125, Italy; \\ nirrera@unime.it (N.I.); cgpizzino@unime.it (G.P.); vaccaro@unime.it (M.V.); \\ varcoraci@unime.it (V.A.); abitto@unime.it (A.B.) \\ 2 Department of Human Pathology, University of Messina, Messina 98125, Italy; rdanna@unime.it \\ 3 Department of Biomedical Sciences, Dentistry and Morphological and Functional Images, \\ University of Messina, Messina 98125, Italy; daltavilla@unime.it \\ * Correspondence: fsquadrito@unime.it; Tel.: +39-090-2213648; Fax: +39-090-2213300 \\ $+\quad$ N.I. and G.P. equally contributed to this paper.
}

Received: 14 April 2017; Accepted: 14 June 2017; Published: 17 June 2017

\begin{abstract}
In women, aging and declining estrogen levels are associated with several cutaneous changes, many of which can be reversed or improved by estrogen supplementation. Two estrogen receptors $-\alpha$ and $\beta$-have been cloned and found in various tissue types. Epidermal thinning, declining dermal collagen content, diminished skin moisture, decreased laxity, and impaired wound healing have been reported in postmenopausal women. Experimental and clinical studies in postmenopausal conditions indicate that estrogen deprivation is associated with dryness, atrophy, fine wrinkling, and poor wound healing. The isoflavone genistein binds to estrogen receptor $\beta$ and has been reported to improve skin changes. This review article will focus on the effects of genistein on skin health.
\end{abstract}

Keywords: genistein; skin; estrogen receptor beta

\section{Introduction}

Aging is associated with a reduction in skin thickness and in the number of epithelial cells, with a concurrent decrease in stromal collagen [1]. The skin ageing process may intensify after menopause, and estrogen deficiency seems to have a direct effect on the epidermis. It seems that estrogen stimulation could encourage the proliferation of keratinocytes, leading to thickening of the epidermis, preventing its atrophy [2]. In the dermis, blood vessels would be stimulated again, and fibroblast production would also be stepped up, thus preserving the components that they secrete (e.g., collagen, elastic fibres, and glycosaminoglycans) [2,3]. Estrogen exerts its actions through two different receptors (estrogen receptors, ERs), also found in the skin. Both ERs are distinct proteins encoded by separate genes on different chromosomes. ER $\beta$ is located on human chromosome 14, whereas ER $\alpha$ is found on chromosome 6. Ligand binding activates the receptors that act as transcription factors by binding conserved estrogen response elements in the regulatory regions of target genes. Interestingly, many genes that are induced by estrogens lack estrogen response elements, including epidermal growth factor, epidermal growth factor receptor, and the cell cycle-associated cyclin D1. These genes belong to the group of secondary estrogen responsive genes, and estrogen affects their transcription/expression through activation of cytoplasmic signaling pathways such as Src/Shc/ERK. These secondary messengers are known to be activated by many transmembrane tyrosine kinase growth factor receptors, suggesting that estrogen effect may sometimes augment growth factor receptor activation [4]. In some cells, ER $\beta$ counteracts $E R \alpha$, in some cases acting as an ER $\alpha$ heterodimer to 
inhibit the transactivating function of $\mathrm{ER} \alpha$, and in other cases acting as a homodimer to regulate specific genes, many of which are anti-proliferative [5]. The binding of estrogens to the ERs triggers specific responses in terms of proliferation activation and apoptosis arrest; nonetheless, some estrogen actions require the intervention of other hormones, such as progesterone and androgens. The variation in the distribution of receptors within the skin suggests that each has a different, cell-specific role. ER $\beta$ is the predominant estrogen receptor in adult human skin; it is strongly expressed in the stratum basale and stratum spinosum of the epidermis [6,7]. The same receptor is also found activated in fibroblasts from female skin, suggesting a strong involvement of ER $\beta$ in maintaining skin homeostasis [7]. Furthermore, estrogens prolong the anagen phase of scalp hair growth by increasing cell proliferation rates and postponing their transition to the telogen phase. ER $\alpha$ expression is limited to the dermal papilla cells of the hair follicle, whereas ER $\beta$ is found in the outer root sheath cells, epithelial matrix cells, dermal papilla cells, and the cells of the specialized bulge region of the outer root sheath [8]. It has been collectively demonstrated that ER $\alpha$ expression is quite low in the epidermis, while ER $\beta$ is abundantly represented.

Considering the presence and distribution of estrogen receptors within the skin and its annexes, it is not difficult to understand that hormone replacement therapy (HRT) can improve the menopause-related alterations. However, given the serious side effects of HRT (e.g., an increased risk of blood clots and certain types of cancer), a number of alternatives have been explored during the last decades, and the most intriguing seems to be the use of phytoestrogens. Isoflavones and lignans are the two main groups of phytoestrogens; their activity is generally weaker than endogenous estrogens, and they are not stored within the tissues [9]. Many phytoestrogens possess both agonist and antagonist estrogen properties, and their estrogenic activity is demonstrated through interaction with both ER $\alpha$ and ER $\beta$ [10]. Genistein (5,7,4'-trihydroxyisoflavone) is an isoflavone abundantly found in soy and other legumes, and acts as a selective estrogen receptor modulator (SERM), mainly binding to $\operatorname{ER} \beta$ [11]. Genistein has a 30-fold higher affinity for $\operatorname{ER} \beta$ than $\operatorname{ER} \alpha$; when genistein is bound to $\mathrm{ER} \beta$, helix 12 does not adopt an agonist conformation, but instead has a position more similar to that seen with an antagonist. This result is unexpected because the molecular shape and volume of genistein and estradiol are very similar, and because genistein is a partial (60-70\% of E2) agonist of $\mathrm{ER} \beta$. The conformation of helix 12 in the ER $\beta$-genistein structure may account for the different effects exerted by genistein compared to estradiol.

Besides the specific estrogen receptor-related effects, genistein also acts as an antioxidant and as a specific inhibitor of tyrosine kinases, affecting many signaling pathways [12]. Genistein-as with all the other isoflavones-exists in two different forms: the glycosylated (genistin) and the aglycone (genistein). The aglycone genistein is absorbed from the intestine and conjugated with glucuronic acid during transport across the intestinal epithelial cells. After transport to the liver, the glucuronide may be excreted in the bile, whereafter it could re-enter the small intestine, allowing genistein to be deconjugated, absorbed, and metabolized for the second time [13]. In vivo bioavailability of genistein and its glycoside genistin indicates that genistein is readily bioavailable, while the glycoside derivative is poorly absorbed in the small intestine due to the higher molecular weight and hydrophilicity [14]. For this reason, the most studied form of genistein is the aglycone, which so far has been used in preclinical and clinical studies. Genistein supplementation in postmenopausal women is quite popular; in fact, several nutraceutical preparations have been launched in the market with very different amounts of genistein alone or combined with other isoflavones. However, the beneficial effects of genistein on menopausal features can be achieved at very specific dosage ( $54 \mathrm{mg} /$ day), and only few preparations fulfill this requirement. In this review article are summarized the main effects of genistein on skin health from preclinical and clinical observations. This is a narrative review focusing on studies performed using genistein in vitro or in vivo to demonstrate an effect on skin. As search terms on Pubmed, we used "genistein and skin", "genistein and fibroblast", "genistein and wound healing", and we selected all those articles with available full text. As a further criterion, articles using mixes of isoflavones without clear indications of genistein's dose were not included. 


\section{In Vitro Evidence}

\subsection{Genistein Effects on Fibroblasts}

Wound healing and scar formation are dynamic biological processes involving numerous cell-cell and cell-matrix interactions in a complex milieu of both local and systemic influences. In abnormal hyper-proliferating fibroblasts from hypertrophic scars, an important role is played by altered growth factors expression, dysfunctional receptors, and tyrosine kinase signaling dysregulation [15]. Fibroblasts obtained from hypertrophic burn scars were cultured with genistein at different concentrations $(25,50,100 \mu \mathrm{mol} / \mathrm{L})$ to inhibit their growth and proliferation through the blockade of the RAS (Rat sarcoma), Raf, ERK, and p38 proteins [16]. Genistein inhibited cell proliferation and activity by affecting nuclear translocation of phosphorylated ERK molecules; ERK mainly regulates cell proliferation, growth, and differentiation, while p38 is mainly related to stress and inflammatory reactions. These pathways are interconnected, and genistein may exert its suppressing effects on cells' proliferation by interfering with them [16].

Abnormal scarring can also result in keloid formation, characterized by an imbalanced extracellular matrix (ECM) synthesis and degradation, a CTGF (connective tissue growth factor)-dependent fibroblast proliferation, and resistance to apoptosis. Normal human dermal fibroblasts (NHDF, Adult) and keloid fibroblasts (KEL FIB) were tested with different concentrations of genistein; the keloid fibroblast culture revealed an increase of CTGF mRNA and an increase of CTGF protein expression compared to normal fibroblast, confirming the contribution of CTGF in keloid fibroblast pathology [17]. Genistein decreased mRNA and protein expression of CTGF in keloid fibroblast in a concentration-dependent manner. Moreover, genistein decreases TGF $\beta 1, \beta 2$, and $\beta 3$ gene expression in keloid fibroblast, but its potential application as an antifibrotic factor in keloids treatment requires further research. Genistein did not induce p53 and p21 expression, and therefore it seems that it does not induce apoptosis in monoculture of keloids fibroblast. However, it revealed a cytoprotective effect, stimulating BCL-2 gene expression [17]. In another study, normal human epidermal keratinocytes (NHEK), NHDF, and KEL FIB were tested to investigate genistein as a potential regulator of C-JUN, C-FOS, and FOS-B of AP-1 subunits expression. C-JUN and C-FOS expression was lower in keloid fibroblast compared to normal fibroblasts cultured in control conditions. The study demonstrated that genistein modulated C-JUN expression in dermal keratinocytes and fibroblasts in a dose-dependent manner. The expression of C-JUN was significantly higher in keratinocytes treated with genistein compared to control cells [18]. These data further corroborate the potential efficacy of genistein for treating keloid scars.

Collagen synthesis by fibroblast is also crucial for maintaining skin homeostasis and healing of wounds. Genistein effects on collagen biosynthesis and the signaling pathways involved in its regulation were investigated in human dermal fibroblasts [19] under the oxidative stress conditions evoked by $t$-BHP ( $t$-butylhydroperoxide). Genistein exerts biphasic effects on collagen biosynthesis; at $1 \mu \mathrm{M}$, it counteracted collagen biosynthesis inhibition evoked by $t$-BHP in fibroblasts. At $10 \mu \mathrm{M}$, it exerted a significantly diminished protective effect on collagen biosynthesis, whereas at $100 \mu \mathrm{M}$, it potentiated the inhibitory action of $t$-BHP. The study suggested that at nutritionally attainable concentrations $(1 \mu \mathrm{M})$, genistein protects human dermal fibroblasts from oxidative stress-induced collagen biosynthesis inhibition. The mechanism of the protective effect of genistein on collagen biosynthesis in $t$-BHP-treated fibroblasts may be due to the prevention of disturbances in the IGF-I receptor-mediated, ERK1/ERK2-associated signaling pathway evoked by the oxidant [19].

\subsection{Genistein Affects Glycosaminoglycan Synthesis}

Aside from the tyrosine kinase inhibitory effect of genistein, another modulating activity was identified on glycosaminoglycan (GAG) synthesis by blocking the tyrosine kinase activity of the epidermal growth factor receptor [20] in fibroblasts from patients with mucolipidosis II (a mucopolysaccharidosis caused by deficiencies in enzymes involved in degradation of GAG). In this experimental condition, genistein-despite causing a significant reduction in GAG synthesis 
rate-ensures the maintenance of sufficient amounts of GAG, which is necessary for the proper functioning of cells and tissues [21]. In another mucopolysaccharidosis, the Sanfilippo disease, genistein was tested and compared to other flavonoids to reduce GAG synthesis and accumulation in fibroblasts obtained by affected subjects [22]. An inhibition of GAG synthesis was found in the presence of all tested compounds, though the most pronounced impairment of production of GAGs was observed in the presence of kaempferol, daidzein, and genistein; thus, the authors concluded by suggesting that these flavonoids alone or in combination might be a safe treatment option for mucopolysaccharidosis [22].

\subsection{Genistein Effects on ultraviolet (UV)-Protection}

Genistein was also tested for protection against UV-light exposure and prevented the UV radiation-dependent expression of cyclooxygenase-2 (COX-2) in HaCaT cells cultures suppressing both the basal and stimulated expression of cyclooxygenase-2, which suggests that it exerts anti-inflammatory activity [23]. In UVB-irradiated BJ-5ta cells are human skin fibroblast cells immortalized with hTERT, and besides reducing COX-2 expression, genistein also induced Gadd45 gene expression, thereby activating the DNA repair system [24]. The protective effect of genistein against senescence-like characteristics was also tested on human dermal fibroblasts (HDFs) following repeated subcytotoxic exposures to UVB to cause senescence. Genistein reversed the senescence process in HDFs, acting as an antioxidant through the down-regulation of p66Shc protein that involves forkhead protein suppression [25]. These data further suggest that genistein could be a good candidate ingredient for protective agents against UV-induced photodamage.

The effects reported by these in vitro studies have been summarized in Table 1.

Table 1. Genistein effects on in vitro experimental models.

\begin{tabular}{ll}
\hline \multicolumn{1}{c}{ In Vitro Experimental Models } & Genistein Effects \\
\hline $\begin{array}{l}\text { Fibroblasts from hypertrophic burn scars, normal human dermal fibroblasts (NHDF) keloid } \\
\text { fibroblasts (KEL FIB), normal human epidermal keratinocytes (NHEK), NHDF stimulated with }\end{array}$ & $\downarrow$ RAS, RAF, ERK, p38 \\
$t$-BHP (t-butylhydroperoxide) [16-19] & $\uparrow$ BCL-2 \\
& $\uparrow$ C-JUN \\
\hline Fibroblasts from patients with mucolipidosis II and Sanfilippo disease [21,22] $\beta 2$ and $\beta 3$ \\
\hline \\
HaCaT, BJ-5ta, and human dermal fibroblasts exposed to UV radiation [23-25] & $\downarrow$ GAG \\
& $\uparrow$ COX-2 \\
& $\downarrow$ Gadd45 gene expression \\
\hline
\end{tabular}

$\uparrow$ and $\downarrow$ respectively indicate an increase or a reduction of the expression of the molecules. RAS, Rat Sarcoma; RAF, Rapidly Accelerated Fibrosarcoma; ERK, Extracellular signal-regulated Kinase; CTGF, Connective Tissue Growth Factor; TGF $\beta$, Transforming Growth Factor beta, BCL-2, B cell leukemia 2.

\section{In Vivo Studies}

\subsection{Genistein Effect on Photodamage}

Most of the in vivo studies carried out in rodents evaluated the effect of genistein alone or combined with other flavonoids in reducing the damages associated with UV-light exposure. In a long-term (8 weeks) dietary supplementation study with isoflavone-rich fermented soybean $(0.2083 \mu \mathrm{g}$ genistein $/ \mathrm{mg}$ of soybean), hairless albino mice exposed to UVB demonstrated a markedly reduced skin inflammation [26]. Topically applied genistein was shown to reduce the incidence and multiplicity of skin tumors in the dimethylbenz(a)anthracene initiated and 12-O-tetradecanoyl phorbol-13-acetate promoted mouse models [27,28]. In the UVB light-induced complete carcinogenesis model, topical pretreatment of mice with $10 \mu \mathrm{mol}$ genistein significantly reduced the formation of $\mathrm{H}_{2} \mathrm{O}_{2}$ and 8-hydroxy-2-deoxyguanosine [27,28]. Because these are the precursors for free radicals, their attenuation is a significant step for chemoprevention.

The possible anti-nitrosative effect of genistein $(10 \mathrm{mg} / \mathrm{kg}$ or $15 \mathrm{mg} / \mathrm{kg}$, i.p.) in the prevention of skin injury and in the modulation of cell proliferation were tested in Hairless HRS/J mice following 
$24 \mathrm{~h}$ of UVB irradiation [29]. The most effective dose $(10 \mathrm{mg} / \mathrm{kg})$ demonstrated a reduction in lipid peroxides and nitrotyrosine, accompanied by upregulation of both PCNA and Ki67, which indicated that prevention of nitrosative skin injury promoted cell proliferation and DNA repair [29]. In another study, genistein potently inhibited the UVB-induced skin carcinogenesis and photodamage in hairless mice. The possible mechanisms of the anticarcinogenic action hypothesized by the authors include scavenging of reactive oxygen species, blocking of oxidative and photodynamic damage to DNA, inhibition of tyrosine protein kinase, downregulation of EGF-receptor phosphorylation and MAPK activation, and suppression of oncoprotein expression [30].

\subsection{Genistein Effect on Wound Healing}

In wound healing models, genistein was tested in excisional and incisional wound models. ICR mice receiving a genistein-enriched diet $(0.025 \%$ and $0.1 \%$ genistein) for 2 weeks before wounding demonstrated a faster wound repair, likely stimulated by a modulated production of reactive oxygen species (ROS), which in turn downregulated the activity of NF- $k B$ and TNF- $\alpha$ within the first $72 \mathrm{~h}$ following wounding. In ovariectomized 10-week-old C57/Bl6 mice, full thickness skin incisional wounds were systemically treated with 17-estradiol $(0.05 \mathrm{mg}$, 21-day, slow-release 17-estradiol pellet, subcutaneously implanted) or genistein $(50 \mathrm{mg} / \mathrm{kg} /$ day $)$. Genistein substantially accelerated wound repair, associated with a dampened inflammatory response. Unexpectedly, co-treatment with the ER antagonist ICI had little impact on the anti-inflammatory and healing promoting effects of genistein $[31,32]$. Thus, the authors speculated that genistein's actions are only partially mediated via classical estrogen receptor-dependent signaling pathways.

In ovariectomized rats chronically (6 months) treated with genistein $(1$ and $10 \mathrm{mg} / \mathrm{kg} /$ day, subcutaneously), full thickness incisional wounds were made and analyzed after 7 and 14 days [33]. Genistein was able to counteract the delayed wound healing, improving extracellular matrix remodeling and turn-over in OVX rats. Moreover, genistein $(1 \mathrm{mg} / \mathrm{kg})$ was more effective than estradiol or raloxifene on all skin parameters tested at days 7 and 14 after wounding [33]. The same authors also evaluated skin aging in 9-month-old OVX rats chronically treated with genistein, raloxifene, and estradiol [34]. OVX rats showed a decrease in TGF-b1, VEGF, MMP-2, MMP-9, tissue inhibitor of metalloproteinase (TIMP)- 1 and TIMP-2 compared with sham OVX rats. All the treatments significantly restored this depressed molecular profile, but genistein $(1 \mathrm{mg} / \mathrm{kg})$ also significantly increased collagen thickness and skin breaking strength [34].

Collectively (Table 2), these data suggest that dosages as low as $1 \mathrm{mg} / \mathrm{kg}$ could be beneficial for treating skin disorders during estrogen deprivation, while higher dosages (up to $50 \mathrm{mg} / \mathrm{kg}$ ) are needed where a normal estrogenic milieu is present.

Table 2. Genistein effects on in vivo experimental models.

\begin{tabular}{|c|c|c|c|}
\hline In Vivo Model & Dose & Outcomes & Genistein Effects \\
\hline $\begin{array}{l}\text { UVB-irradiation and } \\
\text { carcinogenesis models }[26,27,29]\end{array}$ & $\begin{array}{l}\text { Dietary supplementation } 0.2083 \mu \mathrm{g} / \mathrm{mg} \text { of } \\
\text { fermented soy beans } \\
1,5,10,20 \mu \mathrm{mol} \text { genistein topically applied } \\
\text { Intraperitoneal injections } 10-15 \mathrm{mg} / \mathrm{kg}\end{array}$ & $\begin{array}{l}\text { Anti-inflammatory effect in } \\
\text { hairless mice with } \\
\text { photodamage } \\
\text { Reduction of skin } \\
\text { tumorigenesis in mice } \\
\text { Anti-nitrosative effect in } \\
\text { hairless mice }\end{array}$ & $\begin{array}{l}\downarrow \text { skin inflammation } \\
\downarrow \mathrm{H}_{2} \mathrm{O}_{2} \\
\downarrow \text { 8-hydroxy-2-deoxyguanosine } \\
\downarrow \text { lipid peroxides } \\
\downarrow \text { nitrotyrosine } \\
\downarrow \text { tyrosine protein kinase } \\
\downarrow \text { EGF-receptor phosphorylation } \\
\downarrow \text { MAPK activation } \\
\downarrow \text { oncoprotein expression } \\
\uparrow \text { PCNA } \\
\uparrow \mathrm{Ki67}\end{array}$ \\
\hline Wound healing models [31-34] & $\begin{array}{l}\text { Dietary supplementation } 0.25-1 \mathrm{~g} / \mathrm{kg} \\
\text { Subcutaneous injections } 50 \mathrm{mg} / \mathrm{kg} \\
\text { Subcutaneous injections } 1-10 \mathrm{mg} / \mathrm{kg} \\
\text { Subcutaneous injections } 1-10 \mathrm{mg} / \mathrm{kg}\end{array}$ & $\begin{array}{l}\text { Improving wound healing in } \\
\quad \text { intact mice } \\
\text { Wound healing in OVX mice } \\
\text { Wound healing in OVX rats } \\
\text { Skin aging in OVX rats }\end{array}$ & $\begin{array}{l}\uparrow \text { wound repair } \\
\downarrow \text { ROS } \\
\downarrow \text { NF-kB } \\
\downarrow \text { TNF- } \alpha \\
\uparrow \text { TGF- } \beta 1 \\
\uparrow \text { VEGF } \\
\uparrow \text { MMP- } 2 \text { and MMP-9 } \\
\uparrow \text { TIMP- } 1 \text { and TIMP-2 } \\
\uparrow \text { collagen thickness } \\
\uparrow \text { skin breaking strength }\end{array}$ \\
\hline
\end{tabular}




\section{Human Studies}

In addition to all of the aforementioned preclinical evidence, it was also reported that the use of soy isoflavones may induce proliferation of the epidermis and increase dermal collagen [35].

In a double-blind placebo-controlled trial, 26 women in their late 30s and early 40 s were randomly assigned to receive either an oral intake of $40 \mathrm{mg}$ soy isoflavone aglycones per day or placebo for 12 weeks. It was observed that the isoflavones improved fine wrinkles and malar skin elasticity at the end of the study period [36]. Based on this observation, a randomized, double-blind, estrogen-controlled trial evaluated in 36 postmenopausal women the effect of a topical gel applied on the face, containing either isoflavone ( $4 \%$ genistein) or estrogen for 24 weeks [37]. Facial skin biopsies were taken from the preauricular area before and after the 24-week gel treatment; the cutaneous effects of isoflavone gel at six months were limited to an increase in dermal thickness and in the number of blood vessels, but the increments were smaller than those in the estradiol group. Furthermore, no change in hormonal vaginal cytology at 3 and 6 months was observed in comparison to baseline, suggesting the absence of a significant systemic effect [37]. Genistein was also tested in UVB-induced erythema (sunburn) in the dorsal skin of six men with skin type II to skin type IV. Genistein $5 \mu \mathrm{mol} / \mathrm{cm}^{2}$ was topically applied $60 \mathrm{~min}$ before and $5 \mathrm{~min}$ after UVB irradiation. The skin was photographed and quantitated for erythema, and genistein effectively blocked UVB-induced skin burns, suggesting its use as UV-protective agent [30].

Genistein was also used in an open-label study, where a group of 19 children with different subtypes of mucopolysaccharidosis type III, Sanfilippo syndrome, and different degrees of disability received genistein supplementation $(5 \mathrm{mg} / \mathrm{kg} /$ day) for 1 year [38]. Treatment with genistein produced improvement in skin texture and hair morphology, reduced CoQ10, but did not modify GAG urinary excretion or the severity of the underlying disease. The treatment was generally well-tolerated and no secondary effects were observed. This study is of great importance because it demonstrates that genistein can also be safely administered to young subjects [38].

\section{The Problem of Delivery}

Topical delivery should help overcome the discrepancies associated with the relative bioavailability of genistein after oral administration, which has been observed in certain studies [39,40], but not in others [41]. However, genistein use in creams and topical applications may be affected by a low permeability rate, as reported by some authors [42]. To overcome this problem, drug delivery systems based on lipid nanoparticles have been employed. The most widely studied lipid nanoparticles are solid lipid nanoparticles (SLN) and nanostructured lipid carriers (NLC). Both have a solid lipid matrix, but NLC are prepared with a mixture of solid lipid and oil, which can increase drug loading and stability [43]. An experimental study compared SLN and NLC nanoparticles combined with genistein for controlled release in skin, and increased penetration in deeper skin layers. The genistein-NLC formulation was the most effective in favoring the penetration in to the deeper skin layers, suggesting that NLC could be a promising nanocarrier for the topical delivery of genistein [44].

Another well-studied approach exploited the use of polymeric gels-in particular, polyethylene glycol 400 (PEG 400). The formulation was tested for skin permeation from a saturated solution of dry soy extract [45]. Genistein flux increased significantly when pure genistein was used as a suspension in PEG 400, and resulted in significant skin retention. To further investigate the transdermal delivery of genistein, studies were conducted using $\mathrm{pH} 6$ and $\mathrm{pH} 10.8$ buffers and soybean oil as vehicles [46]. Of these, the calibrated deposition of genistein into nude mice and pig skin for a saturated solution in $\mathrm{pH} 6$ buffer was higher than the other vehicles. Pretreatment of skin for $2 \mathrm{~h}$ with either oleic acid or $\alpha$-terpineol as penetration enhancers did not increase the permeation of genistein at $\mathrm{pH} 6$. It was reported that topical delivery was promising for genistein use against photoaging and photodamage, providing a possible application of these gels as skin protection agents for UV-induced damage and chemoprevention of melanoma [47]. 
Another approach exploited to improve isoflavone delivery into the skin was tried with microemulsions. The effects accumulation as well as the solubility of genistein were tested on pig skin using a water/oil-type microemulsion, and a significant protection against UV-induced oxidative damage was observed [48]. Genistein inhibited lipid peroxidation in guinea pig dorsal skin, as well as UV-B-induced erythema formation.

\section{Conclusions}

During menopause, as estrogen levels decrease, testosterone stimulates sebaceous glands to secrete thicker sebum, giving the appearance of oily skin; in addition, some women may develop facial hair, particularly in the chin area. As estrogen levels drop during menopause, fat deposits tend to redistribute over the abdomen and/or on the thighs and buttocks. The result is a loss of supportive fat below the skin of the face, neck, hands, and arms; this allows sagging wrinkles to appear, and the skin over these areas is less easily compressed as it loses its mobility. The lowered estrogen levels also result in less production and repair of collagen and elastin in the dermis (particularly if the skin is exposed to UV rays), resulting in elastosis. Estrogens also temper melanin production; their lack can result in brown "age spots" appearing on the face, hands, neck, arms, and chest of many women. All these changes lead to a faster aging of skin. Estrogen replacement prevents and ameliorates these features, but too many side effects have been related to their use. Over the past decades, genistein has been used as an alternative treatment for menopausal symptoms [49-53], and in light of the effects reported on skin aging (Figure 1), it could be considered as an effective alternative treatment for menopause. In conclusion, genistein might be a new potential therapy for the management of skin disorders as well as age- and menopause-related skin changes commonly observed in postmenopausal women. Considering the bioavailability following oral assumption and the permeability after topical application, it seems that oral administration of at least $50 \mathrm{mg} /$ day of pure genistein without other isoflavones could be the best dosing strategy to obtain clinical efficacy.

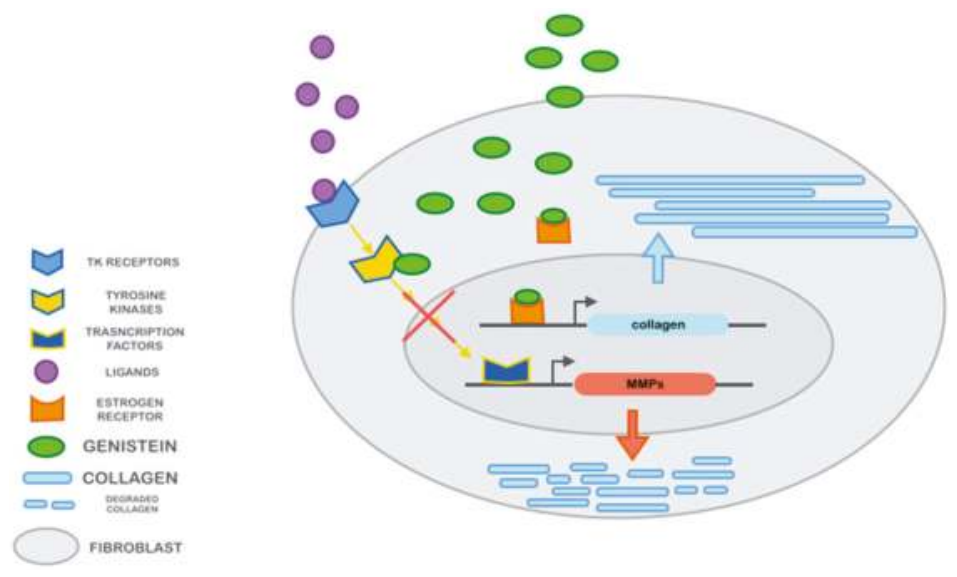

Figure 1. Mechanism of action of genistein in fibroblasts.

Acknowledgments: No specific funds have been received for experiments or covering the costs to publish in open access.

Conflicts of Interest: The authors declare no conflict of interest. 


\section{References}

1. Hall, G.; Phillips, T.J. Estrogen and skin: The effects of estrogen, menopause, and hormone replacement therapy on the skin. J. Am. Acad. Dermatol. 2005, 53, 555-568. [CrossRef]

2. Verdier-Sevrain, S.; Bontè, F.; Gilchrest, B. Biology of estrogens in skin: Implications for skin aging. Exp. Dermatol. 2006, 15, 83-94. [CrossRef]

3. Wolff, E.F.; Narayan, D.; Taylor, H.S. Long-term effects of hormone therapy on skin rigidity and wrinkles. Fertil. Steril. 2005, 84, 285-288. [CrossRef]

4. Albertazzi, P.; Purdie, D.W. The life and times of the estrogen receptors: An interim report. Climacteric 2001, 4, 194-202. [CrossRef]

5. Younes, M.; Honma, N. Estrogen receptor ß. Arch. Pathol. Lab. Med. 2011, 135, 63-66.

6. Thornton, M.J.; Taylor, A.H.; Mulligan, K.; Al-Azzawi, F.; Lyon, C.C.; O’Driscoll, J.B.; Messenger, A.G. Estrogen receptor beta $(\mathrm{ER} \beta)$ is the predominant estrogen receptor in human scalp. Exp. Dermatol. 2003, 12, 181-190. [CrossRef]

7. Thornton, M.J.; Taylor, A.H.; Mulligan, K.; Al-Azzawi, F.; Lyon, C.C.; O’Driscoll, J.B.; Messenger, A.G. The distribution of estrogen receptor beta $(\mathrm{ER} \beta)$ is distinct to that of ER alpha and the androgen receptor in human skin and the pilosebaceous unit. J. Investig. Dermatol. Symp. Proc. 2003, 8, 100-103. [CrossRef]

8. Thornton, M.J. The biological actions of estrogens on skin. Exp. Dermatol. 2002, 11, 487-502. [CrossRef]

9. Fitzpatrick, L.A. Selective estrogen receptor modulators and phytoestrogens: New therapies for the postmenopausal women. Mayo Clin. Proc. 1999, 74, 601-607. [CrossRef]

10. Beck, V.; Unterrieder, E.; Krenn, L.; Kubelka, W.; Jungbauer, A. Comparison of hormonal activity (estrogen, androgen and progestin) of standardized plant extracts for large scale use in hormone replacement therapy. J. Steroid Biochem. Mol. Biol. 2003, 84, 259-268. [CrossRef]

11. Kuiper, G.G.; Lemmen, J.G.; Carlsson, B.; Corton, J.C.; Safe, S.H.; van der Saag, P.T.; van der Burg, B.; Gustafsson, J.A. Interaction of estrogenic chemicals and phytoestrogens with estrogen receptor beta. Endocrinology 1998, 139, 4252-4263. [CrossRef]

12. Akiyama, T.; Ishida, J.; Nakagawa, S.; Ogawara, H.; Watanabe, S.; Itoh, N.; Shibuya, M.; Fukami, Y. Genistein, a specific inhibitor of tyrosine-specific protein kinases. J. Biol. Chem. 1987, 262, 5592-5595.

13. Steensma, A.; Faassen-Peters, M.A.; Noteborn, H.P.; Rietjens, I.M. Bioavailability of genistein and its glycoside genistin as measured in the portal vein of freely moving unanesthetized rats. J. Agric. Food Chem. 2006, 54, 8006-8012. [CrossRef]

14. Cederroth, C.R.; Zimmermann, C.; Nef, S. Soy, phytoestrogens and their impact on reproductive health. Mol. Cell. Endocrinol. 2012, 355, 192-200. [CrossRef]

15. Chin, G.S.; Liu, W.; Steinbrech, D.; Hsu, M.; Levinson, H.; Longaker, M.T. Cellular signaling by tyrosine phosphorylation in keloid and normal human dermal fibroblasts. Plast. Reconstr. Surg. 2000, 106, 1532-1540. [CrossRef]

16. Cao, C.; Li, S.; Dai, X.; Chen, Y.; Feng, Z.; Zhao, Y.; Wu, J. Genistein inhibits proliferation and functions of hypertrophic scar fibroblasts. Burns 2009, 35, 89-97. [CrossRef]

17. Jurzak, M.; Adamczyk, K.; Antończak, P.; Garncarczyk, A.; Kuśmierz, D.; Latocha, M. Evaluation of genistein ability to modulate CTGF mRNA/protein expression, genes expression of TGF $\beta$ isoforms and expression of selected genes regulating cell cycle in keloid fibroblasts in vitro. Acta Pol. Pharm. 2014, 71, 972-986.

18. Jurzak, M.; Adamczyk, K. Influence of genistein on c-Jun, c-Fos and Fos-B of AP-1 subunits expression in skin keratinocytes, fibroblasts and keloid fibroblasts cultured in vitro. Acta Pol. Pharm. 2013, 70, 205-213.

19. Sienkiewicz, P.; Surazyński, A.; Pałka, J.; Miltyk, W. Nutritional concentration of genistein protects human dermal fibroblasts from oxidative stress-induced collagen biosynthesis inhibition through IGF-I receptor-mediated signaling. Acta Pol. Pharm. 2008, 65, 203-211.

20. Piotrowska, E.; Jakóbkiewicz-Banecka, J.; Barańska, S.; Tylki-Szymańska, A.; Czartoryska, B.; Wegrzyn, A.; Wegrzyn, G. Genistein-mediated inhibition of glycosaminoglycan synthesis as a basis for gene expression-targeted isoflavone therapy for mucopolysaccharidoses. Eur. J. Hum. Genet. 2006, 14, 846-852. [CrossRef]

21. Otomo, T.; Hossain, M.A.; Ozono, K.; Sakai, N. Genistein reduces heparan sulfate accumulation in human mucolipidosis II skin fibroblasts. Mol. Genet. Metab. 2012, 105, 266-269. [CrossRef] 
22. Kloska, A.; Jakóbkiewicz-Banecka, J.; Narajczyk, M.; Banecka-Majkutewicz, Z.; Wegrzyn, G. Effects of flavonoids on glycosaminoglycan synthesis: Implications for substrate reduction therapy in Sanfilippo disease and other mucopolysaccharidoses. Metab. Brain Dis. 2011, 26, 1-8. [CrossRef]

23. Isoherranen, K.; Punnonen, K.; Jansen, C.; Uotila, P. Ultraviolet irradiation induces cyclooxygenase-2 expression in keratinocytes. Br. J. Dermatol. 1999, 140, 1017-1022. [CrossRef]

24. Iovine, B.; Iannella, M.L.; Gasparri, F.; Monfrecola, G.; Bevilacqua, M.A. Synergic Effect of Genistein and Daidzein on UVB-Induced DNA Damage: An Effective Photoprotective Combination. J. Biomed. Biotechnol. 2011, 2011, 692846:1-692846:8. [CrossRef]

25. Wang, Y.N.; Wu, W.; Chen, H.C.; Fang, H. Genistein protects against UVB-induced senescence-like characteristics in human dermal fibroblast by p66Shc down-regulation. J. Dermatol. Sci. 2010, 58, $19-27$. [CrossRef]

26. Lee, T.H.; Do, M.H.; Oh, Y.L.; Cho, D.W.; Kim, S.H.; Kim, S.Y. Dietary fermented soybean suppresses UVB-induced skin inflammation in hairless mice via regulation of the MAPK signaling pathway. J. Agric. Food Chem. 2014, 62, 8962-8972. [CrossRef]

27. Wei, H.; Bowen, R.; Zhang, X.; Lebwohl, M. Isoflavone genistein inhibits the initiation and promotion of two-stage skin carcinogenesis in mice. Carcinogenesis 1998, 19, 1509-1514. [CrossRef]

28. Khan, N.; Afaq, F.; Mukhtar, H. Cancer chemoprevention through dietary antioxidants: Progress and promise. Antioxid. Redox Signal. 2008, 10, 475-510. [CrossRef]

29. Terra, V.A.; Souza-Neto, F.P.; Frade, M.A.; Ramalho, L.N.; Andrade, T.A.; Pasta, A.A.; Conchon, A.C.; Guedes, F.A.; Luiz, R.C.; Cecchini, R.; et al. Genistein prevents ultraviolet B radiation-induced nitrosative skin injury and promotes cell proliferation. J. Photochem. Photobiol. B 2015, 144, 20-27. [CrossRef]

30. Wei, H.; Saladi, R.; Lu, Y.; Wang, Y.; Palep, S.R.; Moore, J.; Phelps, R.; Shyong, E.; Lebwohl, M.G. Isoflavone genistein: Photoprotection and clinical implications in dermatology. J. Nutr. 2003, 133 (Suppl. 1), 3811S-3819S

31. Park, E.; Lee, S.M.; Jung, I.K.; Lim, Y.; Kim, J.H. Effects of genistein on early-stage cutaneous wound healing. Biochem. Biophys. Res. Commun. 2011, 410, 514-519. [CrossRef]

32. Emmerson, E.; Campbell, L.; Ashcroft, G.S.; Hardman, M.J. The phytoestrogen genistein promotes wound healing by multiple independent mechanisms. Mol. Cell. Endocrinol. 2010, 321, 184-193. [CrossRef]

33. Marini, H.; Polito, F.; Altavilla, D.; Irrera, N.; Minutoli, L.; Calò, M.; Adamo, E.B.; Vaccaro, M.; Squadrito, F.; Bitto, A. Genistein aglycone improves skin repair in an incisional model of wound healing: A comparison with raloxifene and oestradiol in ovariectomized rats. Br. J. Pharmacol. 2010, 160, 1185-1194. [CrossRef]

34. Polito, F.; Marini, H.; Bitto, A.; Irrera, N.; Vaccaro, M.; Adamo, E.B.; Micali, A.; Squadrito, F.; Minutoli, L.; Altavilla, D. Genistein aglycone, a soy-derived isoflavone, improves skin changes induced by ovariectomy in rats. Br. J. Pharmacol. 2012, 165, 994-1005. [CrossRef]

35. Kessel, B. Alternatives to estrogen for menopausal women. Soc. Exp. Biol. Med. 1998, 217, 38-44. [CrossRef]

36. Izumi, T.; Saito, M.; Obata, A.; Arii, M.; Yamaguchi, H.; Matsuyama, A. Oral intake of soy isoflavone aglycone improves the aged skin of adult women. J. Nutr. Sci. Vitaminol. (Tokyo) 2007, 53, 57-62. [CrossRef]

37. Moraes, A.B.; Haidar, M.A.; Soares Júnior, J.M.; Simões, M.J.; Baracat, E.C.; Patriarca, M.T. The effects of topical isoflavones on postmenopausal skin: Double-blind and randomized clinical trial of efficacy. Eur. J. Obstet. Gynecol. Reprod. Biol. 2009, 146, 188-192. [CrossRef]

38. Delgadillo, V.; O'Callaghan Mdel, M.; Artuch, R.; Montero, R.; Pineda, M. Genistein supplementation in patients affected by Sanfilippo disease. J. Inherit. Metab. Dis. 2011, 34, 1039-1044. [CrossRef]

39. Setchell, K.D.; Brown, N.M.; Desai, P.; Zimmer-Nechemias, L.; Wolfe, B.E.; Brashear, W.T.; Kirschner, A.S.; Cassidy, A.; Heubi, J.E. Bioavailability of pure isoflavones in healthy humans and analysis of commercial soy isoflavone supplements. J. Nutr. 2001, 131, 1362S-1375S.

40. Setchell, K.D.; Brown, N.M.; Desai, P.B.; Zimmer-Nechimias, L.; Wolfe, B.; Jakate, A.S.; Creutzinger, V.; Heubi, J.E. Bioavailability, disposition, and dose-response effects of soy isoflavones when consumed by healthy women at physiologically typical dietary intakes. J. Nutr. 2003, 133, 1027-1035.

41. Zubik, L.; Meydani, M. Bioavailability of soybean isoflavones from aglycone and glucoside forms in American women. Am. J. Clin. Nutr. 2003, 77, 1459-1465.

42. Georgetti, S.R.; Casagrande, R.; Verri, W.A., Jr.; Lopez, R.F.; Fonseca, M.J. Evaluation of in vivo efficacy of topical formulations containing soybean extract. Int. J. Pharm. 2008, 352, 189-196. [CrossRef] 
43. Müller, R.H.; Petersen, R.D.; Hommoss, A.; Pardeike, J. Nanostructured lipid carriers (NLC) in cosmetic dermal products. Adv. Drug Deliv. Rev. 2007, 59, 522-530. [CrossRef]

44. Andrade, L.M.; de Fátima Reis, C.; Maione-Silva, L.; Anjos, J.L.; Alonso, A.; Serpa, R.C.; Marreto, R.N.; Lima, E.M.; Taveira, S.F. Impact of lipid dynamic behavior on physical stability, in vitro release and skin permeation of genistein-loaded lipid nanoparticles. Eur. J. Pharm. Biopharm. 2014, 88, 40-47. [CrossRef]

45. Minghetti, P.; Cilurzo, F.; Casiraghi, A.; Montanari, L. Evaluation of ex vivo human skin permeation of genistein and daidzein. Drug Deliv. 2006, 13, 411-415. [CrossRef]

46. Huang, Z.R.; Hung, C.F.; Lin, Y.K.; Fang, J.Y. In vitro and in vivo evaluation of topical delivery and potential dermal use of soy isoflavones genistein and daidzein. Int. J. Pharm. 2008, 364, 36-44. [CrossRef]

47. Chadha, G.; Sathigari, S.; Parsons, D.L.; Jayachandra Babu, R. In vitro percutaneous absorption of genistein from topical gels through human skin. Drug Dev. Ind. Pharm. 2011, 37, 498-505. [CrossRef]

48. Kitagawa, S.; Inoue, K.; Teraoka, R.; Morita, S.Y. Enhanced skin delivery of genistein and other two isoflavones by microemulsion and prevention against UV irradiation-induced erythema formation. Chem. Pharm. Bull. (Tokyo) 2010, 58, 398-401. [CrossRef]

49. Arcoraci, V.; Atteritano, M.; Squadrito, F.; D'Anna, R.; Marini, H.; Santoro, D.; Minutoli, L.; Messina, S.; Altavilla, D.; Bitto, A. Antiosteoporotic Activity of Genistein Aglycone in Postmenopausal Women: Evidence from a Post-Hoc Analysis of a Multicenter Randomized Controlled Trial. Nutrients 2017, 9, 179. [CrossRef]

50. Bitto, A.; Polito, F.; Squadrito, F.; Marini, H.; D'Anna, R.; Irrera, N.; Minutoli, L.; Granese, R.; Altavilla, D. Genistein aglycone: A dual mode of action anti-osteoporotic soy isoflavone rebalancing bone turnover towards bone formation. Curr. Med. Chem. 2010, 17, 3007-3018. [CrossRef]

51. Marini, H.; Bitto, A.; Altavilla, D.; Burnett, B.P.; Polito, F.; Di Stefano, V.; Minutoli, L.; Atteritano, M.; Levy, R.M.; D'Anna, R.; et al. Breast safety and efficacy of genistein aglycone for postmenopausal bone loss: A follow-up study. J. Clin. Endocrinol. Metab. 2008, 93, 4787-4796. [CrossRef] [PubMed]

52. Marini, H.; Minutoli, L.; Polito, F.; Bitto, A.; Altavilla, D.; Atteritano, M.; Gaudio, A.; Mazzaferro, S.; Frisina, A.; Frisina, N.; et al. Effects of the phytoestrogen genistein on bone metabolism in osteopenic postmenopausal women: A randomized trial. Ann. Intern. Med. 2007, 146, 839-847. [CrossRef] [PubMed]

53. D'Anna, R.; Cannata, M.L.; Marini, H.; Atteritano, M.; Cancellieri, F.; Corrado, F.; Triolo, O.; Rizzo, P.; Russo, S.; Gaudio, A.; et al. Effects of the phytoestrogen genistein on hot flushes, endometrium, and vaginal epithelium in postmenopausal women: A 2-year randomized, double-blind, placebo-controlled study. Menopause 2009, 16, 301-306. [CrossRef] [PubMed]

(c) 2017 by the authors. Licensee MDPI, Basel, Switzerland. This article is an open access article distributed under the terms and conditions of the Creative Commons Attribution (CC BY) license (http:/ / creativecommons.org/licenses/by/4.0/). 


\title{
Influences of Orally Taken Carotenoid-Rich Curly Kale Extract on Collagen I/Elastin Index of the Skin
}

\author{
Martina C. Meinke ${ }^{1, *, \dagger}$, Ceylan K. Nowbary ${ }^{1, \dagger}$, Sabine Schanzer ${ }^{1}$, Henning Vollert ${ }^{2}$, \\ Jürgen Lademann ${ }^{1}$ and Maxim E. Darvin ${ }^{1}$ \\ 1 Charité-Universitätsmedizin Berlin, Corporate Member of Freie Universität Berlin, Humboldt-Universität \\ zu Berlin, and Berlin Institute of Health, Center of Experimental and Applied Cutaneous Physiology (CCP), \\ Department of Dermatology, Charité-Universitätsmedizin Berlin, 10117 Berlin, Germany; \\ ceylan.nowbary@charite.de (C.K.N.); sabine.schanzer@charite.de (S.S.); juergen.lademann@charite.de (J.L.); \\ maxim.darvin@charite.de (M.E.D.) \\ 2 Bioactive Food GmbH, Am Ihlsee 36a, 23795 Bad Segeberg, Germany; henning_vollert@t-online.de \\ * Correspondence: martina.meinke@charite.de; Tel.: +49-30-450-518244 \\ + These authors contributed equally to this work.
}

Received: 1 June 2017; Accepted: 14 July 2017; Published: 19 July 2017

\begin{abstract}
Two differently designed, spatially resolved reflectance spectroscopy-based scanners and two-photon tomography were used for noninvasive in vivo determination of cutaneous carotenoids, and collagen I/elastin aging index of dermis, respectively, in the skin of 29 healthy female volunteers between 40 and 56 years of age. The volunteers received a supplement in the form of a carotenoid-rich natural curly kale extract containing $1650 \mu \mathrm{g}$ of carotenoids in total (three capsules of $550 \mu \mathrm{g}$ ), once a day. Measurements were taken before, after 5 months and after 10 months of daily supplementation. The results showed significantly increased values for the cutaneous carotenoids and the collagen I/elastin aging index of dermis 5 and 10 months after the beginning of the study. The obtained results show that a natural carotenoid-rich extract could prevent the aging-related collagen I degradation in the dermis and improve the extracellular matrix.
\end{abstract}

Keywords: skin aging; two photon tomography; reflectance spectroscopy; noninvasive methods; auto fluorescence; second harmonic generation; nutrition

\section{Introduction}

Nutrition rich in fruit and vegetables is beneficial to human health and wellbeing [1-4]. Secondary plant components, such as antioxidants [5], protect the cells and connective tissue against the development of oxidative stress, which is related to several pathological consequences, e.g., neurological [6], cardio-vascular [7], dermatological diseases [8] or aging [9]. Oxidative stress is induced by various factors, inter alia, solar radiation, smoking, alcohol consumption, lack of sleep, intensive physical activities and psychological stress [10-20], and therefore cannot be avoided in daily life. Oxidative stress is characterized by the generation of large amounts of reactive oxygen species (ROS), whose action is responsible for the development of premature skin aging and photo aging [7,21]. To counteract this effect, antioxidants should be available in sufficient concentration and composition to keep the balance and avoid tissue damage [22]. The majority of the essential antioxidants, including carotenoids, have to be consumed via dietary sources. The uptake and bioavailability of carotenoids can be measured in vivo by resonance Raman and reflectance spectroscopy [23,24]. Furthermore, it could be demonstrated that skin with a high amount of the carotenoid lycopene is less rough and therefore looks younger [21]. The physiologically relevant composition and concentration of antioxidants is important, as at very high concentrations, antioxidants might reverse their properties and become pro-oxidant $[25,26]$. This process is intricate and depends on many factors including oxygen tension and 
further antioxidants such as $\alpha$-tocopherol in the tissue $[27,28]$. Taking this into account, a promising anti-aging strategy could be the physiologically relevant enhancement of carotenoids and other antioxidants in the tissue by nutrition and/or supplements [22].

The beneficial effects of such application on the skin status were studied based on skin parameters such as furrows and wrinkles, elasticity and skin hydration [29]. To understand such effects, more than roughness and wrinkle volume should be measured. The dermal proteins collagen I and elastin are related to the skin age and could serve as reliable parameters of the anti-aging effect of nutrition or a supplement $[30,31]$. Various studies have shown that the collagen I/elastin ratio, as well as the collagen I and elastin structures, change with age [32-35]. The elasticity of the skin increases with atrophy of the collagen I fiber bundles, the related flattening of the rete ridges, and the thickening of the dermal elastic fibers. Previously, biopsies had to be taken, but nowadays, noninvasive two-photon tomography is available to measure the collagen I by second harmonic generation (SHG) and the elastin by auto fluorescence (AF) signals in vivo in the dermis [36,37]. The SHG to AF aging index of dermis (SAAID) is a noninvasive objective parameter for determining the collagen I status in the dermis, which can be used for the description of the intrinsic and extrinsic aging on various skin areas in vivo [38,39], effect of skin protection [40], as well as efficacy of wound healing [41] and identification of disease affected skin [42,43]. The collagen I/elastin index can be quantified with the SAAID, which is defined according to [42]:

$$
\mathrm{SAAID}=(\mathrm{SHG}-\mathrm{AF}) /(\mathrm{SHG}+\mathrm{AF})
$$

SAAID-scoring is a powerful method for analyzing the dermis dependent on AF and SHG signal intensities [43]. Other dermal collagens of types IV, VII and XVII are also known to reduce with age [44], but it is impossible to determine their concentrations in vivo in the skin. This can only be done by applying special algorithms [45].

The hypothesis is that systemically administered carotenoids will increase the collagen I synthesis or at least prevent its decrease, which usually occurs due to oxidative stress during our life, and results in skin aging. In the presented in vivo study, two different cutaneous carotenoid sensors, which were recently developed based on spatially resolved reflectance spectroscopy (SRRS), were applied to monitor the skin carotenoids noninvasively [46]. To increase the skin carotenoids, a vegetable curly kale extract rich in carotenoids, in comparison to a placebo, was systemically administered over a time period of 10 months. The curly kale extract was selected because previous studies had shown its positive effects on the skin in terms of bioavailability and radical scavenging activity [47,48]. The SAAID was measured as an objective skin aging parameter. The measurements were performed before, after 5 , and after 10 months of intake of the carotenoid-rich curly kale supplement.

\section{Material and Methods}

\subsection{Subjects}

29 healthy female volunteers (10 smokers, 19 non-smokers) aged between 40 and 56 years (mean 49.2 years) of skin type II according to the Fitzpatrick classification [49] were enrolled in the study.

Exclusion criteria were subjects with skin diseases, admission to an institution due to an administrative or judicial order (according to $§ 29$ MPG), known drug addiction or alcoholism, and expectant or lactating women. The investigations were carried out in accordance with the ethical guidelines of the Declaration of Helsinki and had been approved by the local Ethics Committee prior to starting the study (EA1/229/14). All volunteers gave their informed written consent.

\subsection{Supplements}

During the uptake study, the volunteers ingested three capsules of a curly kale extract, hereinafter called verum, once a day (provided by BioActive Food, Bad Segeberg, Germany). One capsule contained the following substances: $430 \mu \mathrm{g}$ lutein, $70 \mu \mathrm{g}$ beta-carotene, $30 \mu \mathrm{g}$ lycopene, $20 \mu \mathrm{g}$ 
zeaxanthin, i.e., $550 \mu \mathrm{g}$ carotenoids, analyzed independently by Bioanalyt $\mathrm{GmbH}$ (Potsdam, Germany). As a control, a placebo capsule containing an olive oil without carotenoids was given. The content of the capsules was measured with resonance Raman spectroscopy and the placebo did not show any carotenoid values.

\subsection{Study Protocol}

The volunteers were randomly divided into placebo $(N=15)$ and verum groups $(N=14)$.

The measurements were performed on the palm, the inner forearm and the cheek. Carotenoids can be sensitively determined only at the palm as the stratum corneum is sufficiently thick there and the measurements are not influenced by blood and melanin. The SAAID was measured on the cheek and the inner forearm to have one sun exposed and one sun protected area.

\subsection{Carotenoid Determination}

For the investigations, two different sensors were applied. One optical sensor was based on multiple spatially resolved reflectance spectroscopy (MSRRS). This sensor is commercially available under the tradename biozoom (model Biozoom Portable, Kassel, Germany). It uses several differently positioned light sources and light detectors, exhibiting different distances between light source and detector and different angles for irradiation and detection, and has been described in detail elsewhere $[23,46]$. Briefly, the LED light sources cover a spectral range from approximately $350 \mathrm{~nm}$ to $1000 \mathrm{~nm}$ in wavelength in 16 steps, providing 118 light emitters. The sensor picks up the backscattered light at a total number of 152 light sensitive areas. This combination results in almost 18,000 raw data values, which are picked up several times during one measurement at a sensor surface area of $20 \mathrm{~mm}$ by $20 \mathrm{~mm}$.

The second carotenoid sensor was a CaroLED sensor (Laser-und Medizin-Technologie Berlin, LMTB, Germany). The underlying measuring principle of CaroLED is based on SRRS, and was recently described by Meinke et al. [46]. Briefly, five light emitting diodes (LED) of different wavelengths in the visible and near infrared region illuminate the skin through a measurement window. While light propagates through the individual skin layers, and the spectral signature of carotenoids and of other skin constituents is obtained [50]. Four photodiodes, each of which is placed at a different lateral distance to the illumination site, measure the spectral signature at different depth sensitivities.

The measurements using both sensors were performed five times on the same palm area (thenar eminence) of the right hand for all subjects, and mean values were calculated for each subject and measurement method before the statistical analysis was applied. Because of the different initial values, the data were normalized to the initial values, dividing the values from visit after 5 and 10 months by the initial values.

Both sensors were calibrated before starting the study using resonance Raman spectroscopy on the palm of healthy volunteers in vivo using a resonant excitation at $488 \mathrm{~nm}$ [46].

\subsection{Collagen I/Elastin Index SAAID}

The collagen I and elastin structure of the skin can be characterized by the second harmonic generation (SHG) to autofluorescence (AF) aging index of dermis (SAAID). The SAAID can be determined in vivo using two-photon microscopy by calculation of the corresponding AF and SHG image intensities [51,52]. The SAAID is defined as described in Equation (1). In the dermis, the AF is mainly determined by the elastin concentration, while the SHG is determined by the collagen I concentration. In this nomenclature, the SAAID decreases with photo aging, approaching its minimal value- 1 when the collagen is completely replaced by elastic fibers.

The investigations were carried out using a two-photon tomograph (JenLab GmbH, Jena, Germany) equipped with a femtosecond titanium sapphire laser (Mai Tai XF, Spectra-Physics, Santa Clara, CA, USA). The laser was operated at $760 \mathrm{~nm}$ and generated $100 \mathrm{fs}$ pulses at a repetition rate of $80 \mathrm{MHz}$. For studying the structure of the dermis at different depths of the skin, sensitive photo multipliers 
were applied. The radiation collected from different depths was averaged over slices of $200 \times 200 \mu \mathrm{m}^{2}$. The acquisition time was equal to $7 \mathrm{~s}$ for one slice, thus ensuring a satisfactory measurement stability and picture quality [41].

During the measurements, the laser focus was moved from the skin surface into deeper parts of the skin at $10 \mu \mathrm{m}$ increments. The maximum measuring depth into the skin was $120 \mu \mathrm{m}$. For the whole depth, the SAAID was calculated and the profiles were normalized to the onset of the SHG signal. Four values after the SHG's onset were considered within the mean values to ensure data from the upper part of papillary dermis below the basal layer. The utilized two-photon tomograph has been described in detail elsewhere [40].

The measurements were performed in vivo at the lower inner arm and the cheek. For all subjects, the area at the forearm was selected at $30 \mathrm{~cm}$ from the middle finger tip. The cheek area was selected, as it had the best coupling possibility with the optical measuring head on the line between nose and ear.

\subsection{Statistics}

The data were statically analyzed using SPSS for Windows (IBM ${ }^{\circledR}$ SPSS ${ }^{\circledR}$ Statistics, version 19, Inc., Chicago, IL, USA). The data were analyzed to a normal distribution using explorative data analysis. An unpaired $t$-test was applied to determine differences in the mean values between the groups, and a paired $t$-test for changes within one group. For the correlation, the Pearson correlation coefficient was calculated. Values of $p \leq 0.05$ were considered to indicate a significant difference.

\section{Results}

Although 34 volunteers were enrolled, only 29 completed the study successfully. 5 dropouts occurred, 3 in the verum group and 2 in the placebo group. 2 volunteers quit the study due to problems with the stomach, and 3 did not come to the investigation after the 3rd visit and therefore were not replaced. All 29 volunteers underwent the carotenoid measurements and the two-photon tomography.

\subsection{Carotenoids}

The group-averaged cutaneous carotenoid concentrations measured using both carotenoid sensors are shown in Figure 1. The data are presented normalized to the initial value because the individual values show a high variation. The carotenoid values of the verum group measured with both devices increased significantly within the first 5 months, in which the volunteers ingested the carotenoid-rich extract, and decreased again over the next 5 months. But the values of the verum group after 10 months were still significantly higher than the initial value. The placebo values measured by CaroLED also increased and decreased, but the changes were not significant. The carotenoid concentration in the placebo group remained unchanged for the first 5 months and showed an insignificant decrease after 10 months of supplementation.

\subsection{SAAID}

The SAAID was measured at the inner forearm and at the cheek. The differences from the initial values are shown in Figure 2. After 5 months, all SAAID values had increased significantly $(p<0.05$ for placebo and $p<0.01$ for verum), but after 10 months, the increase had remained significant only for the verum group. The verum group consistently showed higher values than the placebo group, and the difference between the two groups was significant for the cheek after 10 months $(p<0.05)$. 


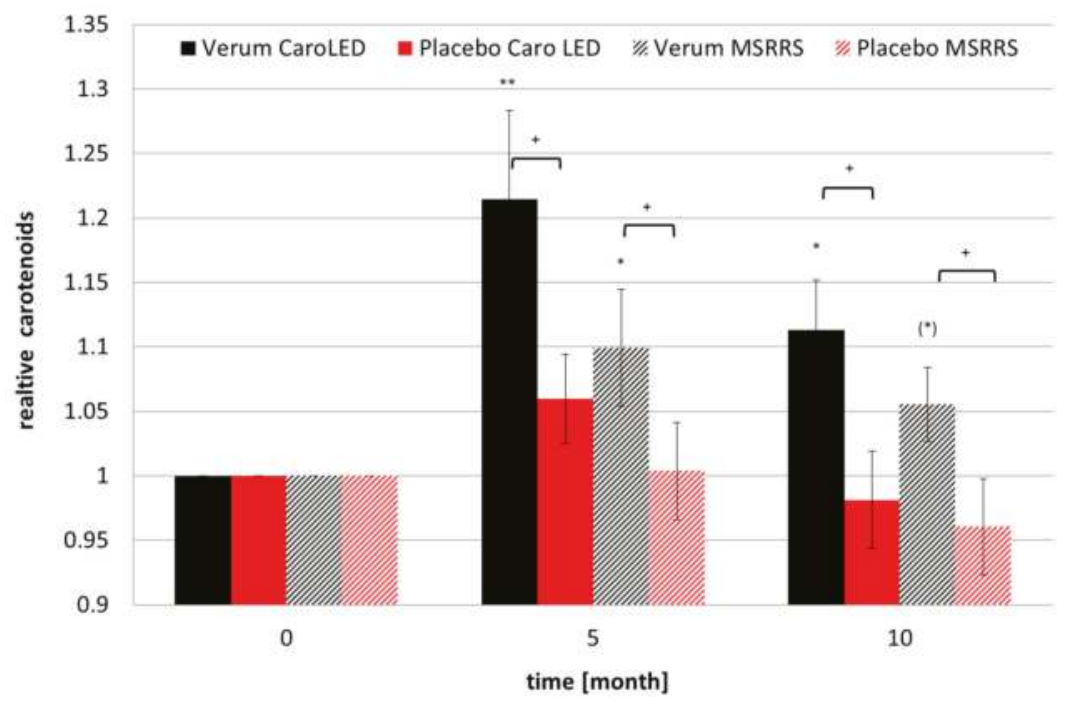

Figure 1. Carotenoid values relative to the initial values measured by two different carotenoid sensors obtained in the palm (mean \pm SEM). $+p<0.05$ between placebo and verum; $\left(^{*}\right) p<0.1,{ }^{*} p<0.05$, ** $p<0.01$ to initial value. $N=15$ for placebo and $N=14$ for verum.

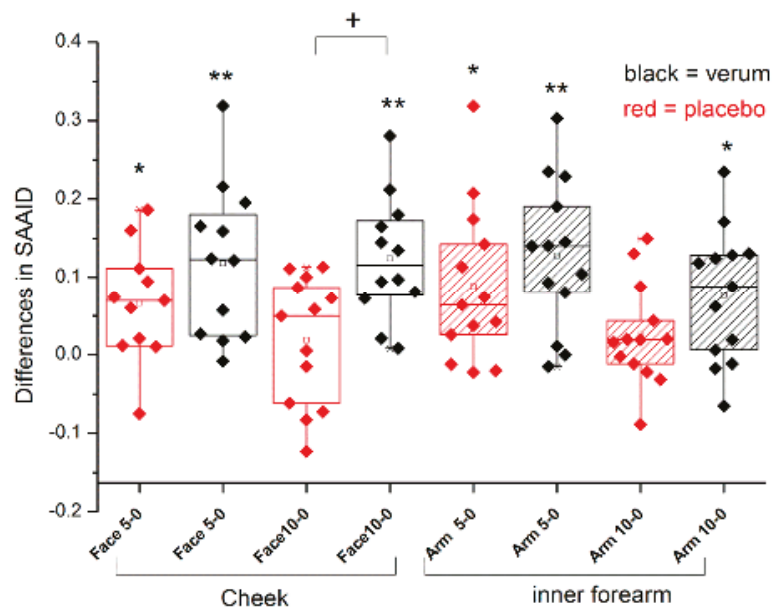

Figure 2. Box plot of the differences of SAAID to the initial values measured at the cheek and inner forearm. $+p<0.05$ between placebo and verum; ${ }^{*} p<0.05,{ }^{* *} p<0.01$ to initial value. The box presents $50 \%$ of the data. The bottom and top of the box are always the first and third quartiles and the solid line box is always the second quartile, the median.

\subsection{Correlations}

If the hypothesis is correct that the SAAID increases during the intake of carotenoids, a correlation of these two values is possible. Therefore, the Pearson correlation coefficient of the SAAID values and the carotenoid values was calculated (Table 1) for all subjects from whom at least one SAAID value (cheek or arm) was obtained for all visits (max. $n_{\text {subjects }}=27$, maximum measured values 
$N=81$ ). As a positive check, the correlation between the two carotenoid values was determined, as well. As expected, the two carotenoid values have a highly significant positive correlation. Furthermore, the two SAAID values show a moderately positive correlation, which is highly significant. The correlations between the carotenoid and the SAAID values are between slightly and moderately positive with an $r$ of about 0.3 , and are significant.

Table 1. Pearson correlation coefficient $\mathrm{r}$ with $p$-values and number of the SAAID and carotenoid values $(N) ;{ }^{*} p<0.05, * * p<0.01$.

\begin{tabular}{cccc}
\hline & SAAID Cheek & SAAID & Carotenoids \\
\cline { 3 - 4 } & & Forearm & CaroLED \\
\hline SAAID forearm & & & \\
\hline Correlation according to Pearson & $0.504^{* *}$ & 1 & \\
Significance & $<0.001$ & & \\
$N$ & 76 & 77 & \\
\hline Carotenoids CaroLED & & & \\
\hline Correlation according to Pearson & $0.279^{*}$ & $0.295^{* *}$ & \\
Significance & 0.014 & 0.008 & \\
$N$ & 77 & 80 & \\
\hline Carotenoids MSRRS & & & \\
\hline Correlation according to Pearson & $0.328^{* *}$ & $0.225^{*}$ & $0.853 * *$ \\
Significance & 0.004 & 0.023 & $<0.001$ \\
$N$ & 77 & 80 & 81 \\
\hline
\end{tabular}

\section{Discussion}

Human nutrition is subject to seasonal variations, and the intake of secondary plant substances such as carotenoids and other antioxidants is related to it. From former investigations, it is known that the carotenoid status in humans is enhanced in the summer and autumn months due to the availability of regional fresh and ripe fruits and vegetables. This has been referred to as "seasonal increase" [53]. In the current study, the first measurements were performed in autumn and the second ones in the springtime. The last visit took place at the end of summer and mainly in autumn. This could explain the observed changes in the placebo group. Furthermore, infections, which occur very often in the wintertime, additionally decreased the carotenoid values in the skin [53].

As expected, the skin carotenoid values increased by 10 to $20 \%$ in the group that received the carotenoid-rich curly kale extract. This increase is below the data obtained in former studies because the dose of carotenoids in this long-term study was lower compared to former studies $[47,48]$. Nevertheless, the obtained enhancement was significantly higher than that of the placebo.

The investigations performed using two-photon tomography have shown that the SAAID also increased at the forearm and cheek in the placebo group. However, the enhancement of the SAAID of the verum group was always higher than the placebo. This indicates new collagen I production or a reduced thickening of the dermal elastic fibers. So far, it has not been observed in vivo that nutrition or supplementation results in such an effect. The more pronounced differences at the solar-exposed cheek compared to the protected inner forearm indicate not only a protection by the antioxidants and less degradation in the verum group, but also an improvement of the extracellular matrix compared to the first visit. Exposure to solar radiation induces free radicals, which interact with the extracellular matrix determinants once a critical threshold is reached [40]. This effect is called photo aging or premature skin aging [54-56]. The radical scavenging activity of the utilized curly kale extract has already been shown in a former in vivo study using electron paramagnetic resonance spectroscopy [48]. The verum group showed a higher radical scavenging activity in the skin and, as a result, an enhanced protection against VIS/NIR irradiation. 
Interestingly, a correlation between the carotenoids and the SAAID values was obtained. These findings also suggest that the significant increase of SAAID values after 10 months could be due to the intake of the carotenoid-rich extract. The tendency towards correlation between SAAID and carotenoids has previously been reported [40], but such a supplementation-induced effect has not been observed so far.

The long-term study indicates that anti-aging effects are not easy to observe because many other factors could also influence the values. The measurements are only snapshots during this long-term investigation. Nevertheless, the SAAID could serve as a reliable parameter for the determination of the skin aging status, which is not so sensitive to short-term changes.

The above-mentioned skin parameters were measured noninvasively and in vivo, and do not interfere with each other.

\section{Conclusions}

In the performed placebo-controlled in vivo study, it was demonstrated with two different optical scanners that oral supplementation of the carotenoid-rich natural curly kale extract (1650 $\mu \mathrm{g}$ of total carotenoids) once a day had caused a statistically significant increase of carotenoids in the skin 5 and 10 months after the beginning of the study. In the cheek and forearm skin areas, the collagen I/elastin ratio (SAAID value) increased significantly in both the verum and placebo groups, although it was higher in the verum group. After 10 months of supplementation, the SAAID value in the verum group had remained significantly higher. Thus, the results show that a natural extract containing a mixture of carotenoids at physiological concentrations could prevent skin aging and improve the extracellular matrix. Therefore, a healthy lifestyle including a diet rich in carotenoids is the best prevention strategy against premature skin aging.

Acknowledgments: The study was supported by the German Federal Ministry of Education and Research (BMBF), funding code $13 \mathrm{~N} 12593$.

Author Contributions: M.C.M. planned, analyzed and wrote the paper, C.K.N. performed the measurements and partly analyzed the data, S.S. partly performed the measurements, H.V. developed and provided the supplement. J.L. designed the study and M.D. analyzed the data und partly wrote the paper.

Conflicts of Interest: Hennig Vollert is owner of the company Bioactive Food. All authors declare no conflict of interest.

\section{References}

1. Fito, M.; Konstantinidou, V. Nutritional Genomics and the Mediterranean Diet's Effects on Human Cardiovascular Health. Nutrients 2016, 8. [CrossRef] [PubMed]

2. Li, S.; Zhu, Y.; Chavarro, J.E.; Bao, W.; Tobias, D.K.; Ley, S.H.; Forman, J.P.; Liu, A.; Mills, J.; Bowers, K.; et al. Healthful Dietary Patterns and the Risk of Hypertension Among Women With a History of Gestational Diabetes Mellitus: A Prospective Cohort Study. Hypertension 2016. [CrossRef]

3. Sun, Y.; Li, Z.; Li, J.; Li, Z.; Han, J. A Healthy Dietary Pattern Reduces Lung Cancer Risk: A Systematic Review and Meta-Analysis. Nutrients 2016, 8. [CrossRef] [PubMed]

4. Dias, I.H.; Polidori, M.C.; Li, L.; Weber, D.; Stahl, W.; Nelles, G.; Grune, T.; Griffiths, H.R. Plasma levels of HDL and carotenoids are lower in dementia patients with vascular comorbidities. J. Alzheimer's Dis. 2014, 40, 399-408. [CrossRef]

5. Polidori, M.C.; De Spirt, S.; Stahl, W.; Pientka, L. Conflict of evidence: Carotenoids and other micronutrients in the prevention and treatment of cognitive impairment. Biofactors 2012, 38, 167-171. [CrossRef] [PubMed]

6. Xin, Y.J.; Yuan, B.; Yu, B.; Wang, Y.Q.; Wu, J.J.; Zhou, W.H.; Qiu, Z. Tet1-mediated DNA demethylation regulates neuronal cell death induced by oxidative stress. Sci. Rep. 2015, 5, 7645. [CrossRef] [PubMed]

7. Varga, Z.V.; Giricz, Z.; Liaudet, L.; Hasko, G.; Ferdinandy, P.; Pacher, P. Interplay of oxidative, nitrosative/nitrative stress, inflammation, cell death and autophagy in diabetic cardiomyopathy. Biochim. Biophys. Acta 2015, 1852, 232-242. [CrossRef] [PubMed] 
8. Moretti, D.; Del Bello, B.; Allavena, G.; Corti, A.; Signorini, C.; Maellaro, E. Calpain-3 impairs cell proliferation and stimulates oxidative stress-mediated cell death in melanoma cells. PLoS ONE 2015, 10, e0117258. [CrossRef] [PubMed]

9. Kandola, K.; Bowman, A.; Birch-Machin, M.A. Oxidative stress-A key emerging impact factor in health, ageing, lifestyle and aesthetics. Int. J. Cosmet. Sci. 2015, 37 (Suppl. S2), 1-8. [CrossRef] [PubMed]

10. Khoubnasabjafari, M.; Ansarin, K.; Jouyban, A. Reliability of malondialdehyde as a biomarker of oxidative stress in psychological disorders. Bioimpacts 2015, 5, 123-127. [CrossRef] [PubMed]

11. Mons, U.; Muscat, J.E.; Modesto, J.; Richie, J.P.J.; Brenner, H. Effect of smoking reduction and cessation on the plasma levels of the oxidative stress biomarker glutathione-Post-hoc analysis of data from a smoking cessation trial. Free Radic. Biol. Med. 2016, 91, 172-177. [CrossRef] [PubMed]

12. Varadinova, M.G.; Valcheva-Traykova, M.L.; Boyadjieva, N.I. Effect of Circadian Rhythm Disruption and Alcohol on the Oxidative Stress Level in Rat Brain. Am. J. Ther. 2015, 23, 6. [CrossRef] [PubMed]

13. Yoshida, A.; Shiotsu-Ogura, Y.; Wada-Takahashi, S.; Takahashi, S.S.; Toyama, T.; Yoshino, F. Blue light irradiation-induced oxidative stress in vivo via ROS generation in rat gingival tissue. J. Photochem. Photobiol. B 2015, 151, 48-53. [CrossRef] [PubMed]

14. Grether-Beck, S.; Marini, A.; Jaenicke, T.; Krutmann, J. French Maritime Pine Bark Extract (Pycnogenol(R)) Effects on Human Skin: Clinical and Molecular Evidence. Skin Pharmacol. Physiol. 2016, 29, 13-17. [CrossRef] [PubMed]

15. Schempp, C.M.; Ludtke, R.; Winghofer, B.; Simon, J.C. Effect of topical application of Hypericum perforatum extract (St. John's wort) on skin sensitivity to solar simulated radiation. Photodermatol. Photoimmunol. Photomed. 2000, 16, 125-128. [CrossRef] [PubMed]

16. Jung, S.; Darvin, M.E.; Chung, H.S.; Jung, B.; Lee, S.H.; Lenz, K.; Chung, W.S.; Yu, R.X.; Patzelt, A.; Lee, B.N.; et al. Antioxidants in Asian-Korean and caucasian skin: The influence of nutrition and stress. Skin Pharmacol. Physiol. 2014, 27, 293-302. [CrossRef] [PubMed]

17. Fluhr, J.W.; Caspers, P.; van der Pol, J.A.; Richter, H.; Sterry, W.; Lademann, J.; Darvin, M.E. Kinetics of carotenoid distribution in human skin in vivo after exogenous stress: Disinfectant and wIRA-induced carotenoid depletion recovers from outside to inside. J. Biomed. Opt. 2011, 16, 035002. [CrossRef] [PubMed]

18. Maeter, H.; Briese, V.; Gerber, B.; Darvin, M.E.; Lademann, J.; Olbertz, D.M. Case study: In vivo stress diagnostics by spectroscopic determination of the cutaneous carotenoid antioxidant concentration in midwives depending on shift work. Laser Phys. Lett. 2013, 10, 105701. [CrossRef]

19. Darvin, M.E.; Sterry, W.; Lademann, J.; Patzelt, A. Alcohol consumption decreases the protection efficiency of the antioxidant network and increases the risk of sunburn in human skin. Skin Pharmacol. Physiol. 2013, 26, 45-51. [CrossRef] [PubMed]

20. Vierck, H.B.; Darvin, M.E.; Lademann, J.; Reisshauer, A.; Baack, A.; Sterry, W.; Patzelt, A. The influence of endurance exercise on the antioxidative status of human skin. Eur. J. Appl. Physiol. 2012, 112, 3361-3367. [CrossRef] [PubMed]

21. Darvin, M.; Patzelt, A.; Gehse, S.; Schanzer, S.; Benderoth, C.; Sterry, W.; Lademann, J. Cutaneous concentration of lycopene correlates significantly with the roughness of the skin. Eur. J. Pharm. Biopharm. 2008, 69, 943-947. [CrossRef] [PubMed]

22. Darvin, M.; Zastrow, L.; Sterry, W.; Lademann, J. Effect of supplemented and topically applied antioxidant substances on human tissue. Skin Pharmacol. Physiol. 2006, 19, 238-247. [CrossRef] [PubMed]

23. Darvin, M.; Lademann, J.; Magnussen, B.; Köcher, W. Multiple Spatially Resolved Reflection Spectroscopy for non-invasive determination of carotenoids in human skin. Laser Phys. Lett. 2016, 13, 095601. [CrossRef]

24. Darvin, M.E.; Meinke, M.C.; Sterry, W.; Lademann, J. Optical methods for noninvasive determination of carotenoids in human and animal skin. J. Biomed. Opt. 2013, 18, 61230. [CrossRef] [PubMed]

25. Palozza, P.; Serini, S.; Di Nicuolo, F.; Piccioni, E.; Calviello, G. Prooxidant effects of beta-carotene in cultured cells. Mol. Asp. Med. 2003, 24, 353-362. [CrossRef]

26. Lambert, J.D.; Elias, R.J. The antioxidant and pro-oxidant activities of green tea polyphenols: A role in cancer prevention. Arch. Biochem. Biophys. 2010, 501, 65-72. [CrossRef] [PubMed]

27. Zhang, P.; Omaye, S.T. DNA strand breakage and oxygen tension: Effects of beta-carotene, alpha-tocopherol and ascorbic acid. Food Chem. Toxicol. 2001, 39, 239-246. [CrossRef]

28. Palozza, P.; Calviello, G.; Bartoli, G.M. Prooxidant activity of beta-carotene under $100 \%$ oxygen pressure in rat liver microsomes. Free Radic. Biol. Med. 1995, 19, 887-892. [CrossRef] 
29. Heinrich, U.; Tronnier, H.; Stahl, W.; Bejot, M.; Maurette, J.M. Antioxidant supplements improve parameters related to skin structure in humans. Skin Pharmacol. Physiol. 2006, 19, 224-231. [CrossRef] [PubMed]

30. Gogly, B.; Godeau, G.; Gilbert, S.; Legrand, J.M.; Kut, C.; Pellat, B.; Goldberg, M. Morphometric analysis of collagen and elastic fibers in normal skin and gingiva in relation to age. Clin. Oral Investig. 1997, 1, 147-152. [PubMed]

31. Yasui, T.; Yonetsu, M.; Tanaka, R.; Tanaka, Y.; Fukushima, S.; Yamashita, T.; Ogura, Y.; Hirao, T.; Murota, H.; Araki, T. In vivo observation of age-related structural changes of dermal collagen in human facial skin using collagen-sensitive second harmonic generation microscope equipped with 1250-nm mode-locked Cr:Forsterite laser. J. Biomed. Opt. 2013, 18, 31108. [CrossRef] [PubMed]

32. Knott, A.; Reuschlein, K.; Lucius, R.; Stab, F.; Wenck, H.; Gallinat, S. Deregulation of versican and elastin binding protein in solar elastosis. Biogerontology 2009, 10, 181-190. [CrossRef] [PubMed]

33. Sanchez, W.Y.; Obispo, C.; Ryan, E.; Grice, J.E.; Roberts, M.S. Changes in the redox state and endogenous fluorescence of in vivo human skin due to intrinsic and photo-aging, measured by multiphoton tomography with fluorescence lifetime imaging. J. Biomed. Opt. 2013, 18, 061217. [CrossRef] [PubMed]

34. Koehler, M.J.; Preller, A.; Kindler, N.; Elsner, P.; Konig, K.; Buckle, R.; Kaatz, M. Intrinsic, solar and sunbed-induced skin aging measured in vivo by multiphoton laser tomography and biophysical methods. Skin Res. Technol. 2009, 15, 357-363. [CrossRef] [PubMed]

35. Karagas, M.R.; Zens, M.S.; Nelson, H.H.; Mabuchi, K.; Perry, A.E.; Stukel, T.A.; Mott, L.A.; Andrew, A.S.; Applebaum, K.M.; Linet, M. Measures of cumulative exposure from a standardized sun exposure history questionnaire: A comparison with histologic assessment of solar skin damage. Am. J. Epidemiol. 2007, 165, 719-726. [CrossRef] [PubMed]

36. Konig, K. Multiphoton microscopy in life sciences. J. Microsc. (Oxf.) 2000, 200, 83-104. [CrossRef]

37. Konig, K. Clinical multiphoton tomography. J. Biophotonics 2008, 1, 13-23. [CrossRef] [PubMed]

38. Sugata, K.; Osanai, O.; Sano, T.; Akiyama, M.; Fujimoto, N.; Tajima, S.; Takema, Y. Evaluation of unique elastic aggregates (elastic globes) in normal facial skin by multiphoton laser scanning tomography. Eur. J. Dermatol. 2015, 25, 138-144. [CrossRef] [PubMed]

39. Koehler, M.J.; Konig, K.; Elsner, P.; Buckle, R.; Kaatz, M. In vivo assessment of human skin aging by multiphoton laser scanning tomography. Opt. Lett. 2006, 31, 2879-2881. [CrossRef] [PubMed]

40. Darvin, M.E.; Richter, H.; Ahlberg, S.; Haag, S.F.; Meinke, M.C.; Le Quintrec, D.; Doucet, O.; Lademann, J. Influence of sun exposure on the cutaneous collagen/elastin fibers and carotenoids: Negative effects can be reduced by application of sunscreen. J. Biophotonics 2014, 7, 735-743. [CrossRef] [PubMed]

41. Springer, S.; Zieger, M.; Bottcher, A.; Lademann, J.; Kaatz, M. Examination of wound healing after curettage by multiphoton tomography of human skin in vivo. Skin Res. Technol. 2017. [CrossRef] [PubMed]

42. Adur, J.; Carvalho, H.F.; Cesar, C.L.; Casco, V.H. Nonlinear optical microscopy signal processing strategies in cancer. Cancer Inform. 2014, 13, 67-76. [CrossRef] [PubMed]

43. Cicchi, R.; Kapsokalyvas, D.; De Giorgi, V.; Maio, V.; Van Wiechen, A.; Massi, D.; Lotti, T.; Pavone, F.S. Scoring of collagen organization in healthy and diseased human dermis by multiphoton microscopy. J. Biophotonics 2010, 3, 34-43. [CrossRef] [PubMed]

44. Langton, A.K.; Halai, P.; Griffiths, C.E.; Sherratt, M.J.; Watson, R.E. The impact of intrinsic ageing on the protein composition of the dermal-epidermal junction. Mech. Ageing Dev. 2016, 156, 14-16. [CrossRef] [PubMed]

45. Shirshin, E.A.; Gurfinkel, Y.I.; Priezzhev, A.V.; Fadeev, V.V.; Lademann, J.; Darvin, M.E. Two-photon autofluorescence lifetime imaging of human skin papillary dermis in vivo: Assessment of blood capillaries and structural proteins localization. Sci. Rep. (UK) 2017, 7, 1171. [CrossRef] [PubMed]

46. Meinke, M.C.; Lohan, S.B.; Kocher, W.; Magnussen, B.; Darvin, M.E.; Lademann, J. Multiple spatially resolved reflection spectroscopy to monitor cutaneous carotenoids during supplementation of fruit and vegetable extracts in vivo. Skin Res. Technol. 2017. [CrossRef] [PubMed]

47. Meinke, M.C.; Darvin, M.E.; Vollert, H.; Lademann, J. Bioavailability of natural carotenoids in human skin compared to blood. Eur. J. Pharm. Biopharm. 2010, 76, 269-274. [CrossRef] [PubMed]

48. Meinke, M.C.; Friedrich, A.; Tscherch, K.; Haag, S.F.; Darvin, M.E.; Vollert, H.; Groth, N.; Lademann, J.; Rohn, S. Influence of dietary carotenoids on radical scavenging capacity of the skin and skin lipids. Eur. J. Pharm. Biopharm. 2013, 84, 365-373. [CrossRef] [PubMed] 
49. Fitzpatrick, T.B. The validity and practicality of sun-reactive skin types I through VI. Arch. Dermatol. 1988, 124, 869-871. [CrossRef] [PubMed]

50. Andree, S.; Reble, C.; Helfmann, J. Spectral in vivo signature of carotenoids in visible light diffuse reflectance from skin in comparison to ex vivo absorption spectra. Photonics Lasers Med. 2013, 2, 3. [CrossRef]

51. Darvin, M.E.; Gersonde, I.; Meinke, M.; Sterry, W.; Lademann, J. Non-invasive in vivo determination of the carotenoids beta-carotene and lycopene concentrations in the human skin using the Raman spectroscopic method. J. Phys. D Appl. Phys. 2005, 38, 2696-2700. [CrossRef]

52. Lin, S.J.; Wu, R., Jr.; Tan, H.Y.; Lo, W.; Lin, W.C.; Young, T.H.; Hsu, C.J.; Chen, J.S.; Jee, S.H.; Dong, C.Y. Evaluating cutaneous photoaging by use of multiphoton fluorescence and second-harmonic generation microscopy. Opt. Lett. 2005, 30, 2275-2277. [CrossRef] [PubMed]

53. Darvin, M.E.; Patzelt, A.; Knorr, F.; Blume-Peytavi, U.; Sterry, W.; Lademann, J. One-year study on the variation of carotenoid antioxidant substances in living human skin: Influence of dietary supplementation and stress factors. J. Biomed. Opt. 2008, 13, 044028. [CrossRef] [PubMed]

54. Baillie, L.; Askew, D.; Douglas, N.; Soyer, H.P. Strategies for assessing the degree of photodamage to skin: A systematic review of the literature. Br. J. Dermatol. 2011, 165, 735-742. [CrossRef] [PubMed]

55. Proksch, E.; Schunck, M.; Zague, V.; Segger, D.; Degwert, J.; Oesser, S. Oral intake of specific bioactive collagen peptides reduces skin wrinkles and increases dermal matrix synthesis. Skin Pharmacol. Physiol. 2014, 27, 113-119. [CrossRef] [PubMed]

56. Manickavasagam, A.; Hirvonen, L.M.; Melita, L.N.; Chong, E.Z.; Cook, R.J.; Bozecc, L.; Festy, F. Multimodal optical characterisation of collagen photodegradation by femtosecond infrared laser ablation. Analyst 2014, 139, 6135-6143. [CrossRef] [PubMed]

(C) 2017 by the authors. Licensee MDPI, Basel, Switzerland. This article is an open access article distributed under the terms and conditions of the Creative Commons Attribution (CC BY) license (http:/ / creativecommons.org/licenses/by/4.0/). 
Article

\title{
Claimed Effects, Outcome Variables and Methods of Measurement for Health Claims Proposed Under European Community Regulation 1924/2006 in the Framework of Maintenance of Skin Function
}

\author{
Daniela Martini ${ }^{1,+}$, Donato Angelino ${ }^{1,+}$, Chiara Cortelazzi ${ }^{2}$, Ivana Zavaroni ${ }^{3,4}$, \\ Giorgio Bedogni ${ }^{5}$, Marilena Musci ${ }^{6}$, Carlo Pruneti ${ }^{7}$, Giovanni Passeri ${ }^{8}$, Marco Ventura ${ }^{9}$, \\ Daniela Galli ${ }^{10}$, Prisco Mirandola ${ }^{10}$, Marco Vitale ${ }^{10}$, Alessandra Dei Cas ${ }^{3,4}$, \\ Riccardo C. Bonadonna ${ }^{3,4}$, Sergio Di Nuzzo ${ }^{2}$, Maria Beatrice De Felici ${ }^{2}$ and Daniele Del Rio ${ }^{1, *}$ \\ 1 The Laboratory of Phytochemicals in Physiology, Department of Food and Drug, University of Parma, \\ 43125 Parma, Italy; daniela.martini@unipr.it (D.M.); donato.angelino@unipr.it (D.A.) \\ 2 Department of Medicine and Surgery, Section of Dermatology, University of Parma, 43125 Parma, Italy; \\ chiara.cortelazzi@gmail.com (C.C.); sergio.dinuzzo@unipr.it (S.D.N.); bea.defelici@gmail.com (M.B.D.F.) \\ 3 Department of Medicine and Surgery, Division of Endocrinology, University of Parma, 43125 Parma, Italy; \\ ivana.zavaroni@unipr.it (I.Z.); alessandra.deicas@unipr.it (A.D.C.); riccardo.bonadonna@unipr.it (R.C.B.) \\ 4 The Azienda Ospedaliera Universitaria of Parma, Division of Endocrinology, 43125 Parma, Italy \\ 5 Clinical Epidemiology Unit, Liver Research Center, Basovizza, 34149 Trieste, Italy; giorgiobedogni@gmail.com \\ 6 Department of Food and Drug, University of Parma, 43125 Parma, Italy; marilena.musci@unipr.it \\ 7 Department of Medicine and Surgery, Clinical Psychology Unit, University of Parma, 43125 Parma, Italy; \\ carlo.pruneti@unipr.it \\ 8 Department of Medicine and Surgery, University of Parma, Building Clinica Medica Generale, \\ 43125 Parma, Italy; giovanni.passeri@unipr.it \\ 9 Laboratory of Probiogenomics, Department of Chemistry, Life Sciences and Environmental Sustainability, \\ University of Parma, 43125 Parma, Italy; marco.ventura@unipr.it \\ 10 Department of Medicine and Surgery, Sport and Exercise Medicine Centre (SEM), University of Parma, \\ 43125 Parma, Italy; daniela.galli@unipr.it (D.G.); prisco.mirandola@unipr.it (P.M.); \\ marco.vitale@unipr.it (M.V.) \\ * Correspondence: daniele.delrio@unipr.it; Tel.: +39-0521-903830 \\ + Authors equally contributed to the study.
}

Received: 31 October 2017; Accepted: 19 December 2017; Published: 22 December 2017

\begin{abstract}
Evidence suggests a protective role for several nutrients and foods in the maintenance of skin function. Nevertheless, all the requests for authorization to use health claims under Article 13(5) in the framework of maintenance of skin function presented to the European Food Safety Authority (EFSA) have received a negative opinion. Reasons for such failures are mainly due to an insufficient substantiation of the claimed effects, including the choice of inappropriate outcome variables (OVs) and methods of measurement (MMs). The present paper reports the results of an investigation aimed at collecting, collating and critically analyzing the information with relation to claimed effects (CEs), OVs and MMs related to skin health compliance with Regulation 1924/2006. CEs, OVs and MMs were collected from both the EFSA Guidance document and from the authorization requests of health claims under Article 13(5). The critical analysis of OVs and MMs was based on a literature review, and was aimed at defining their appropriateness (alone or in combination with others) in the context of a specific $\mathrm{CE}$. The results highlight the importance of an adequate choice of OVs and MMs for an effective substantiation of the claims.
\end{abstract}

Keywords: health claim; outcome variable; method of measurement; skin health 


\section{Introduction}

Skin represents the most external layer of the organism, and forms an effective barrier between the body and the environment [1]. Skin functions are extremely important and fall in several different categories: resistance to chemical and physical insults, defense from parasites and general poisons, regulation of body water, body temperature, oxygen absorption, and excretion of potentially toxic compounds (i.e., urea) [2,3]. All these functions are possible because of the particular structure of the skin, which consists of at least three main layers: (i) The epidermis, the most external layer, in direct contact with the environment, is formed by a stratum germinativum that constantly produces melanin and renews keratinocytes, and a stratum corneum consisting of dead cells, where keratin is stored, and which protects the layers underneath; (ii) The dermis, formed by epithelial tissue containing hair follicles, lymph and blood vessels; (iii) The subcutis, where blood vessels, nerves, glands and muscle fibers reside [1]. The cells in the structure are strictly connected by junctions (tight, gap, desmosomes, etc.), which allow the crosslinking of layers, thereby creating a strict and resistant mesh with selective permeability to nutrients and other compounds.

A wide range of diseases, including psoriasis, dermatitis, burns, ulcers, autoimmune disorders and cancer, can originate from alterations of the skin barrier induced by physical or chemical insults, by microorganisms, and by inflammatory processes that activate the immune system [4].

It has been estimated that at least $20 \%$ of the world population is affected by some sort of skin disorder requiring medical attention, i.e., viral or bacterial eczema, acne and infections [5]. Hay et al. highlighted that skin disorders are the fourth leading cause of non-fatal burden diseases in terms of years lost in disability [6], and statistics evidenced an $8.5 \%$ increase of death from skin disorders, and a 58.4\% increase from malignant skin melanoma during the period 1990-2010 [7].

Lifestyle has been recognized as a relevant factor influencing the onset and the development of skin disorders. A major focus has been given to the relationship of body weight and lipid-related skin disease development. Randomized clinical trials (RCTs) have shown that physical activity and diet are strictly related to a decrease of psoriasis severity [8], as well as an improvement in the "dermatology life quality index" [9]. Dietary patterns, i.e., low glycemic load diets, enhanced the reduction of follicular sebum overflow and skin surface triglycerides, and increased the ratio of saturated/monounsaturated fatty acids [10]. However, as authors have concluded that it was not possible to attribute these effects to diet composition or weight loss, the role of the diet on sebum composition needs to be further investigated.

Some investigations have focused on the role of individual components of food, such as bioactive compounds, in skin health RCTs that have considered a lycopene-enriched tomato paste [11], a high flavanol-3-ols drink [12], or a green tea polyphenol beverage [13], showing significant reductions of erythema formation in healthy volunteers. Nevertheless, evidence of a direct effect of both single components and the whole diet in affecting skin-related outcomes is still extremely weak.

The current scientific evidence has convinced stakeholders to submit requests for authorization of health claims to the European Food Safety Authority (EFSA). Some health claims pertinent to Article 13(1) of the Regulation 1924/2006 have been approved (ec.europa.eu/nuhclaims/), whereas all the requests related to Article 13(5) regarding skin function have received a negative opinion. The main reasons for the negative opinions concern the insufficient characterization of the food item and its constituents, the choice of an inappropriate (e.g., vague) claimed effect and/or target population, and most of all the inadequate substantiation of the claim through well-designed and well-performed RCTs $[14,15]$. Many parameters may affect the quality of an RCT, such as the use of a placebo-controlled approach, the calculation of the sample size, the statistical analysis, and the choice of outcome variables (OVs) and/or their methods of measurements (MMs). For these reasons, the present work aims to collect, collate and critically analyze the information in relation to claimed effects (CEs), OVs and MMs, in the context of maintenance of normal skin function. 


\section{Search Strategy}

This manuscript refers to the critical analysis of OVs and MMs collected from the EFSA Guidance on the scientific requirements for health claims related to bone, joints, skin, and oral health (EFSA 2011), from the requests for authorization of health claims under Articles 13(5) and 14 of Regulation 1924/2006 related to skin health (ec.europa.eu/nuhclaims/), and from comments received during public consultations. Adopting the decision tree described in Martini et al. (2016) [14], 3 claimed effects with $21 \mathrm{OVs}$ were evaluated under Article 13(5). No disease risk reduction claims and no health claims referring to children's development had been proposed under the Article 14. For each OV, a database of references was created on PubMed based on the keywords defined by each OV, allowing a specific critical analysis of the OVs and the MMs (Table 1). The literature databases were reviewed and used for the critical evaluation of each OV and MM. The critical evaluation of OVs and MMs was performed taking into account their relevance in the framework of randomized controlled trials. For MMs, the two main parameters that were considered were (i) being a gold standard method; and (ii) the field acceptance. Moreover, we considered the following parameters in agreement with Fitzpatrick et al. [16].

- Appropriateness

- Reliability

- Validity

- Responsiveness

- Precision

- Interpretability

- Acceptability

- Feasibility

Each OV and related MM was ranked in one of the following categories: (i) appropriate alone; (ii) appropriate only in combination with other OVs or MMs; (iii) not appropriate per se; (iv) not appropriate in relation to the specific claimed effect proposed by the applicant(s); (v) not appropriate alone, but useful as supportive evidence for the scientific substantiation of the claimed effect. The index at the beginning of this paper lists the OVs and the respective MMs from the most to the least appropriate, if a ranking was applicable. The flow chart of the project is shown in Figure 1.

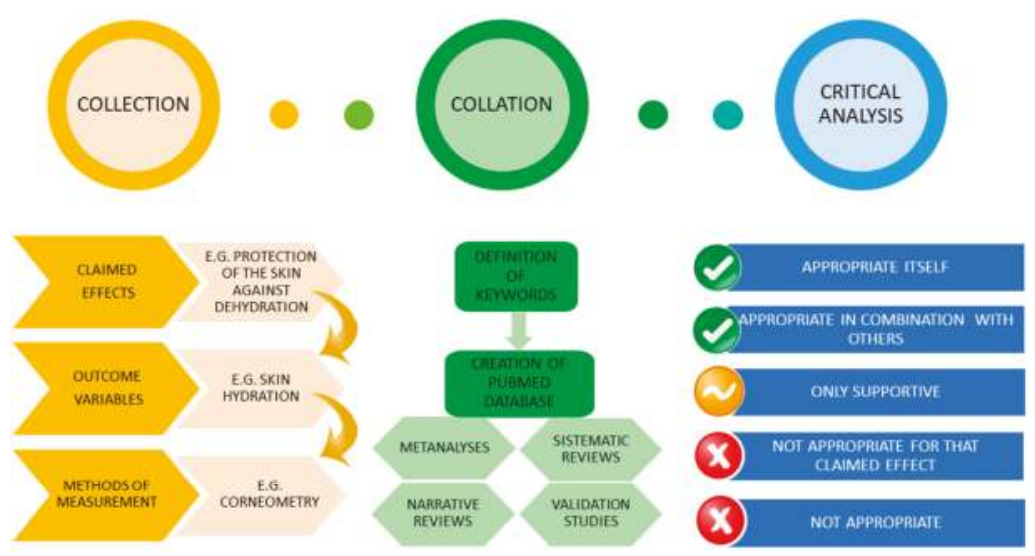

Figure 1. Collection, collation and critical evaluation of information in relation to claimed effects, outcome variables and methods of measurement in the framework of maintenance of skin function: flow chart of the project. 


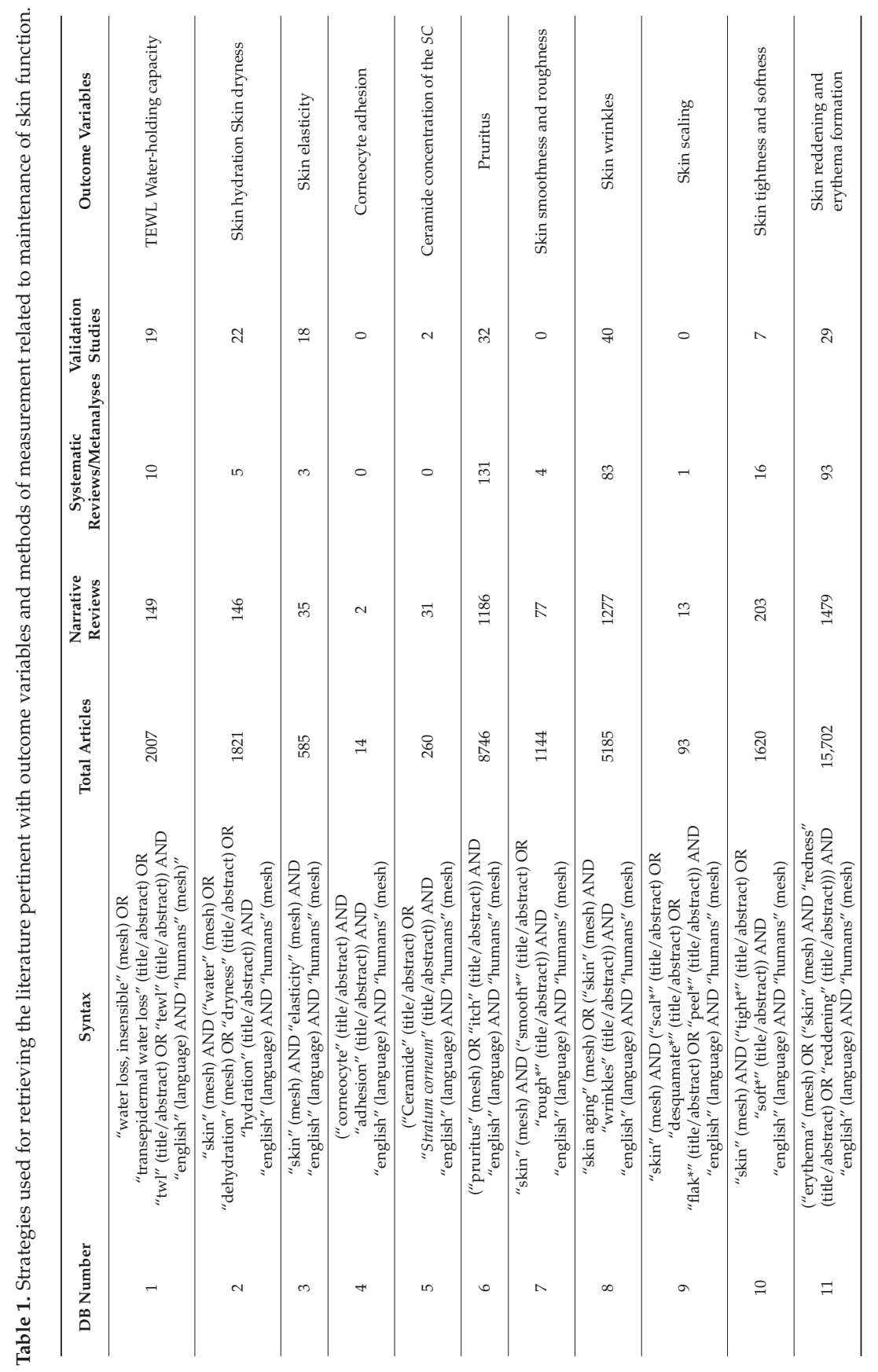




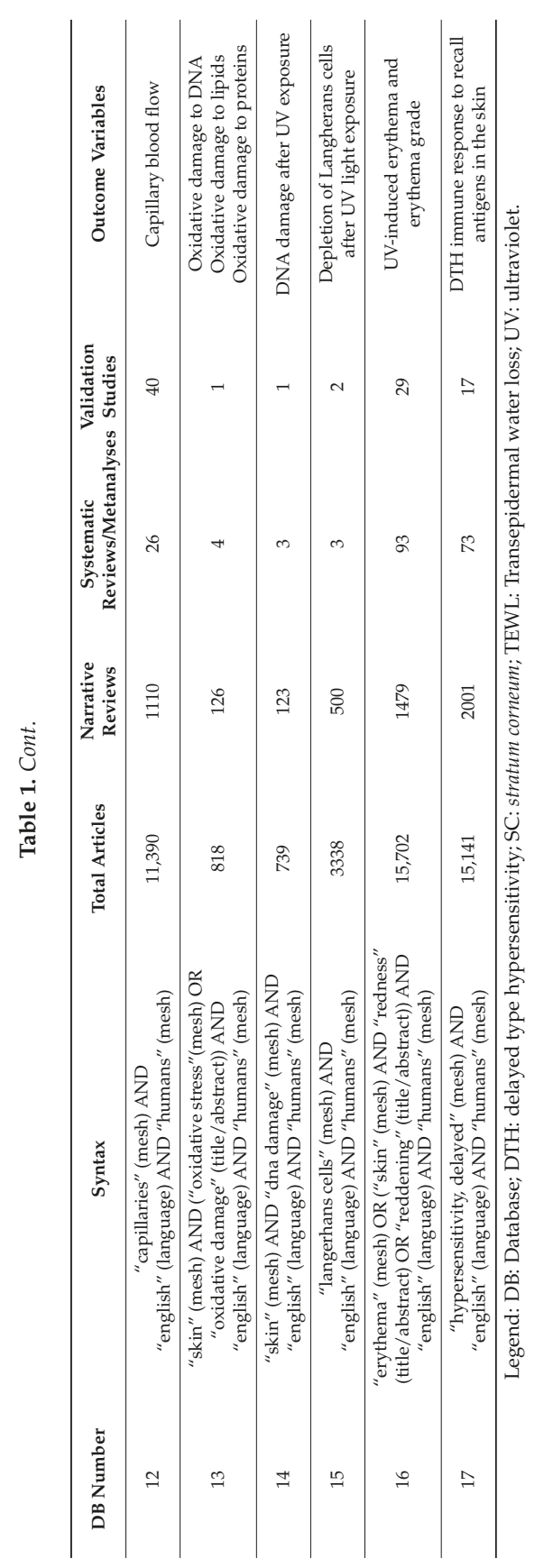




\section{Critical Evaluation for Function Claims 13(5)}

\subsection{Protection of the Skin Against Dehydration}

Skin is the largest organ of our body, and represents a physical barrier capable of holding water, as well as limiting the penetration of chemical substances, microorganisms and radiation from the environment. The ability of the skin to prevent the loss of body fluids is mainly attributed to the role of the stratum corneum (SC), an outer layer of the epidermis, formed by several deposits of dead keratinocytes, which acts as a physical barrier, limiting the loss of water and avoiding skin dehydration [1,17]. In particular, the presence of keratine and ceramides allows the formation of a compact layer that prevents the entrance of external particles and at the same time retains the body fluids under the epidermal layer [17].

Nevertheless, the use of aggressive products, such as detergents and surfactants, or exposure to environmental agents (e.g., sun, wind, cold) may compromise the structure and the function of SC, leading to a visible onset of the appearance of dehydration, e.g., dry and rough skin, desquamation. For these reasons, a compromised skin barrier may enhance the penetration of a wide range of substances/pathogens leading to pathological processes. Consequently, the maintenance of its permeability is essential and highly beneficial.

\subsubsection{Transepidermal Water Loss}

Transepidermal water loss (TEWL) has been recognized for over half a century as an in vivo parameter for assessing the skin barrier function. TEWL can be defined as the outward permeation of condensed water across the SC via diffusion, excluding other forms of water loss, such as perspiration [17]. It is expressed as grams of water per unit area of skin per unit of time $\left(\mathrm{g} / \mathrm{m}^{2} / \mathrm{h}\right)$.

To evaluate the appropriateness of TEWL as an OV for the protection of skin against dehydration, database 1 was generated (see Table 1 ).

TEWL is one of the most important parameters for evaluating the permeability function of the skin. A low TEWL is generally a characteristic feature of an intact skin function and a high hydration of the SC. Conversely, an elevated TEWL value is typically correlated with low hydration of the SC, and with an impaired epidermal barrier. This can be associated to several skin diseases, such as atopic dermatitis, or to contact with aggressive substances, such as solvents and detergents [18].

Based on these considerations, TEWL is an appropriate outcome measure to use for the substantiation of health claims in the context of protection of the skin against dehydration.

Tewameter ${ }^{\circledR}$

Being the flux of condensed water diffusing through the skin, there are no direct methods for measuring TEWL. Nevertheless, there are two different indirect methods of measurement: open-chamber and closed-chamber methods [19]. Open chambers are open to the surrounding atmosphere. The closed-chamber method is a more recent methodology/procedure, developed to avoid the effect of external air convection and turbulence but, compared to the open chambers, have the disadvantage of requiring a purge after each measurement because of the accumulation of humidity and water vapor.

TEWL assessment can be performed using different instruments and, among them, the Tewameter ${ }^{\circledR}$ (Courage-Khazaka Electronic, Cologne, Germany), an open-chamber instrument, is one of the most widely accepted and employed methods for the measurement of TEWL. Therefore, most of the scientific literature on TEWL refers to this apparatus.

Open-chamber methods are preferable for the evaluation of skin barrier function, but must occur in a room with standardized conditions (i.e., with standardized temperature and relative humidity) [19].

In these open-chamber methods, TEWL is calculated by measuring the water vapor pressure (VP) gradient immediately above the surface of the skin, calculated as the difference in VP between two distinct points aligned perpendicularly to the skin surface. 
The measuring principle of the instrument is that the vapor pressure gradient above the skin surface is proportional to the difference between the vapor pressures measured at two different heights located perpendicularly above the skin surface. With this aim, a probe, consisting of an open cylinder, is placed on the skin. The probe indirectly measures the density gradient of the water evaporation from the skin by two pairs of sensors inside the hollow cylinder. A microprocessor analyzes the values, and expresses the evaporation rate in $\mathrm{g} / \mathrm{h} / \mathrm{m}^{2}$.

TEWL is one of the most measured parameters used in cosmetology to assess the efficacy of moisturizing cosmetics. It can be evaluated in combination with the use of irritants like sodium lauryl sulphate, sodium hydroxide or dimethylsulfoxide, to identify irritant reactions.

It is also worth noting that several and various conditions can influence the value of TEWL, such as ambient temperature, relative humidity, topical products, skin damage or diseases, probe position, sweating, smoking, age, sex, and skin sites $[17,18]$. Nevertheless, many of these factors can be controlled or minimized by using a well-developed study design. The Tewameter ${ }^{\circledR}$ allows an accurate and quick measurement of the TEWL. Limitations of the Tewameter ${ }^{\circledR}$ include the slight overestimation of the resulting values in comparison to other methods [17], and the variations of results among different instruments of the same type. Despite these limitations, the Tewameter ${ }^{\circledR}$ is one of the most used instruments in RCT.

In conclusion, the Tewameter ${ }^{\circledR}$ is an appropriate method to use for the measurement of TEWL.

\subsubsection{Skin Hydration}

Skin hydration is defined as the water content of the epidermis and the dermis. An adequate skin hydration is considered a very important factor in skin health. The epithelium remains flexible when it contains 10-20\% water, and several substances may contribute to maintaining a balance in the skin homeostasis, such as natural moisturizing factor and some intercellular lipids of the SC $[20,21]$. All these factors synergize to preserve the adequate skin hydration and the barrier function of the epidermis as well as to prevent TEWL.

Superficial lipids create a filter for interaction with the external environment, and have been found to serve as water modulators in the SC.

The correct amount of water in the SC plays an important role on softness, smoothness and elasticity of the skin and allows maintenance of the typical barrier function of the skin, thus avoiding penetration of substances and microbes. Conversely, dry skin is generally linked to a reduced water content of the SC, and is associated with a rough surface, modifications of the lipid content or profile and of the permeability of the skin [21].

To evaluate the appropriateness of skin hydration as an OV for the protection of skin against dehydration, database 2 was generated (see Table 1).

An appropriate amount of water in the skin is important for the maintenance of its normal structure and properties, including adequate hydration and elasticity of the skin [22]. Moreover, skin hydration has been used extensively as an index of skin barrier function. An altered skin barrier function may modify the permeability function of the skin and facilitate the loss of body fluids and the penetration of chemicals and allergens.

Several diseases are associated to an impaired epidermal barrier, including atopic dermatitis, psoriasis, eczema, ichthyosis, and sensitive skin/rosacea [21]. Skin hydration can also decrease after the contact with aggressive cosmetics or professional substances leading to desiccation of SC with alteration of the normal epidermal barrier.

In conclusion, skin hydration is an appropriate outcome variable to use for the substantiation of health claims in the context of protection of the skin against dehydration. However, the simultaneous measure of TEWL is preferable, and provides a more complete and accurate measure of the hydration state of the skin. 


\section{Corneometry}

Parameters related to skin hydration may be measured by considering the electrical properties that are dependent on the water content of the SC. Many commercially available instruments, based on principles of measuring skin capacitance, conductance, or impedance, have been widely used for this purpose [18,23].

Corneometry is one of the most commonly used techniques for measuring skin hydration, as well as water-holding capacity, as a surrogate measure of skin hydration.

The Corneometer ${ }^{\circledR}$ (Courage-Khazaka Electronic, Cologne, Germany) measures skin hydration by determining the skin capacitance with the use of probes [23]. The measurement is based on the difference between the dielectric constant of water and other substances by measuring the capacitance of a dielectric medium. The Corneometer ${ }^{\circledR}$ measures the change in the dielectric constant due to skin surface hydration by modulating the capacitance of a precision capacitor. The depth of measurement is quite low, as it reaches the first $10-20 \mu \mathrm{m}$ of the SC.

One of the techniques that can be used is the water sorption-desorption test, which consists of the hydration of the skin with water followed by the observation of the subsequent dehydration activity by means of serial recording with electrical instruments.

The instrument delivers values expressed in arbitrary units (AU) varying from 0 to 120, where a higher value indicates a more hydrated skin with values $>40$ indicating adequate skin hydration [24].

Similar to other electrical measurements, corneometry has become very popular because of the relative low cost and ease of use; in fact, corneometry measurements are very rapid, and the modern probes enable temperature stability. In addition, the technique minimizes the subjectivity typical of visual scoring methods, and therefore allows greater accuracy and reproducibility [25,26].

Moreover, although several experimental and instrumentation-related factors also influence measurements (e.g., skin temperature, sweating, ambient temperature and humidity), many of these factors can be controlled or minimized by using a well-developed study design and specific standardized-condition rooms.

In conclusion, corneometry is an appropriate non-invasive method that can be used for the measurement of skin hydration and water-holding capacity, especially if in association with other skin parameter measurements, e.g., TEWL. In fact, as these are all indirect parameters, their association can lead to more meaningful results.

\subsubsection{Skin Dryness}

Skin dryness is a common condition in which skin loses its homeostasis, and there is an impaired epidermal barrier. Many causes can contribute to this condition, such as exposure to aggressive solvents, frequent hand washing, specific occupational activities, or skin diseases (allergic or atopic dermatitis). Aging and hormonal changes can determine skin dryness, too.

Skin dryness is often called "xerosis", a term used in dermatology to indicate any condition of abnormal dry skin [27].

Pruritus is the most important symptom of skin dryness.

To evaluate the appropriateness of evaluation of skin dryness by an expert evaluator as an OV for the protection of skin against dehydration, database 2 was generated (see Table 1).

Daily insults from the environment can lower the water content of the stratum corneum, which impairs the enzymatic function required for normal desquamation, leading to a dry and flaky skin. Therefore, skin dryness can be considered to be an indirect measure of skin hydration.

However, dry skin is associated with major signs such as scaling, roughness, cracks and redness, and thus the evaluation of skin dryness cannot disregard the evaluation of these signs to obtain the overall dry skin score (ODS), a scoring scale combining all these major and minor signs of dry skin [28].

In conclusion, the skin dryness score is an appropriate outcome measure to be used for the substantiation of health claims in the context of protection of the skin against dehydration. 
However, the combination with additional outcome measures such as TEWL is essential to obtain a more reliable and objective result.

\section{Evaluation of Skin Dryness by an Expert Evaluator}

The evaluation of skin dryness can be self-assessed by the subject or performed by an expert evaluator. In the first case, the evaluation can be influenced by subjective feelings, such as itching, while the evaluation by experts is based on objective criteria.

Usually, as recommended in the guidance published by The European Group on Efficacy Measurement of Cosmetics and other Topical Products, the expert evaluator can use a scoring scale combining all the effects of dry skin and judging it from 0 (absent) to 4 (advanced roughness, redness and cracks) [28]. If necessary, a specified symptom sum score can be used, using an evaluation of the main signs of xerosis (scaling, roughness, redness and cracks) in selected anatomical regions. Finally, an evaluation of the whole skin, as well as specifically at different sites (e.g., head/neck, upper and lower extremities, trunk), can be performed.

Moreover, dryness is a condition often associated with several dermatological diseases, in particular atopic dermatitis (also known as atopic eczema). Different scores have been developed to evaluate and classify disease grade: Eczema Area and Severity Index, Scoring Atopic Dermatitis, Objective Component of Score, Investigator Global Assessment, Atopic Dermatitis Severity Index and Body Surface Area [29]. Simultaneous use of two or more scores is a reliable assessment of disease severity.

The evaluation of dry skin by an expert evaluator is preferable compared to self-evaluation, because it is not influenced by subjective feelings such as itching and thus is more reproducible. Nevertheless, most of these scores consider patient's symptoms (as pruritus) and the effect of the disease on quality of life.

In conclusion, the evaluation of dry skin by an expert evaluator is an appropriate method to use for the measurement of skin dryness.

\subsubsection{Skin Elasticity}

Skin elasticity represents the ability of the skin to resume its original shape once it is stretched [30]. This property is determined by elastic and collagen fibers, arranged as a mesh in the dermis, which are responsible for the elastic and mechanical properties of the skin, respectively. Specifically, collagen fibers determine the mechanical stability of the tissue and its resistance to deformation; meanwhile, elastic fibers restore deformed collagen bundles to a more relaxed state. As with all organs, skin health is affected by aging, but in two ways: chrono-aging (the physiological aging of the skin genetically determined) and photo-aging (due to ultraviolet (UV) exposure). These processes are also influenced by many other factors (e.g., smoke, alcohol, pollution), which lead to degradation of collagen and elastin with a more stiffness of the skin [31]. The resulting loss of elasticity, with consequent reduction in moisture and formation of wrinkles, typically occurs in sun-exposed areas, such as facial skin.

To evaluate the appropriateness of skin elasticity as an OV for the protection of skin against dehydration, database 3 was generated (see Table 1).

The measurement of the viscoelastic properties of the skin can be an indicator for the biological age of the skin. Being an elastic material, skin is subject to the mechanical laws defining its properties, which are modified by some factors. One of the most important is the process of skin aging (i.e., intrinsic and extrinsic). In fact, tensile functions of the skin and subcutaneous tissues contribute to the appearance of the aged and photo-damaged skin, and to the effects of various other pathophysiological processes [32]. Moreover, the depth of skin wrinkles can appear deeper in less moisturized skin.

Therefore, skin elasticity is not an appropriate outcome variable to be used alone for the substantiation of health claims in the context of protection of the skin against dehydration. However, it can be used in combination with more significant skin measures, such as TEWL, in order to provide significant and descriptive information about the hydration of the skin. 


\section{Cutometer $^{\circledR}$}

To date, several methods based on different principles have been proposed for evaluating skin elasticity.

Among these, the Cutometer ${ }^{\circledR}$ (Courage-Khazaka Electronic, Cologne, Germany) is probably the most commonly used instrument, and is considered the tool against which innovative or pilot instruments are to be compared. It is a handle device able to measure the viscoelastic properties of human skin using the suction/elongation method [33]. In detail, it can determine the elasticity of the upper skin layer using negative pressure, which is created in the device, and which mechanically deforms the skin. Skin is drawn into the aperture of the probe, and is released after a defined time. The depth of penetration of the skin inside the probe is determined using a non-contact optical measuring system.

The elasticity, i.e., the skin's ability to return to its original position when deformed, is displayed as a strain-time curve that can be analyzed by software. The many parameters that can be measured include the ability to return to the original state, the overall elasticity, and the net elasticity of the skin [34]. In addition, F- and Q-parameters can be measured, giving additional indications on skin age and elasticity [35].

These parameters have been widely used in studies both on normal skin (including cosmetological applications) and in skin disease (e.g., psoriasis and scleroderma), reflecting both the state of the skin and the change in the skin structure.

Because of significant regional variations in the viscoelastic properties of the skin, skin elasticity should be measured in the same area within a RCT [36].

In conclusion, the Cutometer is an appropriate method for the measurement of skin elasticity.

\subsubsection{Corneocyte Adhesion}

The major purpose of epidermal differentiation is generating the stratum corneum (the superficial skin layer), whose primary function is to protect the internal organs from desiccation and from external injuries.

Moreover, stratum corneum functions as a protective barrier against the outside, because of its well-organized structure. The structural integrity of the stratum corneum is guaranteed by corneocytes - dead differentiated keratinocytes, in which the cytoplasm is constituted by specialized proteins (keratins, filaggrin)—while the interstices between the corneocytes are enriched by lipids [37].

Stratum corneum lipids are nearly devoid of phospholipids, and are selectively enriched in sphingolipids (ceramides), free sterols and not-esterified fatty acids, with lesser quantities of nonpolar lipids and cholesterol sulphate. Ceramides are hydrophobic, and are ideal for preventing excess water loss. Corneocyte adhesion is possible because they are bridged together through particularly modified desmosomes, named corneodesmosomes, which ensure the stability and integrity of the stratum corneum. As corneocytes migrate towards the upper layer of the stratum corneum, corneodesmosomes weaken their adhesive function, allowing cells to be disrupted, and promoting the physiological desquamation process for the renewal of the stratum corneum layer. Accelerated corneodesmosome degradation leads to an enhanced desquamation process, leaving the outer layer exposed to the environment, xerosis, desquamation, and possible penetration by microbes or allergens [38]. Abnormality of the normal corneodesmosome structure is also related to common skin disease (e.g., psoriasis, atopic dermatitis, lichen planus).

To evaluate the appropriateness of corneocyte adhesion as an OV for the protection of skin against dehydration, database 4 was generated (see Table 1 ).

Ultraviolet, the outermost epidermal layer, plays a critical role in the physical protection of the body. To maintain a constant SC thickness, as observed in normal epidermis, the continuous generation of corneocytes is balanced by cell shedding at the external surface in the tightly regulated process of desquamation. Cohesion of the SC is largely dependent on modified desmosomes or corneodesmosomes. Corneodesmosome degradation is of major importance in the desquamation 
process [39]. Several skin conditions are related to an altered skin scaling. In xerosis and various hyperkeratotic states, including psoriasis, accumulation of scales is observed, and the number of corneodesmosomes persisting over the corneocyte surface in the upper SC is greatly increased [40]. Moreover, some of the disorders of cornification, such as congenital ichthyosiform erythroderma, shows other alterations of the epidermal features, such as increased TEWL. Perturbed barrier function, abnormal desquamation, and hyperproliferation appear to be intimately linked.

In conclusion, corneocyte adhesion is not appropriate to be used alone for the substantiation of health claims in the context of protection of the skin against dehydration. Nevertheless, it can be used as supportive evidence when combined with other appropriate outcome measures (e.g., TEWL).

\section{Squamometry}

The assessment of scaling and dryness is difficult to standardize. Nowadays, several methods based on different principles have been proposed for the evaluation of skin scaling. The most common technique is based on the sampling of the superficial scaling portion of the stratum corneum using various kinds of adhesive tapes (for example D-Squame ${ }^{\circledR}$, CuDerm, Dallas, TX, USA) [41]. The quantification of scales or squames is made by using low-power imaging techniques [42], which allow the calculation of the squame size, number, optical density, and heterogeneity in relation to different aspects of the desquamation process. Image analysis can be performed using appropriate video-cameras such as the Visioscan ${ }^{\circledR}$ (Courage-Khazaka Electronic, Cologne, Germany), an ultraviolet A (UVA)-light video camera with high resolution. The images show the skin structure and the level of dryness, but it can also be used on spots of hair and scalp. The camera can be connected to the computer, and several skin surface parameters can be determined, including desquamation, sebum production, scaliness, smoothness and roughness [43]. The technique is repeatable and reproducible.

In conclusion, squamometry is an appropriate method to use for the evaluation of corneocyte adhesion.

\subsubsection{Ceramide Concentration of the Stratum Corneum}

In the stratum corneum, the matrix between corneocytes is composed of lipids arranged in numerous lamellar sheets, creating an impermeable barrier against external pathogens, and preventing water loss. More than $50 \%$ of the lipids of the intercellular space is represented by ceramides, which chemically belong to the sphingolipid class, as they are produced following the hydrolysis of sphingomyelin. Ceramides allow the formation of a barrier against cell permeability, and play a role in signal transduction, cell regulation, cell differentiation and immune response [44]. In human cells and tissues, there are commonly three ceramide classes, and human stratum corneum is known to contain even more complex ceramides. At least nine classes of ceramides have been classified on the basis of the characteristic fatty acid chain: non-hydroxy, $\alpha$-hydroxy, ester-linked $\omega$-hydroxy fatty acids, etc. [45] A lower level of ceramides, due to fatty acid hydrolysis, has been related to a loss in permeability of the skin surface, enhancing the skin inflammation by external agents. In fact, the loss of ceramides allows the pauperization of the intercellular lipid content, triggering water loss, as well as inflammation due to parasites and the grime accumulation between corneocytes. Although the causes of the decreased concentration of the ceramides in the stratum corneum due to skin lesions have been established, there is still a debate on the causes of the loss in non-lesioned skin, such as dermatitis, psoriasis or xerosis. However, it is widely recognized that skin disorders involving diminished barrier function show also a decrease in total ceramide content with some differences in the ceramide pattern.

To evaluate the appropriateness of ceramide concentration of the SC as an OV for the protection of skin against dehydration, database 4 was generated (see Table 1).

SC lipids are selectively enriched in sphingolipids (ceramides), free sterols, and free fatty acids, with lower quantities of non-polar lipids and cholesterol sulphate. Ceramide critical components of the barrier are hydrophobic, and are ideal for preventing excessive water loss. For this reason, SC water retention is improved by the addition of ceramides to damaged skin and a loss of these 
lipids causes profound barrier damage with a TEWL increase [44]. However, damage is also due to other mechanisms, such as a simultaneous, passive loss of extracellular calcium and potassium ions, and other lipids, including cholesterol and fatty acids, are also implicated.

In conclusion, ceramide concentration of the SC cannot be used alone as an outcome variable for the substantiation of health claims in the context of protection of the skin against dehydration. However, it can be used as supporting evidence when appropriate outcome measures (e.g., TEWL) are also considered. In fact, all of these values are indirect values, so it is better to combine different measurements for a more reliable and sensitive result.

\section{HPLC (High Performance Liquid Chromatography)}

The most common method for detection and quantification of the different classes of ceramides is based on liquid chromatography techniques [46].

Biological samples that can be used for the analyses include epidermis collected by tape-stripping method, as well as blood or tissue samples (e.g., liver or adipose). Ceramides are chemically derivatized with benzoyl chloride or anhydride, depending on the fatty acid chain bound to sphingosine [47]. This step leads to the production of the $\mathrm{N}$-acyl derivatives, which strongly absorb in the range 230-280 nm, depending on the ceramide type. Then, direct-phase HPLC is performed by using silica gels resins, while the mobile phase for the elution is based on organic solvents, such as hexane, pentane or ethyl acetate. The method is simple, sensitive and accurate. However, due to the instability of the derivatization product, samples should not be stored for prolonged periods, and analysis must be performed just after derivatization. Another limit of the HPLC method is that ceramide classes targeted for quantification are restricted to only few types.

In conclusion, HPLC is an appropriate method to be used for the measurement of the ceramide concentration of the stratum corneum.

\subsubsection{Pruritus}

Pruritus, or itching, can be defined as an unpleasant sensation of the skin causing the desire to scratch the affected area. The mechanisms of pruritus have not yet been totally understood. It may be both localized and generalized and can occur as an acute or chronic condition. Itching can be the result of a dermatological disease (such as atopic dermatitis, eczema or urticaria), but can also be the result of several systemic diseases (e.g., thyroid or liver dysfunctions, important hormonal changes, hematological diseases, and nerve or psychiatric disorders) [48]. Nevertheless, itching can also be quite a common sign of simple skin conditions, such as dehydrated skin, and it can be exacerbated by cold and dry weather or increase with age. Moreover, if severe, itching can affect the quality of life of individuals and it has also important psychological implications [49,50]. Evaluating pruritus is very difficult due to the absolute subjectivity of the symptoms.

To evaluate the appropriateness of pruritus as an OV for the protection of skin against dehydration, database 6 was generated (see Table 1).

Pruritus is the most common symptom in dermatology. As mentioned above, pruritus can be related to several pathological conditions, but it can also indicate a lack of the normal skin integrity and correct hydration. For this reason, it is a frequent symptom in aged people [50]. Due to the large amount of pruritus-associated conditions, to the difficulties in identifying symptoms and the psychological components, and to the difficult quantification for its subjectivity, pruritus cannot be used alone as an outcome variable for the substantiation of health claims in the context of protection of the skin against dehydration. However, it can be used as supporting evidence when appropriate outcome measures (e.g., TEWL) are also considered.

Questionnaires

Itching is a subjective condition difficult to quantify. Moreover, its multifactorial etiology is difficult to characterize. 
Several scales and scores have been proposed, both monodimensional and multidimensional. The former, including the visual analogue scale, numerical rating scale, and verbal rating scale, report information about intensity of itching in a specific moment. Conversely, multidimensional scales can provide different information including affected areas, progression of itch throughout the day and its evolution during the time, disability induced by the pruritus, improvement during treatment. This is why multidimensional scales can also be used as an instrument for evaluating treatment.

One of the most used methods are the visual analog scales (VAS), both vertical and horizontal [51]. Different authors have proposed different categorizations of VAS, such as: $0=$ no pruritus, $>0-<4$ points $=$ mild pruritus, $\geq 4-<7$ points $=$ moderate pruritus, $7-8.9$ points $=$ severe pruritus, and $\geq 9$ points = very severe pruritus. The VAS is a valuable, easy and rapid method for estimating pruritus, and has shown good reproducibility and reliability [52]. In addition, VAS results have proven to be reproducible even in different populations and ethnic groups. However, similar to other monodimensional methodologies, VAS has some drawbacks. For example, it only measures intensity, without considering the impact on quality of life, or other parameters such as duration and distribution of itching. For this reason, a 5-D itch scale has been developed as a multidimensional questionnaire $[53,54]$. The five dimensions are degree, duration, direction, disability and distribution, with a scale specifically designed to facilitate the use of pruritus as an outcome variable in RCTs and clinical trials.

In conclusion, questionnaires are an appropriate method to be used for the measurement of pruritus. The use of VAS is appropriate, although the use of multidimensional methods is preferable, allowing a thorough comprehension of the pruritus condition.

\subsubsection{Water-Holding Capacity}

The water in the skin is present as both free and bound (to macromolecules) water.

The ability of the skin to hold water is primarily related to the SC, which acts as a barrier to water loss. Although the SC is a thin biological membrane, it is an essential part of the body, allowing it to survive even in a dry atmosphere by protecting our body from desiccation. The barrier function and the water-holding capacity of the SC depend on the presence of well-differentiated corneocytes with cornified envelope-associated proteins. These proteins bind the ceramide containing intercellular lipids and large amounts of moisturizing factors mainly consisting of water-soluble amino acids produced by enzymatic degradation of filaggrin [55].

Lipids and keratins play a key role in the formation of the permeability barrier, and are responsible for the water-holding capacity, which can be defined as the ability of the SC to retain water.

Because the SC is the interface between the fully hydrated viable epidermis and the dry atmosphere, there is a gradient of water in this thin biological membrane [56]. However, when the uppermost portion of the SC loses water, even normal individuals develop dry skin. This condition leads to a fine cracking in the SC, which deteriorates its barrier function focally, allowing permeation of external substances as well as TEWL.

To evaluate the appropriateness of water-holding capacity as an OV for the protection of skin against dehydration, database 6 was generated (see Table 1).

Skin hydration can depend on the water-holding capacities of the SC, but it is not a unique parameter for correctly assessing skin hydration. That is why the combination with other variables is important for a more accurate assessment of skin conditions and of potential alterations and changes. In conclusion, the water-holding capacity is not appropriate to be used alone for the substantiation of health claims in the context of protection of the skin against dehydration. However, it can be used as supporting evidence when other appropriate outcome measures (e.g., TEWL) are considered.

\section{Corneometry}

See "Corneometry" in Section 3.1.2. 


\subsubsection{Skin Smoothness and Roughness}

Due to the three-dimensional organization of cutis and subcutaneous tissue, skin surface is not perfectly smooth and is characterized by folds, furrows, orifices, and crests. In particular, some morphological characteristics of the epidermis (thickness of the cornified layer) and dermis (collagen content) can determine skin surface. However, pathological issues such as dermatitis, eczema, and other conditions, such as chronic light exposure, pollution and aggressive substances, may change the balance between skin smoothness and roughness, and may enhance the hardening process of the skin, leading to the coarsening of the skin surface structure with an increase of the wrinkle number and depth and, in general, of the roughness.

Skin smoothness and roughness, together with skin scaling and wrinkles, are considered to be the qualitative and quantitative parameters of skin physiological conditions, and represent the four clinical parameters that, if considered together, allow the Surface Evaluation of the Living Skin (SELS) [57].

To evaluate the appropriateness of skin smoothness and roughness as an OV for the protection of skin against dehydration, database 7 was generated (see Table 1).

With aging, skin texture undergoes several changes. Epidermal thickness decreases with a flattening of dermal-epidermal junction, while wrinkles increase due to loss of collagen content. This process is typical of aging, and it is more evident in photo-exposed areas. As the skin ages, changes in the texture and roughness or smoothness of the skin become apparent [58]. This is the result of loss of barrier integrity, which leads to increased water loss, but it is also due to changes in the collagen-supporting matrices showing visibly on the skin surface [59]. In fact, skin aging is a multifactorial process involving different mechanisms with a modification of different skin parameters, such as an increase in epidermal water loss.

In conclusion, skin smoothness and roughness are not appropriate to be used alone for the substantiation of health claims in the context of protection of the skin against dehydration. However, they can be used as supporting evidence when other appropriate outcome measures are considered (e.g., TEWL). In addition, the simultaneous evaluation of all 4 parameters, allowing the SELS (e.g., skin wrinkles and scaling), is preferable, in order to have a more reliable value.

\section{UV Camera}

Skin topography can be directly evaluated by using appropriate video-cameras. Among these, Visioscan ${ }^{\circledR}$ —a UVA-light video camera with high resolution—has been widely and successfully used as an alternative to the conventional color video cameras.

Visioscan ${ }^{\circledR}$ consists of a black and white video sensor chip, an objective and an UVA-light, and is equipped with two special halogenide lights that illuminate the skin.

Visioscan ${ }^{\circledR}$ can be used to measure the SELS, which has been proposed as a qualitative and quantitative measure of the skin surface. SELS includes evaluation of skin smoothness and roughness, skin scaling and wrinkles [57]. Other parameters can be analyzed with Visioscan ${ }^{\circledR}$, such as skin desquamation and scalp and sebum production. With this method, skin can be optically monitored using an image-digitalization process. The grey level distribution is analyzed, making it possible to calculate the four SELS parameters, both quantitatively and qualitatively. This is useful to describe the skin surface as an index [60].

Visioscan ${ }^{\circledR}$ is small, easy-to-handle, and economical, and makes it possible to have multi-frame pictures of the skin and to store these images for subsequent evaluation; it is therefore useful in elaborating different parameters throughout the time.

In conclusion, UV cameras such as Visioscan ${ }^{\circledR}$ are an appropriate method for measuring skin smoothness and roughness, wrinkles and scaling. However, it is worth noting that none of these outcome measures is appropriate to be used alone for the substantiation of health claims in the context of maintenance of skin function. 


\subsubsection{Skin Wrinkles}

Skin surface is not flat, but is characterized by several grooves, classified according to their depth. For this reason, the skin surface exhibits a network called microrelief or texture. This microrelief is very important for the mechanical properties of the skin, as it forms a complex system of small lines intersecting each other. On fingers and toes, these lines assume a characteristic disposition, and determine the fingerprint. As a result of age and UV exposure, skin microrelief changes, and some lines become more marked-being defined as wrinkles [58]. An example of wrinkles are so called "crow's-feet", which are localized on the forehead or around the eye circles, and may vary in amplitude and marking severity.

Age, diseases (including psoriasis or atopic dermatitis) and the loss of hydration of the skin may alter the physiological desquamation of the skin, giving some roughness to the touch.

As already mentioned, skin wrinkles, together with skin scaling, roughness and smoothness, are considered to be qualitative and quantitative parameters of skin physiological conditions, and represent the four clinical parameters that, if considered together, allow the SELS [57].

To evaluate the appropriateness of skin wrinkles as an OV for the protection of skin against dehydration, database 8 was generated (see Table 1).

Wrinkle formation is a genetically-determined process (a process called "chrono-aging" or intrinsic aging). Several environmental factors contribute to wrinkle formation and/or worsening, such as smoking, UV-exposure, age, pollution ("photo-aging" or extrinsic aging) [58]. These mechanisms lead to a loss of elasticity that contributes to the worsening of wrinkles through decreased skin hydration.

Even if skin wrinkles are a reliable parameter of skin structure, they are an indirect parameter of skin hydration [61]. For this reason, skin wrinkles are not appropriate to be used alone for the substantiation of health claims in the context of protection of the skin against dehydration. However, they can be used as supportive evidence when appropriate outcome measures are also considered (e.g., TEWL).

In addition, the simultaneous evaluation of all 4 parameters that allow the SELS (e.g., skin smoothness, roughness and scaling) is preferable, in order to obtain more accurate results.

\section{UV Camera}

See “UV Camera” In Section 3.1.9.

\subsubsection{Skin Scaling}

Skin scaling, also known as skin peeling or desquamation, is the loss of the outer layer of the epidermis (stratum corneum) in large flakes. This layer consists of 18-20 layers of flattened dead keratinocytes with no nuclei and cell organelles, which have a defensive role against pathogens and environmental insults [62]. Skin scaling is a physiological process occurring when keratinocytes shed in an inappreciable way because of the new ones located in the underneath layer. In contrast, it is considered pathological when induced by disease or pathological causes, such as atopic dermatitis, contact dermatitis/eczema, or extreme cases of severe drug reactions, which can lead to a real exfoliation with loss of several layers of epidermis [63]. In these cases, keratinocytes scale down, leaving the outer layer of the skin unprotected. For these reasons, the pathological peeling of the epidermis may be accompanied by itch, dryness or irritating phenomena, which enhance the loss of the keratinocytes.

As already mentioned, skin scaling, together with skin wrinkles, roughness, and smoothness are considered to be qualitative and quantitative parameters of the skin physiological conditions, and represent the four clinical parameters that, if considered together, allow the SELS [57].

To evaluate the appropriateness of skin scaling as an $\mathrm{OV}$ for the protection of skin against dehydration, database 9 was generated (see Table 1). 
Skin scaling is a sign frequently observed on the skin surface. It usually suggests an alteration in skin homeostasis and an alteration of the stratum corneum. During their transit through the epidermal layers toward the skin surface, keratinocytes follow a well predetermined differentiation program in order to originate the stratum corneum. Several conditions can lead to skin scaling. As cited above, some dermatological diseases are characterized by scaling. However, scaling can be also determined by a transient state of skin dehydration or other particular conditions. For example, being elderly is accompanied by an epidermal barrier impaired with TEWL increase, xerosis and skin scaling [64].

In conclusion, skin scaling is not appropriate to be used alone for the substantiation of health claims in the context of protection of the skin against dehydration. However, it can be used as supporting evidence when appropriate outcome measures are also considered (e.g., TEWL).

In addition, the simultaneous evaluation of all 4 parameters allowing the SELS (e.g., skin smoothness, roughness and wrinkles) is preferable.

\section{UV Camera}

See "UV Camera" in Section 3.1.9.

\subsubsection{Skin Tightness and Softness}

Tightness and softness are skin conditions mainly dependent on skin compactness and elasticity, which may be influenced by several factors: diet; exposure to chemicals such as soaps or detergents; and atmospheric conditions, i.e., sun or wind. Pathological conditions, such as scleroderma, or diseases of collagen and subcutaneous tissue can modify skin tightness and softness [65].

To evaluate the appropriateness of skin tightness and softness as an OV for the protection of skin against dehydration, database 10 was generated (see Table 1).

Skin tightness and softness are features more typical of the deep tissue of the cutis (connective tissue) than the epidermis. They are determined by changes of deep components of the dermis, in particular, collagen and elastic fibers. Intrinsic and extrinsic aging leads to a degradation of collagen and elastic fibers with a consequent intensification of skin wrinkles, dryness and scaling.

However, skin texture can also be modified by pathological conditions of the connective tissue, which is characterized by changes in composition and/or tridimensional organization of collagen. In particular, systemic sclerosis (also known as scleroderma) or morphea (a localized form of sclerosis) are the result of an excessive activation of the repair process known as fibrosis leading to a severe skin tightening [66]. On the other hand, an increased softness is the main feature of elastic fiber disease, such as cutis laxa, in which the skin becomes inelastic and hangs loosely in folds. Patients develop a prematurely aged appearance. Nevertheless, a loss of skin elasticity is often associated with changes in other skin features, such as a decreased water content of the skin.

In conclusion, skin tightness and softness are not appropriate to be used alone for the substantiation of health claims in the context of protection of the skin against dehydration. However, they can be used as supporting evidence when appropriate outcome measures are also considered (e.g., TEWL).

\section{Self-Assessment}

Skin tightness or softness is evaluated by using the Modified Rodnan Score [67]. This score consists of an evaluation of skin thickness rated by clinical palpation using a $0-3$ scale $(0=$ normal skin; 1 = mild thickness; 2 = moderate thickness; 3 = severe thickness with inability to pinch the skin into a fold) for each of the 17 surface anatomic areas of the body: face, anterior chest, abdomen, (right and left separately) fingers, forearms, upper arms, tights, lower legs, dorsum of hands and feet. These individual values are added, and the sum is defined as the total skin score, ranging from 0 to 51 .

Modified Rodnan score is frequently utilized to evaluate the systemic sclerosis (or scleroderma), characterized by a progressive increase in skin tightness [68], and to monitor the efficacy of treatments. Skin thickness is used as a surrogate measure of disease activity, severity and mortality in patients 
with diffuse cutaneous systemic sclerosis, and higher skin thickness progression rates are predictive of internal organ involvement and mortality. This score can be used by physicians, but can also be self-assessed by the subjects, due to the high level of correlation between subject self-assessment and physician-assessment. In conclusion, the self-assessment with Modified Rodnan Score is an appropriate method to be used for the measurement of the skin tightness and softness.

\subsubsection{Skin Reddening and Erythema Formation}

Erythema is a skin condition characterized by redness and is typically caused by vasodilation of superficial capillaries in the dermis [69]. It is a major feature of inflammatory skin reactions elicited by irritants or allergens. Erythema is often associated with an impaired barrier function, such as in the case of "sensitive skin" and rosacea, atopic dermatitis, psoriasis, allergic reactions, autoimmune disease [70]. Erythema is one of the most frequent signs of a pathological skin, with or without an impaired epidermal barrier function.

To evaluate the appropriateness of skin reddening and erythema formation as an OV for the protection of skin against dehydration, database 11 was generated (see Table 1).

If severe, reddening and erythema may lead to the loss of the barrier function of the skin. Moreover, an impaired epidermal barrier (as measured by an increase in TEWL) can be associated with clinical symptoms like redness and erythema.

Nevertheless, erythema is not directly and unequivocally related to an impaired structure or hydration or permeability function of the skin.

Therefore, skin reddening and erythema formation are not appropriate to be used for the substantiation of health claims in the context of protection of the skin against dehydration.

\subsubsection{Capillary Blood Flow}

The total blood flow within the systemic circulation is about $5 \mathrm{~L} / \mathrm{min}$. Most of the cardiac output is received by the gastrointestinal system and the skeletal muscle, while only $\sim 5 \%$ goes to the skin. Cutaneous microcirculation is organized into two horizontal plexuses: one more superficial, situated in the upper dermis ( $1 \pm 1.5 \mathrm{~mm}$ below the skin surface) and one deeper, at the dermal-subcutaneous junction [71]. Several factors can induce an alteration of cutaneous microcirculation with a vasodilation such as drugs or chemicals, UV, cutaneous inflammatory disease (e.g., psoriasis, eczema, allergic reactions, rosacea), and vascular disorders (i.e., Raynaud's phenomenon or peripheral circulating disorders) [72]. A preserved microcirculation is essential for the proper delivery of oxygen and nutritive substances to the biological tissue as well as for the removal of toxins.

To evaluate the appropriateness of capillary blood flow as an OV for the protection of skin against dehydration, database 12 was generated (see Table 1).

A normal blood flow allows an appropriate delivery of nutrients and a simultaneous removal of products of metabolism. This is why an inappropriate removal of these waste products, which may occur in cases of reduced blood flow, could have negative effects on both the body and the skin.

Therefore, the measurement of blood flow might provide useful information on skin health. For instance, blood flow in the capillaries can be directly associated with wrinkle formation by a reduction of the flow and of the delivery of nutritional compounds to the cells [73].

However, there is no strong scientific evidence suggesting that a reduced capillary blood flow is unequivocally related to an impaired structure/hydration or elasticity of the skin. There are several other mechanisms that contribute to unhealthy skin. Erythema and altered blood flow are also not always associated with either an impaired of barrier or with a decrease in hydration.

In conclusion, capillary blood flow is not an appropriate outcome variable to be used for the substantiation of health claims in the context of protection of the skin against dehydration. 


\subsection{Protection of the Skin against Oxidative (Including UV-Induced) Damage}

In physiological conditions, the skin cellular redox processes constantly produce free radicals, such as reactive oxygen species (ROS) and reactive nitrogen species (RNS), which are finely counteracted by endogenous and exogenous systems. When these antioxidant defenses are inadequate to fully inactivate the ROS (because of excessive production of ROS and/or because of inadequate antioxidant defenses), a condition of oxidative stress can occur. Oxidative stress has been widely associated with an increased risk of many acute and chronic diseases, mainly because of its role in altering the molecular structure and function of DNA, proteins and lipids. In addition, these oxidative products may accumulate over the time.

As skin is the most exposed organ to environmental sunlight and pathogens, it represents the major protective interface between the body and the environment. Therefore, it is exposed to many sources of oxidative stress including pollutants, infrared irradiation, xenobiotics and most of all UV radiation. Many mechanisms are involved in UV-induced damage, including up-regulation of gene expression through intracellular signal transduction pathways, suppression of immune reaction and induction of tolerance to antigens. Moreover, UV radiation seems to form a complex interaction with mitochondria, where it may contribute to a vicious circle of increasing damage.

\subsubsection{Oxidative Damage to DNA}

Exposure to UVA and ultraviolet B (UVB) radiation may lead to the oxidation of DNA, proteins and lipids. As a result, several products of oxidation may originate. The main classes of products directly deriving from DNA oxidation are cis-syn cyclobutane pyrimidine dimers (CPDs), followed by pyrimidine-pyrimidone photoproducts [74].

Regarding the DNA products of oxidative DNA damage due to ROS, these mainly consist of a large group of compounds deriving from the DNA bases such as the guanine. One of the main products of DNA oxidation is the 8-hydroxydeoxyguanosine (8-OHdG), a pre-mutagenic lesion in mammalian cells that is considered a ubiquitous marker of oxidative stress. 8-OHdG is generated by hydroxyl radical, singlet oxygen, or direct electron transfer which does not involve any ROS. 8-OHdG may cause mutation (G:C to T:A) at DNA replication. In addition to 8-OHdG, UV radiation may produce many other products of DNA damage, such as protein-DNA crosslinks and single-strand breaks.

To evaluate the appropriateness of oxidative damage to DNA as an outcome variable for the protection of the skin against oxidative damage, database 13 was generated (see Table 1).

Solar UV radiation is one of the most important causes of skin lesions and related diseases. In fact, UV radiation induces a variety of photoproducts in DNA, including CPDs, pyrimidine-pyrimidone photoproducts, thymine glycols, cytosine damage, purine damage, DNA strand breaks, and DNA-protein crosslinks. If unrepaired damage occurs to regulatory genes (e.g., tumor suppressor genes), this may promote the process of carcinogenesis. In this context, gene mutation and activation may be important [75]. Other responses resulting from UV ray exposure of cells include increased cellular proliferation, which could have a tumor-promoting effect on genetically altered cells, as well as changes in components of the immune system present in the skin.

It is well documented that, in contrast to UVB radiation, the less energetic UVA photons may indirectly affect membranes, proteins, and DNA, by producing ROS. Among the most representative DNA oxidation products, $8-\mathrm{OHdG}$ is commonly used as an indirect biomarker of oxidative DNA stress, although several factors (e.g., artifacts during isolation of DNA and its preparation for analysis) should be carefully considered, as they can affect its measurement [76].

To gain a better understanding of the mutagenic and carcinogenic features of UVA radiation, the identification of other products of oxidatively-generated DNA damage requires further investigations. These products include DNA-protein crosslinks and adducts, which may be issued from the covalent binding of reactive aldehydes arising from the decomposition of lipid hydroperoxides to nucleobases. 
In conclusion, direct measures of oxidative damage to DNA, such as CPDs, are appropriate to be used for the substantiation of health claims in the context of protection of the skin against oxidative (including UV-induced) damage. Conversely, indirect products of DNA oxidation such as 8-OHdG are not appropriate for the substantiation of health claims in the context of protection against oxidative damage to DNA when measured alone, but they could be used as supporting evidence.

\section{Skin Biopsy and High Performance Liquid Chromatography}

The in vivo evaluation of direct markers of oxidative damage to DNA must be assessed directly on the target tissue, which should be correctly harvested, treated and stored, prior to being analyzed. Skin biopsy is the procedure used to sample the outer layer of the derma, and may be performed using different techniques, according to the type of analysis, the size and the location of the part which should be sampled. Shave biopsy, performed with a scalpel or a razor blade, allows the harvest of a tangential part of the skin across the target place. Punch biopsy, performed with a circular blade, allows the harvest of a round or ellipsoidal part of the epidermis, dermis and subcutaneous fat close to the target place of the skin. Excisional biopsy is an incisional biopsy where the harvested sample includes the target place with the lesion or the mutation [77]. A particular skin collection may be performed by using stripes that allow the sampling of the lonely dead keratinocytes on the outer layer, or by using cotton pads to collect sebum or skin lipids.

Skin samples collected by biopsy can be used for the evaluation of products of oxidative damage to DNA, such as CPDs, detected by using several methods, including high performance liquid chromatography with electrospray ionization-tandem mass spectrometry, which represents the reference method. In vitro UVR radiation of standard nucleosides is performed to obtain a calibration curve useful for quantifying the products of oxidation, such as cis-syn and trans-syn CPDs, cyclobutane thymine dimers, as well as oxidized nucleosides, i.e., 1,3 dimethyluracile [78].

In conclusion, skin biopsy followed by high performance liquid chromatography is an appropriate method for the measurement of direct markers of oxidative DNA damage like CPDs.

\section{Skin Biopsy and Immunohistochemical Techniques}

The in vivo evaluation of direct marker of oxidative damage to lipids must be assessed directly on the target tissue, and skin biopsy is the most accurate procedure, as described in "Skin Biopsy and High Performance Liquid Chromatography" in Section 3.2.1.

In alternative to chromatographic techniques, oxidative damage to DNA can be measured by semiquantitative immunohistochemical ones.

After the biopsy is assessed, skin samples are washed in saline solutions and fixed in opportune buffers (i.e., formalin, formaldehyde, paraformaldehyde) and included in paraffin. Samples are then sectioned and stained with non-human primary monoclonal or polyclonal antibodies anti-CPD by using a direct or a sandwich enzyme-linked immunosorbent assay (ELISA) technique. In this last case, a secondary antibody, which may be bound to a biotin-streptavidin complex, is required to increase the signal of CPD detection. Alternatively, primary cells harvested from the biopsy samples may be cultured and allowed to grow in vitro. In this way, it is possible to harvest cells from the culture medium, and process them for immunohistochemical analysis with primary or secondary antibodies anti-CPD [79]. It has been demonstrated that cells containing CPDs have a shorter survival and tend to die quicker than non-damaged cells.

Indirect products of oxidation, such as $8-\mathrm{OHdG}$, are also assayed with immunohistochemical techniques. As described previously, the included skin cells are processed for staining with anti-8-OHdG antibodies. Further washing allows the elimination of the remaining sample, and then a secondary anti-primary antibody is added to the well. An enzyme catalyzing the production of a colored substrate is bound to the same secondary antibody. The quantification of the analyte is carried out by measuring the intensity of the immunostaining, and by subtracting the value of the 
background at a cell-free area of the slice. Immunostaining is measured by using microscopes or cameras equipped with computer presenting image analysis software [80].

The method results are more sensitive to both CPD and 8-OHdG than the chromatographic ones, because even very small amounts of antigen are stained with the antibodies. Nevertheless, its results are less specific, particularly when polyclonal antibodies are used, as cross-link phenomena may occur between molecules having similar structure.

In conclusion, based on these considerations, skin biopsy followed by immunohistochemical detection of CPD and 8-OHdG is an appropriate method for measurement of markers of oxidative DNA damage at skin level.

\subsubsection{Oxidative Damage to Lipids}

Lipids are fundamental components of the skin surface and are present as both sebaceous and epidermal lipids. Although the lipid profile is characterized by a large inter-individual variation, squalene, sebaleic acid, linoleic acid, and cholesterol are the most represented.

Skin lipids are susceptible to oxidation through three different mechanisms: free radical chain oxidation, enzymatic oxidation, and non-radical, non-enzymatic oxidation [81].

Skin photo-oxidation, which is a consequence of exposure to UVA and UVB, may result in the production of many oxidation products, including squalene monohydroperoxyde and hydroperoxycholesterol. In addition, lipid peroxidation products such as hydroxyeicosatetraenoic acids (HETEs) and isoprostanes have been found to increase in human skin following UV exposure.

Lipid peroxidation (LPO) products tend to accumulate in the cellular membranes proportionally with the cumulative oxidative stress of the skin. High concentrations of LPO products perturb the integrity of the membranes, and thus of the cells involved. Lipid peroxides can be further decomposed to many reactive aldehydic species, such as malondialdehyde, 4-hydroxynonenal, hexanal, as well as other saturated and unsaturated aldehydes and ketones [82].

To evaluate the appropriateness of oxidative damage to lipids as an outcome variable for the protection of the skin against oxidative damage, database 13 was generated (see Table 1).

UV radiation induces many indirect photo-chemical effects in the skin. In this context, LPO is one of the major pathways by which photo-oxidative stress disturbs cell signaling, and promotes photo-carcinogenesis and photo-aging. Studies have shown a significant linear relation between UVB exposure recorded by the dosimeters and colorimetry parameters of the skin reaction.

An established marker of oxidative damage to cell membranes, which is reliably measured by immunohistochemistry, is represented by F2-isoprostanes. F2-isoprostanes are a series of prostaglandin F2-like compounds produced in vivo independently of cyclo-oxygenase as products of radical catalyzed LPO. Among these F2-isoprostanes, 8-epi-prostaglandin 2a (8-isoprostane) is the most representative. A linear relation was found between the generation of 8-isoprostane in the skin and the dosimeter readouts [83]. 8-Isoprostane has been validated as a marker for oxidative stress in various conditions and in the skin.

In conclusion, products of oxidative damage to lipids, such as isoprostanes, are appropriate outcome variables to be used for the substantiation of health claims in the context of protection of the skin against oxidative (including UV-induced) damage.

Skin Biopsy and Liquid or Gas Chromatography-Mass Technique

The in vivo evaluation of direct markers of oxidative damage to lipids must be assessed directly on the target tissue, and skin biopsy is the most accurate procedure, as described in "Skin Biopsy and High Performance Liquid Chromatography" in Section 3.2.1.

The most common method for detecting and quantifying hydroxyperoxides, such as squalene monohydroperoxide and cholesterol, is based on the liquid or gas chromatography technique [84]. After the collection of the skin, samples are extracted with organic solvents, e.g., acetone or 1-butanol, and prepared for the injection into chromatographic instruments. Chromatographic detection and 
quantification of target compounds may be performed by analyzing their mass, and/or that of their main fragments. Gas chromatography is more frequently used than liquid chromatography, as the mobile phase is cheaper, and because of the volatility of the sampling compounds.

Liquid chromatography techniques can be also used for the detection and quantification of isoprostanes (i.e., 8-Isoprostaglandin F2 $\alpha$ ), leukotrienes (i.e., HETE), hydroperoxyeicosatetraenoic acid (HpETEs) and lipoxin (i.e., lipoxin A4 (LXA4) and B4 (LXB4)) [85]. This is the preferred method, as it is simple, cheap, and very sensitive, as the concentration of these analytes in tissues are very scarce.

In conclusion, liquid or gas chromatography-mass techniques following skin biopsy are appropriate methods to be used for the measurement of markers of lipid oxidation like isoprostanes at skin level.

Skin Biopsy and Immunohistochemical Techniques

The in vivo evaluation of direct markers of oxidative damage to lipids must be assessed directly on the target tissue, and skin biopsy is the most accurate procedure, as described in "Skin Biopsy and High Performance Liquid Chromatography" in Section 3.2.1.

The detection and quantification of isoprostanes (i.e., 8-Isoprostaglandin F2 $\alpha$ ), leukotrienes (i.e., HETE, HpETEs and lipoxin (i.e., LXA4 and LXB4) can be performed by using immunohistochemical techniques, such as ELISA assays [83]. After the biopsy is assessed, skin samples are washed in saline solutions and fixed in opportune buffers and included in paraffin. Subsequently, samples are sectioned and stained with non-human primary antibodies specific for the marker (i.e., polyclonal goat anti-8-epiPGF- $2 \alpha$ antibody). As described in "Skin Biopsy and Immunohistochemical Techniques"in Section 3.2.1, further washing allows the elimination of the remaining sample before a secondary anti-primary antibody is added in the well and an enzyme catalyzing the production of a colored substrates is bound to the same secondary antibody. The quantification of the analyte is carried out by measuring the intensity of immunostaining by subtracting the value of the background at a cell-free area of the slice. Immunostaining is measured by using microscopes or cameras equipped with a computer running image analysis software.

The principal limitation of immunohistochemistry is the need for high-resolution image analysis to obtain reliable results. Furthermore, the technique is more specific when monoclonal antibodies are used for the precise marker compared to polyclonal ones, which may recognize similar epitopes of different isoprostanes. However, monoclonal antibodies are expensive and request long times of production.

In conclusion, skin biopsy, followed by immunohistochemical detection of lipids, is an appropriate method for the measurement of markers of oxidative damage to lipid like isoprostanes in the skin.

\subsubsection{Oxidative Damage to Proteins}

UV radiation can interact with cellular photosensitizers to generate ROS and ROS-mediated oxidative damage to DNA, proteins and lipids. It is well known that UVB rays induce a direct formation of DNA-photoproducts that are generally removed by the nucleotide excision repair system. UVA represents more than $95 \%$ of the incident solar radiation. The effects of UVA reflect the induction of oxidative stress that causes extensive protein oxidation.

Moreover, sustained UV-exposure can lead to a high extent of protein oxidation, which is generally increased in aged tissue [86]. ROS-induced oxidative damage to the structural dermal proteins collagen and elastin can result in changes in the protein conformation and unfolding, leading to modifications in the mechanical properties of skin. Collagen degradation and abnormal elastin accumulation are visible in photo-aged skin. Proteins undergo modifications and subsequent conformational changes when certain amino acids are converted to their oxidized forms.

Several biomarkers have been used to assess the extent of oxidative damage to protein in the skin. Reactive oxygen species (ROS) can lead to protein modifications occurring at the backbone, at amino acid side chains, as well as by the formation of protein carbonyls. Oxidative damage to proteins results 
in a multitude of products, arising from modification of a wide range of amino acids [87]. The various amino acid residues do not have the same susceptibility to oxidative modifications. For instance, histidine, leucine, methionine, and cysteine, as well as phenylalanine, tyrosine, and tryptophan, are more susceptible than others to the presence of thiols or hydroxyl moieties, which are more sensitive to oxidation processes [75].

Conversely, oxidation of proteins containing proline, arginine, lysine and threonine results in the formation of irreversible carbonyl groups. If compared to the deeper layers of the epidermis, these carbonyls have been found to be more concentrated in the SC.

To evaluate the appropriateness of oxidative damage to proteins as an outcome variable for the protection of the skin against oxidative damage, database 13 was generated (see Table 1).

Despite the presence of antioxidant defensive-systems, UV radiations cause extensive protein modification, which seems to be involved in aging processes and disease development [88].

ROS generated from UV exposure is the most important triggering agent of protein oxidation. Hydroxyl-, peroxyl-, nitro-, etc. radicals are able to modify both the carbon skeleton and the $\mathrm{R}$ group of the protein in order to create more unstable products. In detail, cysteine or methionine sulfenic/sulfonic acids, hydroxytyrosine, nitrotryptophan are produced inside the cell, causing the loss of the function of the relative proteins which they constitute. Due to their instability, the next step is the generation of protein carbonyl groups which are usually the resulting marker of protein oxidation, as they represent an irreversible form of protein modification [75].

In conclusion, products of oxidative damage to proteins such as oxidative changes in amino acids are appropriate outcome variables to be used for the substantiation of health claims in the context of protection of the skin against oxidative (including UV-induced) damage.

Conversely, protein carbonyls should be used in combination with direct markers of oxidative damage to proteins in vivo.

Skin Biopsy and Liquid Chromatography-Mass Technique

The in vivo evaluation of direct markers of oxidative damage to proteins must be assessed directly on the target tissue, and skin biopsy is the most accurate procedure, as described in "Skin Biopsy and High Performance Liquid Chromatography" in Section 3.2.1.

A sensitive and specific method for detection of the direct products of protein oxidation is based on the liquid chromatography technique [89]. After the collection of the SC samples by using strips, they are washed and treated to avoid contaminations. Then, the sample is treated to avoid artefacts, and is digested with proteases, i.e., pepsin, trypsin and chymotrypsin, in acid conditions in order to avoid the formation of polymers. Samples are injected into chromatography instruments, and the detection and quantification of target compounds may be performed by analyzing the mass of the target compounds and/or their main fragments.

In conclusion, based on these considerations, skin biopsy followed by liquid or gas chromatography techniques is an appropriate method for the measurement of markers of oxidative damage to proteins at skin level.

\subsection{Protection of the Skin from UV-Induced (Other Than Oxidative) Damage}

\subsubsection{DNA Damage after UV Radiation Exposure}

Exposure of skin to UV radiation has been shown to have a number of deleterious effects, including photo-aging, photo-immunosuppression and photo-induced DNA damage, which can lead to the development of skin cancer [90]. DNA has been shown to be a skin chromophore, and absorption of UV radiation by DNA can result in the formation of thymine dimers.

To evaluate the appropriateness of DNA damage after UV radiation exposure as OV for the protection of the skin from UV-induced damage, database 14 was generated (see Table 1). 
DNA, in vitro and in vivo, is susceptible to being damaged when exposed to high-energy radiation. This damage can be a breaking of one or both of the strands in the DNA helix, a fusing of the two strands to each other, to themselves (dimers), or other types of molecular damage to the nucleotides. Usually, the majority of DNA damage is repaired. However, incomplete or deficient repair may lead to skin cancer, which is a multistep process involving tumor initiation, tumor promotion, and tumor progression, ultimately resulting in visible skin cancer [91]. Based on these considerations, the measurement of DNA damage after UV radiation exposure is an appropriate outcome variable to be used for the substantiation of health claims in the context of protection of skin from UV-induced (other than oxidative) damage.

Immunohistochemical Techniques

Methods for quantifying photoproducts in human skin include DNA extraction analysis and immunohistochemical analysis. In vitro fragmented DNA can be observed directly using conventional techniques such as capillary electrophoresis and the comet assay. The measurement of internal damage typically requires analysis such as high performance liquid chromatographic-mass spectrometry, hydrolysis of DNA followed by chromatographic separation, electrochemical measurements, or the enzymatic conversion of photoproducts into strand breaks [92]. However, in vivo quantification is more appropriate for evaluating UV-induced DNA damage in humans. There are some advantages to using immunohistochemical quantification, compared to DNA extraction. Histology is able to identify the skin compartment in which DNA damage has occurred, to which depth cells are affected, and which subpopulations of cells are damaged. Immunohistochemistry is based on the quantification of the thymine cyclobutane dimer (TT-CPD), the main DNA lesion induced by both UVB and UVA radiations. The level of TT-CPD in DNA may be determined by, immunohistochemical staining of photoproduct positive nuclei of keratinocytes in the epidermis. Manual counting of photoproduct-positive immunohistochemically stained nuclei in the epidermis is a frequently used method for quantification of DNA damage [93].

In conclusion, immunohistochemical analysis is an appropriate method for the measurement of DNA damage after UV radiation exposure.

\subsubsection{Depletion of Langerhans Cells after UV Light Exposure}

Langerhans cells (LCs) are effective antigen-presenting cells that function as "custodians" of the skin, altering the immune system to pathogen entry, but also the tolerance to self-antigen and commensal microbes [94]. LCs are also recognized to play a key role in the induction and maintenance of the immune response against skin cancer. Exposure of human skin to solar UV light induces local and systemic immune suppression. It is known that alterations of numbers and immune functions of LCs mediate this phenomenon [95]. The effect of UV on epidermal LCs has been studied for many years, since the first reports in the 1980s focusing on the deleterious effects of UV on epidermal LCs in humans. Exposure to UV induces a decrease in LCs within the human skin, and this depletion is probably due to both cell death and enhanced migration to regional lymph nodes.

To evaluate the appropriateness of depletion of Langerhans cells after UV-light exposure as OV for the protection of the skin from UV-induced damage, database 15 was generated (see Table 1).

It has been shown that UV radiation induces epidermal LCs to emigrate to draining lymph nodes starting a few hours or a few days after UV exposure. This leads to a decrease in the number of Langerhans cells, which can persist for up to four weeks before recovery of a normal epidermal pool. The consequence is direct damage to the immunological function of the skin. Therefore, decreasing the depletion of Langerhans cells after UV light exposure is beneficial. It has been shown that the protection afforded by sunscreens against photo-immunosuppression must be broad-spectrum with an adequate UVA protection. Based on these considerations, the evaluation of depletion of Langerhans cells after UV light exposure, measured with appropriate techniques, is appropriate for the substantiation of health claims in the context of protection of skin from UV-induced (other than oxidative) damage. 
Histochemical and Immunological Techniques

The measurement of the number of LCs necessarily requires skin samples. The simplest way to obtain a skin sample is a punch skin biopsy, which is generally performed in UV-unexposed skin such as buttock skin or the inner forearm after local anesthesia. This is an easy, minimally invasive and low-cost procedure, which provides the substratum for the subsequent measurement [77].

Langerhans cells cannot be identified in routinely prepared histologic testing, but they can be visualized at the light microscope by histochemical and immunological techniques. Appropriate methods for LC detection in the human skin include histo-enzymatic methods of adenosintriphosphatases, acid phosphatase, alpha-naphthylacetate esterase and the peroxidase-antiperoxidase immunochemistry method with S-100 protein antibody [96]. Based on these considerations, skin biopsy, if followed by a histochemical or immunological technique, is an appropriate method for the measurement of the number of LCs after UV-light exposure.

\subsubsection{UV-Induced Erythema and Erythema Grade (Reddening)}

Erythema or skin reddening is an inflammatory response of the skin to UV-induced molecular and cellular damage [97]. If severe, i.e., sunburn, it may lead to blisters and loss of the several skin functions. Erythema and subsequent pigmentation are immediate responses of normal human skin exposure to UV radiation. Even if it is an indicator of direct UV-induced skin damage, there is no direct evidence of correlation with skin cancer and photo aging [98].

To evaluate the appropriateness of UV-induced erythema as OV for the protection of the skin from UV-induced damage, database 16 was generated (see Table 1).

A reduction in UV-induced erythema (e.g., measured as change in minimal erythema dose (MED) or erythema grade (reddening)) may indicate less UV-induced damage to the skin. However, it can also reflect a reduction in the capacity of the skin to react to molecular and cellular damage, so it does not represent a univocal measure of UV-induced damage at skin level. Moreover, erythema is a poor indicator of immunosuppression [99]. On the basis of these considerations, UV-induced erythema cannot be used alone as an outcome measure for the substantiation of health claims on protection of the skin from UV-induced damage. However, it can be used as supporting evidence when appropriate outcome variables are also used.

Minimal Erythemal Dose Test

The MED test allows the determination of the amount of UV radiation producing minimal erythema (sunburn or redness caused by engorgement of capillaries) of the skin within a few hours following exposure. The individual MED is determined by irradiating small areas of skin (usually sun un-exposed skin) with increasing UV doses. Results are read at $24 \mathrm{~h}$ post-exposure and the lowest dose in the series that only just produced erythema is considered to be the MED [100]. The increasing UV doses are determined in consideration of the specific skin phototype, since this strictly influences the intensity of the resulting erythema. This procedure is simple to perform, and requires only a few minutes. The only possible drawback is that an appropriate evaluation of phototype is fundamental to avoid excessive UV exposure time with consequent burning.

In conclusion, the MED test is an appropriate, simple and low-cost procedure for the measurement of UV-erythema.

\subsubsection{Delayed-Type Hypersensitivity Immune Response to Recall Antigens in the Skin}

The delayed-type hypersensitivity (DTH) reaction is a cell-mediated reaction to several antigens injected in the skin. The DTH skin test is used to evaluate whether prior exposure to an antigen has occurred, and reflects the cell-mediated immunity that provides the main mechanism against fungi, viruses and other hosts. Following the injection of small amounts of antigen, a typical response occurs, including induration, swelling and monocytic infiltration into the site of the lesion within 24 
to $72 \mathrm{~h}$. This reaction has been considered a surrogate for immune response to tumor antigens [101]. Three types of DTH reactions have been described: contact hypersensitivity (e.g., caused by metal ions), tuberculin-type reaction and granulomatous hypersensitivity.

To evaluate the appropriateness of DTH immune response as an outcome variable for the protection of the skin from UV-induced damage, database 17 was generated (see Table 1).

Solar UV radiation has been demonstrated to have suppressive effects on the immune system. UV radiation inhibits antigen presentation and induces the release of immunosuppressive cytokines. This specific immunosuppression is mediated by antigen specific suppressor/regulatory $\mathrm{T}$ cells, which mediates UV-induced inhibition of DTH response in human skin.

However, DTH immune responses to recall antigens in the skin is more a marker of systemic UV-induced damage on the immune system rather than a marker of UV-damage at skin level.

Therefore, based on these considerations, DTH immune responses to recall antigens in the skin cannot be used alone as an outcome measure for the substantiation of health claims on protection of the skin from UV-induced damage. However, it can be used as supporting evidence in combination with appropriate outcome variables.

\section{Multitest Kit Merieux and Mantoux Testing}

Most of the methods for determining the UV-induced immunosuppression are sunburn protection factor-based measures of UVB induced erythema response. The main drawback is the inability of these methods to provide an accurate evaluation of the immune protection. In this scenario, inhibition of DTH response has been suggested as a test for evaluating UV-induced immunosuppression, but the lack of appropriate techniques for evaluating immunosuppression still remains a challenge.

The DTH skin test is used to test if prior exposure to an antigen has occurred. So far, two procedures have been used successfully: Multitest Kit Merieux and Mantoux testing [102]. The Multitest Kit Merieux is a DTH to seven antigens (e.g., Candida albicans, Streptococcus antigens) and provides comprehensive information on the immune status of the human volunteers. Mantoux testing with tuberculin purified protein derivatives provides a possible alternative model of DTH to recall antigen.

Overall, the DTH response is not easy to assess; it requires several weeks, and it is invasive. However, it represents an interesting tool for evaluating photoimmunosuppression in subjects within RCTs.

In conclusion, Multitest Kit Merieux and Mantoux testing are appropriate methods for the measurement of DTH immune response to recall antigens in the skin.

\section{Conclusions}

Insufficient scientific substantiation for a health claim represents the most common reason for a negative response to a request for the authorization of a health claim. In this context, RCTs should be well-designed and well-performed, taking into account many parameters affecting the quality of a RCT, such as adequate sample size, proper study design (including an adequate duration of the intervention), adequate statistical analysis, and choice of appropriate OVs and related MMs. The present report provides a critical analysis of all the OVs and MMs that have been proposed so far in the context of maintenance of skin function, compliant with the European Regulation. This critical analysis could represent a useful tool for applicants during the design or selection of human intervention studies aimed to substantiate health claims related to skin function. Moreover, this information could serve as a basis for EFSA to develop and update the Guidance on the scientific requirements for health claims related to skin health. 
Acknowledgments: This project has received financial support from the European Food Safety Authority (EFSA), Grant GP/EFSA/NUTRI/2014/01. The present article, however, is under the sole responsibility of the authors. The positions and opinions presented in this article are those of the authors alone and do not necessarily represent the views/any official position or scientific works of EFSA. To know about EFSA guidance documents and other scientific outputs of EFSA, please consult its website at: http:/ /www.efsa.europa.eu.

Author Contributions: D.M. was the project developer and wrote the manuscript together with D.A., M.B.D.F., C.C. and S.D.N.; G.B. generated the literature databases and helped with the evaluation of the appropriateness and the validity of the statistics found in the literature studies taken into account; I.Z., M.M., C.P., G.P., M.V., D.G., P.M., M.V., A.D.C., R.C.B. and S.D.N. were expert members of the project team, helped secure the funding, and critically read and revised the manuscript; D.D.R. was the Principal Investigator of the project, critically revised the manuscript and had primary responsibility for final content.

Conflicts of Interest: The authors declare no conflict of interest.

$\begin{array}{ll}\text { Abbreviations } \\ \text { CPDs } & \text { cyclobutane pyrimidine dimers } \\ \text { DTH } & \text { delayed type hypersensitivity } \\ \text { EFSA } & \text { european food safety authority } \\ \text { ELISA } & \text { enzyme-linked immunosorbent assay } \\ \text { HETE } & \text { hydroxyeicosatetraenoic acids } \\ \text { HpETEs } & \text { hydroperoxyeicosatetraenoic acid } \\ \text { HPLC } & \text { high performance liquid chromatography } \\ \text { LCs } & \text { langerhans cells } \\ \text { LPO } & \text { lipid peroxidation } \\ \text { LXA4 } & \text { lipoxin a4 } \\ \text { LXB4 } & \text { lipoxin b4 } \\ \text { MED } & \text { minimal erythemal dose } \\ \text { MM } & \text { method of measurement } \\ \text { OV } & \text { outcome variable } \\ \text { RCT } & \text { randomized controlled trial } \\ \text { ROS } & \text { reactive oxygen species } \\ \text { SC } & \text { stratum corneum } \\ \text { SELS } & \text { surface evaluation of the living skin } \\ \text { TEWL } & \text { transepidermal water loss } \\ \text { TT-CPD } & \text { thymine cyclobutane dimer } \\ \text { UV } & \text { ultraviolet } \\ \text { UVA } & \text { ultraviolet A } \\ \text { UVB } & \text { ultraviolet B } \\ \text { VAS } & \text { visual analog scales } \\ \text { VP } & \text { vapor pressure } \\ \text { 8-OHdG } & \text { 8-hydroxydeoxyguanosine } \\ & \\ \text { HE } & \end{array}$

\section{References}

1. Proksch, E.; Brandner, J.M.; Jensen, J.-M. The skin: An indispensable barrier. Exp. Dermatol. 2008, 17, 1063-1072. [CrossRef] [PubMed]

2. Madison, K.C. Barrier function of the skin: "La raison d'etre" of the epidermis. J. Investig. Dermatol. 2003, 121, 231-241. [CrossRef] [PubMed]

3. Stucker, M.; Struk, A.; Altmeyer, P.; Herde, M.; Baumgartl, H.; Lubbers, D.W. The cutaneous uptake of atmospheric oxygen contributes significantly to the oxygen supply of human dermis and epidermis. J. Physiol. 2002, 538, 985-994. [CrossRef] [PubMed]

4. Maru, G.B.; Gandhi, K.; Ramchandani, A.; Kumar, G. The role of inflammation in skin cancer. Adv. Exp. Med. Biol. 2014, 816, 437-469. [PubMed]

5. Gawkrodger, D.; Ardern-Jones, M. Dermatology, 6th ed.; Elsevier: London, UK, 2016. 
6. Hay, R.J.; Johns, N.E.; Williams, H.C.; Bolliger, I.W.; Dellavalle, R.P.; Margolis, D.J.; Marks, R.; Naldi, L.; Weinstock, M.A.; Wulf, S.K.; et al. The global burden of skin disease in 2010: An analysis of the prevalence and impact of skin conditions. J. Investig. Dermatol. 2014, 134, 1527-1534. [CrossRef] [PubMed]

7. Lozano, R.; Naghavi, M.; Foreman, K.; Lim, S.; Shibuya, K.; Aboyans, V.; Abraham, J.; Adair, T.; Aggarwal, R.; Ahn, S.Y.; et al. Global and regional mortality from 235 causes of death for 20 age groups in 1990 and 2010: A systematic analysis for the global burden of disease study 2010. Lancet 2012, 380, 2095-2128. [CrossRef]

8. Naldi, L.; Conti, A.; Cazzaniga, S.; Patrizi, A.; Pazzaglia, M.; Lanzoni, A.; Veneziano, L.; Pellacani, G. Diet and physical exercise in psoriasis: A randomized controlled trial. Br. J. Dermatol. 2014, 170, 634-642. [CrossRef] [PubMed]

9. Jensen, P.; Zachariae, C.; Christensen, R.; Geiker, N.R.W.; Schaadt, B.K.; Stender, S.; Hansen, P.R.; Astrup, A.; Skov, L. Effect of weight loss on the severity of psoriasis. JAMA Dermatol. 2013, 149, 795-801. [CrossRef] [PubMed]

10. Smith, R.N.; Braue, A.; Varigos, G.A.; Mann, N.J. The effect of a low glycemic load diet on acne vulgaris and the fatty acid composition of skin surface triglycerides. J. Dermatol. Sci. 2008, 50, 41-52. [CrossRef] [PubMed]

11. Stahl, W.; Heinrich, U.; Wiseman, S.; Eichler, O.; Sies, H.; Tronnier, H. Dietary tomato paste protects against ultraviolet light-induced erythema in humans. J. Nutr. 2001, 131, 1449-1451. [PubMed]

12. Heinrich, U.; Neukam, K.; Tronnier, H.; Sies, H.; Stahl, W. Long-term ingestion of high flavanol cocoa provides photoprotection against UV-induced erythema and improves skin condition in women. J. Nutr. 2006, 136, 1565-1569. [PubMed]

13. Heinrich, U.; Moore, C.E.; De Spirt, S.; Tronnier, H.; Stahl, W. Green tea polyphenols provide photoprotection, increase microcirculation, and modulate skin properties of women. J. Nutr. 2011, 141, 1202-1208. [CrossRef] [PubMed]

14. Martini, D.; Biasini, B.; Rossi, S.; Zavaroni, I.; Bedogni, G.; Musci, M.; Pruneti, C.; Passeri, G.; Ventura, M.; Galli, D.; et al. Claimed effects, outcome variables and methods of measurement for health claims on foods proposed under european community regulation 1924/2006 in the area of appetite ratings and weight management. Int. J. Food Sci. Nutr. 2017. [CrossRef] [PubMed]

15. Martini, D.; Rossi, S.; Biasini, B.; Zavaroni, I.; Bedogni, G.; Musci, M.; Pruneti, C.; Passeri, G.; Ventura, M.; Di Nuzzo, S.; et al. Claimed effects, outcome variables and methods of measurement for health claims proposed under european community regulation 1924/2006 in the framework of protection against oxidative damage and cardiovascular health. Nutr. Metab. Cardiovasc. Dis. 2017, 27, 473-503. [CrossRef] [PubMed]

16. Fitzpatrick, R.; Davey, C.; Buxton, M.J.; Jones, D.R. Evaluating patient-based outcome measures for use in clinical trials. Health Technol. Assess. 1998, 2, 1-74. [PubMed]

17. Rogiers, V. Eemco guidance for the assessment of transepidermal water loss in cosmetic sciences. Skin Pharmacol. Appl. Skin Physiol. 2001, 14, 117-128. [CrossRef] [PubMed]

18. Du Plessis, J.; Stefaniak, A.; Eloff, F.; John, S.; Agner, T.; Chou, T.-C.; Nixon, R.; Steiner, M.; Franken, A.; Kudla, I.; et al. International guidelines for the in vivo assessment of skin properties in non-clinical settings: Part 2. Transepidermal water loss and skin hydration. Skin Res. Technol. 2013, 19, 265-278. [CrossRef] [PubMed]

19. Pinnagoda, J.; Tupker, R.A.; Agner, T.; Serup, J. Guidelines for transepidermal water loss (TEWL) measurement. A report from the standardization group of the european society of contact dermatitis. Contact Dermat. 1990, 22, 164-178. [CrossRef]

20. Ezerskaia, A.; Pereira, S.F.; Urbach, H.P.; Verhagen, R.; Varghese, B. Quantitative and simultaneous non-invasive measurement of skin hydration and sebum levels. Biomed. Opt. Express 2016, 7, 2311-2320. [CrossRef] [PubMed]

21. Harding, C.R.; Watkinson, A.; Rawlings, A.V.; Scott, I.R. Dry skin, moisturization and corneodesmolysis. Int. J. Cosmet. Sci. 2000, 22, 21-52. [CrossRef] [PubMed]

22. Verdier-Sevrain, S.; Bonte, F. Skin hydration: A review on its molecular mechanisms. J. Cosmet. Dermatol. 2007, 6, 75-82. [CrossRef] [PubMed]

23. Berardesca, E. Eemco guidance for the assessment of stratum corneum hydration: Electrical methods. Skin Res. Technol. 1997, 3, 126-132. [CrossRef] [PubMed] 
24. Heinrich, U.; Koop, U.; Leneveu-Duchemin, M.C.; Osterrieder, K.; Bielfeldt, S.; Chkarnat, C.; Degwert, J.; Hantschel, D.; Jaspers, S.; Nissen, H.P.; et al. Multicentre comparison of skin hydration in terms of physical-, physiological- and product-dependent parameters by the capacitive method (Corneometer CM 825). Int. J. Cosmet. Sci. 2003, 25, 45-53. [CrossRef] [PubMed]

25. Constantin, M.-M.; Poenaru, E.; Poenaru, C.; Constantin, T. Skin hydration assessment through modern non-invasive bioengineering technologies. Maedica (Buchar) 2014, 9, 33-38. [PubMed]

26. Khazaka, G. Assessment of stratum corneum hydration: Corneometer CM 825. In Bioengineering of the Skin: Water and the Stratum Corneum; Fluhr, J.W., Elsner, P., Berardesca, E., Maibach, H.I., Eds.; CRC Press: Boca Raton, FL, USA, 2005; pp. 249-261.

27. Rudikoff, D. The effect of dryness on the skin. Clin. Dermatol. 1998, 16, 99-107. [CrossRef]

28. Serup, J. Eemco guidance for the assessment of dry skin (xerosis) and ichthyosis: Clinical scoring systems. Skin Res. Technol. 1995, 1, 109-114. [CrossRef] [PubMed]

29. Chopra, R.; Vakharia, P.P.; Sacotte, R.; Patel, N.; Immaneni, S.; White, T.; Kantor, R.; Hsu, D.Y.; Silverberg, J.I. Severity strata for EASI, mEASI, oSCORAD, sCORAD, ADSI and BSA in adolescents and adults with atopic dermatitis. Br. J. Dermatol. 2017. [CrossRef]

30. Rodrigues, L. Eemco guidance to the in vivo assessment of tensile functional properties of the skin. Part 2: Instrumentation and test modes. Skin Pharmacol. Appl. Skin Physiol. 2001, 14, 52-67. [CrossRef] [PubMed]

31. Chung, J.; Cho, S.; Kang, S. Why does the skin age? Intrinsic aging, photoaging, and their pathophysiology. In Photoaging; Rigel, D.S., Weiss, R.A., Lim, H.W., Dover, J.S., Eds.; Marcel Dekker Inc.: New York, NY, USA, 2004.

32. Pierard, G.E.; Grp, E. Eemco guidance to the in vivo assessment of tensile functional properties of the skin-Part 1: Relevance to the structures and ageing of the skin and subcutaneous tissues. Skin Pharmacol. Appl. 1999, 12, 352-362. [CrossRef] [PubMed]

33. Wilhelm, K.P.; Cua, A.B.; Maibach, H.I. In vivo study on age-related elastic properties of human skin. In Noninvasive Methods for the Quantification of Skin Functions; Frosch, P.J., Kligman, A.M., Eds.; Springer: Berlin, Germany, 1993; pp. 190-203.

34. Ohshima, H.; Kinoshita, S.; Oyobikawa, M.; Futagawa, M.; Takiwaki, H.; Ishiko, A.; Kanto, H. Use of cutometer area parameters in evaluating age-related changes in the skin elasticity of the cheek. Skin Res. Technol. 2013, 19, E238-E242. [CrossRef] [PubMed]

35. Qu, D.; Seehra, G.P. Improving the accuracy of skin elasticity measurement by using q-parameters in cutometer. J. Cosmet. Sci. 2016, 67, 37-44. [PubMed]

36. Ryu, H.S.; Joo, Y.H.; Kim, S.O.; Park, K.C.; Youn, S.W. Influence of age and regional differences on skin elasticity as measured by the cutometer. Skin Res. Technol. 2008, 14, 354-358. [CrossRef] [PubMed]

37. Proksch, E.; Jensen, J.-M. Skin as an Organ of Protection. In Fitzpatrick's Dermatology in General Medicine, 8th ed.; Goldsmith, L.A., Katz, S.I., Gilchrest, B.A., Paller, A.S., Leffell, D.J., Wolff, K., Eds.; Mc Graw Hill: New York, NY, USA, 2012.

38. Ishida-Yamamoto, A.; Igawa, S.; Kishibe, M. Order and disorder in corneocyte adhesion. J. Dermatol. 2011, 38, 645-654. [CrossRef] [PubMed]

39. Piérard, G.E.; Hermanns-Lê, T.; Piérard-Franchimont, C. Stratum corneum desquamation. In Agache's Measuring the Skin; Humbert, P., Maibach, H., Fanian, F., Agache, P., Eds.; Springer International Publishing: Cham, Switzerland, 2016; pp. 1-5.

40. Candi, E.; Knight, R.; Melino, G. Cornification of the skin: A non-apoptotic cell death mechanism. In Cell Death; Melino, G., Vaux, D., Eds.; John Wiley \& Sons, Ltd.: Chichester, UK, 2009.

41. Wilhelm, K.P.; Kaspar, K.; Schumann, F.; Articus, K. Development and validation of a semiautomatic image analysis system for measuring skin desquamation with d-squames. Skin Res. Technol. 2002, 8, 98-105. [CrossRef] [PubMed]

42. Pierard, G.E. EEMCO guidance for the assessment of dry skin (xerosis) and ichthyosis: Evaluation by stratum corneum shippings. Skin Res. Technol. 1996, 2, 3-11. [CrossRef] [PubMed]

43. Gao, Y.R.; Wang, X.M.; Chen, S.Y.; Li, S.Y.; Liu, X.P. Acute skin barrier disruption with repeated tape stripping: An in vivo model for damage skin barrier. Skin Res. Technol. 2013, 19, 162-168. [CrossRef] [PubMed]

44. Jackson, S.M.; Williams, M.L.; Feingold, K.R.; Elias, P.M. Pathobiology of the stratum corneum. West. J. Med. 1993, 158, 279-285. [PubMed] 
45. Masukawa, Y.; Narita, H.; Sato, H.; Naoe, A.; Kondo, N.; Sugai, Y.; Oba, T.; Homma, R.; Ishikawa, J.; Takagi, Y.; et al. Comprehensive quantification of ceramide species in human stratum corneum. J. Lipid Res. 2009, 50, 1708-1719. [CrossRef] [PubMed]

46. Cremesti, A.E.; Fischl, A.S. Current methods for the identification and quantitation of ceramides: An overview. Lipids 2000, 35, 937-945. [CrossRef] [PubMed]

47. Gołębiowski, M.; Paszkiewicz, M.; Haliński, Ł.; Stepnowski, P. Hplc of plant lipids. In High Performance Liquid Chromatography in Phytochemical Analysis; Waksmundzka-Hajnos, M., Sherma, J., Eds.; CRC Press: Boca Raton, FL, USA, 2010; pp. 425-452.

48. Tivoli, Y.A.; Rubenstein, R.M. Pruritus: An updated look at an old problem. J. Clin. Aesthet. Dermatol. 2009, 2, 30-36. [PubMed]

49. Zachariae, R.; Lei, U.; Haedersdal, M.; Zachariae, C. Itch severity and quality of life in patients with pruritus: Preliminary validity of a danish adaptation of the itch severity scale. Acta Derm. Venereol. 2012, 92, 508-514. [CrossRef] [PubMed]

50. Garibyan, L.; Chiou, A.S.; Elmariah, S.B. Advanced aging skin and itch: Addressing an unmet need. Dermatol. Ther. 2013, 26, 92-103. [CrossRef] [PubMed]

51. Reich, A.; Heisig, M.; Phan, N.Q.; Taneda, K.; Takamori, K.; Takeuchi, S.; Furue, M.; Blome, C.; Augustin, M.; Stander, S.; et al. Visual analogue scale: Evaluation of the instrument for the assessment of pruritus. Acta Derm. Venereol. 2012, 92, 497-501. [CrossRef] [PubMed]

52. Stander, S.; Augustin, M.; Reich, A.; Blome, C.; Ebata, T.; Phan, N.Q.; Szepietowski, J.C. Pruritus assessment in clinical trials: Consensus recommendations from the international forum for the study of itch (IFSI) special interest group scoring itch in clinical trials. Acta Derm. Venereol. 2013, 93, 509-514. [CrossRef] [PubMed]

53. Elman, S.; Hynan, L.S.; Gabriel, V.; Mayo, M.J. The 5-D itch scale: A new measure of pruritus. Br. J. Dermatol. 2010, 162, 587-593. [CrossRef] [PubMed]

54. Di Nuzzo, S.; Ercolini, E.; Carrozzo, E.; Fante, C.; Casanova, D.; Pruneti, C. Personality, alexithymic and autonomic aspects in psoriatic patients: A preliminary study. Clin. Drug Investig. 2013, 33, S88-S90.

55. Rigopoulos, D.; Tiligada, E. Stratum corneum lipids and water-holding capacity. In Dermatoanthropology of Ethnic Skin and Hair; Vashi, N., Maibach, H., Eds.; Springer: Cham, Switzerland, 2017.

56. Crowther, J.M.; Matts, P.J.; Kaczvinsky, J.R. Changes in stratum corneum thickness, water gradients and hydration by moisturizers. In Treatment of Dry Skin Syndrom; Lodén, M., Maibach, H., Eds.; Springer: Berlin/Heidelberg, Germany, 2012.

57. Tronnier, H.; Wiebusch, M.; Heinrich, U.; Stute, R. Surface evaluation of living skin. Adv. Exp. Med. Biol. 1999, 455, 507-516. [PubMed]

58. Callaghan, T.M.; Wilhelm, K.P. A review of ageing and an examination of clinical methods in the assessment of ageing skin. Part 2: Clinical perspectives and clinical methods in the evaluation of ageing skin. Int. J. Cosmet. Sci. 2008, 30, 323-332. [CrossRef] [PubMed]

59. Makrantonaki, E.; Zouboulis, C.C. Molecular mechanisms of skin aging-State of the art. Ann N. Y. Acad. Sci. 2007, 1119, 40-50. [CrossRef] [PubMed]

60. Piérard, G.E.; Piérard-Franchimont, C.; Piérard, S. Visioscan-driven ulev method. In Non Invasive Diagnostic Techniques in Clinical Dermatology; Berardesca, E., Maibach, H., Wilhelm, K.P., Eds.; Springer: Berlin, Germany, 2014.

61. Choi, J.W.; Kwon, S.H.; Huh, C.H.; Park, K.C.; Youn, S.W. The influences of skin visco-elasticity, hydration level and aging on the formation of wrinkles: A comprehensive and objective approach. Skin Res. Technol. 2013, 19, E349-E355. [CrossRef] [PubMed]

62. Menon, G.K.; Cleary, G.W.; Lane, M.E. The structure and function of the stratum corneum. Int. J. Pharm. 2012, 435, 3-9. [CrossRef] [PubMed]

63. Black, D.; Boyer, J.; Lagarde, J.M. Image analysis of skin scaling using d-squame samplers: Comparison with clinical scoring and use for assessing moisturizer efficacy. Int. J. Cosmet. Sci. 2006, 28, 35-44. [CrossRef] [PubMed]

64. Haroun, M.T. Dry skin in the elderly. Geriatr. Aging 2003, 6, 41-44.

65. Daungkum, K.; Foocharoen, C.; Mahakkanukrauh, A.; Suwannaroj, S.; Thinkhamrop, B.; Nanagara, R. Self-assessment of skin tightness severity by scleroderma patients. Int. J. Rheum. Dis. 2016, 19, 989-995. [CrossRef] [PubMed] 
66. Vitiello, M.; Abuchar, A.; Santana, N.; Dehesa, L.; Kerdel, F.A. An update on the treatment of the cutaneous manifestations of systemic sclerosis: The dermatologist's point of view. J. Clin. Aesthet. Dermatol. 2012, 5, 33-43. [PubMed]

67. Clements, P.J.; Lachenbruch, P.A.; Seibold, J.R.; Zee, B.; Steen, V.D.; Brennan, P.; Silman, A.J.; Allegar, N.; Varga, J.; Massa, M.; et al. Skin thickness score in systemic sclerosis: An assessment of interobserver variability in 3 independent studies. J. Rheumatol. 1993, 20, 1892-1896. [PubMed]

68. Khanna, D.; Furst, D.E.; Clements, P.J.; Allanore, Y.; Baron, M.; Czirjak, L.; Distler, O.; Foeldvari, I.; Kuwana, M.; Matucci-Cerinic, M.; et al. Standardization of the modified rodnan skin score for use in clinical trials of systemic sclerosis. J. Scleroderma Relat. Disord. 2017, 2, 11-18. [CrossRef] [PubMed]

69. Braun-Falco, O.; Plewig, G.; Wolff, H.H.; Burgdorf, W.H.C. Dermatology; Springer: Berlin, Germany, 1996.

70. Habif, T. Clinical Dermatology: A Color Guide to Diagnosis and Therapy, 6th ed.; Saunders: Philadelphia, PA, USA, 2015.

71. Braverman, I.M. The cutaneous microcirculation. J. Investig. Dermatol. Symp. Proc. 2000, 5, 3-9. [CrossRef] [PubMed]

72. Berardesca, E.; Elsner, P.; Maibach, H.I. Bioengineering of the Skin: Cutaneous Blood Flow and Erythema; CRC Press: Boca Raton, FL, USA, 1994; Volume II.

73. Tsukahara, K.; Nagashima, Y.; Moriwaki, S.; Fujimura, T.; Hattori, M.; Takema, Y. Relationship between physical parameters and blood flow in human facial skin. J. Cosmet. Sci. 2003, 54, 499-511. [PubMed]

74. Kim, S.I.; Jin, S.G.; Pfeifer, G.P. Formation of cyclobutane pyrimidine dimers at dipyrimidines containing 5-hydroxymethylcytosine. Photochem. Photobiol. Sci. 2013, 12, 1409-1415. [CrossRef] [PubMed]

75. Rinnerthaler, M.; Bischof, J.; Streubel, M.K.; Trost, A.; Richter, K. Oxidative stress in aging human skin. Biomolecules 2015, 5, 545-589. [CrossRef] [PubMed]

76. Halliwell, B. Why and how should we measure oxidative DNA damage in nutritional studies? How far have we come? Am. J. Clin. Nutr. 2000, 72, 1082-1087. [PubMed]

77. Zuber, T.J. Punch biopsy of the skin. Am. Fam. Phys. 2002, 65, 1155-1158, 1161-1162, 1164.

78. Ravanat, J.L.; Douki, T.; Cadet, J. Direct and indirect effects of UV radiation on DNA and its components. J. Photochem. Photobiol. B 2001, 63, 88-102. [CrossRef]

79. Mori, T.; Nakane, M.; Hattori, T.; Matsunaga, T.; Ihara, M.; Nikaido, O. Simultaneous establishment of monoclonal-antibodies specific for either cyclobutane pyrimidine dimer or (6-4)photoproduct from the same mouse immunized with ultraviolet-irradiated DNA. Photochem. Photobiol. 1991, 54, 225-232. [CrossRef] [PubMed]

80. Ahmed, N.U.; Ueda, M.; Nikaido, O.; Osawa, T.; Ichihashi, M. High levels of 8-hydroxy-2'-deoxyguanosine appear in normal human epidermis after a single dose of ultraviolet radiation. Br. J. Dermatol. 1999, 140, 226-231. [CrossRef] [PubMed]

81. Niki, E. Lipid oxidation in the skin. Free Radic. Res. 2014, 49, 827-834. [CrossRef] [PubMed]

82. Ayala, A.; Munoz, M.F.; Arguelles, S. Lipid peroxidation: Production, metabolism, and signaling mechanisms of malondialdehyde and 4-hydroxy-2-nonenal. Oxid. Med. Cell. Longev. 2014, 2014, 360438. [CrossRef] [PubMed]

83. Schneider, L.A.; Bloch, W.; Kopp, K.; Hainzl, A.; Rettberg, P.; Wlaschek, M.; Horneck, G.; Scharffetter-Kochanek, K. 8-isoprostane is a dose-related biomarker for photo-oxidative ultraviolet (UV) B damage in vivo: A pilot study with personal UV dosimetry. Br. J. Dermatol. 2006, 154, 1147-1154. [CrossRef] [PubMed]

84. Nakagawa, K.; Ibusuki, D.; Suzuki, Y.; Yamashita, S.; Higuchi, O.; Oikawa, S.; Miyazawa, T. Ion-trap tandem mass spectrometric analysis of squalene monohydroperoxide isomers in sunlight-exposed human skin. J. Lipid Res. 2007, 48, 2779-2787. [CrossRef] [PubMed]

85. Fogh, K.; Herlin, T.; Kragballe, K. Eicosanoids in skin of patients with atopic dermatitis: Prostaglandin E2 and leukotriene B4 are present in biologically active concentrations. J. Allergy Clin. Immunol. 1989, 83, 450-455. [CrossRef]

86. Krutmann, J. Skin aging. In Nutrition for Healthy Skin. Strategies for Clinical and Cosmetic Practice; Krutmann, J., Humbert, P., Eds.; Springer: Berlin, Germany, 2011; pp. 15-24.

87. Davies, M.J. Protein oxidation and peroxidation. Biochem. J. 2016, 473, 805-825. [CrossRef] [PubMed]

88. Berlett, B.S.; Stadtman, E.R. Protein oxidation in aging, disease, and oxidative stress. J. Biol. Chem. 1997, 272, 20313-20316. [CrossRef] [PubMed] 
89. Weber, D.; Davies, M.J.; Grune, T. Determination of protein carbonyls in plasma, cell extracts, tissue homogenates, isolated proteins: Focus on sample preparation and derivatization conditions. Redox Biol. 2015, 5, 367-380. [CrossRef] [PubMed]

90. Ichihashi, M.; Ueda, M.; Budiyanto, A.; Bito, T.; Oka, M.; Fukunaga, M.; Tsuru, K.; Horikawa, T. UV-induced skin damage. Toxicology 2003, 189, 21-39. [CrossRef]

91. Kim, Y.; He, Y.Y. Ultraviolet radiation-induced non-melanoma skin cancer: Regulation of DNA damage repair and inflammation. Genes Dis. 2014, 1, 188-198. [CrossRef] [PubMed]

92. Wiczk, J.; Miloch, J.; Rak, J. Dhplc and ms studies of a photoinduced intrastrand cross-link in DNA labeled with 5-bromo-2'-deoxyuridine. J. Photochem. Photobiol. B 2014, 130, 86-92. [CrossRef] [PubMed]

93. Cooke, M.S.; Podmore, I.D.; Mistry, N.; Evans, M.D.; Herbert, K.E.; Griffiths, H.R.; Lunec, J. Immunochemical detection of UV-induced DNA damage and repair. J. Immunol. Methods 2003, 280, 125-133. [CrossRef]

94. Teunissen, M.B. Dynamic nature and function of epidermal langerhans cells in vivo and in vitro: A review, with emphasis on human langerhans cells. Histochem. J. 1992, 24, 697-716. [CrossRef] [PubMed]

95. Simon, J.C.; Krutmann, J.; Elmets, C.A.; Bergstresser, P.R.; Cruz, P.D., Jr. Ultraviolet b-irradiated antigen-presenting cells display altered accessory signaling for T-cell activation: Relevance to immune responses initiated in skin. J. Investig. Dermatol. 1992, 98, 66S-69S. [CrossRef] [PubMed]

96. Kanitakis, J. Anatomy, histology and immunohistochemistry of normal human skin. Eur. J. Dermatol. 2002, 12, 390-399. [PubMed]

97. Soter, N.A. Acute effects of ultraviolet radiation on the skin. Semin. Dermatol. 1990, 9, 11-15. [PubMed]

98. Hruza, L.L.; Pentland, A.P. Mechanisms of UV-induced inflammation. J. Investig. Dermatol. 1993, 100, 35S-41S. [CrossRef] [PubMed]

99. Kelly, D.A.; Young, A.R.; McGregor, J.M.; Seed, P.T.; Potten, C.S.; Walker, S.L. Sensitivity to sunburn is associated with susceptibility to ultraviolet radiation-induced suppression of cutaneous cell-mediated immunity. J. Exp. Med. 2000, 191, 561-566. [CrossRef] [PubMed]

100. Heckman, C.J.; Chandler, R.; Kloss, J.D.; Benson, A.; Rooney, D.; Munshi, T.; Darlow, S.D.; Perlis, C.; Manne, S.L.; Oslin, D.W. Minimal erythema dose (MED) testing. J. Vis. Exp. 2013, 75, 50175. [CrossRef] [PubMed]

101. Moyal, D.D.; Fourtanier, A.M. Broad-spectrum sunscreens provide better protection from the suppression of the elicitation phase of delayed-type hypersensitivity response in humans. J. Investig. Dermatol. 2001, 117, 1186-1192. [CrossRef] [PubMed]

102. Fourtanier, A.; Moyal, D.; Maccario, J.; Compan, D.; Wolf, P.; Quehenberger, F.; Cooper, K.; Baron, E.; Halliday, G.; Poon, T.; et al. Measurement of sunscreen immune protection factors in humans: A consensus paper. J. Investig. Dermatol. 2005, 125, 403-409. [CrossRef] [PubMed]

(C) 2017 by the authors. Licensee MDPI, Basel, Switzerland. This article is an open access article distributed under the terms and conditions of the Creative Commons Attribution (CC BY) license (http:/ / creativecommons.org/licenses/by/4.0/). 


\title{
Nutraceuticals for Skin Care: A Comprehensive Review of Human Clinical Studies
}

\author{
Almudena Pérez-Sánchez ${ }^{1}$, Enrique Barrajón-Catalán ${ }^{1,2, *}$, María Herranz-López ${ }^{1,2}$ and \\ Vicente Micol ${ }^{1,2,3}$ \\ 1 Instituto de Biología Molecular y Celular (IBMC), Universidad Miguel Hernández (UMH), Edificio \\ Torregaitán, 03202 Elche, Spain; almudena.perez@umh.es (A.P.-S.); mherranz@umh.es (M.H.-L.); \\ vmicol@umh.es (V.M.) \\ 2 Ilice Effitech, UMH Scientific Park, 03202 Elche, Spain \\ 3 CIBER: CB12/03/30038, Fisiopatología de la Obesidad y la Nutrición, CIBERobn, Instituto de Salud Carlos \\ III (ISCIII), 07122 Palma Sola, Spain \\ * Correspondence: e.barrajon@umh.es
}

Received: 12 February 2018; Accepted: 21 March 2018; Published: 24 March 2018

\begin{abstract}
The skin is the body's largest organ, it participates in sensitivity and offers protection against microorganisms, chemicals and ultraviolet (UV) radiation. Consequently, the skin may suffer alterations such as photo-ageing, immune dysfunction and inflammation which may significantly affect human health. Nutraceuticals represent a promising strategy for preventing, delaying, or minimising premature ageing of the skin and also to alleviate certain skin disorders. Among them, bioactive peptides and oligosaccharides, plant polyphenols, carotenoids, vitamins and polyunsaturated fatty acids are the most widely used ingredients. Supplementation with these products has shown evidence of having an effect on the signs of ageing and protection against UV radiation ageing in several human trials. In this review, the most relevant human studies on skin nutraceuticals are evaluated and the statistical resolution, biological relevance of their results, and, the trial protocols are discussed. In conclusion, quality and rigorousness of the trials must be improved to build credible scientific evidence for skin nutraceuticals and to establish a cause-effect relationship between the ingredients the beneficial effects for the skin.
\end{abstract}

Keywords: nutraceutical; skin; natural compound; polyphenols

\section{Introduction}

The skin is the body's largest organ, representing one sixth of the total body weight, and its main role is to act as a chemical and physical barrier to protect the body against harmful external environmental agents such as pathogen, ultraviolet (UV) radiation exposure, chemical threats, temperature changes and even dehydration [1-3]. The skin is composed of three main layers with different underlying structures: (a) the epidermis, (b) the dermis and (c) hypodermis or subcutaneous tissue [4] (Figure 1).

The epidermis, of ectodermal origin, is the major protective outer layer and serves as the body's point of contact with the environment. The stratum corneum is the outermost layer of the epidermis, consisting of dead cells or corneocytes and has a thickness between $10 \mu \mathrm{m}$ and $30 \mu \mathrm{m}$. Underneath the stratum corneum, are living keratinocytes, melanocytes and Langerhans cells [2,3,5]. Keratinocytes are the predominant cell type in the epidermis producing keratin, a protein that makes the skin waterproof. Another significant cell group in the epidermis is that composed of the melanocytes. These cells form a heterogeneous group of cells in the human body and are present in the epidermis-dermal junction and hair follicles. Melanocytes produce melanin, a pigment responsible for skin pigmentation and photoprotection. Melanin may have other important physiological effects, including regulatory 
influence of epidermal homeostasis, free radical scavenging to protect against oxidative stress and even antimicrobial activity [6-12]. Langerhans cells are immune dendritic cells protecting against external substances and microorganisms [13-15].

The dermis, of mesoderm origin, is the layer that provides strength and elasticity to the skin. It includes the vascular, lymphatic and neuronal systems. It also contains sweat pores and hair follicles (Figure 1). The dermis is primarily composed of complex extracellular matrix (ECM) proteins, specially collagen fibres. ECM proteins can be categorised as either structural such as collagen and elastin or non-structural (glycoproteins), depending on their function. Integrins are essential compounds of the ECM, in addition to a group of matrix metalloproteinases (MMPs) and growth factors (GFs) $[16,17]$. Dermal collagen represents the most abundant ECM protein constituting $90 \%$ dry weight of the skin. Dermal connective tissue collagen is responsible for the skin's tensile strength and mechanical properties [18]. The dermis also contains abundant immune cells and fibroblasts (the major cell type present in this layer), which are involved in the synthesis of many of the ECM components. Blood vessels which provide nutrients to the skin and help regulate body temperature are also present in the dermis.

The hypodermis or subcutaneous tissue helps insulate the body from heat and cold, provides protective padding and serves as an energy storage area.

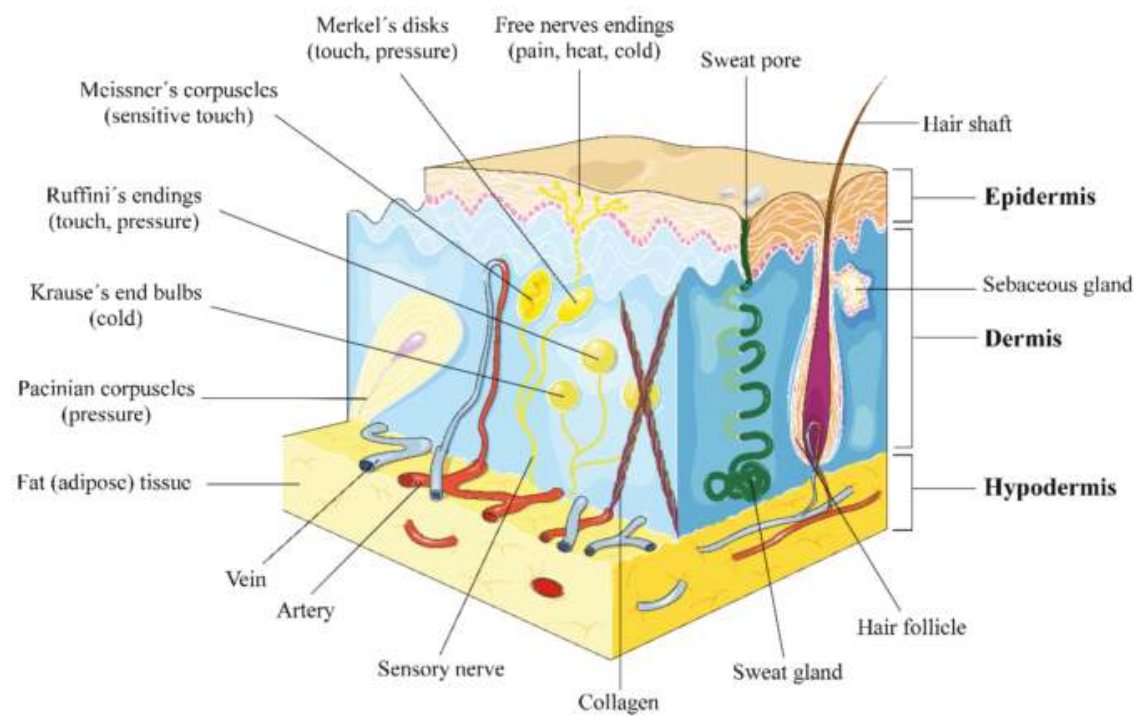

Figure 1. Human skin anatomy. There are three mechanoreceptor categories: tactile, proprioceptors and baroreceptors. The four major types of tactile mechanoreceptors are Merkel's disks, Meissner's corpuscles, Ruffini's endings, and Pacinian corpuscles. The fifth type of mechanoreceptor, Krause's end bulbs, is found only in specialised regions to detect cold. Free nerve endings are sensitive to painful stimuli, to hot and cold, and to light touch. This figure was created using Servier Medical Art [19], licensed under the Creative Commons Attribution 3.0 Unported License (www.creativecommons.org/ licenses/by/3.0/).

UV exposure is a major causative factor for age-related changes including inflammation, degenerative ageing, ECM degeneration and cancer. UV radiation is divided into long wave UVA (320-400 nm), medium wave UVB (280-320 nm), and shortwave UVC (100-280 nm), which is absorbed by the ozone layer. UVB represents only $0.3 \%$ of the sun's emission reaching the ground, and UVA radiation reaches Earth surface almost entirely. UV penetrates the skin in a wavelength-dependent 
manner. UVA (longer wavelength) reaching the dermis and UVB is almost entirely absorbed by the epidermis [20] (Figure 2).

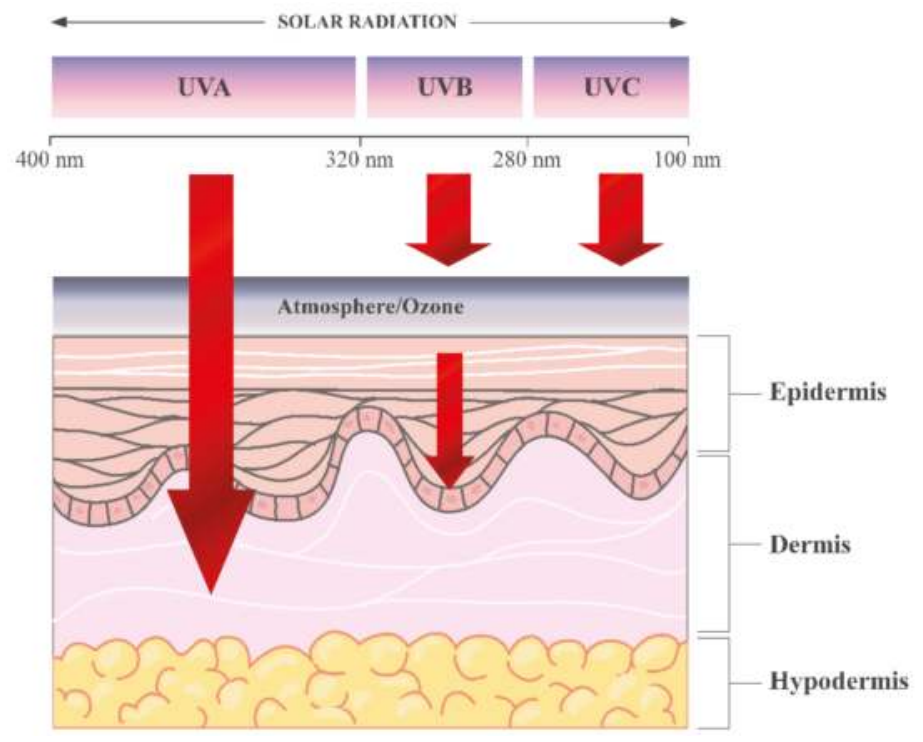

Figure 2. UV penetration into the layers of the skin. The figure was created using Servier Medical Art [19], licensed under the Creative Commons Attribution 3.0 Unported License (www. creativecommons.org/licenses/by/3.0/).

Solar UV radiation can interact with many molecules (chromophores) in different layers of the skin. These interactions may have both positive and negative biological effects, depending on wavelength, radiation exposure and UV sources. The positive effects mainly include vitamin D synthesis and treatment of different skin disorders (Figure 3). It is well known that solar radiation promotes the synthesis of vitamin D precursors at the skin. Most people can synthesise enough vitamin D from being out in the sun daily for short periods with their forearms, hands or lower legs uncovered. However, some populations as Africans, African/black Americans, or those from low sun-exposed areas, cannot synthesise it from limited sun exposure. In these situations, dermatologists recommend to get vitamin $\mathrm{D}$ from diet or vitamin supplements rather than from extra-exposure to UV.

The UVB photons enter the skin and photolyse 7-dehydrocholesterol to previtamin $\mathrm{D}_{3}$, which in turn, is isomerized by body's thermal energy to vitamin $\mathrm{D}_{3}$. Deficiency of vitamin $\mathrm{D}$ causes growth retardation in children and can precipitate and aggravate osteoporosis and osteopenia in adults. However, this is only associated with extremely low sun exposure lifestyles (northern Native people or some Asian populations). In addition, phototherapy is also an option in the treatment of many skin pathologies such as psoriatic and nonpsoriatic (e.g., morphea, scleroderma, vitiligo, atopic dermatitis) disorders [21-23].

The adverse effects of UV-radiation include inflammation, immunosuppression and/or allergy disorders, UV-increased sensitivity by drugs (corticoids), photo-aging, DNA damage, oxidative stress and carcinogenesis (Figure 3). UV-mediated skin inflammation is externally characterised by sunburn or erythema. This situation can be visually identified by skin redness or erythema, which is due to blood flow increase caused by dilation of the superficial blood vessels in the dermis. High UV doses can result in oedema, blistering, peeling and pain after exposure and UVB radiation, which is more erythmogenic than UVA radiation [24]. UVB induces a cytokine cascade, neuroactive and vasoactive mediators in the skin, resulting in inflammatory responses. If the dose of UV exceeds a 
certain threshold, affected keratinocytes respond by activating an apoptotic pathway. Such cells can be identified by their pyknotic nuclei and are known as "sunburn cells" [25-29]. From a molecular point of view, damage signals such as p53 activation significantly alter keratinocyte physiology mediating cell cycle arrest and activating DNA repair. NF- $\mathrm{kB}$ nuclear transcription factor is also activated by UV radiation, leading to the initial steps in the inflammatory process of sunburn reactions that increases the expression of proinflammatory cytokines interleukin (IL)-1, IL-6, tumor necrosis factor (TNF)- $\alpha$ and vascular endothelial growth factor (VEGF) [30].

UV-induced immunosuppression does not affect only an irradiated area but also influences the whole immune system as skin includes all the cell types also present in secondary lymphoid organ such as the spleen, lymph nodes and tonsils [31]. The main cells affected by UV radiation are Langerhans cells and T lymphocytes. Langerhans cells are dendritic cells critical for the presentation of antigens to the immune system. Langerhans cells are located in the epidermis and are responsible for T-lymphocyte activation in response to foreign antigens. Several studies have demonstrated that UVB radiation alters the number of Langerhans cells (decrease density), morphology and immunological function. Also, UVB radiation has been shown to induce T cell tolerance via modulation of the function of antigen-presenting cells like dendritic cells, leading to immunosuppression [32].

Skin ageing is a complex biological process resulting from two synergistic mechanisms: intrinsic and extrinsic factors. On the one hand, intrinsic or endogenous ageing, is an unavoidable phenomenon that includes several factors such as cellular metabolism, genetics, hormone and the passage of time. It is clinically associated with increased fragility and loss of elasticity. On the other hand, extrinsic or exogenous ageing can be avoided and is caused by repetitive exposure of the skin or body to harmful agents, especially UV light (photo-aging), inappropriate diet, pollution, chemicals and toxins [33,34]. UV radiation increases matrix metalloproteinases (MMPs) expression in human skin. MMPs are responsible for degrading ECM proteins such as collagen, fibronectin, proteoglycans and elastin (functional support). In addition, MMPs play an important role in carcinogenesis affecting several processes related to tumour progression such as growth, angiogenesis and metastasis [35-37]. Therefore, photo-aging is characterised by a disturbed equilibrium in the accumulation and degradation of ECM, losing elasticity, irregular pigmentation, dryness and wrinkling [38-46]. Wrinkling and pigmentation are also directly associated with premature photo-aging and are considered the most critical skin events [47].

UV radiation overexposure causes generation of reactive oxygen species (ROS), leading to an oxidative stress status [48,49]. This prooxidative situation has relevant consequences in cell homeostasis such as lipid and protein oxidation, loss of mitochondrial potential and DNA damage. In addition, ROS increase other UV effects such as DNA damage, inflammation and ageing, as they can activate inflammatory responses and up-regulate matrix metalloproteinase (MMP) production and activity, resulting in collagen breakdown. Skin spontaneously responds to high ROS levels by activating detoxifying enzymes such as superoxide dismutase (SOD), catalase (CAT), thioredoxin reductase (TrxRs) and using other antioxidant molecules such as glutathione (GSH), $\alpha$-tocopherol (vitamin E) and ascorbic acid (vitamin C). However, this response may not be effective enough to prevent oxidative damage of cutaneous cells after exposure to carcinogenic agents [50,51] (Figure 3).

UV radiation is only a fraction of the solar radiation; however, it is responsible for most of its carcinogenic activity as UV photons can affect the DNA integrity, homeostasis and induce mutations of genes including oncogenes and tumour suppressor genes. Although UVB represents a minority part of the whole radiation that reaches to the ground, it is the most dangerous and genotoxic component of sunlight, affecting nucleic acids in the epidermis [52,53]. UVB results in the formation of cyclobutane pyrimidine dimers (CPDs) and pyramidine-pyrimidone photodimers that may lead to DNA mutations and cancer $[38,39,54]$. However, UVA direct effect over nucleic acids is scarce, being its genotoxic effect mainly mediated by ROS as described above.

Skin complexion is among the most critical factors of UV sensitivity and skin cancer risk. The "Fitzpatrick Scale", developed in the 1970 s by Dr. T.B. Fitzpatrick, is a semi-quantitative scale made 
up of six pigmentation categories (phototype) that describe skin colour by skin pigmentation and sensitivity to UV radiation. Minimal Erythematous Dose (MED) is a quantitative method to report the amount of UV, particularly UVB, needed to induce sunburn in the skin $24-48 \mathrm{~h}$ after exposure by determining erythema (redness) and oedema (swelling) as endpoints. Low Fitzpatrick phototype correlates with both MED and with melanoma and other skin cancer risk $[55,56]$ (Figure 4).

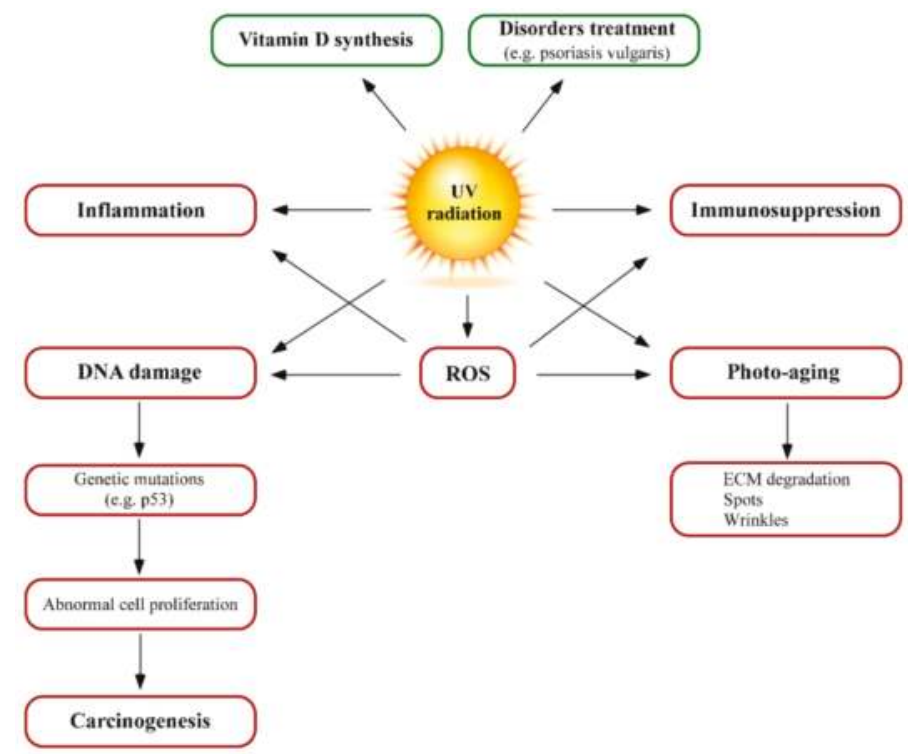

Figure 3. Summary of UV irradiation effects on the skin: positive (green) and adverse effects (red).

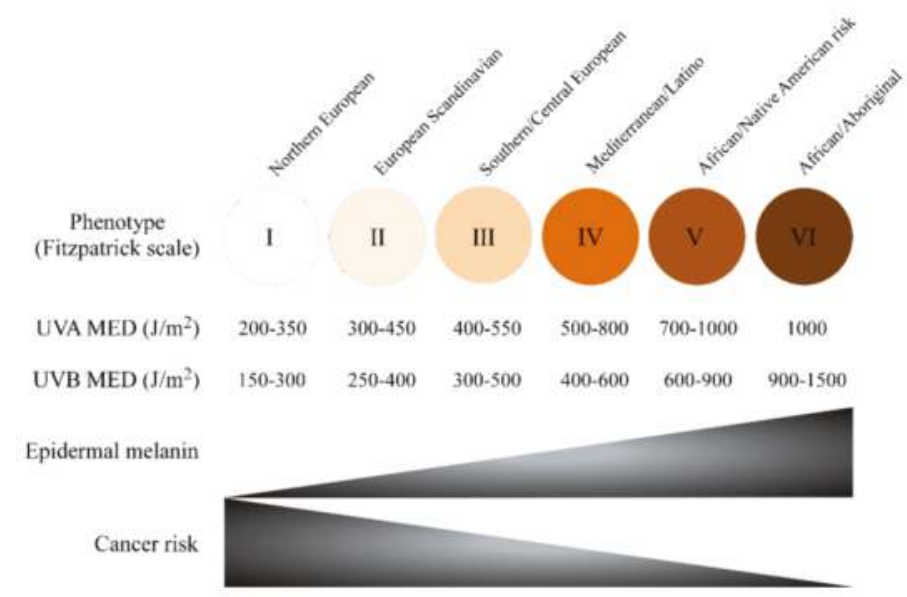

Figure 4. Influence of pigmentation and phototype on skin cancer risk.

Besides biological skin function, the skin plays a role in physical attractiveness. Skin appearance is determined by its texture, colour, and different characteristics such us elasticity, sweat and sebum production. It is accepted that nutritional status concerning both macro and micronutrients is important for skin health and appearance [57]. For example, dermatological signs of vitamin C deficiency include 
skin fragility, alterations in hair texture as corkscrew hairs and bleeding gums as well as impaired wound healing [58-64].

Food and cosmetic industries are developing new strategies to establish the relation between nutrients consumption and skin health. Consequently, the use of food ingredients and supplements that claim to reduce the risk of skin disorders or alleviate skin ageing is increasing [65]. Dietary supplementation with vitamins, minerals or essential fatty acids is proposed to improve skin conditions [65]. Most of the bioactive food compounds responsible for the positive effects on health are predominantly derived from plants while a few are derived from animal sources [66].

The term "nutraceutical" is derived from the combination of "nutrition" and "pharmaceutical" and was established by Stephen DeFelice in 1989, founder and chairman of the Foundation for Innovation in Medicine (FIM), Cranford, New Jersey $[67,68]$. According to DeFelice, nutraceutical can be defined as "a food (or a part of it) that provides medical or health benefits, including the prevention and/or treatment of a disease". Nutraceuticals may be used to improve health, delay the ageing process, prevent chronic diseases such as obesity, increase life expectancy, or support the structure or function of the body.

Nowadays, nutraceuticals have received considerable interest due to their nutritional potential, safety and therapeutic effects. Nevertheless, although nutraceuticals have shown promising results in various complications, their uncontrolled use may not be devoid of secondary effects so they should be strictly regulated as prescription drugs.

The nutraceutical industry's main segments include dietary supplements, herbal/natural products and functional foods [69]. Nutritional supplements and herbal/nature products are the most rapidly growing segments. A recent market research (Variant Market Research, Pune, India), proposed that the global nutraceuticals market is expanding and would reach $\$ 340$ billion by 2024, growing at a Compound Annual Growth Rate (CAGR) of 7.2\% from 2016 to 2024. Several market factors have been related to the significant growth of this sector: increasing healthcare, growing popularity for nutrition and rising demand for nutraceuticals [70].

Nutraceuticals can be classified by several criteria: food source, mechanism of action, chemical nature and specific benefit for health. They may be macronutrients (nutrients salts/chemicals elements required in large ammounts, e.g., potassium, magnesium, calcium and omega 3 acids), micronutrients (nutrients salts/chemicals elements required in small quantities, e.g., vitamins and minerals) and phytochemicals. Food sources used as nutraceuticals are all natural and can be categorised as: dietary fiber, prebiotics, probiotics, polyunsaturated fatty acids, minerals, amino acids and peptides, carotenoids, vitamins, phytochemicals and spices [69,71].

In this review, published data on the effects of nutrients on human skin are summarised. The design of the studies and the results of the different human trials that use prototype or commercial nutraceutical products are discussed to establish a cause-effect relationship between the consumption of the ingredient and the skin effects. The studies are organized according to the different families of ingredients used in the dietary supplements.

\section{Bioactive Peptides}

Peptides are short polypeptidic chains formed by few amino acids and with a low molecular weight, usually under $3 \mathrm{kDa}$. Since some of them carry out critical biological activities, these are generally known as bioactive peptides. Peptides used for cosmetic purposes are typically derived from collagen with improved bioavailability and solubility compared to the whole protein. Bioactive peptides have been used in several nutraceutical formulations claiming antiageing and skin reaffirming properties as described below.

In a double-blind placebo-controlled study, 114 subjects received $2.5 \mathrm{~g}$ of bioactive collagen peptide (BCP) VERISOL ${ }^{\circledR}$ or placebo per day for 8 weeks, 57 subjects in each treatment group. Skin wrinkles were measured before the treatment and after 4 and 8 weeks. The intake of BCP promoted a statistically significant reduction of eye wrinkle volume compared to placebo group after 4 and 8 weeks of 
treatment. In addition, $\mathrm{BCP}$ intake showed a statistically significant higher content of procollagen type I and elastin. Moreover, an increased fibrilin content was detected after BCP treatment, however this increase was not statistically significant. Authors concluded that oral intake of VERISOL ${ }^{\circledR}$ significantly reduced skin wrinkles and had positive effects on skin matrix synthesis $(p<0.05)$ [72].

In a placebo-controlled clinical trial, 60 women were screened, 33 subjects received a placebo or one of two treatments: a specific mixture of collagen peptides of fish origin (Peptan ${ }^{\circledR} \mathrm{F}$ ) or porcine origin $\left(\right.$ Peptan $\left.{ }^{\circledR} \mathrm{P}\right)$. Subjects took a formulated drink that contain either $10 \mathrm{~g}$ Peptan ${ }^{\circledR}$ or $10 \mathrm{~g}$ placebo for 56 days. Facial skin parameters were measured after 4, 8 and 12 weeks of treatment. Oral intake of Peptan ${ }^{\circledR} \mathrm{F}$ after 8 weeks of treatment showed a significant increase of skin moisture (12\%), while Peptan ${ }^{\circledR} \mathrm{P}$ increased skin moisture by $28 \%$ after 4 weeks of treatment and minimised skin micro-relief. In another double-blind placebo-controlled study, 106 women were randomly allocated to either a placebo or Peptan ${ }^{\circledR} \mathrm{F}$ group. This supplement was shown to reduce collagen fragmentation by $31 \%$ after 12 weeks of oral treatment, contributing to an anti-ageing effect [73]. The two fore-mentioned studies included a high number of subjects that provided enough statistical potency to observe statistically significant differences $(p<0.05)$.

In a recent clinical-laboratory study, 41 subjects received CELERGEN ${ }^{\circledR}$, a nutraceutical product containing $570 \mathrm{mg}$ marine collagen peptides (MCPs) derived from deep sea fish, $10 \mathrm{mg}$ grape skin extract, $10 \mathrm{mg}$ Coenzyme Q10 (CoQ10) (Figure 5), $10 \mathrm{mg}$ luteolin and $0.05 \mathrm{mg}$ Se of plant origin for 2 months of pre-treatment followed by 2 months of treatment. Supplementation improved skin elasticity, dermal ultrasonic markers and sebum production but no change was observed in several oxidative stress markers. Although the number of subjects was small, differences were statistically significant $(p<0.05)$. Authors concluded that a combination of MCPs with plant-derived antioxidants could be an effective and safe supplement to improve skin properties without risk of oxidative damage [74].

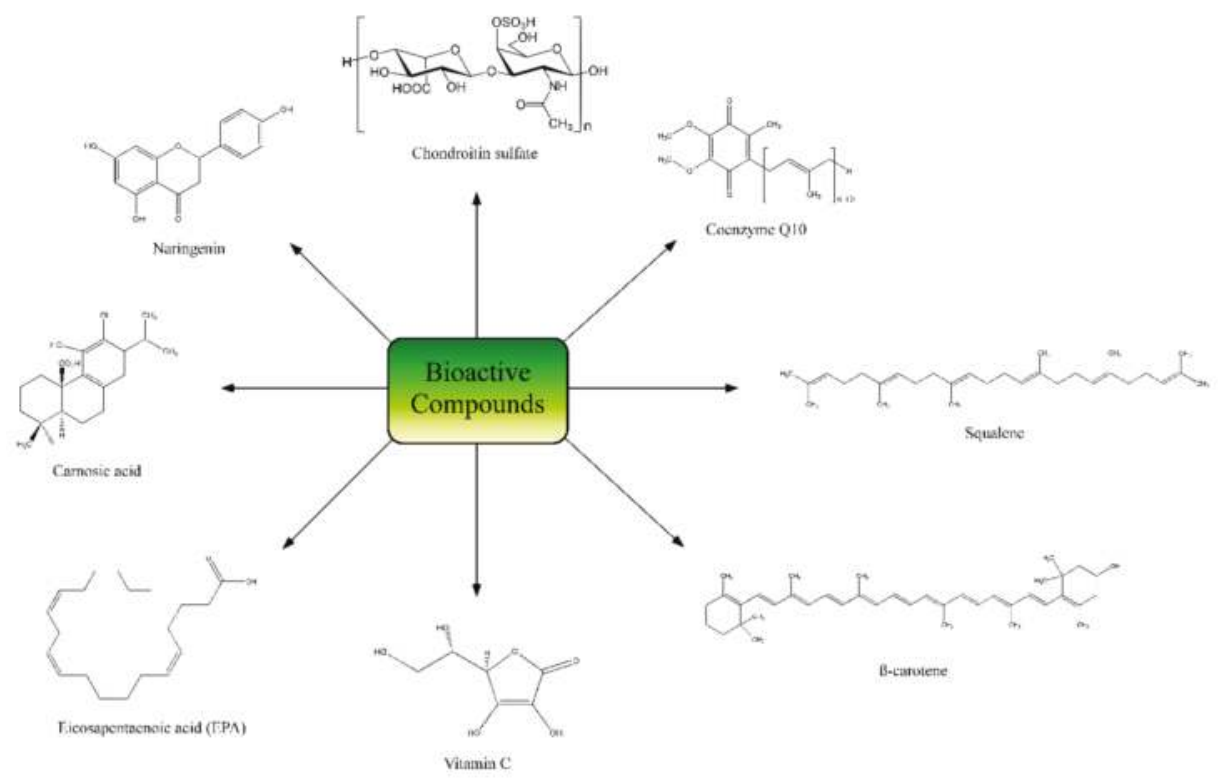

Figure 5. Representative structure compounds of different supplementation products. 


\section{Bioactive Polysaccharides}

Polysaccharides are sugar polymers with both structural and energy storage functions. They are present in plants, animals, fungi and procariota organisms with different structures, monosaccharide compositions and physicochemical properties. As occurred with bioactive peptides, a similar qualifier is used for those polysaccharides that exert biological activities, but in this case, with the peculiarity that it is reserved for those acting in a different organism than in which they were synthetised. Glycosaminocans, especially from marine origin, are the most used for nutraceutical formulations. They are based on an unbranched repeating disaccharide unit of an amino-sugar ( $N$-acetylglucosamine or $N$-acetylgalactosamine) and an uronic acid (glucuronic or iduronic acid). The most relevant human trials are explained below.

Imedeen ${ }^{\circledR}$ Derm One ${ }^{\circledR}$ is a dietary supplement for skin care containing protein fractions and some glycosaminoglycans extracted from marine fish. In addition, this supplement contained vitamin C and zinc gluconate, both relevant ingredients for skin health. This product was used in a trial where 10 women were treated with $500 \mathrm{mg}$ per day of Imedeen ${ }^{\circledR}$ for 90 days. Evaluated parameters included wrinkles, mottles, dryness and brittleness of hair and nails. After 90 days of treatment, all signs were improved, and clinical observations were confirmed by changes in skin elasticity and thickness [75].

On the other hand, Vivida ${ }^{\circledR}$ is a commercially available product containing different active polysaccharides derived from marine fish cartilage. The efficacy and safety of Vivida ${ }^{\circledR}$ and Imedeen ${ }^{\circledR}$ were compared in a double-blind study, in which 15 women were treated with $500 \mathrm{mg}$ of Vivida ${ }^{\circledR}$ per day, and 15 women received $380 \mathrm{mg}$ of Imedeen ${ }^{\circledR}$ per day for 90 days. Both treatments showed statistically significant improvements in skin conditions (epidermal and dermal thickness, elasticity and erythemal index) but Vivida ${ }^{\circledR}$ was more effective than Imedeen ${ }^{\circledR}$ for all parameters [76]. Nevertheless, the low number of subjects in the two previous studies compromise the consistency of the results. In another study with 144 healthy Caucasian subjects that provided significant statistical power, the subjects received Imedeen ${ }^{\circledR}$ tablets containing $105 \mathrm{mg}$ proteoglycan, $30 \mathrm{mg}$ vitamin $C$ and $15 \mathrm{mg}$ zinc gluconate for 12 months. After treatment, Imedeen ${ }^{\circledR}$ showed significant improvement in reduction of fine lines and overall photo-aging, in self-evaluation of skin condition, density, trans-epidermal water loss and skin smoothness. However, the long duration of the intervention study, i.e., 12 months, can raise several concerns. For marketing purposes, the consumer needs to denote positive effects within a reasonable period that may be around 30-60 days, otherwise, the consumer will stop taking the supplement. On the other hand, such a long period of consumption may elicit some safety concerns [77].

Another randomised, double-blind, placebo-controlled clinical study was performed with the ingredient Imedeen ${ }^{\circledR}$. The protocol was well-designed and also provided enough statistical power $(p<0.05) .90$ healthy women received 2 tablets of Imedeen ${ }^{\circledR}$ or placebo daily. Total daily dose contained $210 \mathrm{mg}$ Imedeen ${ }^{\circledR}$ marine complex, $57 \mathrm{mg}$ tomato and grape seed extracts and $60 \mathrm{mg}$ vitamin $\mathrm{C}$ for 4 months. The clinical grading of overall facial appearance improved for both treatment groups, however, the degree of improvement was significantly higher for subjects treated with the anti-ageing skin supplement. Results showed a positive effect on the appearance of skin, decreasing periocular wrinkling, visual and tactile roughness and mottled pigmentation [78].

In a quasi-experimental clinical study lacking a placebo group, 47 male subjects received two tablets of Imedeen ${ }^{\circledR}$ Man.Age.Ment containing per tablet: $105 \mathrm{mg}$ marine protein, $27 \mathrm{mg}$ vitamin C, 13.7 grape seed extract, $2 \mathrm{mg}$ zinc, and 14.38 tomato extract for 6 months. Results showed significant improvements from baseline in skin hydration, dermal ultrasound density and reduction of skin $\mathrm{pH}(p<0.05)$. The photographic assessment showed an improvement in the overall appearance and objective measurements correlated subject's satisfaction by an increase of collagen and elastic fibers [79].

In a double-blind, randomised, placebo-controlled study, 84 Asiatic healthy subjects were divided into two groups receiving control and galacto-oligosaccharides (GOS) respectively. Subjects received GOS ( $1 \mathrm{~g}$ in a capsule) twice a day for 12 weeks. Results showed that the increase in 
corneometer values from baseline to week 12 was significantly higher in the GOS treated group and the transepidermal water loss (TEWL) was reduced significantly $(p<0.05)$. In addition, the differences in total and percentage of wrinkle areas between the two groups were significant after 12 weeks of GOS treatment [80]. The evidence derived from the study supports a substantial improvement of the skin condition (hydration and skin barrier function) in individuals 50 years of age. However, the rationale and the mechanistic aspects of how oligosaccharides exert that action need to be further explored, at least in cell models.

\section{Bioactive Botanical Extracts}

Botanical extracts are complex mixtures of natural compounds with different structures and origins. Their use in cosmetics and skin care is well known since ancient times and have been extensively reviewed before [81,82]. Polyphenols are the main natural compounds with cosmetic applications and include a large variability of different structures and families (Figure 6). Composition and proportion of polyphenols may greatly vary depending on plant family and extraction procedure. The following are the most relevant studies of nutraceutical products based on botanical extracts that have been tested in humans.

Pycnogenol ${ }^{\circledR}$ is a standardised extract of bark of the French maritime Pine bark (Pinus pinaster) rich in flavonoids, such as catechins and procyanidins (B1, B2, B3, B7 C1 and C2), and phenolic acids such as caffeic, ferulic, and $p$-hydroxybenzoic acids. Pycnogenol ${ }^{\circledR}$ and it has been demonstrated to have various biological and health effects such as cardiovascular and cholesterol lowering benefits and antioxidant, antidiabetic and anti-inflammatory activity [83-86]. Studies have proposed that Pycnogenol $^{\circledR}$ is highly bioavailable and the mixture is more effective than individual components (synergistic effect). However, due to low procyanidins absorption in the small intestine and its conversion into smaller flavan-3-ols derivatives by microbiota, the bioavailability in humans is still an unresolved issue. Pycnogenol ${ }^{\circledR}$ oral supplements have been used in 21 Caucasian volunteers (1.1 mg/kg body weight). Results showed the photoprotective effect of this formulation against UV-light induced skin damages [87]. In other study, 20 healthy women were supplemented with three $25 \mathrm{mg}$ tables of Pycnogenol ${ }^{\circledR}$ (75 mg total) for 12 weeks. Pycnogenol ${ }^{\circledR}$ intake showed a significant increase in the mRNA expression of hyaluronic acid synthase (HAS-1) and in gene expression involved in collagen de novo synthesis. Externally, Pycnogenol ${ }^{\circledR}$ supplementation significantly improved hydration and elasticity of skin $(p<0.05)$ [88]. Nevertheless, the low number of subjects in the two previous studies raise doubts about the consistency of the results.

In a double-blind and placebo-controlled study, 62 women were treated with two Evelle ${ }^{\circledR}$ tablets twice a day for 6 weeks. One Evelle ${ }^{\circledR}$ tablet contains $10 \mathrm{mg}$ Pycnogenol ${ }^{\circledR}, 30 \mathrm{mg}$ vitamin C, $50 \mathrm{mg}$ vitamin E, $75 \mu \mathrm{g}$ biotin, $25 \mu \mathrm{g}$ selenium, $7.5 \mathrm{mg}$ zinc as gluconate, $50 \mathrm{mg}$ bio-marine complex, $40 \mathrm{mg}$ horsetail herb extract, $15 \mathrm{mg}$ blueberry extract and $34 \mathrm{mg}$ tomato extract. At the end of study, skin elasticity was found to be statistically significantly increased compared with placebo group. Skin roughness also was shown to be statistically significantly lower compared with the control group after 12 weeks of supplementation [89]. Further, 30 women with melasma were treated with one $25 \mathrm{mg}$ tablet of Pycnogenol ${ }^{\circledR}$ three times a day for 1 month $(75 \mathrm{mg}$ per day). The subjects were evaluated and clinically parameters such as melasma area index and skin pigmentation at the end of treatment, the melasma area and the average pigmentary intensity of the subjects showed a statistically significant decrease. The study conclude that Pycnogenol ${ }^{\circledR}$ is therapeutically effective and safe in patients with melasma [90]. The differences observed were statistically significant $(p<0.001)$ but the number of patients was moderate and no placebo group was included. 


\section{Polyphenols}

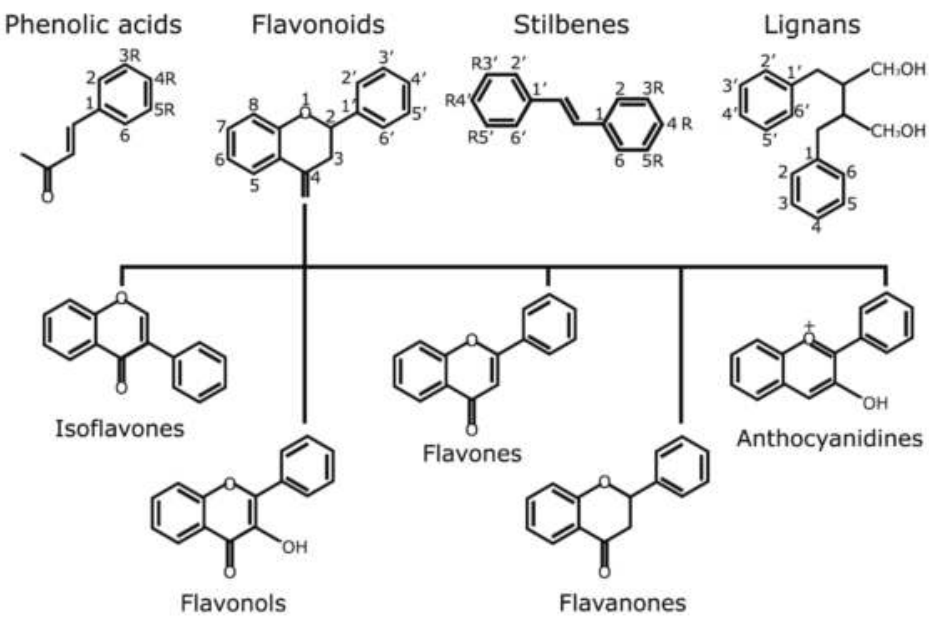

Figure 6. Main classes of polyphenols by structural classification. This image has been created and previously used by author in [91] and is under Creative Commons by Attribution (CC-BY) license (http://creativecommons.org/licenses/by/4.0/).

Oral administration of a different French maritime pine bark extract called Flavangenol ${ }^{\circledR}$, improved the clinical symptoms in photoaged facial skin. This oral supplement was administered to 112 healthy women under 60 years with age spots and multiple symptoms of photo-damaged skin (mottled pigmentation, roughness, wrinkles and swelling). 24 women were treated with $100 \mathrm{mg}$ per day for 12 weeks while 88 women were treated with $40 \mathrm{mg}$ per day for 24 weeks. At the end of the low-dose study, 24 subjects (out of 88 ) were treated with $40 \mathrm{mg}$ per day for an additional 24 weeks to evaluate the long-term efficacy and safety of pine bark extract. In both time courses of $100 \mathrm{mg}$ and $40 \mathrm{mg}$ per day, a significant decrease in clinical grading of skin photo-aging scores such as reduction in the pigmentation of age spots was observed [92].

A randomised, double-blind, placebo-controlled study was performed to evaluate the effect of Aloe sterol supplementation on skin parameters such as elasticity, hydration and the collagen score. 64 healthy women were randomly divided to receive either a placebo or an Aloe sterol supplemented yogurt for 12 weeks. Results showed significance differences in skin moisture, TEWL, elasticity and collagen score between treatment and placebo groups. At the end of supplementation, elasticity and collagen content increased significantly with Aloe sterol intake [93]. In a similar study, 48 healthy men received Aloe sterol for 12 weeks. Results showed that treatment increased melanin index and elasticity while skin moisture decreased [94]. The two previous studies showed statistically significant differences $(p<0.05)$ and had a moderate number of subjects.

NutroxSun ${ }^{\circledR}$, is a standardised formulation of citrus extract, obtained from immature grapefruit (Citus paradisi, enriched in citrus bioflavonoids as naringenin, Figure 5) and rosemary extract (Rosmarinus officinalis, enriched in phenolic compounds and diterpenes such as carnosic acid, Figure 5). To test the photoprotective efficacy of the combined extract, a human intervention study was performed by oral administration to volunteers for whom the minimal erythema dose (MED) was determined after exposure to UV radiation. At the 85th day of treatment, a significant increase in MED was observed $(56 \%)$, indicating that more extended oral treatments can improve UV protection effects [95]. This study provided statistically significant differences in MED between placebo and intervention groups $(p<0.05)$ but the number of subjects was low. In addition, NutroxSun ${ }^{\circledR}$ orally supplement has been used in 
90 Caucasian female subjects to investigate the anti-inflammatory, photoprotective and anti-ageing effects of this combination. After 2 weeks of product consumption, results showed decreasing wrinkle depth and increasing elasticity at 100 and $250 \mathrm{mg}$ extracts dose regimens. At 2 weeks of product use, results showed decreasing wrinkle depth and increasing elasticity at 100 and $250 \mathrm{mg}$ extracts dose regimen [96]. In this second study, number of subjects was high and statistically significant differences were confirmed in MED and also in wrinkle depth, elasticity and skin lipoperoxides between placebo and intervention groups.

The skin photo-protective and anti-ageing effects of a powder extract of red orange fruit (Red Orange Complex ${ }^{\circledR}$ ) were also reported in humans. The dietary supplement contained 2.8-3.2\% $w / w$ anthocyanins, $1.8-2.2 \% w / w$ hydroxycinnamic acids, $8.5-9.5 \% w / w$ flavone glycosides and $5.5-6.5 \% w / w$ ascorbic acid (see Figure 5 for representative structures). A moderate number of Caucasian subjects (20) received $100 \mathrm{mg}$ of extract per day for 15 days. In the evaluation of skin erythema induced by UV irradiation the results showed that red orange extract intake significantly decreased UV-induced skin erythema $(p<0.05)$. In the evaluation of skin appearance homogeneity, subjects were exposed to solar lamp, and age spot pigmentation decreased $20 \%$. The authors postulate that red-orange extract supplementation can present antioxidant activity and improves skin appearance and pigmentation [97].

Another study compared the effect of Polypodium leucotomos/Pomegranate combination (PPmix ${ }^{\circledR}$ ) versus Polypodium leucotomos alone $\left(\right.$ Fernblock $^{\circledR}$ ) on skin biophysical parameters. Both extracts contained different polyphenols specifically ellagitannins such as punicalagin. 40 subjects (20 males and 20 females) received 480 mg PPmix ${ }^{\circledR}$ or Fernblock ${ }^{\circledR}$ per day for 3 months. Six skin parameters were measured: skin sebum, hydration, transepidermal water loss (TEWL), melanin index, erythema index and elasticity. After treatment, elasticity and hydration were improved and TEWL was reduced in both groups. Both treatments reduced erythema index but PPmix ${ }^{\circledR}$ was more effective. Melanin index and skin sebum were decreased only by PPmix ${ }^{\circledR}$. Melanin index and skin sebum were reduced only by PPmix ${ }^{\circledR}$ [98]. A similar P. leucotomos extract was also utilized alone in Heliocare ${ }^{\circledR}$ formulation. A randomised, double blind, placebo-controlled study was developed. Subjects from both sexes and with Fitzpatrick skin types I to IV were randomised to receive $240 \mathrm{mg}$ of $P$. leucotomos extract $(N=20)$ or placebo $(N=20)$ twice a day for two months. The results confirmed $P$. leucotomos extract safety and showed that extract increased MED and reduced ultraviolet-induced erythema intensity $(p<0.05)$ [99].

\section{Carotenoid Supplementation}

Carotenoids are naturally occurring pigments with a lineal tretraterpenoid structure. They can be found in algae, photosynthetic bacteria and plants [100] providing red, orange and yellow colouration. Humans incorporate them from fruits and vegetables sources, with $\alpha$-carotene, $\beta$-carotene, $\beta$-cryptoxanthin, lutein, zeaxanthin, and lycopene being the most common dietary carotenoids [100] (Figure 5). Their main biological activities are related to cardiovascular diseases risk reduction and mentaining optimal visual function maintaining [101]. However, besides these systemic effects, their consumption has been considered beneficious for skin health, especially for photoprotection purposes shown by most of the following human studies.

Several studies have demonstrated the capacity of carotenoids' supplements to prevent UV-induced skin damage in human volunteers. One study demonstrated the effects of the consumption for 6 weeks of a specific probiotic (Skin-Probiotic ${ }^{\mathrm{TM}}$ ) and carotenoids in decreasing early UV-induced skin damage as well as in modulating early skin biomarkers of UV effects [102]. Three different studies using a total of 139 subjects and exposing subjects to UVA, solar radiation and natural sunlight exhibited statistically significant differences $(p<0.05)$ in MED, skin damage biomarkers and skin colour by comparing subjects before and after treatment [102].

Lee et al. investigated the photoprotective effects of a $30 \mathrm{mg}$ carotenoid mixture $(29.4 \mathrm{mg}$ $\beta$-carotene, $0.36 \mathrm{mg} \alpha$-carotene, $0.054 \mathrm{mg}$ lutein) per day in a limited number of subjects ( $11 \mathrm{men}$ and 11 women) for 8 weeks, but no placebo group was used. The concentration of carotenoid supplement 
was enhanced at $30 \mathrm{mg}$ increments to a final dose of $90 \mathrm{mg}$ per day. The results showed a modest, but significant dose-dependent increase in MED with 60 and $90 \mathrm{mg}$ carotenoid per day $(p<0.05)$. In addition, serum $\beta$-carotene concentrations increased after each intake, and serum lipid peroxidation was reduced, although no $\beta$-carotene was found in the skin [103].

In a study by Gollnick et al., 20 healthy young female students received a moderate dose of $\beta$-carotene, $30 \mathrm{mg}$ per day for 10 weeks. After the supplementation period, supplementation continued with exposure to natural sunlight for 13 days. An increased yellow pigmentation of skin surfaces and a reduction of erythema in subjects that had taken $\beta$-carotene was observed. The study concluded that pre-supplementation with moderate dose of $\beta$-carotene before and during sun exposure protects against sunburn by significantly decreasing erythema and increasing Langerhans cells $(p<0.01)$. Results also showed that the combination of systemic and topical photoprotection by sunscreens offers a synergistic effect, effect but the number of subjects was limited and no placebo group was used [104].

Postaire et al. also investigated the beneficial effects of a combination of $\beta$-carotene and other antioxidants. In this study, a limited number of subjects (10) received a supplement providing $13 \mathrm{mg}$ $\beta$-carotene, $2 \mathrm{mg}$ lycopene, $5 \mathrm{mg}$ tocopherol and $30 \mathrm{mg}$ ascorbic acid per day for 8 weeks. Although photoprotection was not directly measured in the study, authors suggest that carotenoids may be photoprotective because of a melanogenesis stimulation [105].

In another study, 20 subjects received $25 \mathrm{mg}$ of carotenoid mix $(23.8 \mathrm{mg} \beta$-carotene, $0.75 \mathrm{mg}$ $\alpha$-carotene, $0.18 \mathrm{mg}$ cryptoxanthin, $0.15 \mathrm{mg}$ zeaxanthin and $0.12 \mathrm{mg}$ lutein) per day and another group received this mix and $335 \mathrm{mg} \alpha$-tocopherol for 12 weeks. After this period, both groups showed a yellowing of the skin and elevated concentrations of $\beta$-carotene in serum and skin. The degree of erythema was highest in the group that received the carotenoids mix only $(p<0.01)$, so, the authors concluded that vitamin E might increases carotenoid protection against UV irradiation [106].

The effect of a mixture of the three main dietary carotenoids, beta-carotene, lutein and lycopene ( $8 \mathrm{mg} /$ day each), compared to beta-carotene $(24 \mathrm{mg} /$ day from an algal source) was evaluated in the erythema-protective effect. Thirty-six subjects were randomly assigned to three groups of 12 subjects in a placebo-controlled, parallel study design. The first group of treatment received $24 \mathrm{mg}$ of $\beta$-carotene per day for 12 weeks, the second group received the carotenoid mix and the third group received placebo. Although the number of subjects was low, the results showed that the intensity of erythema $24 \mathrm{~h}$ after irradiation was significantly decreased in both groups treated with carotenoids after 12 weeks of treatment, in correlation with the increase of serum carotenoids $(p<0.001)$. An increase of serum and skin concentration of carotenoids was determined in groups 1 and 2 throughout the study. Therefore, both carotenoid based treatments ameliorated UV-induced erythema in humans in a similar way [107].

\section{Vitamin Supplementation}

Vitamins are organic compounds which are essential nutrients for humans. They are required in limited amounts from diet and belong to different structural families. Vitamins also play a significant role in skin health [108], exerting different actions including antioxidant activity, sebum and keratinisation regulation, collagen synthesis, ECM homeostasis and photoprotection [108]. Several nutraceutical products containing vitamins as their main ingredient have been tested in humans as detailed below.

A mixture of vitamins derived from fermented papaya (Carica papaya L.) and an antioxidant cocktail was tested in 60 healthy subjects. Subjects received $4.5 \mathrm{~g}$ per day of fermented papaya preparation (FPP, final composition per $100 \mathrm{~g}$ : $90.7 \mathrm{~g}$ carbohydrates, $17 \mu \mathrm{g}$ vitamin B6, $2 \mu \mathrm{g}$ folic acid, $2.5 \mathrm{mg}$ calcium, $16.9 \mathrm{mg}$ potassium, $240 \mu \mathrm{g}$ niacin, $4.6 \mathrm{mg}$ magnesium, $14 \mu \mathrm{g}$ copper, $75 \mu \mathrm{g}$ zinc, $16 \mathrm{mg}$ arginine, $6 \mathrm{mg}$ lysine, $5 \mathrm{mg}$ histidine, $11 \mathrm{mg}$ phenylalanine, $9 \mathrm{mg}$ tyrosine, $18 \mathrm{mg}$ leucine, $9 \mathrm{mg}$ isoleucine, $5 \mathrm{mg}$ methionine, $13 \mathrm{mg}$ valine, $11 \mathrm{mg}$ glycine, $8 \mathrm{mg}$ proline, $37 \mathrm{mg}$ glutamic acid, $11 \mathrm{mg}$ serine, $8 \mathrm{mg}$ threonine, $27 \mathrm{mg}$ aspartic acid, and $2 \mathrm{mg}$ tryptophan) and an antioxidant cocktail (10 $\mathrm{mg}$ trans-resveratrol, $60 \mu \mathrm{g}$ selenium, $10 \mathrm{mg}$ vitamin $\mathrm{E}$ and $50 \mathrm{mg}$ vitamin C) for 90 days. Results showed 
a significant improvement in skin elasticity, moisture and antioxidant capacity $(p<0.05)$ with both the papaya preparation and the antioxidant cocktail [109].

In another study, 33 subjects received 100 or $180 \mathrm{mg}$ per day of vitamin C or placebo for 4 weeks. After treatment, results showed that $100 \mathrm{mg}$ of orally administered vitamin $\mathrm{C}$ increased radical scavenging activity of the skin in $22 \%$ while $180 \mathrm{mg}$ dose increased antioxidant activity by $37 \%$, compared to baseline [110].

In a double-blind trial, 16 healthy subjects received vitamins and trace elements to investigate the photoprotective effects of these compounds. The first group received $200 \mu \mathrm{g}$ selenium, $16 \mathrm{mg}$ copper sulfate, $14 \mathrm{mg} \alpha$-tocopherol and $2700 \mu \mathrm{g}$ retinol; the second group only received trace elements ( $200 \mu \mathrm{g}$ selenium and $16 \mathrm{mg}$ copper sulfate); the third group received only vitamins (14 $\mathrm{mg} \alpha$-tocopherol and $2700 \mu \mathrm{g}$ retinol) and the last group received placebo for 3 weeks. Supplementation in all treatments with active elements showed protection against sunburn cells at a low UV irradiation dose compared with the placebo group, but total number of subjects was very limited for three groups. However, treatment with both trace elements and vitamins showed an additional reduction in the number of sunburn cells [111].

The photoprotective effects of vitamins $\mathrm{E}$ and $\mathrm{C}$ have been intensely studied. A combination of vitamins $\mathrm{E}$ and $\mathrm{C}$ showed a protective effect in a double-blind, placebo-controlled study with 20 subjects. Ten subjects received $2 \mathrm{~g}$ of vitamin $\mathrm{C}$ and $1000 \mathrm{IU}$ of vitamin E per day or placebo for 8 days. The sunburn reaction before and after 8 days of the treatment was assessed by MED and by measuring the cutaneous blood flow of irradiated skin. Combined vitamin $C$ and $E$ reduce the sunburn reaction, UV-induced skin damage and cutaneous blood flow, whereas it increased in the placebo group [112]. However, the low number of subjects provided a low statistical resolution.

Fuchs and Kern also demonstrated a photoprotective effect of a combination of vitamins $\mathrm{E}$ and [113]. In this study, 40 healthy subjects received $2 \mathrm{~g}$ vitamin E per day, $3 \mathrm{~g}$ vitamin $\mathrm{C}$ per day, a combination of both vitamins and placebo for 50 days, so only 10 subjects were used per group. MED increased after intake slightly in subjects who received either vitamin alone or placebo. Nevertheless, the combination of both vitamins showed more pronounced photoprotective effect. Authors suggest that vitamin $E$ and $C$ act synergistically in suppression of sunburn reaction [113].

Placzek et al. investigated the effect of long-term oral administration of a combination of vitamin $\mathrm{C}$ and vitamin $\mathrm{E}$ in subjects on UVB-induced epidermal damage. Eighteen volunteers (12 males and 6 females) received supplementation with $2 \mathrm{~g}$ vitamin C and $1000 \mathrm{IU}$ vitamin E per day for 3 months. Blood vitamin concentrations were measured at the beginning and every 30 days during the study. After 3 months of treatment, the intake of vitamin $C$ and vitamin $E$ significantly reduced sunburn reaction to UVB irradiation. In addition, thymine dimers induced by UVB irradiation present in the skin was significantly reduced [114]. Again, the low number of subjects per group provided low statistical resolution.

\section{Coenzyme Q10 (CoQ10)}

CoQ10 is a natural compound of food and is often used in both functional foods and supplements. It is an endogenous lipophilic compound, essential component of mitochondrial energy metabolism, an effective antioxidant and presents a range of putative benefits for human health [115-118]. In a double-blind, placebo-controlled study, 33 healthy subjects received $50 \mathrm{mg}$ or $150 \mathrm{mg}$ CoQ10 $\left(\mathrm{Q} 10 \mathrm{Vital}{ }^{\circledR}\right)$, or placebo for 12 weeks. Results did not show significant changes in the MED. However, supplementation with CoQ10 limited deterioration of viscoelasticity $(p<0.05)$ and decreased some visible signs of ageing such as wrinkles and micro-relief lines $(p<0.05)$ and improved skin smoothness [119].

\section{Polyunsaturated Fatty Acids (PUFAs)}

PUFAs have been traditionally considered beneficious for human health $[120,121]$ and many health agencies and institutions have recommended their consumption. Structurally, PUFAs are 
divided into an omega-3 and an omega-6 series, depending on the position of their double bonds [122]. Their biological activity is especially relevant to cardiovascular and other inflammatory diseases, but also over skin heath [123-126]. Some studies have been developed in humans using PUFAs-based nutraceutical formulations as described below. However, most studies used a low-to-moderate number of subjects that result in low statistical resolution.

To test the photoprotective capacity of fish oil, ten subjects supplemented their diets with ten capsules per day of fish oil containing each $280 \mathrm{mg}$ eicosapentaenoic acid (EPA) and $120 \mathrm{mg}$ docosahexaenoic acid (DHA), and 10 subjects received ten placebo capsules per day. After 2 weeks, no significant effect of fish oil supplementation was observed in the measured parameters. However, after 4 weeks a small statistically significant increase of MED was seen in the fish oil treated group. These authors conclude that a low dose of fish oil (EPA and DHA) in a short period may be photoprotective [127]. In a study by Rhodes et al., 15 female Caucasian subjects supplemented their diets with $10 \mathrm{~g}$ of fish oil per day (18\% EPA and 12\% DHA) for 6 months. After 6 weeks of treatment, an increase in MED was observed but at 10 weeks MED decreased again. Although fish-oil intake reduced UV-induced erythema, the lipid peroxidation of skin increased due to the unstable nature of $n$-3 fatty acids [128]. In another study, the protective effect of EPA supplementation was investigated. For that purpose, 28 subjects received $4 \mathrm{~g}$ EPA (98\%) or oleic acid (98\%) per day for 3 months. UVB irradiation-induced erythema and p53 induction decreased in the EPA-treated group while no significant changes were found in the oleic acid group [129].

SemoSqualene ${ }^{\circledR}$, is an oral supplement rich in squalene (Figure 5), a polyunsaturated aliphatic hydrocarbon, and has been used in 40 healthy female subjects ( $>50$ years of age). A high dose of squalene (13.5-27 g per day) for 90 days reduced facial wrinkles, decreased skin reactivity to UV as shown by increased MED, increased type I procollagen gene expression and reduced UV-induced DNA damage and apoptosis. These effects may be attributed to its antioxidant capacity [130].

Several human studies have been performed to test the capacity of fish oil to alleviate eczema or psoriatic lesions. Ziboh et al. determined the benefits of fish oil supplementation in 13 psoriasis patients receiving a supplement ranging from 60 to $75 \mathrm{mg}$ (18\% EPA and 12\% DHA per gram) for 8 weeks. At the end of treatment, results showed that 8 patients demonstrated mild-to-moderate improvement in their psoriatic lesions which correlated with a high EPA-DHA ratio into the sera, epidermal and neutrophil lipids. Results suggested that increase in the ratio of leukotriene $\mathrm{B}_{5}\left(\mathrm{LTB}_{5}\right)$ was responsible for the reduction in inflammation [126]. In a similar study, ten patients with severe chronic psoriasis received $12 \mathrm{~g}$ EPA per day for 6 weeks. After treatment, a reduction in erythema was observed in $80 \%$ of patients [131]. A much lower dose of fish oil extract which provided $1.8 \mathrm{~g}$ EPA (MaxEPA ${ }^{\circledR}$ ) was administered daily to 28 psoriasis patients for 12 weeks. After 8 and 12 weeks, itching, erythema, and scaling decreased in the active treatment group, with a trend towards an overall decrease in body surface area affected, while no changes occurred in the placebo group [132].

Only two studies performed on patients with psoriasis or atopic eczema used a large number of subjects. First, 80 patients with chronic and stable psoriasis were supplemented with $1122 \mathrm{mg}$ EPA and 756 mg DHA for 8 weeks. Results showed an improvement in the overall skin condition and individual disease such as pruritis, scaling, induration and erythema [133]. Second, 99 subjects (60 adults and 39 children) with atopic eczema received primrose seed oil (EPO) for 12 weeks. Adults were supplemented with $1440 \mathrm{mg}$ linolenic acid and $190 \mathrm{mg} \gamma$-linolenic acid per day. Children received $720 \mathrm{mg}$ linolenic acid and $90 \mathrm{mg} \gamma$-linolenic per day. Results showed a moderate improvement in clinical signs of atopic eczema after treatment [134].

\section{Discussion}

The nutraceuticals market focused on skin health is increasing driven by consumer demand but strong scientific evidence for the products is still scarce. On the one hand, the increasing demand of naturally based products points to a promising future and plenty of opportunities for companies. 
On the other hand, scientific evidence about these products must increase considerably to improve products credibility.

First, although some studies include plasmatic measurements of the main compounds and metabolites derived from the administered formulations, most of the studies did not provide this information. The correlation between plasmatic concentrations of the metabolites and the observed biological effects would allow for the establishment of a cause-and-effect relationship and would improve claim substantiation. Bioavailability studies are deficient for different reasons. In some cases, either the bioavailability of a single pure compound is studied and not that of the same compound in the presence of the total formulation. In other cases, only in vitro absorption studies are performed, which are difficult to extrapolate in vivo. Besides bioavailability studies, mechanistic studies in skin cell models must also be performed to provide with a rationale of the observed biological effects.

Another difficulty when trying to compare different studies is related to the big differences in doses, additives and galenic formulations for the same active ingredient that prevents appropriate comparisons. Formulations are optimised by authors to improve solubility, absorption or bioavailability and to be technologically feasible. Therefore, even when similar doses of the same ingredient are used, a large variability is obtained between studies due to the formulation, besides the different baseline skin characteristics of the subjects.

However, the most important issue of the human trials using nutraceuticals for skin purposes is the low statistical resolution for some of these studies and, therefore, the low significance of the results. In most cases, as shown in this review, differences between treated and placebo groups are statistically significant, but their clinical significance is quite low due to the low number of subjects. In other cases, the biological significance is poor due to measurements based on questionnaires or personal perceptions. Some studies even lack a placebo group, which makes it difficult to counteract other effects such as diet, season or lifestyle. It might be the time for standardisation and including a minimum number of subjects and skin measurements in order to provide adequate statistical resolution when a human trial protocol is submitted to an Ethics committee.

This review tries to compile the most relevant human trials based on nutraceutical products related to skin and cosmetic issues. The human trials reviewed here cover many different ingredients: proteins, oligosaccharides, lipids, vitamins, polyphenols, and also pure ingredients or complex formulations. In any case, the strengths and weaknesses of the reviewed studies must be highlighted in order to be improved in the future.

Some human trials with collagen peptides did not use a placebo group or used a complex mixture into the dietary supplement [74], hence, establishing a cause-effect relationship for the ingredient was difficult. Regarding bioactive polysaccharides, despite statistically significant differences observed in some of the studies performed with Imedeen ${ }^{\circledR}$ ingredient, the small number of subjects utilised and the absence of a placebo group may cause doubts about the results obtained $[75,76]$. The authors also reported that five patients in the Vivida ${ }^{\circledR}$ group developed transient, mild pimples during the first weeks of treatment. However, trials made with galacto-oligosaccharides seemed to show consistent results on the improvement of hydration and skin barrier function [80].

French maritime Pine bark extract, Pycnogenol ${ }^{\circledR}$, has been studied in at least five different human trials. However, the low to moderate number of subjects provides low statistical resolution, the lack of placebo group in some cases and the inclusion of Pycnogenol ${ }^{\circledR}$ into complex mixtures prevent establishing a cause-effect relationship, suggesting that some of the results on Pycnogenol ${ }^{\circledR}$ ingredient for skin health [87-89] must be taken with caution and further research is required. Other studies based on procyanidin-based polyphenolic extracts provided conclusive results on the inhibition of UV-induced age spots due to the high number of subjects utilised [92]. The efficacy of rosemary and citrus polyphenols to reduce skin redness and MED and to improve skin elasticity and decrease wrinkle depth in correlation to a decrease in skin lipid oxidation was also consistently proven [96], and a mechanistic rationale was also provided in skin cell model [95]. A red orange extract enriched in anthocyanins [97] and Polypodium leucotomos extract $[98,99]$ also exhibited evidence of improvement in 
several skin signs related to photo-aging (erythema, hydration, elasticity and pigmentation), but with a much lower number of subjects, which compromises the consistency of the results.

Available studies on nutraceuticals containing carotenoids have shown a modest increase of MED with doses of 30-90 mg/day, but again, too small a number of subjects was used in most studies to be conclusive [103-107]. Human studies on vitamins, especially vitamin E and vitamin C, exhibited photoprotective properties through the increase of skin elasticity, moisture and antioxidant capacity in correlation with a MED increase [109-114]. Finally, studies that used PUFAs-based nutraceuticals showed inconsistent results [127-129]. Nevertheless, a reduction of erythema and inflammation was noticed in patients with psoriatic lesions or eczema taking omega-3 based nutraceuticals [126,131-133].

\section{Conclusions}

A number of natural ingredients have been shown to be potentially effective to alleviate the signs of skin ageing and some skin diseases. The rationale for the putative beneficial effects of an ingredient must be primarily demonstrated in skin cell models to elucidate the possible mechanism of action. Although nutraceuticals are under food regulation, the EU cosmetic legislation (2013/674/EU: Commission Implementing Decision of 25 November 2013) banned in the marketing of cosmetic ingredients or products which have been tested on animals. Therefore, the only options to verify the efficacy of a cosmetic ingredient are either in vitro skin cell models or human trials. To date, available human trials are far from conclusive in many cases for different reasons. In some cases, the ingredient tested is poorly characterised or it is part of a complex mixture that prevents establishing a cause-effect relationship between the ingredient and the biological effects. In other cases, clinical trials show poor statistical power due to insufficient number of subjects or the lack of a placebo group. Moreover, human trials only determine macroscopic skin characteristics (redness, spots, elasticity, wrinkles, etc.) and do not measure skin molecular markers (oxidative stress, enzymatic activity, gene expression or metabolites) that provide a mechanistic base. Therefore, in vitro and human trials on skin nutraceuticals must be designed to overcome all these limitations. Skin nutraceuticals are expected to shortly become a very profitable market. Nevertheless, more and better human trials must be performed in order improve the scientific basis of skin nutraceuticals and their credibility.

Acknowledgments: Some of the investigations described in this review have been partially or fully supported by competitive public grants from the following institutions: AGL2011-29857-C03-03 and IDI-20120751 grants (Spanish Ministry of Science and Innovation), projects AGL2015-67995-C3-1-R, AGL2015-67995-C3-2-R and AGL2015-67995-C3-3-R from the Spanish Ministry of Economy and Competitiveness (MINECO); and PROMETEO/2012/007, PROMETEO/2016/006, ACOMP/2013/093, ACIF/2010/162, ACIF/2015/158, ACIF/2016/230 and APOTIP/2017/003 grants from Generalitat Valenciana and CIBER (CB12/03/30038, Fisiopatologia de la Obesidad y la Nutricion, CIBERobn, Instituto de Salud Carlos III).

Author Contributions: A.P.-S. and E.B.-C. gathered all the information and wrote the first version of the manuscript. M.H.-L. reviewed and added new information about polyphenols to the manuscript. E.B.-C. and V.M. coordinated all the authors and edited and reviewed the different versions of the manuscript.

Conflicts of Interest: The authors declare no conflict of interest.

\section{References}

1. Pullar, J.M.; Carr, A.C.; Vissers, M.C.M. The roles of vitamin C in skin health. Nutrients 2017, 9. [CrossRef]

2. Madison, K.C. Barrier function of the skin: "La raison d'etre" of the epidermis. J. Investig. Dermatol. 2003, 121, 231-241. [CrossRef] [PubMed]

3. Shindo, Y.; Witt, E.; Han, D.; Epstein, W.; Packer, L. Enzymic and non-enzymic antioxidants in epidermis and dermis of human skin. J. Investig. Dermatol. 1994, 102, 122-124. [CrossRef] [PubMed]

4. Katiyar, S.; Elmets, C.A.; Katiyar, S.K. Green tea and skin cancer: Photoimmunology, angiogenesis and DNA repair. J. Nutr. Biochem. 2007, 18, 287-296. [CrossRef] [PubMed]

5. Menon, G.K. New insights into skin structure: Scratching the surface. Adv. Drug Deliv. Rev. 2002, 54 (Suppl. 1), S3-S17. [CrossRef] 
6. Slominski, A.; Tobin, D.J.; Shibahara, S.; Wortsman, J. Melanin pigmentation in mammalian skin and its hormonal regulation. Physiol. Rev. 2004, 84, 1155-1228. [CrossRef] [PubMed]

7. Slominski, A.; Paus, R.; Schadendorf, D. Melanocytes as "sensory" and regulatory cells in the epidermis. J. Theor. Biol. 1993, 164, 103-120. [CrossRef] [PubMed]

8. Kalka, K.; Mukhtar, H.; Turowski-Wanke, A.; Merk, H. Biomelanin antioxidants in cosmetics: Assessment based on inhibition of lipid peroxidation. Skin Pharmacol. Appl. Skin Physiol. 2000, 13, 143-149. [CrossRef] [PubMed]

9. Mackintosh, J.A. The antimicrobial properties of melanocytes, melanosomes and melanin and the evolution of black skin. J. Theor. Biol. 2001, 211, 101-113. [CrossRef] [PubMed]

10. Meyskens, F.L., Jr.; Farmer, P.; Fruehauf, J.P. Redox regulation in human melanocytes and melanoma. Pigment Cell Res. 2001, 14, 148-154. [CrossRef] [PubMed]

11. Double, K.L.; Ben-Shachar, D.; Youdim, M.B.; Zecca, L.; Riederer, P.; Gerlach, M. Influence of neuromelanin on oxidative pathways within the human substantia nigra. Neurotoxicol. Teratol. 2002, 24, 621-628. [CrossRef]

12. Wang, A.; Marino, A.R.; Gasyna, Z.; Gasyna, E.; Norris, J., Jr. Photoprotection by porcine eumelanin against singlet oxygen production. Photochem. Photobiol. 2008, 84, 679-682. [CrossRef] [PubMed]

13. Natarajan, V.T.; Ganju, P.; Ramkumar, A.; Grover, R.; Gokhale, R.S. Multifaceted pathways protect human skin from UV radiation. Nat. Chem. Biol. 2014, 10, 542-551. [CrossRef] [PubMed]

14. Cichorek, M.; Wachulska, M.; Stasiewicz, A.; Tyminska, A. Skin melanocytes: Biology and development. Postepy Dermatol. Alergol. 2013, 30, 30-41. [CrossRef] [PubMed]

15. Singh, T.P.; Zhang, H.H.; Borek, I.; Wolf, P.; Hedrick, M.N.; Singh, S.P.; Kelsall, B.L.; Clausen, B.E.; Farber, J.M. Monocyte-derived inflammatory langerhans cells and dermal dendritic cells mediate psoriasis-like inflammation. Nat. Commun. 2016, 7, 13581. [CrossRef] [PubMed]

16. Labat-Robert, J. Information exchanges between cells and extracellular matrix. Influence of ageing. Biol. Aujourdhui 2012, 206, 103-109. [CrossRef] [PubMed]

17. Kim, S.H.; Turnbull, J.; Guimond, S. Extracellular matrix and cell signalling: The dynamic cooperation of integrin, proteoglycan and growth factor receptor. J. Endocrinol. 2011, 209, 139-151. [CrossRef] [PubMed]

18. Quan, T.; Fisher, G.J. Role of age-associated alterations of the dermal extracellular matrix microenvironment in human skin ageing: A mini-review. Gerontology 2015, 61, 427-434. [CrossRef] [PubMed]

19. Smart Servier Medical Art. Available online: https://smart.servier.com/ (accessed on 29 September 2017).

20. D'Orazio, J.; Jarrett, S.; Amaro-Ortiz, A.; Scott, T. UV radiation and the skin. Int. J. Mol. Sci. 2013, 14, 12222-12248. [CrossRef] [PubMed]

21. Holick, M.F. Sunlight, UV-radiation, vitamin D and skin cancer: How much sunlight do we need? Adv. Exp. Med. Biol. 2008, 624, 1-15. [PubMed]

22. Juzeniene, A.; Moan, J. Beneficial effects of UV radiation other than via vitamin D production. Dermatoendocrinol 2012, 4, 109-117. [CrossRef] [PubMed]

23. Gupta, A.; Avci, P.; Dai, T.; Huang, Y.Y.; Hamblin, M.R. Ultraviolet radiation in wound care: Sterilization and stimulation. Adv. Wound Care (New Rochelle) 2013, 2, 422-437. [CrossRef] [PubMed]

24. Diffey, B.L. Solar ultraviolet radiation effects on biological systems. Phys. Med. Biol. 1991, 36, $299-328$. [CrossRef] [PubMed]

25. Slominski, A.; Wortsman, J. Neuroendocrinology of the skin. Endocr. Rev. 2000, 21, 457-487. [CrossRef] [PubMed]

26. Slominski, A.; Wortsman, J.; Luger, T.; Paus, R.; Solomon, S. Corticotropin releasing hormone and proopiomelanocortin involvement in the cutaneous response to stress. Physiol. Rev. 2000, 80, 979-1020. [CrossRef] [PubMed]

27. Slominski, A.T.; Zmijewski, M.A.; Skobowiat, C.; Zbytek, B.; Slominski, R.M.; Steketee, J.D. Sensing the environment: Regulation of local and global homeostasis by the skin's neuroendocrine system. Adv. Anat Embryol. Cell Biol. 2012, 212,1-115.

28. Clydesdale, G.J.; Dandie, G.W.; Muller, H.K. Ultraviolet light induced injury: Immunological and inflammatory effects. Immunol. Cell Biol. 2001, 79, 547-568. [CrossRef] [PubMed]

29. Matsumura, Y.; Ananthaswamy, H.N. Toxic effects of ultraviolet radiation on the skin. Toxicol. Appl. Pharmacol. 2004, 195, 298-308. [CrossRef] [PubMed] 
30. Abeyama, K.; Eng, W.; Jester, J.V.; Vink, A.A.; Edelbaum, D.; Cockerell, C.J.; Bergstresser, P.R.; Takashima, A. A role for NF-kappaB-dependent gene transactivation in sunburn. J. Clin. Investig. 2000, 105, 1751-1759. [CrossRef] [PubMed]

31. Norval, M. The mechanisms and consequences of ultraviolet-induced immunosuppression. Prog. Biophys. Mol. Biol. 2006, 92, 108-118. [CrossRef] [PubMed]

32. Gerlini, G.; Romagnoli, P.; Pimpinelli, N. Skin cancer and immunosuppression. Crit. Rev. Oncol. Hematol. 2005, 56, 127-136. [CrossRef] [PubMed]

33. Gilchrest, B.A. Age-associated changes in the skin. J. Am. Geriatr. Soc. 1982, 30, 139-143. [CrossRef] [PubMed]

34. Cevenini, E.; Invidia, L.; Lescai, F.; Salvioli, S.; Tieri, P.; Castellani, G.; Franceschi, C. Human models of ageing and longevity. Expert Opin. Biol. Ther. 2008, 8, 1393-1405. [CrossRef] [PubMed]

35. Quan, T.; Qin, Z.; Xia, W.; Shao, Y.; Voorhees, J.J.; Fisher, G.J. Matrix-degrading metalloproteinases in photoaging. J. Investig. Dermatol. Symp. Proc. 2009, 14, 20-24. [CrossRef] [PubMed]

36. Kim, J.; Lee, C.W.; Kim, E.K.; Lee, S.J.; Park, N.H.; Kim, H.S.; Kim, H.K.; Char, K.; Jang, Y.P.; Kim, J.W. Inhibition effect of gynura procumbens extract on UV-B-induced matrix-metalloproteinase expression in human dermal fibroblasts. J. Ethnopharmacol. 2011, 137, 427-433. [CrossRef] [PubMed]

37. O'Grady, A.; Dunne, C.; O'Kelly, P.; Murphy, G.M.; Leader, M.; Kay, E. Differential expression of matrix metalloproteinase (MMP)-2, MMP-9 and tissue inhibitor of metalloproteinase (TIMP)-1 and TIMP-2 in non-melanoma skin cancer: Implications for tumour progression. Histopathology 2007, 51, 793-804. [CrossRef] [PubMed]

38. Naylor, E.C.; Watson, R.E.; Sherratt, M.J. Molecular aspects of skin ageing. Maturitas 2011, 69, $249-256$. [CrossRef] [PubMed]

39. Gonzaga, E.R. Role of UV light in photodamage, skin ageing, and skin cancer: Importance of photoprotection. Am. J. Clin. Dermatol. 2009, 10 (Suppl. 1), 19-24. [CrossRef] [PubMed]

40. Farage, M.A.; Miller, K.W.; Elsner, P.; Maibach, H.I. Intrinsic and extrinsic factors in skin ageing: A review. Int. J. Cosmet. Sci. 2008, 30, 87-95. [CrossRef] [PubMed]

41. Kammeyer, A.; Luiten, R.M. Oxidation events and skin ageing. Ageing Res. Rev. 2015, 21, 16-29. [CrossRef] [PubMed]

42. Zouboulis, C.C.; Makrantonaki, E. Clinical aspects and molecular diagnostics of skin ageing. Clin. Dermatol. 2011, 29, 3-14. [CrossRef] [PubMed]

43. Anderson, A.; Bowman, A.; Boulton, S.J.; Manning, P.; Birch-Machin, M.A. A role for human mitochondrial complex II in the production of reactive oxygen species in human skin. Redox Biol. 2014, 2, 1016-1022. [CrossRef] [PubMed]

44. Meadows, C.; Morre, D.J.; Morre, D.M.; Draelos, Z.D.; Kern, D. Age-related NADH oxidase (arNOX)catalyzed oxidative damage to skin proteins. Arch. Dermatol. Res. 2014, 306, 645-652. [CrossRef] [PubMed]

45. Quan, C.; Cho, M.K.; Perry, D.; Quan, T. Age-associated reduction of cell spreading induces mitochondrial DNA common deletion by oxidative stress in human skin dermal fibroblasts: Implication for human skin connective tissue ageing. J. Biomed. Sci. 2015, 22, 62. [CrossRef] [PubMed]

46. Gilchrest, B.A. Skin ageing and photoageing: An overview. J. Am. Acad. Dermatol. 1989, 21, 610-613. [CrossRef]

47. Ganceviciene, R.; Liakou, A.I.; Theodoridis, A.; Makrantonaki, E.; Zouboulis, C.C. Skin anti-ageing strategies. Dermatoendocrinol 2012, 4, 308-319. [CrossRef] [PubMed]

48. Bowden, G.T. Prevention of non-melanoma skin cancer by targeting ultraviolet-B-light signalling. Nat. Rev. Cancer 2004, 4, 23-35. [CrossRef] [PubMed]

49. Afaq, F.; Adhami, V.M.; Mukhtar, H. Photochemoprevention of ultraviolet B signaling and photocarcinogenesis. Mutat. Res. 2005, 571, 153-173. [CrossRef] [PubMed]

50. Rabe, J.H.; Mamelak, A.J.; McElgunn, P.J.; Morison, W.L.; Sauder, D.N. Photoaging: Mechanisms and repair. J. Am. Acad. Dermatol. 2006, 55, 1-19. [CrossRef] [PubMed]

51. F'Guyer, S.; Afaq, F.; Mukhtar, H. Photochemoprevention of skin cancer by botanical agents. Photodermatol. Photoimmunol. Photomed. 2003, 19, 56-72. [CrossRef] [PubMed]

52. Trautinger, F. Mechanisms of photodamage of the skin and its functional consequences for skin ageing. Clin. Exp. Dermatol. 2001, 26, 573-577. [CrossRef] [PubMed]

53. Cavinato, M.; Jansen-Durr, P. Molecular mechanisms of UVB-induced senescence of dermal fibroblasts and its relevance for photoaging of the human skin. Exp. Gerontol. 2017, 94, 78-82. [CrossRef] [PubMed] 
54. Afaq, F.; Mukhtar, H. Botanical antioxidants in the prevention of photocarcinogenesis and photoaging. Exp. Dermatol. 2006, 15, 678-684. [CrossRef] [PubMed]

55. Scherer, D.; Kumar, R. Genetics of pigmentation in skin cancer-A review. Mutat. Res. 2010, 705, 141-153. [CrossRef] [PubMed]

56. Ravnbak, M.H. Objective determination of Fitzpatrick skin type. Dan. Med. Bull. 2010, 57, B4153. [PubMed]

57. Park, K. Role of micronutrients in skin health and function. Biomol. Ther. (Seoul) 2015, 23, 207-217. [CrossRef] [PubMed]

58. Talarico, V.; Aloe, M.; Barreca, M.; Galati, M.C.; Raiola, G. Do you remember scurvy? Clin. Ter. 2014, 165, 253-256. [PubMed]

59. Alqanatish, J.T.; Alqahtani, F.; Alsewairi, W.M.; Al-kenaizan, S. Childhood scurvy: An unusual cause of refusal to walk in a child. Pediatr. Rheumatol. Online J. 2015, 13, 23. [CrossRef] [PubMed]

60. Peterkofsky, B. Ascorbate requirement for hydroxylation and secretion of procollagen: Relationship to inhibition of collagen synthesis in scurvy. Am. J. Clin. Nutr. 1991, 54, 1135S-1140S. [CrossRef] [PubMed]

61. Ellinger, S.; Stehle, P. Efficacy of vitamin supplementation in situations with wound healing disorders: Results from clinical intervention studies. Curr. Opin. Clin. Nutr. Metab. Care 2009, 12, 588-595. [CrossRef] [PubMed]

62. Ross, R.; Benditt, E.P. Wound healing and collagen formation. Ii. Fine structure in experimental scurvy. J. Cell Biol. 1962, 12, 533-551. [CrossRef] [PubMed]

63. Hodges, R.E.; Baker, E.M.; Hood, J.; Sauberlich, H.E.; March, S.C. Experimental scurvy in man. Am. J. Clin. Nutr. 1969, 22, 535-548. [CrossRef] [PubMed]

64. Hodges, R.E.; Hood, J.; Canham, J.E.; Sauberlich, H.E.; Baker, E.M. Clinical manifestations of ascorbic acid deficiency in man. Am. J. Clin. Nutr. 1971, 24, 432-443. [CrossRef] [PubMed]

65. Boelsma, E.; Hendriks, H.F.; Roza, L. Nutritional skin care: Health effects of micronutrients and fatty acids. Am. J. Clin. Nutr. 2001, 73, 853-864. [CrossRef] [PubMed]

66. Kussmann, M.; Affolter, M.; Nagy, K.; Holst, B.; Fay, L.B. Mass spectrometry in nutrition: Understanding dietary health effects at the molecular level. Mass Spectrom. Rev. 2007, 26, 727-750. [CrossRef] [PubMed]

67. Brower, V. Nutraceuticals: Poised for a healthy slice of the healthcare market? Nat. Biotechnol. 1998, 16, 728-731. [CrossRef] [PubMed]

68. Kalra, E.K. Nutraceutical-definition and introduction. AAPS PharmSci. 2003, 5, E25. [CrossRef] [PubMed]

69. Das, L.; Bhaumik, E.; Raychaudhuri, U.; Chakraborty, R. Role of nutraceuticals in human health. J. Food Sci. Technol. 2012, 49, 173-183. [CrossRef] [PubMed]

70. Variant Market Research. Available online: https:/ /www.variantmarketresearch.com/report-categories / food-beverages / nutraceuticals-market (accessed on 3 October 2017).

71. Chavez-Mendoza, C.; Sanchez, E. Bioactive compounds from mexican varieties of the common bean (Phaseolus vulgaris): Implications for health. Molecules 2017, 22. [CrossRef] [PubMed]

72. Proksch, E.; Schunck, M.; Zague, V.; Segger, D.; Degwert, J.; Oesser, S. Oral intake of specific bioactive collagen peptides reduces skin wrinkles and increases dermal matrix synthesis. Skin Pharmacol. Physiol. 2014, 27, 113-119. [CrossRef] [PubMed]

73. Asserin, J.; Lati, E.; Shioya, T.; Prawitt, J. The effect of oral collagen peptide supplementation on skin moisture and the dermal collagen network: Evidence from an ex vivo model and randomized, placebo-controlled clinical trials. J. Cosmet. Dermatol. 2015, 14, 291-301. [CrossRef] [PubMed]

74. De Luca, C.; Mikhal'chik, E.V.; Suprun, M.V.; Papacharalambous, M.; Truhanov, A.I.; Korkina, L.G. Skin antiageing and systemic redox effects of supplementation with marine collagen peptides and plant-derived antioxidants: A single-blind case-control clinical study. Oxid. Med. Cell. Longev. 2016, 2016, 4389410. [CrossRef] [PubMed]

75. Lassus, A.; Jeskanen, L.; Happonen, H.P.; Santalahti, J. Imedeen for the treatment of degenerated skin in females. J. Int. Med. Res. 1991, 19, 147-152. [CrossRef] [PubMed]

76. Eskelinen, A.; Santalahti, J. Natural cartilage polysaccharides for the treatment of sun-damaged skin in females: A double-blind comparison of vivida and imedeen. J. Int. Med. Res. 1992, 20, 227-233. [CrossRef] [PubMed]

77. Kieffer, M.E.; Efsen, J. Imedeen in the treatment of photoaged skin: An efficacy and safety trial over 12 months. J. Eur. Acad. Dermatol. Venereol. 1998, 11, 129-136. [CrossRef] 
78. Stephens, T.J.; Sigler, M.L.; Hino, P.D.; Moigne, A.L.; Dispensa, L. A randomized, double-blind, placebocontrolled clinical trial evaluating an oral anti-ageing skin care supplement for treating photodamaged skin. J. Clin. Aesthet. Dermatol. 2016, 9, 25-32. [PubMed]

79. Costa, A.; Pegas Pereira, E.S.; Assumpcao, E.C.; Calixto Dos Santos, F.B.; Ota, F.S.; de Oliveira Pereira, M.; Fidelis, M.C.; Favaro, R.; Barros Langen, S.S.; Favaro de Arruda, L.H.; et al. Assessment of clinical effects and safety of an oral supplement based on marine protein, vitamin C, grape seed extract, zinc, and tomato extract in the improvement of visible signs of skin ageing in men. Clin. Cosmet. Investig. Dermatol. 2015, 8, 319-328. [CrossRef] [PubMed]

80. Hong, Y.H.; Chang, U.J.; Kim, Y.S.; Jung, E.Y.; Suh, H.J. Dietary galacto-oligosaccharides improve skin health: A randomized double blind clinical trial. Asia Pac. J. Clin. Nutr. 2017, 26, 613-618. [PubMed]

81. Baumann, L.; Woolery-Lloyd, H.; Friedman, A. "Natural" ingredients in cosmetic dermatology. J. Drugs Dermatol. 2009, 8, s5-s9. [PubMed]

82. Zillich, O.V.; Schweiggert-Weisz, U.; Eisner, P.; Kerscher, M. Polyphenols as active ingredients for cosmetic products. Int. J. Cosmet. Sci. 2015, 37, 455-464. [CrossRef] [PubMed]

83. Blazso, G.; Gabor, M.; Schonlau, F.; Rohdewald, P. Pycnogenol accelerates wound healing and reduces scar formation. Phytother. Res. 2004, 18, 579-581. [CrossRef] [PubMed]

84. Iravani, S.; Zolfaghari, B. Pharmaceutical and nutraceutical effects of pinus pinaster bark extract. Res. Pharm. Sci. 2011, 6, 1-11. [PubMed]

85. Rohdewald, P. A review of the French maritime pine bark extract (pycnogenol), a herbal medication with a diverse clinical pharmacology. Int. J. Clin. Pharmacol. Ther. 2002, 40, 158-168. [CrossRef] [PubMed]

86. Maimoona, A.; Naeem, I.; Saddiqe, Z.; Jameel, K. A review on biological, nutraceutical and clinical aspects of French maritime pine bark extract. J. Ethnopharmacol. 2011, 133, 261-277. [CrossRef] [PubMed]

87. Saliou, C.; Rimbach, G.; Moini, H.; McLaughlin, L.; Hosseini, S.; Lee, J.; Watson, R.R.; Packer, L. Solar ultraviolet-induced erythema in human skin and nuclear factor-kappa-B-dependent gene expression in keratinocytes are modulated by a French maritime pine bark extract. Free Radic. Biol. Med. 2001, 30, 154-160. [CrossRef]

88. Marini, A.; Grether-Beck, S.; Jaenicke, T.; Weber, M.; Burki, C.; Formann, P.; Brenden, H.; Schonlau, F.; Krutmann, J. Pycnogenol(r) effects on skin elasticity and hydration coincide with increased gene expressions of collagen type i and hyaluronic acid synthase in women. Skin Pharmacol. Physiol. 2012, 25, 86-92. [CrossRef] [PubMed]

89. Segger, D.; Schonlau, F. Supplementation with Evelle improves skin smoothness and elasticity in a double-blind, placebo-controlled study with 62 women. J. Dermatol. Treat. 2004, 15, 222-226. [CrossRef] [PubMed]

90. Ni, Z.; Mu, Y.; Gulati, O. Treatment of melasma with pycnogenol. Phytother. Res. 2002, 16, 567-571. [CrossRef] [PubMed]

91. Losada-Echeberria, M.; Herranz-Lopez, M.; Micol, V.; Barrajon-Catalan, E. Polyphenols as promising drugs against main breast cancer signatures. Antioxidants (Basel) 2017, 6. [CrossRef] [PubMed]

92. Furumura, M.; Sato, N.; Kusaba, N.; Takagaki, K.; Nakayama, J. Oral administration of French maritime pine bark extract (Flavangenol ${ }^{\circledR}$ ) improves clinical symptoms in photoaged facial skin. Clin. Interv. Ageing 2012, 7, 275-286. [CrossRef] [PubMed]

93. Tanaka, M.; Yamamoto, Y.; Misawa, E.; Nabeshima, K.; Saito, M.; Yamauchi, K.; Abe, F.; Furukawa, F. Effects of aloe sterol supplementation on skin elasticity, hydration, and collagen score: A 12-week double-blind, randomized, controlled trial. Skin Pharmacol. Physiol. 2016, 29, 309-317. [CrossRef] [PubMed]

94. Tanaka, M.; Yamamoto, Y.; Misawa, E.; Nabeshima, K.; Saito, M.; Yamauchi, K.; Abe, F.; Furukawa, F. Aloe sterol supplementation improves skin elasticity in Japanese men with sunlight-exposed skin: A 12-week double-blind, randomized controlled trial. Clin. Cosmet. Investig. Dermatol. 2016, 9, 435-442. [CrossRef] [PubMed]

95. Perez-Sanchez, A.; Barrajon-Catalan, E.; Caturla, N.; Castillo, J.; Benavente-Garcia, O.; Alcaraz, M.; Micol, V. Protective effects of citrus and rosemary extracts on UV-induced damage in skin cell model and human volunteers. J. Photochem. Photobiol. B 2014, 136, 12-18. [CrossRef] [PubMed]

96. Nobile, V.; Michelotti, A.; Cestone, E.; Caturla, N.; Castillo, J.; Benavente-Garcia, O.; Perez-Sanchez, A.; Micol, V. Skin photoprotective and antiageing effects of a combination of rosemary (rosmarinus officinalis) and grapefruit (Citrus paradisi) polyphenols. Food Nutr. Res. 2016, 60, 31871. [CrossRef] [PubMed] 
97. Puglia, C.; Offerta, A.; Saija, A.; Trombetta, D.; Venera, C. Protective effect of red orange extract supplementation against UV-induced skin damages: Photoaging and solar lentigines. J. Cosmet. Dermatol. 2014, 13, 151-157. [CrossRef] [PubMed]

98. Emanuele, E.; Bertona, M.; Biagi, M. Comparative effects of a fixed polypodium leucotomos/pomegranate combination versus polypodium leucotomos alone on skin biophysical parameters. Neuro Endocrinol. Lett. 2017, 38, 38-42. [PubMed]

99. Nestor, M.S.; Berman, B.; Swenson, N. Safety and efficacy of oral polypodium leucotomos extract in healthy adult subjects. J. Clin. Aesthet. Dermatol. 2015, 8, 19-23. [PubMed]

100. Ross, A.C.; Caballero, B.; Cousins, R.J.; Tucker, K.L.; Ziegler, T.R. Modern Nutrition in Health and Disease, 1th ed.; Wolters Kluwer Health/Lippincott Williams \& Wilkins: Philadelphia, PA, USA, 2012; pp. 1-1616.

101. Oregon State University. Available online: http://lpi.oregonstate.edu/mic/dietary-factors/phytochemicals/ carotenoids (accessed on 14 December 2017).

102. Bouilly-Gauthier, D.; Jeannes, C.; Maubert, Y.; Duteil, L.; Queille-Roussel, C.; Piccardi, N.; Montastier, C.; Manissier, P.; Pierard, G.; Ortonne, J.P. Clinical evidence of benefits of a dietary supplement containing probiotic and carotenoids on ultraviolet-induced skin damage. Br. J. Dermatol. 2010, 163, 536-543. [CrossRef] [PubMed]

103. Lee, J.; Jiang, S.; Levine, N.; Watson, R.R. Carotenoid supplementation reduces erythema in human skin after simulated solar radiation exposure. Proc. Soc. Exp. Biol. Med. 2000, 223, 170-174. [CrossRef] [PubMed]

104. Gollnick, H.P.M.; Hopfenmüller, W.; Hemmes, C.; Chun, S.C.; Schmid, C.; Sundermeier, K.; Biesalski, H. Systemic Beta Carotene plus topical UV-sunscreen are an optimal protection against harmful effects of natural UV-sunlight: Results of the berlin-eilath study. Eur. J. Dermatol. 1996, 6, 200-205.

105. Postaire, E.; Jungmann, H.; Bejot, M.; Heinrich, U.; Tronnier, H. Evidence for antioxidant nutrients-induced pigmentation in skin: Results of a clinical trial. Biochem. Mol. Biol. Int. 1997, 42, 1023-1033. [CrossRef] [PubMed]

106. Stahl, W.; Heinrich, U.; Jungmann, H.; Sies, H.; Tronnier, H. Carotenoids and carotenoids plus vitamin E protect against ultraviolet light-induced erythema in humans. Am. J. Clin. Nutr. 2000, 71, 795-798. [CrossRef] [PubMed]

107. Heinrich, U.; Gartner, C.; Wiebusch, M.; Eichler, O.; Sies, H.; Tronnier, H.; Stahl, W. Supplementation with beta-carotene or a similar amount of mixed carotenoids protects humans from UV-induced erythema. J. Nutr. 2003, 133, 98-101. [CrossRef] [PubMed]

108. Shapiro, S.S.; Saliou, C. Role of vitamins in skin care. Nutrition 2001, 17, 839-844. [CrossRef]

109. Bertuccelli, G.; Zerbinati, N.; Marcellino, M.; Nanda Kumar, N.S.; He, F.; Tsepakolenko, V.; Cervi, J.; Lorenzetti, A.; Marotta, F. Effect of a quality-controlled fermented nutraceutical on skin ageing markers: An antioxidant-control, double-blind study. Exp. Ther. Med. 2016, 11, 909-916. [CrossRef] [PubMed]

110. Lauer, A.C.; Groth, N.; Haag, S.F.; Darvin, M.E.; Lademann, J.; Meinke, M.C. Dose-dependent vitamin $\mathrm{c}$ uptake and radical scavenging activity in human skin measured with in vivo electron paramagnetic resonance spectroscopy. Skin Pharmacol. Physiol. 2013, 26, 147-154. [CrossRef] [PubMed]

111. La Ruche, G.; Cesarini, J.P. Protective effect of oral selenium plus copper associated with vitamin complex on sunburn cell formation in human skin. Photodermatol. Photoimmunol. Photomed. 1991, 8, 232-235. [PubMed]

112. Eberlein-Konig, B.; Placzek, M.; Przybilla, B. Protective effect against sunburn of combined systemic ascorbic acid (vitamin C) and D-alpha-tocopherol (vitamin E). J. Am. Acad. Dermatol. 1998, 38, 45-48. [CrossRef]

113. Fuchs, J.; Kern, H. Modulation of UV-light-induced skin inflammation by D-alpha-tocopherol and L-ascorbic acid: A clinical study using solar simulated radiation. Free Radic. Biol. Med. 1998, 25, 1006-1012. [CrossRef]

114. Placzek, M.; Gaube, S.; Kerkmann, U.; Gilbertz, K.P.; Herzinger, T.; Haen, E.; Przybilla, B. Ultraviolet B-induced DNA damage in human epidermis is modified by the antioxidants ascorbic acid and D-alpha-tocopherol. J. Investig. Dermatol. 2005, 124, 304-307. [CrossRef] [PubMed]

115. Crane, F.L. Biochemical functions of coenzyme Q10. J. Am. Coll. Nutr. 2001, 20, 591-598. [CrossRef] [PubMed]

116. Mellors, A.; Tappel, A.L. The inhibition of mitochondrial peroxidation by ubiquinone and ubiquinol. J. Biol. Chem. 1966, 241, 4353-4356. [PubMed]

117. Bentinger, M.; Brismar, K.; Dallner, G. The antioxidant role of coenzyme Q. Mitochondrion 2007, 7, S41-S50. [CrossRef] [PubMed]

118. Littarru, G.P.; Tiano, L. Clinical aspects of coenzyme Q10: An update. Nutrition 2010, 26, 250-254. [CrossRef] [PubMed] 
119. Zmitek, K.; Pogacnik, T.; Mervic, L.; Zmitek, J.; Pravst, I. The effect of dietary intake of coenzyme Q10 on skin parameters and condition: Results of a randomised, placebo-controlled, double-blind study. Biofactors 2017, 43, 132-140. [CrossRef] [PubMed]

120. Simopoulos, A.P. Omega-3 fatty acids in health and disease and in growth and development. Am. J. Clin. Nutr. 1991, 54, 438-463. [CrossRef] [PubMed]

121. Ruxton, C.H.S.; Reed, S.C.; Simpson, M.J.A.; Millington, K.J. The health benefits of omega-3 polyunsaturated fatty acids: A review of the evidence. J. Hum. Nutr. Diet. 2004, 17, 449-459. [CrossRef] [PubMed]

122. Wiktorowska-Owczarek, A.; Berezinska, M.; Nowak, J.Z. Pufas: Structures, metabolism and functions. Adv. Clin. Exp. Med. 2015, 24, 931-941. [CrossRef] [PubMed]

123. Capella, G.L. Strategies for leukotriene modulation in dermatology: Even more visionary perspectives? An update. Anti-Inflamm. Anti-Allergy Agents Med. Chem. 2012, 10, 407-417. [CrossRef]

124. Kendall, A.C.; Nicolaou, A. Bioactive lipid mediators in skin inflammation and immunity. Prog. Lipid Res. 2013, 52, 141-164. [CrossRef] [PubMed]

125. Kowal-Bielecka, O.; Distler, O.; Neidhart, M.; Kunzler, P.; Rethage, J.; Nawrath, M.; Carossino, A.; Pap, T.; Muller-Ladner, U.; Michel, B.A.; et al. Evidence of 5-lipoxygenase overexpression in the skin of patients with systemic sclerosis: A newly identified pathway to skin inflammation in systemic sclerosis. Arthritis Rheum. 2001, 44, 1865-1875. [CrossRef]

126. Ziboh, V.A.; Miller, C.C.; Cho, Y. Metabolism of polyunsaturated fatty acids by skin epidermal enzymes: Generation of antiinflammatory and antiproliferative metabolites. Am. J. Clin. Nutr. 2000, 71, 361s-366s. [CrossRef] [PubMed]

127. Orengo, I.F.; Black, H.S.; Wolf, J.E., Jr. Influence of fish oil supplementation on the minimal erythema dose in humans. Arch. Dermatol. Res. 1992, 284, 219-221. [CrossRef] [PubMed]

128. Rhodes, L.E.; O'Farrell, S.; Jackson, M.J.; Friedmann, P.S. Dietary fish-oil supplementation in humans reduces uvb-erythemal sensitivity but increases epidermal lipid peroxidation. J. Investig. Dermatol. 1994, 103, 151-154. [CrossRef] [PubMed]

129. Rhodes, L.E.; Azurdia, R.M.; Dean, M.P.; Moison, R.; Steenwinkel, N.J.; Beijersbergen van Henegouwen, G.M.J.; Vink, A.A. Systemic eicosapentaenoic acid reduces UVB-induced erythema and p53 induction in skin, while increasing oxidative stress, in a double-blind randomised study. Br. J. Dermatol. 2000, 142, 601-602.

130. Cho, S.; Choi, C.W.; Lee, D.H.; Won, C.H.; Kim, S.M.; Lee, S.; Lee, M.J.; Chung, J.H. High-dose squalene ingestion increases type i procollagen and decreases ultraviolet-induced DNA damage in human skin in vivo but is associated with transient adverse effects. Clin. Exp. Dermatol. 2009, 34, 500-508. [CrossRef] [PubMed]

131. Maurice, P.D.; Allen, B.R.; Barkley, A.; Stammers, J. Effects of dietary supplementation with eicosapentaenoic acid in patients with psoriasis. Adv. Prostaglandin Thromboxane Leukot. Res. 1987, 17B, 647-650. [PubMed]

132. Bittiner, S.B.; Tucker, W.F.; Cartwright, I.; Bleehen, S.S. A double-blind, randomised, placebo-controlled trial of fish oil in psoriasis. Lancet 1988, 1, 378-380. [CrossRef]

133. Lassus, A.; Dahlgren, A.L.; Halpern, M.J.; Santalahti, J.; Happonen, H.P. Effects of dietary supplementation with polyunsaturated ethyl ester lipids (angiosan) in patients with psoriasis and psoriatic arthritis. J. Int. Med. Res. 1990, 18, 68-73. [CrossRef] [PubMed]

134. Wright, S.; Burton, J.L. Oral evening-primrose-seed oil improves atopic eczema. Lancet 1982, 2, 1120-1122. [CrossRef]

(C) 2018 by the authors. Licensee MDPI, Basel, Switzerland. This article is an open access article distributed under the terms and conditions of the Creative Commons Attribution (CC BY) license (http:/ / creativecommons.org/licenses/by/4.0/). 



\section{MDPI}

St. Alban-Anlage 66

4052 Basel

Switzerland

Tel. +41616837734

Fax +41 613028918

www.mdpi.com

Nutrients Editorial Office

E-mail: nutrients@mdpi.com www.mdpi.com/journal/nutrients

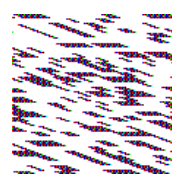



MDPI

St. Alban-Anlage 66

4052 Basel

Switzerland

Tel: +41 616837734

Fax: +41 613028918 